\title{
GENERAL BOTANY
}

\section{DENSMORE}



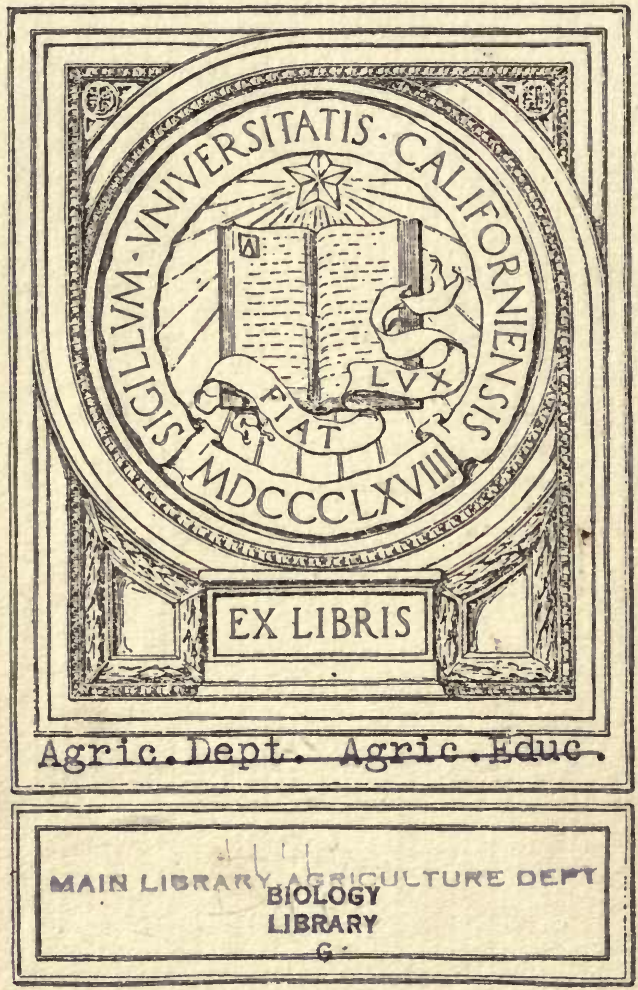





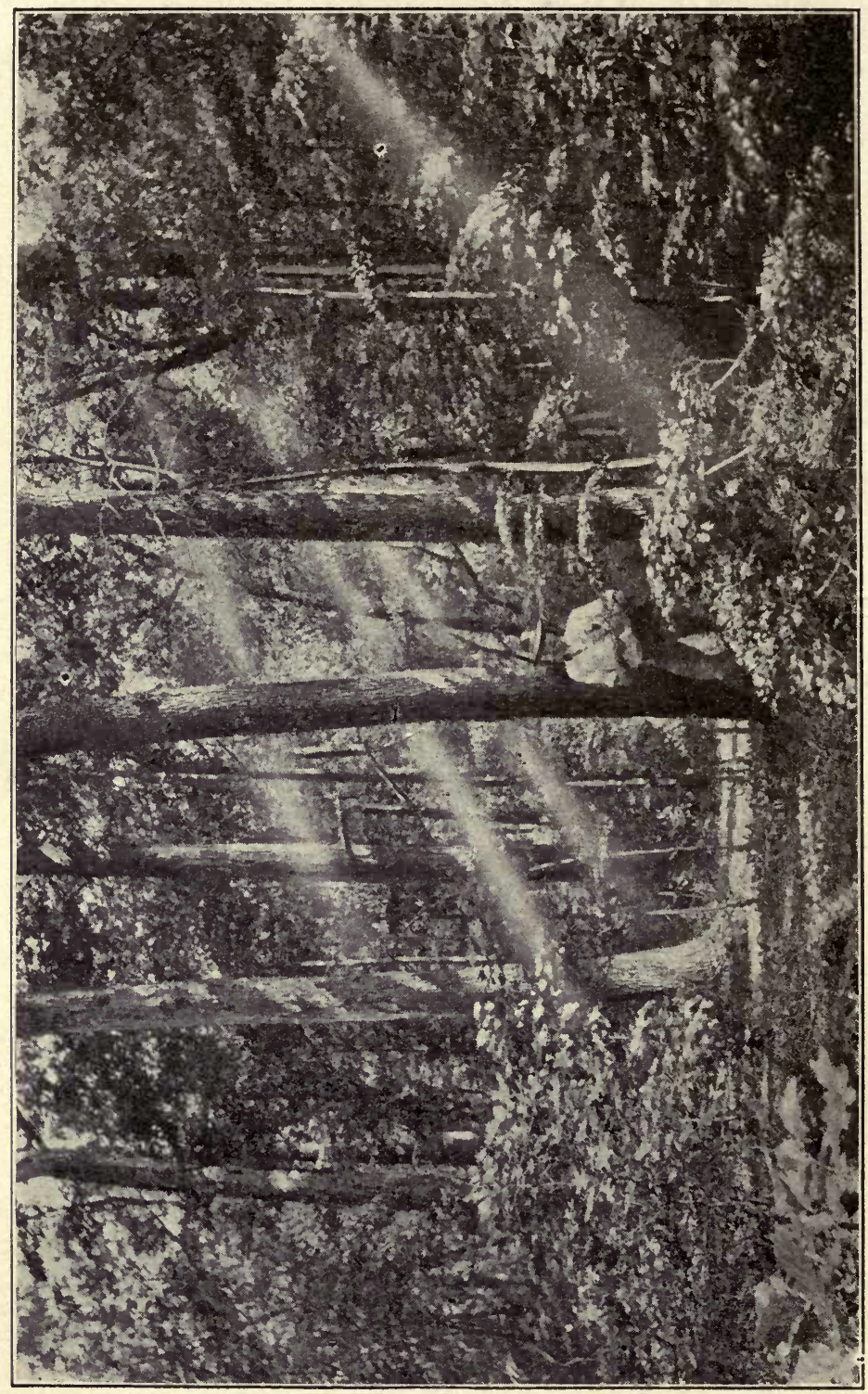

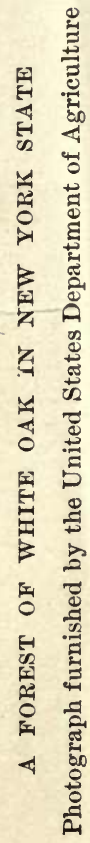




\section{GENERAL BO'TANY}

FOR

\section{UNIVERSITIES AND COLLEGES}

BY

HIRAM D. DENSMORE, M.A.

PROFESSOR OF BOTANY AT BELOIT COLLEGE, BELOIT, WISCONSIN

WITH ORIGINAL ILLUSTRATIONS BY

THE AUTHOR AND BY M. LOUISE SAWYER, M.S., FORMERLY INSTRUCTOR . IN BOTANY A'T BELOIT COLLEGE

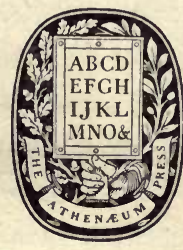

GINN AND COMPANY

BOSTON - NEW YORK - CHICAGO - LONDON

atlanta - DALLAS - COLUMBUS - SAN FRANCISCO 


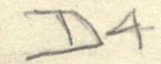

\section{BIOLOEY}

LIBRAR

G

ENTERED AT STATIONERS' HALL

COPYRIGHT, 1920, BY

HIRAM D. DENSMORE

ALI RIGHTS RESERVED

320.10

AGRIE DEPT. Agric. Education

PHIQ LIBRARY-RGRICULTUKE DEF'. 


\section{PREFACE}

This textbook is an outgrowth of the author's long experience in giving to college students an introductory course in botany. It has been used by the writer, in a briefer typewritten form, with several classes at Beloit College and by botanical instructors in three other institutions. It has thus been adapted to the needs of students as a result of practical experience and criticism.

The author's aim in writing the book has been to furnish the student with clear statements, properly related, of the essential biological facts and principles which should be included in a first course in college botany or plant biology. It has been assumed that the text would be supplemented by lectures and readings to adapt it to particular needs in different institutions. Such topics as relate to the economic aspects of botany have consequently been treated concisely, with the idea that they would be elaborated and extended variously by different instructors. In all cases, however, the biological principles underlying such practical aspects of botany have been supplied in the text. The author hopes, therefore, that the book will furnish both students and instructors with a helpful, connected statement of the more important facts and principles of modern botany.

Content and use. The subject matter of the text is divided into three parts, which are so arranged that they may be used together, as the basis for a year's course in general botany, or separately, for courses of one term or one semester in length.

Part $I$ is intended to present the biological aspects of plant life from the standpoint of structure and function, on the basis of studies of the higher and more familiar seed plants. Three main themes are considered in this part of the text, namely, the relations and adjustments of the higher plants to other organisms 
and to the inanimate forces and materials of their environment; the cellular structure of plants and its relation to growth, reproduction, and the anatomy of woody and herbaceous plants; the phenomena of reproduction, and their relation to crossing, hybridization, and plant breeding. The author has used this portion of the text, with some selections from Part II, as a basis for a semester course in plant biology.

Parts I and II could, with equal advantage, be combined as the basis for a course on the higher plants during the latter portion of the college year.

Part II deals with the morphology, life histories, and evolution of the main plant groups. In the chapter devoted to the fungi emphasis is placed upon the nature of enzymes and fermentation and upon the relations of these processes to parasitism, disease, and decay. This chapter also gives an introduction to the important aspects of fungi related to plant diseases and plant pathology. In the treatment of the higher spore-bearing and seed plants the author has given nearly equal attention to the evolution of structure and to reproduction, instead of placing the main stress upon reproductive features, as is often done in elementary textbooks. In the parts relating to structure the teachings of the newer anatomy are followed. This method of treatment has been found to be simpler pedagogically, and more in accord with modern botanical knowledge, than the usual presentation based upon the older anatomy.

Part III is intended to serve as an introduction. to field work and to the study of the interesting biological and economic aspects of a few important families and species represented in the spring flora. To this end considerable space is allotted to the study of trees and their importance to man. The main problems of forestry are emphasized concretely in connection with the life of a few selected species of forest trees. The herbaceous species of the monocotyledons and the dicotyledons are also treated from their biological and economic aspects, in order to indicate, if possible, the point of view from which additional species may be studied. 
A brief chapter on plant associations is appended to the studies of families and species, in order to stimulate thought and observation along these lines. No attempt has been made to present plant ecology in a formal manner, since the entire treatment of families and species in Part III is ecological in its nature and thus presents the elements of ecology in a concrete manner in connection with field studies.

Part III is therefore not primarily taxonomic, but rather biological, economic, and ecological in its point of view, and is thus in harmony with the treatment of plants in the first two parts of the text.

Distinctive features. Plants are presented throughout the text as living, active organisms, comparable to animals and with similar general physiological life functions.

The purely scientific and descriptive portions of the text are directly linked with the theoretical and economic aspects of biology which are of immediate human interest. 'Thus, the cellular structure of organisms is directly related to growth, - to the structure and life of trees, and to the economic value of wood; the chapter on vegetative and sexual reproduction is followed by a presentation of the essential facts and theories relating to hybridization, breeding, and evolution. The topic of evolution, however, is not presented as a theory by itself, but rather as nature's method of plant breeding and improvement, closely related to similar processes carried on by man.

The chapters on plant physiology are summarized and closely correlated with the seasonal life of such common plants as the bean, clover, and locust. Physiological processes are thus made directly and concretely applicable to the seasonal life of wellknown plant species. More space is devoted to the cell, mitosis, and cell structure than is usually accorded such topies in an elementary textbook.

Two reasons seem to the author to justify giving this larger space to cellular biology. The first is derived from experience in presenting these subjects to beginning students, who usually manifest more interest in them than in most other aspects of 
botany. The second relates to the increasing importance of firsthand knowledge of the cell and mitosis to a proper understanding of the modern literature and popular discussions concerning genetics, heredity, and breeding. This part of biology is also of fundamental importance in psychology, physiology, and sociology. In case teachers do not care to give much time to laboratory work on mitosis, the outline and figures of the text should furnish a valuable basis for class discussion of this important topic.

The presentation of plant structure from the viewpoint of modern anatomy is also new in an elementary textbook, but such treatment is justified by the author's experience in teaching this aspect of the subject to beginning students in botany. It is hoped also that the outline figures and the simple treatment in the text will enable instructors not familiar with this phase of botany to present the subject from the standpoint of modern plant anatomy.

The laboratory directions, which are printed under separate cover, are intended to stimulate interest and observation without giving detailed guidance in laboratory work.

Acknowledgments. In closing the author desires to acknowledge his indebtedness to former students and assistants, as well as to other botanists and friends, who have materially aided him in the completion of this book.

First acknowledgements are due to Miss M. Louise Sawyer, instructor in botany at Knox College and a former student and instructor at Beloit College. Miss Sawyer's criticisms and suggestions were invaluable in the early stages of writing the text and while it was being tested with classes in Beloit College. In the making and reproduction of the original drawings Miss Sawyer deserves equal credit with the author for whatever of originality or helpfulness they may be found to possess. She reproduced all of the original drawings in ink and made the camera drawings on the cell, mitosis, and anatomy from slides in the author's laboratory. 'The drawings on the pollen tubes and spermatogenesis in Iris are taken from an original paper published by Miss Sawyer. 
Special acknowledgment is also made for the privilege of using in this text various illustrations from Bergen's "Foundations of Botany," Bergen and Caldwell's "Introduction to Botany" and "Practical Botany," and Bergen and Davis's "Principles of Botany."

Acknowledgments are due to Dr. H. S. Conard of Grinnell College for the critical reading of the preliminary manuscript of Parts I and II and for criticisms and suggestions based upon the use of the text, in typewritten form, with his own classes.

The author is also indebted to Dr. E. C. Jeffrey of Harvard University for some of the microphotographs of woody and herbaceous stems used in the text, and to Dr. W. J. V. Osterhout of Harvard for valuable suggestions. 



\section{CONTENTS}

PART I. BIOLOGY OF THE HIGHER SEED PLANTS

\section{Section I. Plants and the Environment}

CHAPTER

I. The Relations of Plants to the Environment . . . 3

II. The Form and Adjustments of the Plant Body to the Environment . . . . . . . . . . . . . 14

Section II. Cell Structure and Anatomy

III. The Cellular Structure of Plants . . . . . . . 45

IV. Historical Sketch (The Cell and the Cell Theory) 54

V. Growth and Cell Division. . . . . . . . . . 60

VI. The Structure and Functions of Stems, Roots, and Leaves . • . . . . . . . . . . . 83

Section III. Physiology

ViI. Nutrition and Seasonal Life of Plants . . . . 117 Vili. The Relation of Plants to Water . . . . . . 137

Section IV. Reproduction

iX. Vegetative and Sexual Reproduction . . . . 154

X. Plant Breeding and Evolution . . . . . . . . 174

XI. Historical Development of Botany and the Biological Sciences. . . . . . . . . . . . . . 212

\section{PAR'T II. THE PLANT GROUPS}

XII. The Alge . . . . . . . . . . . . . . 219

Xili. The Fungi . . . . . . . . . . . . . . . . . . . 242

XIV. Bryophytes (Liverworts and Mosses) . . . . . 287

XV. Pteridophytes (Ffrns, Equiseta, and Club Mosses) • 297

XVI. Gymnosperms . . . . . . . . . . . . . . . . 321

XVII. Angiosperms (Dicotyledons) . . . . . . . . 337 
PART III. REPRESENTATIVE FAMILIES AND SPECIES OF THE SPRING FLORA

CHAPTER

XVIII. Descriptive Terms.

PAGE

XIX. Trees, Shrubs, and Forests . . . . . . 366

XX. Herbaceous and Woody Dicotyledons . . . . . 396

XXI. Monocotyledons . . . . . . . . . . . . 416

XXII. Plant Associations . . . . . . . . . . . . . . 435

INDEX . . . . . . . . . . . . . . 447 


\section{GENERAL BOTANY}

\section{PART I. BIOLOGY OF THE HIGHER SEED PLANTS}





\section{SECTION I. PLANTS AND THE ENVIRONMENT}

\section{CHAPTER I}

\section{THE RELATIONS OF PLANTS TO THE ENVIRONMENT}

Plant biology, or elementary botany, is chiefly concerned with the structure and activities of plants and with their relations to the living and lifeless objects and forces which surround them and constitute their environment. It is evidently impossible at the outset of our study to present more than a general survey of the relations and functions of plants in nature, but it is hoped that such a survey will stimulate the interest of the student in the larger aspects of botany and will serve to give him a new appreciation of the great importance of plants in the world of living things.

In order to present this more comprehensive idea of plant life clearly and concisely we shall need to consider first the peculiar relations of the world of vegetation to inanimate nature, including the soil, the air, and such forces of its environment as light and gravity. This discussion can then be logically followed by considering the relation which plants sustain to animate nature, including man and other animals. With this brief introduction we may proceed directly to the consideration of these topics.

\section{THE INANIMATE ENVIRONMENT}

The forces. Light and gravity, which impinge upon the bodies of plants from without, are used by the higher plants in such a way as to secure the advantageous placing or adjustment of their leaves, roots, stems, and flowers in the soil 
and the air. Fig. 1, $a$, illustrates this placing of the organs of a young, growing plant in the positions usually assumed by them, and we shall find that these positions are, in part at least, directly traceable to the effects of environmental forces acting on the plant body. We may note in the figure that the main taproot grows vertically downward toward the center

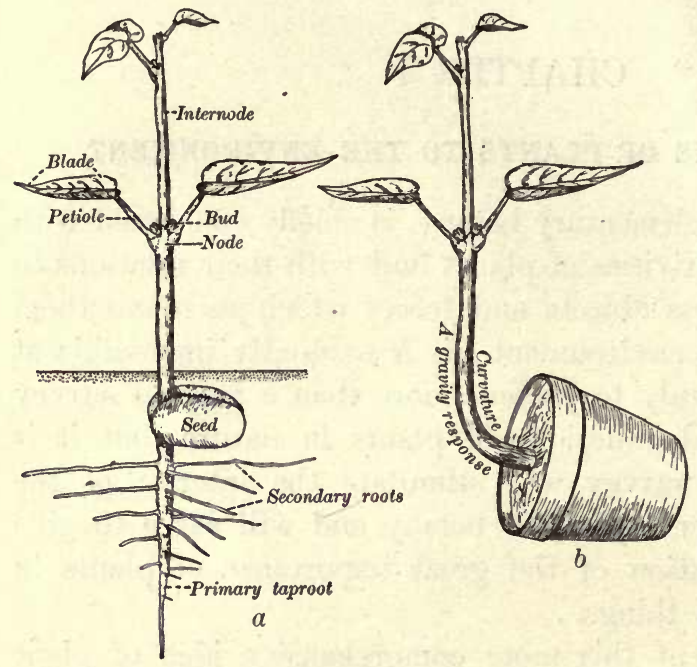

Fig. 1. Two seedlings of the scarlet-runner bean

$a$, a seedljng growing in a normal position with its organs properly adjusted to light and soil; $b$, the stem (hypocotyl) has adjusted itself by curvature in response to gravity acting as a stimulus of the earth, but that the lateral, or secondary, roots assume quite a different position, growing at more or less definite angles from the primary taproot. 'The main stem likewise grows in a direction exactly opposite to that of the main root, as it should do in order to perform its assigned task of displaying the leaves to the sunlight and the flowers to wind and insects.

The student has doubtless noticed also that the leaves of plants may assume the normal horizontal position illustrated in the figure, or, in the case of plants before a window, the leaf blades may take an oblique position, so that they face the maximum light. If the plant represented in Fig. 1, $a$, had been grown in a flowerpot laid on its side, as in $b$, or if it had started its growth on a steep hillside, the roots, stem, and leaves would have turned so as finally to assume the most favorable relation to sunlight, air, and soil. Botanists have demonstrated the fact, which we shall explain more in detail later, that growing roots, 
stems, and leaves are sensitive to such forces as light and grav. ity, and that they have the ability to respond to them by definite growth movements which are dependent upon the nature of the organ concerned. This sensitiveness and response enables the organs of plants to adjust themselves to their environment by placing themselves in proper positions for the absorption of foods and energy. For example, the root system secures a wide distribution in the soil, since its primary and lateral roots are able to respond to the gravity stimulus by growth in different directions with reference to the stimulating force. Similarly, the main stem and its branches respond to gravity and light, and leaves are able to adjust themselves to light coming from different angles.

Plants are, therefore, sensitive organisms which sustain a definite relation to the forces of their environment, and they are able to use these forces in assuming the various attitudes and positions which are most advantageous to them in a given place and time.

The energy of the sunlight is also absorbed and transformed into heat and manufacturing energy, to be used in the making of sugar, starch, and cellulose by the green portions of plants.

The materials. Green plants are likewise able to use the material substances of the air and the soil as no other organisms can do, and thereby to build up food for themselves and for the animal world, for we shall find that all animals are ultimately dependent upon plants for sustenance and continued existence.

This peculiar relation of green plants to the inorganic substances of their surroundings is easily understood from the diagrammatic drawing (Fig. 2).

The root system of the plant represented in Fig. 1, $\alpha$, has been admirably distributed through a wide area of soil by means of the sensitiveness of the roots to gravity. From this soil the roots absorb water (composed of hydrogen and oxygen $\left(\mathrm{H}_{2} \mathrm{O}\right)$ ) and soil salts containing the various substances, such as nitrogen, sulphur, phosphorus, and potash, which the plant uses in building its own foods and its living substance. The leaves take in carbon dioxide $\left(\mathrm{CO}_{2}\right)$ from the air, and practically 
all parts of the plant body may absorb oxygen. These lifeless substances of its environment are therefore continually streaming into the plant from its surroundings through its roots, stems, and leaves. The striking and characteristic thing about the green plant is that it can take these simple, lifeless substances of the air and the soil and construct food from them both for itself and for animals, and that, like animals, it can build these inert foods into living substance, endowed with the unique and characteristic properties of life. Within recent times chemists have been able, in the chemical laboratory, to compound such food substances as sugar and even simple forms of nitrogenous substances or proteins; but no chemist has yet been able to do what the plant does daily, namely, to convert lifeless matter into living matter. The power of green plants to make organic compounds is due to the fact that they have a green substance, chlorophyll, within their tissues which enables them to absorb and use the energy of light in, the making of starch and sugar. When the streams of carbon dioxide pour into the leaf from the air, they meet there the water derived by the roots from the soil. In the presence of the green pigment, chlorophyll, the living substance of the leaf is then able to utilize the outside energy of the sun and unite the carbon of the inflowing carbon dioxide $\left(\mathrm{CO}_{2}\right)$ with the hydrogen and oxygen of the water $\left(\mathrm{H}_{2} \mathrm{O}\right)$ to make sugar and starch. The sugar is then combined with the nitrogen, sulphur, and phosphorus, derived from soil salts which are absorbed by the roots, into nitrogenous foods and ultimately into the living substance of the plant body.

A part of these foods, or, more probably, the living substance itself, is then broken down by union with the oxygen which enters the plant at various points in the plant body and brings about oxidation, or respiration, as in our own bodies. This oxidation process resembles somewhat the oxidation which occurs when coal is burned in a furnace or in a stove, and results in the formation of cell energy and certain waste products in the plant comparable to the heat, gases, and ash produced by the combustion of coal and wood in a stove. This waste material 
is often cast out of the plant in much the same form in which it came into it, namely, carbon dioxide and water; but more frequently these substances are used over again to rebuild living substance and plant food. The energy released by oxidation is used by the plant in its vital processes of growth, reproduction, and repair.

The peculiar relation which nonliving matter in the form of foods sustains to the living matter composing the bodies of animals and plants may be expressed in various ways. Cuvier and Huxley put this relation in a striking manner by comparing living beings to a whirlpool.

The whirlpool is permanent, but the particles of water which constitute it are constantly changing. Those which enter it on the one side are whirled around and temporarily constitute a part of its individuality; and as these leave it on the other side their places are made good by newcomers.

It is undoubtedly true that the green plant illustrates the whirlpool conception more correctly than any other organism, since it alone of all living forms has the power of converting the simple lifeless compounds of the earth and air into living matter. When we study the next topic, we shall see that animals and man are not so intimately related to inorganic nature as green plants are.

\section{THE ANIMATE ENVIRONMENT}

Most plants are intimately associated with other plants and also with certain forms of animal life which affect their lives in various ways and so constitute a part of their natural environment.

Thus, a plant on a lawn, in a meadow, or in a forest is closely surrounded by its neighbors, which compete with it for light, air, and soil space in which to expand and obtain food. Tall plants shade shorter ones, and elosely matted plants, like grass, are likely to occupy the soil space to the exclusion of less social plants. In a forest the competition of trees is always evidenced by the death of those which fail to keep pace with 
their neighbors in the upward growth toward the light. A tree developing in the open always has a more symmetrical form and a fuller leafage than one of the same species growing in a forest. In these and various other ways the environment of any given individual or species of plant is vitally affected by neighboring plants of the same community or society.

Animals are also factors in the plant's environment in that they tend to injure or destroy plant life for food or for protec-

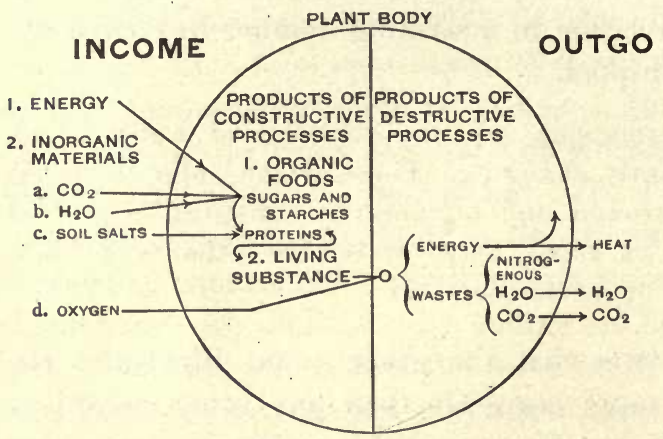

Fig. 2. Diagram illustrating the nutrition of a green plant

At the extreme left the income in energy and inorganic materials is shown; within the circle the main organic substances constructed within the plant body are indicated. At the extreme right the outgo in energy and inorganic materials is indicated; within the circle the energy and waste products of respiration (destructive process) are shown tion. Some plants, like the cacti and thistles, are armed and thus protected against the higher forms of animals; but even these exceptional forms are subject to attack by smaller forms, which may infest the roots or bore into the stem or leaves and either kill or injure them.

So, while the plant draws upon its inanimate environment for energy and foods, it must needs compete, in a hard struggle for its existence, with the other living organisms, including both plants and animals, by which it is surrounded. These living forms constitute its animate environment.

\section{RELATIONS OF PLANTS TO ANIMALS}

Plants help man and other animals by giving them food and protection and by creating an environment favorable to their needs and comfort. Plants are also of the greatest industrial and commercial importance on account of the food, shelter, 
and energy which they furnish for the maintenance and welfare of man in the home and in the industries.

Food relations of plants and animals. The food relations of plants to man and animals can be most easily understood by comparing the income and outgo of a common green plant with that of a man, or of an animal similar to man in its feeding habits.

For this comparison Figs. 2 and 3 may be used. The circle in each figure may be taken to represent the plant or animal body containing the living substance that both does the feeding and produces the energy for the living organisms. These figures illustrate the fact that the animal bears the same general relation as the plant to lifeless matter, to the production of energy, and to the elimination of wastes. Life-

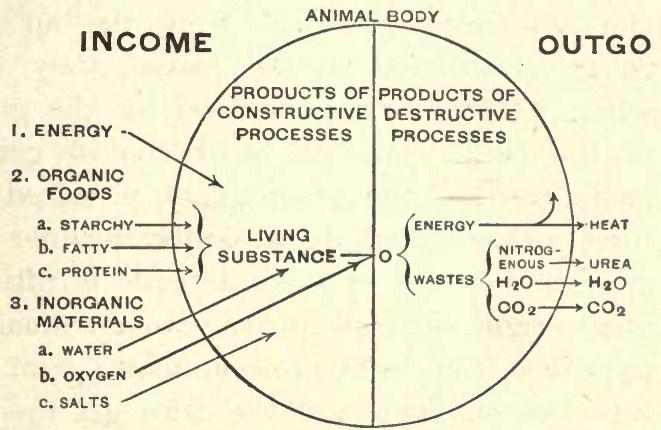

FIG. 3. Diagram illustrating the nutrition of an animal

The income, outgo, and products of constructive and destructive processes are shown as for the green plant in Fig. 2. Compare Figs. 2 and 3 and consult the text for further discussion less matter, in the form of foods, - bread, butter, and meat, - is taken into the body, digested and assimilated by the living substance of the animal body, and converted temporarily into living matter as illustrated in the figure. The living matter thus produced from lifeless foods is then oxidized, and this process yields energy for the ordinary animal activities. The waste materials produced by this oxidation are then cast out of the body in the form of carbon dioxide, water, and nitrogenous wastes; and there is some loss. of energy with the heat that escapes from the body.

A real difference between the animal and the plant, however, lies in the nature of the lifeless matter taken in. In the animal 
the bread, butter, and meat (which are taken as representative of animal foods) are real organic food materials, which have been previously built up from raw, inorganic materials by green plants.

Thus, bread, as we all know, is made of flour, which consists of finely ground kernels of wheat. The starch and the smaller quantities of nitrogenous gluten of which flour is largely composed were built up, as we have already learned, in the leaves of wheat plants from carbon dioxide, water, and soil salts, absorbed from the air and the soil. After being constructed in the leaves, they were passed into the wheat kernels as reserve food for the growth of the embryos in the seed when the seed should germinate. This readymade food of the green plant is seized upon by animals for their maintenance. In a similar manner the meat which constitutes the food of some animals is ultimately traceable to a plant origin, since even carnivorous animals (meat eaters) derive their food from herbivorous animals (plant eaters). For example, cats live on mice, and the mice get their food from grain or other plant parts; and cattle, pigs, chickens, and fish, which are the most common sources of meat for man's use, depend upon grass, hay, grain, and water plants for their food supply. All of these animals would starve in a comparatively short time without the continued supply of food which green plants build from carbon dioxide, water, and soil salts.

This general relation of the lifeless world of matter and force to the living world of animals and plants is illustrated diagrammatically in Fig. 4 . It is only necessary to add to the explanation already given the well-known fact that the death of animals and man is frequently caused by colorless plants known as bacteria. Entire crops are also often destroyed by parasitic fungi such as the rusts of grains, and all decay is induced by these colorless fungous plants. This decay finally converts the bodies of the dead animals and plants into gases and other chemical substances, which filter into the soil and form a part of the lifeless matter which is absorbed as raw food material by green plants. The most familiar 
instance of this fact is that of the farmer fertilizing his fields with fertilizers composed of the remains of plants and the excrements of animals.

We have now completed the general survey of the relation of green and colorless plants to that part of nature which

\section{INCOME}

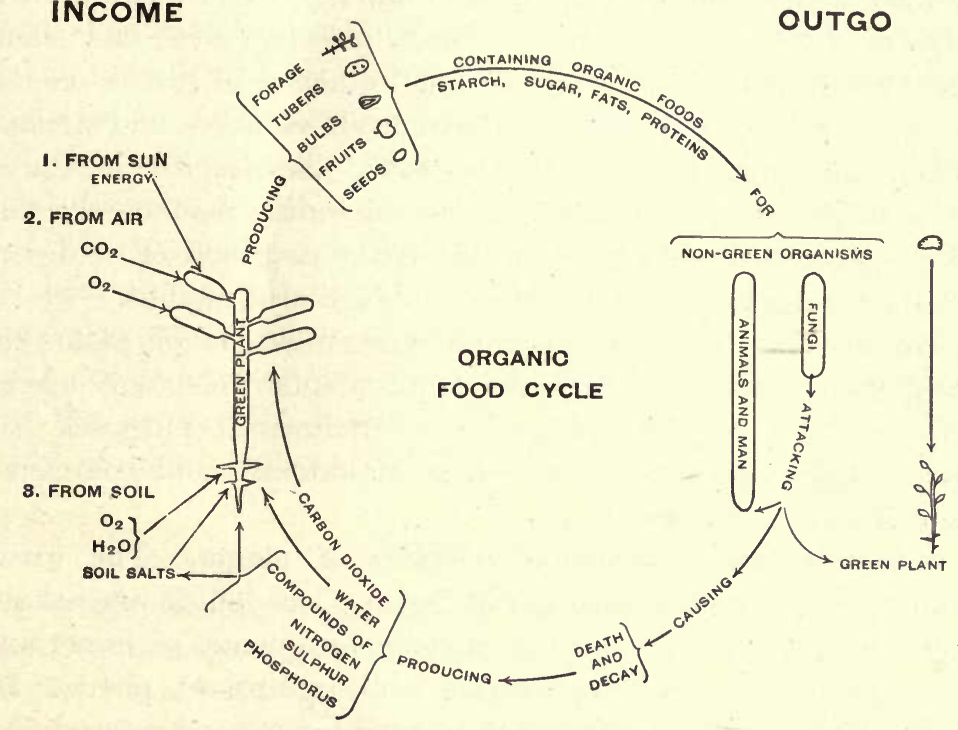

FIG. 4. Organic food cycle

What three classes of organisms are represented as concerned with the construction and use of foods in the world? What part does each play in the maintenance and consumption of its food supply? What distinctive relation has the green plant to the maintenance of the world's food supply?

constitutes their environment. The details of the picture will be filled in as we proceed in later chapters with the structure and work of plants.

Plants and the environment of animals. We are not accustomed to think of the earth as it would be without the plant life which both clothes and beautifies it. The protective, environmental function of plants is perfectly obvious, however, without lengthy comment or discussion. The great forests form a necessary environment for hundreds of species of animals, ranging from the larger forms, which make their homes in 
the trees, to the smaller and humbler forms, like insects, which inhabit the crevices of decaying logs and bark and the leaf mold created by the decay of fallen leaves. Winter to these creatures of the forest is less severe than it would be in an open and barren region, and summer brings an abundance of food, with protection from heat. Similarly, an intimate knowledge of the life of our common birds, insects, fishes, and mammals would reveal the all-important influence of plants on the environment of these animals in the meadows, lakes, and streams which they inhabit. The great deserts, likewise, are habitable by a limited number of animal forms, including man, on account of the protection and food supplied by the eacti and other desert plants which have become adapted to such arid regions.

On account of these environmental relations of plants to man, the culture of certain kinds of plants which are useful for food and for lumber and for orramental purposes has assumed a larger and larger place in. industry and commerce as civilization has advanced.

Industrial and commercial relations of plants. The great importance of modern industrial botany lias led to the establishment, by our national and state governments, of important departments for the investigation and culture of plants. In these departments hundreds of expert botanists are studying those aspects of plants and plant culture which are deemed to be of the greatest importance to man.

Agriculture, and its relation to plants, is one of the great lines of endeavor connected with practical botany. Forestry, although not yet so fully developed in our own country, bids fair to receive an increasing amount of attention as our forests become depleted and the supply of lumber decreases.

Plant breeding, or the production of better kinds of fruits, grains, and ornamental plants, is receiving wide attention and interest at the present time. As a result of this great activity on the part of individuals and of our national and state governments, many improved kinds of food and forage plants have already been bred, and the future promises even greater improvements in both agricultural and horticultural varieties. 
Plant breeding, aside from its great practical importance, is of equal theoretical interest to students of evolution and genetics, since the practical breeding of plants is based upon theoretical principles which form the basis for generalizations in these subjects.

The study of plant diseases, or plant pathology, is also of the greatest practical interest to the growers of all kinds of plants. It has been found that plants are not only subject to diseases produced by such colorless plants as rusts and smuts of grain and tree-killing fungi but that they are also subject to bacterial diseases, as is the case with man and other animals. To eraclicate these diseases and save the great losses produced by them annually, our government has now organized a very large department of plant pathology. It is not necessary to point out in greater detail the importance of plants in nature and the consequent interest and importance attached to the study of plant biology and botany.

\section{SUMMARY}

1. Plants use the forces of their environment - light, heat, and gravity - in adjusting their organs properly, in the air and in the soil, for the absorption of raw food materials.

2. Green plants are unique among living objects in being able to build foods for themselves and for animals out of simple chemical substances which occur in the air and soil.

3. Green plants are therefore intermediates between inorganic, lifeless nature and the more highly organized animals.

4. Plants are also important to animals and man in forming a proper environment for their protection and welfare.

5. Plants are of the greatest importance to man industrially and commercially. 


\section{CHAPTER II}

\section{THE FORM AND ADJUSTMENTS OF THE PLANT BODY TO THE ENVIRONMENT}

We learned in the previous chapter that the organs of the plant body - roots, stem, leaves, and flowers - were so arranged and adjusted to the materials and forces of the environment as to secure abundant food and energy for the maintenance of life. This necessary arrangement and adjustment of plant organs to their surroundings is secured in part by an inherited plan of architecture, which governs the formation and growth of organs in the embryo and in the adult organisms, and in part also by powers of movement, called tropisms, by which growing plant organs place themselves in proper relations to soil, light, and air.

\section{THE FORM AND PLAN OF THE PLANT BODY}

If we observe the plant body of most plants, we shall see that it consists of a main stem and root, which constitutes its central axis, and of lateral organs in the form of leaves, flowers, branches, and lateral roots. More careful observation of such a plant will also show that the lateral organs are not placed on the main axis in an indefinite manner, but that they have a definite order and arrangement inherited from a long line of plant ancestors. It will soon become evident also that by this inherited plan of the plant body the organs are so related to each other and to air, light, and soil as to make the plant as a whole a good working organism in its efforts to secure food for sustenance and growth. These facts will become more and more obvious as we proceed to study the relations of leaves, branches, and secondary roots to each other and to the main axis. 


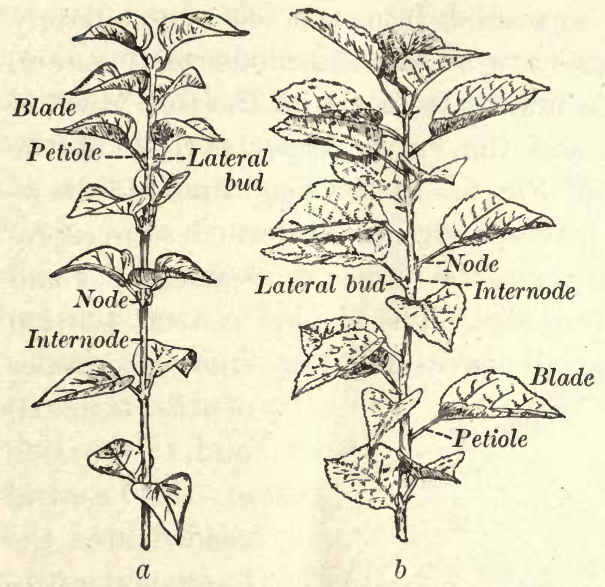

Fig. 5. Body plan of the lilac and apple

$a$, lilac twig with cyclic leaf arrangement; $b$, apple twig with spiral leaf arrangement. Compare Fig.5, $a$ and $b$, with Fig. $7, a$ and $b$

The leaves of plants (Fig. 5) spring from definite points on the main axis, which are called nodes, and the nodes are separated by definite spaces, or intervals of stem, called internodes. The leaves also grow from definite points at the nodes and are so arranged on adjacent nodes that a leaf at one node never stands immediately above the leaf at the node just below it. By this arrangement the shading of the green tissues of one leaf by another above it is avoided. The leaves on a stem are therefore arranged in mathematical order, which usually conforms to one of two types of arrangement, namely, the cyclic arrangement and the spiral arrangement.

In the cyclic leaf arrangement two or more leaves occur at each node, and the leaves of adjacent nodes alternate with each other. In such plants as the lilac and catnip two leaves are placed opposite each other at each node, alternating with the pairs of leaves at the nodes immediately above and below them (Figs. $5, a$, and 6 ). The entire leafage of such a stem is thus arranged in four

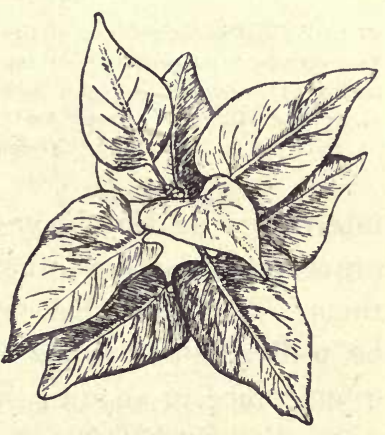

FIG. 6. Leaf arrangement on a lilac twig seen from above

Note the alternating leaf pairs, providing for maximum light exposure. Compare Figs. 6 and 7, $a$ vertical rows of leaves up and down the stem, each row being separated from its neighbor by an angle of $90^{\circ}$. Each leaf in 
any one row is therefore separated from tne leaf immediately above or below it in the same row by two internodes of the stem. If one observes a stem or a branch from above, the four rows of leaves are plainly visible, and the entire upper portion of the shoot has the appearance of Fig. 6. In the diagrammatic drawings (Fig. 7), in which the leaves, nodes, and internodes are represented as if projected on a plane, the circles represent nodes and the intervening spaces internodes. The shaded central portion is meant to represent the small leaves and very short internodes

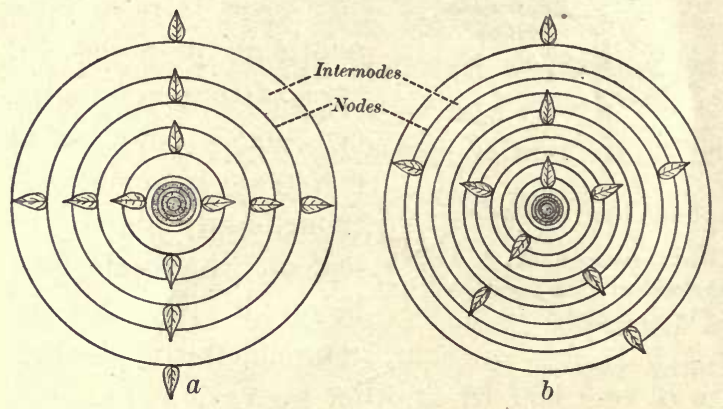

Fig. 7. Diagrams showing cyclic and spiral leaf arrangements

$a$, cyclic leaf arrangement of the lilac, reduced to one plane. The circles represent nodes, and the spaces between internodes. The leaves are seen to be in four longitudinal rows along the stem. $b$, spiral leaf arrangement of the apple, reduced to one plane. Nodes and internodes as in $a$ of the terminal bud. Outside of this central shaded area the internodal spaces widen gradually toward the periphery of each figure to represent the gradual lengthening of the internodes on a stem from its apex to its base. This lengthening of the lower internodes as the leaves grow in surface is of advantage in preventing shading of the lower leaves by large leaves above them. The cyclic leaf arrangement (Fig. 7, a) is thus seen to be well adapted to the exposure of leaves to sunlight without interference or shading by their neighbors on the same shoot.

In the spiral leaf arrangement (Figs. 5, $b$, and 8) only one leaf is placed at a node, and the leaves spring from the nodes so as to effect a spiral distribution along the twigs and young shoots. Since any given leaf is always separated from a leaf above or below it in the same straight line by two or more internodes (Fig. 7,b), according to the type of spiral arrangement in any particular case, the same advantages as regards sun exposure 
are secured by the spiral as by the cyclic leaf arrangement. The spiral arrangement of leaves is often distorted by growth and is therefore difficult to trace on mature shoots. It is consequently more clearly evident at the apex of a branch, where the twisting effects of growth are less evident.

Since the buds and lateral branches arise from the axils of the leaves of the season, it is evident that the entire branch system must follow either the spiral or the cyclic plan upon which the leaves are arranged (Fig. 8). The general form and leaf exposure of an adult plant wii! therefore be largely determined by the above body plans and by the manner in which they are worked out through growth as the plant matures.

The root systems of plants are less definite in their arrangement than the branch sys-

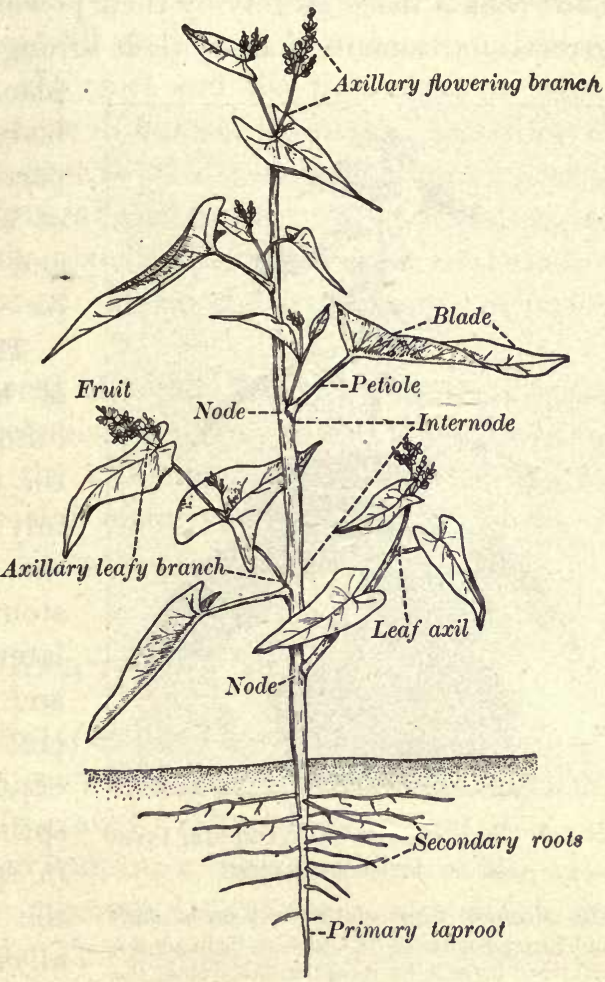

Fig. 8. Body plan and arrangement of organs in the buckwheat (Fagopyrum)

Note that the branches spring from the angle (axil of the leaf) between the leaf and stem, and are therefore disposed spirally on the stem, like the leaves. The adjustment of leaves and roots to light and soil is evident tems, probably owing to the fact that their soil environment is more uniform and less exacting than the forces and elements to which the aërial portions of the plant body are subjected. Thus, there may or there may not be a primary central taproot corresponding to the central stem axis aboveground. The secondary 
roots usually arise in an orderly succession, but there are no definite nodes and internodes. We shall learn later that the successful distribution of roots in the soil is determined in most plants more largely by their powers of adjustment through growth movements than by their arrangement on any fixed body

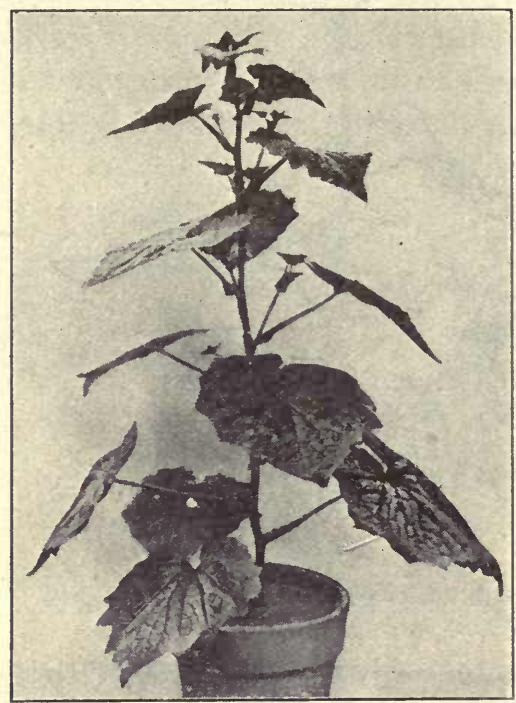

Fig. 9. Body plan and pyramidal form of an herbaceous plant

The ultimate form of this cineraria plant and the exposure of its leaves to light are determined largely by its spiral body plan, the alternation of nodes and internodes, and the continued growth of the terminal bud. Photograph by Fuller, from Cowles's "Ecology"

plan. The relation of the body plan of aërial plant parts to the ultimate form of some common herbaceous and woody plants will now be considered.

The form and development of herbaceous plants. In a number of herbaceous plants the terminal bud grows rapidly during the early part of the season and produces a stout central stem, while the lateral buds remain dormant and thus fail to produce lateral branches to any considerable extent. The leaves spring from the nodes either in spiral or in cyclic order, the lower leaves being usually somewhat larger and having longer petioles than the upper and younger ones. The result of such growth is a plant of pyramidal form (Fig. 9), in which the entire leafage is admirably exposed to sunlight.

The root system of such plants is variously arranged, but without the definite plan of the aërial parts, owing to the fact that the main root has no definite nodes from which lateral roots spring. Since, however, the soil salts and water are usually distributed equally on all sides of the roots, and since there is no danger from shading, it is evident that roots do not need 
the more accurate plan of the stem, leaves, and branches. In other herbaceous plants the lateral buds may all produce lateral branches, and in that case the plant assumes a symmetrical pyramidal or rhomboidal form like the ragweed (Ambrosia), aster, and Russian thistle. All gradations in symmetry and regularity of form between the erect and the pyramidal or rhomboidal types mentioned occur in herbaceous plants, according to the relation between the growth of the main axis and the later branches. Where great irregularity in the growth of the lateral branches occurs, the body plan is often obscured in the adult plant but is evident in youth and in the arrangement of leaves and buds on the branches.

The form and development of trees. Among trees there is the same general relation between the body plan, the growth of buds and branches, and the ultimate form of the tree and its adaptation to the forces and materials of the environment as we have noted above in herbaceous plants.

\section{The Erect Tree Type}

The pines. In erect tree types like the common pine (Fig. 10) the main axis terminates each season in a twig which bears the buds for the next season's growth. These buds consist of a vigorous terminal bud, which continues the elongation of the axis, and of from three to five vigorous lateral buds clustered at the base of the terminal bud. 'The remaining buds of the terminal twig are latent and rarely if ever grow into lateral branches.

When spring arrives the vigorous apical bud elongates and produces the terminal twig of the season with its spirally arranged scale and needle leaves. Meanwhile the vigorous lateral buds elongate and produce a whorl of branches which are separated from each other by inconspicuous internodes. Since these branches arise from buds which are really arranged spirally in the axils of minute scale leaves, they are called false whorls, to distinguish them from true whorls, which could only arise on trees with a cyclic body plan. The newest false whorl of each season is separated from the false whorl of the previous season, 
immediately below it, by that portion of the terminal twig which bore latent buds and is hence devoid of branches (Fig. 10, a). Since but one false whorl of branches is produced each season in the manner described above, the trunk of an adult pine tree presents a series of false whorls of branches from base to apex,

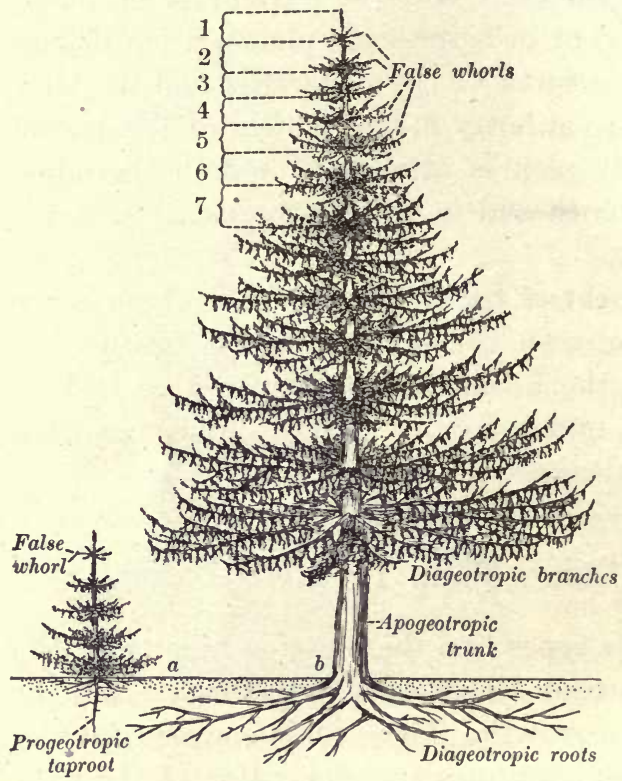

Fig. 10. Pine trees illustrating the erect type $a$, a young pine; $b$, a mature pine. Observe the excurrent trunk, the false whorls of branches, and the pyramidal form. Consult the text for the main factors concerned in the seasonal growth (1-7) and the development of these external features of the pine separated by smooth portions of the trunk (Fig. 10, b). These smooth, branchless portions of the trunk are not internodes, as they are sometimes thought to be, but rather represent those portions of the terminal twigs of each season where the latent buds failed to produce branches. Since the branches at the apex of the tree are younger and so shorter than those toward the base, the result is a pyramidal tree with a strong central excurrent trunk.

The needle leaves and reproductive cones are always produced by buds at the ends of the lateral branches, and on account of their spiral arrangement they are so placed as to be admirably exposed to the sunshine, which aids the leaves in their foodmaking, and to the wind, which helps to scatter the winged seeds of the pine cones. The body plan and the method of bud growth, therefore, combine in the pines to produce a tree of great beauty and of nice adaptation to environmental conditions. 


\section{Spreading Tree Types}

The elm. In trees of the spreading type, like the elm, poplar, oak, and hickory, the same general plan of development can be traced as that outlined for the pine, except that the terminal bud of the main axis is replaced by a lateral bud after a few years, so that no main central excurrent trunk is continued throughout the life of such trees. In the elm the terminal bud is replaced each season by a lateral bud, which produces a main central excurrent trunk for a few years (Fig. 11,e). Ultimately, as in all such trees of the spreading type, a few lateral branches gain the ascendancy and form all of the spreading crown of the adult tree (Fig. 11, f).

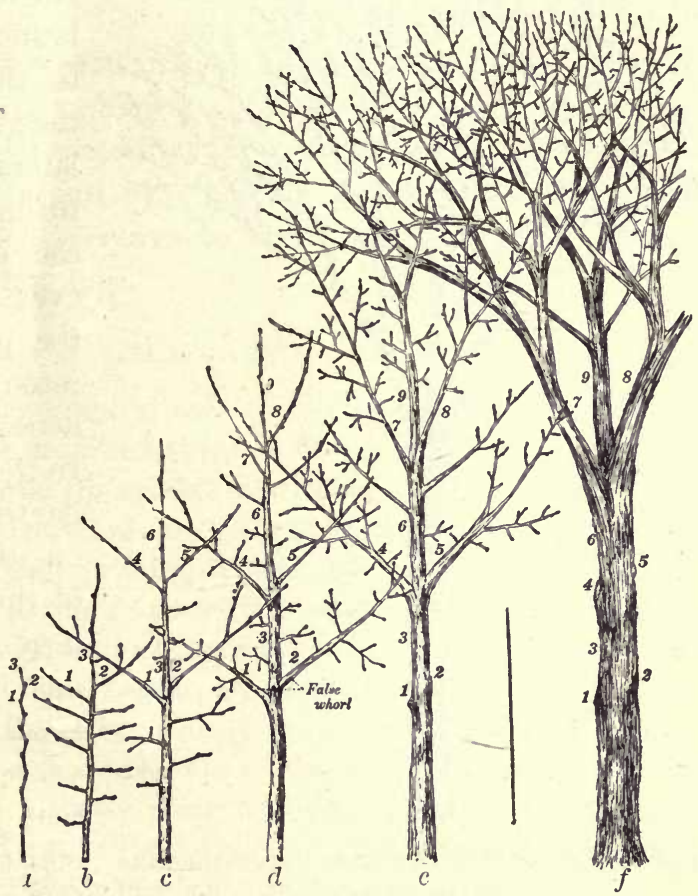

Fig. 11. Growth of the American elm, an illustration of the spreading type of trees

The letters from left to right show several stages in the development of the elm. The ultimate form is determined by body plan, the method of bud growth, and pruning effects. The corresponding letters on each figure indicate the vigorous (and so successful) branches produced each season. For further discussion consult the text

So long as the central axis continues to grow in length, its method of growth, as well as that of the vigorous lateral branches, follows the general plan already outlined for the pine. The terminal twigs at the ends of the main branches produce each year 
clusters of vigorous buds, as in the pine. In the elm the subterminal bud of each cluster early replaces the true terminal bud and continues the growth of the main axis of the branch or, in the young tree, of the central trunk. Two or three vigorous buds

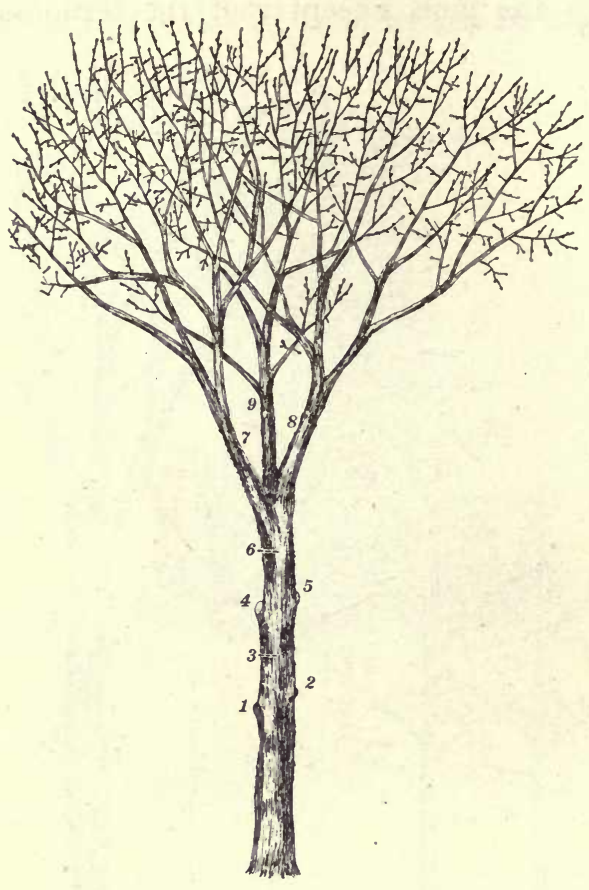

FIG. 12. A mature elm tree illustrating the spreading habit

The numbers on the main trunk and branches are similar to those in Fig. 11 below the subterminal one form vigorous lateral branches corresponding to the false whorls of the pine. These vigorous lateral branches constituting a false whorl in the elm are not, however, so numerous as in the pine and are separated by longer internodes (Figs. 11 and 12). In the elm and other similar trees some of the buds on the terminal twigs of the season below the terminal cluster of vigorous buds usually form small dwarfed twigs, while others remain latent. These smaller lateral twigs are ultimately self-pruned, owing to the fact that they are shaded by their more vigorous competitors, so that the mature portions of the trunk and branches ultimately present a condition similar to that which exists in the pines, with alternating false whorls of lateral branches and naked segments of the central axis. The false whorls in the elm are represented, however, by not more than two or three successful branches in a whorl, instead of by many as in the pine.

Often, as the elm grows older, a single vigorous branch grows, each season, just below the bud which continues the main axis, 
so that a forking appearance is produced in the upper branches and the terminal twigs of the crown.

In other hardwood trees, like the hard maple and Carolina poplar, false whorls of branches are formed, resembling very closely those of the pine in distinctness and in the number of branches in a whorl.

The leaves of the season in the elm and other hardwood trees are disposed as in the pine, at the ends of the terminal twigs of the season. In the spreading type of tree they present an immense surface to the sun for photosynthesis, while their spiral or cyclic arrangement secures to them adequate light without the danger of overlapping.

\section{SUMMARY}

From the above accounts, illustrated by the development of the pine and the elm, it is seen that three main factors determine the ultimate forms of trees and the successful display of their foliage, fruits, and seeds. These factors are the body plan, the unequal growth of buds and branches, and pruning effects due to competition in the crown of the tree. Of these factors the unequal growth of the buds is certainly the most important, since by this factor the pyramidal or the spreading form of the tree is determined, as well as the nature and disposition of the false whorls of vigorous lateral branches. In the spreading-tree types the use of the term false whorls is only permissible in order to make clear the close similarity which exists between the mode of annual bud growth in the pyramidal pines and that in the spreading types of deciduous trees.

In herbaceous plants the same general principles obtain in the development of the mature plant as in trees, and the forms assumed by them correspond, as we have seen, to the erect pyramidal type and the spreading type of the pine and the elm.

\section{ADJUSTMENTS TO THE ENVIRONMENT BY TROPISMS}

It has just been shown that the inherited plan of the plant body of our common plants is favorable to the proper placing of leaves, roots, and stems for the absorption of food materials and energy from the soil, air, and sunlight. It will be quite evident, however, to the critical observer of plants, 
that the growth of branches and leaves at regular nodal intervals, and their cyclic and spiral arrangements on the main stem and its branches, are not all that is necessary for the proper adjustment of these organs to the environment. This fact is most strikingly illustrated in the growth of young plants from the seed, where the parts of the embryo, originally folded in

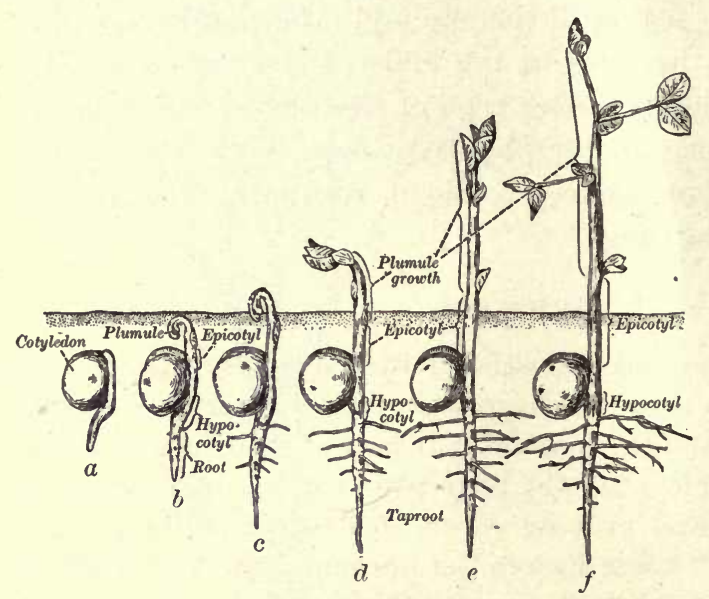

Fig. 13. Stages in the development of a seedling of the garden pea

$a-c$, emergence of the embryo from the seed and of the plumule from the soil; $c-e$, erection of the plumule and growth of the lateral roots; $f$, mature seedling with leaves and roots adjusted to light and soil the seed, must gradually expand and adjust themselves to the air, light, and soil for theabsorption and use of raw food materials. The growth and development of the embryo and seedling of the common pea furnishes a good illustration of the movements which take place in the adjustment of a young plant to the new conditions presented to it as it emerges from the seed and the soil.

The seed of the pea is composed of a dense outer covering, or seed coat, inclosing the embryo proper, which entirely fills the seed coats. This embryo consists of two fleshy cotyledons, or food-storing leaves, which comprise the bulk of the embryo. Between the two cotyledons are found two or three minute leaves, constituting the first bud of the young plantlet, and a stemlike body, the hypocotyl, which bears the cotyledons and the plumule. These parts are all shown in their proper relation in the early stages of germination of the pea (Fig. 13, a), just after the plumule and hypocotyl have broken through the seed coat. 
As the embryo emerges from the seed the plumule is curved and the leaves are seen to be borne on the first internode of the stem above the cotyledons, which is known as the epicotyl. The hypocotyl has meanwhile elongated and is continued as the primary root. The curved condition of the plumule protects its delicate leaves from being broken off as it pushes up through the soil. The curvature is not due to gravity but to unknown internal causes.

As soon as the embryo emerges from the soil, the plumule, now composed of several nodes and internodes, straightens and finally assumes an erect position. As the leaves expand they respond to the stimulus of light and take up a favorable position for the reception of the maximum amount of light for the manufacture of starch.

The roots, meanwhile, respond to gravity in such a manner that the lateral roots grow out at an angle from the vertical taproot and so permeate a large area of soil from which to draw water and soil salts.

The mature seedling (Fig. 13, $f$ ), through these various adjusting movements, is thus admirably adapted to securing from the environment the energy, gases, water, and salts necessary to its growth and development.

It is quite evident that the above movements and changes in the position of the various organs of the growing seedling are quite independent of the division of the stem into nodes and internodes and of the cyclic or spiral arrangement of the leaves. These structural arrangements are, however, closely correlated to the adjusting movements, so that the architectural plan and the unfolding movements work together for a better final adjustment of the organs in the older seedling.

Mature plants do not usually manifest such definite and obvious movements as seedlings, and yet there is abundant evidence in adult plants of all kinds that the ultimate position of their growing organs, and their more perfect adjustments to the environment, are brought about by a correlation of body plan with adjusting movements which are caused by external and internal stimuli. 
One of the principal reasons why such adjusting movements are necessary is the fact that the foods and energy which must be absorbed by green plants for their maintenance are usually quite unequally distributed in the air and soil about an individual plant. The plant must therefore turn its parts toward the most favorable source of supply to reap the maximum benefit of any given condition. Thus, the two sides of a plant before a window are exposed to very unequal intensities of light, and in

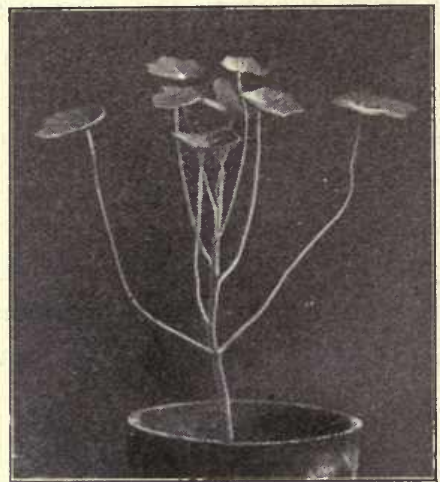

$a$

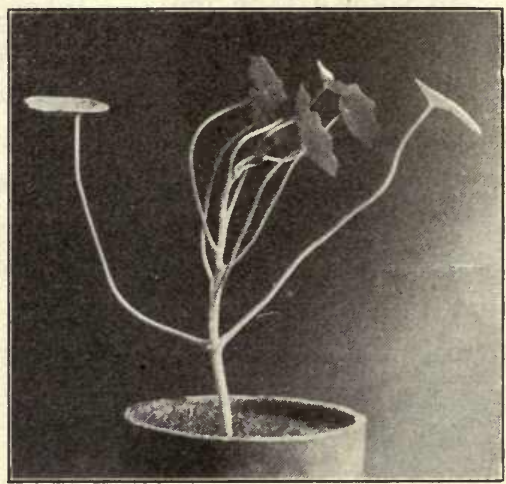

$b$

FIG. 14. Phototropic response in leaves of Nasturtium tropaeolum $a$, a plant grown under normal illumination in a greenhouse; $b$, the same plant after exposure for six hours to lateral illumination. From Cowles's "Ecology"

order to receive enough of the sun's energy for effective work in making starchy foods it must turn or swing its leaves in such a manner that their broad green surfaces shall be exposed directly to light coming in from the outside. We are all familiar with the fact that plants under such circumstances are able to turn the entire leaf surface from the normal horizontal position to an oblique position (Fig. 14), so as to face the sources of maximum light supply. Under these circumstances the separation of the leaves by internodes, and their cyclic or spiral arrangement, is still effective in preventing shading, but the nicer adjustment of the leaves to unequal light exposure on the two sides of the organ is made through the power of the leaves to respond to light acting as a stimulus and to turn their 
faces toward the most abundant light supply. In a similar manner, roots which are growing in soil where the amount of water or food varies on different sides of the root system are able to turn toward the maximum food or water supply, as when roots grow into old wells and water pipes, attracted by the excess of moisture. These turnings in response to stimuli are called tropisms.

It is not surprising, therefore, that plants, probably on account of their stationary habit; have developed a wonderful sensitiveness to the forces and agents of their environment, which enables them to adapt themselves and their fixed architectural plan to the variations in their surroundings which might otherwise harm them.

Investigation has shown that plants are sensitive to gravity, sunlight, moisture, soluble substances in the soil, and various other stimulating forces and materials. Indeed, this ability to adjust their organs is more marked in plants than in most animals. While plants are sensitive to a great variety of stimuli in their surroundings, certain forces and agents are more prominent as directing stimuli than others, and these will therefore receive the most attention in the following discussion.

Stimulus and response. Plant stimuli, as indicated above, are usually the external forces and materials of the environment. Any difference in the intensity of such external stimuli or in the direction of their application to a plant organ is capable of bringing forth a response in the form of growth, food building, adjusting movements, or even the death of the organism. We are here concerned only with that form of response to stimuli that results in definite movements which adapt the plant more effectively to the daily and seasonal changes in its surroundings. A moment's consideration will enable the student to realize the great difference between the higher plants and the higher animals as regards both the reception of an external stimulus and the method, or mechanism, by which the two classes of organisms respond to stimuli. The higher animals are furnished with special sense organs, such as the eye for the reception of light and the ear for sound; in plants the entire surface of a leaf or 
a stem usually receives the stimulus of light or of a mechanical agent. The nearest approximation to sense organs in plants are certain groups of cells in root tips, and in the stem tips of some particular plants, which are endowed with the property of receiving gravity or light stimuli. In general, however, plants have no special sense organs with nerve endings, like animals,

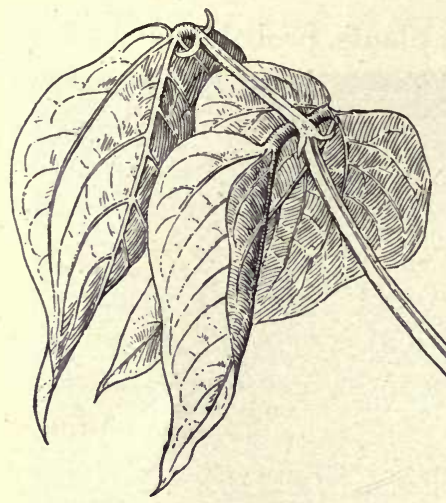

Fia. 15. A compound leaf of the bean with pulvini

The pulvini appear at the base of each leaflet. Note also a large pulvinus at the base of the main petiole. After Sachs with which to receive external stimuli.

The mechanism, or method of response to stimuli, is likewise very different in the higher plants and the higher animals, since plants have no muscles, attached to a jointed skeleton, for effecting movements. The most common method of response in plants is that of unequal growth on the two sides, or on regularly alternating sides, of an organ which has been excited by an external stimulus. Thus, in the pea seedling (Fig. 13) the erection of the epicotyl from its curved position was effected by the greater elongation of the tissues on the concave side of the epicotyl than on the convex side. The horizontal spread of the leaves is likewise brought about by the more rapid growth of the tissues on one side of the young leaves than on the other. The curvatures of roots and stems, with which we shall have to deal later, are also brought about in the same manner. This method of securing movements in plants by growth is necessarily slower than the corresponding movements of animals, which are results of nerve stimulation and muscular contraction, but it is nevertheless well suited to the nature of stationary organisms in which a new set of leaves and new growths of stems and roots are produced each year. 
In the case of roots the older portions of the root systems are already fixed in their position by the surrounding solid earth; but the new roots which grow from the old ones each spring are enabled to move and penetrate into new soil regions in order to absorb foods and water. In a similar manner each new annual crop of leaves can adjust itself to the conditions confronting it in the season in which it must do its work. Flowers are likewise able to adjust their positions so that they may secure the visits of pollinating insects, and fruits assume positions suitable for dissemination by wind or animals. The older parts, which have lost the power of growth, and therefore of movement, thus become supporting, storing, and conducting organs of plants, while the annual growth of new roots, leaves, and flowers enables the organisms to adjust themselves to any ordinary change which may take place in

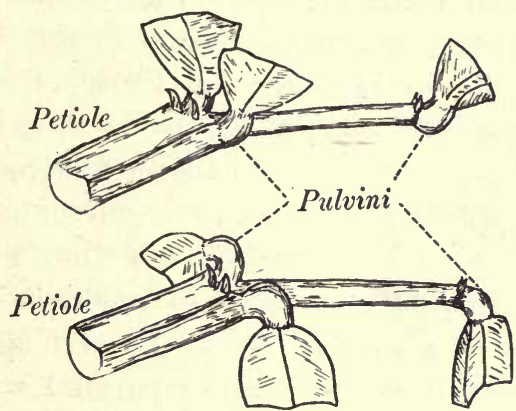

Fig. 16. Pulvini of the bean

Magnified. After Sachs the environment, and thus to adapt themselves to the usual seasonal changes and other requirements of their surroundings.

Special motor organs exist in some plants, however, which enable certain organs to execute more rapid and exact movements than those described above. Examples of such motor organs are found in the leaves of peas and their near relatives. These special organs are termed pulvini (singular, pulvinus) (Figs. 15 and 16). They are highly modified portions of the petioles at the base of each leaflet and often at the base of the petiole itself, where it joins the main stem or a branch. The quick curvature of these pulvini serves to swing the leaves into positions which enable them to secure a better adaptation to light or a more complete protection from too great loss of water. Internal and unknown stimuli also affect plant movements, in some instances quite as profoundly as the external 
stimuli discussed above. Thus, the arching of the epicotyl of the pea seedling, already referred to, is not due to external stimuli, but to internal conditions which bring about unequal growth of the organ on two opposite sides, resulting in an adaptive curvature. Some of the adaptive movements of flowers and fruits are apparently due to similar internal conditions which appear to affect the organism like external stimuli. These latter are, however, special cases applying to particular plant groups or species and do not affect the general principle that plant movements are usually effected by unequal growth in response to external stimuli.

In designating the kinds of stimuli and the nature of the response of plant organs to them botanists have adopted certain terms which are useful in designating stimulus and response as applied to any given organ of a plant. Thus, organs may be said to be protropic when they grow toward the source of the stimulating force, and apotropic when they grow away from such a source. Protropic and apotropic organs will evidently have their main axes parallel with the direction from which a given stimulus acts. Diatropic organs, on the contrary, do not grow toward the source of a directive force, but place their main axes at some angle to the direction of a stimulating agent. If we apply these definitions to the pea seedling (Fig. 13), the main root would be protropic and the stem apotropic with reference to gravity. The secondary roots would, however, be diatropic with reference to gravity, and the leaves diatropic with reference to light. If it is desired to combine the name of the stimulus with the nature of the response, other terms may be combined with protropic, apotropic, and diatropic. Thus, organs stimulated by gravity are said to be geotropic; if stimulated by light, they are designated as phototropic. Progeotropic, apogeotropic, and diageotropic may therefore be used to indicate the responses to a gravity stimulus, on the one hand, and prophototropic, apophototropic, and diaphototropic to indicate the responses to a light stimulus, on the other. Similar combined terms are used in connection with other stimuli, but these need not be considered in our brief account. 
The gravity sense. It will be of special interest to the student at this point to learn something of the method by which some of the great botanists of the past laid the foundation for our present understanding of the nature of plant movements and of the relation which exists between external stimuli and the response of the plant to them. For this purpose the classical experiments of Thomas Andrew Knight, Julius von Sachs, and Charles Darwin on the gravity seuse of plants and its relation to plant movements may well serve as an illustration.

Gravity is the most universal and constant external force acting upon the living plant world, and it is not surprising, therefore, that of all the outside forces this is found to be the most potent in directing the adjustments of plant organs to their environment by movements or tropisms.

'The general nature of the response of plants to gravity is suggested by the fact that the stems and roots of all plants take up the same position with reference to the earth's center at all points on the earth's surface. Thus, plants on opposite sides of the earth are found to have the main root growing toward the earth's center and the main stem away from it. Likewise, growing plants which have been prostrated by storms, or which happen to grow on steep hillsides, always tend to place their stems in a vertical position with reference to the earth's center. This suggests the general law that roots respond to gravity by growing toward the earth's center, while stems tend to grow in the opposite direction in response to the same stimulus.

This general law was first tested out in 1806 by. Thomas Andrew Knight, an English botanist, who conceived the idea of substituting centrifugal force for gravity, to see how roots and stems would respond to other forces than gravity. Knight attached boxes, in which young plants were growing, to the circumference of rapidly rotating wheels. When the wheels were rotated rapidly enough, he observed that the roots grew toward the circumference of the wheels, with the acting centrifugal force, while the stems grew toward the center of the wheels, or against the acting force. If the wheels were rotated less rapidly, the 
roots and stems took up an intermediate position which was a resultant of the response of the plant to the two forces, gravity and centrifugal force, acting separately.

Knight concluded, therefore, that roots and stems respond to centrifugal force acting as a stimulus. His simple appliances are now replaced by more perfect pieces of apparatus, on which disks can be rotated with extreme rapidity and accuracy. If kernels of germinating seeds of corn are placed on such a disk
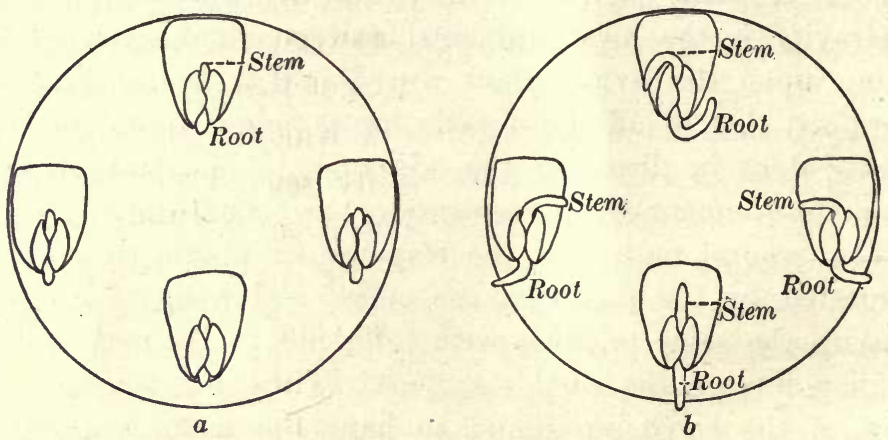

Frg. 17. Diagram illustrating the principle of an experiment by Thomas Andrew Knight (1806)

$a$, position of seeds, roots, and plumule (stem) at the beginning of the experiment; $b$, position of root and plumule (stem) after rapid rotation. Further discussion in explanation of $a$ and $b$ in the text

in the positions indicated in Fig. 17, $a$, and the disk is then rotated for twenty-four hours, the elongating root and stem will gradually assume the positions indicated in $b$. The roots will all grow toward the circumference of the wheels, while the stems will all grow toward its center.

By employing a force which he could control and modify, Knight was thus able to show that the root and the stem could be caused to curve and to take up various positions as a result of their response to this force acting as a stimulus. Since seeds germinating in the soil seemed to behave toward gravity as they did toward centrifugal force in his experiment, he concluded that gravity, acting as a stimulus, directed the growth of the root toward the earth's center and the stem away from it, and that 
the opposite positions assumed by these organs on the earth's surface were due to gravity acting as the stimulating agent.

It is a matter of common experience that if germinating seeds are placed horizontally in soil or with the root pointing upward and the stem downward, the growing organs turn and adjust themselves to gravity, as they do to centrifugal force on rotating disks.

Lateral organs such as branches and lateral roots have also been found to be governed very largely, in the position which they finally assume, by gravity, although other forces are often influential in the ultimate adjustment.

Knight's early conclusions were proved to be correct by the great German botanist Julius von Sachs in 1879. Sachs used a different method for proving that gravity acts upon plants
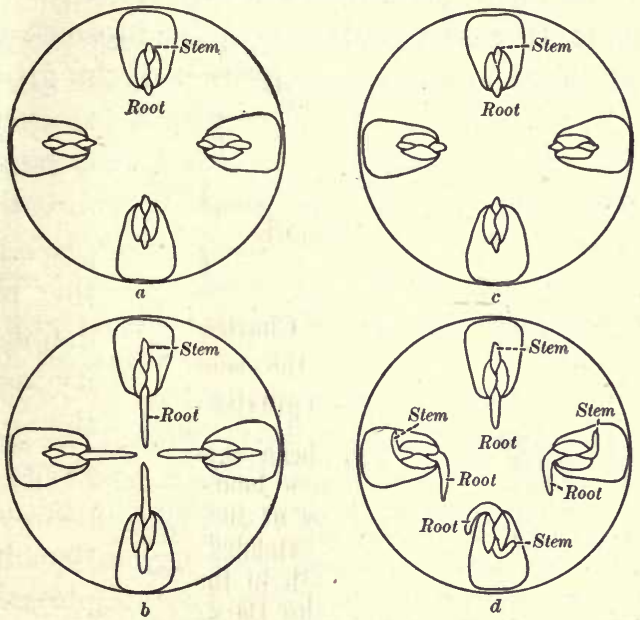

FIG. 18. Diagrams illustrating the principle of an experiment by Julius von Sachs (1879)

$a$, the position of germinating seeds of corn on a disk, which is then slowly rotated for several hours in a vertical position ; $b$, the positions assumed by roots and plumule (stem) after twenty-four hours of rotation; $c$, the position of germinating seeds of corn on a stationary disk at the beginning of the experiment; $d$, the positions assumed by roots and plumule (stem) after twenty-four hours without rotation. Consult the text for a discussion of this experiment

as a directive stimulating force. He placed growing seedlings on slowly rotating vertical wheels or disks (Fig. 18). As the wheels revolved, the stimulus of gravity continued, but the effect of gravity on the growing stem and root was practically eliminated, since it acted for too short an interval of time on any given side of these organs to secure a reaction in the form of a curvature. Plant organs usually have to remain in a position of stimulation from thirty minutes to several hours in order to 
result in curvature. If, therefore, seedlings are rotated so that opposite sides of the stem and root are alternately placed in a position of stimulation for shorter periods than are required for a reaction, the organs will fail to respond to gravity. 'The time during which an organ like a root must be continuously stimulated on one side in order to secure a reaction is called presentation time. In Sachs's experiment the presentation time to gravity was too short in any given position of the growing stem and root tip
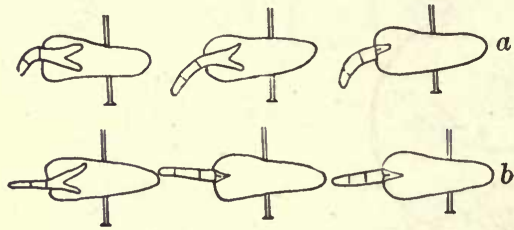

Fig. 19. An experiment by Charles Darwin, designed to locate the sensitive portion of the root to gravity

$a$, uninjured roots of the bean extended horizontally for twenty-three hours and thirty minutes; $b$, root tips of the bean touched with caustic and extended horizontally for the same length of time as

those in $a$. After Charles Darwin to secure curvature, and these organs therefore grew in any direction in which they were placed at the beginning of the rotation. This proved definitely the effect of gravity as a directive force in the growth of roots and stems in the normal vertical position. It will thus be seen that Knight and Sachs employed quite different methods in their experiments. Knight aimed to substitute another force for gravity in order to note its effect on root and stem growth, while Sachs sought to neutralize and so eliminate the effects of gravity. Knight's experiment proved that roots and stems respond to an external force which can be controlled and its effects therefore proved, while Sachs's experiment showed conclusively that gravity was necessary for the downward growth of roots and the upward growth of the stem.

Darwin's work entitled "The Power of Movement in Plants" greatly extended the observations already made on the sensitiveness of the root to gravity and other stimuli. His most important contribution to the subject was the elaboration of the idea, already discovered by Sachs, that the sensitive zone is located in the very apex of the root. Fig. 19 illustrates Darwin's method of locating the sensitive, or perceptive, zone of the root in the root tip of the common garden bean (Vicia faba). Darwin's 
conclusion, given in a summary of his chapter on the "sensitiveness of the radicle (root) to contact and other irritants," indicates the nature of his contribution to the general subject of the response of the root to various stimuli, including gravity, in its natural growth through the soil.

The peculiar form of sensitiveness which we are here considering is confined to the tip of the radicle for a length of from $1 \mathrm{~mm}$. to $1.5 \mathrm{~mm}$. When this part is irritated by contact with any object, by caustic, or by a thin slice being cut off, the upper adjoining part of the radicle, for a length of from 6 or 7 to even $12 \mathrm{~mm}$., is excited to bend away from the side which has been irritated. Some influence must therefore be transmitted from the tip along the radicle for this length. The curvature thus caused is generally symmetrical. The part which bends most apparently coincides with that of the most rapid growth. The tip and the basal part grow very slowly, and they bend very little.

Considering the several facts given in this chapter, we see that the course followed by a root through the soil is governed by extraordinarily complex and diversified agencies, - by geotropism acting in a different manner on the primary, secondary, or tertiary radicles; by sensitiveness to contact, different in kind in the apex and in the part immediately above the apex; and apparently by sensitiveness to the varying dampness of different parts of the soil. These several stimuli to movement are all more powerful than geotropism, when this acts obliquely on a radicle which has been deflected from its perpendicular downward course. The roots, moreover, of most plants are excited by light to bend either to or from it; but as roots are not naturally exposed to the light, it is doubtful whether this sensitiveness, which is perhaps only the indirect result of the radicles' being highly sensitive to other stimuli, is of any service to the plant. The direction which the apex takes at each successive period of the growth of a root ultimately determines its whole course; it is therefore highly important that the apex should pursue from the first the most advantageous direction; and we can thus understand why sensitiveness to geotropism, to contact, and to moisture all reside in the tip, and why the tip determines the upper growing part to bend either from or to the exciting cause. A radicle may be compared with a burrowing animal such as a mole, which wishes to penetrate perpendicularly down into the ground. By continually 
moving his head from side to side, or circumnutating, he will feel any stone or other obstacle, as well as any difference in the hardness of the soil, and he will turn from that side; if the earth is damper on one than on the other side, he will turn thitherward as a better hunting-ground. Nevertheless, after each interruption, guided by the sense of gravity, he will be able to recover his downward course and to burrow to a greater depth.

Darwin's contributions to our knowledge of the sensitiveness of the root tip have been confirmed by later researches and have
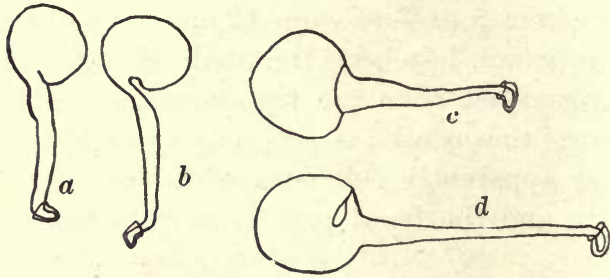

Fig. 20. Drawings illustrating experiments by Czapek, designed to locate the sensitive zone of the root to gravity acting as a stimulus

The roots of the bean were grown into glass slippers and then placed so as to expose the last few millimeters of the tip to gravity at different angles from the vertical. a, position of the root tips at the beginning of the experiment; $b$, position assumed by the above root tips twenty hours later; $c$, root tips placed vertically at the beginning of the experiment: $d$, position assumed by the same roots eighteen hours later. Further discussion in the text. How do you explain the difference in the position assumed by ronts in $b$ and $d$ ? After Czapek

done much towards elucidating our ideas concerning the nature of stimulus and response as applied to plants. Fig. 20 illustrates the principle of some ingenious experiments by Czapek, who confirmed Darwin's general idea that the sensitiveness of the root to gravity is located mainly in the root tip. Czapek forced roots to grow into bent tubes, as illustrated in the figure, so that the last millimeter of the root was at right angles to the body of the root. Such roots, when placed in the position indicated in Fig. 20, a, produced a curvature like that in $b$. Root tips placed in a position like that of $c$, however, failed to curve in response to gravity, as indicated in $d$, since the sensitive tip was in the normal progeotropic position. When, therefore, the terminal millimeter of the root tip is placed in a position of stimulation (Fig. 20, a), curvature of the root occurs ; but when the terminal millimeter is placed vertically (c), no curvature results. We may conclude, therefore, with Czapek, that the last millimeter of the 
root tip is the most sensitive part of the root and is the perceptive region for the gravity stimulus which causes curvature.

We may now profitably turn to a few common garden and field plants for a practical application of the principles established above with reference to the adjustment of the plant parts in response to stimuli and consider briefly the part which these responses play in determining the attitudes and ultimate form of these plants.

\section{ADJUSTMENTS IN SOME COMMON PLANTS}

Caladium and clover. The power of two quite different plants to adjust their organs to the environment may be seen by contrasting the ultimate positions assumed by the organs of caladium and red clover (Figs. 21 and 22). These two plants have very different forms of roots and leaves, and yet each plant, on account of its ability to respond in its own way to the forces of its environment, has succeeded in placing its roots and leaves in the position most favorable to its own maintenance and growth. As may be seen from Fig. 21, the caladium is what is known as a surface feeder, spreading its roots out horizontally and absorbing foods and moisture from the surface layers of the soil. Many desert plants arrange their roots in this manner in order to avail themselves of the light rains and heavy dews which occur in arid regions at certain times of the year. The leaves of the caladium are also favorably adjusted to light as a result of their ability to turn in response to the light stimulus. The roots are evidently diageotropic and the leaves diaphototropic in their response.

The common red clover (Trifolium pratense) (Fig. 22) presents a strong contrast to the caladium not only in the form of its organs but also in its responses to light and gravity. The strong taproot is here progeotropic and bores deeply into the soil, so that the lateral diageotropic roots absorb food and moisture from much deeper areas than the similar roots of the caladium. The leaves of the clover, like those of the bean, are furnished with pulvini (b) and thus adjust themselves rapidly and effectively to changes in the external environment. During the day they 
are expanded to the sun, while at night they fold up (Fig. 23), assuming the so-called nyctitropic, or sleep, position.

The mechanism of this curvature is supposed to consist in an unequal absorption of water on two opposed sides of the pulvinus as a response to different intensities of light, as in the

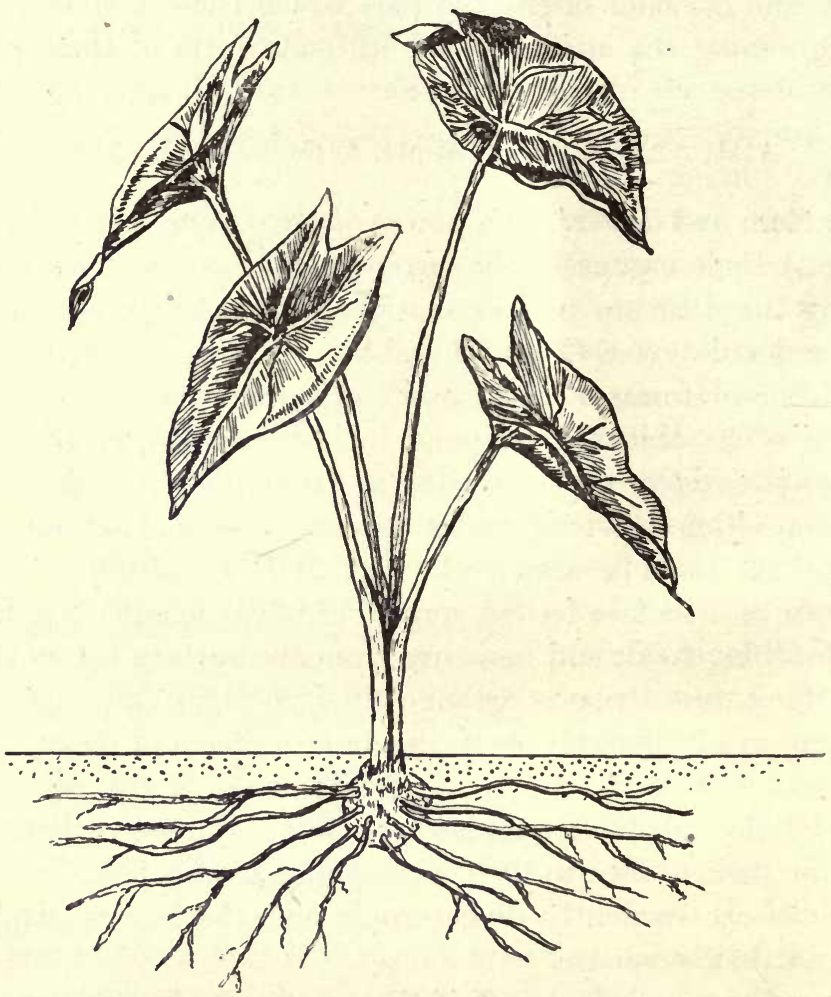

Fig. 21. Positions assumed by the leaves and roots of a mature caladium plant in response to light and gravity. After Koerner

leaves of bean plants, which may be seen to fall and assume a vertical, hanging position at night, owing to the greater increase in length of the upper side of the pulvinus as compared with the lower side, thus inducing curvature and the downward bending of the leaves (Fig. 16). This method of curvature is not unlike that of roots and petioles already described, except that in these 
cases curvature is due to slow, unequal growth on opposite sides of a part, while in pulvinar movements the curvature is due to rapid temporary growth caused by the unequal inflation with

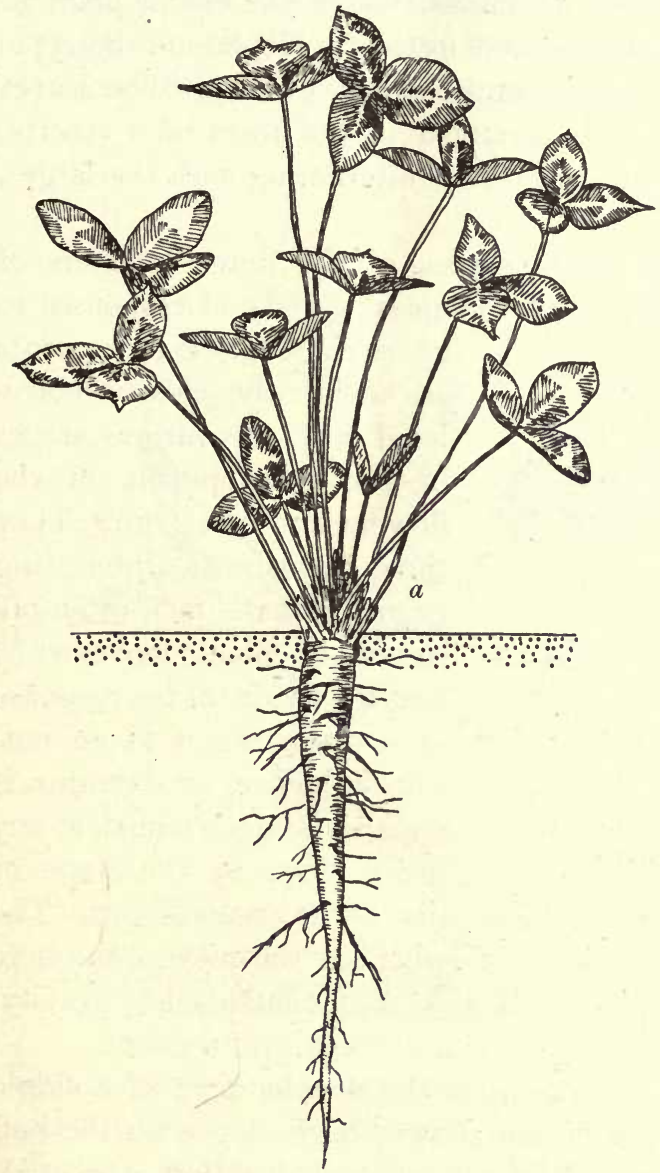

Fig. 22. Positions assumed by the leaves and roots of a young plant of red clover in normal light $a$, the entire plant; $b$, three leaflets with pulvini

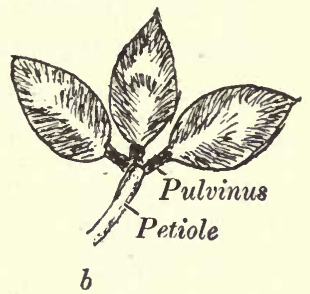

water of the cells on the upper and lower sides of the pulvinus. This highly specialized type of leaf movement by pulvini, observed above in the bean and the red clover, is characteristic of the entire pea family (Leguminosae), to which they belong. Alfalfa, the common clovers, locust, peas, beans, and the sensitive plant ( $M i$ $m o s \alpha$ ) are other wellknown members of this very important family of plants. The contrast between the caladium and the caladium and clover plants outlined above emphasizes the fact that each plant in any given environment is able to adjust its organs by movements so as to adapt them to surrounding conditions. The amount and 
manner of adjustment varies in different plants, but all plants secure a fair adaptation to the environment in which they live.

The dandelion. In the dandelion (Taraxacum officinale) (Fig. 24) the root system resembles that of the clover plant in the form of the main taproot and in the protropic and diatropic responses of the main and lateral roots to gravity. The leaves are diaphototropic and are arranged in the form of a rosette, with the smaller leaves in the center alternating with the larger, outside leaves.

The scapes, or supporting stalks, of the flower clusters of the dandelion, however, manifest a great variety of responses to
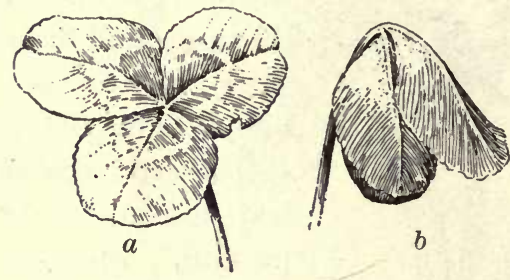

Fig. 23. Corresponding positions assumed by the leaflets of red clover during the day and at night.

$a$, position of the leaflets in normal daylight; $b$, positions of the same leaflets at night. Note that the position in $b$ is assumed by curvature of the pulvini gravity, and these movements are evidently closely correlated with the various stages in the development of the flowers and the fruit. They thus furnish an interesting example of the fact, often observed in plant movements, that the nature of the response of a given organ to an outside influence, or stimulus, is apparently determined, at any given time, by the stage of development and the internal condition of the organ or part. The dandelion scapes are sensitive to light, but the movements here figured and discussed are, for the most part, controlled by gravity, and hence the light stimulation is not taken into account.

In Fig. 24 the various stages in the development of a single flower cluster and its fruit are shown, beginning with the bud stage $(a)$ and ending with the final seed-shedding stage $(h)$. By placing the plant upon a slowly rotating disk connected with a clinostat it can be determined that the various positions assumed by the scapes in the figure are a result of the response of these scapes to gravity. The scapes are therefore either apogeotropic or diageotropic at different stages of development. In the early bud stage (a) the scape is short and the bud is 
apogeotropic and erect. As the bud grows the scape elongates and becomes temporarily diageotropic, as indicated in $b$ and $c$. When the flowers open, the scape and flower cluster become apogeotropic, and the erect flowers are thus exposed favorably for cross-pollination by insects. Curiously enough, in the dandelion the necessity for cross-pollination seems to have been lost in its

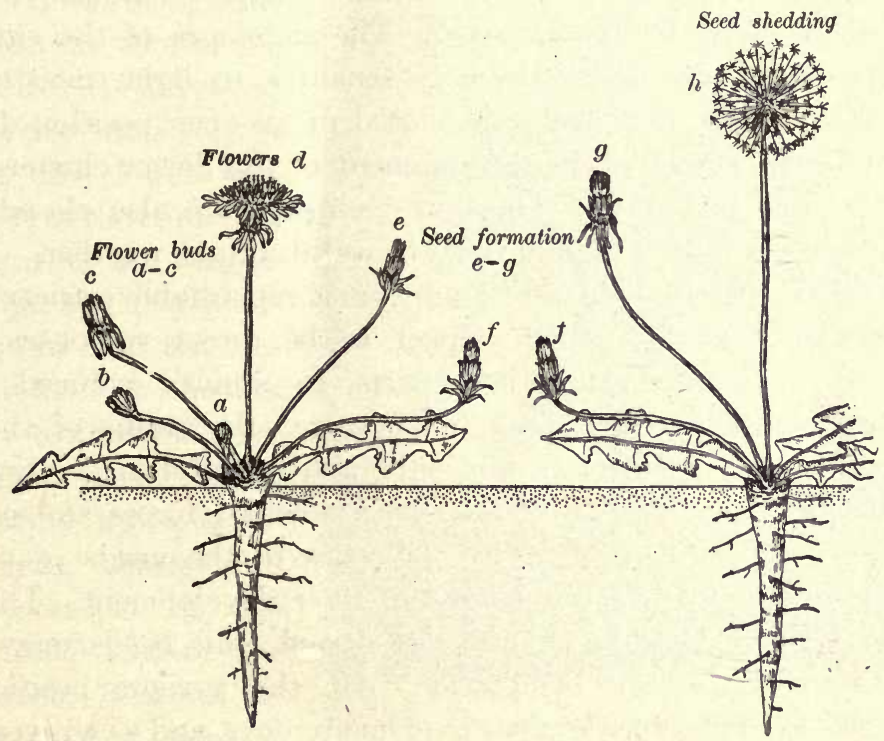

Fig. 24. The response of the organs of the dandelion to light and gravity

Note and be able to explain the positions assumed by the leaves and roots. The different positions of the flower stalk (scape) of the flowering head are indicated by the letters $a-h$. Consult the text for further discussion

later history, since it has been found that its seeds may develop without fertilization, though insect pollination, and its useful effects, is not thereby precluded. When the seeds begin to form, the scape moves downward ( $e$ and $f$ ), becoming again diageotropic in its response to gravity, so that the seeds develop near the ground $(f)$, in a more protected position than the upright, apogeotropic position of the open flowers (d). When the seeds are ripe and ready to be shed, the scape once more changes its responses to the gravity stimulus from the diageotropic position $(f)$ and 
quickly moves upward again to the apogeotropic position (h), which is favorable to the distribution of the seeds. Each seed, thus exposed to air currents, is furnished with a parachute apparatus composed of fine hairs which grow out from the end of a long, stalklike beak projecting from the seed. This hairy parachute is also sensitive to moisture, closing up in wet weather and opening out on dry days, when the seeds may more easily be disseminated. The envelopes of the entire flower cluster are also extremely sensitive to light, moisture, and temperature and assume a closed or an open position during different stages in the development of the flower cluster, as may be seen in Fig. 24. The flower clusters are also closed at night and on cold days, and open in warm, bright weather.

We see, therefore, in the dandelion a remarkable variety of movements, most of which appear to be direct responses of the various organs and their parts to stimuli induced by gravity, light, moisture, and temperature. By means of these responses the organism is not only able to adjust its organs to the daily fluctuations of the environmental forces and conditions but can also adapt its responses to the needs of particular organs at different stages in their development. Thus, the opening and closing of the flower and fruit heads may be a daily response and adaptation, but the various positions assumed by the scapes extend over many days and even weeks and are determined by some unknown coördination between the state or condition of the organ and its power of response to gravity. Many other instances of similar adjustments of floral parts to environmental conditions might be mentioned, but they are all similar, in their general nature, to that of the dandelion and need not be considered here.

Trees and shrubs. In trees and shrubs, as we have already learned, the ultimate form is assumed as a result of the general plan of the plant body combined with adjusting movements. The conelike aspect of a pine tree, for example, is an excellent illustration of the truth of the above statement. In the pine (Fig. 10, b) the main stem remains apogeotropic and continues its vigorous growth during the life of the tree. The lateral branches, 
which arise at regular intervals determined by the mathematical spiral arrangement of the leaves and buds, are diageotropic and diaphototropic and thus assume a horizontal position.

The influence of light in modifying the attitude of the pine tree and its branches is also well illustrated in the figure. At the apex of the tree the young branches assume a more or less uniform upward direction, due to the combined effect of gravity and light. On the lower portions of the trunk the branches are diageotropic and nearly horizontal near the tree trunk, but farther out toward the apex they curve rather sharply upward, becoming nearly prophototropic at the extreme end of the branch. Careful inspection of such a tree will demonstrate the fact that these

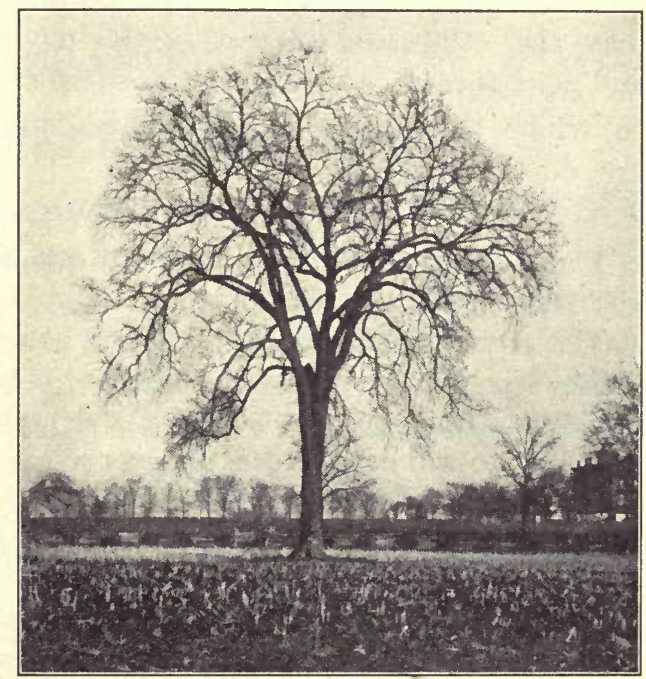

Fig. 25. The form and position of the branches of the American elm

Note the mixed response of the branches to light and gravity. Consult the text for a discussion of this figure various attitudes assumed by the branches of the pine, due to adjusting movements in the response to light and gravity stimuli, secure the best possible exposure of the terminal tufts of needle leaves to light. In a similar manner the ripening seeds in the pine cones are exposed to air currents for dissemination.

Exactly similar phenomena may be observed in young and adult trees of the spreading type (Fig. 25), where the ultimate form of the branches and the placing of the leaves are determined by responses to light and gravity acting as stimuli. The form of all trees and the proper exposure of their leafage 
and fruit are, therefore, determined by the three factors so frequently referred to in the previous pages, namely, body plan, bud growth, and adjusting movements, or tropisms.

These few illustrations of the adjusting movements of some common plants will enable the student to interpret similar phenomena in the great variety of plant forms which enter into his daily experience. The simple account given above may also stimulate interest in the movements of plant parts, and will furnish a clearer realization of the important factors to which the ultimate forms and attitudes of common plants are due.

\section{SUMMARY}

Plants, like animals, are sensitive to stimuli acting upon the living substance of the plant organism.

The nervous mechanism of plants is, however, lodged in the living substance of growing organs, and there is no evidence of the differentiation within the plant body of a definite nervous system corresponding to the nerve fibers and nerve cells of the higher animals. The reception of stimuli and the transmission of impulses in plants take place, therefore, through the living substance of plant organs, which is undoubtedly so connected as to establish a continuity of living substance throughout sensitive and moving parts.

The mechanism of movement also differs in plants and animals, being confined in plants to growing parts in which the unequal growth on different sides of an organ can effect curvature and movements such as we have observed above in roots, stems, and leaves.

The function, or use, of the sensitiveness of plants and their power to react to stimuli is found in the adjustment of their organs to the environment. For this reason plants have developed a special sensitiveness to such stimuli as light and gravity, which enables them to adjust their organs to advantageous positions for the absorption of raw materials, the manufacture of food, and the production of flowers, fruits, and seeds. Highly sensitive living substance for the reception of stimuli is found in root tips, in some stem tips, and possibly in some leaf surfaces. Specialized motor organs are also developed in some plant families. Plants as a whole, however, while sensitive to a greater variety of stimuli than animals, are far below the latter in special sense organs and in nervous organization for receiving and responding to external and internal stimuli. 


\section{SECTION II. CELL STRUCTURE AND ANATOMY}

\section{CHAPTER III}

\section{THE CELLULAR STRUCTURE OF PLANTS}

The bodies of all plants and animals have been found to be composed of organic units termed cells. Each cell unit in its. simplest form is a boxlike structure with bounding cell walls inclosing the cell cavity in which is lodged the living substance of the organism. These structural units of the plant and animal body were first called cells on account of their fancied resemblance to the cells of a honeycomb or of a prison.

The lowest forms of plants and animals are unicellular in structure, but the higher forms are multicellular, consisting of innumerable cells of the most varied form and function. Thus muscles and bones, roots, stems, and leaves, are all built up of cells, which are adapted in their form and structure to the peculiar functions of each particular organ or part.

Just as bricks or stones are used as structural units with which to build a wall or a tower, so we may conceive of the bodies of the higher animals and plants as being built up of living cell units. It has been found also that nearly all organisms have their origin in a single cell, the fertilized egg cell.

In the building of the complex organism from this single egg cell the egg first divides into a multitude of simple cells like itself, and these similar cells are then gradually transformed into the different kinds of cells which make up the various tissues and organs of the adult body.

This conception that all living organisms, however diverse in their character, are composed of similar structural cell units and of a common living substance, protoplasm, is now known as the 
cell theory. It is needless to say that this generalization was the result of prolonged scientific observation and research, extending over many years and engaged in by a host of scientific men, each of whom added his mite to the complete conception.

\section{PLANT CELLS AND THEIR PARTS}

The cell structure of plants outlined above, and the general structure of the plant cell, can be most easily demonstrated by
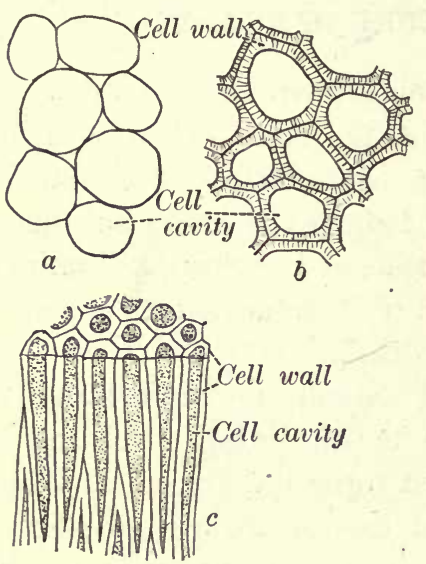

Fig. 26. Cellular structure of pith and celery

$a$, note the intercellular spaces and thin cell walls; $b$, cells in transverse section; $c$, same in both transverse section and long section. The cell cavity is shaded in $c$, and the thickened portions of the cell wall are left light. The primary, or first, wall is indicated by a middle line. Consult the text for the method of cell-wall thickening in such a case as this the student in thin sections cut from the softer parts of plants and examined under a compound microscope.

If such sections are cut from dead tissues like bottle cork or pith, the honeycomblike appearance of the sections will be very obvious (Fig. 26, $a$ ). The entire section will appear, like the honeycomb, to be made up of boxlike units termed cells, each cell unit being composed of a cell wall and a cell cavity inclosed by it. In cork tissue the cells fit closely without spaces between them, exactly like honeycomb cells; but in pith and various other plant tissues the cells round up as they grow, and leave spaces, especially at the junctions of the cells with each other. These spaces serve in many instances for the passage of air through the plant and are called intercellular spaces.

If sections similar to the above are cut from living tissues, as from a root (Fig. 27), the same general cellular structure will be observed as that seen in sections of dead tissues. The cell cavities of the living cells will, however, appear to be filled 
with a semifluid, viscid substance, not unlike the honey in a honeycomb in consistency and in general appearance. 'This substance, which fills the cell cavities of all living plant cells, is the living substance of the plant body, to which Von Mohl first gave the name protoplasm. If sections of roots or of other living parts of plants are properly stained, this living substance, protoplasm, within the cell cavities will be found to be composed of a darker central body, called the nucleus, and a less dense part surrounding the nucleus, called the cytoplasm. For convenience in designating the parts of a living cell the entire mass of living protoplasm within one cell cavity is termed the protoplast, which, as we have already seen, is composed of two distinct parts, the cytoplasm and the nucleus.

The protoplast in young plant cells ( $\alpha$ ) usually bears a somewhat different relation to the other parts of the cell from what it does in older cells $(b-d)$. In young cells
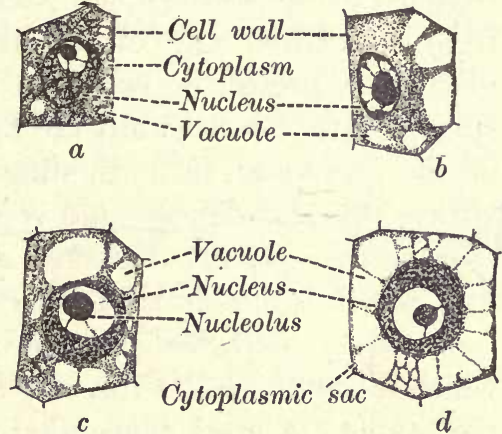

Frg. 27. Camera drawings, greatly magnified, of root tip cells containing cytoplasm, nuclei, and vacuoles

The figures are designed to illustrate the cell parts and the gradual formation of vacuoles. $a$, a young eell with small vacuoles ; $b-d$, progressive vacuole formation as it occurs during the enlargement of a cell by growth. Consult the text for further discussion it fills the cell cavity with a dense mass of protoplasm, while in the older cells it contains water - in the form of water drops, called vacuoles. As the cells grow, these water drops, or vacuoles, enlarge and unite until they finally accumulate as a large central water drop, vacuole, or sap cavity in the center of the cell. In such instances the solid protoplast of the young cell becomes gradually forced outward as the cell grows by the accumulation of water in the large central vacuole. The cytoplasmic portion of the protoplast then forms a thin layer, lining the cell wall and inclosing the central vacuole. It is now properly called the cytoplasmic sac $(d)$. The nucleus in these older 
cells with a large central sap cavity is either suspended in the center of the cell by fine cytoplasmic fibrils, or strands, extending from the nucleus to the cytoplasmic sac, or it lies embedded in the cytoplasmic sac next to the cell wall. In any case a dense envelope of cytoplasm surrounds the nucleus.

The cell wall of all plant cells is formed by the living protoplasm, or protoplast, which finally occupies the cell cavity surrounded by the secreted cell wall. In the development of a plant from a fertilized egg cell the first wall is secreted by its protoplast as a protective envelope for the living protoplasm. All subsequently formed walls are laid down between the two halves of the protoplast in a dividing cell. The wall first formed is always thin andelicate and is composed of pectose. Upon this there is immediately deposited a whitish substance called cellulose, which is closely related to starch and sugar in its chemical composition. Bleached celery is composed largely of the cellulose which forms the cell walls, and a bleached celery stalk will give the student a good conception of the general appearance and nature of this cell-wall substance of plant cells.

Although the first wall is always thin, it may become greatly changed in its character as the cells of the plant body differentiate to form the plant organs and tissues. These modifications of the cell wall are brought about by two distinct methods: first, by cell-wall thickening; second, by changes in its chemical character.

The thickening of the cell wall may be effected by the deposit of new layers of cellulose on the primary thin wall by the cell protoplast, in much the same way as a new layer of plaster might be added to the wall of a house; or the new wall substance may filter into the cell wall and be deposited between the cellulose particles of the original cell wall. The thick-walled cells which form the supporting layers of stems and leafstalks (Fig. 26, $b$ and $c$ ) are illustrations of thick-walled cells whose walls are composed largely of cellulose, while the wood and bast fibers (Figs. 52 and 53) from which wood pulp, linen, and hemp are made are illustrations of thick-walled cells in which the chemical character of the cell-wall substance has been changed by 
infiltration of lignin or a similar substance made by the living protoplasts of these cells. In these highly modified cells the protoplast usually disappears after the cell wall is fully formed, and they become dead cells in which the cell cavities are filled with air.

The skeleton, or supporting framework, of plants is thus composed of the cell walls of the individual cells making up the plant body. In the case of the higher plants these cell walls become thickened and hardened, as indicated above, and form a firm supporting skeleton for trees and other plants of large size. This skeleton of cell walls divides the living substance (protoplasm) of the entire plant body into separate units, or protoplasts, each protoplast being surrounded by its own skeletonlike cell wall. This is quite unlike the condition found in animals, where the cells often have no definite cell walls and are supported by an internal body skeleton, as in higher animals, or by a crustlike external skeleton, as in insects and crayfish.

The plastids are minute granules of denser protoplasm which occur in most living plant cells. They occur in the cell cytoplasm and vary in form from minute granules to very elaborate bands and disks, such as are found in some algæ. Some plastids are green, others are composed of colorless cytoplasm, while still others are tinted with yellow and orange pigments. They all agree in being composed of living protoplasm. They are classified according to their color into chloroplastids, which are green; leucoplastids, which are colorless; chromoplastids, which are variously tinted.

Chloroplastids are found in all parts of plants which have a green color, such as leaves and the outer parts of some stems. The green color in such organs is due to a green pigment, chlorophyll, which is secreted within the numerous plastids contained within the cells of such structures (Fig. 28, $a$ and b). Each chloroplastid $(b)$ is composed of a cytoplasmic granule, or disk, and the green pigment which gives it its color. In such thin leaves as those of mosses or of the water weed Elodea the plastids are plainly visible under low powers of the microscope, embedded in the cell cytoplasm. In Elodea the plastids are often carried around the cells in the moving cytoplasm, like boats in 
a stream, and serve to indicate the rate and direction of cytoplasmic movement in the leaf cells of this plant. The green pigment, chlorophyll, can be removed from the cytoplasmic body of the plastids by treating the leaves with alcohol, when the green pigment dissolves out, leaving the colorless plastid behind in the cytoplasmic sac. We have already learned that the green pigment enables the chloroplastids, with the aid of the sun's energy, to build and store starch made from carbon dioxide and water. The chloroplastids are thus of the greatest importance in making the necessary starchy food for the plant.
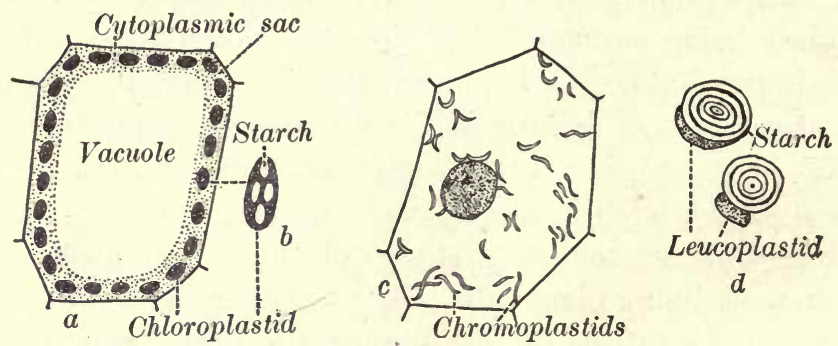

Frg. 28. Three kinds of plastids and the formation of starch grains $a$, a mature cell with chloroplastids embedded in the cytoplasmic sac; $b$, a single chloroplastid containing light-colored starch grains ; $c$, chromoplastids; $d$, leucoplastids of begonia forming starch grains

Leucoplastids (Fig. 28, d) are similar to chloroplastids except that they lack the green pigment, chlorophyll, and are therefore unable to manufacture starch from raw food elements such as carbon dioxide and water. They are able, however, to transform sugar into starch, and are therefore present in all such special storage organs as potato tubers and seeds, as well as in ordinary stems and leaves where starch is stored away from direct sunlight. They are more difficult to demonstrate microscopically than chloroplastids, and hence are less frequently seen than the latter. Leucoplastids are often transformed into chloroplastids by the secretion of green pigments when they are exposed to sunlight. This is frequently observed when potato tubers are uncovered and turn green in the part of the tuber which is directly exposed to the sun. 
Chromoplastids (Fig. 28, c) are plastids which secrete pigments other than green, which tint the plastids and the organs in which they are located yellow, orange, or red. Flowers and fruits often owe their color to the abundant chromoplastids developed in the cytoplasm of their cells. Familiar examples are the yellow petals of nasturtium flowers, the red color of the tomato, and the orange yellow of the berry of bittersweet.

\section{FUNCTIONS OF CELL, PARTS}

The cell wall, as has been stated, serves as a protection for the delicate protoplasts and as a skeletal framework for the entire plant body. The protoplasts of the plant body have been shown to be connected in some plants by delicate strands of protoplasm, which pass through fine pores in the separating cell walls. The protoplasts in these plants are not, therefore, isolated units of living substances, but are closely joined in a living body of protoplasmic units. Such a continuity of the protoplasts by means of connecting strands might conceivably be of direct service in the passage of foods from cell to cell or in establishing a nervous connection between the various tissues and organs of the plant body. Both of these hypotheses have been advanced by reputable botanists, but there is as yet no conclusive proof that either is true.

The cytoplasm and the nucleus undoubtedly represent a division of labor in the living substance of the protoplast by which it is enabled to do its complex work more satisfactorily.

The cytoplasm is supposed to be largely concerned with the building, storage, and use of foods and with the reception of impressions (stimuli) from the outside. We have already seen that the green chloroplastids are portions of the cytoplasm which build starch, and we shall learn that the starch in potatoes and in seeds is stored in the cytoplasm of the cells of those structures by the agency of leucoplastids. It is equally evident that the cytoplasm must be the first to feel impressions from outside of the cell.

The vacuole inclosed by the cytoplasmic sac is filled with water in which various substances formed by the cytoplasm finally 
accumulate. This water and the substances - sugar, acids, and salts - which diffuse into it from or through the cytoplasmic sac are together called the cell sap. In the cells of the sugar beet, sugar is the main constituent of the cell sap, while in fruits both sugar, acids, and salts are found in considerable quantities. The cell sap thus becomes a storehouse for the water and foods which may be needed by the cell and the plant.

The nucleus is also very important in the life of the cell and the organism, being very closely connected with fertilization and with the formation of new cells by division. The most important act of fertilization seems to be the union of the nucleus of the male cell or gamete with that of the female cell or gamete, while the complex changes undergone by the chromatin of the nucleus in cell division indicates its importance in that process. Although the nucleus and cytoplasm are thus separated structurally and seem to have certain specialized functions, it has been conclusively shown that they are coworkers in the various cell activities and are mutually dependent upon each other for continued existence.

The following table summarizes the relations of the constituents of the cell in terms of living and lifeless parts as discussed above.

\section{SUMMARY}

\section{Lifeless parts of plant cells :}

The cell wall: outer membranous covering of the living protoplast of a cell.

Vacuoles and cell sap: the cavities, or spaces (termed sap cavities) in the protoplasts of plant cells, and the contained cell sap, composed of water and other substances in solution within the vacuole.

Metaplasmic bodies: solid waste and food particles embedded in the protoplast.

2. Living parts of plant cells :

Protoplast: the entire living protoplasm of the cell, including the nucleus.

Cytoplasm: all protoplasm of the cell exclusive of nucleus and nucleolus. 
Cytoplasmic sac: the term used to designate the cytoplasm of the cell when it takes the form of a sac lining the cell wall and bounding the central water vacuole.

Nucleus : a denser differentiated protoplasmic body within the cell bounded by the nuclear membrane.

Nucleolus: a darker dense mass of protoplasm within the nuclear cavity. It is usually surrounded in plant cells by a nuclear vacuole.

Plastids : differentiated grains or bodies of cytoplasm of different color and function. 


\section{CHAPTER IV}

\section{HISTORICAL SKETCH (THE CELL AND THE CELL THEORY)}

\section{EARLY DISCOVERIES AND THEORIES}

Cell wall. The first discovery of the cell structure of plants is attributed to Robert Hooke, an Englishman, who, about the year 1665, observed the cellular structure of plant tissue in sections of the wood, bark, and leaves of plants. Hooke was impressed with the fact that such sections, when viewed under a strong microscope, presented the same appearance as the cells of a honeycomb. He therefore applied the term cell to the cavity which he saw inclosed by the conspicuous cell walls, which form the cell boundary of most plant cells. This application of the word cell to the cavity, and not to its contents, is now regarded as a misnomer, since we know that the most important part of plant and animal cells is the living substance, protoplasm, which is contained in the cell cavity. Long usage has so firmly established the term, however, that it is still in use, although its meaning is now extended to include, with the cell wall, the substances within the cell cavity, the most important of which is the living protoplasm.

The protoplasm and nucleus. The observation of the living substance within the cell cavities of plant and animal cells, and the recognition of its true nature and significance, was not understood for fully two centuries after Hooke made his discovery of the cellular structure of plants. This was due in part to faulty observations and in part to the imperfect microscopes with which the observations were made.

Robert Brown (1831), an English botanist, discovered a dark central body within the cells of orchids, which he named the cell nucleus. Other observers of cellular structure saw a substance outside of the cell nucleus in plant cells, which 
they termed variously gum, mucilage, and sarcode. The real nature of this substance within the cell wall was not, however, positively known until about the middle of the last century, when its true significance as the essential living substance of the cell and of the organism was recognized. 'The name protoplasm, which it now bears, was first given to this living substance in animal cells by Purkinje (1839-1840) and in plant cells by Von Mohl (1846). Von Mohl's conception of the nature of the living substance of plant cells, including the nucleus, is embodied in the following quotation, taken from his work on "The Vegetable Cell."

In the center of the young cell, with rare exceptions, lies the so-called nucleus cellulae of Robert Brown. It is usually of very considerable size in proportion to the magnitude of the yourg cell, so that in particular cases - for example, in the cells of jointed hairs - it almost fills the cavity. The remainder of the cell is more or less densely filled with an opaque, viscid fluid of a white color, having granules mingled in it, which fluid I call protoplasm.

Von Mohl and his contemporaries, Schleiden and Nägeli, not only clarified the current conceptions of the nature of the cell and of protoplasm but also established the fact of cell differentiation, or change in the form and structure of cells to serve different functions in the plant. 'They discovered that the stems, roots, and leaves of plants are composed of a great variety of cells, varying in form, structure, and function, which enable the plant organism to do its work more effectively than it otherwise could.

The cell theory. The idea that all living plants and animals, however diverse in their nature, are composed of similar cell units was first published by Schleiden, a botanist, and Schwann, a zoölogist, in 1839. Later investigations have not only corroborated the truth of this generalization but have greatly extended its meaning and emphasized its importance. It has been found also not only that animals and plants are similar in general cellular structure but that the living substance is not essentially different in the two kingdoms of life. The 
latter idea was first formally established and published by Max Schultze in 1863. This essential identity in cellular structure, and in the nature of the living substance of all organisms, explains the great similarity which has long been known to exist between the lowest plants and animals. Indeed, this similarity is so great that certain organisms are to-day claimed by botanists as belonging to the plant kingdom and by zoölogists as belonging to the animal kingdom. The great importance of the cell theory can only be realized by the student as we proceed to study more intimately the biology of the higher and the lower plants, in which we find exactly the same processes performed by very different structures and organs of the plant body.

\section{LATER DISCOVERIES AND THEORIES}

The later developments of the cell theory were the result of researches and discoveries made by a notable group of biologists during the latter part of the nineteenth century. These later observers applied the early ideas and discoveries concerning the structure of the cell and protoplasm to problems concerned with the ultimate structure of protoplasm and nucleus, cell divisions, fertilization, and development, and to the fundamental processes of plant and animal physiology. Their discoveries and conclusions in these various fields can only be outlined here, but the following brief survey is given in order that the student may realize, in part at least, the full significance of the cell theory to modern biological thought and discovery.

Cell division. The general process of cell division in the higher plants was first correctly interpreted by Karl Nägeli in 1846, although. Schleiden and Von Mohl made large contributions to the final solution of the problem.

Basing their observations on those of Nägeli, Von Mohl, and Schleiden, the later botanists of the nineteenth century, under the leadership of Eduard Strasburger, the great German botanist, worked out in much greater detail the mechanism and the meaning of the division process now designated as mitosis. 
Fertilization. The process of fertilization was first observed in Spirogyra, one of the lower water plants, in 1879, and in the higher plants by Strasburger in 1884 .

The essential act of this fertilizing process was found to consist in the union of two cells, male and female, termed gametes or, more frequently, sperm cell and egg cell (Fig. 29). Later studies seem to indicate that the union of the male and female nuclei is the most important if not the only essential part of the process.

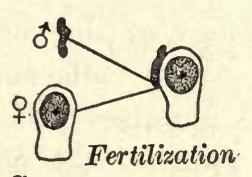

Gametes

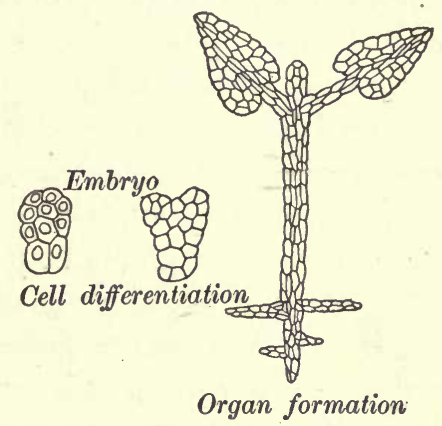

Fig. 29. A diagram illustrating the main cell processes occurring during the development of a plant

Consult the text for further discussion

The two gamete cells, when united in fertilization, form a new cell, the zygote cell, which is a double cell in the sense that it is composed of two cells from two distinct individual plants or from separate portions of one plant. This double nature of the zygote cell will be found, in our later studies in reproduction, to be of great significance in heredity, since it contains the fundaments of two sets of characters, one set brought in by the male gamete and one by the female gamete.

Embryology. The development of a higher plant from the zygote was first completely worked out by Hanstein in 1869 . The development process of the higher plant, like that of a higher animal, was found to embrace three important stages, or phases; each phase including processes of cell division and growth. The first stage, or phase, is concerned with the division of the single-celled zygote into many cells, thus giving rise to 
a cellular embryo. In the second phase of development the uniform cells of the early cellular embryo begin to differentiate to form the first organs and tissues of the early embryo. In the third phase this early embryo forms the seedling, in which the parts outlined in the early embryo take on the form of the parts of the mature plant.

'The mature form and structure of the plant is derived from the seedling by the growth and differentiation of cells located in particular growing regions, called meristems and cambiums, at the tips of the stem, roots, and branches and in the internal growing layers (cambiums) of the plant body.

Physiology. The physiology of both plants and animals has been shown to be dependent upon the physiology of the constituent cells of the plant and animal body. Nerve cells and their processes are known to receive and send impulses which cause muscle cells to contract and move the parts of the animal body. In a similar manner the cells of plants cause rapid or slow movements in roots, stems, and leaves. In the sensitive plant (Mimosa) the movements caused by specialized cells at the base of the leaves and leaflets are as rapid as those of some animals, but the slower movements of the parts of plants have been shown to be no less cellular in their mechanism than those of Mimosa and in animals. Similarly, food-building and the circulation and use of foods are ultimately traceable to the activities of the constituent cells of plants and animals. In the higher plants food-building takes place in the green leaf cells provided with chloroplasts; circulation of foods, water, and salts occurs in long chains of cells (ducts and sieve tubes); while assimilation goes on in all living cells of the plant body. In the light of these facts the organism is seen to be a great and highly differentiated eolony of cells, which, like the members of a civilized community or of a factory, are organized to maintain the welfare of the community as well as that of each individual member. In the studies which follow, the student will be interested in gaining at first hand a knowledge of the cell structure and cell activities of plants upon which the above generalizations of the modern cell theory are based. 


\section{SUMMARY}

1. The cell diseovered and named as a unit of organic structure in plants (Robert Hooke, 1665).

2. The nucleus discovered in orchidaceous plants (Robert Brown, 1831).

3. The term protoplasm applied to living substance of plant cells (Von Mohl, 1846).

4. Cell theory announced (Schleiden and Schwann, 1839).

5. Cell division correctly interpreted (Nägeli, Von Mohl, and Schleiden, about 1846).

6. Cell differentiation and tissue structure explained (Von Mohl and contemporaries, 1840-1850).

7. Later developments of the cell theory:

a. Fertilization in Spirogyra (1879); in higher plants (Strasburger, 1884).

b. Development of plant embryo (Hanstein, 1869).

c. Cytology (1873-1900). Minute strueture of eytoplasm and nucleus; details of nuclear and cell division. Strasburger the leading botanist of this period in eytology.

d. Physiology of plants (1860-1900). Sachs and Pfeffer the most prominent plant physiologists.

The above summary indicates the general course of the investigations and discoveries which have established the cell theory and have furnished us with our present knowledge of the structure and development of organisms. The student who has followed this development of the cell theory will be better fitted to understand the bearing of the more detailed account of the cell structure, development, and activities of plants which now follows in the text. 


\section{CHAPTER V}

\section{GROWTH AND CELL DIVISION}

The growth of the higher plants is very different from that of the higher animals. In animals growth ceases after a relatively short period in the life of the organism; plants, on the contrary,

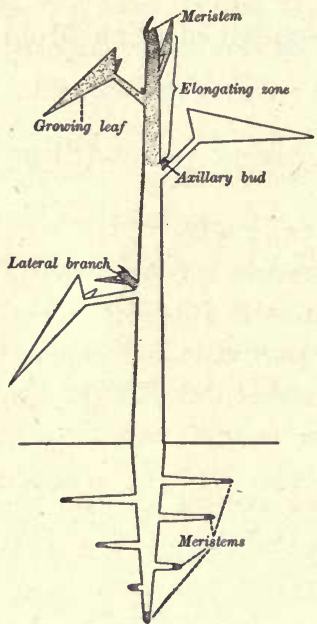

Fig. 30. Growing areas of a buckwheat plant. The growing parts are stippled; the permanent portions are white continue to grow throughout their whole life, which, as in the case of long-lived trees, may extend over hundreds of years.

In the early stages of the growth of a higher plant from the egg cell all parts of the embryo grow until the seedling is thoroughly established for self-support. In annual and biennial plants growth may continue to be general over a large part of the plant body, although it usually becomes localized as maturity approaches. In perennials, however, including our common trees and shrubs, growth and its accompanying cell division is largely limited to the tips of the roots and shoots and to the cambiums, or growing cell layers, which produce annually new layers of wood and bark. Buds are familiar examples of this localization of growth at the branch tips, and we shall find a similar provision at the tips of roots. Fig. 30 illustrates diagrammatically the growing zones of an ordinary herbaceous plant in which growth has become localized.

Since growth is one of the most important phenomena in the life of plants, we can profitably devote a considerable space to an account of a concrete case of cell and organ growth, which will furnish a foundation for understanding all growth more clearly. 
For this the root tip furnishes the best material both for observations of growth in a living object and for the study of its more detailed features as revealed in its cellular organization.

\section{GROWTH AND STRUCTURE OF THE ROOT}

The elongation of the root is easily observed in living roots by selecting the first or primary root, which springs from a seed during its early stages of germination. In order to locate the exact region in which growth in length takes place the tip of the root should first be marked with fine lines, evenly spaced, for several millimeters back of the apex of the root tip. In Fig. 31 these lines have been made at intervals of two millimeters in $b$, and the separation of the lines in $c$ indicates where the root grew in length for the first twenty-four hours after it was marked. It will be noted by

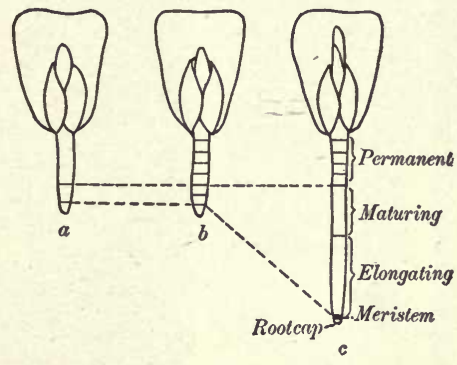

Fig. 31. Growth in length of the root of corn

The separation of the millimeter spaces indicates the place of greatest elongation consulting the figures that the root elongated in the second and third millimeter spaces back of its apex, and that the first millimeter space at the very apex of the root tip and the millimeter spaces just back of the elongating area have remained unchanged. In the first millimeter or two, at the apex of the root, two distinct groups of cells exist, with which we shall be concerned as we proceed to study the method of growth in the root tip. One of these is apparent in living roots as a light-colored, conical mass of cells constituting the apical point of the root. This light-colored point is the protective rootcap, which incloses a disk of cells, not more than a millimeter in length, which is invisiblc in fresh roots but is easily recognized in sections of growing root tips. This latter disk of cells is the meristem, or dividing cell zone, which plays a very prominent part in root growth, since it furnishes most of the new cells for the rootcap 
and elongating zones. For our convenience in describing the growth of the root the tip may therefore be divided (Fig. 31, c) into the following cell zones: the rootcap, the meristem (just above the rootcap and inclosed by it), and the elongating, maturing, and permanent zones. The term permanent zone, as here used, signifies all that portion of the root tip in which the cells have ceased to grow. In the lower portion of this zone the cells

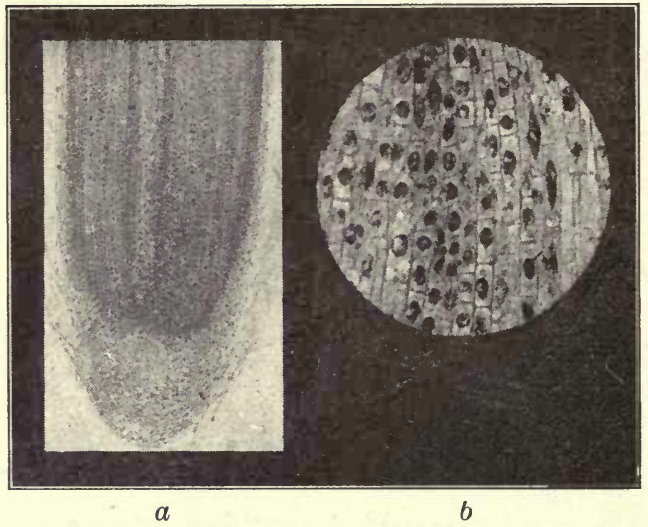

Fig. 32. Microphotographs illustrating the cellular structure of the root tip

$a$, a long section through a growing root tip of the false Solomon's seal (Smilacina) (the lighter cells of the rootcap, the darker meristem zone, and the longer cells of the elongating region are plainly shown); $b$, cells of a portion of the elongating zone, greatly magnified have not yet become fully differentiated into the different tissues of the mature root.

Structure. Before proceeding with the more detailed aspects of the growth of the root tip it will be necessary to consider the cellular organization of the root at its apex. For this purpose thin sections which have been especially prepared for microscopic study are necessary. First the roots are embedded in paraffin wax; then the sections are cut lengthwise of the roots with special section-cutting machines, or microtomes. The sections thus obtained are fastened on glass slides and are stained by special methods for microscopic observation. In sections so prepared the student can easily determine the main cell areas of the growing root tip which have already been observed in living roots, and can also relate the cell structure of these areas to the processes of cell and organ growth.

The cellular structure of the root tip is illustrated in Fig. 32, $a$ and $b$, which are microphotographs of a portion of a root tip and 
of some of its cells highly magnified. Fig. 33 is a diagrammatic drawing of a median long section of a root in connection with which are shown camera lrawings of separate groups of cells taken from the rootcap, the meristem, the elongating zone, the maturing zone, and the permanent zoine. These latter drawings not only indicate the actual form of the cells of the above regions of the root but, since they are all drawn to the same scale, they show also the increase in size of the roottip cells from the meristem to the permanent zone, where they cease to grow in length.

The rootcap is a conical mass of lightcolored cells that terminates the root tip and forms a protective mantle for the actively dividing and growing meristem cells which lie just back of it. The outer cells of the cap are large, thin-walled,

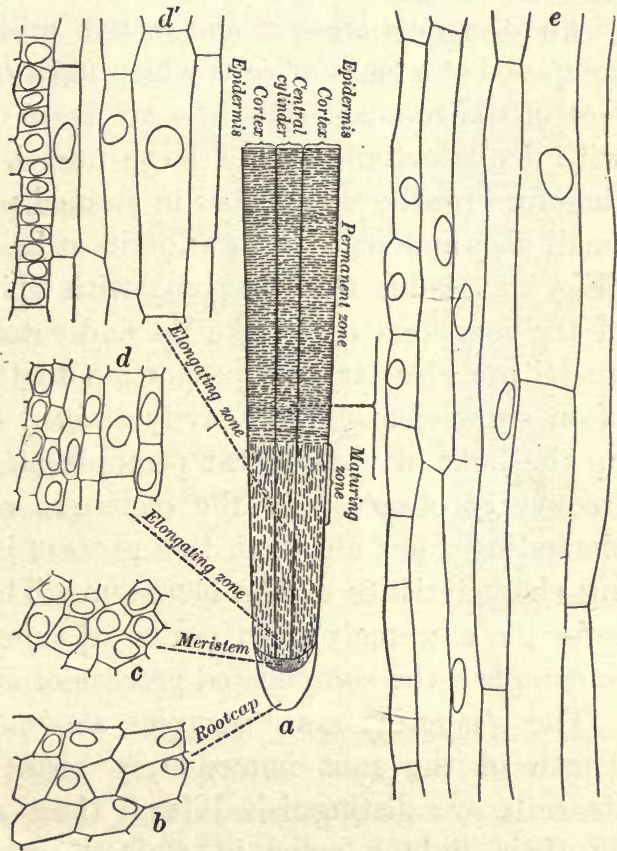

Frg. 33. The growth of cells in a root tip

$a$, the main areas of the root tip shaded; $b-e$, camera drawings of cells from the various regions of the root as indicated in the figure. Note the changes in form and size of the cells as growth proceeds. The nuclei grow with the cells, but they occupy a proportionately smaller part of the cell cavity as the cells enlarge

and loosely joined together. Their lighter color is due to the large vacuoles which nearly fill the cell cavity of each cell. These outer cells are being constantly worn off as the tip of the root is forced through the soil by the elongation of portions of the root above or behind the cap cells. The inner cells merge insensibly into the cells of the meristem above them and, 
like the latter, have the power of active cell division, thus producing new cells to replace those worn off at the apex by contact with the particles of soil. There is no danger, therefore, that the root will be deprived of its protective cap, even though it is constantly losing its outer cells by death and abrasion from the soil.

The meristem layer is one or two millimeters in length and is composed of a mass of cells which fit into the upper, concave surface of the rootcap. Its cells are more or less cuboidal in form, with dense cytoplasm and large nuclei. The meristem layer is therefore readily recognized in stained preparations, both by the small size and regular form of its cells and by its darker color (Fig. 32, a), due to the activity with which the dense protoplasts of the meristem cells take up and retain artificial stains. The nuclei are also large in proportion to the size of the cells and often show clearly the heavily stained chromatin which makes up the bulk of the nuclear protoplasm. Cell division is not so frequently observed in the meristem cells as in those of the elongating zone, although this process is one of the distinguishing characteristics of the meristem. The reason for this seems to be the extremely short period of time required by these cells to complete the complicated process of cell and nuclear division.

The elongating zone ${ }^{1}$ occupies about four millimeters of the length of the root immediately back of the meristem zone. Its cells are distinguished from those of the meristem region by their lighter color in stained preparations and by their greater average length. The lighter color of its cells is due to their less dense cytoplasm as compared with that of the meristem cells, and to the gradual accumulation in their protoplasts of absorbed water in the form of vacuoles, which increase in number and size toward the upper limit of the elongating zone. We consequently find that the cells in the lower half of the elongating zone resemble closely those of the meristem, into which they graduate insensibly at the junction of the two cell areas, while the cells. of the upper half are longer and lighter-colored, with large vacuoles.

1 The upper part of the elongating zone, in which the cells are beginning to differentiate into permanent tissue, is here designated as maturing. 
The permanent zone merges gradually and indistinguishably into the elongating zone below it and into the mature portions of the root immediately above it. In general, however, its cells are characterized by their great length and large central vacuoles, or sap cavities. The cyptoplasm in the longer cells is usually in the form of a delicate cyptoplasmic sac, in which the nucleus is embedded. The nuclei appear to be relatively smaller in these cells, but accurate measurements indicate that they usually maintain a slow growth as long as the cells increase in size, and that they do not diminish in volume in the cells in which growth is completed.

Elongation. In a growing root the meristem layer gives rise to hundreds of new cells. As these new cells are formed part of them remain meristem cells, while others in the upper portion of the meristem begin to elongate and form a part of the elongating zone, as shown in Fig. 34. At the same time the cells in the upper part of the elongating

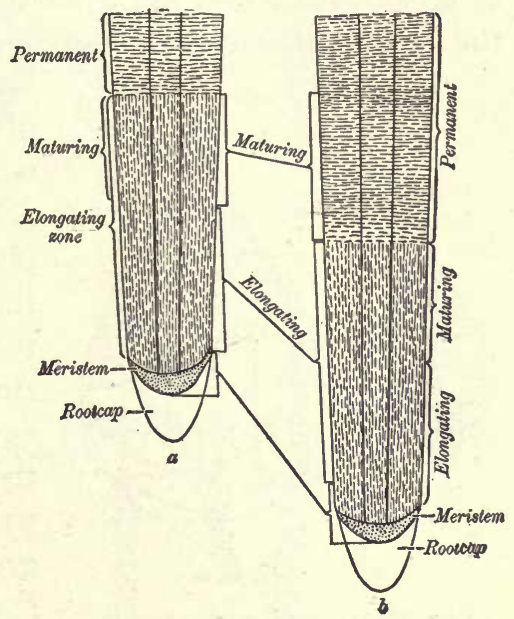

Fig. 34. A diagram illustrating the method of elongation of a root

$a$, shows the main zones of the root shaded as in Fig. 33, $a$; in $b$. the same zones are shown after the root has elongated. Note that the meristem and elongating zones remain of the same length in $a$ and $b$ as growth proceeds zone are reaching their definite size and will add a new disk of cells to the length of the permanent zone of the root. It will therefore be clear that by this process the permanent part of the root becomes continually longer by successive additions from the elongating zone, while the length of the latter zone is kept constant by a similar number of cells derived from the division of the meristem cells. By these two processes - namely, cell division and cell elongation - the root thus grows in length and its tip advances through the soil. 


\section{GROW'TH OF OTHER PLANT ORGANS}

The same phenomena which we have outlined in the growth of the root obtain in the growth of all other plant organs, including leaves, buds, and stems.

Cell division and cell enlargement are the two main phenomena of all growth which are demonstrable by scientific methods. In the case of plants the enlargement of cells in growth seems to be mainly due to the absorption of water and the inflation of the cells by osmotic pressure. By this

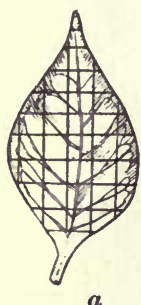

$a$

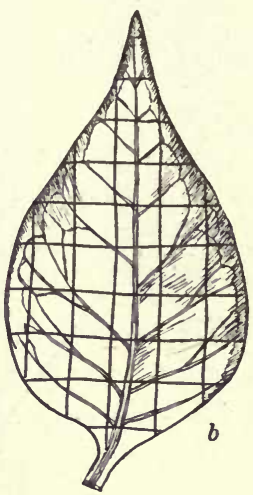

Fig. 35. Growth of leaves of the lilac

The spread of the squares indicates a uniform growth of the leaf over its entire surface means very rapid growth can take place without the expenditure of the energy necessary to manufacture great quantities of living substance with which to fill the cell cavities of the expanding cells. In the slower-growing animals the water-inflation method of cell growth does not exist to any such extent as it does in plants, since the latter need to adapt themselves quickly to the changing seasons, which necessitates the rapid production of extensive root and leaf surfaces for the absorption of water and gases at the opening of each warm season. The growth of leaves in spring is a good illustration of this aspect of growth in plant organs. A large tree will produce in a few days in spring many square yards of leaf surface; this involves the production and inflation of thousands of new cells. It is obvious that the tree would be wholly unable in this short time to manufacture enough new protoplasm to fill the tremendous space caused by the expansion of the cell cavities of its leaf cells. The water-inflation method is therefore both economical and necessary in all rapidly growing plant organs, such as leaves, stems, and roots. 
The place where growth takes place varies in different organs. In most leaves (Fig. 35) growth is uniform over the entire leaf surface, although in the case of long, narrow leaves it may continue longer at the base than at the apex. In stems growth is more localized than in leaves; it continues for a longer period and extends over a larger portion of the stem than it does in the root tip just studied. In herbaceous stems (Fig. 36) growth may continue at the apex of the main shoot or its branches for several weeks or for the entire growing season, as in the root. This growth, like that of the root, is due to an active meristem at the stem tip, within the apical buds, in which active cell division furnishes new cells for continuous growth in the elongating zone, which, as indicated above, may extend over several.internodes at the apex of the stem.

In woody stems growth in length takes place in

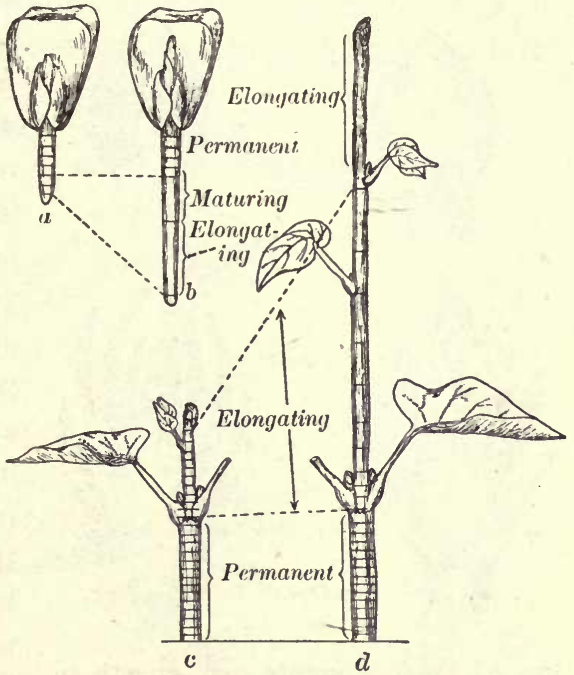

Fig. 36. Growth in length of a root of corn and of the stem of a bean seedling

The spread of the markings in $b$ and $d$ indicates the places of greatest growth. Observe the greater area over which growth takes place in the stem as compared with the root terminal or in lateral buds, as in herbaceous stems. The buds of woody stems, however, usually contain most or all of the parts of the next season's shoot formed in advance, so that its growth in the spring is completed more quickly than in herbaceous stems, and the plant is thus able to expand its leaves in a comparatively short time when conditions favorable to spring growth occur. In the lilac (Fig. 37) the bud $a$ is composed of the leaves of the next season, arranged on the very short axis of the future branch. The internodes separating the successive 
pairs of leaves at the nodes are very short, as shown in the long section of the bud $b$. The meristem terminates the axis of the bud, all of which will constitute the elongating zone when growth first starts in the spring. The expansion of the internodes by growth separates the leaves ( $c$ and $d$ ), which at first grow more rapidly on their inner than on their outer surfaces,
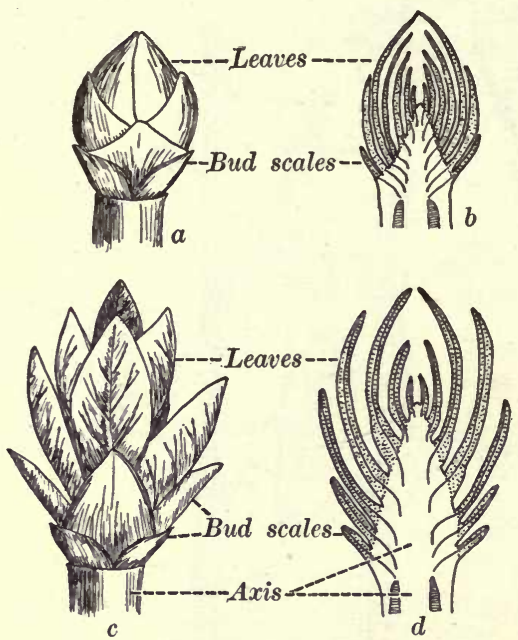

Fig. 37. Bud structure and growth in the lilac

$a, b$, surface and sectional views of a bud in the resting condition; $c, d$, similar views of the same bud in spring when it first begins to grow and so unfold and spread out horizontally to the light. As growth proceeds the cells within the lower internodes reach their definite length and thus add new segments to the permanent portions of the young stem, as in the case of roots. In this permanent portion the cells differentiate and the main tissues of the mature stem are formed. Before growth in length ceases, the buds for the next season become apparent and proceed to form the young leaves for the growth of the following spring. Indeed, the growing points, or meristems, for these buds are apparent, in the lilac, in the wintering bud in the axils of its rudimentary leaves. In the autumn, therefore, when the leaves fall, the lilac shoot of the season presents the aspect shown in Fig. 38, $b$, with all the buds laid down and protected by bud scales.

In all these changes incident to growth the cells and organs of plants are subject to the effect of environmental forces. A certain amount of water and heat, varying with the type of organism, is necessary for growth. Light usually has a retarding effect on growth, although this too is a variable factor in its influence. In general it may be said that extremes 
of heat, cold, moisture, and food supply are detrimental to growth, while mean conditions in the case of any given factor are likely to be favorable to it.

\section{SUMMARY}

Growth in plants comprises three main phases or stages. The first is that of cell division, in which the cells of the entire structure, or of specialized parts termed meristems or cambiums, undergo rapid multiplication by mitosis. The second phase is that of enlargement, during which the new cells formed by mitosis increase greatly in size, and so cause the growth of the organ or part concerned. The third is the phase of differentiation and maturation, in which the enlarged cells are modified to form the different tissues of the permanent plant organs.

Plants differ from animals principally in the second and third phases of both cell and organ growth. Plant cells increase in size very largely by the absorp-

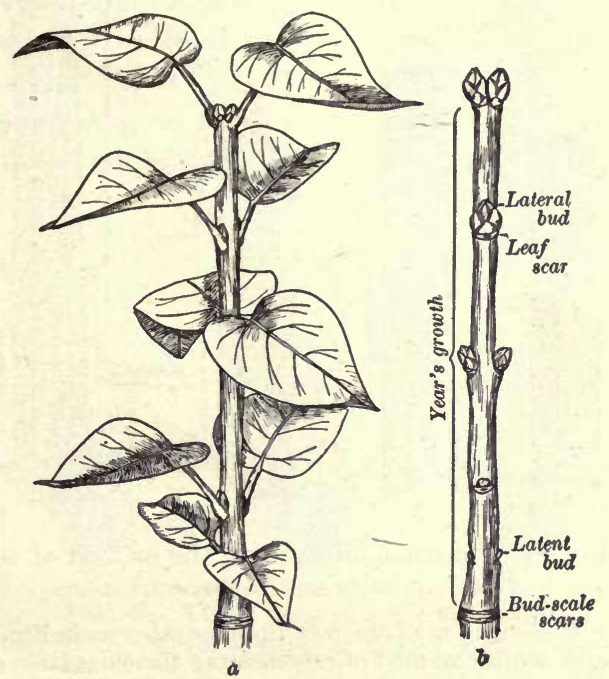

Frg. 38. Lilac twigs in summer and winter conditions

$a$ and $b$ represent the season's growth of the bud shown in Fig. 37 tion of water, which collects inside the cells in the form of water drops, or vacuoles. In animals the increase in the size of the cells is largely due to actual increase in the amount of living substance. The differentiation and maturation stages of cell growth are also necessarily different in plant and animal cells, on account of the different kinds of permanent tissue formed in plant and animal organs.

Plant organs differ in their methods of growth according to the nature of the organ or part and the length of time during which enlargement continues. Organs which have a short period of growth, 
like leaves, fruits, seeds, and tubers, usually grow more like animals, - by a uniform increase in the size of cells throughout the entire structure. Cylindrical organs, on the contrary, like roots and stems, which have periods of enlargement often extending over many years, have definitely located growing masses, or layers, of cells, called meristems and cambiums. These meristem and cambium cells renew their cell divisions each season, in the case of perennial organs,

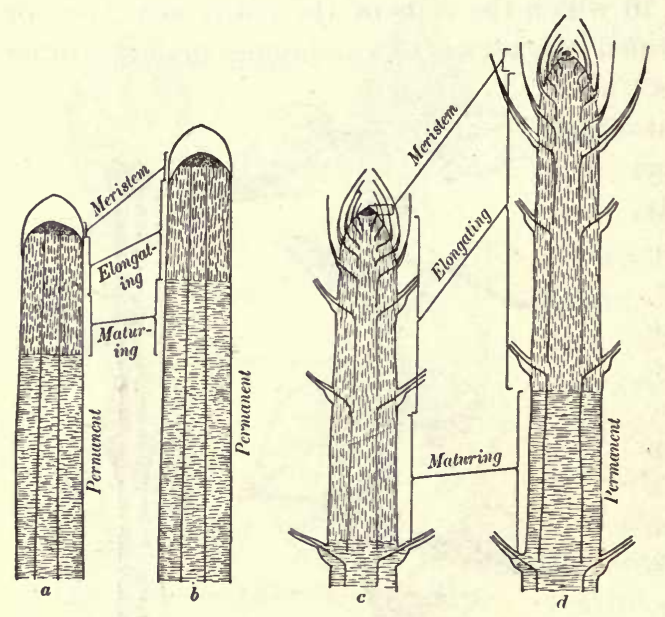

F1G. 39. Diagrams illustrating the method of elongation of roots and herbaceous stems

$a, b$, elongation of the root tip illustrated as in Fig. 34; $c, d$, similar method of representing the elongation of a herbaceous stem like the bean (Fig. 36). Consult the text for further discussion and contribute new cells, which then pass through the second and third phases of growth to form new tissues and organs. The apical meristems which terminate the roots, the stems, and the branches have already been studied. The cambiums are cylindrical layers of meristem cells lying between the wood and the bark in stems and roots and between the outer cork and inner bark. These cambiums enable roots and stems to grow in diameter and also

to form new annual tissue layers for protection and for the conduction and storage of foods, water, and soil salts. The apical meristems also enable plants to send out new leaves and roots each season for the absorption of raw food elements and the compounding of these raw materials into organic foods.

The above general statements relative to the growth of stems and roots by means of apical meristems and cambium layers will be more readily understood by reference to the following diagrammatic figures: Fig. 39, $a$ and $b$, is a repetition of Fig. 34, illustrating the method of growth of roots in length by means of an apical meristem. Fig. 39, $c$ and $d$, illustrates in a similar manner the growth of a herbaceous 
stem, represented in Fig. 36, $e$ and $d$. In the latter cases the meristem is practically identical in form and structure with that of the root tip. It does not, however, produce a protective rootcap, since it is here protected by the enveloping leaves of the terminal bud.

Growth in length takes place in the stem, as in the root, by the continuous transformation of cells produced by the division of $\mathrm{tl}$. meristem cells into cells of the elongating zone. Later these become permanent tissue and form the mature nodal and internodal tissue of the older portions of the stem. At the same time a cylindrical cambium layer, in the region indicated by the dark lines in the figure, begins to divide to form the new water-conducting and food-conducting tissue of the vascular, or woody, cylinder of the plant.

Fig. 40 represents the elongation of a woody stem by a large terminal bud. In this instance the general structure of the meristem and the method of elongation are the same as in the herbaceous stem. The differences between the two arise from the fact that the herbaceous stem has a more continuous growth throughout the season than the woody

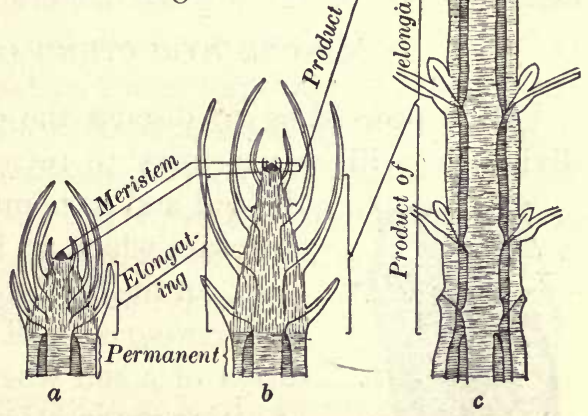

Fig. 40. Three diagrams showing methor of elongation of a lilac bud

$a$, bud in winter condition, similar to Fig. $37, b$; $b$, elongation stage in spring, similar to Fig. 37, $d$; $c$, permanent condition, similar to Fig. 38. Meristem, elongating, and permanent zones shaded as in Figs. 33,34 , and 39 stem, and from the fact that the buds of the herbaceous stems contain only a small part of the season's growth in miniature, while those of the woody stem contain all of the nodes, internodes, and leaves for the next year, laid down in advance. When such a bud (a) begins to grow in the spring, the tissues of the elongating zone, laid down the year before, produce the terminal twig of the season $(c)$, while the meristem produces a new bud, shown in $c$, resembling the mother bud $(a)$ in structure and function. The tissues of the elongating zone in $a$ are already partially differentiated, but 
with their great increase in length, $b$ and $c$, they gradually form the tissues of the bark, cortex, and woody cylinder of the fully formed twig $(c)$. While this extension and differentiation is proceeding, a new cylindrical cambium, formed within the wood cylinder, adds new conducting tissue to the stem in a manner to be explained later.

From the above discussion it is apparent that roots and stems grow in essentially the same manner, with slight modifications due to the nature of the particular organ and its environment.

Growth also plays a prominent part in the movements of plant organs and in the ultimate form of the plant body, as shown in an earlier chapter.

\section{THE CELL AND CELL DIVISION}

\section{Minute Structure of the Cell}

Before proceeding to discuss the complicated process of cell division it will be necessary to review briefly the structure of

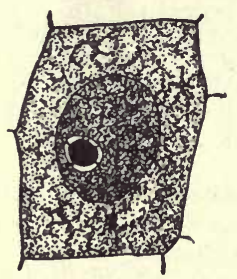

Fig. 41. A cell of a root tip in the resting stage

Note the distributed condition of the dark chromatin net the cell and to point out certain details of cell structure which we have not heretofore considered. In the brief discussion which follows, the term resting cell will be used to indicate the condition of a cell when it is neither dividing nor in active preparation for cell division. Fig. 41 is a camera drawing of a resting cell of a root tip, in which it may be seen that the protoplasm of such a cell presents the aspect of a mesh, or network, inclosing light spaces. In the living state the meshwork would be made up of living cytoplasm, and the light spaces would correspond to minute vacuoles. This appearance of the living substance in artificial preparations has received various interpretations, which cannot be discussed in an elementary textbook.

The simplest conception for the beginning student is to regard the living substance as spongy in structure, resembling the structure of a fine bath sponge, with lamellæ, or plates, of living protoplasm surrounding minute spaces filled with cell sap. Such a spongework, when cut in thin sections, would appear like the 
network of the cell protoplasm in our figure. The nuclear wall, or membrane, is a delicate bounding membrane which in the living condition of the cell is composed of cytoplasm. Within this membrane the nuclear protoplasm is seen to have essentially the same structure as the cytoplasm, except that the bounding walls of the meshes in the nuclear protoplasm are heavier and more granular and stain more deeply in permanent preparations. This deeply staining protoplasm of the nucleus is called chromatin (from chroma, meaning "color") on account of its avidity for various stains used in preparing cells for microscopic study. The chromatin substance is very essential in cell division and in reproduction, and its distribution in the nucleus of the resting cell is thus of extreme importance. In addition to the chromatin network the figure shows a conspicuous nucleolus surrounded by a characteristic nuclear vacuole. Under high powers of the microscope the nucleolus appears to be suspended in this vacuole by delicate strands of nuclear substance which connect the nucleolus with the chromatin network, or spongework. With this preliminary review of the minute structure of a resting cell we may proceed to consider the stages of cell division.

\section{Cell Division - Mrtosis}

Function. We have learned that the cells in the meristem and the elongating zones of growing root and stem tips increase in number by cell division and that the cells thus produced grow into the mature organs and tissue of the plant. The same thing happens also when the single egg cell of the plant forms a manycelled embryo by division. The embryo then differentiates and grows into a new organism. The multiplying, or increase in number, of cells in an organism for purposes of growth is therefore the most obvious use of cell division in plants.

Although this production of new cells appears at first sight to be the main object of mitosis, biologists in recent times have called attention to the remarkable precision with which the chromatin substance of the nucleus is divided and to the complicated mechanisms of mitosis by which this equal division is effected. 
The goal of cell division, therefore, is probably not simply a division of a mother cell into two essentially equal daughter cells but also the distribution of exactly equivalent masses of chromatin, called chromosomes, to each daughter nucleus of the newly formed cells. We shall learn that this equal division and distribution of the chromatin substance to all the cells of a complex organism is the basis for the modern theories concerning reproduction and inheritance. We may therefore anticipate with interest the discussion of these important and complicated processes, concerned with the division of the cell and the nucleus, which are comprehended under the general term mitosis.

Process. It is customary, for the sake of clearness, to describe the various processes of mitosis under certain phases or stages (Fig. 42). These phases, taken in the order of their occurrence, are the prophase, metaphase, anaphase, and telophase. The student should bear in mind, however, that the processes included under the above phases are continuous processes, and that one phase graduates insensibly into the next phase, which follows it in orderly sequence. Some of these phases, such as the metaphase, are undoubtedly longer than others, which are passed through more quickly. This is indicated by the fact that in prepared slides certain phases are much more prominent than others, for the probable reason that their longer duration makes it easier to fix a larger number of nuclei in these stages. All the phases of mitosis are passed through rapidly, however, and the entire process of cell and nuclear division never occupies more than a few hours.

The prophase is the preparatory phase of nuclear division, during which changes take place in the nucleus and the cell preparatory to the equal division of the chromatin substance, which is the principal goal of nuclear division. These preparatory changes involve two structures which play an important part in the ultimate division of the nucleus. These structures are the chromatin, which we have learned is a permanent portion of the nucleus, and the spindle, which is a temporary structure apparently designed as a framework on which the chromatin gathers and finally becomes distributed in equal amounts to the 
daughter nuclei. Although the changes which the chromatin undergoes during prophase are coincident witl the building of the spindle, it is easier and clearer to discuss the chromatin changes and the origin of the spindle separately. We are already
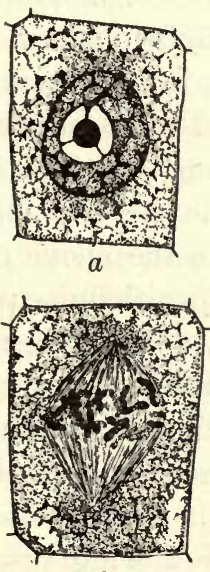

e

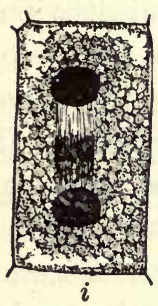

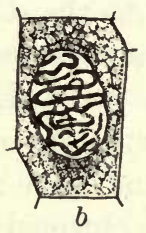
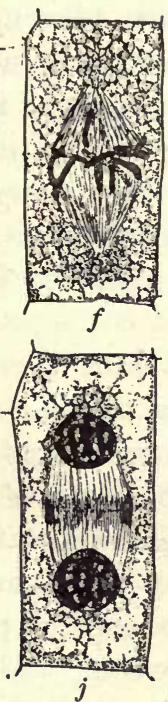

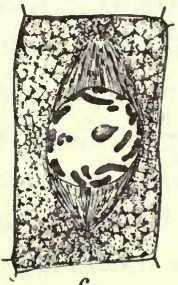

c
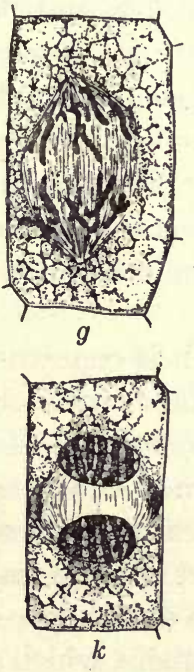
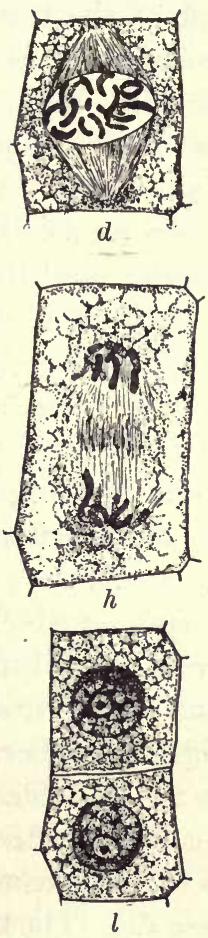

FIG. 42. Drawings illustrating the main stages in mitosis $a-e$, prophase ; $f$, metaphase ; $g$, anaphase ; $h-i$, telophase ; $j-k$, cell division ; $l$, daughter nuclei

familiar with the fact that the chromatin in the nucleus of a resting cell is in the form of a net, or meshwork. In early prophase, when the nucleus begins its preparation for division, it may be noted that the chromatin granules begin to accumulate along definite portions of the resting network, thus giving rise to denser masses of chromatin at certain points $(a)$. These dense masses of chromatin, which are at first irregular, ultimately assume a 
more definite form and arrangement within the nuclear cavity. In some instances they seem to form a continuous band of chromatin substance, which is called the spireme $(b)$. In other cases definite rodlike masses, called chromosomes (c), emerge directly from the nuclear network. In either case the conversion of the network of the resting nucleus into the definite masses known as chromosomes is most easily understood by supposing that after the chromatin granules have condensed to form either the spireme or the isolated chromosomes, the remaining portions of the original network break down and leave the chromosomes free.

As soon as the chromosomes are formed in the above manner they shorten and thicken and finally take up a definite position at the periphery of the nuclear cavity next to the nuclear membrane. The chromatin rods are now ready for the equal division of their substance preparatory to the formation of daughter nuclei. Since the further changes in the chromosomes during prophase are concerned with the spindle, they will be described in connection with the following discussion of that structure.

The spindle which is concerned with the equal distribution of the chromatin substance in nuclear division may for convenience be termed the first spindle, to distinguish it from the second spindle, which is concerned with the building of the cell wall which divides the cell as a whole. In such cells as those found in growing root tips the first spindle makes its appearance as two fibrous masses of cytoplasm at the opposite ends, or poles, of a nucleus in prophase $(c)$. The fibers, which are composed of living cytoplasm, become more conspicuous as the spindle continues its formation at either pole of the nucleus. Finally two half spindles are formed, each with a conical apex and a broad base. The base of each half spindle fits over one pole of the nucleus like a skullcap, and the fibers composing these half spindles appear to extend from its apex, or pole, to the nuclear membrane $(d)$. When the half spindles are fully formed, the nuclear membrane gradually disappears, beginning at the poles of the nucleus, and the half spindles elongate across the nuclear cavity. They ultimately unite to form a complete single spindle, with a bulging equatorial 
region and conical poles (e). The spindle fibers are supposed finally to pass through the nuclear cavity from pole to pole of the complete spindle. When the spindle began to form, the chromosomes occupied the periphery of the nuclear cavity next to the nuclear membrane. As the half spindles elongate across the nuclear cavity with the disappearance of the nuclear membrane the chromosomes appear to be pushed into the equatorial region of the nucleus between the two half spindles, forming what is sometimes called the equatorial plate. The chromosomes of this equatorial plate ultimately move toward the outside of the completed spindle and become arranged in a definite radiate manner at its periphery, attached to the outer spindle fibers. On account of their radiate appearance at this stage it is often called the mother star stage $(f)$. The particular fibers to which the chromosomes are attached are called the traction fibers, since, as we shall learn, they appear to contract and separate the two halves of each chromosome in the next stage of mitosis. The remaining fibers of the spindle are termed the central spindle fibers, or supporting fibers. With the completion of the spindle and the arrangement of the chromosomes in the mother star stage the events of the prophase are completed and those of the metaphase are ushered in.

The metaphase stage $(f)$ is concerned with the equal longitudinal division of the chromosomes into half chromosomes and with the separation of these half chromosomes preparatory to the migration of the chromosomes to the poles of the spindle to form the daughter nuclei. In this process each chromosome splits throughout its entire length into two equal halves, each of which appears to be attached to a traction spindle fiber. The half chromosomes then begin to separate, as though pulled apart by the shortening traction fibers. The final separation of the half chromosomes marks the end of the metaphase stage.

The anaphase is the stage $(g)$ in which the half chromosomes continue their migration toward the poles of the spindle, where they finally arrange themselves in a more or less radiate manner resembling somewhat the mother star stage of metaphase. This radiate arrangement of the daughter half chromosomes is therefore called the daughter star stage and marks the end of anaphase 
and the beginning of telophase $(h)$. During their migration to the poles the chromosomes assume various forms in the nuclei of different plants and in those of the same plant in different kinds of cells. In the root tip the chromosomes during anaphase are usually greatly elongated and often hooked like a shepherd's staff, while in the germ cells of the same plant they are more often V-shaped and greatly shortened and thickened. In nuclei in root tips the traction fibers appear to be attached to the bent chromosomes at the curve of the hooked chromosomes, while in the germ cells they are attached, at the point of the V. It should perhaps be stated that there is no evidence that the traction fibers actually contract and pull the chromosomes to the poles, beyond the facts of their apparent attachment and the peculiar appearance of the chromosomes at this period, when they look like plastic rods being pulled poleward. It is quite possible that the chromosomes move to the poles by virtue of their own inherent power of movement, or else by attraction exerted at the poles during anaphase.

The telophase $(h, i)$ is the final phase of mitosis and includes the organization of the daughter nuclei and the division of the cell into two daughter cells. The formation of the daughter nuclei occurs after the daughter star stage, which marks the close of anaphase. The daughter chromosomes, in the stage immediately following the daughter star arrangement, draw together and adhere to form a dense mass of chromatin at either pole of the nucleus. A new nuclear membrane is now formed around each chromatin mass by the cytoplasm. Within each daughter nucleus thus initiated a nuclear vacuole arises; the chromatin mass begins to loosen up, and the outlines of the daughter chromosomes reappear. The chromosomes then begin to spread out and unite by new anastomosing branches of their chromatin substance, while vacuoles appear in increasing number within each chromosome. By the formation of these vacuoles and new anastomosing branches the chromosomes are soon reduced to the form of a network, or meshwork, quite similar to that of an ordinary resting nucleus. With the growth of the chromatin net the nuclear sap increases in volume and apparently inflates the nuclear membrane, which 
thus augments the size of the nuclear cavity. In the nuclei of root tips and similar vegetative plant parts the two daughter nuclei finally rcach the stage of perfect resting nuclei, with chromatin net, nuciear sap, and nucleolus. The nucleolus grows gradually with the chromatin net, but its origin is still obscure. It should not be overlooked by the student that the above processes, which lead to the formation of the new chromatin net of the daughter nuclei by vacuolization and anastomosis of the daughter chromosomes, is exactly the reverse of the processes by which the chromatin net of a resting nucleus is transformed into chromosomes. Condensation of a chromatin net to form the chromosomes of the mother nucleus is always followed by expansion of the chromosomes to form the resting net of the daughter nuclei in the vegetative cells of plant organs. In germ cells a slightly different procedure is usually manifested at certain stages in their division processes.

Cell division is initiated by the formation of a dense spindle $(j)$ which we have called the second spindle, in the space occupied by the central spindle fibers of the first spindle during anaphase and early telophase. It soon becomes barrel-shaped $(k)$, with very dense outer peripheral fibers, which stain heavily with cytoplasmic stains. This second spindle unites the forming daughter nuclei, and has for its function the formation of the new cellulose wall, which completes the separation of daughter nuclei and the protoplast of the mother cell into two daughter cells. The new separating cellulose wall is secreted by a dense cell plate of cytoplasm, which apparently arises as thickenings of the fibers of the second spindle at the center of each fiber. These fiber thickenings increase in size and finally unite to form a solid cytoplasmic disk, or plate ( $l$ ), extending across the equator of the spindle. The spindle at the same time increases in diameter and stretches entirely across the cell from wall to wall, dividing its protoplast into two equal parts. The cell plate then splits, the split beginning at its center and extending to the junction of the cell plate with the cellulose walls of the mother cell. The new cell wall is formed by the deposit or secretion of cellulose particles between the halves of the cell 
plate. When the wall is complete, the halves of the cell plate form the outer layer of cytoplasm of the newly formed daughter cells, next to the new cell wall. Meanwhile the meshed structure of the cytoplasm appears between each daughter nucleus and the new cellulose wall, which marks the completion of cell division and the formation of the two daughter cells. When one considers that all of the above phases of mitosis are necessary for the formation of each pair of new cells in a growing organism, some conception is gained of the immense constructive activity going on in every growing plant or animal.

\section{Reduction Division AND Reproduction}

The method of cell division, which we have traced above in the cells of root tips, obtains in all vegetative parts of plants, including roots, stems, and leaves, and is hence called vegetative
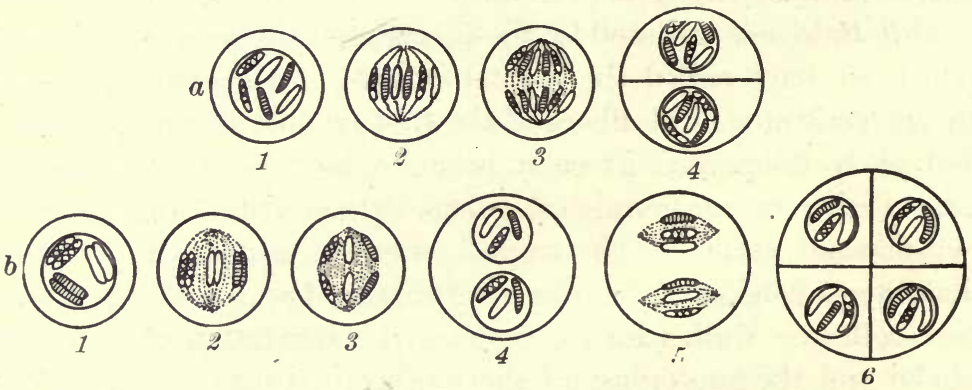

FIg. 43. Diagrams designed to show the difference between vegetative and reduction (heterotypical) mitosis

$a$, ordinary vegetative mitosis in root-tip cells with equal division of chromosomes, as in Fig. $43 ; b$, mitosis with reduction of chromosomes to one half in each daughter nucleus. Further discussion in the text

cell division (Fig. 43, a). In this type of mitosis the chromosomes which are formed in a cell in prophase (1) line up on the equator in metaphase (2) and split longitudinally to form daughter chromosomes. These daughter chromosomes then migrate to the poles of the spindle (3) and form the daughter nuclei of two new daughter cells (4). By this method of mitosis all cells of the vegetative plant body are supplied with an equal number of chromosomes. 
In all of the higher plants a different kind of cell division, called the reduction division (Fig. 43, b), occurs in the spore mother cells which give rise to the spores from which the gametes, egg and sperm cells, are ultimately derived. In such a spore mother cell - for example, the mother cell of a pollen grain - the chromosomes become associated in pairs in prophase (1) and are thus arranged on the equator of the spindle as double chromosomes in metaphase (2). These paired chromosomes do not split in metaphase as they do in vegetative mitosis, but the

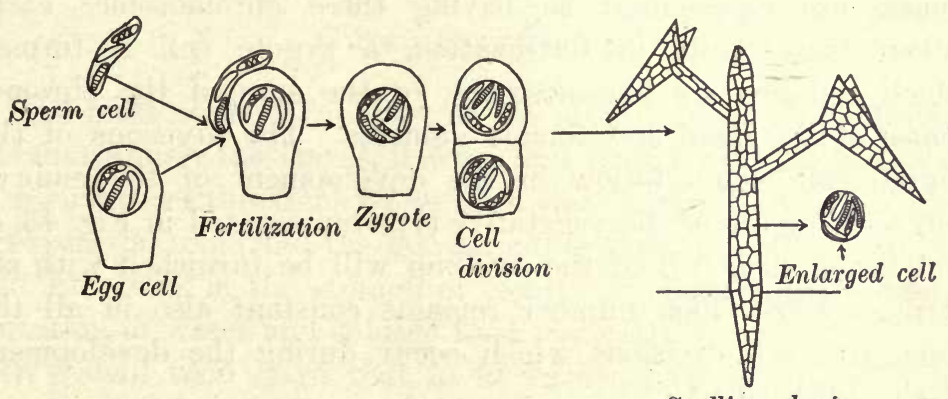

Seedling plant

Fig. 44. A diagram illustrating the history of the chromosomes in the development of a plant

Note the following important facts brought out in the diagram: The chromosomes are three in number in each gamete; they are doubled in the zygote by fertilization; in the succeeding vegetative mitoses the number of chromosomes remains the same as in the zygote cell. Consult the text for a discussion of this figure

two chromosomes of each pair separate as whole chromosomes and migrate to opposite poles of the spindle in anaphase (3) to form the chromosomes of the new daughter nuclei (4). As a result each daughter nucleus receives one half of the number of chromosomes contained in the original mother cell, and so one half of the number contained in all of the vegetative cells of the plant body.

This method of cell division is termed reduction division, and it is of the greatest importance in reproduction and in the life history of plants. In the formation of spores in the higher plants the daughter nuclei divide at once, by the ordinary vegetative method without reduction (5), to form four nuclei, which remain associated in groups of four called tetrads (6). Each member 
of the tetrad then becomes a spore, from which the gamete cells, egg and sperm, are ultimately derived. These spores and the gametes derived from them will consequently have the reduced number of chromosomes characteristic of the reproductive cells of the higher plants.

Fig. 44 illustrates the relation of the reduction division to sexual reproduction and the development of a plant organism. In the figure the male and female gametes, which have been derived from the spores with the reduced number of chromosomes, are represented as having three chromosomes each. When these unite in fertilization, a zygote cell is formed which contains six chromosomes, or the sum of the chromosomes of the male and female gametes. The divisions of the zygote cell which follow in the development of the embryo and seedling are of the vegetative type represented in Fig. 43, a, and hence each cell of the seedling will be furnished with six chromosomes. This number remains constant also in all the vegetative cell divisions which occur during the development of the adult plant.

It is now known that every species among the higher plants has the same chromosome history as that sketched above, in that the chromosomes are always doubled when fertilization occurs and are reduced to one half the vegetative number in the spores and the gametes. A similar reduction division occurs in animals during the formation of the egg and sperm cells. It is probable also that all of the lower plants have a reduction division at some point in their life history which corresponds to that described above for the higher plants. It is easily seen that if reduction in the number of chromosomes did not occur at some point in the life cycle of each individual organism, the chromosomes would ultimately become innumerable in the cells of all of the higher plants. Reduction division is also supposed to have an important bearing on the method of inheritance of parental characters, which will be discussed in a later chapter. The great precision and regularity with which the vegetative and reduction cell divisions are carried out in the life of each organism is a sufficient guaranty of their fundamental biological importance. 


\title{
CHAPTER VI
}

\section{THE STRUCTURE AND FUNCTIONS OF STEMS, ROOTS, AND LEAVES}

\author{
WOOIY STEMS
}

\section{Gross Structure}

The most evident function of the stem is that of displaying advantageously the leaves, flowers, and fruit for the performance of their proper functions. The main stem is also an intermediary between the roots and the leaves, and as such it performs important functions in the storage of reserve foods and in the transportation of water and soluble food materials.

We shall soon learn that in its external features and in its internal structure the stem is a living, active organ in which structure and function are admirably correlated. In our study of the woody stem we shall consider first its gross external features and then its more minute internal structure. It may interest the student to know also that woody stemmed plants are now regarded by some botanists as the forerunners of the soft-stemmed, herbaceous plants of the present day. It is therefore appropriate to reverse the usual order of presentation and consider the woody stem first.

External features. Fig. 45 represents the external features of a shoot of a lilac which has been produced by a season's growth, as described in the last chapter. In the specimen selected for the illustration, both the main shoot and the smaller lateral shoot have two lateral buds which have replaced the terminal bud. This latter condition is the more common one in the lilac, except in the sucker shoots which spring directly from the roots of the old plants. In the figure it may be seen that the fallen leaves have left definite scars, the leaf scars, below each lateral 
bud, and that the lower limit of the season's growth on both the main and the lateral shoots is marked by a ring of bud-scale scars.

The brown bark which gradually covers over the early green bark of the growing shoot is also seen to be broken by minute

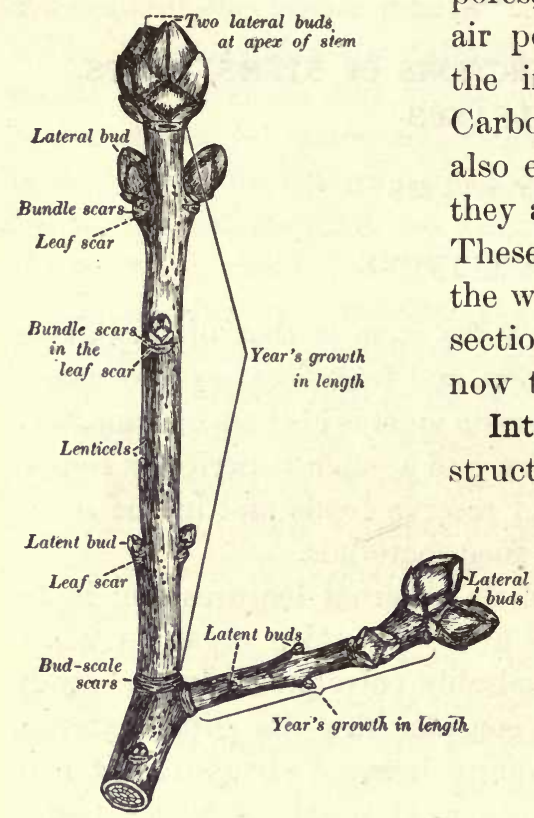

Fig. 45. External features of a lilac twig in winter

In the lilac the terminal bud dies early and is not usually present in mature twigs. The spring growth is produced from the last two lateral buds formed at the end of the twig of the season, as in the figure pores, the lenticels, through which air penetrates from the outside to the internal tissues of the shoot. Carbon dioxide and water vapor are also eliminated from the lenticels as they are from the stomata of leaves. These same structures, together with wood cylinder, will appear in the stem to which we will

Internal structure. The gross structure of the shoot is represented in Fig. 46 as it appears in a transverse section. The epidermis and the brown bark are not distinguishable in gross sections and appear as a single brown layer covering the outside of the entire section. This layer is designated as the brown bark, within which is the green bark, composed of cells which contain green chlorophyll; within the green bark there is an inner lighter layer, called the inner bark, or phloëm. The wood cylinder is clearly marked, but the cambium, or growing layer, cannot be clearly distinguished from the inner lighter bark. Its position at the junction between the bark and the wood is indicated by a line. The pith occupies the center of the section. Within the wood cylinder two annual rings of wood are represented as having been formed. The wood rays are also shown extending 
as radial lines in the wood. The annual rings arise from the cambium layer and increase the diameter of the stem annually, thus stretching and cracking the bark jacket of older trees. The wood of each annual ring is divided into two zones, called spring wood and summer wood. The spring wood is distinguishable by being porous; that is, it is made up of cells with larger openings, which give greater porosity to the spring wood than to the summer wood.

These larger cells, or pores, are the cavities of the large water ducts, which are usually more numerous in spring wood, since it is formed when a large supply of water is needed for the growth of buds, leaves, and flowers. The summer wood, on the contrary, is made up of thick-walled cells (which are smaller than those of the spring wood) and has fewer water ducts. Its chief functions for the stem seem to be mechanical and storing. Since the last-formed and

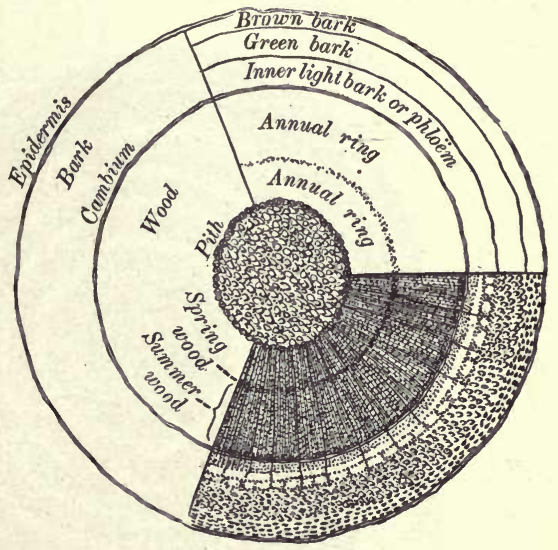

Fig. 46. Gross structure of a lilac shoot two years of age

The limits of the pith, wood, bark, and epidermis are shown in the sector of the stem at the left. The principal tissue layers in each of the above subdivisions are shown at the right in the upper and lower sectors denser summer wood of one season abuts upon the first-formed porous spring wood of the next season, the junction line of the annual rings is usually clearly marked. The width of the annual ring for any given season is determined in part by the age of the tree and in part by external conditions. Periods of drought or the loss of leaves by frost or by insects check the annual growth and may even result in a double ring of wood in one season if growth is resumed in the latter part of the summer after such a checking process. Most trees also grow slowly in extreme youth or old age, while growth is most rapid in the middle life. 
In sections cut across more mature stems of trees like the oak and the alder the above layers will be found to be considerably changed in their relative width and structure (Fig. 47). In such sections the outer, brown barkis much thicker and is often cracked or seamed by the great increase in the diameter of the central wood cylinder. The green bark has also usually disappeared in mature stems and forms a thin layer of crushed cells between the outer corky bark and the inner light bark, or phloëm.

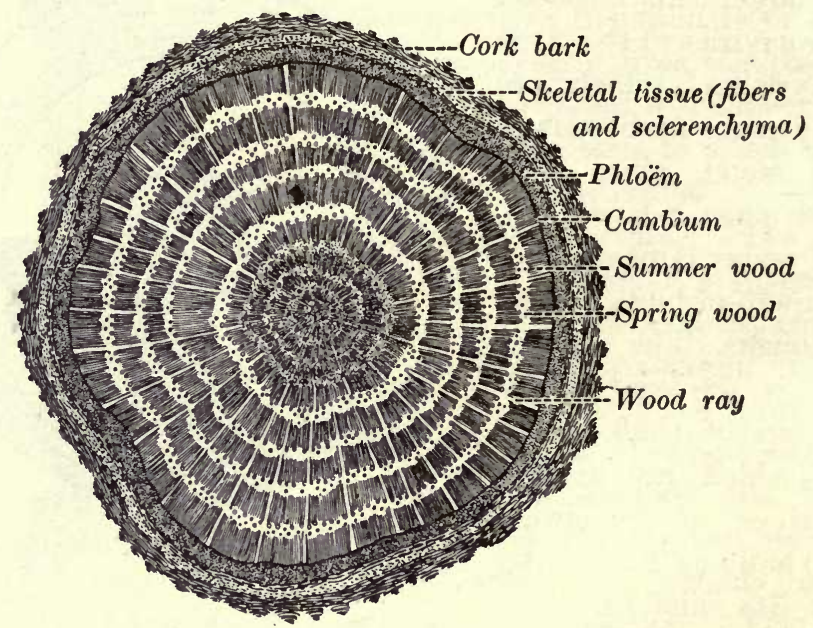

Fig. 47. Transverse section of an oak stem eight years old. Diagrammatic The central darker dotted portion is heartwood; the outer wood layers, with alternating light spring wood and dark summer wood, belong to the sapwood

The wood cylinder is also differentiated into an outer light area of sapwood composed of several of the latest-formed annual rings of growth, and of a darker central portion, the heartwood, made up of the first-formed wood in the center of the tree. The sapwood contains living cells in the form of wood rays and wood parenchyma and is active in the conduction of water and food and in the storage of food reserves. The heartwood is dead and serves a purely mechanical function in supporting the tree. Its cell walls are impregnated with various substances which change their color and increase their strength. 
The wood rays are more numerous than in young shoots and vary greatly in length and width according to their position and the time of formation. 'This is due to the fact that as the wood increases in circumference new rays are started from the cambium at various points year by year. The new rays supply a
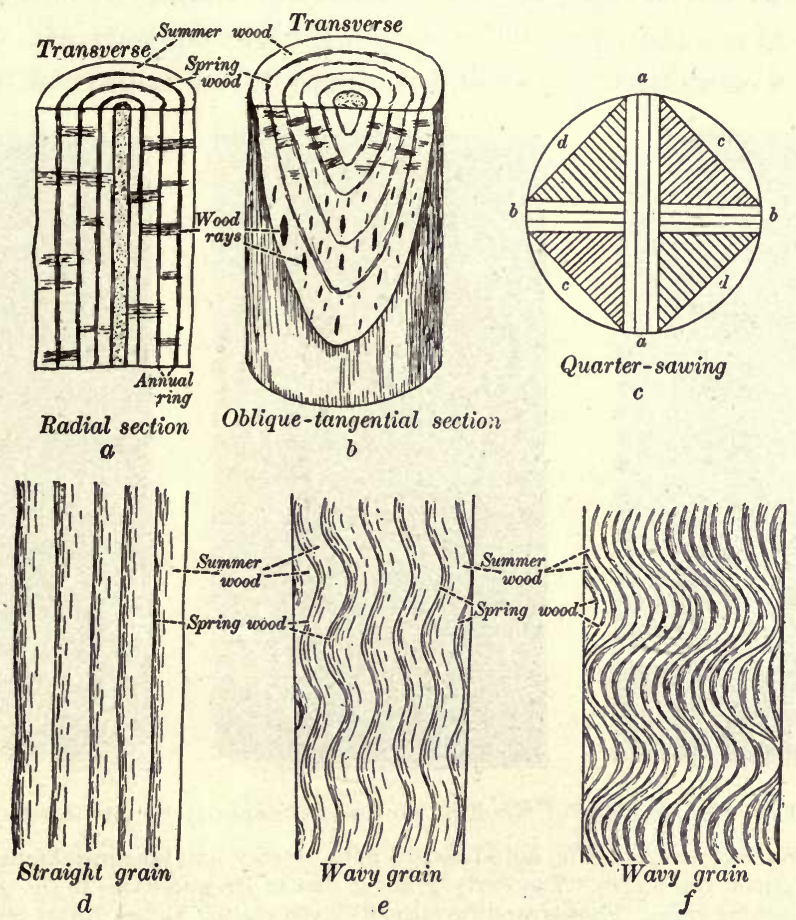

Fig. 48. Various cuts of wood drawn to show the grain and other structural features

Consult the text for an explanation of these figures

larger food-storage system for the tree, which, with the disappearance of the pith and cortex, comes to be lodged more and more in the wood rays and wood parenchyma of the wood cylinder. The pith usually disappears very early and is marked by a dark spot in the center of the tree.

The above tissues and tissue layers have a very different appearance when observed in wood blocks or in thin sections cut in 
transverse, radial, and tangential sections. Fig. 48 illustrates the appearance of the annual rings and of the spring and summer wood as they appear in transverse and longitudinal sections of stems four and five years of age. In the transverse cuts at the upper end of Fig. 48, $a$ and $b$, the spring and summer wood appear as in the oak (Fig. 47), but in the radial (a) and oblique (b) sections they present a very different appearance. In the radial section the spring and summer wood appear as narrow
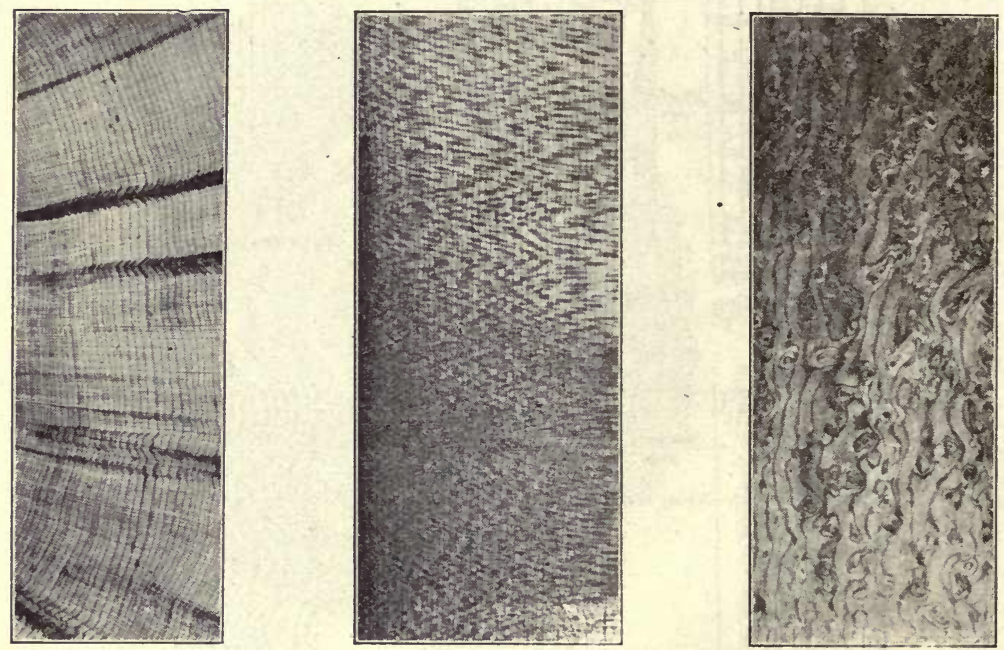

Fig. 49. Sections of wood of bird's-eye maple (Acer saccharum)

Transverse section at the left, radial section in the center, and tangential (curly grain) at the right in the figure. The curly grain is due to irregularities in the growth of the annular rings. Photograph furnished by the United States Forest Service

and wide vertical lines or bands, while the wood rays run across the grain as darker horizontal bands. In the oblique-tangential section the rays have a similar appearance in the upper semiradial portion of the section, but in the lower tangentially cut portion they show as vertical lines of varying height and width. This difference in appearance in the wood rays is due to their shape and their radiate arrangement in the tree trunk. The "silver grain" in finished woods, notably in quarter-sawed oak, is due to the wide wood rays, which have a shining, 
bandlike appearance when viewed in radial sections. Fig. 48, c, illustrates the method of preparing and sawing logs for boards having the quarter-sawed effect. The logs are first stripped of their bark and squared as in the figure. The first boards are then cut in the plane $a a$, which gives a radial cut. Shorter radial cuts are afterward secured by sawing in the planes $b b$ and $c c$. It is evident that the first cuts through the plane $a \alpha$ give the widest and most valuable quarter-sawed boards.
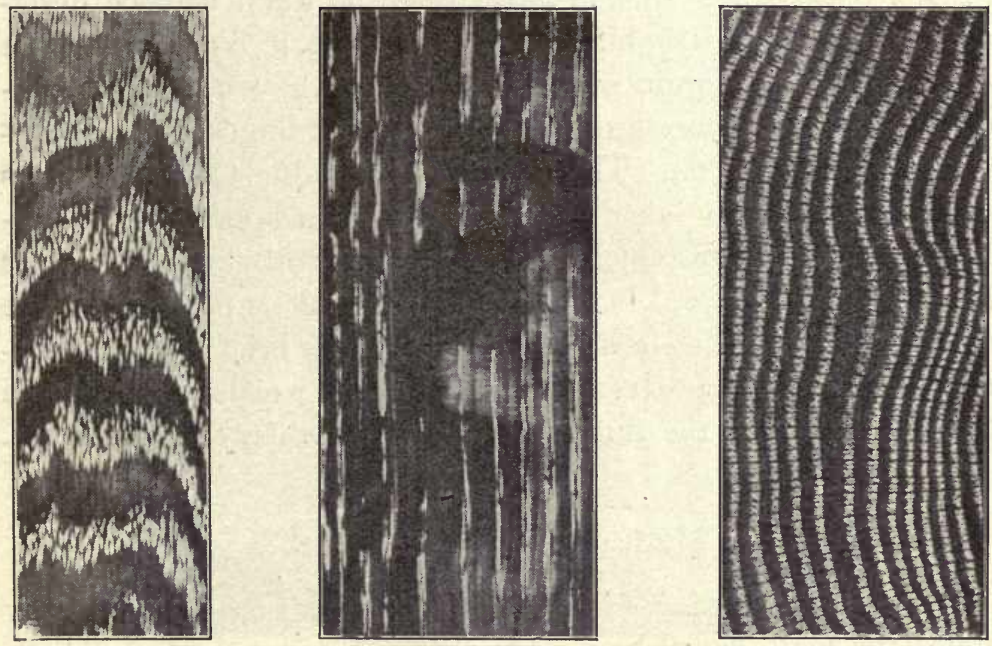

Fig. 50. Section of red oak (Quercus rubra)

Transverse section of oak wood at the right, showing wood rays and annual rings composed of porous spring and dense summer wood. Radial section in the center, with dark wood rays running across the section. Tangential section at the left, showing porous spring and dense summer wood. Photograph furnished by the United States Forest, Service.

The above structures, seen in gross sections of woody stems, are more evident in finished woods and produce the chief ornamental effects of wood furnishings. This is especially true of the contrasts between the heartwood and sapwood, fall and spring wood, and between the wood proper and the wood rays. Heartwood and sapwood are easily distinguishable from annual rings in furniture or room finishings for the reason that each of the former includes several annual rings of growth, each of which has its 
layers of summer and spring wood. The annual rings determine the grain of wood, which is said to be coarse if the annual rings are wide, and fine if the rings are comparatively narrow. Texture, which refers to the coarseness or fineness of the wood elements, also enters into the designation of grain as coarse or fine. Again, the grain is straight if the woody elements of the annual ring run straight up and down (Fig. 48, d), or it is wavy or curly if these elements take an undulating course (Fig. 48, $e$ and $f$ ). In this latter case the grain may be designated as curly, in curly birch, or as bird's-eye, in bird's-eye maple (Fig. 49). Silver grain of wood is due to the wood rays, especially when the latter are cut radially, as is the case in quartersawed oak (Fig. 50). The grain, whether due to annual rings or to wood rays, is also greatly modified in appearance in finished woods by different modes of cutting or sawing the original $\log$ or piece from which the finished product is prepared. The student should in all cases supplement this brief text description with critical observations of finished woods displayed in the furniture and the finishings of the laboratory or the home.

\section{Microscopic Structure}

In the above discussion of the gross structure of woody stems the main layers have been outlined under the terms.bark, cambium, wood, and pith. It remains, therefore, to discuss these layers somewhat more in detail as they are seen in thin section under a compound microscope. In such sections it may be observed that each of the above gross subdivisions of a stem is composed of one or more groups of highly differentiated cells called tissues. Each tissue is a group of cells, similar in structure and in function, which has differentiated from the products of the meristem cells formed in the bud.

The bark (Fig. 52) is a complex layer made up of dead tissue elements, which serve for protection and mechanical support, and of living, active cells, which are concerned with the life processes of the stem. It is composed of the following tissues : epidermis, cork, cortex, and phloëm. 
The epidermis, in most plant stems, consists of a single layer of uniform cells, which serve to protect the young stem until the cork layers appear. As soon as the cork is formed the epidermis is sloughed off and the cork functions in its place. When present the epidermal cells may be seen to be of uniform size

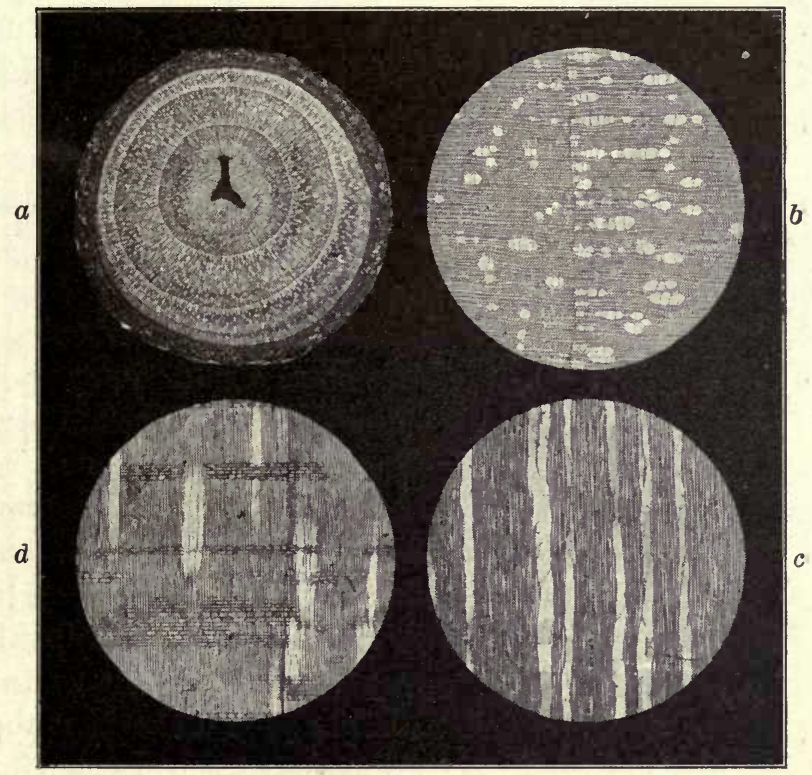

Fig. 51. Microphotographs of sections of the stem and wood of the alder (Alnus mollis)

$a$, transverse section of a stem of Alnus three years old, showing spring and summer wood, pith, and bark (microphotograph by Dr. E. C. Jeffrey); $b$, transverse section of the wood of Alnus; $c$, tangential section; $d$, radial section. Note the large light water ducts in all sections and the difference in the appearance of the wood rays in the tangential and radial sections

and structure, with their cell walls greatly thickened on the outer exposed surface. This outer cell wall is usually waxy and impervious to water.

* The cork layer is composed of thin-walled cells which have the properties of ordinary bottle cork. In young stems they form a thin layer of brown bark, which increases in thickness as the stem matures. 
$\alpha$ The cortex is composed of thin-walled cells which serve a storage function. These cells are living during the early life of a tree, but they are ultimately crushed by the growth in diameter

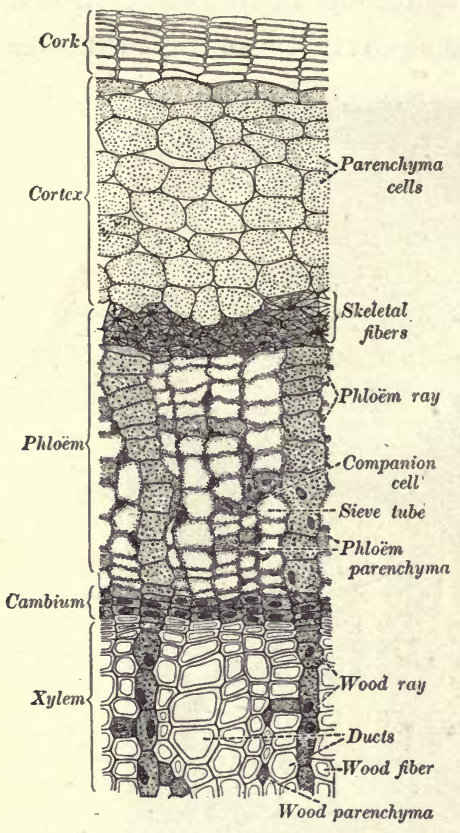

Fig. 52. Microscopic structure of the stem of the alder (Alnus) in transverse section

The figure represents the cambium in the fall and winter condition, after the year's growth was completed. Note the regular rows of xylem, phloëm, and wood-ray tissue cells, originating from definite portions of the cambium layer of the stem or branch, and then they form a part of the dead tissue of the bark.

The phloëm is early differentiated into two distinct layers in most common trees and shrubs. The outer layer is composed of cells with greatly thickened walls, which are termed phloëm fibers, since in long sections these thick-walled strengthening cells are seen to be long, pointed, fibrous cells. These fibers are of special interest, since it is from such cells that the commercial fibers of flax, hemp, etc. are derived. Their great strength and flexibility make them invaluable to the plant as well as to man. We shall learn also that they are always favorably located for the greatest efficiency in supporting delicate stems and soft tissues. The inner phloëm layer is composed of large conducting cells, joined end to end, called sieve tubes, and between them smaller living cèlls called phloëm parenchyma. The chain of cells which together constitute each sieve tube is so named on account of the fine perforations in the transverse walls of the cells, composing the sieve plates, which are supposed to facilitate the flow of soluble food materials up and down the stems. The small cells between the sieve tubes, being also living cells, serve both for the storage of starch and for the 
vertical conduction of sugar through the stem. The inner phloëm as a whole is therefore primarily a food-conducting layer, as distinguished from the wood, which conducts water and soil salts.

The wood, or xylem, is much more complex than the phloëm and is composed of a great variety of living and lifeless cells which perform storage, conducting, and mechanical functions for

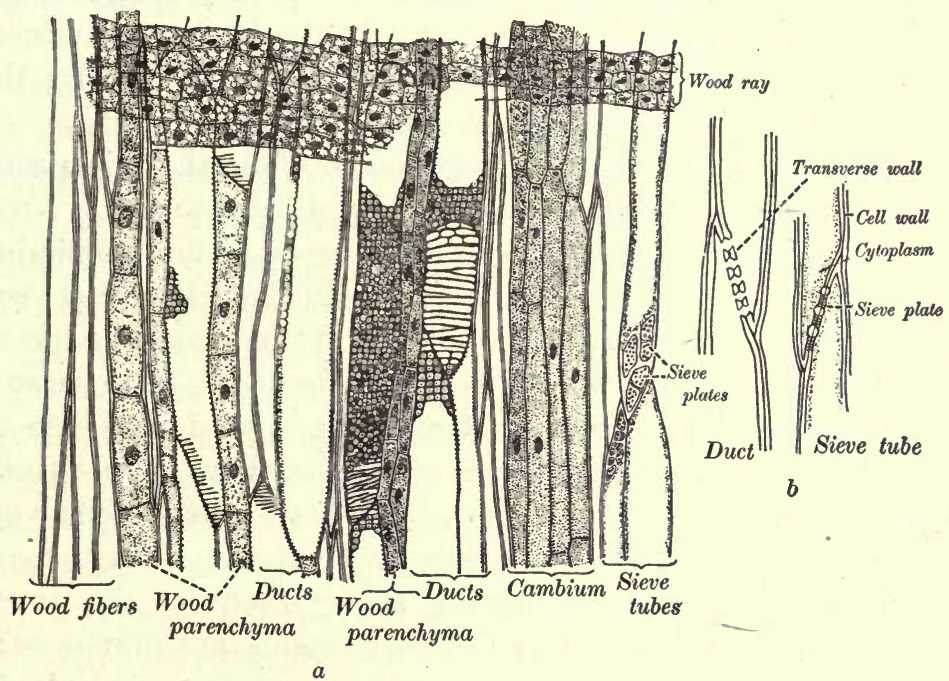

FIG. 53. Microscopic structure of the wood of alder in radial long section $a$, a drawing of a radial section of the wood of the alder. The living tissues have the protoplasm and nuclei dotted. The sieve tubes have the sieve plates on the transverse walls separating adjacent cells of the tubes. $b$, sectional views of a duct and of a sieve tube. Compare the sectional views of the transverse walls of the duct and sieve tube in $b$ with the surface views of these same transverse walls shown in $a$.

the plant. Only the more important of these tissue cells need be described here. The water ducts, or tracheo,, are the most conspicuous elements of the wood; these, as we have already learned, are characteristic of the porous spring wood. In stems like the alder the water ducts appear like large light holes in thin sections of the wood (Fig. 51, a). Microscopic observation will show, however, that they are greatly enlarged cells with thickened walls and without living contents. In long sections (Fig. 51, $c$ and $d$, and Fig. 53), the ducts will be found to be 
composed of chains of cells in which the transverse walls have wholly or partly disappeared, thus forming a long tube for the conducting of water. These water ducts are usually not more than three or four inches in length, although they may be from three to six feet in length in oaks and many feet in length in some climbing plants. The first-formed ducts are also characterized by thickenings on their lateral walls in the form of spirals, rings, and netlike or reticulate thickenings. In the later-formed ducts these walls are often marked with peculiar pits, which give the name dotted ducts to such water vessels. These thickenings on the wall of a duct serve to strengthen it, while the thin places between the thickenings allow water and salts to move laterally through the duct wall. The ducts are therefore admirably adapted for strength and for the rapid conduct of water. Between the ducts are the smaller fibrous cells of the xylem, some of which are living, while others are dead. The living cell elements are termed wood parenchyma; they either serve for storage of reserve foods during periods of inactivity or they conduct foods locally to and from the wood rays. These wood-parenchyma cells are usually thick-walled, with fine perforations in the cell walls, through which protoplasmic strands connect the protoplasts of adjacent cells. The lifeless elements are fibrous cells resembling closely those of the phloëm and serve mainly to strengthen the stem. In some trees, however, they remain living for a considerable time and serve for storage, while in others they conduct water and soil salts. The walls of wood fibers are usually thick, with minute pits or pores which mark thinner places in the cell wall.

The wood rays are composed of thin-walled living cells with their long axes running radially in the stem. In a tangential section of wood the rays are very numerous and are made up of more or less elliptical masses of living cells. In radial view they are plates of cells running across the section. Their primary function is the conduction and storage of organic food, although they serve as lateral water carriers in some plants. 
The Cambium Layer and Annual Growth in Thickness

We have already become familiar with the structure of the cambium and of the annual layers of phloëm and xylem which are formed by it each season. We may now proceed to discuss more in detail the formation and development of the tissue elements which comprise the phloëm and xylem.

The cambium is a cylindrical layer of actively dividing cells, which functions much like the apical meristems of the root and the stem. The cells of the cambium layer divide repeatedly during the spring and summer months, and the products of this division are gradually transformed into the permanent sieve tubes, ducts, and other tissue elements of the annual layers of the phloëm and xylem.

The cambium in Fig. 52 is only a few cells in width, owing to the fact that the section from which the drawing was made was cut from a twig gathered late in the autumn. The cambium is bounded on its inner side by the regular rows of cells comprising the tissue elements of the xylem, and on the outside by the sieve tubes and accompanying cells of the phloëm.

When active growth begins in the spring, the cells of this narrow cambium zone increase greatly by cell division, so that the cylinder becomes several cells in thickness. The outer layers of new cells thus produced then become gradually transformed into the sieve tubes and the living and fibrous elements of the phloëm, while the inner layers, in a similar manner, become transformed into the ducts and other tissue elements of the xylem. Since, however, many more layers of cambial cells become transformed into xylem elements than into phloëm during one growing season, the rings of xylem are much wider than those of the phloëm. This process, repeated each year, finally produces, in old trees, a thick cylinder of wood covered by a comparatively nărrow layer of bark. The usual period for the production of wood and phloëm by the cambium seems to range, in temperate climates, from about the fifteenth of April to the fifteenth of August or the first of September, although phloëm may be formed after these dates in some trees. 
The development of the tissue cells of the phloëm and xylem follows very closely the stages of cell growth and differentiation already traced in the root tip. The cell-division stage takes place very largely in the cambium layer (Fig. 54), corresponding to the meristem of the root tip. This cell-division stage is followed by a period of growth in diameter and by changes in cytoplasm and

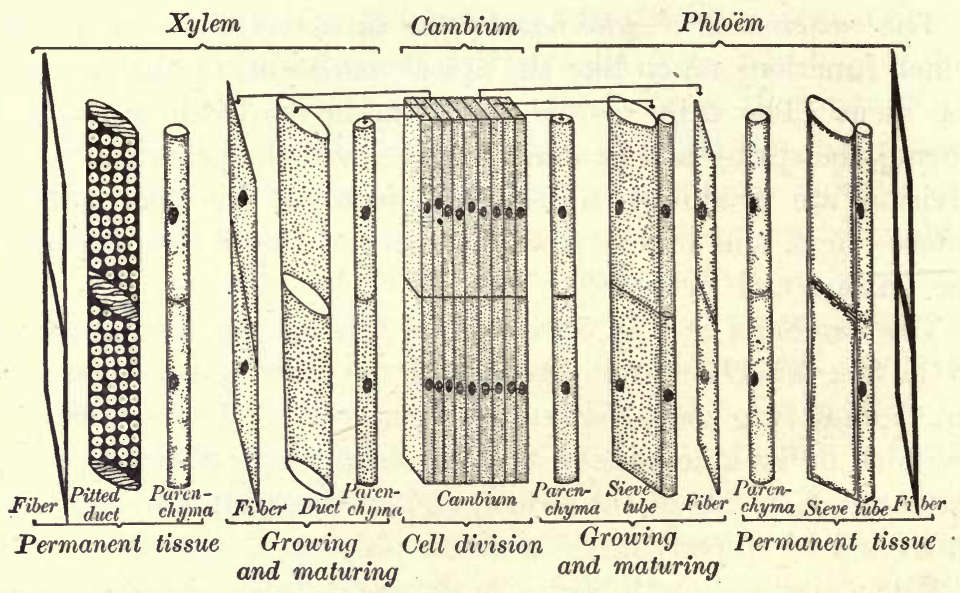

Fig. 54. The development of sieve tubes, fibers, ducts, and parenchyma from the cambium. Diagrammatic

Each of the above elements of the phloëm and xylem is represented in three stages, namely, as a part of the cambium, as a growing and maturing cell or cell chain, and as a permanent element of either the xylem or the phloëm. The cambium is regarded as a cylindrical meristem layer, which, like the apical meristems, produces new cells by division. Consult the text discussion of this figure

cell walls which may be designated as the growth and maturing stages for both the phloëm and the xylem elements. The growth and maturing stages are followed in each tissue cell or cell complex by the permanent tissue elements.

We may now consider in greater detail the changes which occur during the growth and maturing stages of the living cell elements, the fibrous elements, and the ducts.

Xylem elements. In the case of the cells which will ultimately form the living storage parenchyma cells very little change takes place during the growth and maturing stage, except a slight 
thickening of the cell walls and some increase in diameter. The cells destined to form the strengthening fibers of the wood undergo much greater changes in form and in the thickening of their cell walls to produce the pointed, thick-walled permanent fibers. These fibrous cells also lose their living contents and are finally useful only as mechanical tissue elements of the wood.

The vertical rows of cells produced by the cambium which are to form the ducts undergo very great changes in size, in cell contents, and in the character of the cell walls. Unlike the fibrous cells the ducts are compound structures formed of a vertical row, or chain, of cambium cells joined end to end. Each one of these cells enlarges greatly in diameter during the maturing stage, and the protoplasm begins to deposit new cell-wall substance in definite patterns on the thin cell wall of the original cambium cell. These new cell-wall deposits may take the form of spiral or reticulate (netlike) thickenings, or they may take place over most of the cell wall, leaving thinner places, called simple pits, on the cell wall. These simple pits then become arched over, forming what are called bordered pits. When the growth in diameter and the thickening of the walls of the duct are completed, the protoplasm dies and the transverse walls between the constituent cells partially or wholly disappear. These changes finally result in a long, tubular duct, which allows the easy vertical flow of water and soil salts. The thin portions of the lateral walls also allow of lateral migration of fluids, while the thickened parts secure strength to the tree and prevent the collapse of the duct when the stem is bent by the wind or by other agencies.

Phloëm elements. The fibers and living cells of the phloëm not directly connected with the sieve tubes have much the same history as that just outlined for similar elements of the xylem. The sieve tubes, however, have a somewhat different series of developmental stages. The mother cells of sieve tubes, which are formed by the cambium, divide once after their formation, giving rise to a future sieve-tube cell and a living companion cell. The companion cell undergoes very little further differentiation, but the sieve-tube cells, like the cells composing the ducts of the 
xylem, increase greatly in diameter and undergo marked changes in the character of their cell walls. This latter change consists in the formation of the so-called sieve plates by permanent perforations of the transverse walls between a vertical row of cells which unite to form the sieve tube.

The sieve tubes in the permanent condition are therefore like water ducts in being composed of a vertical chain of greatly enlarged cells adapted to the rapid conduction of materials for the use of the plant. In the case of the sieve tubes the materials conducted are soluble nitrogenous foods, with probably some sugars and other substances. The perforations in the sieve plates allow this material to move with less obstruction than would otherwise be possible. The sieve tubes, unlike the water ducts, retain their cytoplasmic contents during their active life but lose their nuclei. They are connected with the companion cells by perforated areas in the side walls, resembling somewhat the sieve plates.

Wood rays. The wood-ray cells arise from the cambium, like the other tissues of the xylem and phloëm, but undergo less change in form and structure than the sieve tubes and the ducts. They are really horizontal cells of the phloëm and xylem, corresponding in the main, in structure and function, to the living cells already described.

The above account illustrates the similarity between the growing layers, or meristems, in all parts of the plant body and the developmental stages of cells which become transformed from the meristem condition into the permanent tissues of the plant.

\section{The General Functions of the Stem Tissues}

Storage. If transverse sections of living twigs or stems are cut in late autumn or winter and stained with iodine solution, the abundant storage of starch can easily be demonstrated. This starch storage occurs mainly in the thin-walled cells of the cortex, phloëm, xylem, and pith. In the xylem, or wood, the starch appears abundantly in the wood-ray cells and in the wood parenchyma. These cells often form continuous bands of 
tissue connected with the wood rays and with the ducts. This distribution of the starch-storage cells in the wood enables the living cells to transport the sugar, which forms from the starch in the spring, along almost continuous paths to the cambium layer and the phloëm. The other reserve foods, such as fats and nitrogenous substances, are less easily demonstrated in wood, but such reserves are laid up with the starch in trees in the thinwalled tissues mentioned above. Toward spring, when the growth of the tree begins, the solid reserves are converted into soluble sugars, fats, and proteins, and migrate by osmosis to the cambium, growing buds, and root tips, where they are converted into protoplasm and cell walls.

Conduction. The soluble foods just mentioned move horizontally along the wood rays and upward or downward in the wood parenchyma and the phloëm, as the case may be. The nitrogenous substances appear to move mainly in the sieve tubes, while the soluble sugars migrate in the phloëm parenchyma. The method of movement is by osmosis, as already indicated, and the direction may be upward toward the terminal buds, downward toward the root tips, or outward into horizontally placed branches and lateral buds. Mass movement also occurs in sieve tubes when the bending of a tree by the wind squeezes the sieve tubes and forces the food in them to move along. Sugar also moves in the ducts in certain trees like the maple and birch. In a tree or other plant there is not, therefore, any definite circulation of foods, but rather a general movement from places of storage to places of growth, or from places of manufacture to storage tissues. The water and soil salts move upward, in a stem, from the roots to the leaves. The main channels are the great water ducts, although in many trees some of the fibrous cells, called tracheids, serve as conductors with the duets. In trees of the pine family these shorter wood cells (tracheids) are almost the sole conductors of water. The great water and food streams in a tree have therefore quite different paths, so that they do not interfere with each other. In summer, when the foods are being constructed in the leaves, the soluble foods move downward in the phloëm to be stored in the wood rays, 
pith, phloëm, and cortex. The main water stream at the same time moves upward to supply the leaves with water and prevents them from drying up by the constant loss of water vapor.

In early spring and summer the food stream moves in reverse directions to supply the growth needs of the tissues, while the water stream again moves upward and supplies water for the inflation of the growing cells in buds and in the cambium layer.

Supporting and protective function. We have already learned that the early formation of cork and bark in the outer layers of twigs and shoots soon replaces the epidermis as a protective external layer. The continued formation of cork and of the supporting layers of the phloëm finally produce in old trees a highly protective bark which shields the delicate tissues within the tree from mechanical injury and guards against excessive loss of water and sudden changes in temperature.

As we have already seen, the supporting, or mechanical, function is provided for by thick-walled cells or fibers, both in the phloëm and in the wood. In older trees and shrubs the thickwalled cells of the wood cylinder are the real supporting tissues, while the mechanical layers of the phloëm serve to strengthen the outer bark jacket.

\section{General Structure and Physiology of Trees}

\section{SUMMARY}

Structural features of the tree in longitudinal section. The main facts noted in the previous pages can now be summarized and correlated by using diagrammatic drawings (Fig. 55, $A$ and $B$ ), as illustrations of the main structural features involved in the growth and development of a tree. In Fig. 55, $A$, the tissues of the main trunk and branches of a tree are so drawn as to display in their proper relations the annual rings of growth, the wood rays in radial view, and the differentiation of the woody cylinder into a central area of heartwood and an outer lighter zone of sapwood. In addition to these structures the figure shows the close connection of the wood and pith of the lateral branches with similar structures of the main axis. The student will also observe that each so-called annual ring of wood is really a cone-shaped cylinder laid down by the cambium 


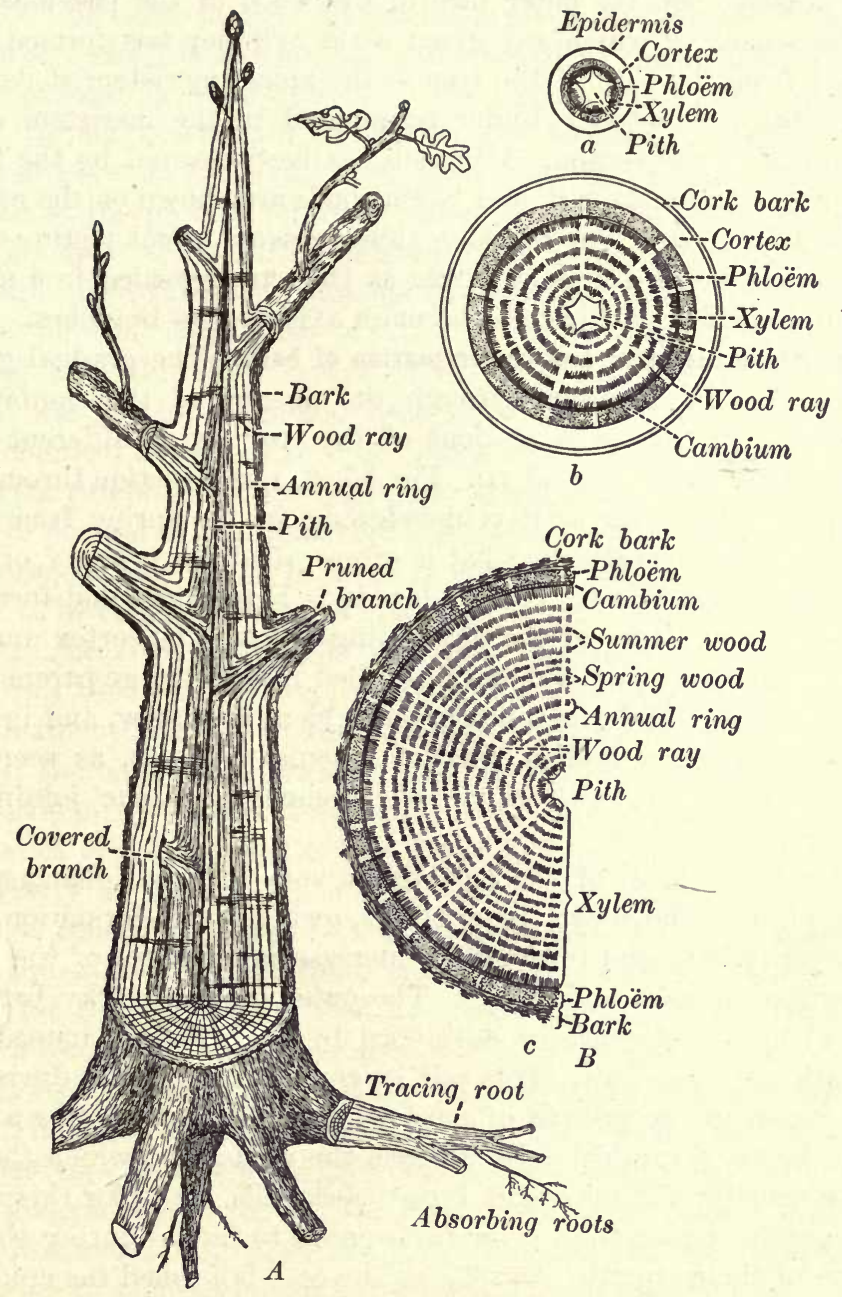

Fí. 55. The structure and growth of a tree

$A$, a diagram illustrating the gross anatomy of an oak tree in long section. Note the relation of the annual rings, the junction of the branches with the main trunk, and the covering of pruned branches. $B$, transverse sections of the tree shown in $A$, at three levels. These latter figures are designed to illustrate the early, or primary, structure $(a)$ and the secondary changes $(b, c)$ due to the production of wood and bark by the cork and the fascicular cambiums. Consult the text for a discussion of these changes 
each season upon the outer face of the wood of the previous year, In the season in which any given wood cylinder was formed it extended from the base of the tree to the apical meristem at its apex, where the new wood cylinder terminated in the meristem of the apical bud of the season. A branch has been covered by the formation of new tissue over it, and lateral buds are shown on the exterior of the tree above. The drawing thus presents a real picture of the main structural features of a tree as they are revealed in a median longitudinal section through the main axis and its branches.

Growth in diameter and the formation of bark. The gradual growth of the tree in diameter through the agency of the cambium is illustrated in the three sections of the tree cut at different levels of the main trunk (Fig. 55, $B$ ). Fig. 55, $B, a$, is a section through the young terminal twig as it is developing in the spring from a terminal bud, and represents what is often called the primary, or early, structure of the shoot. The epidermis is here intact and forms the only protective layer of the developing shoot. The cortex and pith are wide and are composed of thin-walled living storage parenchyma. The vascular cylinder of phloëm and xylem is narrow, and in many instances forms a broken ring of vascular bundles, as seen in a transverse section, with bands of parenchyma tissue joining the pith and cortex.

At a lower level of the tree trunk very material changes have taken place in the above tissue layers, owing to the formation of an outer corky bark and to the great increase in diameter of the vascular ring of phloëm and xylem. The outer layer of corky bark was formed by a cork cambium developed by the cell layer immediately beneath the epidermis. This cell layer begins to divide during the first season in the growth of most young shoots and forms a cylindrical layer of cambial cells beneath the epidermis, which then becomes transformed into dead, brown cork cells. It is for this reason that shoots change their color from green to brown during the first seasion of their growth. As soon as the cork is formed the epidermis dies for lack of water and is finally sloughed off. A shoot in the condition shown in Fig. 55, $B, b$, would present the external brown bark, the green bark, or cortex, and the inner light bark, or phloëm, outside of the wood ring, as shown in the section of the lilac (Fig. 46).

Structure of the tree at maturity. Fig. $55, B, c$, shows the structural features of the old stem well down toward the base of the tree. The outer brown bark has here been replaced by a thick bark jacket 
composed of three layers. The outer layer is the greatly thickened cork layer, which is now seamed and cracked by the pressure exerted by the expanding wood cylinder. The middle layer is the remnant of the former green bark, or cortex, now composed of dead tissue squeezed between the outer cork layer and the phloëm. The outer portions of the phloëm form the inner layer of the dead bark, made up of layers of thick-walled phloëm fibers alternating with crushed and dead sieve tubes and phloëm parenchyma. This outer portion of the phloëm constitutes a kind of exoskeleton for the tree, forming with the other layers of the bark an effective protection against the loss of water and heat, as well as a means of warding off mechanical injury. The inner phloëm is replaced each year by a new layer produced by the cambium. This new annual layer is the effective portion of the phloëm in conducting and storing food, since the average life of the sieve tubes in most trees is limited to a single season. The central portion of the wood cylinder has been converted into dead heartwood, and the pith has disappeared. The sapwood now forms the living, active portion of the wood, furnished with the three main systems of tissues discussed in the preceding pages: namely, the conducting water ducts; the storage system of living cells, made up of wood rays and xylem parenchyma ; and the mechanical fibrous elements constituting the endoskeleton.

Longevity of the tree. The tree, unlike any other known organism, is so constituted as to be able to perpetuate its life through an almost indefinite period of time. A new crop of leaves is formed each season for the making of food by photosynthesis, while the roots invade a constantly increasing area of soil for the absorption of water and solutes in the form of soil salts. The supporting trunk is enabled, through its outer and inner cambium layers, to increase the thickness and effectiveness of its protective bark and also of its inner skeleton of xylem and phloëm fibers. At the same time, as the central wood becomes transformed into dead mechanical elements, the cambium forms new layers of conducting and storing tissues in the sapwood, which keep pace with the increase of its absorbing and manufacturing surfaces. This power of constant growth, throughout a period extending over hundreds of years in the case of the longest-lived trees, is wholly due to the fact that certain cell groups, which we have called meristems and cambiums, retain a condition of perpetual youth and activity, which enables them to supplement dead and useless tissues with an annual growth of new and active ones. The long period 
necessary for the growth of a young tree from the seed is thus compensated for by its extraordinary life period when it is once established.

Physiology of the tree. During the summer months water is being absorbed by the roots and passed up the great water ducts to the living tissues of the tree trunk and to the leaves, where a small part is used in the manufacture of food and a very large part is evaporated. During the entire summer season the leaf is making food in the form of sugar, starch, and soluble nitrogenous food substances. A part of this food is used at once by the living tissues of the leaf, but most of it is transported back through the phloëm into the stem and stored in the wood rays and the wood parenchyma. The food moves down the veins and vascular tissue of the leaf petiole into the phloëm of the branches and main tree trunk. In the phloëm the sieve tubes serve to convey the greater part of the nitrogenous food materials, while the sugars move in the phloëm parenchyma and companion cells of the sieve tubes. When the food stream reaches the wood rays in the phloëm, a portion of it passes horizontally along the rays to the xylem, where it is usually transformed into starch and stored in the rays themselves and in the living wood-parenchyma cells of the sapwood. If the tree is young and still retains a living pith and cortex, a portion of the food may pass into these tissues and be stored. Trees in which the stored food is mainly in the form of starch are often called starchy trees, to distinguish them from trees like the linden, or basswood, in which the reserved food is mainly composed of fats. In either case the reserve food is converted into sugar, by digestion, before it is circulated and used for growth and repair. When spring growth begins, before the advent of the leaves, the food currents are reversed and pass outward from the rays and wood parenchyma to supply the cambium for its growth. In a similar manner food streams move upward and downward in the phloëm to supply the growing buds and root tips with food at the extremities of the tree. We see, therefore, that the tree, while its tissues provide adequately for the transportation of water and foods and for food storage, has no circulation such as that provided by the heart, arteries, and veins of the higher animals. Foods move up or down, outward or inward, according to. the needs of the tissues in any given part. Water, on the contrary, moves mainly upward, although some lateral movement is necessary in order to supply the living cells of wood, phloëm, pith, and cortex. 


\section{HERBACEOUS STEMS}

\section{Dicotyledons}

In the following account of the structure of herbaceous dicotyledons the stem of a common cultivated sage (Salvia) will be taken to illustrate the usual structure of one type of herbaceous stems. In studying sections through portions of young stems of Salvia (Fig. 56, a) and through the older basal portions (Fig. 56,b) the student will be impressed with certain broad distinctions between such a type of herbaceous stem and the woody stems already considered. These general distinctions are necessarily concerned with the main tissue areas common to all stems; namely, the bark, cortex, and pith, the cambium, and the vascular cylinder.

The bark is evidently quite lacking in Salvia, if by this term we refer only to the thick jacket of bark which clothes the outside of woody-stemmed plants. The epidermis is therefore persistent throughout the season and serves its usual functions of checking the loss of water from the delicate tissues beneath it and of protecting the stem from insects and fungi.

The cortex of the Salvia stem is a wide layer, as in most strictly herbaceous stems, and in the younger portions its cells contain green chloroplasts which function in photosynthesis. The cortex cells are also differentiated into a narrow outer thickwalled strengthening cylinder and a wider inner cylinder made up of more delicate cells, which perform the work of photosynthesis and storage. The pith is relatively larger than in woody stems and serves the usual storage function.

The vascular cylinder is perhaps the most distinctive feature of the stem of Salvia as compared with the thick vascular cylinder of trees and shrubs. In sections of young stems (Fig. 56,a) the vascular tissue, composed of phloëm and xylem, is seen to occur in isolated strands, called vasscular bundles, united by thin bands of fiberlike cells, easily distinguished from the adjacent cells of the pith and cortex by their smaller size in transverse section and by their considerable length as seen in long sections. These connecting bands or plates of fibrous tissue, together with the 
vascular bundles, form a complete cylinder separating the pith from the cortex and comparable to the thicker phloëm and
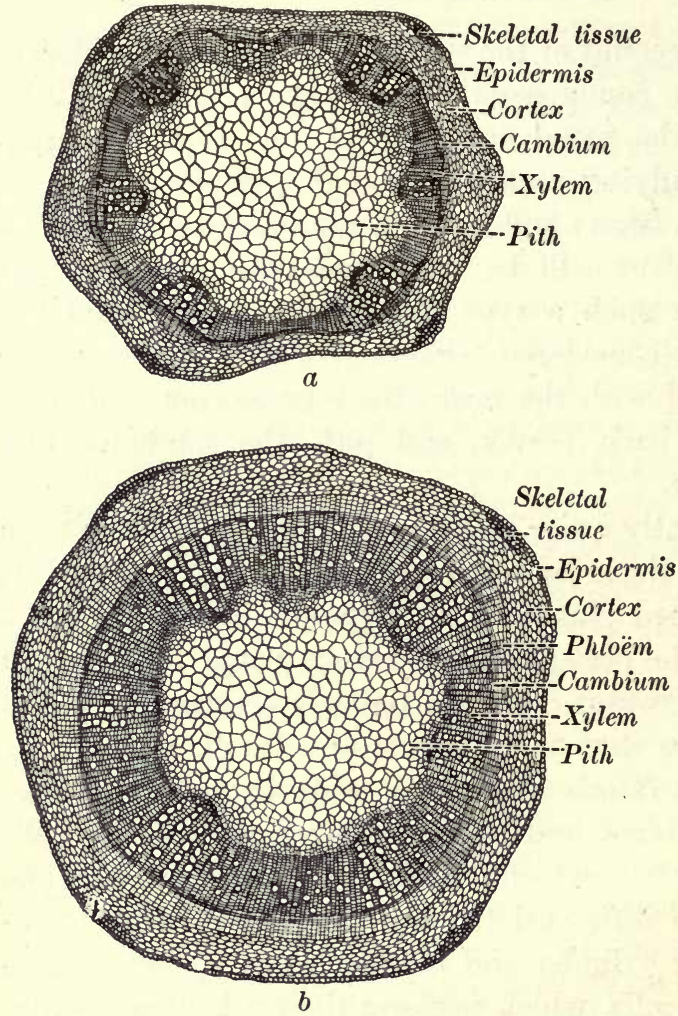

Fig. 56. Structure of the young and mature stem of a common sage (Salvia)

$a$, a transverse section of a young stem of Salvia. The section illustrates the structure of herbaceous stems as compared with wood stems, like that of the alder and oak; $b$, a transverse section of an older stem of Salvia. Secondary thickening by means of the cambium has taken place, forming a wider zone of phloëm and xylem xylem cylinder of woody plants.

The structure of the young stem of Salvia outlined above is very similar to that observed in young, growing stems of herbaceous and woody plants. In some herbaceous plants the vascular bundles remain completely isolated throughout the life of the plant, even in older portions of the stem, and within these bundles very little secondary growth of xylem and phloëm takes place from the cambium. In older stems of the Salvia (Fig. 56, b) a solid vascular ring of xylem, phloëm, and cambium is to be seen, resembling closely the thicker vascular cylinder of woody plants. This complete cylinder of vascular tissue in the older stems of Salvia is produced by the cambium, which, as the stem matures, forms across the fibrous tissue bands separating the primary bundles, and starts the 
production of new secondary phloëm and xylem in these regions. This new phloëm and xylem, which is common in the older portions of most herbaceous stems, is composed of the same kinds of tissue elements as those produced by the cambium in woody stems. Some of the tissue elements - for example, ducts and wood rays - have certain features characteristic of herbaceous stems, but these differences are not significant for our general treatment.

It is also evident that no annual rings of wood can be produced in an annual herbaceous stem like Salvia. The above distinctions between Salvia as a type of herbaceous stem and the woody stems of trees previously studied may be taken as generally applicable to annual herbaceous plants and perennial tree types. It should be remembered, however, that between the two extremes all gradations in stem structure occur, so that it is impossible to establish distinctive types of herbaceous and woody stem structure.

We have indicated above that in some herbaceous plants the vascular strands remain isolated during the entire seasonal life of the plant. In Salvia and its relatives there always exists a complete cylinder of tissue, including the vascular-strands, which corresponds to the thick vascular cylinder of trees. In larger herbaceous plants, like the castor bean and the sunflower, a thick vascular ring is developed, approaching the condition found in shrubs like the roses, currants, and barberry. These latter plants again present a graduated series in stem structure which bridges the gap between the larger herbaceous types and the strictly woody types of forest trees.

The stem, like other parts of the plant body, is thus seen to illustrate the principle of evolution in a complete series of closely related forms. Some botanists now maintain that the course of evolution in stems has been downward from the trees of early geologic times to the herbs of the present day. If this is true, then the vascular cylinder of Salvia is simply a greatly reduced vascular cylinder of earlier tree forms, in which the mature stem of the herbaceous type approximates in structure to young twigs of a tree before the outer corky bark and the thick vascular 
cylinder have had time to develop. In other words, the herbaceous stem remains permanently in the twig stage of its woody progenitors.

With this brief discussion of the intermediate types of stem structure, we may return to a summary of the differences between strictly herbaceous stems like that of Salvia and the pure woody types represented in our common trees.

\section{SUMMARY}

1. The corky bark of trees is absent in Salvia, and the epidermis is continuous throughout the seasonal life of the plant.

2. The cortex is wide and the pith is correspondingly large in the herbaceous type in order that they may serve as storage areas. In woody stems storage is more largely in the wood rays and parenchyma of the vascular cylinder, since the pith and cortex die early in the life of such perennial plants.

3. The vascular cylinder is thin in herbaceous plants, particularly in the upper and younger parts of the stem.

4. A cambium layer is present in most herbaceous stems, which (as in Salvia) produces a complete vascular cylinder of xylem and phloëm in the older portions of the stem.

5. Annual rings of growth are absent in the aërial stems of these plants, since their life is limited to a single season.

6. Wood rays are present in the xylem and phloëm produced by the cambium, but in many herbaceous stems they differ in structure from those found in woody plants.

\section{Monocotyledons}

The monocotyledonous stems, including corn, the cereals, the grasses, and the palms, have a very different type of stem from that of the dicotyledons described above.

The distinctive features of the mature stem of monocotyledons may be summarized as follows:

The bundles (fibrovascular bundles) mentioned in the herbaceous dicotyledon as forming parts of a dissected phloëm and xylem ring are scattered throughout the stem in typical adult stems of monocotyledons (Fig. 57, A). The bundles themselves have also lost the 
cambium layer, so that such stems have no power of increasing the diameter of the stem by cambial growth. There is no distinctive pith, since the bundles from the leaves arch into the center of the stem and occupy portions of the pith region, as indicated in the figure. In many monocotyledons the cortex is also difficult to distinguish, since the vascular bundles occur in it and no sharp division line exists between cortex and pith. In these unusual stem types the storage, mechanical, and conducting functions are provided for in much the same general way as in dicotyledons, except that the tissues are differently disposed in the stem and in the bundles. The structure of the stem in the seedling of a monocotyledon often resembles that of the dicotyledons, so that botanists are agreed that the monocotyledonous type of stem originated from the dicotyledonous type.
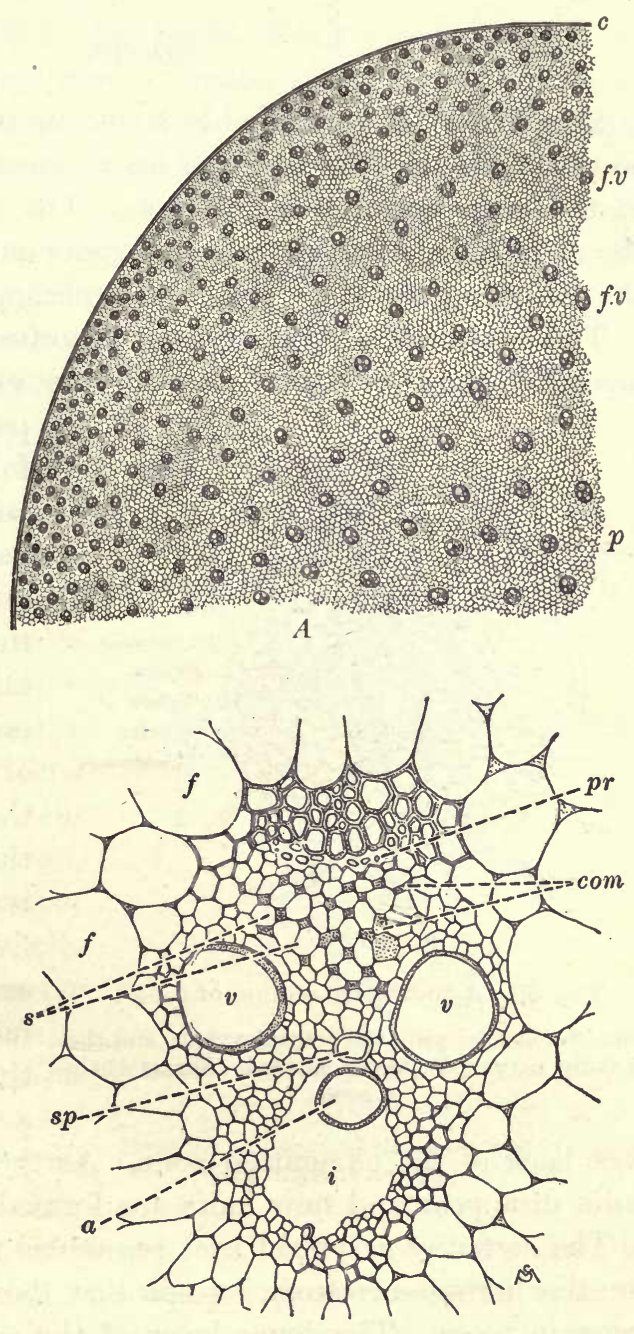

$B$

Fig. 57. Structure of the stem of corn (Zea mays) $A$, gross structure of the corn stem: $c$, epidermis; $f v$, vascular bundles; $p$, pith; $B$, microscopic structure of a vascular bundle : $f$, parenchyma ; $p r$, early phloëm (protophloëm); $s$, sieve tubes; com, companion cells; $v$, large water ducts; $s p$, spiral duct; $a$, annular duct; $i$, intercellular passage 


\section{ROOTS}

Structure. Roots resemble stems in their general structure, so that little need be repeated here concerning the cell elements of the main tissue areas of roots. The essential differences in the arrangement of the tissues in roots and stems is indicated in the following summary and figure relating to root structure.

The epidermis of roots (Fig. 58) is often termed the piliferous layer, for the reason that its cells may elongate in the younger

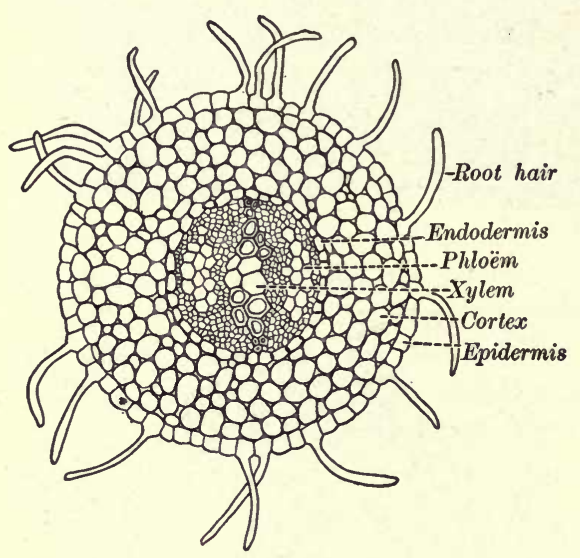

Fig. 58. $\Lambda$ transverse section of a root

Note the lack of pith, the central xylem, and the radially arranged phloëm masses, characteristic of roots portion of the root to form so-called root hairs, which are the most important absorbing area of roots. It is estimated that as many as three hundred hairs may grow on one square millimeter of root surface, and that the absorptive surface of this portion of the root is thereby increased from five to ten times that of an equal naked root surface, or even more than that. These hairs cover

a limited surface of the root back of the elongating zone. As the root grows older the hairs disappear and new hairs are formed nearer the tip.

The cortex of a typical root resembles that already described for the herbaceous stem, except that there is no strengthening layer in roots. The inner layer of the cortex, called the endodermis, is, however, much better developed than it is in most stems. This endodermis in roots, like the outer epidermis of stems, is practically impervious to water (except in certain places where the cells are not of the usual impervious type), thus keeping the water and food streams of the phloëm and xylem confined and hindering them from flowing laterally into the cortex. 
The pericycle, which is the layer of cells just beneath the endodermis, is very important in roots, since it is the place of origin of cork and of lateral roots. When older roots begin to form cork, it does not arise from the epidermis or from the cortex, as in stems, but from the pericycle. The cells of the pericycle therefore act as a cork cambium and thus originate cork layers within the root by cell division and cell differentiation into cork. When one or more cork layers are formed, the cortex and the epidermis dry up and peel off, leaving the cork as the outer brown bark of the root.

Lateral roots also arise from the pericycle and bore their way through the cortex and epidermis. They thus differ in their origin from the leaves and branches, which arise by the division and differentiation of cells in the epidermis and outer cortex.

In young roots the xylem and phloëm have the arrangement which is represented in the figure. The

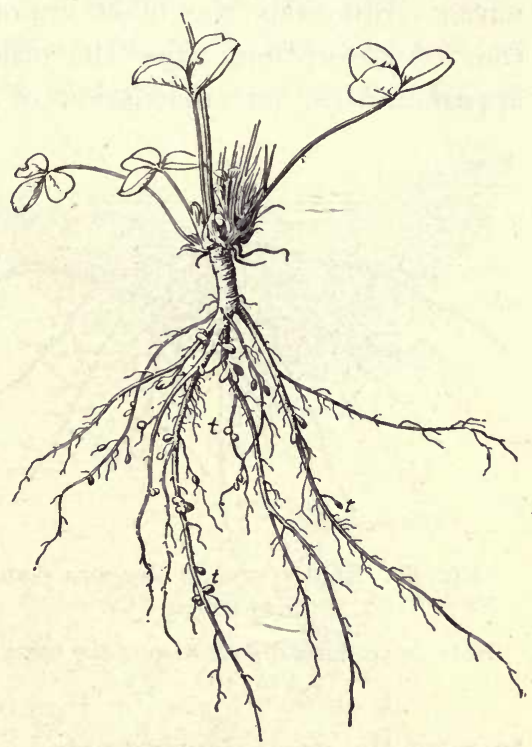

Fig. 59. Root system of a plant of red clover (Trifolium pratense)

Note the tendency of the main roots to penetrate to great depths in the soil xylem occupies the center of the root, thus excluding a definite central pith. The phloëm alternates with the radiating arms of the xylem and so gives rise to what is often termed the radial arrangement of phloëm and xylem.

The cambium arises outside of the xylem and inside of the phloëm masses. Its general method of developing tissues by cell division is identical with that of the cambium of stems, already described. The ultimate structure of old roots is therefore very similar to that of stems, except that the center of a mature root. 
is not occupied by pith or a pith cavity. This is due to the fact, already mentioned, that the xylem of a young root occupies the center of the root, excluding the pith.

Functions. Roots serve various functions, such as anchorage in the soil for the aërial portions of the plant body, the transportation of food and water, storage, and absorption. Of these various functions the most important for our consideration is that of absorption, since the other functions are more or less apparent after our discussion of similar functions in stems.

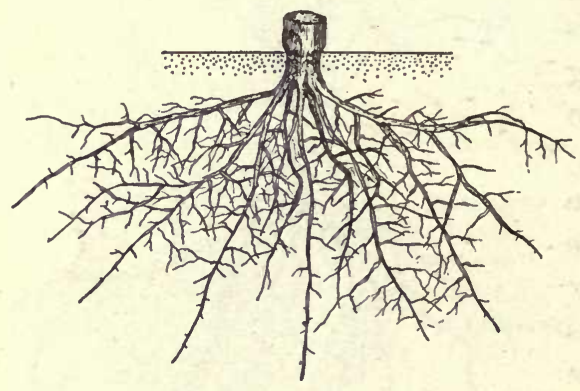

Fig. 60. Root system of the corn plant (Zea mays)

Note the uniform distribution of the roots in the soil

We have already learned that the root system is able to adjust itself by tropistic movements so as to place its members in favorable relations to the soil particles and soil water. This adjustment, together with the great extent of the root system, enables both herbaceous and woody plants to absorb the large quantities of water necessary to meet the great demands made upon them by the evaporation of water vapor from the leaves. Some plants, like the clovers, have deeply penetrating roots (Fig. 59), while others (for example, many of the cereal grains and some trees) have surface roots. In alfalfa the root system is said to penetrate to a depth of 31 feet, and in mesquite, a desert plant, to that of 60 feet. Corn (Fig. 60) has been estimated to have a root system which reaches a total length of over 1300 feet, and the roots of a single plant of oats may be as much as 150 feet in length. In many trees the root system quite equals the branch system in horizontal extension, and its absorbing root tips are placed where they receive the drip from the outer leaf surface of the tree. In order to understand the very important absorptive function of roots it will be necessary to discuss in some detail 
the phenomena of osmosis by living cells. It seems wiser, therefore, to consider absorption by roots later, in connection with the absorption and movement of water in plants.

\section{LEAVES}

Structure. Leaves are flat expansions of the plant body, designed to present a large surface of green leaf tissue to air and sunlight for the purpose of food-making. The leafstalk, or petiole, connects the leaf blade, or expanded green portion of the leaf, with the conducting and storage portions of the main stem. It also serves to display the leaf effectively to sunlight and air. The lamina, or blade, of the leaf (Fig. $61, a)$ is composed largely of green tissue cells called mesophyll, supported and subdivided into small areas by the intricately branched veins. The relation of veins and mesophyll is best appreciated by holding the leaf of an ordinary plant toward the light. The veins not only
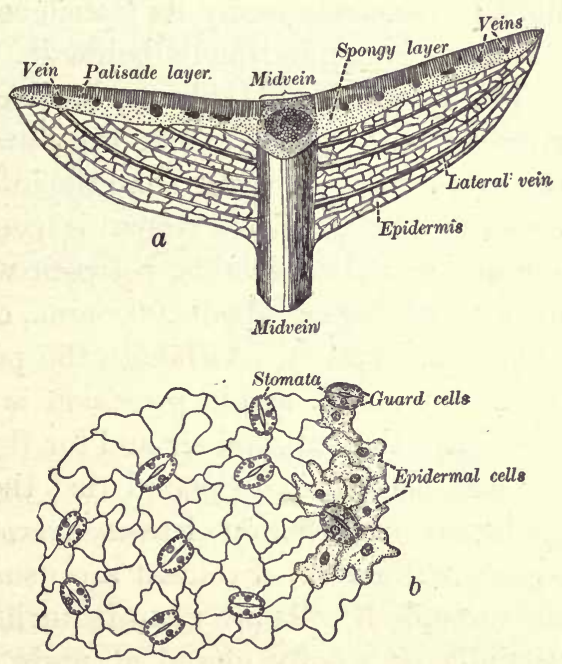

Fig. 61. Gross structure of a leaf of the milkweed (Asclepias)

$a$, surface and sectional views of a milkweed leaf; $b$, microscopic structure of the epidermis form a supporting framework for the leaf but also serve to irrigate the mesophyll cells with water and to remove sugar and other foods which must be transported back into the stem from the manufacturing mesophyll cells. The leaf, like other organs of the plant, is therefore composed of layers and groups of cells called tissues, each cell layer, or group, having a special function to perform for the leaf and for the entire body. The principal leaf tissues are the epidermis, the mesophyll, and the veins. 
The epidermis (Fig. $61, b$ ) is usually composed of a single layer of cells without green chloroplasts, which inclose and protect the more delicate mesophyll cells over the entire surface of the leaf. Its cells are distinctive in that their outer walls are covered with a waxy secretion which makes them almost completely impervious to water and air. The epidermis thus forms an effective protection against excessive evaporation from the mesophyll cells, which would wither and destroy the leaf. The lack of green chloroplasts in the epidermis also enables the sunlight to penetrate easily its translucent cell walls and illuminate the green mesophyll cells below it.

The stomata are highly modified cells of the epidermis which regulate to a certain extent the flow of gases into the leaf and the evaporation of water from the internal air spaces between the mesophyll cells. Each stoma is composed of two guard cells containing chloroplastids, between which there is a narrow slit, or pore, averaging about .008 mm., or $\frac{1}{3000}$ of an inch, in width (Figs. 62, $b$ and $c$ ). Although the pore between the guard cells is so very small, ample provision is made for the exchange of gases with the external air and for the evaporation of water from the leaf, since it is estimated that the number of stomata in the epidermis of common leaves averages from 100 to 700 per square millimeter. Stomata are usually open in daylight, when the mesophyll cells are manufacturing sugar and starch, and are partially or wholly closed at night, during the period of leaf inactivity. The exact mechanism for the control of stomata is not thoroughly understood, although the guard cells are known to be sensitive to light.

The mesophyll cells vary, in size, form, and arrangement, in different kinds of leaves. In the more typical horizontal leaves (Fig. 62,a) the mesophyll cells of the upper surface next to the epidernis are greatly elongated, with their long axes perpendicular to the leaf surface. On account of their palisadelike form and arrangement this layer of mesophyll cells is termed the palisade layer. In vertical or erect leaves, such as those of narcissus and many lilies, the palisade layer often extends entirely around the leaf as a continuous layer beneath 
the epidermis. The remaining cells of the mesophyll are called spongy cells, since they are more loosely arranged than the palisade layer, with large intercellular spaces like the canals and pores of a sponge. This canal system of intercellular spaces within the mesophyll of the leaf contains air, with water vapor and gases. Since the composition of the air within the leaf differs from the external air in the relative amount of water and gases contained in it, we may properly designate it as the

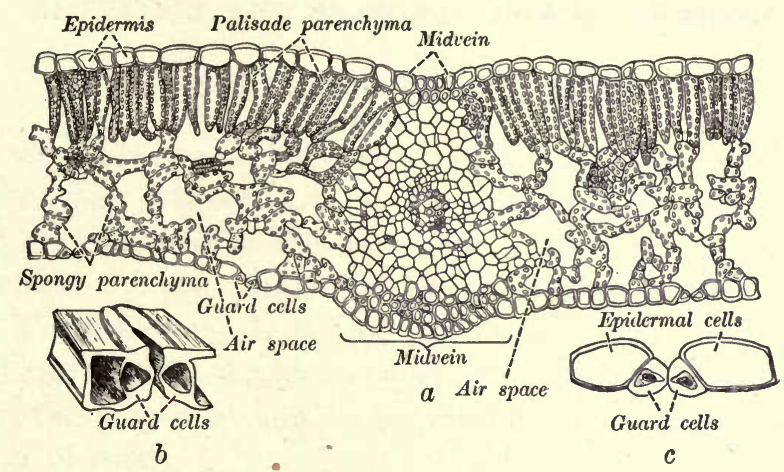

Fig. 62. Microscopic structure of a leaf of the milkweed (Asclepias)

$a$, a transverse section of a milkweed leaf; $b$, a diagrammatie drawing illustrating the structure of the guard cells; $c$, the guard cells of the milkweed enlarged

internal atmosphere of the leaf. The gases and water of this internal atmosphere come in contact with the external atmosphere outside the leaf through the stomata, with which the intercellular spaces of the leaf are directly connected. All of the mesophyll cells are also living cells, in which green chloroplastids are embedded in a living cytoplasmic sac surrounding the large central water vacuole. The chloroplastids are thus placed at the outside of the cell, where they are exposed to sunlight and to the constituents of the internal atmosphere. The importance of these internal arrangements of the leaf will be more fully appreciated when we consider its function in starch manufacture and in the evaporation of water.

The veins are composed of two kinds of tissue cells; namely, supporting and conducting cells. The thick-walled supporting 
cells flank the veins above and below to prevent the collapse of the conducting cells and to strengthen the entire framework of the leaf. The conducting cells are in the form of vascular bundles, which are continuations of the vascular bundles of the stem and root. They have the same general character as those of the stem, except in the smaller veinlets, where the conducting tissue is reduced to a few cells. The vein tissue thus puts the manufacturing mesophyll cells in direct connection with the main axis of the plant. 


\section{SECTION III. PHYSIOLOGY}

\section{CHAPTER VII \\ NUTRITION AND SEASONAL LIFE OF PLANTS}

\section{PHOTOSYNTHESIS AND RESPIRATION}

The student has now become familiar with the form of plants, and with their adjustments to the environment. He has also studied the structure of the organs and tissues of the plant body, upon which a proper understanding of its various functions is based. The following discussion will therefore be confined to the main physiological processes concerned with nutrition; namely, photosynthesis, respiration, transpiration, assimilation, and the digestion of foods.

\section{Photosynthesis}

If a green leaf which has been exposed to bright sunlight for a few hours is bleached with alcohol and then tested for starch with iodine, the green mesophyll areas will be tinted blue or bluish black. This will be found to be due to minute starch grains, which are formed in the green chloroplastids of the mesophyll cells during the exposure of the leaf to the sunlight. The starch grains are formed in such numbers throughout the green tissues of the leaf that the latter is tinted, owing to the reaction of the iodine on the abundant starch.

This simple experiment indicates the principal function of the leaves, which is to manufacture sugar and starch for the plant. This sugar and starch then becomes a basis for the manufacture of other foods, such as the fats and the nitrogenous foods, or proteins. It is probable that the leaf is the main center for the compounding of these nitrogenous foods from the sugar which 
it manufactures within its green mesophyll cells and the nitrogenous salts which come up to the leaf from the roots in the water stream. The leaves, through their green cells, are therefore the manufacturing organs for the other members of the plant body, which are dependent upon them for nourishment.

The term photosynthesis means literally the uniting, or compounding, of substances by means of light. When applied to the work of a green leaf it signifies the making of sugar from the simple raw materials, carbon dioxide $\left(\mathrm{CO}_{2}\right)$ and water $\left(\mathrm{H}_{2} \mathrm{O}\right)$, by means of energy supplied to the leaf by sunlight. These raw materials for photosynthesis are supplied from the soil through the roots and stem and from the air through the stomata. The carbon dioxide is absorbed by diffusion into the internal atmosphere of the leaf from the external air, and is then taken up by the chloroplasts located in the mesophyll cells surrounding the intercellular spaces of the leaf. The water which combines with the carbon dioxide, although ultimately supplied by the roots, is immediately absorbed for sugar-building from the cell sap of the mesophyll cells. Since carbon dioxide, like other gases, tends to move from a point where it is abundant, or concentrated, to a point where it is less abundant, we must picture it as constantly flowing through the stomata into the leaf during the day, to take the place of that which is absorbed from the i:1ternal atmosphere of the leaf in the intercellular spaces by the mesophyll cells for the making of starch and sugar. The excess of oxygen which is liberated during photosynthesis likewise diffuses out of the leaf or is partly used in the process of respiration, which goes on both day and night in the leaf as in other living parts of plants.

One of the first products of photosynthesis is undoubtedly sugar, but the excess of sugar produced, which is not used by the living cells for growth and repair, is usually transformed into starch within the plastids of the leaf cells themselves. The excess of sugar and starch formed in the leaf is later transported in the form of sugar into the special storage tissues of the stem, roots, fruits, and seeds. During the day this excess of starch accumulates in the leaf cells, as can be demonstrated by testing 
the leaf with iodine, but it is finally transported back into the other organs of the plant, either for immediate use or for storage.

The process of photosynthesis is not fully understood, but it is supposed to involve the union of carbon dioxide and water to form carbonic acid $\left(\mathrm{CH}_{2} \mathrm{O}_{3}\right)$. Under the influence of sunlight and chlorophyll the carbonic acid is reduced to form a compound, possibly formaldehyde $\left(\mathrm{CH}_{2} \mathrm{O}\right)$, which is then multiplied or condensed into sugar $\left(\mathrm{C}_{6} \mathrm{H}_{12} \mathrm{O}_{6}\right)$. A part of the original molecule of carbonic acid is at the same time given off in the form of free oxygen, which represents the excess of that gas not needed for the building of sugar molecules. The reduction of the carbonic acid is accomplished, in some manner which is not fully understood, by the sunlight acting upon this substance in the presence of the green pigment (chlorophyll) of the leaf plastids. It is estimated that under ordinary circumstances this decomposition would require the production of energy "equivalent to $1300^{\circ}$ of heat," and yet the green leaf, through the agency of chlorophyll, is able to do this work without high temperatures or elaborate machinery.

The importance of photosynthesis to both plants and animals can hardly be overestimated, since its first products, sugar and starch, form the basic food for plant and animal nutrition. Not only are these products the chief forms of reserve foods in the special storage organs and cells of plants but they also function as the most important material around which other kinds of organic foods are constructed. Thus, the water stream from the roots brings up soil salts and deposits them in the mesophyll cells of the leaves. The nitrogen, sulphur, and phosphorus of these soil salts is then combined with the sugar molecules formed by photosynthesis into nitrogenous foods, such as the gluten of wheat and other forms of protein food material. Without the sugar furnished by photosynthesis this formation of available protein food, upon which both plants and animals depend for sustenance, would not be possible. We know also that the fats and oils of such seeds as flax, hemp, and castor beans are derived from sugar in some unknown manner, and that they are reconverted into sugar and starch during the germination of such 
seeds and the growth of the embryo. Fats and oils, therefore, like nitrogenous foods, are primarily derived from the products of photosynthesis. All organic food for both plants and animals is thus composed of the products of photosynthesis, or of food substances which have been built around these products. In a similar manner the skeletal and supporting structures of the plant in the form of cell walls are immediately derived from sugar and starch formed by photosynthesis. The cellulose which forms the bulk of the cell-wall substance of plant tissue is closely related to starch in chemical composition and is undoubtedly constructed from sugar molecules by the living protoplasm of plant cells. This cellulose framework of the plant body also comprises the bulk of the fuel, in the form of wood, coal, and combustible oils, upon which mankind depends, either directly or indirectly, for heat and light. We see, therefore, that a large part of the food supply for the organic world, the skeletal structures of plants, and the energy derived from fuel in the form of light and heat are dependent upon the process of photosynthesis by green plants.

\section{RESPIRATION}

The process by which living organisms secure energy by oxidation for carrying on their life activities is termed respiration. Unlike photosynthesis, respiration is not confined to the cells of the plant body which contain green chlorophyll, but takes place in every living cell of the organism. On account of its similarity to combustion the respiratory process is most easily understood by beginning students when compared with the burning of wood or coal in a stove or a furnace. When thus compared it is found that respiration and combustion are alike in that free oxygen is absorbed and energy is liberated, together with certain waste products which depend for their complexity upon the nature of the substance oxidized. In the burning of coal the oxygen unites directly with the pure carbon of the coal, and the products are heat energy and carbon dioxide, which may be represented by the following general equation :

Coal $(\mathrm{C})+$ oxygen $\left(\mathrm{O}_{2}\right)=$ carbon dioxide $\left(\mathrm{CO}_{2}\right)+$ energy 
In the burning of wood the cellulose of the wood-cell walls and the stored sugar, starches, and nitrogenous foods are chemically more complex than coal. As a consequence of this complex chemical character of the substances oxidized or burned the final products are more complex than in the burning of coal, and in addition to the energy released we have also carbon dioxide, water, and other substances given off or thrown down as by-products. In a somewhat similar manner we may picture the respiration or oxidation processes which go on in the living cells of germinating seeds or in other active cells of the plant body of a growing plant. This combustion of more complex compounds may also be represented by a generalized formula as follows:

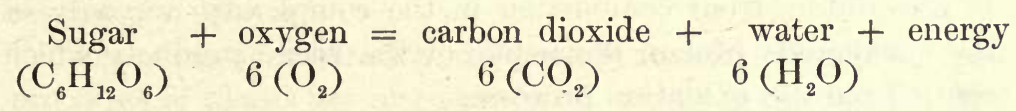

In the living cells of plants the compounds which are finally oxidized are very complex and therefore yield more complex final products than is the case in the oxidation of coal or wood. Some investigators believe that the sugars and similar. substances are directly oxidized in living cells, much as they are in a piece of wood containing these substances as reserve foods or as constituents of cell walls. Others suppose that the protoplasm, or living substance, is gradually decomposed during the respiratory process and that oxygen plays an important part in the process, which results in the release of energy and in the production of carbon dioxide, water, and nitrogenous wastes; for example:

$$
\begin{aligned}
\text { Proteins }+ \text { oxygen }=\text { carbon dioxide } & + \text { water } \\
& + \text { nitrogenous wastes } \\
& + \text { energy }
\end{aligned}
$$

It appears, therefore, that while the energy released is comparable in combustion and respiration, the final waste products are more elaborate in the oxidations which take place in living cells than they are in ordinary combustion, for the reason that more complex compounds, possibly including protoplasm itself, are decomposed and partially oxidized in plant respiration. 
Another striking difference between the oxidations in the living cells of plants and animals and the combustion of coal or wood is found in the temperatures at which the two processes take place. In animals respiration goes on at the normal temperature of the animal body, which does not exceed $100^{\circ}$ Fahrenheit. The temperatures at which combustions are made possible are known to be much higher than that at which living matter could continue to exist.

The above comparison of the combustion of coal or wood and respiration in living organisms may be summarized as follows:

Respiration is like combustion in that oxygen is necessary for both processes and both processes yield energy and carbon dioxide, or energy, carbon dioxide, and water, as final by-products. It also differs from combustion in the complexity not only of the compounds broken down but of the waste products which result from the oxidation processes.

'The function, or use, of respiration is much the same as combustion in an engine, in that it liberates energy which can be used directly or can be transformed so as to furnish power for work of various kinds. In the living cells of seeds, leaves, stems, or roots the energy released by respiration is used for making new protoplasm, for cell division, for protoplasmic streaming, and for other vital processes necessary to the life of the plant organism.

'This energy, as the student will recognize, is different in origin and in function from the external energy absorbed from sunlight by the chlorophyll and used in photosynthesis for the building of sugar and starch. The latter energy enables green plants to make sugar, which forms the basic organic food for all plant and animal life, while the energy released by the respiratory process is necessary for maintaining the vital processes of all cells, whether they are furnished with green chlorophyll or not.

The mechanism of plant respiration is very different from that of animals in that the plant is not furnished with lungs and an elaborate blood system for absorbing and distributing oxygen to the living tissues of the plant body. The living cells which we have observed in wood rays and in the wood proper are 
comparable to the living muscle and nerve cells in animals in their need for a certain amount of oxygen for respiration, but there is no definite circulating system in plants to carry this oxygen to these cells from the external air. In plants the oxygen moves through the intercellular system, which penetrates to all parts of the plant body, by the slow process of gas diffusion. 'The oxygen enters the plant through leaf stomata and through the lenticels which we have already observed in the bark of twigs and stems. The slow diffusion of oxygen suffices, however, for the production of sufficient energy for the less active tissues of higher green plants. In the case of more active plants, like bacteria and yeasts, and in the active tissues of growing buds and flowers, plant cells often equal or exceed animals in the energy of the respiratory process.

Comparison of respiration and photosynthesis. These two vital processes of plants are often confused, owing to the fact that the same gases are involved in both processes and that they may go on at the same time in one organ, as, for example, the green leaf.

Photosynthesis is a food-building process in which carbon dioxide is absorbed from the external air by cells containing green chloroplasts and combined with water to make sugar and starch. During this process the excess oxygen, contained in water and carbon dioxide, which is not needed for making sugar, is liberated as free oxygen into the intercellular system of the plant. This absorption of carbon dioxide and the accompanying liberation of oxygen can only go on in the daytime, when the chlorophyll can absorb the sun's energy for the photosynthesis process. No gaseous exchange, therefore, which is due to photosynthesis can go on at night or in darkness.

Respiration is exactly opposed to photosynthesis in its need for and use of oxygen and carbon dioxide. In this process oxygen is absorbed, carbon dioxide is liberated, and energy is formed by all living cells of the plant body, regardless of whether they contain chloroplasts or not. Furthermore, oxygen is absorbed and carbon dioxide is liberated by plant cells at all times of day and night as long as they live and need energy for maintaining their vital functions. 
It will occur to the student that the oxygen liberated by photosynthesis during the day may be used by the green leaf cells for respiration, and that the carbon dioxide liberated in respiration may likewise be built by photosynthesis into sugar and starch. While this is true, it does not change the fundamental distinction between the two processes as to their nature and use in the living plant organism. In addition to photosynthesis there are other processes, notably fermentation and what is termed anaërobic respiration, which are closely related to normal, or aërobic, respiration. These processes will be considered more appropriately, however, in connection with the life of the fungi, in a later chapter.

\section{DIGESTION AND ASSIMILATION}

\section{Digestion}

The nature of the digestive process is the same in both plants and animals, and consists in the conversion of foods from an insoluble into a soluble condition fitting them for circulation and final assimilation by the tissue cells of the body. The starch which is stored in leaves, wood rays, tubers, and seeds is a good illustration of an insoluble food which must be converted into soluble sugar by digestion before it can be circulated or used by the living cells of growing parts. In like manner the gluten or protein of wheat and the fat of seeds like flax and the castor bean are insoluble and unusable until they are digested at the time of seed germination to form soluble proteins and fats for the growth of the embryo. The place where digestion occurs is very different in the higher plants and animals. Since there are in plants no specialized digestive organs like the alimentary canal of animals, digestion takes place in the cells of storage organs in any part of the plant body where reserve foods exist. In the mesophyll cells of leaves digestion is probably going on at all times, since starch formed by photosynthesis is continually being converted into sugar for immediate use by the leaf cells or for transport along the veins and the phloëm of the stem to the wood-ray cells and other storage tissues of the stem. In germinating seeds 
digestion takes place in the cells of the storage endosperm or in the embryo itself, where reserve foods were laid down during the growth of the seed from the ovule. Digestion may therefore be said to be distributed throughout the plant body, and for the most part takes place within the cell cavities in the higher plants instead of being localized in a special digestive tract.

The agents of digestion are the so-called enzymes, or ferments, familiar to us in the secretions of the digestive glands of man, such as the saliva, gastric juice, and intestinal secretions. In plants these ferments are usually formed by the protoplasts in the cells where digestion goes on (Fig. 63); but in some seeds, like the grasses and cereal grains, special glandular layers of secreting cells exist where digestive ferments are formed and then excreted into neighboring tissue cells for digestive purposes.

The method of digestion is chemical in nature and consists, in most cases, in adding water to the insoluble food molecules, thus rendering them soluble, as in the conversion of reserve starch to sugar:

Starch + water + diastase ferment $=$ sugar + diastase $\left(\mathrm{C}_{6} \mathrm{H}_{10} \mathrm{O}_{5}\right)_{n} \quad \mathrm{H}_{2} \mathrm{O}$

$\left(\mathrm{C}_{12} \mathrm{H}_{22} \mathrm{O}_{11}\right)$

In this process, which is termed hydrolysis, the exact rôle of the ferment is unknown, since it is not apparently destroyed or diminished by the process, as indicated in the formula.

\section{Assimilation}

The conversion of foods rendered soluble by digestion into the living protoplasm of plant cells is termed assimilation. Since this conversion is impossible unless the food is in the proper state, digestion and assimilation are closely linked processes in plant nutrition.

SEASONAL LIFE OF ANNUALS, BIENNIALS, AND PERENNIALS

We shall now attempt to apply to the seasonal life of a few common plants the principles of nutrition already laid down.

In order to make this application as comprehensive as possible, plants will be selected which live under quite different conditions 
and thus have a very different organization and mode of life. We shall thus secure not only a summary of the principles already learned but also a fundamental study of the relations which plants sustain to their environments.

\section{An Annual: the Garden Bean}

The bean plant is typical of the most abundant and common forms of plant life that live in medium conditions on land. It is likewise representative of the so-called annual plants, which complete their life cycle in a single season and then die down, leaving the seed as the wintering and hibernating structure to perpetuate the race the next season. The life of an individual bean plant for a season (Fig. 64) will thus give us a general idea of the seasonal life and activities of common annual land plants.

Food storage. As indicated above, the seed is the wintering stage of the bean plant and is composed of an embryo plantlet in which is stored an abundance of food for the growth of the embryo until it becomes self-supporting. This food, however, is stored up in the bean in the form of solid grains of starch and protein. Fats are also stored in a condition unsuitable for immediate use by the embryo. In order that the growing embryo plantlet may use this solid food, therefore, it must first be transformed into soluble foods by digestion.

Digestion and respiration. Digestion in the bean is not very different from the same process carried on in a growing animal fed upon beans, whole or ground into meal. In the case of the animal the digestive juices are poured into the digestive tract and mixed with the food in the stomach and intestine; in the bean seed the cells of the cotyledons in which the food is stored secrete the digesting substances or ferments, which transform the solid starch and protein into soluble sugar and protein. The fat is likewise ultimately transformed into sugar before being used by the growing plantlet. In Fig. 63 these facts are graphically illustrated in connection with the cotyledons of a growing bean seedling. In such a seedling the life processes are unusually active, as in animals. The reason for this is that growth in the 
plant involves the building of new living substances, the formation of new cells by innumerable cell divisions, and the expansion of cells in the plant body by means of absorbed water.

Respiration is also very active in germinating seeds and growing plants, as can easily be demonstrated if germinating

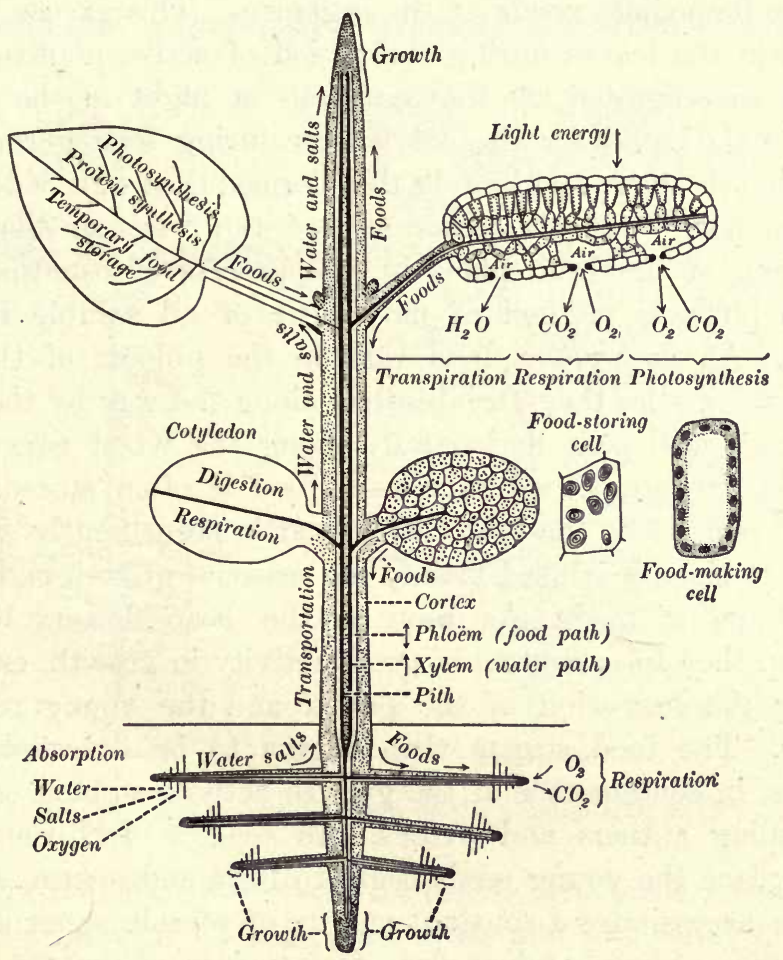

FIG. 63. The main physiological activities of a bean plant illustrated diagrammatically

seeds or growing seedlings of beans are placed in a thermos bottle under proper precautions. A thermometer thrust among them will quickly demonstrate the evolution of heat, which is an index of the respiratory activity of the growing plant parts.

Photosynthesis and migration of foods. The leaves as they unfold begin to absorb carbon dioxide and the energy of sunlight. The roots also absorb water and soil salts, which pass up 
the ducts and are combined by the leaf with the carbon dioxide from the air to form starch, sugar, and nitrogenous foods. As long as the plant is young and growing this leaf-made food will be used for immediate growth, but as the leafage increases, an excess of food will be formed daily over and above that used for the immediate needs of the organism. This excess of food, stored in the leaves during the period of active photosynthesis by day, is digested by the leaf cells at night in the manner already indicated for the cotyledons during seed germination. The soluble sugar and protein thus formed then moves down the phloëm portion of the veins and of the vascular bundles of the stem of the bean plant by the process of osmosis, which is the physical method of movement of all soluble foods in plants. As they move downward in the phloëm of the main vascular bundles they are absorbed along the way by the cortex cells and also pass horizontally along the wood rays toward the pith, where, as we have seen, food is often stored. In an annual plant, like the bean, little food is permanently stored in the stem since it is used mainly for seasonal growth and for the production of seeds. As soon as the bean flowers begin to develop they form centers of great activity in growth, especially during the formation of the pollen and the young seeds, or ovules. The food stream then begins to be diverted to the flowers, in consequence of the growth activities going on in the developing anthers and ovules. As soon as fertilization has taken place the young seeds begin to form endosperm, and this process necessitates a constant supply of soluble sugar and protein. Since osmosis takes place from points of greater concentration to those of less concentration for any given substance, the cells of the growing cotyledons of the bean must needs convert the sugar and soluble protein into insoluble starch and protein grains in order to reduce the concentration of soluble foods in their water vacuoles; otherwise the flow of food toward these cells would soon cease. This conversion of soluble sugar into insoluble starch is done by leucoplastids in the cells of the cotyledons, while storage protein granules are formed in the general cytoplasm of the cells. Fats and oils also seem to be 
made from sugar in small quantities by the general cell cytoplasm. When the seeds are fully formed and stored with food, they are shed from the mother plant and begin their long winter rest preparatory to starting the new bean plant of the next season. When winter comes on, the living substance of the plant body is killed by frost, and the cellulose framework falls to the ground and is gradually converted into carbon dioxide and

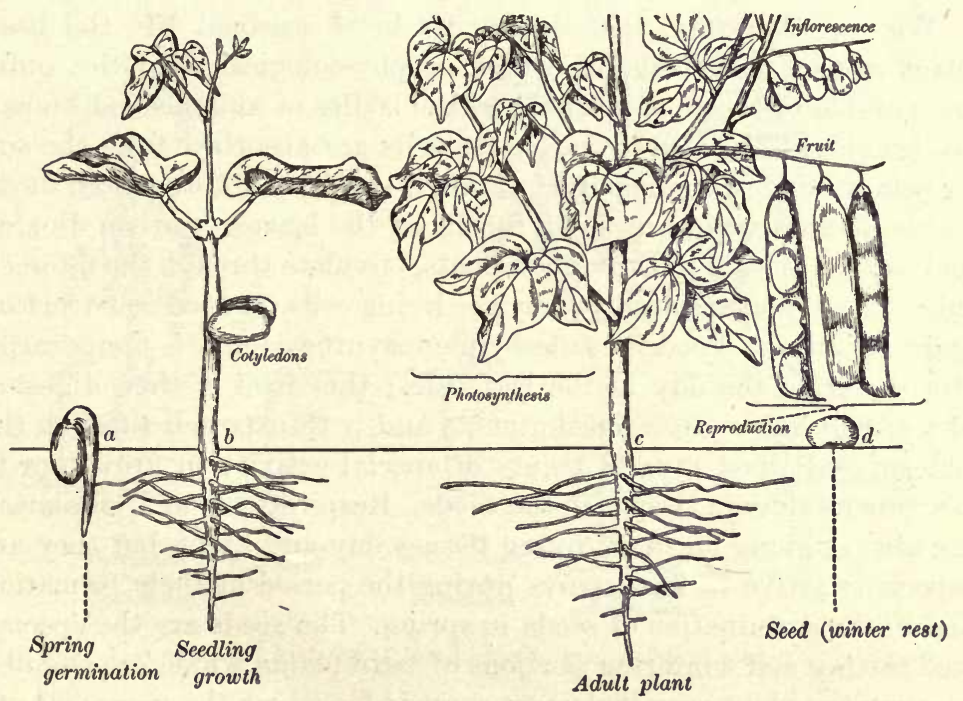

Fig.64. The seasonal history of the annual bean plant

water by fungi and by bacterial ferments. The seeds, meanwhile, are furnished with resistant seed coats and rest safely upon or in the soil until the next spring, when they germinate and produce a new generation of bean plants.

Assimilation. The conversion of food into living protoplasm is constantly taking place as the bean plant grows and produces new organs and tissues. This conversion of food into living protoplasm is called assimilation. A considerable bulk of cellulose in the form of new cell walls is also formed to serve as the skeleton, or framework, for the mechanical support of the living substance of the plant body. 
Movements. During these various internal activities the external organs of the growing plant are actively engaged in adjusting themselves to the environment; but this indispensable phase of the plant's life has already been dwelt upon at sufficient length and need not be reviewed at this point.

\section{SUMMARY}

We see, therefore, that during its brief seasonal life the bean plant carries on a complex series of physiological activities quite comparable to those maintained by the bodies of animals and human beings (Fig. 63). Water and crude salts are absorbed from the soil by osmosis and are transported through specially differentiated ducts to the inanufacturing mesophyll cells of the leaves. Carbon dioxide and oxygen stream in through stomata, circulate through the intercellular spaces, and are absorbed by the living cells for food construction and respiration. Food is made by photosynthesis and is temporarily stored during the day in the leaf cells; this food is then digested at night by active digestive ferments and is transported through the phloëm and wood rays to points of special activity in growth or to permanent storage tissues in the seeds. Respiration and assimilation are always going on in all living tissues day and night, but they are especially active in floral parts during the period of their formation and in the germination of seeds in spring. The seeds are the specialized resting and wintering portions of bean plants which are adapted to earry the plant over inclement periods for which the more delicate working plant body is unfit, and in them, therefore, rests the assurance of a new generation of bean plants in each successive season.

\section{A Biennial: the White Sweet Clover}

If we compare a biennial plant like white sweet clover with that of the annual bean plant described above, we shall find the seasonal and physiological history of the biennial quite different from that of the annual. The general physiological processes concerned with seed germination, the absorption and movement of water, photosynthesis, and respiration will be similar in the two plants; but the seasonal history and the handling and storage of foods are quite unlike in the two, on account of the biennial 
habit of the white sweet clover. In such a plant the first season is devoted largely to the manufacture and storage of food, and this process takes place for the most part in the more or less fleshy taproot. The processes concerned with the migration of food into this taproot and its storage in specialized storage tissues are identical with those already described for the bean. The special storage portions of the root are found in tho very broad wood rays and cortex, which become gradually filled with starch and protein

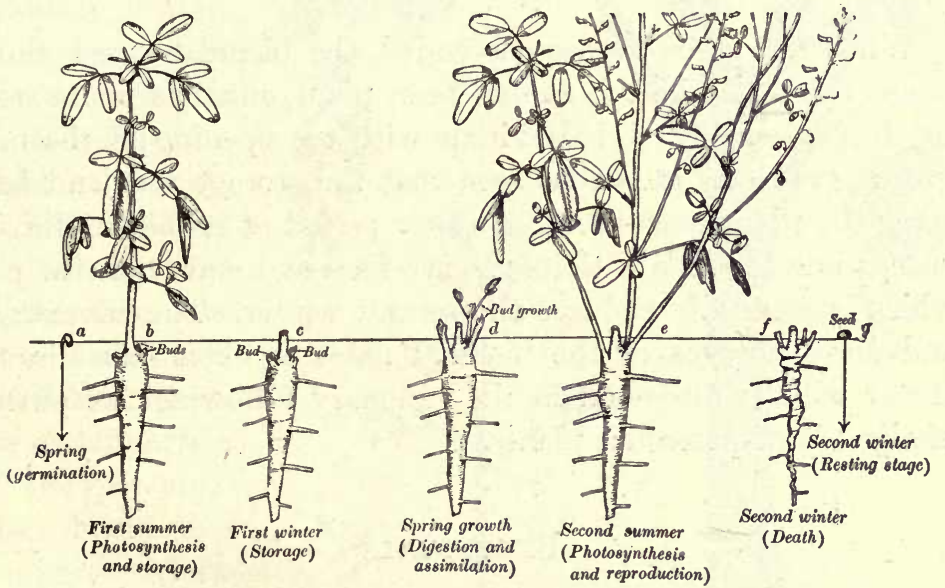

Fig. 65. The seasonal history of the biennial white sweet clover (Melilotus)

during the late summer and autumn, after the plant has reached its growth for the season. During this period also the buds are laid down on the upper broad crown of the main taproot for the early growth of stems and leaves in the spring of the second season. These buds and the taproot then pass the winter in the condition shown in Fig. 65. The following spring, when these buds start to grow, wood-ray cells convert their reserve food by digestion into soluble sugar and protein, which move at first horizontally along the rays, then upward in the phloëm into the expanding buds. In these buds the concentration of soluble foods in the cells is kept low by its constant conversion into new cellulose walls and new protoplasm for the cells and tissues formed in the growing leaves and internodes. The food streams 
are thus able to flow continually from the root cells to the bud cells until the new stems and leaves are formed for the season. When these are fully grown, the entire energy of the biennial clover plant is devoted to the production of flowers, fruits, and seeds. It is not necessary for us to trace the physiological processes involved in the formation and maturing of these structures, since they are exactly like those which have already been recounted in the production of seed by the annual bean plant.

When the second season is ended, the biennial sweet clover plant dies and, like the annual bean plant, intrusts to its seeds the formation of new individuals with the opening of the next growing season. It is thus seen that the storage root and buds carry the plant over the first winter period of its biennial existence, while the seed with its reserve food and embryo is the part which successfully endures the second winter. The advantages and disadvantages of the biennial habit as exemplified in the clover will be discussed in the summary following the outline of the life of perennial plants.

\section{Perennials}

The plant body in perennial plants continues its life from year to year, varying in the length of its existence with the kind of plant and the nature of the surroundings. Since the same plant body continues to live through several seasons, we should expect that perennial plants would manifest distinct adaptations to seasonal changes which are not necessary in annuals and are less marked in biennials. In discussing the life of perennials, therefore, particular stress should be laid on those characteristics which are connected with the perennial habit.

The seasonal life of the herbaceous perennial for the first two years is like that of the white sweet clover. The difference between the two is that the roots and underground stems of the perennial, when once established, serve for storage and the production of aërial shoots for many years instead of for the second season only as in biennials. Many plants live from year to year 
by means of an underground stem which is the perennial part of the plant. Each year an aërial annual part, like the stem of the bean, is sent up to construct food and to produce flowers and fruit. During the warm months of summer the green leaves and stem of this aërial part manufacture and distribute foods to the perennial storing underground stem and to the developing flowers, fruits, and seeds. The summer life of these perennials, therefore, is like that of the annuals and of the biennials. But when cold weather comes on, the aërial portion of the perennial dies down, and the protected underground portion stored with food lies dormant until spring, when it again sends up an aërial annual

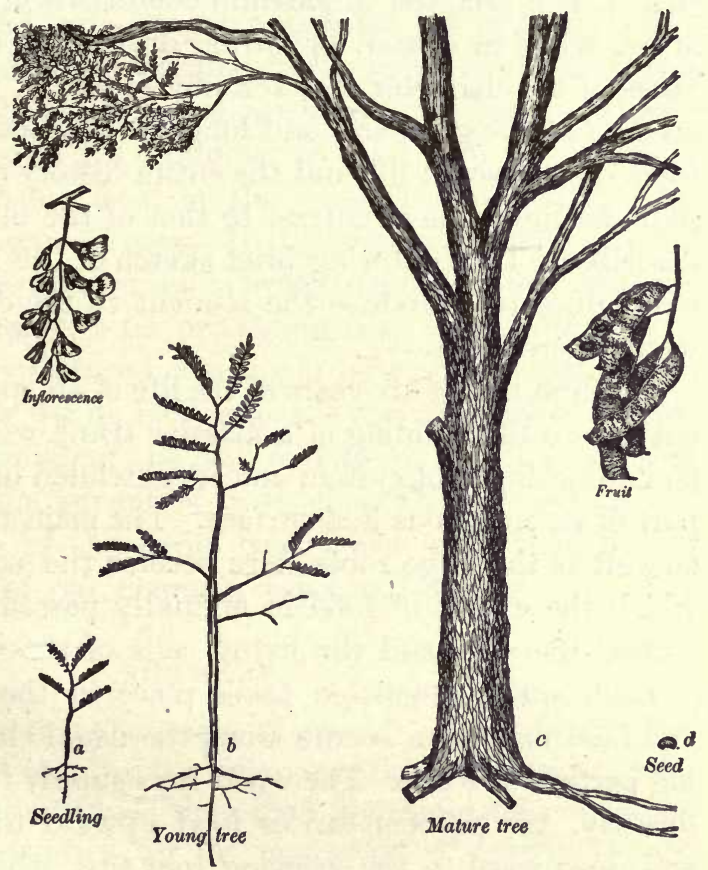

Fig. 66. The seasonal history of a perennial woody plant, the locust (Robinia)

shoot for food-making and reproduction. In the case of grasses some of the old annual foliage often survives the winter, but the really effective aërial and annual leafage is produced each season from an underground stem, or rhizome, which serves the function of storage and hibernation during inclement periods.

In perennial woody plants (the trees and shrubs of the temperate zones) (Fig. 66) the aërial plant body has become adapted to changing seasons and differences in climate, so that it does not die down, as in herbaceous perennials, with each inclement seasonal 
period. This adaptation is secured by the woody character of the stem, by the development of a protective cork jacket of bark, and, except in pines and their relatives, by the seasonal shedding of the delicate leafy portions of the plant with the advent of frost or drought. In summer a woody plant is essentially a mesophyte, that is, it is adapted to medium conditions of water and temperature, while in winter, or during drought in dry regions, it partakes of the character of a xerophyte, that is, a desert plant. On account of the great size and long life of woody plants, especially trees, the seasonal life and the entire history of such a plant presents an interesting contrast to that of the other plants thus far described. The following brief sketch of the life of an apple tree will suffice to introduce the student to the characteristic life of woody perennials.

The first five or six years of the life of an apple tree are devoted entirely to the building of a massive trunk with its great feeding and absorbing root system and its extended branches for the support of an enormous leaf surface. The main trunk and branches, as well as the large roots, here become the main storehouses into which the excess of food is annually passed and stored in the cortex, the rays, and the living cells of the wood and phloën.

Each spring digestion takes place in these storage eenters, and food migration occurs along the usual channels to the growing parts of the tree. The wood rays supply food to the cambium directly, the phloëm carries food upward to the swelling buds and downward to the growing root tips, while later movements take place in all directions to growing cells in the phloëm and the wood. In this way the apple tree expands its leafy shoots and forms new roots each spring for the season's work. When the tree is well established, reproduction begins with the annual production of flowers, which results in the formation of seeds and fruit. After the reproductive phase begins in the life of a tree, all excess food produced in the first months of each season by the leaves, and a part of that previously stored in the trunk and branches, goes largely into the forming of fruit. In the apple tree, as in the sweet clover, the food streams are therefore diverted from the storage tissues in the stem, the branches, and the roots, 
along the flower peduncles, into the ovaries and seeds which are to form the apple crop for the current season. In addition to the sugar and other soluble foods which enter the fruit and seeds water must also be absorbed, particularly by the rapidly expanding cells of the apples, since we know that the enlargement of cells is almost entirely dependent upon water for the growing water vacuoles. If the trees are prevented from overproduction of fruit in any one season, they may repeat essentially the same reproductive history each season for a number of years, and this is one of the methods now being used in the scientific fruit culture for securing a uniform annual crop. If the trees overproduce in one season, they must rest for a time in order to store up new reserves in the trunk and branches for producing fruit in abundance.

After the fruit is matured, the apple lays up a store of food in the usual manner for its next season's spring growth of buds and roots. The ripening of the fruit and seeds, and the shedding of the leaves with the advent of frost, close this interesting and active seasonal life of the apple tree, which may be taken as typical of the life of our common trees and shrubs.

\section{SUMMARY}

We have now seen that the origin of annuals, biennials, and perennials from the seed involves the same essential physiological processes. These processes include the active digestion and circulation of reserve foods, respiration for the production of growth energy, and, finally, adjusting movements for the proper placing of roots, stems, leaves, and flowers in the soil, air, and sunlight.

All of these plants have also an active summer period during which food is manufactured, flowers are produced, and fruits and seeds are matured. The differences between annuals, biennials, and perennials consist largely, therefore, in the length of life of the plant body and the consequent necessity of adapting this vegetative body to seasonal and climatic changes. In this adaptation of the plant to the environment, annuals have a certain advantage in maturing their seeds in one season, since the seed is a favorable structure for distribution and for withstanding cold, drought, and other environmental factors unfavorable to plant life. The annual death of the plant body is therefore of no consequence as far as 
perpetuation of the life of the species is concerned. The plant body, being of importance for the warm season only, ean consequently be delicate and small, so that the entire energy of the organism may be concentrated on the reproductive structures. Annuals, on the other hand, are not able to hold their ground against such perennials as dandelions and grass, since these plants retain a position once gained and spread out vegetatively from year to year. Annuals are therefore good immigrant plants, which find new places and occupy them temporarily. They are able to do this by means of their seeds, which are produced abundantly each year and are readily disseminated by wind, water, and animals; but in the end they are usually crowded out of their places by the hardier and longerlived perennial plants. Biennials have an advantage in specializing the first season on the production and storage of a large amount of food and in devoting this food storage during the second season to the maturing of fruits and seeds. The biennial habit is especially adapted to regions with recurring dry and wet seasons. In such localities the rainy season, which is usually short, is sufficient for the production of a new plant body and the storage of a rich food supply. During the dry season such plants lose their leaves and hibernate in the form of underground rhizomes, bulbs, or tubers. During the second rainy season flowers, fruit, and seeds are produced, by means of which the species is preserved and disseminated. Herbaceous perennials are also adapted to such climatic changes as those indicated above, and have the additional advantage of the perennial habit. In the temperate regions of the United States, perennials also represent the dominant herbaceous types, since they easily adapt themselves to medium, dry, and wet situations and hold the territory once gained from the less enduring annuals.

Trees and shrubs, although adapted to endure great variations in the environment, are not the equals of the herbaceous perennial grasses and allied plants in adapting themselves to wide ranges of climate and soil. This is shown by the fact that the great deserts, the plains, and the high mountain areas are not their usual habitats. The prophecy has therefore been made that the future vegetation of the earth will be derived from the herbaceous perennial type of plants. The tree type had its origin in the remote past, as our coal deposits testify. It may have given rise to the herbaceous perennials of to-day, and it may succumb in the future to the younger and more progressive herbaceous perennial and its offspring. 


\title{
CHAPTER VIII
}

\section{THE RELATION OF PLANTS TO WATER}

\author{
THE MECHANISM OF ABSORPTION
}

\section{Osmosis}

The particles of the soil from which roots absorb water and soil salts are surrounded by delicate films of water (Fig. 69) in which the dissolved portions of the soil necessary to plant life are held in very dilute solution; namely, from .0001 to .03 per cent. In order to understand the method by which roots absorb this soil water and its dilute salt solutions, the student must first understand something of the laws of osmosis, upon which all absorption and much of the movement of fluids in the plant depend. A simple experiment illustrated in Fig. 67, $a$, will serve to give the necessary data for understanding the application of osmosis to the movement of water and soil salts into and through the plant. Fig. 67 shows a parchment tube which is not unlike a root hair in its form and in its osmotic properties. If now the parchment tube has been filled with a strong solution of common salt before being placed in the distilled water, the results of osmosis will shortly begin to be manifest to the observer. The water will be seen to rise slowly in the glass tube, until a column several feet in height is attained. At the same time it will be found, by chemical analysis or by the taste of the water in the jar, that minute quantities of salt have flowed out of the parchment tube into the pure water.

In the above experiment we have illustrated the essential facts regarding osmosis, or the diffusion of substances in solution through an osmotic membrane, which separates two solutions of different composition. In such cases the substance (for example, salt) dissolved in a liquid (for example, water) is called a solute, 
and the water in which it is dissolved is termed a solvent. The solutes are usually said to be of a certain concentration, which means the relative amount of the substance dissolved in the water (the solvent) in a unit of volume.

In the experiment, therefore, if the salt and water are assumed to occupy equal portions of the space in the diffusion shell, the salt is of greater concentration inside the shell than outside in

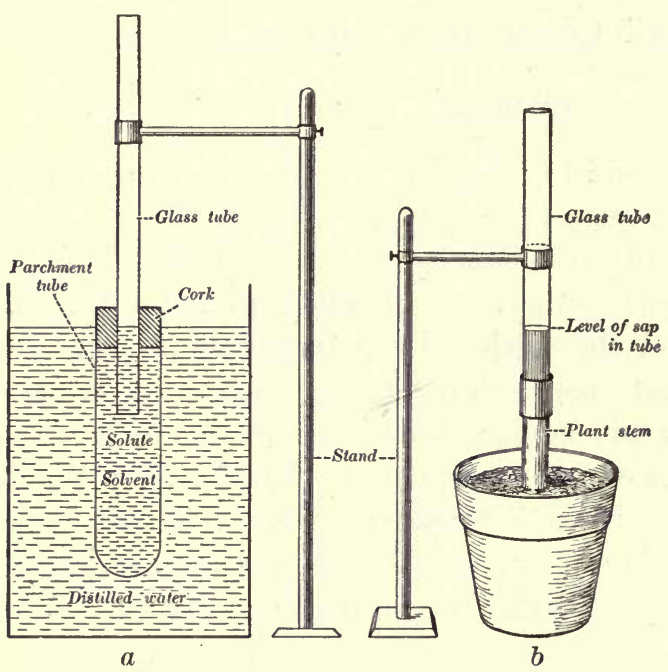

Fig. 67. Experiments in osmosis and root pressure $a$, a diagram illustrating an experiment in osmosis; $b$, a diagram of an experiment illustrating the exudation of water from a cut stem the distilled water, where it would be nil; and the water is greater in amount per unit of space in the jar than inside the shell. Neglecting for the moment the physical explanations for the movements of the solvent (water) and the solute (salt), we have seen that each tended to flow from a point of greater to a point of less concentration, and this result may be taken as a common law, or tendency, of substances of a liquid nature and of different concentration separated by a membrane. Since, however, the parchment membrane in the experiment allowed the water to pass in freely, being permeable to it, and hindered the outgo of salt, the result was a great increase in the volume of the water in the parchment tube, and a corresponding increase of pressure (called osmotic pressure) which tended to overdistend the tube. As the glass tube furnished an easy exit for the water, and a relief, as it were, from the osmotic pressure in the parchment tube, the water rose against gravity, thus giving rise 
to a water column in the glass tube. It is quite probable that the real cause of the forcible inflow of water into the parchment tube, and of the osmotic pressure thus developed, may be found in the attraction which the wall of the tube and the particles composing the solute have for the water molecules themselves. We are not here so much concerned, however, with the physical explanation of osmosis as we are with its results, which we need to study in order to understand the work of the plant in absorbing and circulating water and foods. These results, as we have indicated above, are, first, the tendency of solvents and solutes to equalize through a separating membrane, and, second, the development of a considerable osmotic pressure within a closed membrane into which an excess is thus induced to flow.

\section{Absorption by Roots}

The absorption of soil water and soil salts by roots is governed by processes very similar to those indicated above in the experiment with a parchment tube. The root hair, which is the most important absorbing portion of the root, is a tubular extension of a single epidermal cell (Fig. 68). Like most plant cells it is furnished with a delicate cell wall and a lining cytoplasmic sac composed of living protoplasm. The center of the root-hair cell is occupied by the water vacuole, containing a solution of organic acids, salts absorbed from the soil, and in many instances sugar, all dissolved in the water of the vacuole. The cell wall is permeable to most substances, but the cytoplasmic sac resembles closely the parchment membrane of a parchment tube in being more permeable to the water than to the solutes dissolved in it. It differs from the parchment membrane in being composed of living substance and in being thus able to control to a certain extent its permeability to substances outside in the soil and also within its water vacuole. For instance, in sugar beets the cells of the root are able to retain from 14 to 18 per cent of sugar within the water vacuoles of the root cells, while no sugar exists in the soil water in which the roots are bathed. Beet roots at the same time allow minute quantities of soil salts, amounting 
on the average to from .0001 to .03 per cent, to pass into the water vacuoles of the root cells from the soil by osmosis. In general, absorption by root-hair cells is undoubtedly to be explained as an osmotic process following the laws already laid down regarding the movement of solvents and solutes through a parchment membrane. The actual phenomena of absorption and of movement of water and soil salts through the root will be more readily understood by reference to Fig. 69 , which indicates a portion of a long section of a root, showing root hairs,

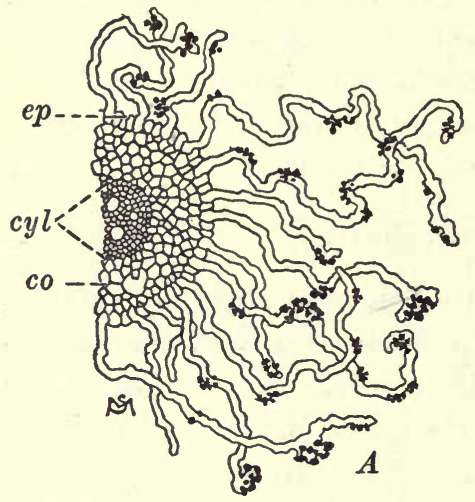

Fig. 68. The structure of root hairs

$A$, a transverse section of a root with hairs; $B$, a single
hair with adhering soil particles a portion of the cortex, and water ducts. The lower root hair is represented surrounded by the soil, which is made up of soil particles (solid black), water films (concentric lines), and air (light spaces surrounding the soil particles). If now nitrogen in the form of a nitrate is in solution in the soil

water in greater concentration than it is in the water vacuole of the root hair, the laws of osmosis already enunciated will insure the inflow of the needed nitrogen salt into the root-hair cells, and thence, by the same physical law, into the cortex cells which surround the duct. Water will likewise tend to flow from the soil water into the water vacuoles of the root hair and root cortex cells as long as these water vacuoles contain more solutes, and so less water per unit of volume, than the soil water outside. The result will be a continued flow of certain soil salts into the root hairs from the soil, and a great pressure developed inside of the root hair and cortex cells by the forcible inflow of large quantities of water into the water vacuoles of these cells. This 
latter fact may be practically demonstrated by the student in an experiment similar to that illustrated in Fig. 67, $b$. If the stem of a proper plant be cut off close to the root, as in the figure, and a glass tube be fitted over the cut end of the stump by means of rubber tubing, water will soon begin to well out of the ducts, which have been opened by cutting the stem. This water, in an active plant such as a coleus or a begonia, will often rise to a height of several feet in a small glass tube, or to that of from 40 to 50 feet in the case of some trees. This phenomenon (erroneously called root pressure) is partially explained by osmosis, but the ultimate explanation is as yet unknown. In nature the outflow of water from wounds usually occurs in the spring before the leaves unfold; it ceases as soon as the leaves expand and begin active transpiration.

The soil salts taken into the roots by osmosis move with the water into the ducts and up the stem to the leaves, where, as we
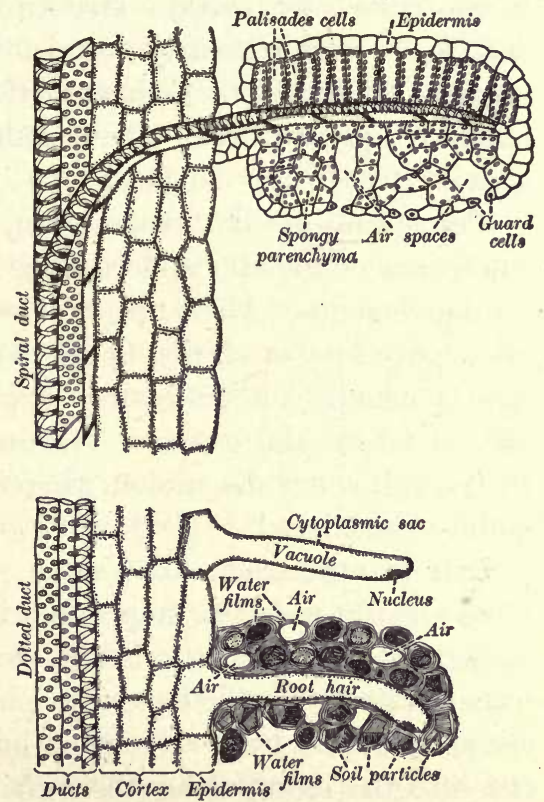

Fig. 69. The path of water in the plant

The lower portion of the figure shows the structure of the soil and the relation of root hairs to the soil particles and to the water films; the upper portion illustrates the connection of the leaf tissues with the ducts of the stem have already noted, the salts are combined with the sugar, which results from photosynthesis, to form the basic nitrogenous foods for the entire plant. Nitrogenous foods are undoubtedly formed also in other living cells of the plant body in the same manner as in the leaves. 


\section{Transpiration and Water Ascent}

Transpiration is a term used to indicate the loss of water from leaves and other exposed organs of the plant. It differs from the evaporation of water from a free water surface in that it is controlled by certain structural features of the epidermis and bark, which greatly restrict the loss of water from these organs. Thus, Sachs estimated that a given area of sunflower leaves evaporated only about half as much water as a similar area of free water surface.

Transpiration, like evaporation, is also controlled by external conditions of the atmosphere, such as temperature, humidity, and air movements. Although the loss of water takes place from all exposed parts of plants by evaporation, the term transpiration is usually understood to apply to the loss of water from leaves, where the greatest amount of evaporation takes place. In the following discussion, therefore, leaf transpiration will be mainly considered.

Leaf transpiration. The same structural features which we have already noted as important in the gaseous exchanges concerned with photosynthesis and respiration are also important in transpiration. The delicate mesophyll cells of the leaf (Fig. 62, $a$ ) are surrounded by a system of intercellular spaces which open out into the external air through innumerable stomata. Therefore the water which is supplied to these mesophyll cells from the veins tends to evaporate from their cell walls into the internal air within the intercellular spaces of the leaf, from which it diffuses, like a gas, into the external air through the stomata. If this evaporation of water is too great, the leaf wilts and the plant is in danger. It is thus seen that the need for structural adaptations in the leaf to facilitate gaseous exchange is often an element of danger, since they may also lead to an undue loss of water by transpiration. Therefore leaves often effect a compromise in their structure between the need for gaseous exchange and that of controlling water loss which might endanger the life of the plant. Some of the important structural adaptations designed to control transpiration are the following: 
Leaves in dry climates, in a location where soil water is not readily available, are wont to be smaller than in regions and localities where water is abundant. This contraction of the leaf results in a diminution in size of the intercellular spaces, which thus reduces the danger of excessive evaporation into the intercellular spaces from the mesophyll cells. The outer walls of the epidermal cells may also become greatly thickened and coated over with a waxy secretion called the cuticle. This prevents all loss of water except through the stomata. The stomata in most plants are also able to limit the amount of transpiration by effecting a closure when the loss of water from the mesophyll cells is not balanced by that received from the veins. This opening and closing of the stomata is partly explainable on physical grounds, but is not as yet fully understood. Then again many plants, like the mullein, have leaves in which the epidermal cells grow out into a thick coating of hairs which prevent loss of water (Fig. 72). These are only a few of the innumerable structural devices for controlling the excessive loss of water from leaves by transpiration.

Control. The external factors which control transpiration are light, heat, humidity, air currents, and the available water in the soil. Light affects transpiration largely through its control of the stomata, which, as was stated above, are usually open during sunlight and closed in darkness. Temperature plays an important rôle in water loss on account of its effect on the leaf tissues and on the water content of the air. If the leaf tissues are heated by the sun's rays, the result is an increased evaporation from the mesophyll cells and an acceleration of diffusion from the internal atmosphere of the leaf through the stomata into the external air. This external air will also take up more moisture when heated than when cool. These facts are confirmed by experience with plants grown in warm, dry living rooms in the home, where the greatest care must be exercised to prevent them from wilting on account of the excessive loss of water. It is a well-known fact also that plants in humid regions lose very little water by transpiration, on account of the high relative humidity of the air. For the same reason there is 
very little transpiration during rain or fog, when the humidity of the air approaches 100 per cent, while the transpiration is less on a moderate day, with the humidity at 70 per cent, than it is on a dry day, with the humidity at 50 per cent. Air currents, by removing the water as fast as it evaporates from the stomata, are also important factors in causing excessive evaporation, especially when vegetation is parched by a combination of low humidity and high winds. In this case the external coatings of hairs, already mentioned, is an important factor in preventing loss of water, since it helps to maintain a cushion of moist air over the entire leaf surface, protected from evaporation by the hair layer.

It is evident also that the amount of water available from the soil may modify transpiration from the leaves through their tendency to wilt as soon as evaporating overbalances absorption, and so to cause the closure of the stomata, with a consequent check on transpiration. It is quite probable also that the leaf cells have some control over their own loss of water in a vital way, although the nature of this control is only indicated by recent experiments, which need elaboration and confirmation.

Water ascent. The path of water ascent has already been explained as occurring in the great water ducts which form a part of the conducting and supporting vascular system of the plant. The forces necessary to accomplish the task of lifting water from the roots of tall trees to the crown can best be appreciated after a brief statement of the volume of water transpired and the rate at which it moves up the ducts in the wood of plant stems. The volume of water exhaled from the leaves of ordinary plants is indicated by the rate of transpiration from the leaves. Ganong estimates that the average daily transpiration from a square meter (10 $\frac{3}{4}$ square feet) of leaf surface is 50 grams per hour in daylight and 10 grams per hour in darkness. A birch tree with 200,000 leaves is supposed to give off from 300 to 400 kilograms (from 660 to 880 pounds) of water on a hot day in summer. Sachs estimates that a sunflower plant the height of a man would evaporate from 800 to 1000 cubic centimeters (about 1 quart) of water from its leaves on an average July day. The rate at which this water moves up the ducts varies in different plants, as the 
following figures, derived from the experiments of Sachs, will show. Sachs states that a particle of water may travel as much as 100 centimeters ( 40 inches) per hour in the vessels of woody plants. He found that in one species of willow the water moved 85 centimeters per hour, in corn plants from 30 to 42 centimeters, in the sunflower 70 centimeters, and in the grape 98 centimeters. With these facts in mind we may now turn to the forces available in the plant for transporting these great volumes of water, at the rates indicated, from the roots to the crown of woody and herbaceous plants.

In herbaceous plants it is conceivable that capillarity, or the rise of water in the ducts due to root pressure, might accomplish the work involved in water ascent. It has been found, however, that capillarity is not effective for this purpose in tubes as large as the ducts of our common plants, and that root pressure, while important in the spring, before transpiration begins, is practically in abeyance during the periods of vigorous transpiration. In tall trees these same forces would be much less adequate than in the smaller herbaceous plants. As indicated above, no theory has ever been advanced to explain satisfactorily all the aspects of water ascent, but there are certain known physical factors which should be mentioned as furnishing a partial explanation for the phenomena. These factors, taken together, constitute what is often called the cohesion theory for water ascent. The cohesion theory is based upon the cohesive power of small columns of water, which is known to be very great when subjected to a straight pull and is variously estimated at from 10 to 150 atmospheres. If this cohesion of a water column applies to the water in the condition in which it exists in the ducts and stems of plants, a scientific explanation of water ascent is conceivable on this basis. The entire column of water in a tree trunk would then act like a rope or chain and could be drawn upward as a whole if a sufficient lifting force were applied at the top. This lifting force is believed by the advocates of the cohesion theory to exist in the osmotic suction of the mesophyll cells bordering on the veins of a leaf. As these cells evaporate water into the intercellular spaces of the leaf the cell sap becomes concentrated 
in them, and a higher osmotic pressure is thus developed. This concentration of cell sap and the increased osmotic pressure produce a suction force on the less concentrated water content of the adjacent cells of the veins. The result would be the withdrawal of sufficient water from the vein cells at the top of the water column to supply the mesophyll cells, which would cause a corresponding deficiency of water at the base of the water column in the roots. This deficiency in the roots would then be supplied by absorption from the soil and by the forcible filtration of water into the ducts by the cortex cells of the root. This is in brief the conception of the cohesion theory of water ascent, which, while not entirely satisfactory, has the virtue of dealing with known physical principles. Two of the physical principles involved - namely, leaf-cell suction and filtration, due to osmotic pressure in the cortex cells of the root - are known to be operative in the plant. The third principle of cohesion, while valid for water columns in general, may not be applicable when applied to water columns as they exist in the ducts of plants.

The importance of water ascent in plants is also a question concerning which there is considerable difference of opinion. Some writers consider transpiration and water ascent as real functions of the plant, designed to supply water and soil salts to the living mesophyll cells of leaves as well as to other cells of the plant body. Others regard it as a dangerous process which is necessitated by the structural adaptations of the leaf for photosynthesis and respiration. The great intercellular spaces of the leaf and the stomata are certainly a menace to the plant when considered from the viewpoint of conservation of water. We shall doubtless find that both conceptions of transpiration are partly true.

\section{Ecological Relations of Plants to Water}

Mesophytes. The plant structures thus far described are those which pertain to plants living under medium conditions of moisture and temperature. Such plants are termed mesophytes, and they include the great plant populations which inhabit most of the temperate regions of the earth. 
From the above discussion it will be evident to the student that the amount of water available for the use of the plant must exercise a profound effect upon its form and structure. This available water is dependent also, in the case of land plants, first, upon the amount of water in the soil available for the roots and, secondly, upon the atmospheric conditions, such as temperature

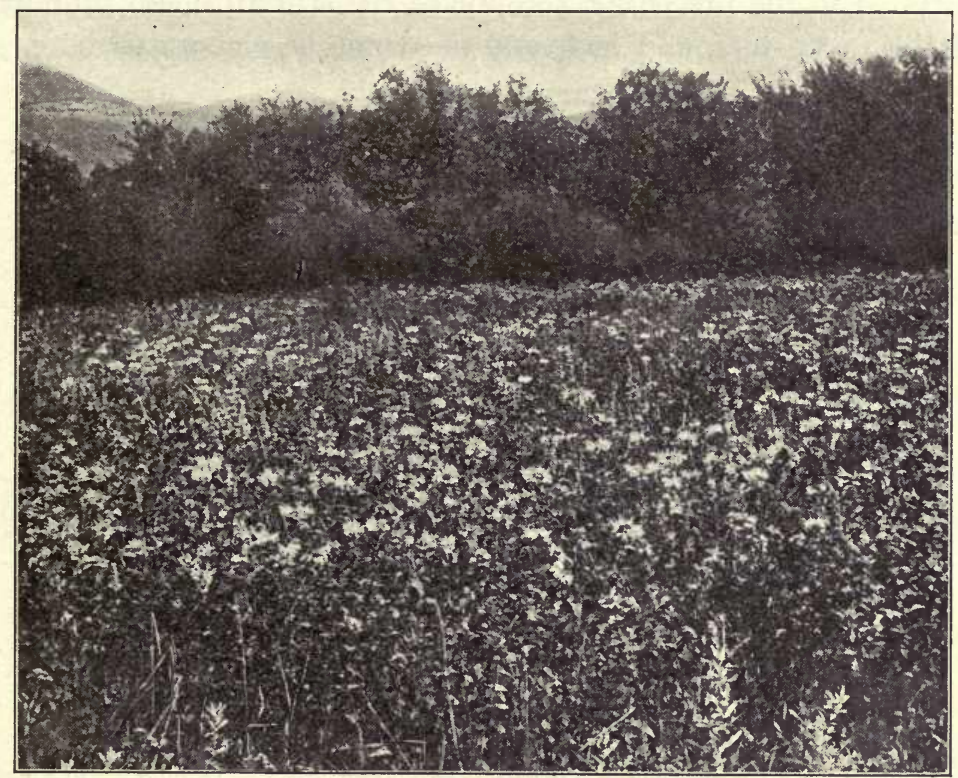

Fig. 70. Mesophytic vegetation

Meadow vegetation occuring in zones, - herbaceous plants of mint (Monarda) in the foreground, scrub and woodland in the background. After Clements

and relative humidity, which determine the amount of evaporation of water vapor from the leaves. If the amount of available water in the soil is abundant and the atmospheric conditions are such as to restrict excessive evaporation, a medium type of plant life is developed, which is usually designated as mesophytic.

The mesophytes include the broad-leaved forest trees, shrubs, and herbs with which we are most familiar, and constitute the typical vegetation of the great forests of the temperate and tropical zones as well as that of the more productive lowland and 
plain regions inhabited and eultivated by man (Fig. 70). These so-called mesophytes thus furnish the principal plant environment of the civilized races of mankind, from which have been derived the main food, forage, and fuel plants which minister to man's comfort and progress. The form, structure, and physiology characteristic of the typical mesophytes have been sufficiently outlined in the preceding account of the structure and function of the root, stem, and leaf, and need not be elaborated here.

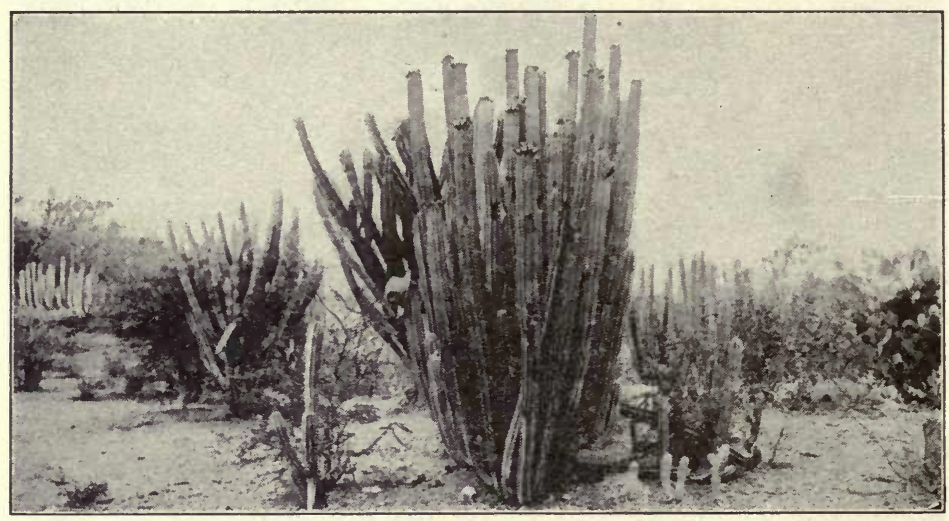

Fig. 71. Xerophytic vegetation

Desert vegetation made up principally of " ornamental cacti." Photograph furnished by the United States Department of Agriculture

Xerophytes. The traveler in desert regions of the United States, or in sandy areas like the peninsula of Florida, is at once impressed with the unusual forms assumed by the characteristic native plants of these regions. In the desert conspicuous forms of plant life are mostly of the contracted, cactuslike type, which is in strong contrast to the expanded, broad-leaved types which clothe the more productive mesophytic areas inhabited by man. Plants of this type are called xerophytes (Fig 71). The reason for the difference is obvious if we consider for a moment the statement made at the outset of this topic, namely, that the water supply available to the plant is dependent upon the available soil water and upon the conditions controlling evaporation in any given plant habitat. In the American deserts during the 
dry season the amount of available water in the sandy or alkaline soil is very small, and the roots, which often extend to great depths, are wholly unable to provide a large volume of water for the aërial stem and leaves. The atmosphere also, in these regions, is dry and hot during the long, dry season, and the plant is thus in danger of losing the small amount of water available from the roots. Under these extreme conditions all plants of the expanded mesophytic type are likely to be destroyed, and only

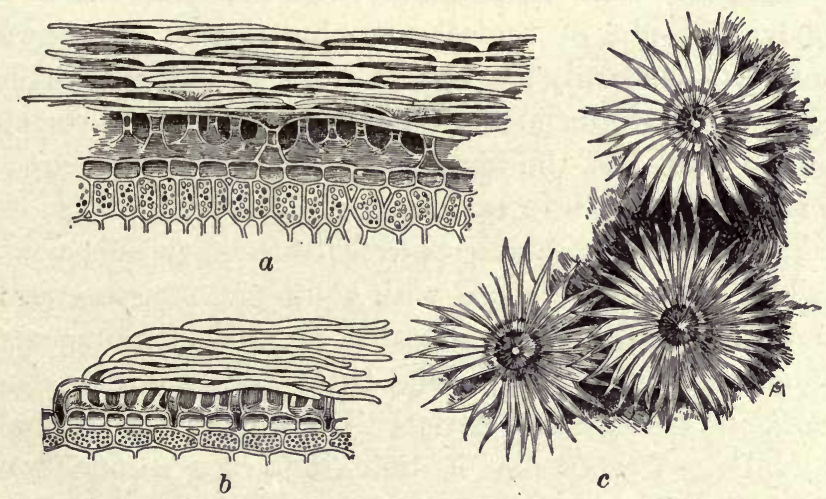

Fig. 72. Leaves of xerophytes protected by hairs from excessive loss of water

$a$, hairs of wormwood ; $b$, of Convolvulus ; $c$, of Elaeagnus. After Körner

those contracted xerophytic forms survive which are adapted to the peculiar conditions existing in the desert. These xerophytic desert plants are characterized not only by their contracted form and restricted leaf surface but by peculiarities in their structure as well. The protective epidermis in such plants is wont to be coated with wax or cutin, or is supplied with abundant hairs to protect the plant from excessive surface evaporation. The internal tissues are also more compact, with fewer and smaller intercellular spaces into which water vapor can pass from the living cells and then into the external atmosphere. Many plants of this character are known also to have dense cell sap, which enables them to hold water vapor and thus prevent its loss by evaporation. These and various other modifications looking toward the 
conservation of water characterize the typical dry-land xerophytes of desert regions. In regions like the Florida peninsula and the coastal regions of the American continent sandy soil and brackish salt water often bring about a condition approximating that of the desert, since the roots of plants in these regions are unable to secure a large amount of water from the dense soil solution. Similar conditions exist in undrained freshwater bogs and marshes.

Tropophytes. The student must not entertain the idea, from the above account of typical xerophytes, that xerophytes and mesophytes are sharply marked off from each other in all regions. Dry conditions obtain in most mesophytic areas at certain periods of the year, and the xerophytic and mesoplyytic areas often graduate insensibly into each other. Thus, our common broadleaved trees are typical mesophytes during those parts of the year when they are supplied with abundant moisture and when temperature conditions are suitable for the development of the more delicate leaves, flowers, and fruit. When winter comes on, however, the frozen soil restricts the absorption of water by the roots, and the frost makes it difficult for leaves and flowers to survive. These tree mesophytes then become practically winter xerophytes, in which evaporation is restricted and temperature changes are modified by a thick coating of bark on trunks and branches (Fig. 25). The buds of trees, likewise, in temperate and arctic regions, are adapted to the xerophytic conditions of winter and are protected by the highly modified (indurated and hairy or resinous) bud scales common in oaks, poplars, and evergreens.

Herbaceous plants likewise hibernate largely underground in the form of xerophytic storage roots or stems, while the more delicate mesophytic aërial parts die down with the advent of frost and are reproduced each year at the beginning of the warm season. It will thus be seen that some plants have become typical dry-land plants and retain permanently a xerophytic habit and structure. Others, which are called tropophytes, adapt themselves to the changing seasons and take on alternately a typically mesophytic or xerophytic form which is adapted to the water supply at a given seasonal period. 
The graduation of a mesophytic vegetation into a xerophytic one is epitomized in many mountainous regions as one ascends from the base to the summit of a high mountain. At the base and along the watercourses of the mountain streams in such regions typically mesophytic condutions often prevail, in which broad-leaved plants are the dominant type. As one ascends to

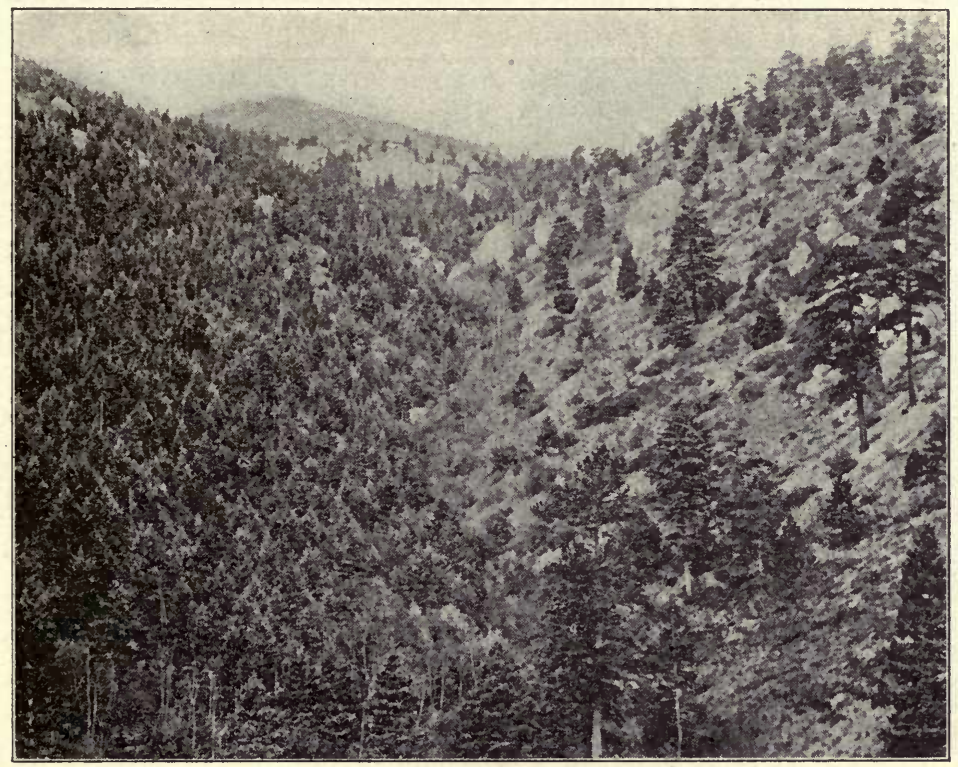

Fig. 73. The effect of exposure, slope, and moisture on vegetation

"North slope covered with dense forest of fir (Pseudotsuga); exposed south slope, with oak scrub and pine." After Clements

higher altitudes the sterility of the soil, combined with the lack of water and the drying effect of winds, produces a distinct type of xerophytic plants, which differ markedly from true desert xerophytes. The high-altitude xerophytes of mountainous regions are usually either herbs with thick, leathery leaves or low, straggling shrubs and dwarfed trees. In the typical pine forests which frequently inhabit mountain slopes (Fig. 73) one notices that the more resistant and hardy species are found high up, near the tree lines, while less hardy forms clothe the 
lower slopes and the borders of streams. It is thus often possible to see on a single mountain slope all gradations between mesophytes and xerophytes, and to gain a more definite idea of the factors which control the great mesophytic and xerophytic plant associations of the earth's surface.

Hydrophytes. Hydrophytes are plants which are wholly or partially submerged in ponds, lakes, and streams and are thus

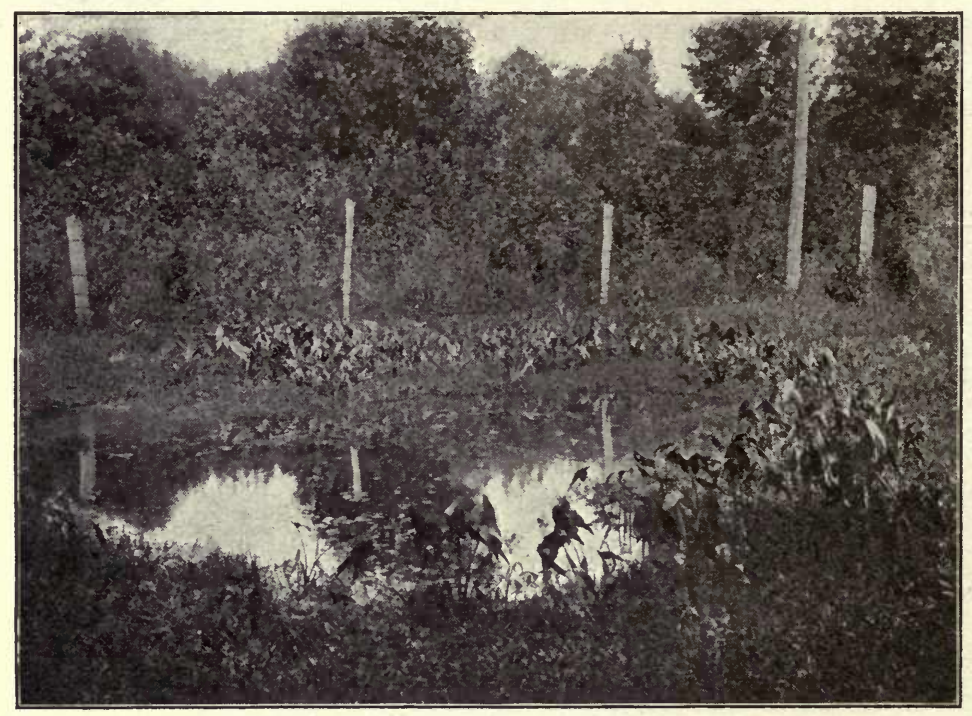

Fig. 74. Hydrophytic vegetation

Pond lilies, with mesophytic vegetation in the background. From Bergen and Caldwell's "Practical Botany"

subjected to conditions very different, as regards water supply, from those on land. Like the typical xerophytes, they are subjected to extreme conditions, which profoundly affect their form and internal organization. In a typical submerged hydrophyte, like the Elodea or the pondweed (Potamogeton), the stem and leaves are of a very delicate nature, since the plant is protected by its surrounding water medium.

Partially submerged water plants, including water lilies, pickerel weed, and some grasses and sedges, partake more nearly 
of the character of mesophytes. There are, therefore, as in the case of xerophytes, all gradations between true submerged hydrophytes and mesophytes. This is readily observed along the borders of streams and lakes, where the vegetation is often divided into more or less clearly defined zones (Fig. 74). In the water will be found both floating and attached hydrophytes of a typical character, while along the shore line a zone of amphibious plants may graduate into the typical grasses, sedges, and cat-tails of a marshy area. Farther back from the stream or lake typical lowland mesophytes often pass insensibly into grass and tree associations, inhabiting the drier hills or uplands bordering the water and marsh areas. The limitations of the text will not admit of a more extended treatment of the wonderful and interesting adaptations in the form and structure of plants to the water conditions of the soil and the atmosphere. Enough has been said, however, to indicate the profound effect of water as a factor in producing the types of vegetation which inhabit the various climatic regions of the earth's surface. The problem of the way in which water as a causal agent has been able to mold plant life is one for the students of variation, adaptation, and evolution to solve. We know simply that the fittest; plants for each particular water environment in any given habitat have been selected for survival in the struggle for existence. 


\section{SECTION IV. REPRODUCTION}

\section{CHAPTER IX}

\section{VEGETATIVE AND SEXUAL REPRODUCTION}

Reproduction is a general term used to designate the various processes by which a parent organism gives rise to new organisms, called offspring or children. Reproduction is fundamentally a cellular process and consists in all cases in the separation of single cells or cell masses from a parent or parents, which possess the power of growth and differentiation to form new individuals. Two distinct kinds of reproduction occur in the ligher plants, which are designated as vegetative and sexual reproduction. In vegetative reproduction the cell masses which give rise to new organisms are usually much less highly specialized than in single sex cells which unite in sexual reproduction. The results of the two processes are also quite different in their nature and importance to man, as we shall observe in the discussion which follows.

\section{VEGETATIVE REPRODUCTION}

Vegetative reproduction in the higher plants takes place exclusively by means of vegetative structures. These may be parts of ordinary roots, stems, and leaves or they may be highly modified parts of the plant body, represented by bulbs, tubers, corms, etc.

\section{STEMS}

One of the simplest forms of vegetative reproduction is that of budding and layering, in which ordinary buds and shoots form the starting points for the production of new individuals. In the black raspberry (Fig. 75) and the strawberry (Fig. 76), layering 
is a natural process, since ordinary branches in the black raspberry and specialized runners in the strawberry take root and form new

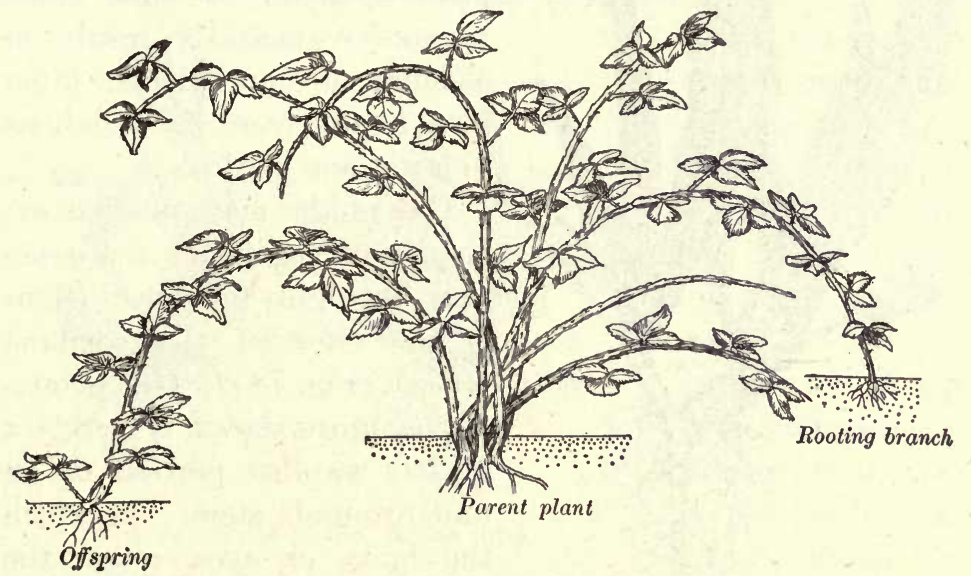

Fig. 75. Vegetative reproduction in the black raspberry

The tip of a branch, or cane, has taken root and formed a new plant

plants. This natural process is imitated artificially by man in propagating many plants, such as the gooseberry, grape, etc.

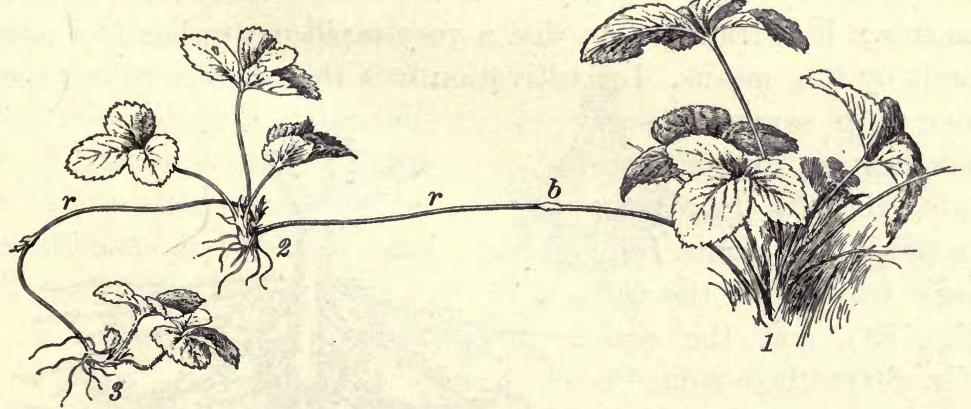

Fig. 76. Vegetative reproduction by runners in the strawberry (Fragaria) 1 , mother plant; 2,3 , daughter plants; $r$, runner; $b$, bud on the runner

Multiplying branches are often formed, as in common wild plantain (Fig. 77). In such instances the branches, which are at first connected by the mother stem axis, may become separated 


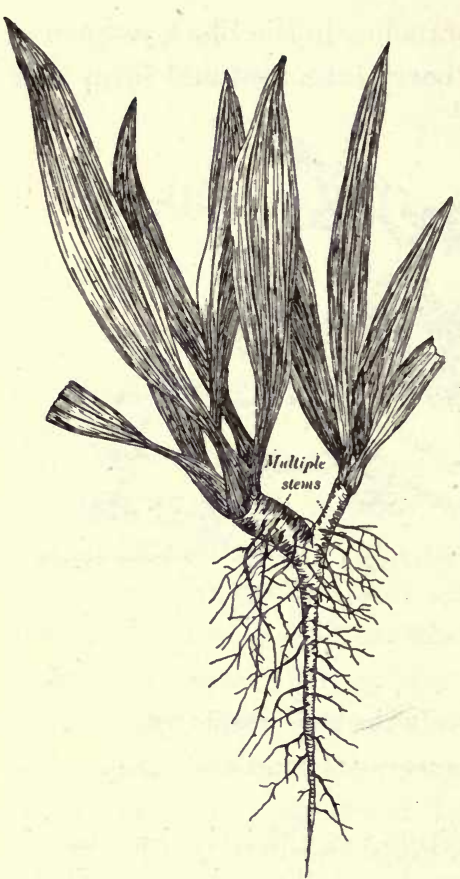

Fig. 77. Multiple stems in plantain

by mechanical injury or by decay, leaving two separated plants instead of one. Such vegetative methods result in clusters of new plants, often seen in the case of dandelions in lawns and gardens.

One of the most familiar examples of vegetative reproduction by highly modified stems is the tuber of the common potato (Fig. 78). The potato, as the figure shows, is merely a greatly swollen portion of an underground stem, in which the buds, or eyes, retain the power of growth into new plants when placed under proper conditions. Since each eye can form a new plant if it remains in contact with some of the stored food within the cells of the tuber, it is readily seen that a very rapid multiplication may result by this means. In cultivation it is the practice to cut the tuber into several pieces, each bearing one or more buds, thereby increasing the output of plants from single tubers. In the bulb (Fig. 79) and the corm (Fig. 80) the stem is greatly shortened and the leaves are highly modified scales. Bulbs and corm thus resemble ordinary buds in which the stem axis ceases to grow in length

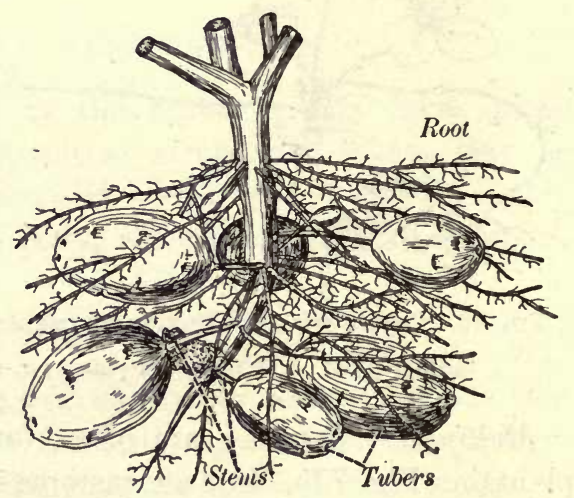

Fig. 78. Production of tubers in the potato 
and either becomes distended with reserve food or serves as an attachment for scalelike leaves and roots. Buds spring from the axils of the scale leaves as they do from the leaves of an ordi-

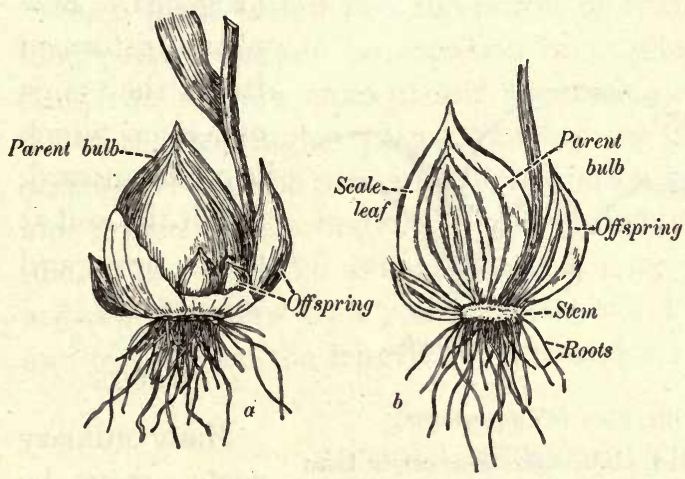

Fig. 79. Bulbs of the garden tulip

$a$, surface view of a large bulb, showing the origin of smaller bulbs in the axils of bulb scales; $b$, sectional view of the same bulb, showing stem, roots, scales, and a lateral bulb (offspring) nary branch, and these grow into new bulbs or new corms. Tubers, corms, and bulbs are favorite reproductive structures of plants in dry regions or in climates where a dry season prevails for a portion of the year. When the dry season comes on, the aërial green part of the plant dies down, and the underground bulb, tuber, or corm is able to live without perceptible injury from drought. These fleshy stems, with great stores of food, have been changed and improved for man's use by high cultivation and selection. In nature their production illustrates the ability of plants to adapt themselves to various environmental conditions by variation and selection.

\section{LEAVES}

Ordinary leaves, such as those of the cultivated begonia

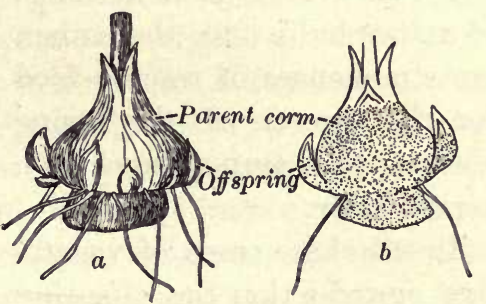

Fig. 80. Corm of gladiolus

$a$, surface view of a large corm with smaller corms; $b$, sectional view of the same corm of the greenhouses, may reproduce new plants vegetatively when properly treated. The leaves are usually cut and placed in moist sand, when new plants spring from the cut surfaces of the veins by budding. The buds take 
root and in a few weeks may be severed from the propagating leaf as new, independent plants. In the walking fern (Camptoso-

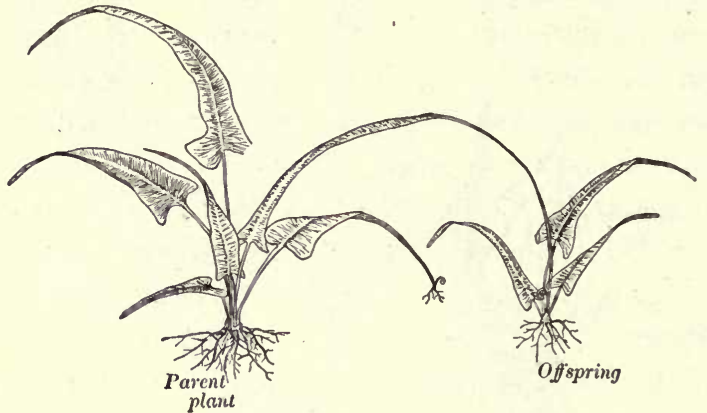

Fig. 81. The walking fern (Camptosorus)

The figure shows how new fern plants take their origin from the tips of leaves

rus rhizophyllus) (Fig. 81) new plants are formed from the ends of leaves, which bend downward, touch the soil at their tips, and take root.

\section{Roots}

Many ordinary roots may be made to reproduce vegetatively in a manner quite similar to that outlined above for the begonia leaf. On the other hand, roots, like stems, may become highly modified for vegetative reproduction. Common examples of this are the roots of the dahlia and of the sweet potato (Fig. 82), which, like the bulb and the tuber, are storehouses of reserve food for the growth of the young plants which spring from them vegetatively.

In all these cases of vegetar tive reproduction the offspring resemble the parent very closely, since the cells which produce the new plants by growth are all derived from a single parent.

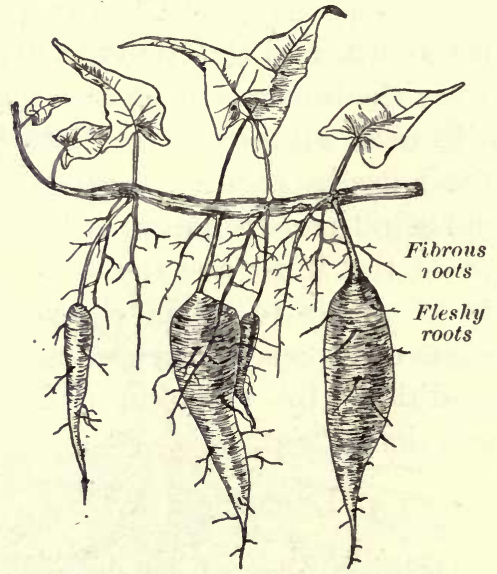

Fig. 82. Enlarged edible roots of the sweet potato (Ipomoea) This is often a very distinct advantage to man, since it enables him to perpetuate a favorable set of characters in a new fruit or vegetable much more easily than could be done by sexual 
reproduction. It is well known that most of the finest fruits are now propagated vegetatively by budding or grafting, and seedless fruits are necessarily perpetuated in this manner.

Such plants often lose the power of sexual reproduction after long cultivation and propagation by vegetative means. It is evident from the few examples of vegetative reproduction indicated above that the higher plants, much more largely than the higher animals, retain the power of reproduction by the cells in all parts and organs of the body. This is probably due to the fact that plants, quite unlike animals, have a long-period of growth and are able to renew each year the leaves and flowers of the season and to increase the length of roots and stems throughout life.

\section{SEXUAL REPRODUCTION}

Sexual reproduction differs from vegetative reproduction in the following important particulars. The reproductive cells in sexual reproduction are not the ordinary unmodified cells of the plant body which serve for vegetative reproduction, but are rather highly specialized naked cells termed gametes. These gamete cells fuse to form a new double cell, the zygote, and the zygote produces a new plant by cell division. When the zygote cell is formed by the union of the two gamete cells, male and female, derived from different parents, the new organism which grows from the zygote is quite certain to be unlike either parent, since it inherits through the male and female gametes two sets of hereditary characters. The sexual process, therefore, instead of producing offspring like a given parent, as in vegetative reproduction, is quite certain to produce a variety in offspring. In nature the production of new kinds of organisms is apparently advantageous to any given species, or kind, of plant in meeting the requirements of a changing environment and the struggle for existence to which all organisms are subjected. Some of the new kinds of offspring resulting from sexual union are quite certain to have new and advantageous combinations of characters which will enable their possessors to win out in the battle of plants for food and light. Man has taken advantage of this tendency in 
the offspring resulting from the sexual process to produce new plants which are either pleasing on account of their beauty or are useful for food, forage, or fuel.

Other advantages have been attributed to the sexual process besides those which accrue to a species by the production of a varied offspring, but these are as yet unproved.

In the higher plants the male gametes are produced in the socalled pollen tube, which is an outgrowth from the pollen grain. They are small, naked cells with a conspicuous nucleus and a

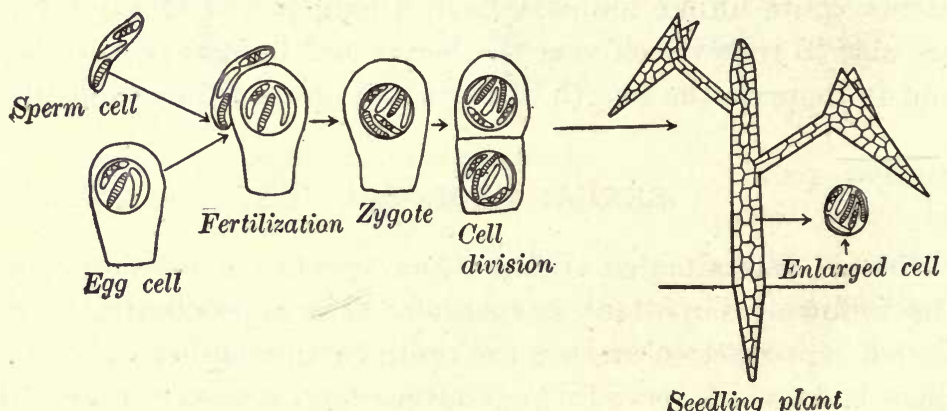

Fig. 83. Sexual reproduction and doubling of the chromosomes in fertilization

very thin sheath of cytoplasm. The end of the pollen tube ruptures when it reaches the vicinity of the egg in the ovule, and frees the male gametes. The female gamete is larger than the male gamete and is furnished with a more conspicuous nucleus and a larger amount of cytoplasm.

When the two naked gamete cells come together, the male and female nuclei, called pronuclei, approach each other and finally unite to form a new double nucleus, - the conjugate nucleus, or fusion nucleus. This process of fusion of male and female sex cells is called fertilization (Fig. 83).

The zygote which results is a double cell structurally and functionally; hence the embryo and the young plantlet in the seed must possess all of the characters of the parents which entered into the zygote cell through the gametes. Since the male gametes are produced in the pollen grains and the female gamete is deeply buried in the ovule of a higher plant, a complicated 
apparatus is necessary in order to make sure that the male gametes will reach the female gametes and fertilize them.

To insure this union of the sex cells the flower has been evolved, with its complicated apparatus for attracting insects and for holding the pollen brought to the stigma by wind or insects.

\section{The Flower and its Parts}

The flower is the reproductive apparatus of the higher plants, designed to insure sexual union of the gametes and the production of embryos in the seed. The early history of the flower shows that it is a modified shoot or bud in which the parts have been changed to meet the needs of a highly organized reproductive apparatus. No attempt will be made here to trace the steps in the development of the flower or to give its manifold variations in the different orders of plants. We shall rather study the parts of a typical flower and then endeavor to trace the processes of pollination and fertilization.

The simple flower of the mandrake (Fig. 84) will be used to illustrate the following general discussion of the parts of a typical flower and its fruit. See also the flowers of the marigold and the buttercup in Part III.

Peduncle and receptacle. Most flowers are borne on a slender stalk, or peduncle, which is enlarged at its apex to form the receptacle. The floral parts have their origin on this receptacle, which corresponds to the apex of the floral branch.

The perianth. The perianth is usually composed of two distinct parts : the calyx, composed of separate leaflike parts called sepals, and the corolla, composed of individual parts called petals. The petals are frequently highly colored and constitute the showy part of common cultivated and wild flowers. The calyx springs from the receptacle just below the corolla; in the mandrake it is composed of six sepals, which fall off as soon as the flower opens from the. bud. In addition to its function as a flag apparatus to attract insects the perianth serves as a protective envelope for the essential organs of the flower, the stamens and the pistil. In the bud stage these organs are completely inclosed 
in the perianth, and many flowers retain for some time the power of opening and closing the calyx and corolla in response to light, temperature, and moisture. They are thus able to serve as a daily protection to the essential organs during the entire flowering period.

Essential organs. The essential organs of the flower (so named for the reason that they bear the pollen and the ovules, which are
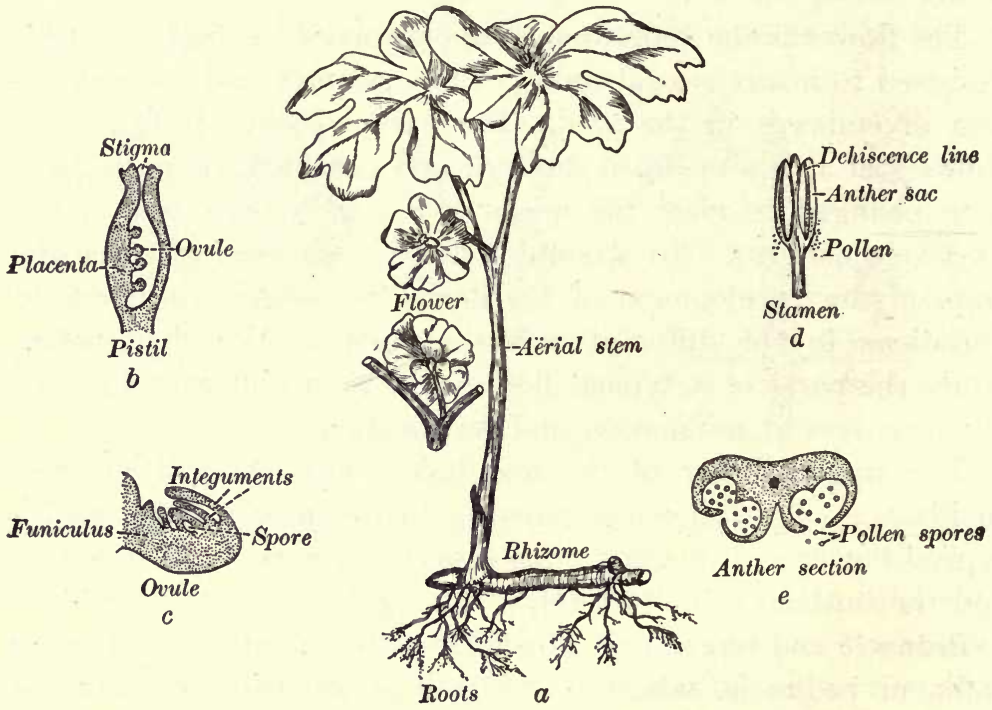

Fig. 84. Habit of the mandrake (Podophyllum), with flowers and floral parts $a$, a mandrake plant with a flower; $b$, a pistil in section, showing the origin of the ovules on the placenta; $c$, an ovule, highly magnified to show its parts; $d$, a stamen, with anther, showing the lines of dehiscence and the pollen; $e$, a transverse section of the anther

necessary to the production of seed) are the stamens and the pistil. The stamens in the simple types of flowers arise above the petals, constituting one or more whorls, the number varying in different kinds of flowers. Each stamen is composed of a delicate stalk, or filament, which bears at its apex the anther, composed of two pollen sacs. The pollen grains or spores are developerl within the pollen sacs. When the pollen grains are ripe, each anther splits along two lines, called the lines of dehiscence, and the 
pollen sacs gape open, thus enabling the pollen to escape. The pollen is then free to sift out and to be deposited by the wind or by insects upon the stigma of the same or a different flower. The deposit of pollen on the stigma is termed pollination and is essential to fertilization and the setting of seed.

The pistil may be borne singly on the receptacle, as in the mandrake, or there may be a cluster of separate pistils in a single flower, as in the buttercup. The pistil is composed of a sac, or flask-shaped lower portion, called the ovary, and of a terminal portion, called the stigma. The stigma is usually roughened, irregular, or furnished with hairs or a sticky fluid for the retention of pollen brought to it during pollination. In many cases the stigma is joined to the ovary by a narrow neck called the style. The style is sometimes lacking, and then the stigma is said to be sessile. The ovary bears the ovules on a cellular outgrowth, or ridge, called the placenta. The ovules (Fig. 84, $b, c$ ) develop into the seeds after fertilization (Fig. 86, $c, d$ ).

\section{Gametogenesis, Fertilization, and Development}

Gametes, or sex cells. The general facts concerning the structure of the sex cells, or gametes, and their union in fertilization have already been discussed under the head of sexual reproduction. It remains, therefore, to give more in detail the origin and development of the sex cells within the pollen grain and ovule, and to explain more fully how fertilization is effected within the ovule through the agency of the pollen tube.

In describing the formation of the gametes it will be necessary to include the structure of pollen and ovules, in order to make clear the processes by which the gametes are developed within these structures.

The young pollen grain is a single cell with a thick cell wall inclosing a dense granular protoplast with a large nucleus (Fig. 85, a). The thickened cell wall, which is designed to protect the pollen grain against desiccation, is divided into a thick outer layer and a thin inner layer. The outer thickened layer is protective, and the inner layer is important in the 
formation of a pollen tube. The single nucleus of the pollen grain (a) divides into two nuclei $(b)$, around one of which a cell is organized which is to form the two male gametes, the other nucleus being left free in the cytoplasm. The newly organized cell divides to form the two male gametes within the pollen tube $(c)$, and the free nucleus becomes what is called the

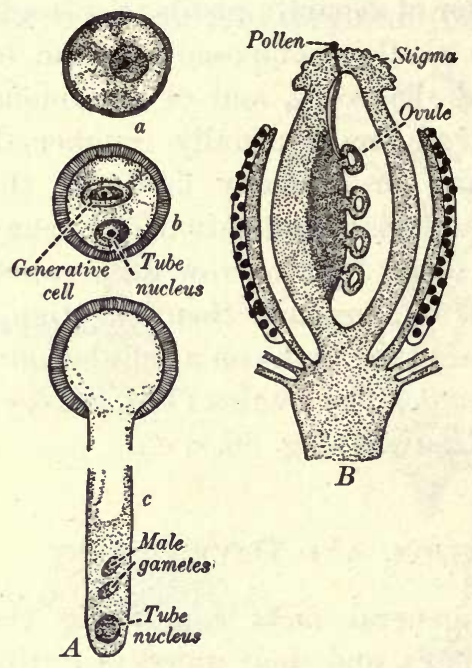

Figs. 85. Germination of pollen and pollination

$A$, pollen germination and the male gametes; $B$, diagrammatic figure of the pistil and anthers, with growth of pollen tube through the style to an ovule tube nucleus, which is concerned with the growth of the pollen tube. The above changes may be completed while the pollen is resting on the stigmatic surface after pollination, but the male gametes may not be completely organized until the pollen tube begins to grow down through the style just before fertilization.

While the above changes are going on in the pollen the female gamete, or egg cell, is being formed in the ovule. Sections of young ovules and of mature ovules at the period of fertilization (Fig. 86, $a, b$ ) show that the body of the ovule is covered by two integuments made up of layers of cells which inclose the central body of the ovule except at one point, where the ovule coats do not quite come together. This failure of the ovule coats to meet leaves a pore, called the micropyle, leading to a large central cavity within the mature ovule (Fig. 86,b) called the embryo sac. Within this embryo sac the egg apparatus is formed, which is composed of the egg cell, or gamete, and two associated cells, called the synergids. Two free nuclei, the polar nuclei, may also be seen near the center or at one end of the sac at this time. The end of the sac opposite the egg apparatus also contains three cells, the antipodals, with which we are not now concerned. After the male 
gametes are formed in the pollen grain on the stigma and the female gamete is developed in the embryo sac, the plant has before it the problem of getting the gametes together. This difficulty has been solved in the seed plants by the production of the pollen tube, which forms a canal down which the male gametes move toward the egg located in the embryo sac. Very soon after the pollen grain is deposited on the stigma its outer coat ruptures and its thin elastic inner coat begins to extend in the form of a tube, the pollen tube, which grows down into the tissues of the style. The tube nucleus and the male gametes pass out of the pollen grain soon after the pollen tube starts to elongate, and move down the tube with its growth and extension through the style. When the pollen tube reaches the ovary
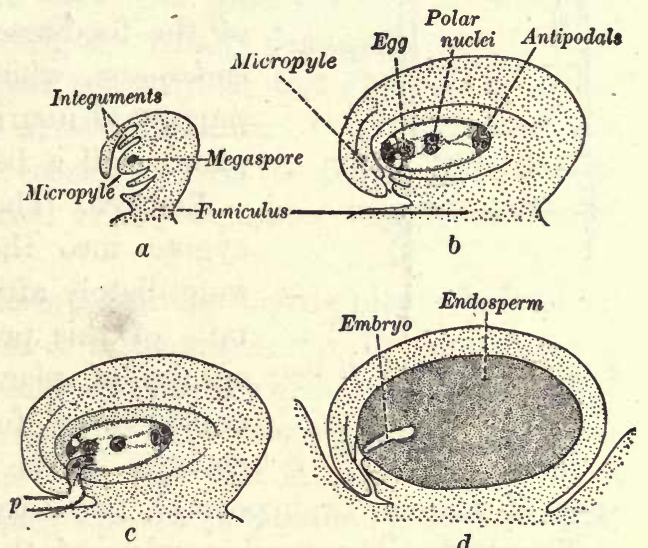

Frg. 86. Ovules, fertilization, and seed of the mandrake

$a$, young ovule with contained spore (megaspore); $b$, mature ovule with an embryo sac containing an egg and synergidæ at the left, two polar nuclei in the center, and three antipodal cells at the right; $c$, fertilization with the entry of the pollen tube into the embryo sac; $d$, the seed with an embryo (light) and endosperm (dotted). All diagrammatic

cavity, it is apparently attracted by some chemical substance secreted from the micropyle of the ovule, since it turns sharply and makes its way into this pore between the ovule coats. When it reaches the embryo sac, it comes in contact with the egg apparatus and the egg cell, which always lies at the base of the micropylar canal. The pollen tube then ruptures at its thin end, and the tube nucleus and male cells enter the embryo sac in the immediate vicinity of the egg, as illustrated in Fig. 86, $c$.

Fertilization. One of the male gametes, or its nucleus, then unites with the female gamete to form the zygote, or fertilized egg 
cell. This union of the male and female gametes constitutes the real act of fertilization. The nucleus of the second male gamete unites with one or both polar nuclei; at least, this is what hap-

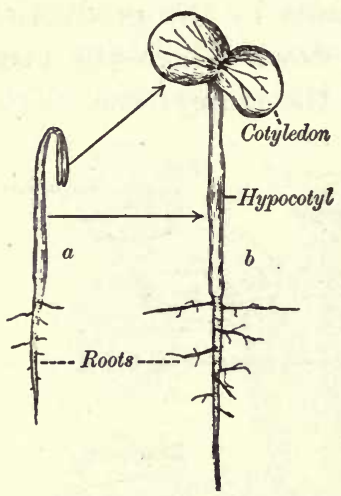

FiG. 87. Seedlings of the mandrake

Redrawn from Holm pens in a large number of instances which have been investigated. This second union of a male nucleus with the polar nuclei results in the formation of the food-reserve material, called the endosperm, which is developed for the purpose of nourishing the young embryo plant until it becomes self-supporting.

Embryo. The development of the zygote into the embryo takes place immediately after fertilization. The details of this process differ in different species of plants and cannot be discussed here for the mandrake. The embryo in the seed (Fig. 86, d) consists of a stem, or hypocotyl, two cotyledons, and a plumule.

The seed. The seed consists of the embryo, the endosperm, and the integuments, which become transformed into the hard seed coats of the ripe seed. The seedlings, which result from the germination of the seed, are illustrated in Fig. 87.

The fruit. The fruit is the ripened ovary, in which the walls and the placenta become fleshy and constitute the edible fruit of the mandrake (Fig. 88).

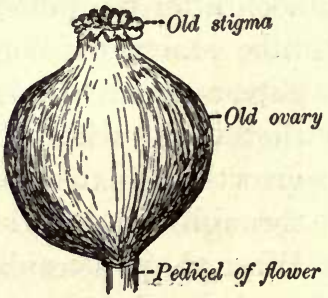

Fig. 88. Fruit of the mandrake The seeds are finally liberated by the decay of the fruit and lie dormant until conditions favorable for germination occur.

\section{Pollination}

Pollination is the term used to designate the transfer of pollen from the anther of a flower to the stigma. In this text, in the discussion pertaining to pollination, the following terms will be used to discriminate between different kinds, or degrees, of pollination. 
Kinds of pollination. The term self-pollination will be used to indicate the transfer of pollen from the anthers of a given flower to the stigma of the same flower. Close-pollination will be interpreted as the transfer of pollen from the anthers of one flower to the stigma of another flower or flowers on the same plant. Close-pollination thus defined is often designated as cross-pollination; but since the practical effects of closepollination in plant breeding are usually different from those of cross-pollination as defined below, it is thought best to retain the above definition of close-pollination. Cross-pollination will be used to designate all cases in which the pollen from flowers on one plant is transformed to the stigma or stigmas of flowers on another plant.

Since pollination is essential in the higher plants before fertilization can take place, it is necessary for the perpetuation of any given race or species of plants which is not adapted to maintaining itself by vegetative reproduction. The researches of Darwin also established the fact that cross-pollination is of distinct advantage to many species in producing stronger and better offspring. It is not surprising, therefore, that nature has evolved a great variety of novel and interesting devices for insuring both self-pollination and cross-pollination in flowers. In the following section the papilionaceous flowers of the pea family have been selected to illustrate some devices for insuring selfpollination and cross-pollination.

Inflorescence and pollination. In some species the flowers are borne singly from the axils of ordinary leaves, but in a large number of plant species the flowers are clustered, and such flower clusters are termed inflorescences (Fig. 90,d). This flower cluster is evidently a modified branch system, in which the central axis, termed the axis of inflorescence, corresponds to the central stem of a shoot. The leaves have been reduced to small bracts, and the flowers replace branches which ordinarily spring from the axils of the leaves. This agrees with the statement made above that flowers are really modified branch buds. The distinct advantage of such an inflorescence as that of the locust (Fig. 90, d) in securing pollination is easily understood if one 
watches a bee seeking for nectar, or pollen, in its flowers. The bee will be seen to go rapidly from one flower to another on the inflorescence, probing for nectar at the base of each flower and so dusting its body abundantly with pollen. When one inflorescence is exhausted, the bee moves to another and repeats the process. It is quite evident that abundant close-pollination will thus be effected by such a bee between flowers of the same plant, and that cross-pollination will be effected if the bee visits successively inflorescences borne on different plants. Moreover, many more pollinations will occur than could possibly be secured if the flowers were borne separately from the axis of the ordinary leaves of the plant. In discussing devices for insuring abundant pollination the inflorescence is therefore of prime importance as an aid in securing frequent close-pollination and cross-pollination. The head of the common white and red clovers and the large flower clusters of the sweet pea and bean are other familiar instances of inflorescences in the pea family which are of advantage in securing cross-pollination of the flowers of these species.

Pollination devices in papilionaceous flowers. Structure of the flower. The flowers of the pea family are very highly specialized, and some are adapted to self-pollination and some to crosspollination. They are usually called papilionaceous flowers, from their fancied resemblance to butterflies of the genus Papilio. The general relations of the floral parts as they appear in the garden pea are illustrated in Fig. 89. The perianth is composed of both calyx and corolla, each having five parts designated respectively as sepals and petals. The calyx is nearly regular, but the corolla is very highly modified and irregular. The largest petal is called the standard, since it projects prominently, like a standard, from the rest of the flower. The standard petal overlaps two lateral petals, or wing petals, and these inclose two united keel petals, which together form the boat-shaped keel. In the normal condition of the flower the stamens and pistil are inclosed and concealed from view by the keel. In Fig. 89, c, a flower is shown in which the petals on one side, including one half of the keel, have been remored so as to expose the stamens and the pistil in their natural position. 
The stamens are united by the lower part of the filaments, which form a membranous sheath, or stamen tube (d), enveloping the ovary like a sac. Nine stamens are usually thus united, leaving the tenth stamen free.

The pistil resembles closely the familiar pod of the garden pea and is composed of the inflated ovary, the style, and the stigma. The ovary forms the pod or fruit, and the slender

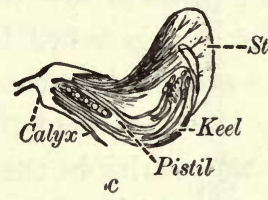

c
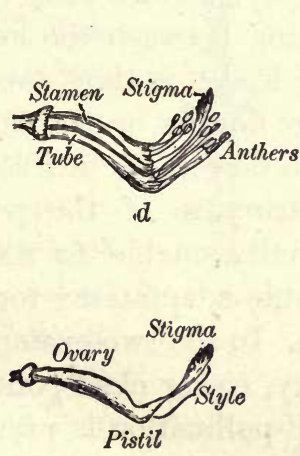

e.

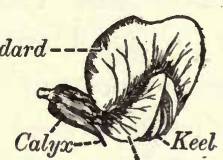

a Wing petal

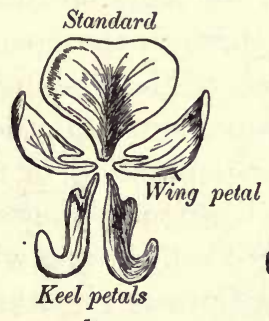

$b$
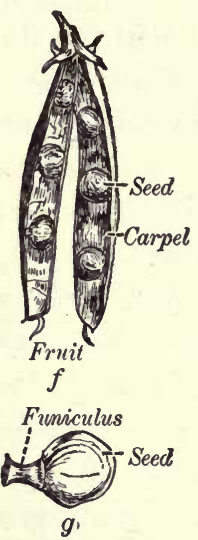

Fig. 89. Structure of the papilionaceous flower of the pea (Pisum)

$a$, flower; $b$, irregular petals ; $c$, stamens and pistil exposed; $d$, relation of stamens and stigma ; $e$, pistil $; f$, fruit ; $g$, seed ; $h$, embryo and cotyledons

style bends sharply at its junction with the ovary, thus taking up a position at right angles to the latter structure. The style is terminated by the somewhat enlarged and roughened stigmas.

Mechanism of pollination. The flowers of a large number of species belonging to the pea family are definitely adapted to securing either close-pollination or cross-pollination through the agency of insects which visit these flowers for nectar or pollen. In some instances, however, the flowers, like those of the garden pea and the sweet pea, are so constructed that self-pollination may habitually occur. In these species the pollen ripens in 
conjunction with the maturing of the stigma, and the anthers are so placed that the pollen is dusted onto the hairs of the stigmatic surface when the anthers dehisce. Self-pollination is thus insured, even though insect visitors are artificially excluded. On the other hand, these flowers are admirably adapted, in many respects, to close-pollination or cross-pollination. The conspicuous standard serves as a flag apparatus, and the nectar is so located at the base of the flower that visiting insects are tempted to probe into the flower for it. The wing petals serve to support the visiting insect; and since they are attached to the keel, this structure is certain to be depressed by the weight of the insect's body. The depression of the keel petals results in the exposure of the anthers and the hairs on the style, which snap up against the insect's body through the opening between the keel petals. The hairy abdomen of the visiting insect is thus covered with pollen, which may be borne to other flowers on the same plant, thus effecting close-pollination, or to flowers on different plants, effecting cross-pollination. The structure of the pea flower, like that of species which are wholly, unable to secure selfpollination, thus manifests remarkable adaptations for securing close-pollination or cross-pollination. In the two examples which follow, selected from the pea family, either close-pollination or cross-pollination is assured, and self-pollination is prevented by the structure of the flower and by the relative positions of the anthers, the style, and the stigma.

The locust and red clover. The flower of the common locust may be used, in contrast with that of the pea, as a concrete example of an elaborate adaptive mechanism in a flower of the pea family, designed to insure either close-pollination or crosspollination. The general arrangement and shape of the floral parts in the locust flower are so similar to those of the pea blossom just described that no additional description is necessary (Fig. 90,e). The special devices for insuring close-pollination or cross-pollination are concerned, as indicated above, with the structure of the perianth, the peculiarities of the pistil, and the relations of the anthers to the style and the stigma. The style, as in the pea blossom, bends so as to make a right angle with the 
ovary at its point of junction with the latter. The stigma terminates the style and is surrounded at its margin by a circlet of hairs which point obliquely upward. Below the stigma is a hairless space, about one fourth of a millimeter in length, which separates the bristles encircling the stigma from the stylar brush. 'This stylar brush is composed of a zone of hairs on the style from one to two millimeters long. The bristles arranged in a circle around the stigma are often called protective bristles, since they are supposed to keep the pollen of the stamens away from the stigma of the same flower. The hairs of the stylar brush are termed collecting
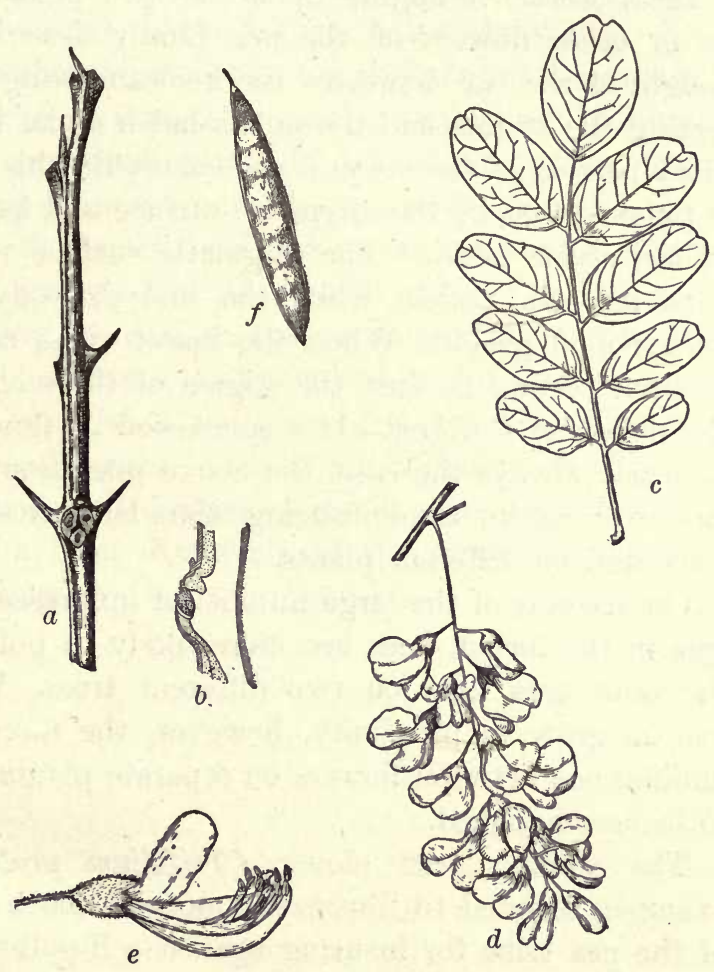

Fig. 90. Black locust (Robinia pseudo-acacia)

$a$, winter twig; $b$, section through a lateral bud; $c$, compound leaf; $d$, inflorescence; $e$, flower enlarged, with stamens and pistil exposed by removal of part of the corolla ; $f$, fruit, or legume. After Otis, from "Michigan Trees" hairs, since they collect and hold the pollen which is discharged upon them by the early opening of the adjacent anthers. The stigma, which is protected from its own pollen by the protective circlet of hairs, is sticky and remains receptive to foreign pollen long after the pollen from the anthers in the same flower has been shed and removed. The wings and the keel are yoked together and 
are depressed at the same time by a visiting bee. The anthers in the locust remain within the keel during insect visitation and finally wither away and disappear. When an insect visits a locust flower, it alights upon the keel petals and the wings, as in other flowers of the pea family described above. The weight of the bee depresses the keel and wing petals, thus projecting the stigma and the pollen-laden stylar brush against the hairy portion of the insect's abdomen. By this act the abdomen is touched first by the stigmatic surface and later by the pollen of the stylar brush. The stigmatic surface is thus protected from its own pollen, while the insect's body is well dusted with pollen grains. When the insect visits another flower, he is quite certain to dust the stigma of the second blossom with the pollen of the first. If a succession of flowers is visited, as is nearly always the case, the above operation is repeated over and over again, thus insuring abundant crosses both on the same and on different plants.

On account of the large number of inflorescences on the same tree in the locust, bees are more likely to pollinate flowers on the same tree than on two different trees. Where groups of locusts grow in proximity, however, the more effective crosspollinations between flowers on separate plants is almost certain to be accomplished.

The common red clover (Trifolium pratense) is another example, selected to illustrate a definite mechanism in a flower of the pea tribe for insuring against self-pollination by insects. Darwin states that one hundred flower heads on plants of red clover, protected by a net, did not produce a single seed, while one hundred heads on plants growing outside, which were visited by bees, yielded approximately 2720 seeds. This species is thus largely, if not entirely, dependent upon either close-pollination or cross-pollination for the production of seed, and thus for self-preservation and dissemination. The general structure of the flower of the red clover is similar to that of the garden pea and locust already described, and the parts are similarly arranged to facilitate the dusting of the abdomen of visiting insects with pollen. 
A very large number of species in the different families of flowering plants have their flowers adapted to cross-pollination and close-pollination. In some cases these adaptations are effected, as in the pea family, by irregularities in the shape of the corolla and by the relative position of the anthers below the stigma. In other instances the anthers ripen before the maturing of the stigma in the same flower, or vice versa, thus insuring against self-pollination. Again, there are some plants in which the pollen of a given flower will not germinate and form a pollen tube on the stigma of the same flower, but will do so on stigmas of other flowers of the same species. His observation of these various arrangements led Darwin to suspect that cross-pollination was in some way beneficial to plants, and resulted in his well-known experiments which established the general theory of the beneficial results of cross-pollination in the vegetable kingdom. These experiments and results will be considered in some detail in the next chapter, after a brief discussion of the terms commonly used in plant breeding. 


\section{CHAPTER X}

\section{PLANT BREEDING AND EVOLUTION}

\section{CROSSING AND HYBRIDIZING}

Pollination, in order to be effective in the production of new and better kinds of plants, must be followed by the union of the gametes in fertilization and by the production of seed. It is a common usage, therefore, in practical work, to substitute the terms self-fertilization, close-fertilization, and cross-fertilization for the corresponding terms already defined, which are used to designate different kinds, or degrees, of pollination. It is of course understood in each case that pollination has preceded the union of the gametes in fertilization.

Inbreeding is a term usually employed by horticulturists and practical breeders of plants to include all cases of self- and closcpollination and self- and close-fertilization, since it has been found that self-fertilization and close-fertilization are essentially the same in their effect upon the offspring. In other words, it makes very little difference in the character of the offspring whether the pollen from a given flower falls upon the stigma of the same flower or upon that of a different flower on the same plant.

Crossing is the term applied to the processes of pollination and fertilization where the pollen and the male gametes derived from a flower on one plant effect pollination of the stigma and fertilization of a female gamete borne by a flower of a different plant of the same kind, or species. Thus, if pollen derived from a locust flower on one tree is transferred to the stigma of a flower on another locust tree of the same kind, or species, both the transfer of pollen and the resultant fertilization are designated by the term crossing. The term crossing, therefore, includes both cross-pollination and cross-fertilization. 


\section{Darwin's Experiments in Inbreeding And Crossing}

Darwin laid the foundation for all modern experimentation in cross-pollination and cross-fertilization of plants in his book on "Cross- and Self-Fertilization in the Vegetable Kingdom." A detailed account is there given of painstaking and elaborate experiments which he performed on a great variety of plants. Darwin's methods were essentially similar to those pursued to-day in the breeding of plants by cross-pollination and hybridization. Some flowers were either self-pollinated (with their own pollen) or close-pollinated (with pollen from another flower on the same plant). Others were cross-pollinated by placing the pollen from one flower on the stigma of a flower on a different plant of the same species. The plants were then protected from cross-pollination by insects or wind by means of fine cloth stretched over frames so as to cover the plants and protect them from insect visitors. In modern practice the anthers are removed from the flowers on the pollinated plants, and they are then inclosed in parchment or paper bags to exclude pollen borne by insects or wind (Fig. 93).

After the plants had formed their seeds, Darwin collected the seeds from the self-fertilized and cross-fertilized species and estimated their relative number and weight in all cases. The seeds from the self-fertilized and the cross-fertilized plants were then sown, and the relative vigor of the offspring derived from the two kinds of seeds was noted and accurately measured. Darwin proved by these laborious methods that fifty-seven species, or kinds, of plants produced more vigorous offspring as a result of cross-fertilization than resulted from close-fertilization of plants belonging to the same species.

The nature of Darwin's methods and results is so important that the following tables and diagram have been selected from his book, with an extract from his summary of results.

The first diagram and the accompanying conclusions relate to an experiment with the common morning-glory (Ipomoea purpurea), in which ten generations of plants were crosspollinated. The second table and summary give similar results 
and conclusions concerning experiments in cross-pollinating and self-pollinating plants of the lupine (Lupinus perennis). In this case plants of the second generation were used for the experiment. In his experiments with the garden pea (Pisum sativum), illustrated in the last table, Darwin secured results which differed from those obtained in the majority of plants with which he worked. This, as we shall see, is in harmony with some recent results obtained by East, Shull, and other scientists.

Morning-glory. The mean height of the self-fertilized plants in each of the ten generations is also shown in the accompanying

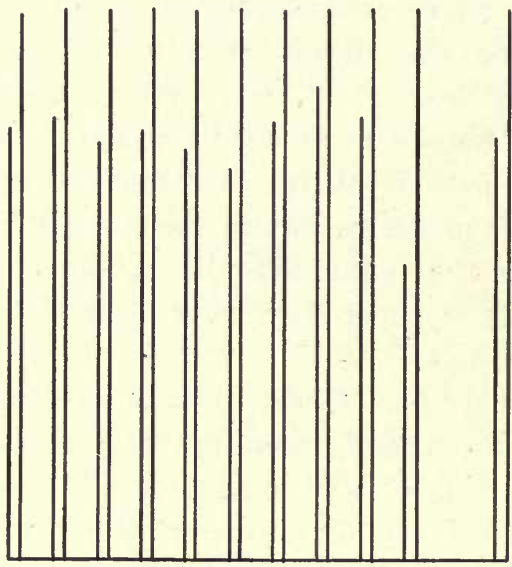

Diagram showing the mean heights of the crossed and self-fertilized plants of Ipomoea purpurea in the ten generations, the mean height of the crossed plants being taken as 100 . On the right hand the mean heights of the crossed and selffertilized plants of all the generations taken together are shown diagram, that of the intercrossed plants being taken at 100 ; and on the right side we see the relative heights of the seventy-three intercrossed plants and of the seventythree self-fertilized plants. The difference in height between the crossed and self-fertilized plants will perhaps be best appreciated by an illustration: If all the men in a country were on an average 6 feet high, and there were some families which had been long and closely interbred, these would be almost dwarfs, their average height during ten generations being only 4 feet $8 \frac{1}{4}$ inches.

It should be especially observed that the average difference between the crossed and self-fertilized plants is not due to a few of the former having grown to an extraordinary height or to a few of the self-fertilized having surpassed their self-fertilized opponents, with the following few exceptions : the first occurred in the sixth generation, in which the plant named Hero appeared; two in the eighth generation, but the self-fertilized plants in this generation were in an anomalous condition, as they grew at first at an 
unusual rate and conquered for a time the opposed crossed plants ; and two exceptions in the ninth generation, though one of these plants only equaled its crossed opponent. Therefore, of the seventythree crossed plants sixty-eight grew to a greater height than the self-fertilized plants to which they were opposed.

Lupine. When the seedlings were only four inches in height, the crossed plants had a slight advantage over their opponents. When grown to their full height, every one of the crossed plants exceeded its opponent in height. Nevertheless, the self-fertilized plants in all three pots flowered before the crossed. The measurements are given in the following table:

\section{LUPINUS LUTEUS}

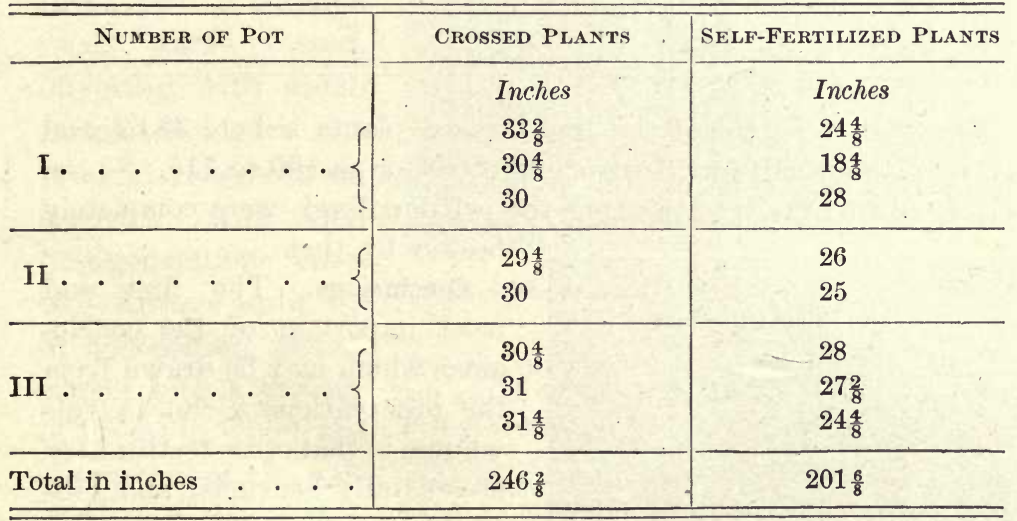

The eight crossed plants here average 30.78 and the eight selffertilized plants 25.21 inches in height, or as 100 to 82 . All of these plants were left uncovered in the greenhouse to set their pods, but they produced very few good ones, perhaps in part owing to few bees visiting them. The crossed plants produced nine pods, containing on an average three seeds, so that the seeds from an equal number of plants were as 100 to 88 .

Garden pea. In 1867 I covered up several plants of the Early Emperor pea, which was not then a very new variety, so that it must already have been propagated by self-fertilization for at least a dozen generations. Some flowers were crossed with pollen from a distinct plant growing in the same row, and others were allowed to fertilize themselves under a net. The two lots of seeds thus obtained were 
sown on opposite sides of two large pots, but only four pairs came up at the same time. The pots were kept in the greenhouse. The seedlings of both lots when between six and seven inches in height were equal. When nearly full grown they were measured as in the following table:

\section{PISUM SATIVUM}

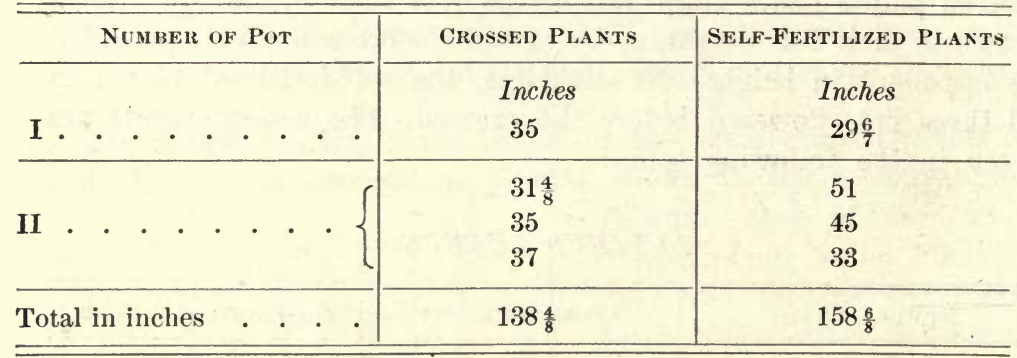

The average height of the four crossed plants is here 34.62 , and that of the four self-fertilized plants 39.68 , or as 100 to 115 . So the crossed plants, far from beating the self-fertilized, were completely

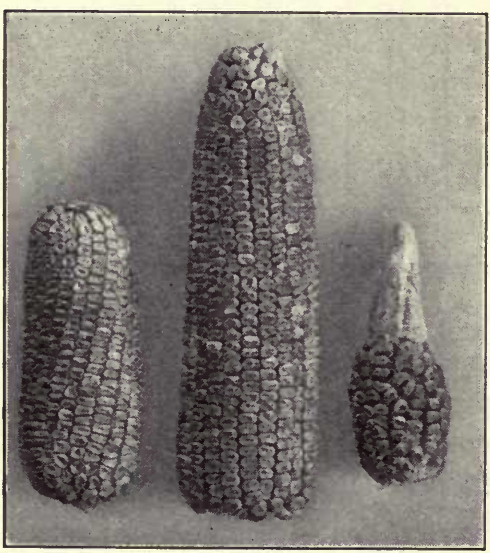

Fig. 91. Increased vigor due to crossing in corn

The two small ears at the right and left represent two pure strains of corn derived by continued self-pollination and selffertilization; the middle ear represents the first-generation hybrid, derived by crossing the two strains. After East beaten by them.

Conclusions. The first and most important of the conclusions which may be drawn from the observations given in this volume is that cross-fertilization is generally beneficial and selffertilization is injurious. This is shown by the differences in height, weight, constitutional vigor, and fertility of the offspring from crossed and selffertilized flowers, and in the number of seeds produced by the parent plants.

Darwin's early experiments were in many instances somewhat faulty, but his conclusions have been found to be in the main correct for 
both wild and cultivated species of plants and animals. A great majority of cultivated species apparently profit by crosspollination, and many species actually deteriorate if continually self-pollinated. Common field corn has been shown by the experiments of East and Shull to belong to this class of plants. These investigators selected and isolated two strains of corn (Fig. 91), which were then close-pollinated for several generations. The result was, in each case, a greatly weakened race, with the ears reduced to nubbins. But when these two weakened races were crossed, offspring with much increased vigor and productiveness were secured. Any such first-generation cross will always split up in succeeding generations into a variety of forms in which the characters of the parents will be differently combined, and

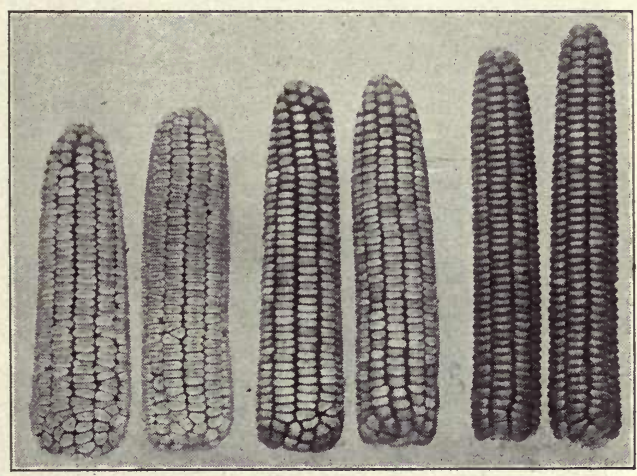

Frg. 92. Increased yield of first-generation crosses in corn

Showing two ears of 'Tyler's White-Cap Dent at the left and of Burwell's Yellow Flint at the right. The first-generation cross of these two varieties (represented by the two ears in the center) has consistently yielded more than the dent, the higher-yielding parent. Photograph furnished by the Connecticut Agricultural Experiment Station the importance of such crosses to agriculture and horticulture is therefore somewhat diminished. In other species, as in Darwin's experiment with peas, no advantage arises from crossing, and the plants are evidently adapted to continuous self-pollination and self-fertilization. Among the species which are known to be adapted to continuous self-pollination, without detrimental effects, are many of the cereals, including wheat, barley, and oats, as well as peas, soy beans, potatoes, tomatoes, flax, and tobacco.

Darwin's pioneer experiments were, nevertheless, of fundamental importance in emphasizing the value of cross-pollination and cross-fertilization. 


\section{Hybridization and the Production of New Varieties}

Hybridization is the crossing of plants of more distant relationship than the members of one species. Cross-fertilization

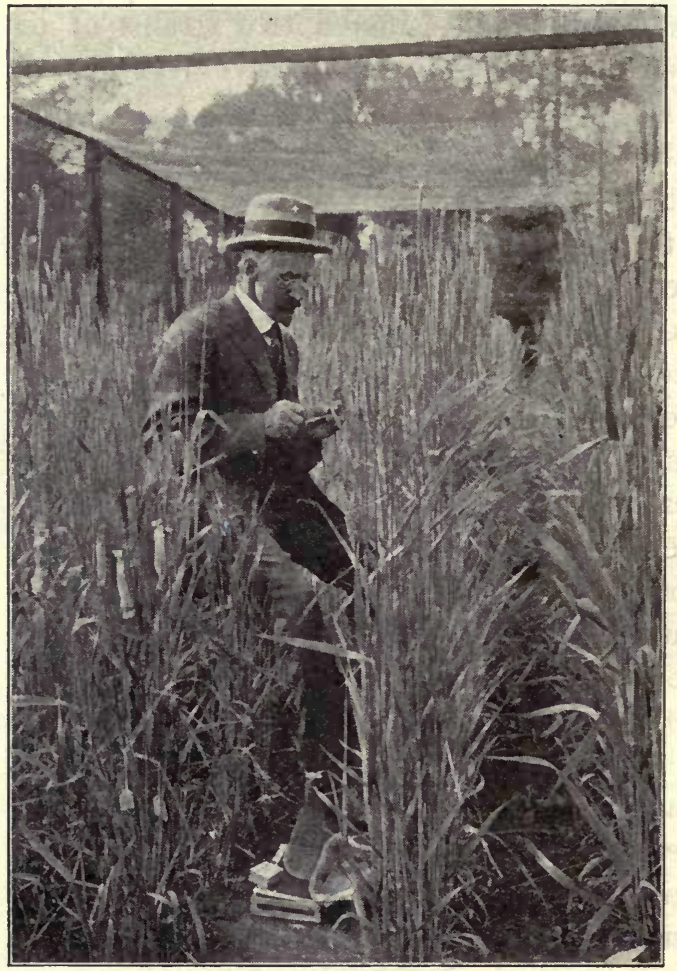

Fig. 93. Hybridizing wheat

Note the operator with his tools : namely, a box containing strips of paper and pins for covering the wheat heads; tags for labeling; alcohol for sterilizing hands and instruments; forceps and scalpel. The wrapped heads of grain have already been pollinated and labeled. From Babcock and Clausen's "Genetics in Relation to Agriculture." Photograph by William C. Matthews between different varieties of cultivated corn, wheat, barley, apples, and carnations results in what are known as variety hybrids. Crossings between different species of plants, for example those between raspberries and blackberries, yield what are termed species hybrids. These are apt to be sterile, which accounts for the fact that of cultivated fruits and vegetables comparatively few have resulted from hybridization. Unless the cross-pollination and fertilization take place between plants which are too far removed from each other in relationship, the offspring will usually inherit increased vigor and also display a greater variety of characters than is attainable by self-fertilization or close-fertilization. This result is exactly what we should expect 
from our knowledge of the nature of the fertilizing process. If pollen from a white flower is placed on the stigma of a red one, the germ cells which unite to form the zygote are going to transfer to the zygote cell, and to the embryo which grows from it, the white and the red characters of the two parents. Exactly the same thing may happen with reference to any of the different characters of two parents which are crossed. The offspring are certain, therefore, to inherit the double set of characters received from the parents. Experiment has shown that the parental characters may be variously combined in the different offspring of a given cross. We are familiar with all of these phenomena in the human race, where the children in a family inherit in different ways and in different degrees the characters of their parents.

If, therefore, a sufficiently large number of crosses are made and the offspring are carefully observed, almost any combination of parental characters may be obtained

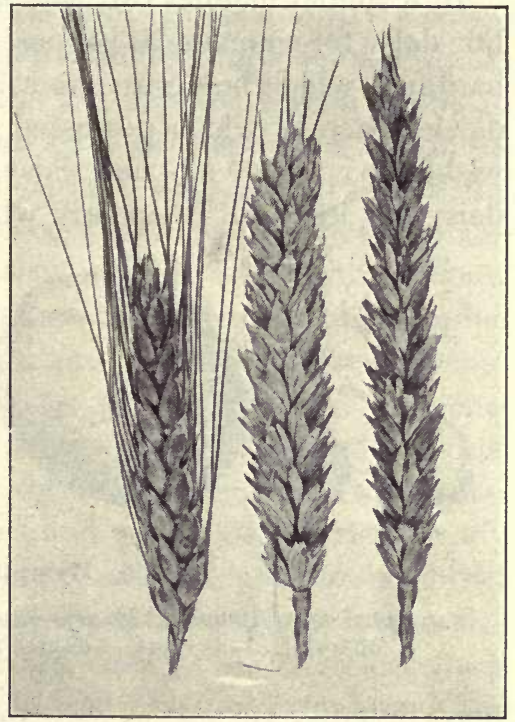

Fig. 94. A hybrid wheat and the parents

Parents at the right and left; hybrid in the center. The size and awn characters of the hybrid are intermediate; the grains and covering bracts resemble the parent at the left. Photograph by the Minnesota Agricultural Experiment Station. From Bergen and Caldwell's "Practical Botany" by the experienced plant breeder. Two instances selected from the work of Burbank will suffice to illustrate the truth of this statement. Burbank selected a wild blackberry, with white or cream-colored fruit, which was too small to be valuable for eating. He crossed this white blackberry with a large edible blackberry, the Lawton, in an endeavor to secure a white edible blackberry. Among the hundreds of hybrids which resulted from extensive crossing a few showed the desired combination 
of characters of the wild and the cultivated parents. Burbank was then able by careful selection to perpetuate a new race of white edible blackberries. He had caused a reshuffling of parental characters, which resulted in a new combination of characters.

In a similar manner Burbank set out to secure a large and prolific daisy for commercial purposes. To secure the size, luster, and hardiness which he desired he selected and crossed three kinds of daisies, each of which possessed one of the characters which he wished to combine in a new daisy. He therefore crossed a French daisy, for its beautiful pearly white luster, with a large English

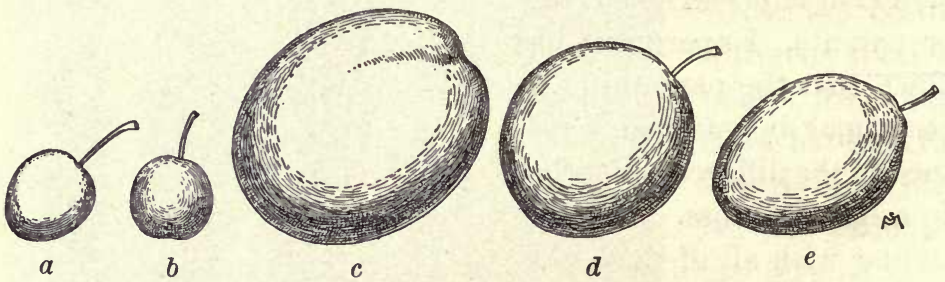

Fig. 95. Hybridization in plums

$a$, a stoneless wild plum which was crossed with an edible French prune; $b-e$, offspring of the cross. Adapted from a photograph by Burbank

daisy and with the common prolific American daisy. Luster, large size, and abundant flower production were the characters which Burbank sought to combine in a single new race of daisies. By careful selection from the offspring of repeated crossings he finally secured a new daisy, the Shasta, in which all of these three desired characters were combined in one plant.

In considering these results of crossing, it should be stated that Burbank did not create or secure any new characters in his daisies. The union of the gametes in his repeated crosses resulted in the mingling of the characters of the three daisy parents in the zygotes in various combination, and in some zygotes the combinations which Burbank sought chanced to come together. When the zygotes grew into plants, they manifested in their external appearance the luster of the French daisy, combined with the size of the English daisy and the abundant flowering 
habit of the American daisy. This favorable chance combination was selected as the Shasta daisy, a well-known garden variety.

The most marked results from the hybridization of cultivated varieties have been obtained among the ornamental plants, which are most highly protected from competition and from the adverse effects of soil and climate. The brilliant effects due to the colors of flowers and foliage among such plants as the common phlox, pansies, tulips, and foliage plants are in most instances due to the mixing of races and varieties by a long series of hybridizations similar to those recounted above in the production of the Shasta daisy by Burbank. Among fruits and vegetables there are fewer successful hybrids than among ornamental plants. Almost no important hybrids are recorded among garden vegetables. Among the fruits there are some hybrid grapes and pears, but in apples, peaches, plums, cherries, and currants there are no important recognized commercial hybrids. Among blackberries there are hybrids between blackberries and dewberries, and between the black and white blackberries already indicated. Among raspberries there are hybrids between the red and the black varieties. Perhaps the most notable hybrids are those obtained among citrous fruits, such as the tangelo (which is a hybrid between the pomelo and the tangerine) and the citranges (which are hybrids between the sweet orange and the hardy hedge orange (Citrus trifoliata)).

In crosses between plants which are too distantly related the unfavorable effect of infertility is often partially obviated in cultivation by propagating the offspring vegetatively. The various methods were discussed earlier under vegetative reproduction, and need not be repeated here. It is sufficient to repeat that by budding, grafting, and propagation by means of bulbs, tubers, and runners favorable varieties obtained by hybridization are now perpetuated for long periods of time. Hybridization, therefore, is one of the most potent of the methods available to the modern breeder for the production of new kinds of ornamental and useful plants. 


\section{Mendel's Principles of Heredity}

It will be evident to the student that in the crossing of plants for increased vigor and for new combinations of characters there is a large element of chance in the methods of Darwin, Burbank, and other plant breeders. The results attained are indeed re-

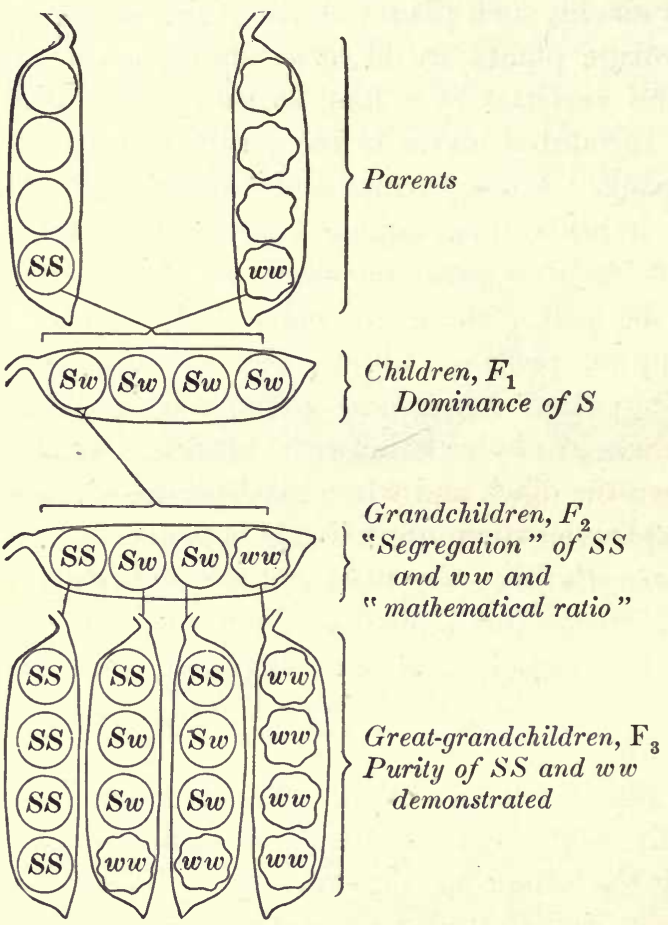

Fig. 96. Diagram illustrating Mendel's results in crosses between smooth and wrinkled peas markable and are of the greatest value in the improvement of plants for man's use, but they lack the certainty and the definiteness of a scientific method. The breeder of plants needs to be in the position of the chemist with reference to the characters of the plants which he wishes to combine by crossing. The chemist can combine the atoms and molecules of two substances in a chemical flask, and by reason of known laws of chemistry he can predict with certainty the new compound or compounds that will result. In a similiar manner the breeder of plants needs to know both the nature of the characters of the plants with which he deals and the laws by which these characters will combine in the zygote to form the kind of offspring which will meet his need or his desire. Mendel's principles of heredity now supply something like this scientific basis for plant breeding and improvement. 
Gregor Mendel was an Austrian monk who in 1865 began experiments on heredity in the gardens connected with the monastery at Brünn, Austria. He experimented on peas, and in his studies he selected only certain easily recognizable characters for observation in his experimental plants. Some of these characters related to the seeds, such as seed color, the smooth or wrinkled character of the seed coat, and the sizes of seeds; tallness and shortness of stems and hairiness and smoothness in leaves were other characters relating to the plant body which Mendel selected for observation.

Mendel's method of observing a few characters only, instead of attempting to observe all the characters in the plants under observation, is the most distinctive feature of his work. It marked a new epoch in the study of heredity, the importance of which we can hardly overestimate. When he crossed two parents which possessed such definite contrasting characters as tall and short stems, or smooth and wrinkled seeds, Mendel found that the appearance of these characters in the offspring followed definite laws, or principles. These laws, or principles, which are now called Mendel's principles of heredity, may be illustrated by giving Mendel's results, obtained in an experiment with seed characters in peas. Mendel chose peas because they are self-pollinating, so that it was unnecessary to pollinate the stigmas artificially where self-pollination was desired. In the experiment illustrated in Fig. 96 he chose two pure strains of peas, which he had previously tested as to their purity. In one of these strains, used as either a male or a female parent in the experiment, the seeds were all smooth and round $(S S)$, while in the other parent the seeds were all wrinkled $(w w)$. When plants grown from these parent seeds were crosspollinated artificially, the seeds thus produced were all smooth like one parent. Since, however, these hybrid children ( $S w$ ) had received both the smooth and the wrinkled character from the two parents, Mendel concluded that the character in the germ which determined the smoothness of the seeds was dominant over the wrinkling character. He therefore called the smooth character dominant and the wrinkling character latent or recessive. 
Dominance is indicated in the figure by the large size of the letter $S$, as compared with the small size of the symbol $w$, used for the wrinkling character.

The Mendelian ratio. Mendel's prediction that the characters for both smooth and wrinkled seeds existed in the hybrid children $(S w)$ was proved to be correct by his next experiment. Ile allowed the plants produced by the hybrid children $(S w)$ to closc-polinate

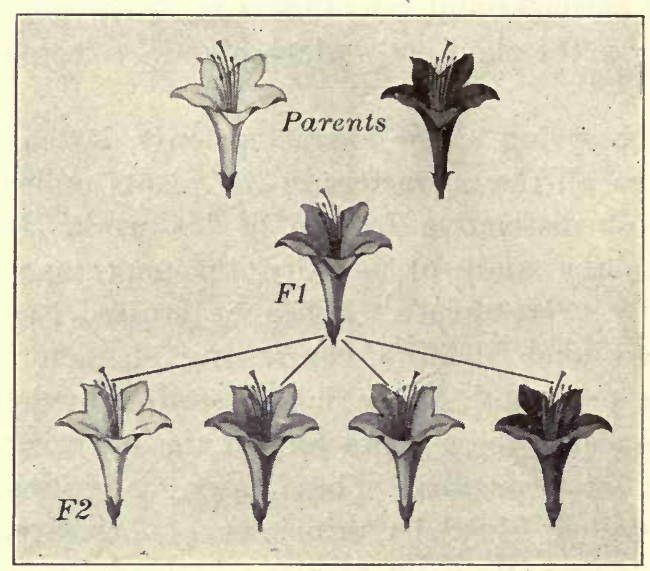

FIG. 97. Diagram illustrating crossing in Mirabilis

The cross between the white-flowered and red-flowered Mirabilis jalapa (four-o'clock) gives an intermediate pink in $F_{1}$. The usual Mendelian ratio is shown in $F_{2}$. After Morgan and produce seeds, the grandchildren of Fig. 96. By observing and counting a large number of these seeds he found that the total number of smooth sceds bore the same ratio to the total number of wrinkled seeds as that represented in the single pod in the figure; namely, as 3 to 1 . This is now known as the Mendelian ratio for the hybrids of the first filial generation $\left(F_{1}^{\prime}\right.$, children) resulting from a cross between parents that have distinctive characters, as in peas and Mirabilis (Fig. 97).

Furthermore, he found that plants grown from the ww seeds always bore pure ww seeds, represented as great-grandchildren, indicating that the original parental recessive strain had reappeared in the second hybrid generation ( $F$, grandchildren) in its original purity.

When, however, the smooth seeds were planted, the plants thus produced were found to contain some pure, smooth-seeded forms (SS), like the original dominant parent, and some hybrid forms $(S w, S w)$. The grandchildren, therefore, when judged by the seeds (Fig. 96), contained two strains ( $S S$ and $w w$ ) which 
exactly resembled the original parents ( $S S$ and $w w$ ). These two strains, when inbred, proved to be pure sirains, as indicated by the great-grandchildren ( $S S$ and $w w)$. 'The grandchildren also carried a hybrid strain ( $S w)$, as indicated above, which by inbreeding split up into pure strains ( $S S$ and $w w$ ) and into a hybrid strain $(S w)$.

This is clearly shown in the great-grandchildren offspring of $S w$ in the figure. Moreover, these pure and hybrid grandehildren occurred in a definite mathematical ratio of $\frac{1}{4}$ pure $S S, \frac{1}{4}$ pure $w w$, and $\frac{1}{2}$ hybrid $S w$. From this it follows that, on the average, out of every four grandchildren one would be pure $S S$, one would be pure ww, and two would be hybrid Sw. This ratio, established by Mendel in experiments with peas, has been found to hold true for a large number of plant and animal characters. In the case of other characters there is still much doubt concerning the applicability of Mendel's ratio. The fact remains, however, that Mendel's method of working with single characters, and his demonstration of a possible law for the combination of these characters in the offspring, has proved to be of immense theoretical and practical value to experimenters in heredity and plant breeding. 'These practical applications of Mendel's theories will be discussed at the end of the chapter.

Gametic purity. Mendel's interpretation of such results of crossing as we have just described will be made clear by Fig. 98 and the following brief explanation. Menclel assumed that the original parents ( $S S$ and $w w$ ) produced male and female gametes, each of which carried but one of the characters $S$ and $w$. In other words, the two characters which united at the time of fertilization, and which appeared to occur together in tlie seeds and adult plants, always separated in the gametes, so that the gametes (1) were pure and not mixed in composition, so far as the two characters were concerned. This is known as the law of gametic purity, which is perhaps the most important of Mendel's theoretical conclusions.

Since, moreover, the male gametes are borne by the pollen grains and the female gametes are formed in the embryo sacs of the ovules, chance pollination of the stigmas of a series of 
flowers would be almost certain to bring about all possible combinations of $S$ and $w$, when fertilization, or union, of the gametes occurred to produce the $F_{1}$ children.

Mendel assumed also that when the hybrid children ( $S w$ )

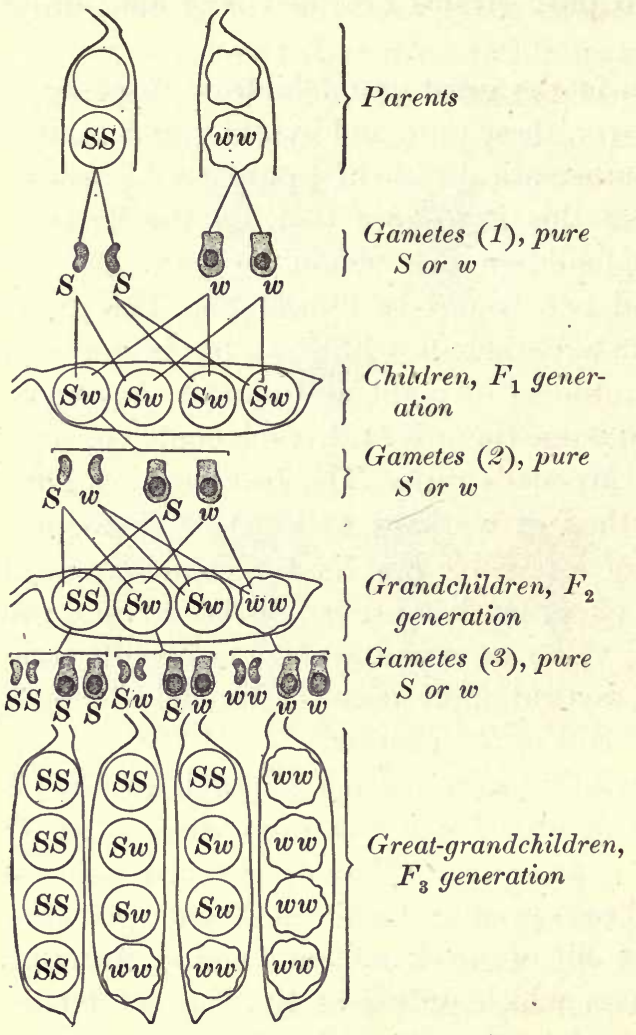

Frg. 98. Diagram illustrating Mendel's law of purity of gametes formed pollen and embryo sacs, the determiners for $S$ and $w$ would again segregate and occur singly in each germ cell. The gametes (2) would therefore carry $S$ and $w$ in the manner indicated in Fig. 98. Chance pollination and fertilization would then result in the combinations of $S$ and $w$ shown in the $\left(F_{2}\right)$ grandchildren. Gametes (3), formed by these grandchildren might then combine to produce pure and hybrid offspring in the ratio shown in the $F_{3}$ greatgrandchildren. If, therefore, we are willing to accept Mendel's idea of the purity of the gametes, we are prepared to understand how the offspring of hybrids may occur in mathematical ratios similar to those obtained by Mendel.

Paired contrasting characters. Mendel-also crossed peas in which the parents differed in two pairs of contrasted characters. In Fig. 99 the results of such an experiment are indicated by drawings and letters similar to those employed in Fig. 98. 
In this instance, however, Mendel crossed peas which bore smooth yellow seeds ( $S Y$ ) with peas having wrinkled green seeds $(w g)$. The large letters $(S Y)$ (Fig. 99) show that these characters were dominant in the offspring, wherever they occurred, and the small letters $(w g)$ in a similar manner indicate recessive characters. The children of this cross were hybrids in, which the two pairs of characters brought in by the parents ( $S Y$ and $w g$ ) were supposed to be mingled as indicated in the figure. These children were all smooth and yellow, like one parent, on account of the dominance of $S$ and $Y$ over $w$ and $g$. When close-pollinated or inbred, these children produced grandchildren of four different classes: smooth yel-

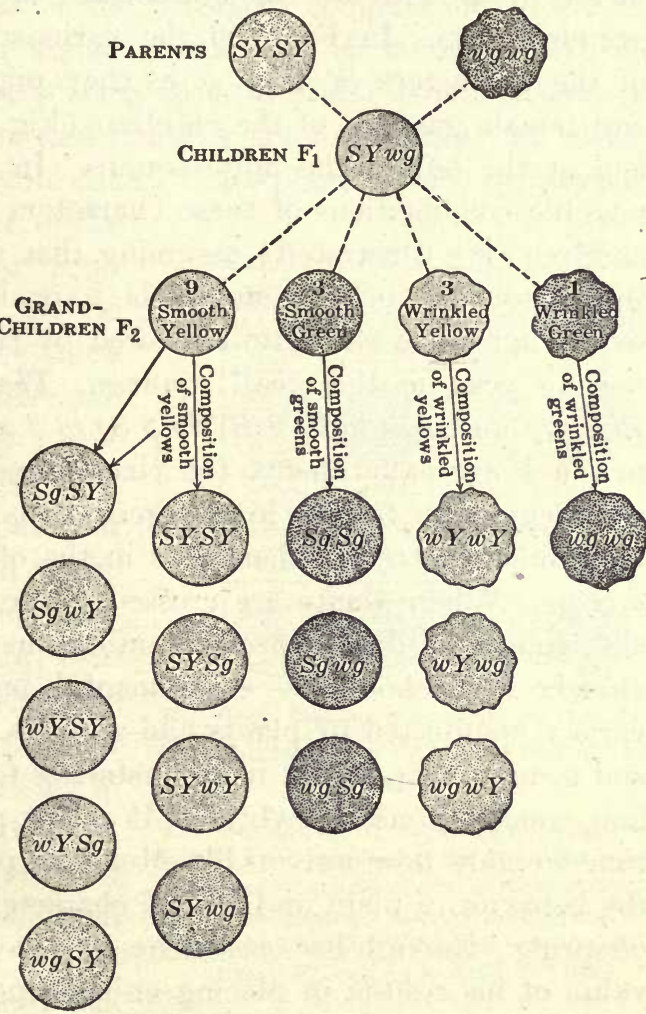

FıG. 99. Diagram illustrating the results derived from crossing peas with two pairs of contrasting characters

$S Y$ (smooth yellow) are dominant over $w g$ (wrinkled green). The figure shows the proportionate number of individuals in each class of the grandchildren $\left(F_{2}\right)$ and also the composition of each class in terms of $S Y w g$. low $(S Y)$, smooth green $(S g)$, wrinkled yellow ( $w Y)$, and wrinkled green $(w g)$. It will be noted that two of these ( $S Y$ and $w g$ ) resemble exactly the original parents, while two ( $S g$ and $w Y$ ) are new kinds of offspring due apparently to a new combination of the original 
parental characters $(S, Y, w, g)$. If we accept Mendel's conclusions as to the stability of plant characters and their separation in the germ cells, we can understand the above four classes of grandchildren. In Fig. 100 the various possible combinations of the characters $S, Y, w, g$, as they might occur in the male and female gametes of the children (Fig. 99), are shown above and at the left of the large square. In the small squares the possible combinations of these characters in the zygotes (grandchildren) are illustrated; assuming that wherever $S$ or $Y$ occur they would dominate $w$ and $g$, the four classes of grandchildren would appear in the ratio indicated by the small circles, representing peas, in the small squares. These ratios are shown in Figs. 99 and 100 to be $9 \mathrm{SY}$ to $3 \mathrm{Sg}$ to $3 \mathrm{wY}$ to $1 \mathrm{wg}$. According to the above experiments the characters of plants seem to be represented by factors in the germ cells which are capable of reproducing parental characters in the offspring resulting from a cross. When plants are crossed, these factors are reshuffled and appear in different combinations in the offspring. It has already been shown by experimental breeders that the great variety manifested by plants and animals, as regards color, form, and structure, is due in many instances to these chance Mendelian combinations in hybrids. It is not possible at the present time to state how universally Mendel's principles will apply to the behavior of plant and animal characters in hybrids and their offspring. Enough has been done, however, to indicate the great value of his results in placing animal and plant breeding upon a more accurate scientific basis.

Practical applications. The results of Mendel's work which are most important to the plant breeder are as follows: First, the breeder may find out by observation and experiment the various desirable and undesirable characters of plants which he wishes to cross. Second, he is certain that the desirable characters will not be contaminated or lost in crossing, although they may appear to be so in the children of the first parents. Third, he knows also that by proper care in self-fertilizing children of the first cross the characters of the original parents will reappear in all possible combinations in the grandchildren and their progeny 
if properly bred and cared for. When the combinations which he desires appear, as in Burbank's Shasta daisy and hybrid walnut, he can proceed to preserve the favorable combination which he knows is composed of stable characters. A favorite method, already indicated, of preserving such desirable combinations of characters is by grafting and budding the new variety on hardy but otherwise less valuable stock. Since plants grown by asexual means always come true to the original parents, a new combination of characters can be preserved indefinitely by these means. In other instances the new combinations may prove to breed true by seeds when the germ cells were pure for the characters combined in the new type. The type may also be maintained by careful continued selection. The scientific breeder is thus able to forecast results and plan his crossing in accordance with definite laws. In the above brief survey of Mendel's work all details and exceptions have been necessarily omitted, but enough has

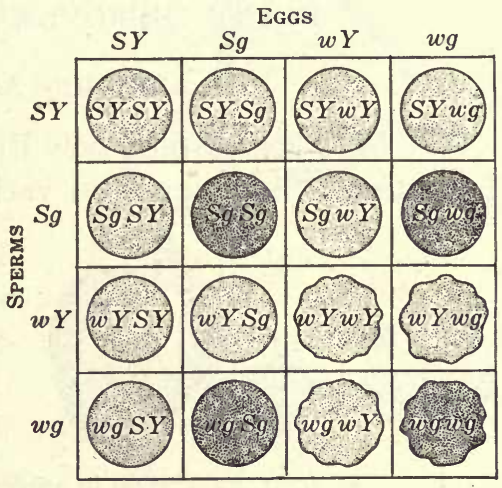

FIG. 100. Diagram showing method of determining the composition of zygotes shown in Fig. 99

The four classes of female gametes above and of male gametes at the left. The possible combinations of the gamete factors shown in squares. Considering $S$ and $Y$ dominant wherever they occur, we get the ratio $9 S Y$ to $3 S g$ to $3 w Y$ to $1 \mathrm{wg}$ been given to indicate to the student that, in the future, plant breeding is likely to become a science and not a game of chance, with results of profound importance to the improvement of plants and animals for man's uses. If this prediction proves to be correct, Mendel will always be known as the founder of a scientific method in plant and animal breeding. It is more than probable also that nature repeats, in the wild, the same experiments which man has learned to do in his culture beds and experiment plots. Nearly related species in nature are known to hybridize, and, such being the case, the hybrids must combine the characters of the parents in new mixtures, and then these mixtures 
must continually segregate in accordance with Mendel's laws in future generations. Much of the great and pleasing variety in nature would thus be produced, so that many of the so-called varieties and species of wild plants have doubtless arisen by these multifarious combinations and segregations of which we have had a glimpse in the above sketch.

\section{PLANT IMPROVEMENT BY SELECTION}

\section{Variation and Selection}

We have just learned how Burbank and other plant breeders have been able to produce variations, or differences, in plants
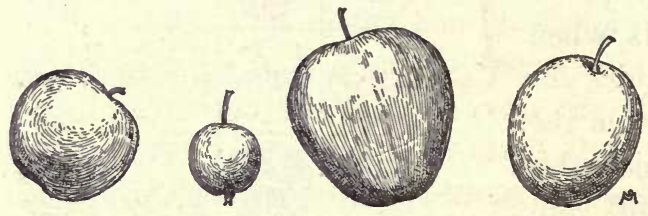

FIg. 101. Variation in apples

Four varieties of the Early Williams apple, selected from thirty-six varieties borne on seedlings. Modified from photograph by Burbank by crossing and hybridizing, owing to the fact that new combinations of parental characters are wont to appear in the offspring of a cross. Out of these differing offspring the cultivator selects those plants which best suit his needs or his desires, and endeavors to perpetuate their kind by vegetative means or by collecting and sowing seed.

This is the essential principle in all plant improvement by the selection of variations, and the general method is familiar to everyone. It is not always necessary, however, to resort to the tedious process of artificially crossing plants in order to secure the desired variations with which to start a new, improved race, since all plants normally produce offspring which vary among themselves in almost every conceivable manner. In a field of corn, for instance, some plants are tall and others short; in some the ears are produced well up on the stalk, while others are borne nearer the ground. The ears likewise vary in length, in the number of rows of kernels on the ear, and in the nature of the stored food in the kernel. Some of these differences in 
corn are due to natural crossing in the field, since corn is normally cross-pollinating. There is evidence also that every field of corn of the same kind or variety is made up of minor races (elementary species), which differ among themselves in some or all of the characters mentioned above. These races cross and produce the same kind of variations as arise from artificial crossing and hybridizing. In other instances the variations are due to differences in soil, water supply, climate, and tillage. Out of these multitudinous differences the corn breeder selects the plant or plants which suit his ideal, and proceeds to secure an improved race by careful selection and cultivation. Fig. 103 illustrates the great variability in ears of corn in the same field, due to the indiscriminate crossings between plants bearing poor and good ears. Fig. 104 illustrates the great improvement in uniformity and yield which can be secured by selecting the best ears for seed and by preventing crosspollination with inferior types for a series of years.

The gradual increase in yield of corn in Illinois, Iowa, and other great corn-producing states

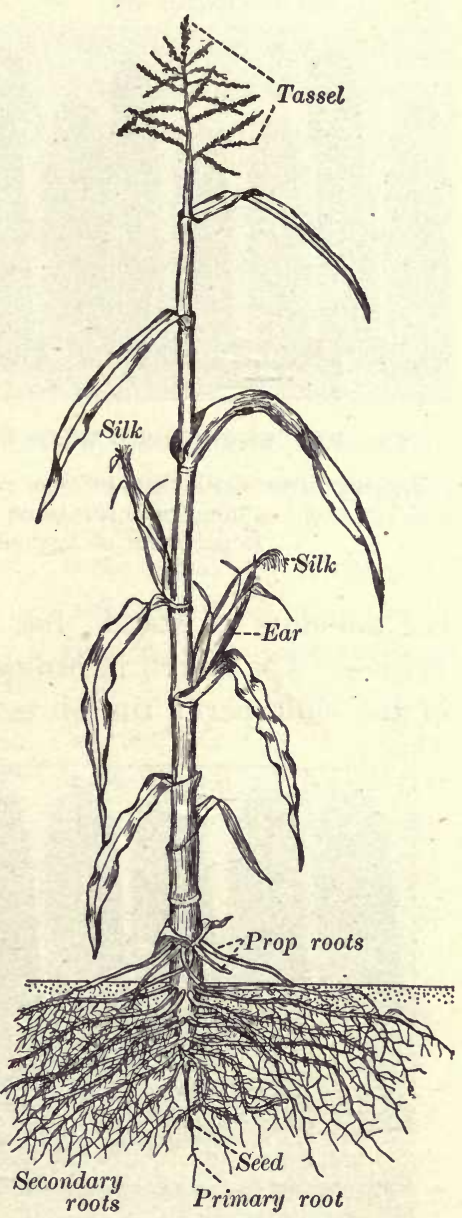

Fig. 102. A plant of Indian corn

Showing male inflorescence (tassel) and ears ready for pollination during the past decade is due in large measure to the fact that corn breeders have exercised more care than they formerly did in studying variations in corn and in selecting proper seed for 
planting. It has also been found that not only can the size and shape of the ear be thus improved by selection but also the

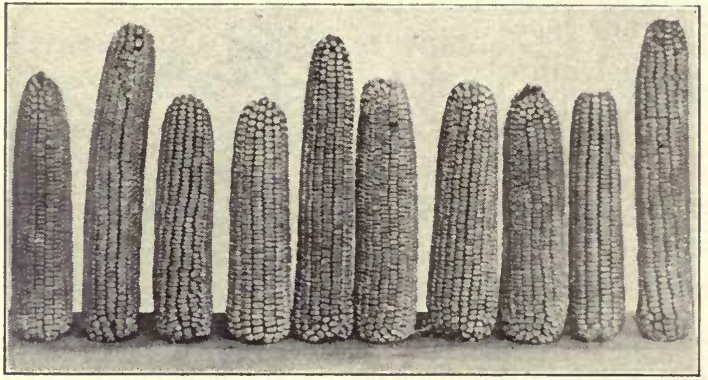

Fig. 103. Boon County White Corn, unselected

"Showing great variability in type resulting from failure to select seed." Photograph furnished by the United States

Department of Agriculture contents of the kernel, including the valuable commercial products derived from corn, such as starch, oil, and protein. In the Illinois agricultural experiment station work was started in 1896 to produce strains of corn with different amounts of starch, fat, or protein as reserve food in the kernels. 'The starch in ordinary varieties of corn forms the bulk of the endosperm, the oil is located largely in the embryo, and

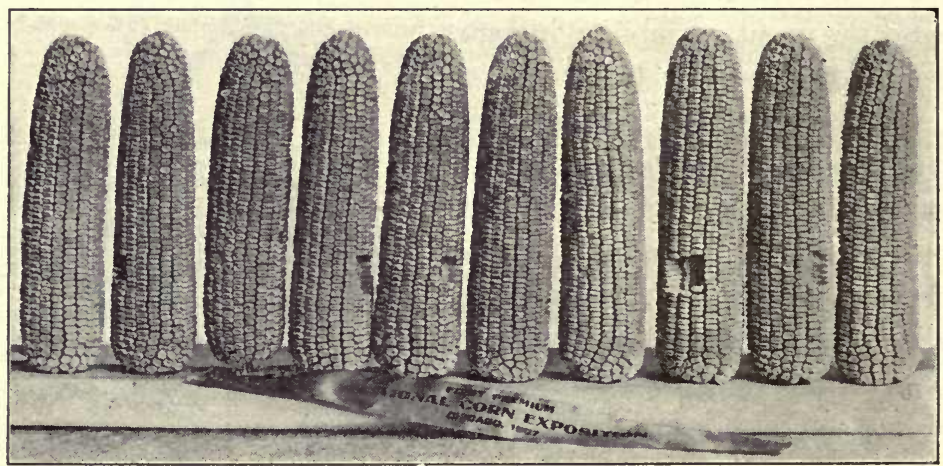

Fig. 104. Boon County White Corn, selected

Showing uniformity in type due to thirty-two years of seed selection. Photograph furnished by the United States Department of Agriculture

the protein is found in the outer portion of the endosperm next to the seed coat. By sectioning kernels on the ears of different plants the amount of starch, fat, or protein can be judged by 
the amount of starchy endosperm, the width of the protein layer, or the size of the embryo (Figs. 105 and 106). In this manner

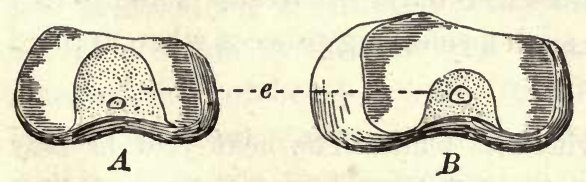

" one hundred sixty-three ears of Burr White Maize from the 1896 crop were analyzed and the proper selections in each case planted in their respective

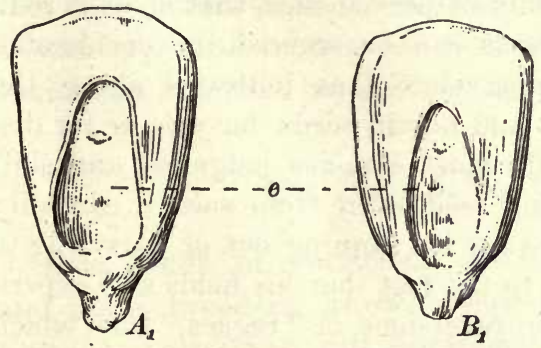

Fig. 1C5. Kernels of corn with high and low percentage of oil

$A, A_{1}$, transverse section and face view of kernels with large embryo having high oil content; $B, B_{1}$, same views of kernels with small embryos having low oil content. From Bulletin 87, University of Illinois Agricultural Experiment Station plots. In each plot from twelve to twenty-four rows were planted, and each row contained only the kernels from a single ear. In succeeding years a hundred or more ears were analyzed from each breeding plot, and those ears from the high-protein plot which showed the highest per cent of protein were saved to plant the next year's high-protein plot, while those from the low-protein plot were saved to plant the low-protein plot of the next season. Like methods were followed in the high-oil and lowoil breeding plots."

By this method the oil, which is called corn oil, was increased in six years in a strain of corn from 4.7 per cent to nearly 7 per cent, while the protein used in making hominy grits and for

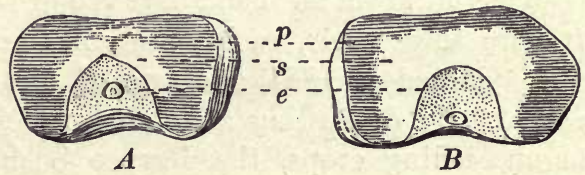

Fig. 106. Kernels of corn with high percentage of protein

$A$, high-protein kernel; $B$, low-protein kermel; $e$, embryo; $s$, starchy area; $p$, horny, or protein, layer. From Bulletin 87 , University of Illinois Agricultural Experiment Station feeding stock was increased from 10.6 per cent to 16 per cent.

The same general procedure outlined above for the production of improved types of corn by the selection of favorable 
variations is followed in the improvement of other kinds of fruit, forage, and food-producing plants. The following extract from "Plant Breeding," by Professor Bailey, illustrates some of the detail and care necessary in such a selecting process when applied to the cultivation of beans.

He [the breeder] starts with one plant. The next year he may have only two. If he has ten or twenty good ones, then the task is easy, for the variety has elements of permanence, that is, of hereditability, in it. As soon as seeds can be secured in considerable amount from a strain of beans, selected as indicated above, the grower can plant a large plot and obtain seeds for sale or for distribution to other breeders. He must exercise judgment and skill each year, however, in selecting seed, even from such a carefully improved race, if he is to prevent its running out or reverting to the original type. This is due to the fact that his fields and experimental plots will always contain reversions, or " rogues," from which seed must not be taken if his improved strain is to be kept true to type. In the common dwarf, or bush, beans of the gardens, for instance, there is always a tendency to revert to the ancestral climbing variety. These rogues with a tendency to climb must, therefore, be eliminated if the bush habit is to be perpetuated.

Mr. Palmer's dwarf lima originated in 1883, when his entire crop of large white (pole) limas was destroyed by cutworms. He went over his field to remove the poles before fitting the land for other uses, but he found one little plant, about ten inches high, which had been cut off about an inch above the ground, but which had rerooted. It bore three pods, each containing one seed. These three seeds were planted in 1884, and two of the plants were dwarf like the parent. By discarding all plants which had a tendency to climb, in succeeding crops, the Burpee bush lima, as we now have it, was developed.

Difficulties. Two main difficulties are experienced by breeders in their attempt to improve plants by the selection of variations. One of these difficulties has already been referred to, namely, the tendency of varieties and races thus produced to revert to the condition of ancestors of the less desirable type, a phenomenon expressed by the term running out. A second point of importance is that races built up by the continued selection and 
accumulation of variations are limited in the degree to which they can be improved. Thus, the sugar beet has been cultivated and selected for a hundred years or more, but it has not been found possible to increase the average sugar production of a selected field of beets beyond from 16 to 18 per cent. The Illinois experiment station has had a similar experience in attempting to raise the percentage of oil and protein in corn beyond the high percentages mentioned above. A similar condition seems to prevail among all cultivated varieties, where ordinary variations are used as a basis for selection.

\section{Two Kinds of Variations}

Two kinds of variations are now recognized by scientists and plant breeders, known respectively as fluctuating (or continuous) variations, and mutations (or discontinuous variations). Fluctuating variations are the ordinary differences observed in plants of the same kind in a field or a garden. They are due to the environment, such as rich or poor soil, abundance or lack of water, sun exposure or shade, and many other environmental influences to which plants growing in the open are subjected. They are furthermore inconstant,

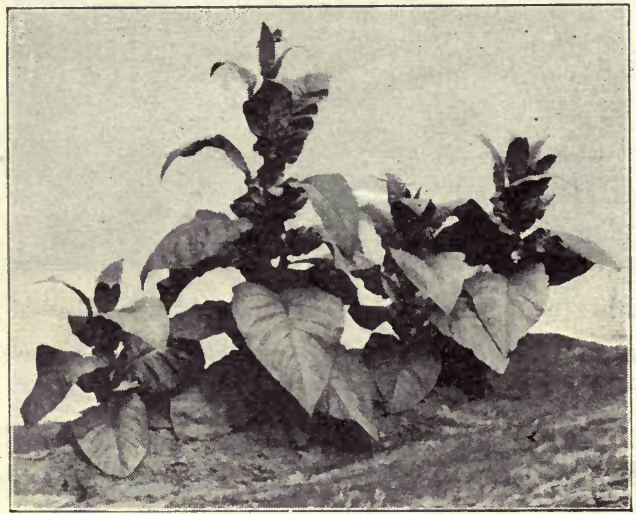

Fig. 107. Connecticut broad-leaf tobacco, unselected

Showing variation in tobacco due to change of soil and climate. Photograph furnished by Connecticut Agricultural Experiment Station

not tending to be perpetuated in the offspring of plants manifesting them: We all know, for instance, that seeds from a large, healthy tomato plant, produced by rich soil and careful culture in a garden, will not bear similar offspring unless they 
grow under like conditions. It seems probable, therefore, that the running out of selected races of corn, beans, and other cultivated plants mentioned in the previous pages is due to the selection, on the part of cultivators and breeders, of plants with these fluctuating variations. Fluctuating variations can be accumulated to a certain extent, and a better race may thus be

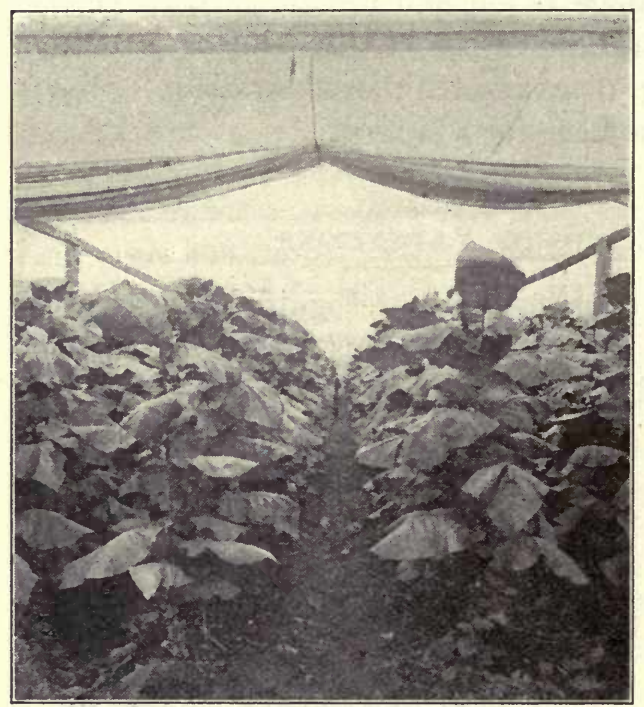

Fig. 108. Uncle Sam Sumatra tobacco, selected Showing uniformity of type in tobacco due to selection of seed. Photograph by the United States Department of Agriculture

produced; but such a race will always remain inconstant and limited in range or in the degree to which it can be improved. Thus, in the tobacco plant (Fig. 107) great variation has been induced in certain varieties by planting in the soil and climate of Connecticut and other Northern states seed obtained in Sumatra and Florida. Some of these variations are of the fluctuating variety, and so will not form the basis for a stable improved race produced by selection. In other instances the variations induced in tobacco by the change in climate and soil are apparently stable from the outset, and form the basis for new constant races. The Uncle Sam Sumatra tobacco (Fig.108) is supposed to have had such an origin by mutation.

The Florida-Sumatra tobacco was grown from seed secured from the island of Sumatra. When the Florida-grown seed was taken to Connecticut, the plants grown from it varied in a marked degree, and several new types developed that did not exist in Florida and, according to the best information obtainable, did not exist in Sumatra. 
The Uncle Sam type appeared among these variations and has proved to be constant when grown from seed. In tobacco, therefore, as in many other plants, two kinds of variations frequently occur which are of very different value and importance in the improvement of these plants by breeding. The inconstant, or fluctuating, variations are not of great value for selection in the making of an improved race, while the stable variations called mutations are of the greatest interest and importance to breeders of new kinds of plants and animals.

\section{The Mutation Theory}

Certain varieties of plants, like the Uncle Sam Sumatra tobacco, have long been known to have arisen suddenly as a result of variations affecting one or more parts of the plant body. The plants manifesting these new variations have usually been called sports in agri-

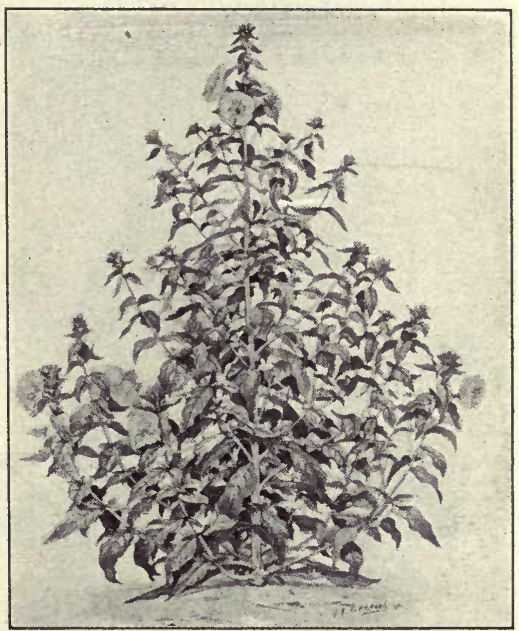

Fig. 109. Enothera lamarckiana

This is the mother plant of mutants discovered and produced by De Vries. From Babcock and Clausen's "Genetics in Relation to Agriculture." After De Vries culture and horticulture, but are now called mutants. The variations which distinguish them from the parent species are called mutations. Among the best known of such sports, or mutants, are the following: the moss rose and the nectarine, which are bud sports from a cultivated rose and from the peach; the various cut-leaved varieties of the willow, maple, and birch; many white-flowered varieties springing from plants with colored flowers; and probably many varieties of vegetables, forage plants, cereals, and fruits whose history, when traced back, indicates a sudden origin from wild species. The mutation theory for the origin of new varieties by sudden constant 
variations has been brought into prominence in recent years through the publications of the great Dutch botanist, Hugo de Vries. De Vries first noticed some mutants of the evening primrose known as Lamarck's evening primrose (Enothera lamarckiana) (Fig. 109) growing in a waste field near Hilver-

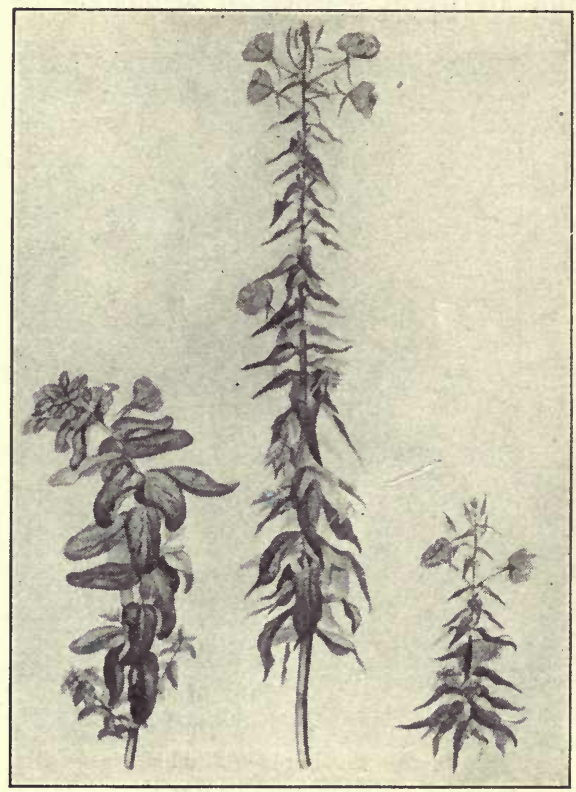

Fig. 110. Enothera lamarckiana and two of its mutants, $\mathscr{E}$. lat $\alpha$ and $\mathscr{E}$. nanella

E. lamarckiana in the middle; lata at the left; and nanella at the right. From Babcock and Clausen's "Genetics in Relation to Agriculture." After De Vries sum in Holland. In 1886 he collected seeds from the mother plant and from the two new varieties produced from it by mutation, and sowed them in the botanic gardens at Amsterdam. He has since carried on extensive culture experiments with the primroses and their offspring, and has succeeded in producing several new varieties which differ from the original mother plant in all of the various organs of the plant body. One is a dwarf species (Fig. 110), while another is a giant form with greater vegetative vigor and larger flowers than the mother plant. Others vary in the form and color of the leaves, in the character of the seedlings (Fig. 111), and in the nature of the reproductive organs, including both the flower and the fruit. Since all of these new primroses came suddenly, by one large variation or mutation, and have bred true to seed, De Vries believes that they indicate the way in which other cultivated and wild varieties have arisen whose history indicates a similar method of production. This sudden origin of cultivated forms is well illustrated by the 


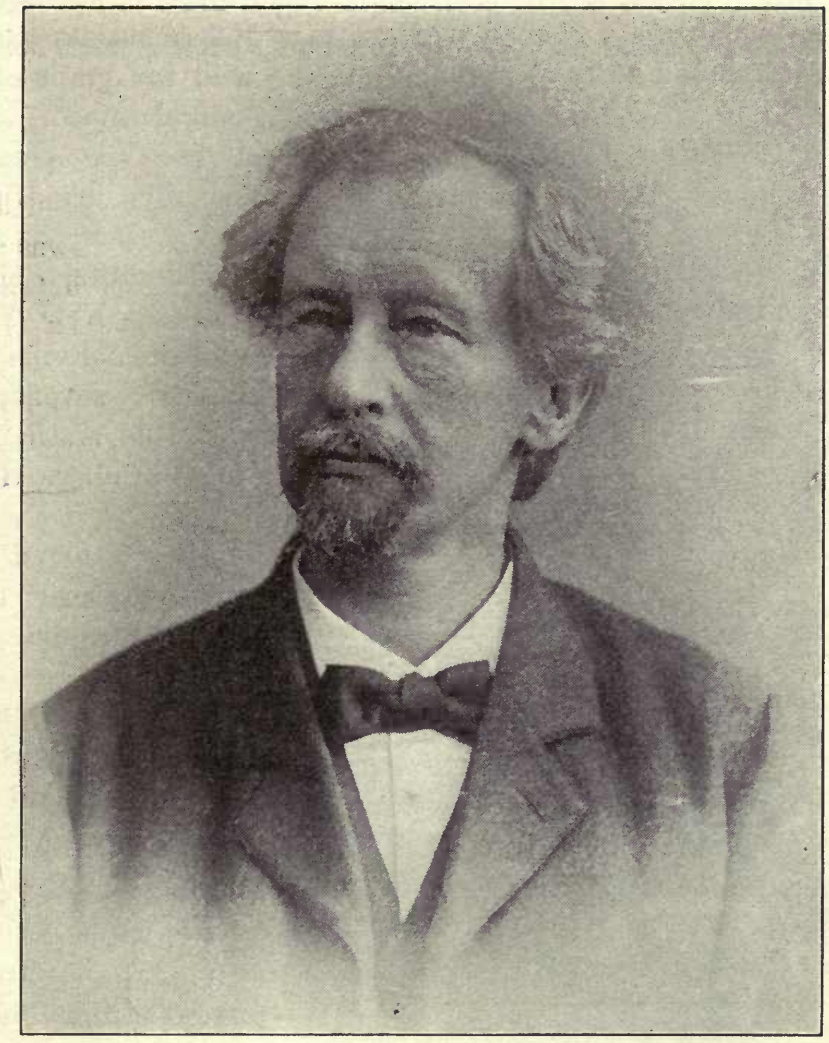

HUGO DE VRIES

Hugo de Vries is director of the Botanic Garden in Amsterdam, Holland. He is the greatest living exponent of the origin of species by sudden constant variations, or mutations. His early investigations on variation, which convinced him of the truth of the mutation theory, extended over a period of nearly twenty-six years and involved experiments and breeding tests with some hundred species of wild plants. His experiments and theories are set forth in his greatest work, "Die Mutations Theorie," and in numerous succeeding books and articles. In his method of work and in the importance of his results De Vries is perhaps more nearly comparable to the great Darwin than any other living investigator 
history of certain species of grapes recounted by Professor Bailey in his "Evolution of our Native Fruits."

The Concord was a chance seedling in a Massachusetts garden, and it is supposed to have sprung from the wild fox grape of the

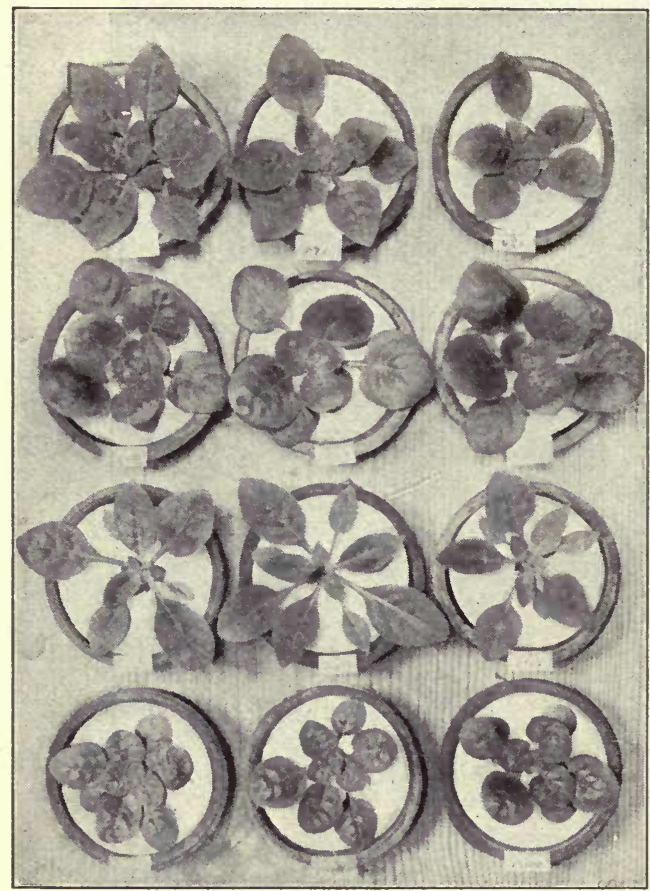

FIG. 111. Seedlings of $E$. lamarckiana and three of its mutants, showing constant differences in rosettes and leaf characters

Upper row, typical lamarckiana; second row from top, E. gigas; third row, EE. rubrinervis; bottom row, $Q E$. lata. Photograph furnished by Dr. George H. Shull neighborhood.

The Clinton came up where a handful of grape seeds was sown at Hamilton College, Clinton, New York; and the old vine, now nearly seventy-five years old, is still growing on College Hill.

The Norton's Virginia was found wild in 1835 near Richmond, Virginia.

The best American gooseberries, the Houghton and the Downing, are supposed, like the Concord and Clinton grapes, to have originated from chance seedlings. At the Swedish experiment station, located at Svalöf, Sweden, new kinds of grains, forage crops, and vegetables have been discovered in the field and garden which are believed to have arisen by mutation, and similar discoveries have been made in different experiment stations in this country. Many plant breeders and botanists believe, therefore, that the origin of new forms of plants by mutations, or sudden variations, is a widespread phenomenon 
in nature, which bids fair to be of inestimable value in the improvement of wild and cultivated ornamental and food plants.

Selection of mutations. According to the above theory a field of oats, corn, timothy, or beans is likely to be made up of plants

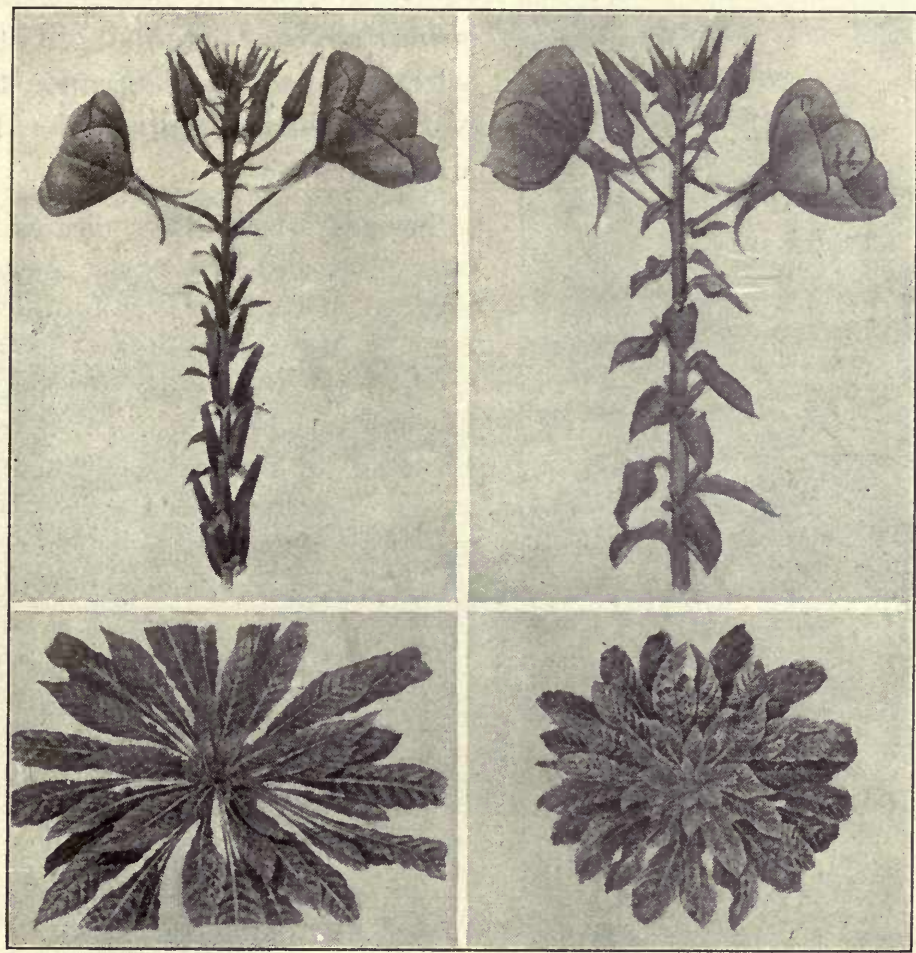

FIG. 112. Inflorescences and rosettes of $E$. lamarckiana and $E$. gigas

Left, E. lamarckiana; right, $E$. gigas. Above, inflorescences; below, young plants (seedlings). From Babcock and Clausen's "Genetics in Relation to Agriculture"

which differ from each other in almost every conceivable manner. Some of these differences would certainly be due to fluctuating variations, and some to previous hybridizations. In other instances, if the experience of the Swedish and American experiment stations is a criterion for judgment, the differences are due to mutations, possibly induced by changes in soil, climate, or culture methods. 
The new forms thus produced by mutation are constant small species, or "elementary species," termed mutants. If these new plants can be recognized and selected, and the seeds sown in

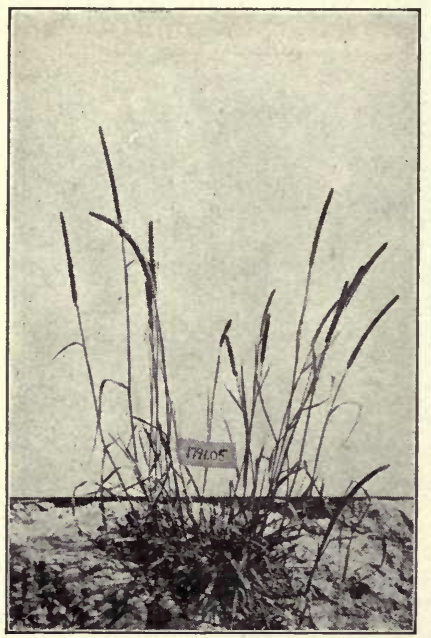
isolated breeding plots, it is possible to secure a new constant strain at once without further selection. In reporting upon the work of the Swedish station De Vries says :

Besides wheat, the cultures of oats, peas, and vetches are seen to produce sports from time to time at Svalöf. Here also the sports are sudden and without preparation or intermediates, each of them at once constituting a new type, which is as distinct from its allies as any new form found in the fields.

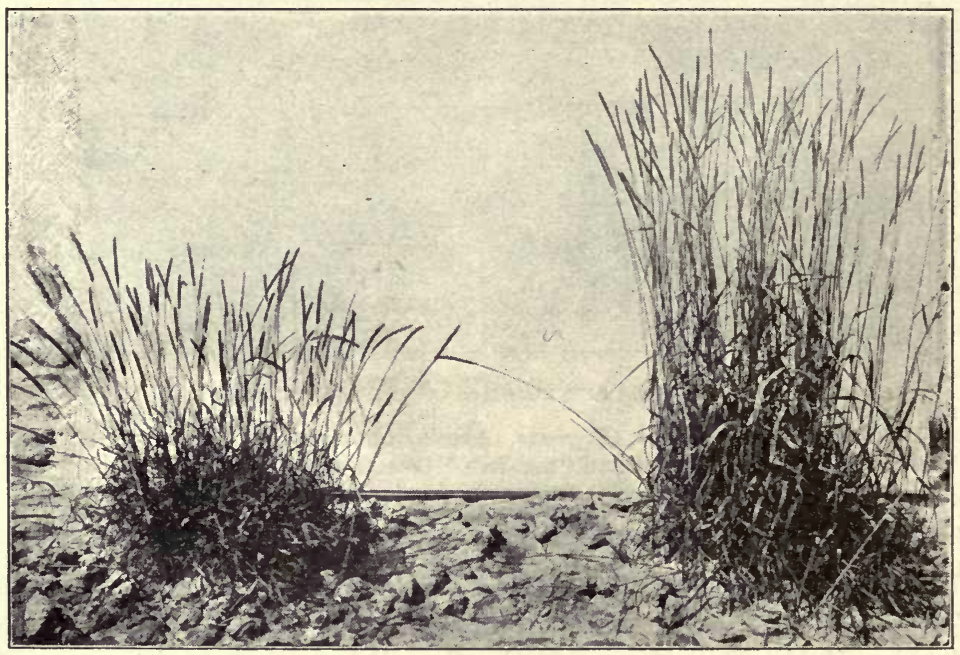

Fig. 113. Variation in timothy

The two lower plants grew side by side from the same seed. Desirable type at the right; dwarf plant at the left. Dwarf plant with large spikes in the upper figure. Photograph by New York Agricultural Experiment Station 
In this manner improved races of cereals and forage crops, adapted to the varying soil and climatic conditions of the country, have been obtained in Sweden at the Svalöf station.

This method of selecting seeds from one plant and growing them in isolated culture plots (Fig. 114), so that the progeny of single individuals can be observed and recorded, is called pedigree culture. Pedigree culture has been practiced for many years in the breeding of animals, but its application to plant breeding

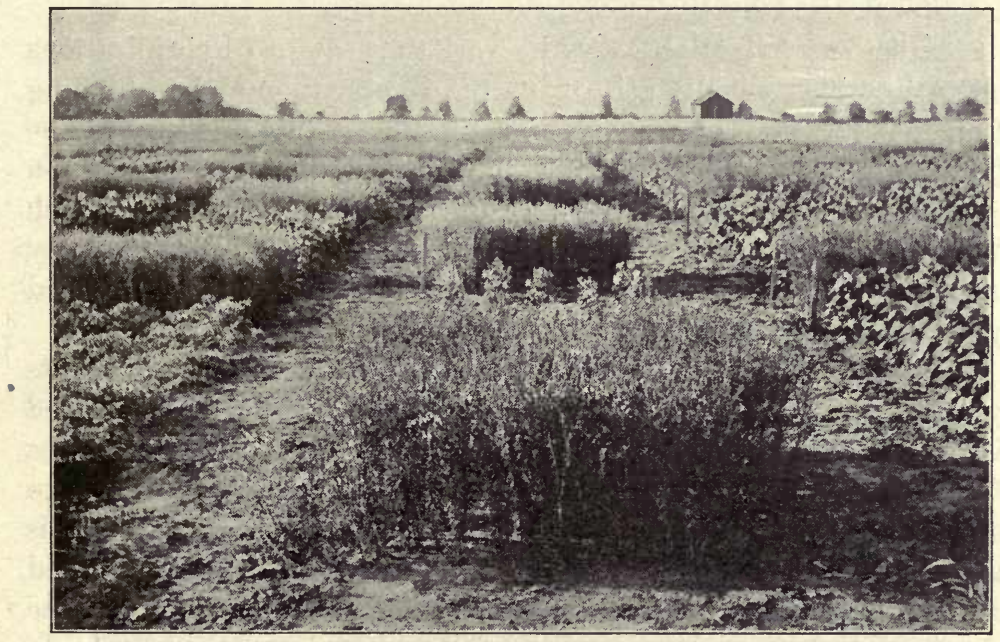

Frg. 114. Centgener plots of flax and beans alternating

One hundred seeds are planted in each centgener plot. Photograph furnished by the United States Department of Agriculture

has been greatly stimulated in the experiment stations of this country by the experiences of the Swedish station mentioned above. Pedigree culture is thus very different from the older mass-culture method, by which seed is collected from a considerable number of the best plants in a field and then sowed en masse, without any attempt to trace or record the progeny of one particular plant. The mass-culture method is often used for selecting the better varieties from a general field, and is then followed by pedigree culture applied to a few best individuals selected from the mass-culture plots. 


\section{SUMMARY}

Variations. Variations form the materials from which new and improved races of plants are built up, either in wild nature or in culture. These variations arise in three ways; namely, by crossing and hybridization, by induced or spontaneous fluctuating variations, and by mutation.

Selection is the method employed in all cases for the isolation of a new race, or for the gradual accumulation of variations in the direction desired by the cultivator.

Selection and hybridization. The variations due to hybridizations and to crossings usually arise from the combination and reshuffling of characters which originally existed in the parents of a cross. The offspring produced by a cross split in succeeding generations, in accordance with Mendel's laws, into a great variety of forms, in which the parental characters are differently combined. Out of the variety thus produced new and improved races can be selected and multiplied by pedigree culture.

Selection of fluctuating variations. The improvement of plants by the continuous selection of forms which vary in the desired direction is one of the oldest methods of securing improved races of cultivated plants. "Nature gives successive vairiations; man adds them up in certain directions useful to himself. ... The key is man's power of accumulative selection." Darwin thus expressed the essential facts relating to the improvement of plants by the selection of fluctuating variations. The points of disadvantage in the method are, first, that races and varieties thus produced are unstable, tending to run out when selection ceases; and, secondly, that they are limited in the degree to which they can be improved by selection.

Selection of mutations and pedigree culture. Mutations are variations in one or more characteristics of a plant which arise suddenly and are stable from the beginning. These mutations are either small, creating the impression of a mere variation in leaf, flower, or fruit, or they may be large enough to produce at once a new kind of plant, which will be recognized by botanists as a new variety or small species, now called an elementary species. The recognition and selection of mutations, especially those large enough to constitute a new elementary species, is one of the surest and best methods for securing an improvement in any variety of plant. The reason 


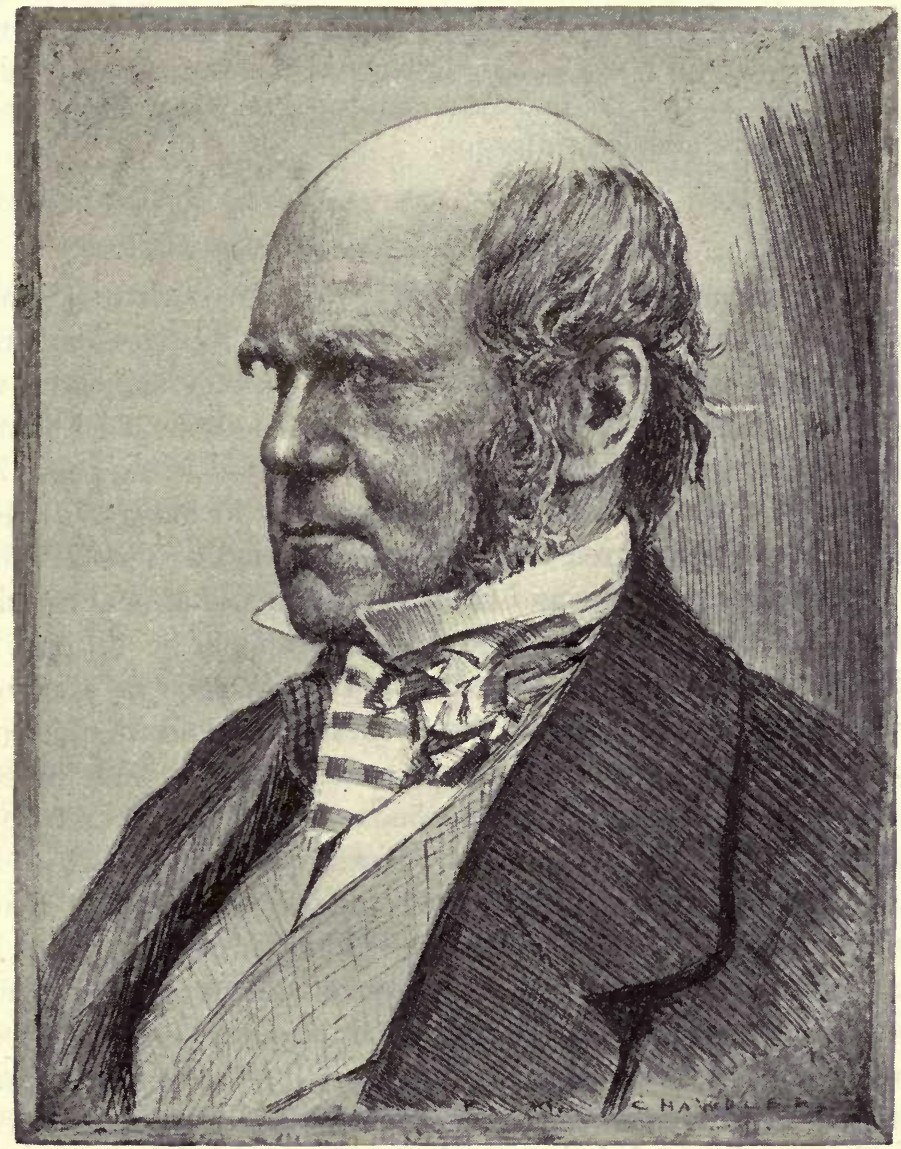

\section{CHARLES DARWIN}

Charles Darwin published his first great book, "On the Origin of Species," in 1859. This book was the outcome of over twenty years' work spent in collecting data on problems relating to variation, heredity, competition, and survival in organisms. Darwin's later publications and investigations were designed to vindicate and extend the theories and facts contained in the "Origin of Species." It is safe to say that no other scientific man ever exercised a greater influence on the thought and progress of mankind. From Bergen and Caldwell's "Introduction to Botany" 
for this is that the forms thus selected remain true in cases where the new race is produced by hybridization. The detection and cultivation, by pedigree-culture methods, of mutations occurring in field and garden crops is one of the most modern phases of plant improvement and is an important addition to the older methods of mass culture and hybridization.

\section{Evolution}

The facts and processes relating to the evolution of plants cannot be dealt with at any considerable length in an elementary textbook. The beginning student should understand, however, the close relation which exists between the methods used by man in the improvement of plants outlined in the preceding pages and the process of evolution among wild species in nature. Indeed, Charles Darwin, the greatest of all students and writers on evolution, took the experience and results of plant and animal breeders as the basis for his theories and investigations in evolution.

Selection of fluctuating variations. Darwin thought that new species arose in wild forms of life by the gradual accumulation of fluctuating variations, in much the same way as a plant breeder now produces a larger fruit or a more beautiful flower by constantly selecting the best plants from those which tend to vary in a given direction. Darwin also endeavored to show that a process of selection takes place in wild nature not unlike that exercised by man, due to the competition and struggle occurring among plants, caused by the overproduction of seeds. "Can it then be thought improbable," says Darwin, "seeing that variations useful to man have occurred, that other variations, useful in some way to each being in the great and complex battle of life, should occur in the course of many successive generations. If such do occur, can we doubt (remembering that many more individuals are born than can possibly survive), that individuals having any advantage, however slight, over others, would have the best chance of surviving and procreating their kind. This preservation of favorable individual differences and variations, and the destruction of those which are injurious, I have called natural selection, or the survival of the fittest." 
The ideas concerning selection through struggle for existence, emphasized in the above extract from the "Origin of Species," can be most easily understood from concrete illustrations. The writer once made an estimate of the number of seeds borne by the common dandelion in a single season. Rather large, healthy plants produced on the average about ten fruiting scapes, and each seed ball on a scape averaged from 160 to 260 seeds. This would mean an average of from 1600 to 2600 seeds borne on each dandelion plant in a single season. The Russian thistle has been estimated to produce from 20,000 to 200,000 seeds on a single plant. If all of these seeds gained a foothold and grew into offspring, it can readily be understood that the earth might be entirely populated with dandelions or Russian thistles in a comparatively few years, since both plants are well adapted for distributing their seeds. It must be remembered, however, that the plants with which the dandelion and Russian thistle have to compete for soil space, air, and light are also producing seeds and offspring at corresponding rates, and that some of their competitors, like the grasses, have vegetative means for spreading which are quite as effective as seed production. It is conceivable, therefore, as Darwin has said, that in the fierce competition among plants, due to overproduction, only those individuals would survive in a given environment which possessed structural and physiological characteristics best adapted to the conditions of that environment. The remainder would die, and the bestadapted plants would live to perpetuate the race, just as the particular races of cultivated plants now in existence are survivors from plants formerly selected by man. In this manner any given race would gradually improve, and so evolution would take place. This selective principle is also easily understood if we contemplate extreme conditions such as obtain in deserts and in lakes or ponds. The vegetation of these two habitats is very distinct, for the reason that extreme drought, on the one hand, and excessive water supply, on the other, destroy all seeds, seedlings, or mature plants which are not definitely adapted to a xerophytic or a hydrophytic habitat. Each year seeds from other habitats are distributed over deserts and lakes, but only those 
best adapted to the conditions survive, so that the cacti, the bulrushes, and the algæ are in a true sense the fittest survivors, selected by the extreme conditions of their environment.

These habitats are not unlike a very exacting plant breeder, who ruthlessly discards all plants in his culture plots which do not conform to a rigid ideal which he has started out to attain. In the development of cultivated races from fluctuating variations it has been found, however, as has already been stated, that such races are both unstable and limited in the degree to which a given variation or tendency can be developed. If the experience of the future confirms that of the past, it would seem to furnish a formidable objection to Darwin's conception that in wild nature fluctuating variations may be accumulated until a new species is formed.

Selection of mutations. Darwin was aware of the fact that sports, or mutations, occasionally arose in nature, but he did not think that they occurred with sufficient frequency or in sufficient numbers to be of great importance in the evolution of wild species. The experiments and writings of De Vries, however, and the experience of breeders and botanists who have been stimulated by his discoveries, seem to indicate that mutations may be a more important factor in evolution than Darwin supposed. Variation and selection would here, as in evolution through fluctuating variations, be the fundamental principles concerned in the origin and perpetuation of improved varieties or species. "Species," says De Vries, "are derived from other species by means of sudden small changes, which, in some instances, may be scarcely perceptible to the inexperienced eye. From their first appearance they are uniform and constant when propagated by seed ; they are not connected with the parent species by intermediates and have no period of slow development before they reach the full display of their characters." Some young species will be better fitted for their life conditions than others, and the struggle for life will induce a selection among them by which the fittest survive. And again: "Thus we come to the conclusion that natural selection is as active as Darwin assumed it to be, and is as preëminent a factor in evolution. It causes the 
survival of the fittest; but it is not the survival of the fittest individuals, but that of the fittest species, by which it guides the development of the plant and animal kingdoms."

\section{SUMMARY}

Whether we accept the theory of Darwin or that of De Vries, it will be evident to the student that the production and survival of improved races of plants has proceeded along the same general lines in culture and in wild nature. Variations, either of the nature of fluctuating variations or of constant mutations, are necessarily the starting points for the origin of a new species or variety. Selection of the best-adapted plants for a given human purpose, or to fit the conditions of a given environment, are then made by man, or by nature, through competition and struggle.

The survival of the fittest, in nature, means that certain plants possess characters, produced by variation, which enable them to live in certain habitats in competition with their neighbors, while other plants of the same species or kin are unable to do so. The survival of the fittest, in culture, means that those plants live and perpetuate their kind which most nearly meet the needs and desires of man in different countries and regions. They are not necessarily the plants best adapted to wild life in any given environment, since man creates a new environment for the plants of his choice by irrigation, by fertilizers, and by cultivation. In other words, man adapts the environment to the plants of his choice.

The double roses, chrysanthemums, and peonies are not the fittest plants to survive in their native habitats. They survive under culture because they are propagated and cultivated in an artificial environment created by the horticulturist and the plant breeder. Despite these discrepancies in the final results, evolution in nature and plant improvement in culture are based upon the same general laws of variation and heredity, and are dependent upon the same general principle of selection. 


\section{CHAPTER XI}

\section{HISTORICAL DEVELOPMENT OF BOTANY AND THE BIOLOGICAL SCIENCES}

Biology in its broadest sense includes all studies pertaining to the form, structure, and activities of living plants and animals. In many American colleges and universities, however, the term biology is used in a restricted sense, to designate an introductory course in biological science for beginning students. In such an introductory course the general facts and principles of the science are presented comprehensively from the standpoint of both plant and animal life, in order either to lay a foundation for more extended studies in botany and zoölogy or to serve as a basis for general reading and culture. In the actual work of the teacher or the investigator it has been found necessary, however, to subdivide the science of biology into botany, the science of plants, and zoölogy, the science of animals. These two sciences are again subdivided into many lesser branches, often termed sciences, since no one mind can any longer master more than a small corner of the ever-widening field of biology. This subdivision of biological science into its smaller branches has come about in a natural way as the knowledge of animals and plants has gradually increased by study and research.

Classification, systematic biology, or taxonomy. The early studies of both botanists and zoölogists were made in an attempt to classify plants and animals into groups based upon external resemblances. These early attempts at grouping organisms gave rise to a definite branch of biology now known as classification, systematic biology, or taxonomy, represented in our many manuals of botany and zoölogy, which are intended to enable students to secure a clearer idea of the relationships and general attributes of common plants and animals. In botany Asa Gray was the greatest early systematic botanist in the United States, and we still 
have Gray's manuals as our principal aids to a better knowledge of the classification of our common wild flowering plants. Since, however, a correct classification of any group of organisms, such as oaks, grasses, or fishes, is based not only upon external characters but upon details of structure and development as well, it is quite obvious that we can never make a reliable classification of any group of living things until we have a more or less complete knowledge of the comparative structure and development of the individual members of such a group. Such a knowledge of the great groups of animals and plants which are now classed together is not yet available, and classification, although the oldest, is consequently a more or less artificial branch of biological science to-day.

Morphology. As the name indicates, morphology is the science of the form of the animal or plant body; but it has come to include the structure and development, as well as the form, of liṿing organisms. It may include simply the study of the general form, structure, and development of a single organism or it may refer to a comparative study of the main organs, such as leaves, stem, roots, and flowers, of a group of related organisms. Applied to such organisms as pine trees and their allies, it would comprehend the common conelike habit of pines and the gross structure and form of their branches, leaves, roots, and reproductive cones. These factors, taken together, determine the characteristic form, appearance, and mode of reproduction of members of the pine family. Since morphology has come to include the structure and development as well as the form of organisms and their parts, special terms are now used to designate the more detailed studies of structure and development which have grown out of the older and grosser morphology.

Anatomy. The term anatomy is usually applied to the more general studies of the structure of the tissues and organs of both animals and plants. In plants anatomy is necessarily microscopic, while in animals it includes the grosser observations of the organs and tissues which can be acquired by dissections. Thus, the anatomy of a pine tree would include the microscopic structure of sections of the trunk, leaves, branches, 
and reproductive organs, while the anatomy of a cat would include the gross structure of the tissues and the main organs of the body.

Histology. In plants histology is easily confused with anatomy, since each includes the microscopic structure of the tissues and organs, although histology in plants comprehends more detailed microscopic structures of the cell units which make up the tissues and organs than is the case in plant anatomy. In animals, however, histology is distinct from anatomy as it is generally understood, since histology in animals means the microscopic structure of the cell units of the parts of the animal body, which are observed in gross in anatomical studies.

Cytology. Cytology is the newest of the branches of biology yet mentioned, and includes the minute microscopic structure of the cell units of living organisms and also of the ultimate structure of living matter.

Embryology. As the term is commonly understood, embryology applies to the processes of development of an organism from the early divisions of the fertilized egg to the adult stage, in which the various organs and tissues have reached their ultimate form and structure. Embryology, like morphology, may include simply the processes and stages in the development of an individual organism, or it may be comparative in its nature and include a comparisonof the developmental processes of a series of related organisms.

Physiology. Opposed to morphology and its subdivisions is physiology, which deals with the functions of organs and parts of single individuals or with the activities of organisms in general. The field of physiology is rapidly extending to-day into the older fields of biology, as is indicated by the newer studies in experimental morphology, ecology, embryology, and evolution. In all of these new fields of study the facts accepted by older systematic and morphological biology are being tested by physiological experiments and observations.

Ecology. The newest of the main subdivisions of biological science is ecology, which comprehends the relation of plants and animals to their environment and the relation which exists between form, structure, and environmental influence. Applied to plants it includes the effects of light, heat, moisture, and soil 
on the ultimate form, structure, and reproductive processes of plants in a given home, or place of growth. In its wider applications ecology includes the plant associations in different localities, and the way in which environment affects migration and the distribution of organisms on the earth's surface. In addition to these more or less definitely conceived branches of biology there are many others, of great theoretical and practical importance to mankind, which are difficult to classify on account of their close interrelation and overlapping.

Evolution. Ever since the time of Charles Darwin the term evolution has included all studies in the method of origin and development of the higher forms of plant and animal life from lower forms. The idea of evolution, since its acceptance, has determined the aims and methods of study in all branches of biological science, and may properly be said to dominate all studies in comparative morphology, embryology, physiology, and ecology.

Closely connected with evolution are such subjects as the dispersal and distribution of organisms, and the modern studies in heredity, variation, and breeding, now being included under genetics.

Industrial and applied biology. Under industrial and applied biology is included the application of such purely scientific biological pursuits as bacteriology, mycology (the study of fungi), physiology, and genetics to medicine, sanitary science, agriculture, horticulture, and forestry. This application of economic biological principles to the problems of agriculture, horticulture, and forestry is mainly centered around the departments of agriculture and forestry of the national and state governments.

Plant and animal pathology. The most important of these new applications of biology to the diseases of domesticated animals and cultivated plants are plant and animal pathology. Millions of dollars are saved to the state and national governments annually as a result of the efforts now being made to counteract the effect of fungous and bacterial diseases in plants and animals which are economically important to man. Plant and animal pathology are therefore important adjuncts to the new scientific agriculture and forestry. 
Plant and animal breeding. Another important field for research and experiment, closely allied, as already indicated, to the modern studies being made in genetics and beredity, is plant and animal breeding. This attempt to produce new and better forms of plant and animal life for man's use has followed closely upon the generalizations of Darwin, Mendel, and De Vries concerning the nature of evolution, variation, and heredity.

The field of biology is thus an ever-widening one, in which the purely scientific and theoretical studies of the scientist are being constantly applied to arts and professions which are immediately concerned with the welfare and progress of society.

The following table will help the student to comprehend clearly the scope of biology as outlined above and to understand the interrelations of its various branches and applications.

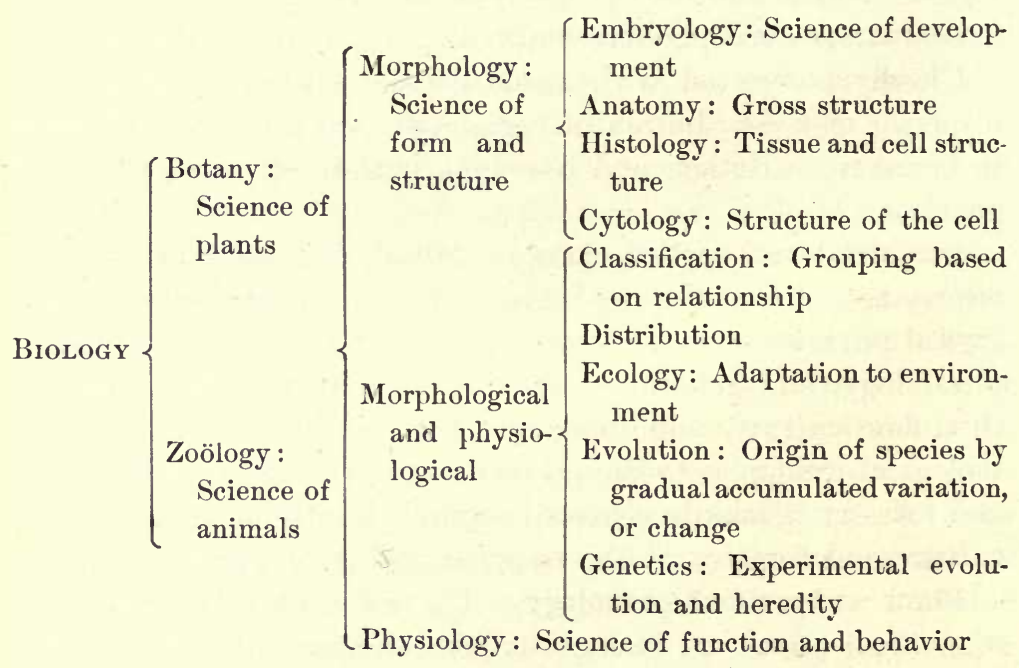

$\underset{\text { trial Biology }}{\text { Applied and Indus- }}\left\{\begin{array}{l}\left.\begin{array}{l}\text { Breeding } \\ \text { Pathology } \\ \text { Agriculture } \\ \text { Forestry } \\ \text { Horticulture } \\ \text { Medicine } \\ \text { Sanitation }\end{array}\right\} \text { Elant and animal } \\ \text { Sarts and professions related to biology }\end{array}\right.$ 
PART II. THE PLANT GROUPS 



\section{CHAPTER XII}

\section{THE ALGEE}

The algæ are the simplest of the green plants and constitute, with the fungi, the group known as thallophytes, or plants without true roots, stems, and leaves. The fresh-water algæ inhabit fresh-water streams, lakes, and ponds, as well as wet banks and the bark of trees. They usually occur in simple colonies in which the cells unite to form cell chains, nets, or spherical aggregates. Like the higher plants, the form which the colony assumes is usually closely related to its needs and to its mode of life.

The immense growth of algæ in ponds and streams, due to their rapid methods of reproduction, is often of the greatest importance in producing food for fish and minute aquatic animals. The term plankton is applied to the great mass of minute living algæ and other organisms floating on the surface of our lakes and ponds in summer. This plankton is of vital importance to the great fisheries which supply European countries and our own country with fish food. The Illinois State Survey, under Dr. Forbes, estimated that the Illinois River plankton produces annually about $150,000,000$ pounds of fish food, and it has been estimated that the plankton of the Rhone River comprises 8000 different species of microscopic plants and 800 species of microscopic animals. This immense number of forms is largely due to their rapid methods of asexual reproduction during the summer months.

Water supplies are also affected by the very rapid multiplication of algæ during the warm season. In this case the difficulty is due not so much to the dangerous nature of the algæ themselves as to the fact that their decay furnishes food for bacteria which are inimical to life if taken into the system with drinking water. The presence of algæ in any considerable abundance in a water supply is therefore a sign, and an indirect cause, of danger to those using the water. 
The marine algæ inhabit the salt water of the ocean and the brackish water of salt marshes and ponds. The lowest and simplest forms resemble closely the green fresh-water algæ, to which they are closely related. Among the higher forms, however, the plant body attains to much greater complexity than any known fresh-water species, and the colors are often very striking, ranging from bright reds to dull browns and greens. Some larger brown algæ may attain to a length of a hundred feet or more, and are attached at the base by large and strong holdfasts resembling roots. These large forms are usually long, flattened, strapshaped bodies which float out upon the surface of the water by means of special air cavities, or spaces within their tissues, called floats. The form thus assumed and the floating habit are both adapted to the needs of these organisms in their manufacture of foods by photosynthesis. The red and brown colors are due to pigments secreted in the chloroplasts, which partially or wholly mask the green chlorophyll pigment; but the significance of these additional pigments is not yet fully understood.

\section{PROTOCOCCUS}

Protococcus occurs in the form of single cells or of loose colonies growing on bark and moist surfaces of all kinds, such as boards, stones, brick walls, etc. Its genetic relationship to the other algæ is not certainly known, since it is a xerophytic type whose form and life history has become greatly modified during its gradual adaptation to its present habitat. It is used here for our initial study of the simplest fresh-water algæ on account of its wide distribution and availability for class use.

Structure and mode of life. Each cell of Protococcus (Fig. 115) has a cellulose cell wall which contains a many-lobed chloroplast and a nucleus embedded in the cell cytoplasm. The plants grow in masses, forming green incrustations on the surfaces which they inhabit. They are essentially air plants, absorbing carbon dioxide and oxygen from the air and taking up soil salts from the dust particles dissolved in the rain or dew which moistens their surface. In this way they are able to subsist without the 
complex organs which are always associated in higher plants with the processes of nutrition. The single cell of which the plant body of Protococcus is composed is thus a very general cell as regards its life functions, in comparison to the highly specialized wood, bark, or leaf cells of higher plants. These cells have become differentiated to perform particular kinds of work in a complex organism, like a single worker in a large factory. The Protococcus cell, on the contrary, is more like the general workman, since it is able to perform all of the life functions by means of its single highly differentiated protoplast. All of the simpler algre resemble Protococcus in this respect; that is, in being composed of cells which are highly organized but independent of each other in the performance of their life functions.

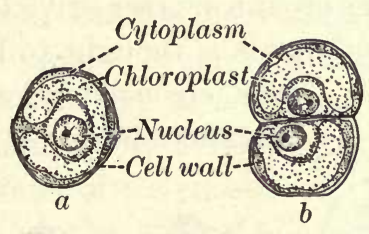

Fig. 115. Protococcus

$a$, single Protococcus plant; $b$, division of the plant into two cells by cell division; $c$, four cells formed by cell division

Reproduction. In Protococcus reproduction takes place solely by the vegetative method. Its reproduction is thus comparable to that of higher plants which reproduce by means of buds, bulbs, and runners. When Protococcus is about to reproduce vegetatively, each cell divides into two daughter cells which are furnished with one half of the original protoplast, plastid, and nucleus. This cell division takes place by mitosis in a manner similar to that already described for root-tip cells. The daughter cells thus formed then divide again, and this process may be repeated many times. The cell colonies thus produced usually remain united for a time, forming the granular incrustations commonly seen on trees and stones inhabited by Protococcus. These minute cell colonies ultimately separate into individual plants, which are light enough to be disseminated by the wind. Water may also be a factor in the distribution of Protococcus where they are located on soil or rocks. The above facts account for the wide dissemination of Protococcus in nature. 


\section{CHLAMYDOMONAS}

In the genus Chlamydomonas, the species of which are common in stagnant water, each plant (Fig. 116) is a single cell with a thin cell wall through which two delicate protoplasmic filaments, called flagella, can be seen to protrude. These flagella are minute extensions of the outer layer of the protoplast of the Chlamydomonas cell, and it is by the contractile, whiplashlike movements of these flagella that the organism is propelled through the water. These movements are often seen to be toward a source of light, indicating that the organism is sensitive to light of different intensities. This sensitiveness is thought to be located in a brick-red

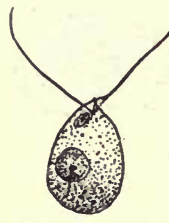

The plant

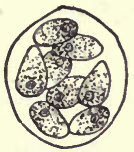

Gamete formation spot, erroneously called the eyespot, which contains a brick-red coloring matter by which

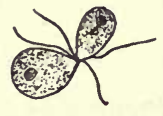

Conjugation

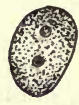

Zygotes

Chlamydomonas and its near relatives are often recognizable.

Reproduction takes place, as in Protococcus,

Fig. 116. Sexual reproduction of Chlamydomonas

by cell division ; but the cell division in Chlamydomonas results in a cleavage of the protoplast within the mother-cell wall into two or more separate protoplasts, which then round up, form flagella, and develop either asexual reproductive cells, called zoöspores, or sexual cells, called gametes. The gametes are frequently produced in greater numbers than the zoöspores, and hence are smaller. The gametes are liberated in the water by the rupture of the cell wall of the original Chlamydomonas cell, and conjugate in pairs to form a zygote cell. This zygote cell then enlarges, secretes a protective cell wall, and undergoes a period of rest. It germinates under favorable conditions to form asexual zoöspores similar to the gametes in appearance, which enlarge to form new free-swimming plants. In some species the gametes are of unequal size, foreshadowing the differentiation in the size of gametes which obtains in higher forms of algæ. In asexual reproduction the zoöspores simply enlarge and form a new plant. 
The free-swimming forms of algæ, like Chlamydomonas, are undoubtedly more primitive types of plants than nonmotile forms like Protococcus, which have apparently become adapted to a dry habitat with the loss of motility.

\section{SPIROGYRA}

Despite its specialized character, Spirogyra has been selected as the first type of a filamentary alga to be studied, on account of its availability and the ease with which its cellular structure and reproductive processes can be demonstrated. It forms a considerable part of the green scum frequently seen on the surfaces of stagnant pools in the summer and is easily recognized by its slippery feeling, due to the gelatinization of the outer layer of the cell walls of its constituent cells.

Structure. Each cell of the filament is a cylinder with a cellulose wall and a thin cytoplasmic sac surrounding a large central vacuole. The large nucleus and its nucleolus are suspended in the center of each cell by strands of cytoplasm extending from the cytoplasm surrounding the nucleus to the cytoplasmic sac (Fig.117, A). The nucleus is thus bathed by the nutrient cell sap of the water vacuole.

Unlike the cells of higher plants, there is no difference between the cells composing the filaments of Spirogyra, and no differentiation into tissues or organs. Each cell, therefore, like the independent cells of Protococcus, is capable of performing all of the functions of life. Absorption, photosynthesis, digestion, assimilation, and respiration are thus carried on by each cell independent of all the others. It follows that if Spirogyra filaments are broken up by the waves so as to separate a cell or a group of cells from its fellows, such cells or cell groups will grow and produce new plants by repeated division and elongation. Since each cell performs all of its own functions, we shall expect to find its different cell structures highly complex. This is particularly true of the chloroplasts, which, in addition to the function of photosynthesis, serve as a temporary storehouse for the starch formed by it during the hours of daylight. 
The chloroplast is by far the most striking structure in the cells of Spirogyra, when viewed with a compound microscope. These chloroplasts are in the form of bands, one or more in each cell (Fig. 117), wound spirally around the cell just beneath the cell wall. Each chloroplast is in reality a differentiated portion of the cytoplasmic sac and corresponds, therefore, in origin and
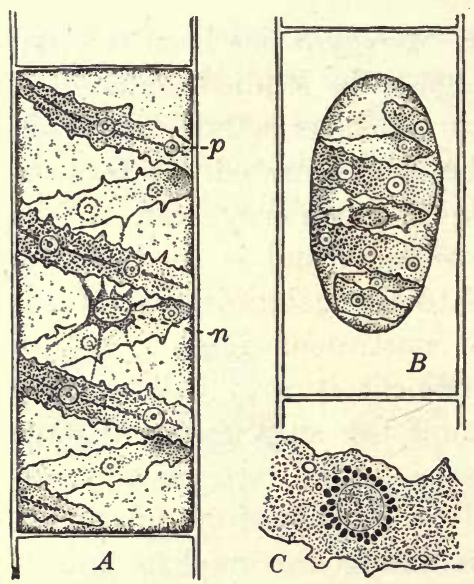

Fig. 117. Cell structure of Spirogyru

$A$, single cell of Spirogyra with nucleus (n) and spiral chloroplast containing pyrenoids $(p) ; B$, cell with the cytoplasmic sac contracted by treatment with salt solution ; $C$, pyrenoid in a small portion of a chloroplast. From Bergen and

Davis's "Principles of Botany" substance, to the granular plastids of higher plants. In sections of Spirogyra cells (Fig. 118) these bandlike chloroplasts are seen to be thickened and to project as ridges of cytoplasm into the cell vacuole slightly more than the intervening portions of the sac.

Under proper magnification each chloroplast band is also seen to be dotted at regular intervals with a series of diskshaped bodies called pyrenoids, the entire series of pyrenoids being connected by a delicate strand of cytoplasm which is devoid of green pigment. Upon investigation with the high power of the microscope the pyrenoid is found to be differentiated into a darker central granule and a lighter surrounding sheath, called the starch sheath. The function of the central granule is not definitely known, but the starch sheath is a storage place for the excess of starch not used by the cell during the daylight, when the chloroplast is forming sugar and starch by photosynthesis. At night this starch is digested or transformed into sugar and is used for the nutrition of the cells during the hours when photosynthesis is not going on. The starch sheath is therefore a temporary storage structure of the cell and of the chloroplast. The above function of the pyrenoid 
can be tested by treating some plants exposed to light and some kept in darkness with iodine solution. The plants exposed to light will give the blue reaction for starch in the starch sheath; the plants which have been in darkness for some hours will fail to give this test, showing that the stored starch has been digested and used. In this case, if the plants are reëxposed to light they will be found to form starch again in a very few minutes.

Physiology. Spirogyra, like Protococcus, is not materially different from higher plants as regards the essential processes of nutrition, cell division, and reproduction. The cell sap contains an abundance of dissolved inorganic food materials, which the cells readily absorb, like a root-hair cell, by osmosis. Carbon dioxide and oxygen are likewise easily obtained from the water in which the plants float, or it may be obtained directly. from the air when large masses of these algæ rise to the surface of ponds or lakes, buoyed up by the gas bubbles liberated during photosynthesis.

Digestion of the excess starch formed and temporarily stored in the pyrenoids during the day is known to take place at night, as in the leaf cells of higher plants. Since it is also known that the cells of

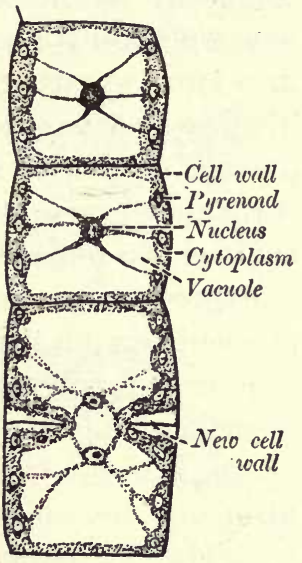

Fig. 118. Cell strueture and cell division in Spirogyra

The cells are shown às they appear in a longitudinal section. Observe the relation of pyrenoids, cytoplasm, and nucleus Spirogyra usually divide and elongate during the night, it is probable that this is a period of active assimilation and respiration in the individual cells of the filaments.

Through the above processes Spirogyra cells grow and attain the maximum size fixed for each species of Spirogyra plant. When this maximum size is reached, each cell may divide and form two new cells by a division wall which is laid down by the cytoplasm across the middle of the cell. This cell division takes place in the following manner: The new dividing wall first forms a thickening, ringlike ridge of cellulose on the old cell 
wall at the middle of the mother cell (Fig. 118). As the ridge grows by the deposit of new cellulose on its inner edge it projects farther and farther into the cell like a thin disk, or plate, except that the center of the disk is not yet completed. As a result of this growth of the cell wall the protoplasmic sac is pushed in and constricted before the advancing wall. Finally the entire sac is cut off by the completion of the disk, and the new wall forms a complete plate across the old cell, dividing it into two new daughter cells. During this process the nucleus divides into two equal parts, each of which organizes a new nucleus in one of the daughter cells. The daughter cells and their nuclei then grow to the size of the mother cell, and in this manner the filaments elongate.

Reproduction. Vegetative reproduction occurs when the delicate filaments are broken up by the waves or are separated into single cells or cell fragments. Since each cell of Spirogyra is capable of maintaining life and growth independently of the other cells of the filament it follows that individual cells or fragments of filaments are able to elongate into new plants and thus to multiply the number of individuals indefinitely.

Sexual reproduction takes place regularly either in the early spring or in the fall, before the plants enter upon the winter period of rest. Bright sunshine and abundant rainfall appear to be factors in inducing Spirogyra to resort to sexual reproduction, while the opposite conditions, with abundant nutriment in the water in which the plants live, tend to prolong the growth period for vegetative reproduction. This is doubtless due to a stimulus exerted by light and soluble food salts upon the delicate protoplasts of the filaments, which respond by initiating reproductive or vegetative growth processes, just as the cells of a root may respond by tropistic movements to the influence of gravity.

Gametophyte is the term applied to the plant filaments of Spirogyra which reproduce and form new offspring by means of gainetes. When such plants are about to reproduce sexually, two filaments which lie near each other become united by tubular outgrowths formed by protrusions from their cells (Fig. 119, a). 
These protrusions finally meet and unite to form tubes connecting the cells of the conjugating filaments in pairs $(b)$. When the cell wall between the united protrusions is absorbed, the protoplasts of each pair of cells come into immediate contact, which initiates in them certain changes resulting in their conversion into the female and male gametes. This conjugation of the filaments thus marks the initial stage of sexual reproduction in Spirogyra.

Gamete formation is a very simple process consisting in the shrinkage of entire protoplasts in the cells of two filaments united by conjugating tubes. Each gamete thus consists of the original cytoplasm, nucleus, and chloroplast of an ordinary Spirogyra cell contracted into a rounded mass of protoplasm. In most species of Spirogyra, gametes are formed in one of the conju-

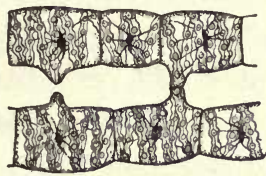

$\boldsymbol{a}$

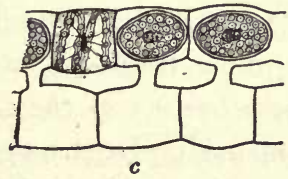

Fig. 119. Sexual reproduction in Spirogyra

$a$, early conjugation of the filaments (the pairs of uniting cells are gametangia in each case) ; $b$, gametes forming and uniting in conjugation to form zygotes; $c$, zygotes with thick cell wall, pyrenoids, and conjugate nucleus; $d$, germination of the zygote to form a new plant gating filaments before the protoplast of the other filament begins to contract. Since these first-formed gametes are the ones which move through the conjugating tubes during fertilization to unite with the stationary gametes of the adjacent filament, they are usually considered to be the male gametes. Such species of Spirogyra are therefore said to be dicecious, which means that one filament bears only male gametes and the other only female.

Gametangium is a term applied to the entire cell in which the protoplast contracts to form a gamete. This cell may be termed either the male or the female gametangium, according as it forms a male or a female gamete.

Fertilization consists in the union of the motile male gamete with the stationary female gamete of the opposite filament. In 
this process the cytoplasm of the two gametes fuse first, and the two gamete nuclei, called pronuclei, fuse later to form the one double conjugate nucleus. As soon as the gametes unite, the double cell formed by the union of the gametes rounds up and secretes a cell wall, which completes the formation of the zygote (c). Since this zygote in Spirogyra is designed to carry the plant over inclement periods of drought or cold, it develops a very heavy protective cell wall, which is divided into three layers. The outer layer is thick and protects the protoplast from mechanical injury and sudden changes of temperature; the middle coat is impervious to water and prevents its loss from the protoplast during drought if the pond or stream in which it lives happens to dry up in summer; the inner coat is thin and elastic. Pyrenoids are also abundant in the mature zygote and, with the aid of the microscope, are easily distinguished from the nucleus by the central granule and surrounding starch sheath.

After its rest period germination of the zygote $(d)$ takes place, owing to environmental stimuli. In the germination process the zygote absorbs water, and the swelling of its contents results in the rupture of the two outer protective layers of the cell wall, which allows the inner elastic layer to protrude and elongate into a filament. This elongation of the zygote cell is accompanied by cell and nuclear divisions which result in an offspring in the form of a minute Spirogyra plant in which the chloroplasts are early reorganized and the nucleus takes up its central position in each cell. The embryo plant thus begins to develop by growth and cell division into an adult Spirogyra plant, which completes the process of reproduction, and the new plant enters upon a period of independent vegetative life.

\section{LIFE HISTORIES OF ALGE}

Every kind of organism, during its life, passes through certain stages and processes which, taken together, are termed its life history. The life history may therefore be conveniently considered and graphically illustrated as a cycle of events in the life of an individual organism which follow each other in orderly sequence. 
It is evident that the simplest life history in plants would be presented by such a simple organism as Protococcus, which reproduces by the division of a mother cell into two daughter cells, each of which, by growth and separation, forms a plant like the mother. The very rapid reproduction and spreading of Protococcus, bacteria, and yeasts is effected by this mode of vegetative reproduction.

The life history of a plant with sexual reproduction alone or with sexual reproduction combined with vegetative reproduction, as in Spirogyra, also presents a comparatively simple cycle of stages. In Spirogyra the nutritive and growth phases of the life cycle are amply provided for by the highly organized cells of the filaments, which may also be multiplied by vegetative reproduction. The nutritive phases usually continue throughout the summer months, when food is plentiful in the waters in which these plants are habitually found. The sexual phase is inaugurated by the formation of gametes, and terminates with the maturing of the zygote resulting from the fertilizing act. After its normal period of rest the zygote gives rise to an offspring in the form of a new nutritive plant, which brings the life cycle back to its starting point. In Chlamydomonas there is added to the vegetative and sexual phases of reproduction an asexual phase, represented by zoöspores. In such plants the asexual zoöspore stage usually has the function of rapid reproduction of the species, while the zygote, which results from sexual union, functions as a resting cell during inclement periods or seasons, so that there is a division of labor between the asexual and sexual phases in the life history. The relation of the different phases in the life history of a plant may be indicated as in the following outline of the life history of Spirogyra. The student should substitute simple diagrams for the written designations of the various stages.

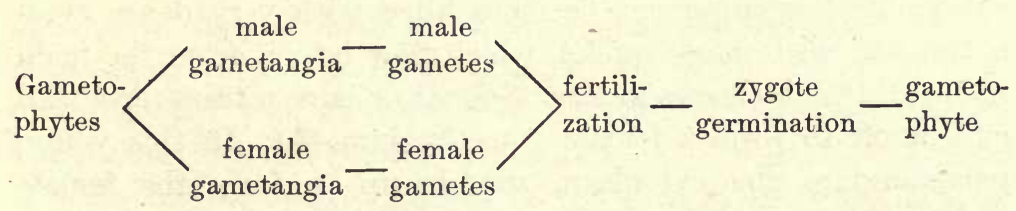




\section{VAUCHERIA (GREEN FELT)}

Various species of Vaucheria, or green felt, are common, either growing on wet soil in greenhouses and on muddy banks or floating free with Spirogyra and other algæ on the surface of ponds and lakes. The species selected for our immediate study is usually found growing on very wet soil in dense green masses or mats, closely interwoven by the branching of the filaments making up the main portions of the plant body.

Sexual reproduction. 'The gametophyte of Vaucheria sessilis is made up of filaments like Spirogyra, but these filaments are highly branched and are not divided by cell walls, so that the entire plant body of an individual plant is composed of one very large multinucleate cell. 'This long and highly branched cell has the same general structure as that already indicated for adult cells in the higher plants, since it is furnished with a cell wall of cellulose lined by a thin cytoplasmic sac, which is kept in place against the cell wall by a huge water vacuole.

Within the cytoplasmic sac are numerous minute nuclei and small granular chloroplasts, as well as many oil drops, which represent the reserve food of the filament. This structure of the gamete plant of Vaucheria is thus quite unusual among the fresh-water algæ in that the plant body, while filamentary and highly branched, is yet composed of a single multinucleate cell.

The gametangia and gametes (Fig. 120) are formed from specialized branches of the plant body, which arise at irregular intervals along the filaments at the time when sexual reproduction is about to occur. These reproductive branches at the outset resemble ordinary young vegetative branches, but they soon begin to differentiate (a) into a swollen subspherical female branch and a cylindrical curved male branch. Each subspherical enlargement. becomes filled with very dense cytoplasm and with many nuclei, which flow into it from the main filament as it increases in size. Sooner or later a transverse wall cuts it off to form a female gametangium (b). In this young gametangium the cytoplasm rounds up to form the female 
gamete, all but one of the nuclei disappear, and a projecting beak is formed, filled with clear cytoplasm free from green chloroplasts. With these changes the gametangium and its inclosed gamete are made ready for the reception of the male gamete at the time of fertilization. The male gametangium, which began as a cylindrical outgrowth of the reproductive branch, elongates and cuts off an end cell, which forms the male gametangium (b). The dense granular cytoplasm of the male gametangium, instead of forming a single gamete, as in the
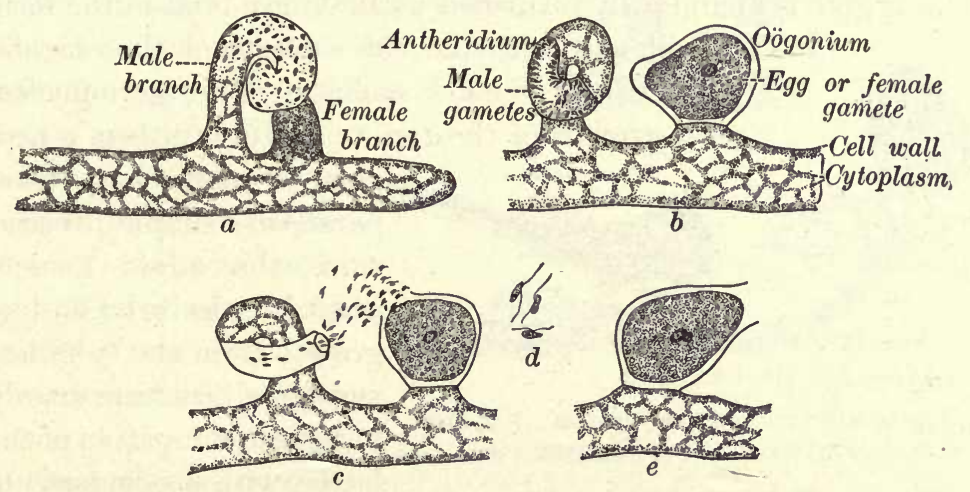

Frg. 120. Sexual reprodaction in Vaucheria

$a$, portion of a filament with male and female reproductive branches; $b$, antheridium and oögonium, with sperms and egg; c, liberation of sperms, and sperms swarming around the receptive spot of the egg; $d$, sperms; $e$, fertilized egg, with union of male and female pronuclei

female, divides into a very large number of naked protoplasts, each of which becomes a motile male gamete. These minute male gametes are finally liberated in the water by the softening and rupture of the cell wall at the end of the male gametangium (e) and are ready to effect a union with the eggs in the female gametangia.

Fertilization results from the union of the cytoplasm and nucleus of one of these small male gametes with that of the large female gamete (Fig. 120,c). In order to effect this union the cell wall at the end of the beak of the female gametangium softens and (e) finally breaks down so that a free passage is made for the motile gamete to the cytoplasm of the female 
gamete. Many botanists hold that the female gamete secretes some substance, such as cane sugar or an organic acid, which attracts the male gamete to the female and thus insures fertilization by directing the free motile male gamete to the stationary egg.

The zygote, or fertilized female gamete, remains in the female gametangium (Fig. 121, a) and undergoes a period of rest. During this rest period it is protected by the old gametangium wall, which remains in its former position around the egg cytoplasm. The zygote is abundantly furnished with reserve food in the form

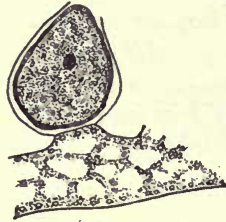

a of oil and with the chloroplasts of the original unfertilized female gamete. The germination and growth of the zygote cell $(b)$ to form a new

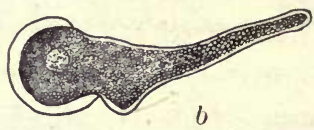

Fig. 121. Germination of the zygote in Vaucheria

$a$, zygote still attached to a filament ; $b$, zygote sending out a new filament gamete plant is a comparatively simple process, since the adult gamete plant is unicellular and its growth from the spherical zygote cell consists mainly in a great elongation of the latter, with accompanying branching of the filament thus produced. During this elongating and branching process new nuclei and chloroplasts are being formed by repeated divisions of the chloroplasts and conjugate nucleus of the zygote. 'The resulting gamete plant resembles exactly the original mother plant, and in time, after a period of vegetative activity, reverts to reproduction.

Asexual reproduction. In Vaucheria sessitis another mode of reproduction occurs which is asexual in its nature. In this process the end of a filament swells up and becomes separated into a distinct cell by a transverse cell wall (Fig. 122, $a$ ). The cytoplasmic contents of the terminal cell then rounds up and sends out minute cytoplasmic projections called cilia, thus forming a large motile asexual naked cell, or zoöspore (b). By the rupture of the end of the enveloping wall this zoöspore becomes a free-swimming cell $(c)$, which later comes to rest and grows into a new plant, clothed with a cell wall (Fig. 122, $d, e$ ). 
Life history. The life history of Vaucheria thus presents a marked contrast to that of Spirogyra or Protococcus. Its gametangia are unlike and are often for this reason termed the antheridium, or male gametangium, and the oögonium, or female gametangium.

The female gamete is a large, stationary cell abundantly stored with nutriment for the production of a new gamete plant. The male gametes are small and actively motile, and are produced in great numbers to insure fertilization of the nonmotile egg. The gamete plant results from the germination of the zygote produced by the union of the male and female gametes.

Asexual reproduction is also provided for by
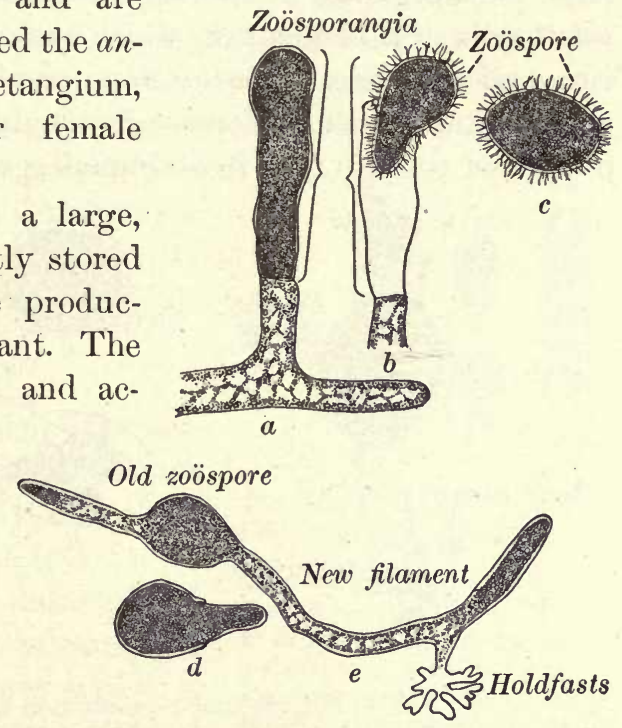

Fig. 122. Asexual reproduction in Vaucheria $a, b$, formation and expulsion of the zoöspore $c$; $d$, early germination of the zoöspore; $e$, new filament formed. $a, b$, and $c$, after Strasburger; $d$ and $e$, after Sachs highly specialized motile asexual bodies called zoöspores. The life history of Vaucheria, therefore, presents the same stages of sexual and asexual reproduction as Chlamydomonas.

\section{EDDOGONIUM}

Edogonium is one of the highest types of the filamentary algæ, in which the filaments are composed of many cells. The general facts regarding its life history are similar to those of Vaucheria in that it reproduces by both the sexual and the asexual method. The female gametangium is called an oögonium and the male gametangium an antheridium, as in Vaucheria. Both organs arise from ordinary cells of the filament. 
The female gametangium (Fig. 123, a) is a greatly enlarged cell of the filament, in which the protoplast differentiates as a large female gamete. The male gametangia are developed from small cells of a filament, in which each protoplast usually forms two male ganetes. Fertilization results in a resting zygote, which, after a period of rest, germinates by the division of its protoplast to form four free-swimming zoöspores (Fig. 123, c, d).
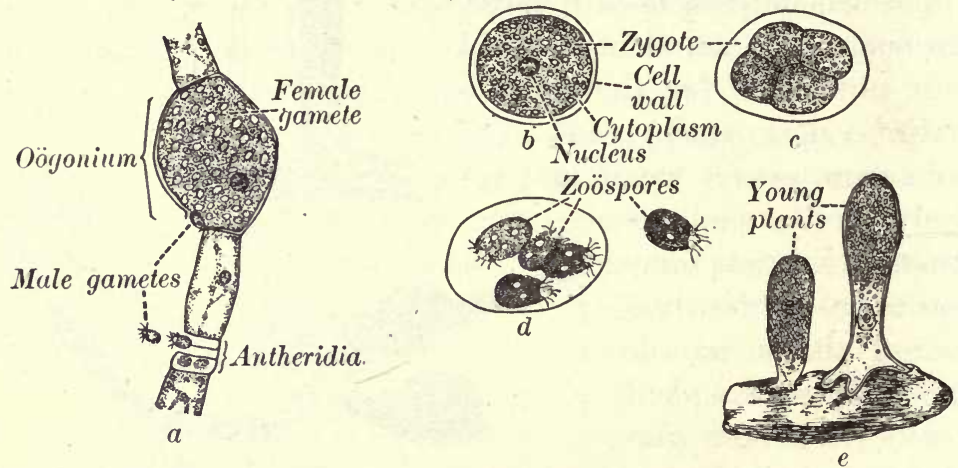

Fıg. 123. Sexual reproduction in Edogonium

$a$, gametes and fertilization; $b$, zygote with heavy cell wall in resting condition; $c$, cell division of the protoplast to form four zoöspores; $d$, zoöspores formed from the divisions of the protoplast in $c$; $e$, growth of the zoöspores into young plants after becoming attached to a rock in the water. $a$, after Coulter; $b-d$, after Hirn; $e$, after Juranyi

These zoöspores become free-swimming by the disorganization of the old zygote cell wall, come to rest on a support, and begin to elongate into a new filament. This new plantlet (e) is at first attached but later becomes a free-floating filament like the parent organism.

In asexual reproduction zoöspores are formed much as in Vaucheria except that they arise from protoplasts of cells within the filament (Fig. 124). Each zoöspore becomes free from the mother plant by the rupture of the mother-cell wall and swims freely in the water for a short period. It then loses its cilia, becomes attached to a support, and forms a new filament, exactly as in the case of zoöspores developed within the zygote. 
Life history. The life history of Edogonium is therefore similar to that of Chlamydomonas and Vaucheria in having an asexual phase, represented by motile zoöspores, and a sexual phase with gametes. The asexual phase, as in the other algæ mentioned, enables the alga to increase rapidly during periods adapted to rapid growth, while the sexual phase enables the plant, by means of the highly protected zygote, to pass inclement seasons without danger.

\section{FUCUS VESICULOSUS (BLADDER WRACK)}

Habit. Fucus grows along the seashore and constitutes a conspicuous part of the marine flora of these coastal regions. The color is due to a brown pigment secreted by the chloroplasts, which masks, wholly or in part, the green chlorophyll pigment which is also secreted by the chloroplastids of Fucus. The plant body is admirably adapted to photosynthesis, since it is flattened like a leaf and forms, at regular intervals, air cavities, or floats (Fig. 126), which buoy the plant up in the sea water and expose its flattened branches to the sunlight. The plants elongate by means of growing points at the branch tips, which fork in a dichotomous manner, thus producing numerous flattened branches all lying in one plane. They are attached to rocks or pier posts along the ocean shores by means of rootlike holdfasts, which serve to anchor the plant body but do not serve for the absorption of raw food materials, since these foods can be absorbed by the entire epidermal surface.

Fucus is much more complex in structure than Spirogyra, being composed of cells differentiated into an epidermis, a brown cortex, and a central cylinder, or medulla, composed of elongated conducting cells in the form of strands or chains. Fucus is thus structurally adapted to withstand the buffeting of the ocean waves. 


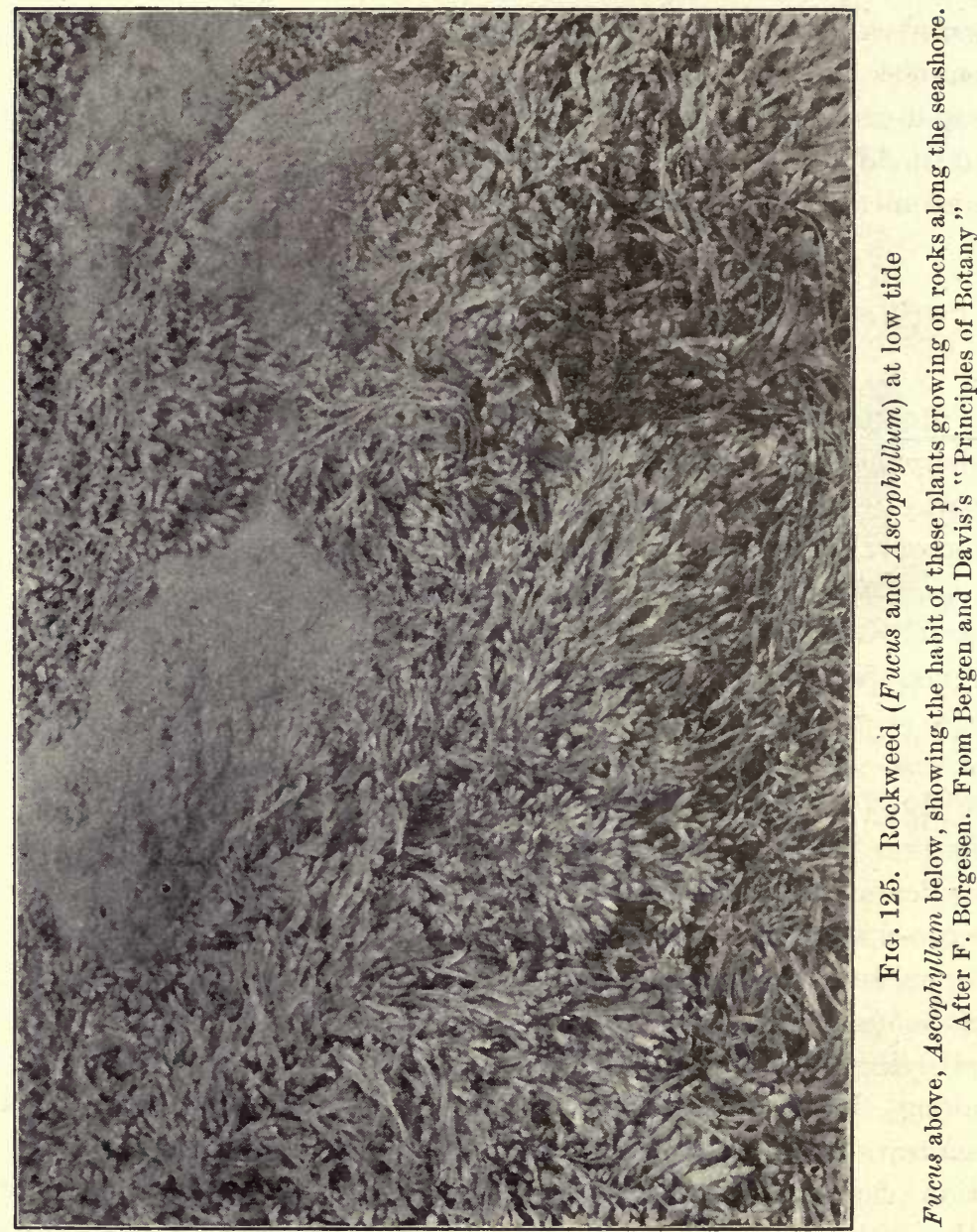


Reproduction. In Fucus, as in Spirogyra, reproduction is accomplished largely by the sexual process, although vegetative reproduction may take place by the growth of parts of the plant body which chance to be broken off by the waves or by other means. The male and female gametangia are borne on separate plants within distinct male and female branches (Fig. 126).

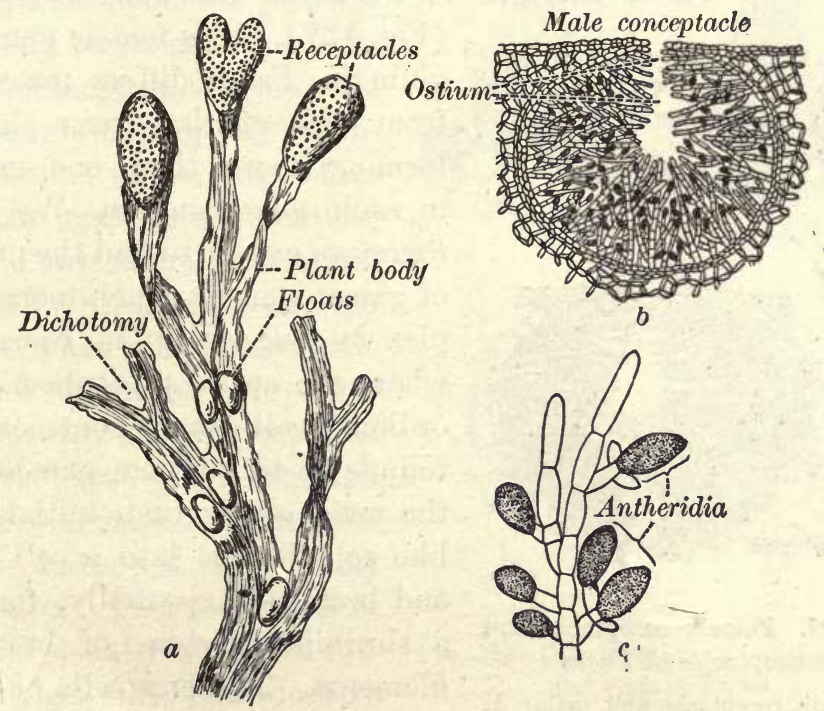

Fig. 126. Plant body and male reproductive organs of Fucus

$a$, plant body, male receptacles, and floats: $b$, male conceptacle with antheridial branches; $c$, antheridial branch with antheridia

Like Spirogyra, Fucus is therefore dicecious, and the male gametes seek the females in the open sea during the fertilization period. Both male and female gametangia are borne in flaskshaped eavities, - the reproductive eavities, or conceptacles (b). Within the reproductive branches each cavity opens to the surface by means of a narrow neck and pore, through which the gametes are finally expelled into the sea water at the time of fertilization. When the male gametes are being shed into the water they give the male reproductive branches a yellowish color, while the eggs impart a greenish tint to the female branehes. 
Both male and female gametangia arise from the cells bounding the walls of the male and female reproductive cavities as hairlike cellular outgrowths, not unlike a root hair growing from an epidermal cell of a root tip.

In the case of the female gametangia each initial hairlike gametangium cell divides to form one or more stalk cells and a terminal cell, which enlarges to form the gametangium proper

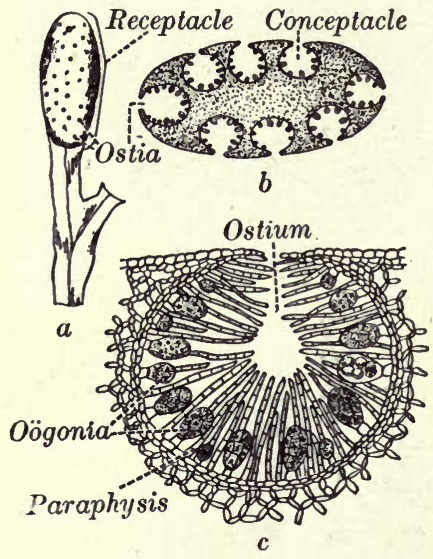

Fig. 127. Female receptacle and conceptacles of Fucus

$a$, female receptacle and ostia; $b$, transverse section of $a$, showing conceptacles; $c$, enlarged conceptacle, oögonia, and paraphyses

(Fig. 127). This female gametangium in Fucus differs materially from that of the green algæ in forming more than one gamete in each gametangium. We shall therefore expect to find the process of gametogenesis much more complex in Fucus than in Spirogyra, where the entire protoplast of an ordinary cell simply contracts and rounds up to form one gamete. In the male cavity each initial hairlike cell divides into a cell chain and branches repeatedly, forming a shrublike system of branched filaments. The end cells of these branches develop the gametangia, in which numerous male gametes are formed during gametogenesis.

Gamete formation takes place by similar processes in both male and female gametangia, although the gametes which result are very different, both in number and in size, in the two cases. Three essentially distinct processes are involved in this formation of the male and female gametes, namely, nuclear division, cell division, and gamete differentiation (Fig. 128, $a-c$ ).

Nuclear division takes place first in each gametangium, forming eight free nuclei in the female gametangium and sixty-four in the male gametangium. These nuclei soon become uniformly distributed throughout the cytoplasm of each large gametangium cell, and then an internal process of cell division follows which results 
in the separation of the mother cells of the future gametes by membranous walls. In this internal cell division a cleavage of the cytoplasm around each nucleus is followed by the simultaneous secretion of the membranous walls between the mother cells. The protoplast of each mother cell then rounds up in the female gametangium (Fig. 128, $b$ and $c$ ), the dividing membranous walls are absorbed, and the gametes are ready to be expelled from the gametangium. In the male gametangium (Fig. 129) similar processes occur, except that the male gametes differentiate to form two laterally attached cilia and a brick-red eyespot in a more or less pear-shaped male gamete. When the gametes are fully formed, they are expelled into the sea water through the pore of each reproductive cavity embedded in a slimy substance. This expulsion of gametes usually occurs at low tide, when the reproductive branches dry and contract in the sun, thus squeezing out the contents of the reproductive cavities. When first ex-
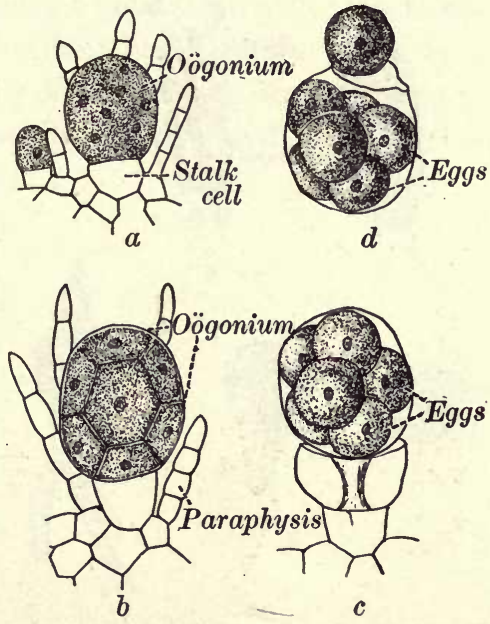

Fig. 128. Development of the oögonium and eggs in Fucus

$a$, young oögonial cell with eight free nuclei; $b$, older oögonium divided to form eight eggs; $c$, eggs in the oögonium rounded up preparatory to expulsion as in $d$. After Thuret pelled, the male and female gametes are both inclosed in the inner membranous layer of the gametangium wall, but this soon ruptures and the individual gametes become free-floating cells (Figs. 128, $d$, and 129, b). With the return of the tide the gametes from the male plants are mixed freely with the female gametes in the sea water, and abundant fertilizations occur, as is evidenced by the great number of these plants in locations favorable to their existence.

In the process of fertilization the female gametes float impassively in the water, but the male gametes swarm in great 
numbers around them, probably attracted by a secretion from the female gamete. When one male gamete has succeeded in penetrating the outer cytoplasmic layer of the female gamete, the other swarming male gametes either disappear or die in contact with the egg. Cytoplasmic and nuclear fusions complete

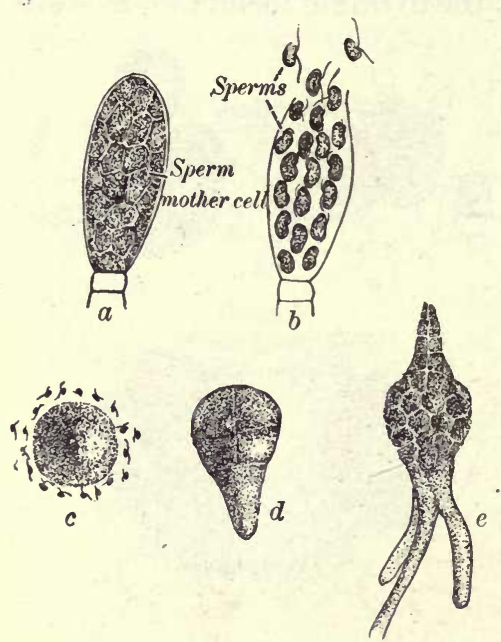

Fig. 129. Development of the male gametes, fertilization, and development of the embryo in Fucus

$a$, antheridium with sperm mother cells; $b$, expulsion of free sperms; $c$, swarming of sperms around an egg preparatory to fertilization; $d$, the cellular embryo; $e$, more advanced embryo of Fucus. After Thuret the act of fertilization, and the fertilized egg becomes the $z y$ gote, which quickly secretes a cellulose wall and begins to divide to form a new Fucus offspring (Fig. $129 d, e$ ).

Germination of the zygote follows immediately upon the fertilizing act instead of after a long rest period as in Spirogyra and most fresh-water algæ. This early germination of the zygote is undoubtedly connected with the fact that Fucus lives in the sea, where the danger of extermination in winter or in dry periods is much less than in fresh-water ponds and lakes. Winter is thus passed safely in the vegetative condition, and the highly protected resting zygote is not a necessity in its life cycle. Germination of the zygote results in a many-celled embryo, which differentiates to form a growing point and a holdfast by which the young offspring becomes anchored to a stone or other object. Elongation and repeated forking of the original growing point follows, which finally results in a new offspring like the parent.

Life history. The life history of Fucus does not differ materially from that of Spirogyra except in the fact that no resting zygote is formed to tide over inclement periods. Asexual reproduction is 
also very limited in Fucus, as it may well be on account of the abundant production of male and female gametes and the greater security of the offspring in the more permanent and less variable habitat in the ocean. The graphical history of a Fucus plant will therefore differ very little from that of Spirogyra except in the details of structure and in processes concerned with reproduction.

On account of nuclear phenomena connected with the formation of the gametes in Fucus the plant body cannot properly be called a gametophyte. The details of this matter do not, however, belong in an elementary course in biology and are therefore passed over with this brief note. 


\section{CHAPTER XIII}

\section{THE FUNGI}

Mode of Life. The fungi differ from all the plants thus far studied in that they lack the green pigment chlorophyll, which enables the algæ and the higher spore and seed plants to manufacture their own organic foods from carbon dioxide, water, and salts derived from the soil. The fungi are therefore dependent plants, living either as saprophytes, upon lifeless organic matter, or as parasites, upon living animals and plants.

Saprophytes. The greater number of fungi, including the familiar mushrooms, molds, and yeasts, are saprophytes, which feed upon and consume lifeless organic bodies or their products. Such fungi serve a useful purpose as scavengêrs, since they tend to dispose of the dead remains of plants and animals, which might otherwise hinder the growth or imperil the life of other living organisms. This is especially evident in a forest, where saprophytic mushrooms, leaf molds, and bacteria convert the annual fall of leaves, tree trunks, and branches into the ingredients of leaf mold, which is a characteristic component of the forest soil. The accumulation of this annual leaf fall would be disastrous to the living trees and plants of the forest if it did not constantly disappear by decay through the agency of saprophytic fungi.

Parasites. The parasitic fungi are less numerous than the saprophytes. They include such forms as the bacteria of disease, the parasitic rusts and smuts of cereal grains, and the treekilling fungi. Such parasites cause immense damage annually through the death of animals and the destruction of valuable crops and timber upon which they prey.

Classification. The fungi have undoubtedly sprung from the algæ by the loss of chlorophyll and chloroplasts, since they so 
closely resemble these plants in their structure and reproduc tion. They have nevertheless become profoundly modified by their parasitic and saprophytic habits and are therefore usually classified into plant groups distinct from the green algæ.

The following simple classification will serve to illustrate something of the great variation in form, structure, and reproduction represented among the fungi.

1. Bacteria, including the simplest of the fungi, which occur in the form of single cells or in loose colonies (Fig. 134).

2. Algal fungi, including the common black molds (Figs. 139 and 140) and water molds infesting dead fish and flies in ponds, lakes, and streams. These fungi resemble closely the green algæ, from which they have sprung, in both structure and reproduction.

3. Sac fungi, including the yeasts, the common blue and green molds (Figs. 143-145), and the blights found on the leaves of trees and various plants. These, with the exception of the yeasts, are moldlike fungi, which form spores in large, saclike cells called asci. These spore sacs, or asci, are usually inclosed in characteristic fruit bodies.

4. Higher fungi, including mushrooms, puffballs, rusts, and smuts of cereal grains (Figs. 145-155). These higher fungi are characterized by the production of conspicuous fruit bodies for the production and scattering of asexual spores.

\section{YEAST}

The origin and relationship of the yeast plants to the other fungi is not definitely known, although they are usually classified with the sac fungi. They are for the most part single-celled plants resembling spores and are widely distributed in nature, since they reproduce rapidly and are easily blown about in the open by the wind or by currents of air in dwellings. They therefore occur on almost all exposed surfaces, particularly on those which are moist and contain sugar or acids, so that grapes, apples, and other fruits, when exposed to the air, are sure to have some of these little plants lodged on the skin. 
When the juice of such fruits is pressed out, as in the making of cider, grape juice, or homemade wines, the wild yeast plants are certain to get into the expressed juice and cause the fermentation which is so common under such circumstances. Sweet solutions of all kinds, when exposed to the air, become infected with wild yeasts, which causes them to ferment.

The cultivated yeasts used in the making of yeast cakes and in the brewing industries are simply selected varieties of wild

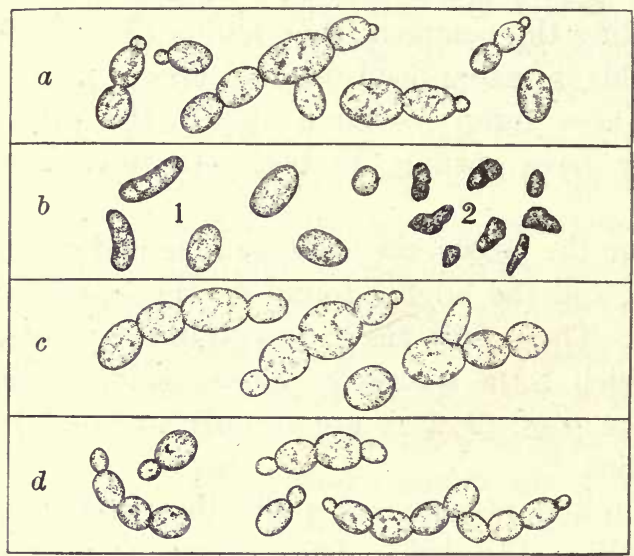

Fig. 130. Various kinds of wine and beer yeasts $a, b$, wine yeast (S. ellipsoideus) ( $a$, young and vigorous; $b$, old (1) and dead (2)); $c, d$, beer yeast (S. cerevisix) (c, bottom yeast; $d$, top yeast). After Marshall yeasts which have been found especially adapted to the raising of bread or to the making of various sorts of fermented liquors. These cultivated yeasts are also of many kinds, each particular kind having its peculiar characteristic effect on sugary solutions in which they are placed. Thus, in making beer the people of some of the European countries use what is called bottom yeast (Fig. 130), which is capable of producing only a moderate supply of alcohol by fermentation. Top yeast, which is used in making English beers and compressed yeast cakes, produces a larger amount of alcohol than bottom yeast. Distillers' yeasts, used in making rum and brandy, are also capable of producing large quantities of alcohol by fermentation. The various kinds of wines likewise owe their peculiar flavors to the kind of yeast used in them. All these cultivated yeasts have been gradually selected by man for particular uses, just as various kinds of fruit trees, cereals, and flowers have been selected and improved by cultivation. 
Structure. The yeasts are composed of minute spherical or slightly elongated cells varying from $\frac{1}{2} \frac{1}{500}$ to $\frac{1}{10000}$ of an inch in diameter. They usually occur as single cells, but may adhere to form cell chains or loose temporary colonies. The yeast cell (Fig. 131) has the same general structure as that of plant cells with which we are familiar. The cell wall is a thin membrane composed of two or more layers, and the cytoplasm is filled with granules of various sizes, some of which are supposed to be composed of fatty material, probably useful as a reserve food. The nucleus can only be observed in material which has been specially stained for that purpose, and is found in close proximity to the large central vacuole which fills the greater part of the cavity of the mature yeast cells.

Reproduction. If yeast plants are placed in a sugary solution, such as

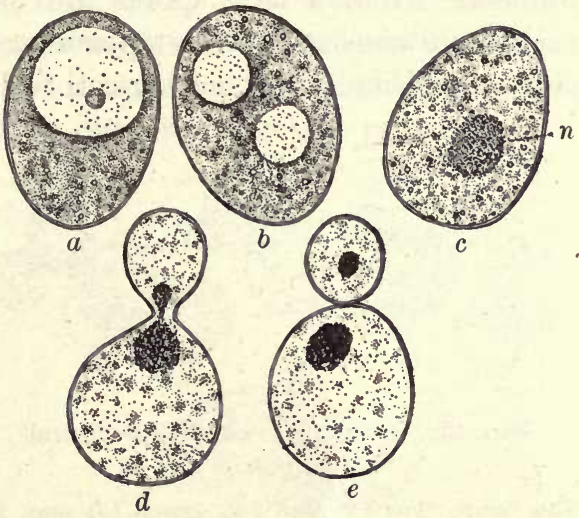

FIG. 131. Yeast cells highly magnified $a$ and $b$, showing vacuoles and granular cytoplasm; $c$, showing nucleus; $d$, budding yeast cell with dividing nucleus; $e$, the bud cut off from the mother cell with its portion of the nucleus. After Conn beer wort, at a temperature of from $25^{\circ}$ to $30^{\circ}$ centigrade, they begin to reproduce new plants by the process known as budding. Budding consists in an outgrowth, or protrusion, of the cell wall of the yeast cell into which the cytoplasm flows, thus forming a new cell. The outgrowth is very minute at first, but by growth it finally reaches the size of the mother cell from which it grew. Finally a cell wall is formed between the mother cell and the bud, and the two cells split apart, thus forming two new yeast plants (Fig. 131, $d$ and $e$ ). Division of the nucleus accompanies the budding process in such a manner that each new bud which is formed into a new yeast plant is furnished with a nucleus. Since the budding process goes on with extreme rapidity 
at the proper temperature, new plants are formed in almost incredible numbers in a comparatively short time when yeast is placed in a favorable medium, such as bread sponge or beer wort.

When young, vigorous yeast cells are grown at the proper temperature and moisture but are lacking in an adequate food supply, they sometimes form internal spores (Fig. 133) by division of the nucleus and aggregation of the cell cytoplasm around each new nucleus. From 4 to 8 spores are usually formed in each cell, each spore consisting of cytoplasm and nucleus surrounded by a cell wall. Finally the mother-cell wall breaks down, and the lib-

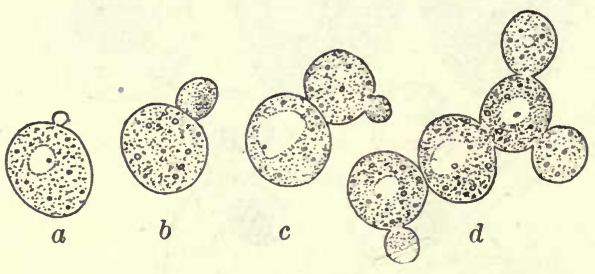

Fig. 132. Growing yeast with buds and cell chains

The figure shows how a cell group $(d)$ may be formed in actively growing yeast if successively formed buds remain attached. After Conn erated spores form new yeast plants. The process of spore formation in yeast is apparently designed to preserve the yeast during inclement conditions, rather than as a method for more rapid reproduction or dissemination.

Fermentation. The importance and interest of yeasts for man is due largely to their power of causing alcoholic fermentations in sugar solutions, since it is upon this power that the modern baking and brewing industries are based.

If yeast plants, in the form of commercial yeast cakes or in a free state, are placed in a sugar solution under the proper temperature $\left(25^{\circ}-30^{\circ} \mathrm{C}\right.$.), bubbles of carbon-dioxide gas will soon rise to the surface of the liquid, indicating that the process of fermentation has begun. Proper chemical tests will also reveal the presence of alcohol, which is formed in the fermenting solution at the same time as the carbon dioxide. It has been found that the carbon dioxide and alcohol are produced by the splitting up of the sugar molecule by the yeast ferment called zymase.

The carbon dioxide which rises to the surface is utilized in the process of bread-making to make the bread light, while the 
alcohol is retained in the commercial manufacture of beer, wine, and all fermented liquors. The splitting of the sugar by the ferment is represented by the following equation:

Grape sugar + zymase $=$ alcohol + carbon dioxide + zymase.

The above fermentation process was for a long time thought to be due to the activity of the protoplast of the living yeast cells, which were therefore called living ferments. It remained for Buchner, a great German scientist, to demonstrate that the active body in the process of fermentation is not the protoplast of the yeast cell but rather a product of the cell protoplasm in the form of a secretion or fermenting substance, which is now termed zymase. Buchner ground yeast cells in fine infusorial earth and filtered the juice pressed from the yeast cells through a porcelain filter. The filtered extract thus obtained caused alcoholic fermentation in sugar
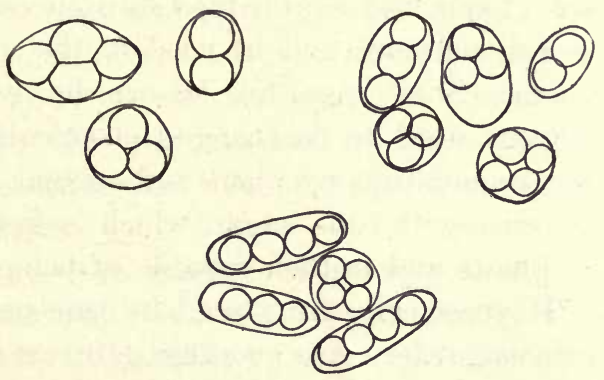

Fig. 133. Spore formation in yeast

After Conn

solutions without the presence of living yeast cells. As a result of this experiment Buchner concluded that an active ferment was secreted by the yeast cell, which passed through his filters and caused fermentation in the sugar solutions to which it was added. This ferment is not liberated from the yeast cell in normal fermentation, but the sugar is first absorbed into the cell and is then attacked by the zymase. Such a ferment, or enzyme, which acts within the cell which secretes it, is called an endoenzyme or ferment. In many other instances living cells form exoenzymes, which are first liberated into the surrounding medium before they cause active fermentation.

The process of fermentation in yeasts is not clearly understood. A distinctive feature of the process is that the ferment is neither diminished in amount nor injured for further use by its activity. 
At the end of a fermenting process, therefore, the same amount of ferment is left over as there was present at the beginning. This is indicated in the above equation for the fermentation of grape sugar, in which the zymase appears on both sides of the equation. It is estimated that zymase can convert as much as 100,000 times its own volume of sugar into alcohol and carbon dioxide without being diminished in volume or in the power to cause renewed fermentation in a sugary solution. Other ferments behave similarly, acting like the so-called catalytic agents among inorganic substances.

Digestion has already been defined as the transformation of solid foods into soluble food substances which can diffuse through cell membranes and be used in the making of new living substance. Some cases are known, however, in which soluble substances need to be charged chemically before they are of use for assimilation by plant and animal cells. This appears to be the case with cane sugar, which is first changed to grape sugar in plants and is then capable of being used in assimilation.

If yeast cells are placed in cane-sugar solutions, they secrete cane-sugar ferments (maltase or invertase), which change the cane into a grape sugar. Alcoholic fermentation yields energy which is available to the yeast cell, while digestive fermentation prepares a food for assimilation by the cell protoplasm. In both cases the active agent in the chemical reactions involved is a secretion of the yeast cell, which is called an enzyme or a ferment.

Cane sugar + water + cane-sugar ferment

$$
\text { = grape sugar + cane-sugar ferment. }
$$

Grape sugar + zymase ferment

= alcohol + carbon dioxide + zymase.

Ferments may be classified, therefore, into energy-forming and digestive ferments, according to the results of their activity.

All cases of digestion in animals and plants are caused by the presence of active ferments, which are secretions of plant or animal cells. In the higher animals these secreting cells usually occur in the form of glands, but in higher plants they may be 
ordinary leaf cells, wood-ray cells, or root cells. Among the higher fungi, as we shall learn later, a great variety of digestive and energy-forming ferments are secreted, which aid both parasitic and saprophytic fungi in securing their food.

The use of fermentation to the yeast plant, beyond the digestion of the small amount of food needed for reproduction and growth, is not certainly known. It is supposed that it uses some of the energy produced by fermentation for its life processes, as the higher plants and animals use the energy resulting from respiration. This idea seems probable, for the reason that certain yeasts can live and grow for a considerable time in a sugar solution without the presence of oxygen for normal respiration. Other organisms, namely, bacteria and certain molds, are able to produce energy by fermentation in the absence of oxygen. This has led to the classification of organisms as aërobic and anaërobic, according to their method of securing energy for the life processes.

Aërobic organisms secure this energy by means of oxygen used in normal respiration, while anaërobic organisms are able to secure it by fermentations in the absence of oxygen. Some of the yeasts are able to accommodate themselves to either mode of life, and are thus either aërobic or anaërobic, according to the nature of the medium in which they are placed.

Bread making. In the making of bread some active yeast, composed of starch mixed with some sugar and water, is added to bread sponge. The action of the yeast on the sponge is a double one, caused by the secretion of a digestive cane-sugar ferment and of an alcoholic energy-forming ferment, zymase. The cane-sugar ferment transforms the cane into grape sugar, as explained above, while the zymase completes the transformation of grape sugar into alcohol and carbon dioxide. The carbon dioxide is liberated in the sponge and causes the rising of the bread. The almost imperceptible amount of alcohol produced disappears in the baking. The two formulæ given above under digestion would therefore indicate in general terms the action of the yeast ferments in bread making. The cane sugar upon which the yeast acts must be supplied in the sponge. 
Beer and wine making. In the making of beer and wine the ferments and the processes involved are identical with those just described for bread making. The difference in the final practical result is that in beer and wine making the alcohol is saved and the carbon dioxide is largely allowed to escape. The sugar for the fermentative process may be supplied directly or it may be obtained from sprouted barley (called malt), from expressed grape juice, or from the juices of other fruits. . The special flavors of different wines and beers are due in part to the kind of yeast plants used and in part to the source from which the sugar solution is derived.

In the preparation of malt from barley the grain is first sprouted, in order that the stored starch of the barley seed may be digested and so transformed into a cane sugar. This digestion is due to the secretion of digestive ferments by the storage cells of the seeds. The cane sugar (maltose) thus formed is dissolved out in water from the dried and crushed seeds, and furnishes the sugar for the action of the beer yeasts. These yeasts then transform the cane sugar into grape sugar, and this again into alcohol and carbon dioxide, exactly as described above in bread making.

The great value of the yeasts in the industries is due to the rapid reproduction of the yeast cells by budding, and to the peculiar nature of the secreted ferment, which is able to transform large volumes of sugar into alcohol and carbon dioxide without losing its active properties.

\section{BACTERIA}

Bacteria are plants of the greatest interest and importance to man on account of their relation to disease, to the destruction of food, and to the maintenance of certain modern industries. It is not generally known that bacteria, as well as yeasts, are plants which belong to the great group of fungi which we are now studying. Botanists classify them as fission fungi, or Schizomycetes, on account of their mode of multiplying by simple cell division, or fission. They are derived, like the yeasts, from 
certain of the lower groups of fungi, although neither the relationship of the bacteria nor their classification into definite groups is as yet understood even by specialists in bacteriology. Bacteria are usually grouped, for convenience, under certain form types, illustrated in Fig. 134.

Structure. The cells of the bacteria are the smallest of which we have any definite knowledge, ranging from $\frac{1}{1000}$ to $\frac{50}{50} \frac{1}{0}$ of
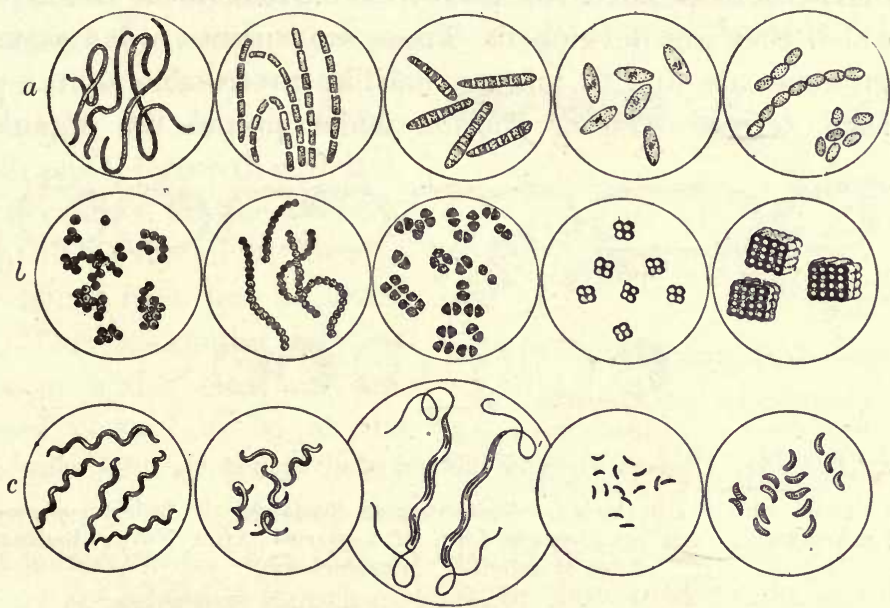

Fig. 134. Form types of bacteria

$a$, types of bacilli ; $b$, types of micrococci ; $c$, types of spirilla. After Williams. Redrawn from Marshall's "Microbiology",

an inch in diameter. The rod bacteria, represented in Fig. 134, $a$ and $c$, vary from $\frac{1}{500 \overline{0}}$ to $\frac{1}{500 \overline{0}}$ of an inch in length.

The bacterial cell differs somewhat from the ordinary plant cell in the structure of the cell wall, in the chemical nature of its protoplasm, and in the nature of the nucleus. The cell wall is composed of two membranes, the outer of which may become gelatinous, thus enabling the cells to adhere and form simple colonies.

The protoplasm is dense and contains more nitrogen than that of ordinary plant cells. The nucleus, if present, has no nuclear membrane, and some bacteriologists deny the presence of a nucleus in the cells of bacteria, while others claim to have 
found the nucleus in division, with spindles resembling those of the higher plants. For the general student the most important thing to realize is that bacteria are unicellular organisms with a cell structure essentially like that of yeasts and other simple plants. This being the case, we shall expect to find that the life processes and activities of bacteria are quite similar to the physiological processes of other fungi.

Many bacteria have the power of movement in the liquids in which they are developed. These movements, when actually progressive, are due to minute, hairlike protrusions of the protoplasm, termed cilia or flagella, which propel the organisms
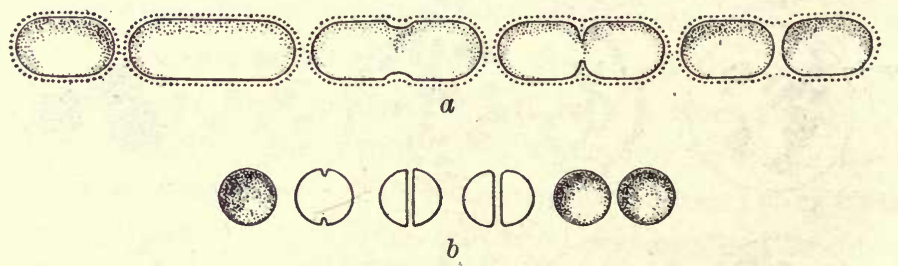

FIG. 135. Diagram showing method of division in bacterial cells

$a$, division of a rod bacterium (Bacterium or Bacillus); $b$, division of a cell of a coccus or of a micrococeus form of bacteria. After Novy. Redrawn from Marshall's "Microbiology"

by simple or complex whiplashlike movements. The cilia are variously distributed, being usually either terminal or uniformly distributed over the cell surface. The vibratory movements of bacteria often observed under the microscope are not due to vital activity of cilia or flagella, but resemble the so-called Brownian movements characteristic of fine inorganic particles of various kinds in liquids.

Reproduction. The bacteria reproduce either by simple division and separation of the two daughter cells or by spore formation. The former process is very rapid and is the usual method of reproduction; this accounts for the ability of bacteria to multiply with such startling rapidity as is often witnessed in the case of diseases and in decaying or putrefying organic material.

When a bacterial cell is about to divide (Fig. 135), the cytoplasm constricts in the middle of the cell from the cell wall 
inward. This constriction may take place in any axis in the spherical bacteria, but in the rod forms it always occurs across the long axis of the cell. The divided protoplast then secretes a cell wall between its two halves, the new wall splits, and the two cells thus formed separate to form two new plants. In a short time the two daughter plants thus formed will reach maturity and divide again. Unless this division process is stopped by lack of food or by other unfavorable conditions, the rapid multiplication mentioned above will result in thousands of new plants in a few hours. It has been estimated that under the most favorable conditions for growth and division seventeen million bacteria might be produced in twentyfour hours from one bacterial cell. This maximum rate of division under such circumstances is said to be at the rate of one division every twenty or thirty minutes; but

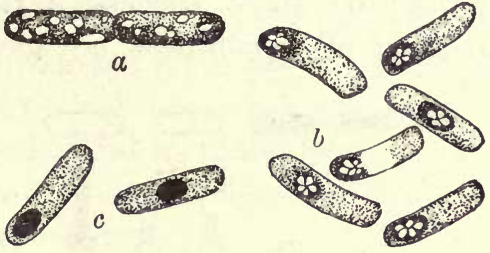

FIG. 136. Spore formation in bacteria $a$, two bacterial cells; $b$, granules collecting to form spores; $c$, black spores in two cells. After Fisher. Redrawn from Marshall's "Microbiology" such a maximum, it is safe to say, is never realized in nature for any considerable length of time, or the world would be overrun with bacteria.

Spore formation in bacteria (Fig. 136) takes place within a bacterial cell which acts as a spore mother cell. One spore only is formed in each cell, and the process begins by the collection and rounding up of the protoplast in the middle, or at the end, of the mother cell, which often bulges at this point with the accumulation of the protoplast. A new cell wall is then formed around the protoplast within the old mother-cell wall, and the spore is complete, although still within the cavity of the mother cell. These spores remain in a resting condition until favorable conditions for growth arise, when they germinate to form a new plant. In this process the wall of the mother cell is split and the spore wall and protoplast protrude and expand into the form of the bacterium from which the spore sprang (Fig. 137). In the case of the rod bacteria the splitting of the 
mother cell takes place either at the end or in the middle of the cell. As indicated above, one of the principal functions of the spore is to carry bacteria over periods which endanger the life of the ordinary vegetative bacterial cells.

Sterilization. On account of the great resistance of the bacterial spores to extremes of temperature and drought the greatest difficulty is experienced in destroying them in food or in cultures. One method of killing the spores of bacteria is to subject the material to be sterilized to flowing steam at $100^{\circ} \mathrm{C}$. for a short period on three successive days. This is the discontinuous method of sterilization advanced by Tyndall. If milk or any

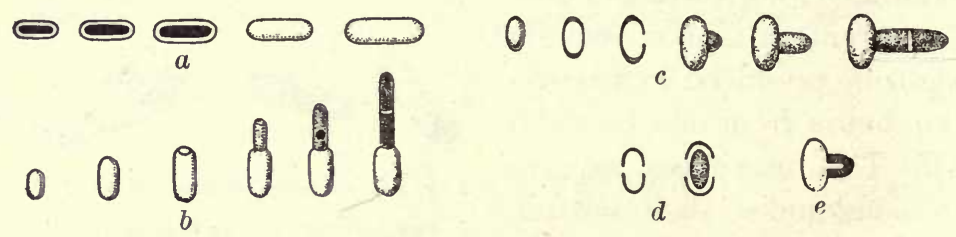

Fig. 137. Different methods of spore germination

$a$, "direct conversion of a spore into a bacillus without the shedding of the spore wall " in B. leptosporus; $b$, polar germination of a spore in B. anthracis; $c$, equatorial spore germination in B. subtilis; $d$, equatorial germination in B. megatherium; $e$, same with horseshoe appearance. After Novy. From Marshall's "Microbiology"

other material is to be freed from bacterial spores by this method, it is first heated in flowing steam at $100^{\circ} \mathrm{C}$. for fifteen minutes, which is sufficient to kill all living vegetative yeast, mold, or bacterial cells in the substance, except certain resistant bacterial spores. Any spores which may remain will germinate if left for a few hours at the proper temperature. The material to be sterilized is consequently allowed to stand after the first heating until any spores which remained have had time to germinate and form vegetative bacteria, when a second heating will kill all of the bacterial cells thus produced. A third heating is usually necessary after an interval, in order to kill any bacteria. which may have been produced by spores which had not yet germinated at the time of the second heating. It is now thought that the highly resistant spores are also gradually weakened and finally killed during the prolonged period of heating. 
Another common method of sterilization is to subject the material to be sterilized to steam at a very much higher temperature $\left(120^{\circ} \mathrm{C}\right.$. $)$ at the outset than that indicated above. Then a single heating is usually sufficient to kill the spores.

In pasteurization no attempt is made to kill the more resistant spores, but simply to kill the vegetative cells of certain disease germs. For this purpose milk or other substances to be pasteurized are heated to about $65^{\circ}$ or $75^{\circ} \mathrm{C}$. By this means fermentation or any other change induced by active bacteria in the pasteurized material is temporarily checked, and the pasteurized milk or food will thus remain unchanged for a longer period of time. The ordinary disease germs which occur in milk, as well as the bacteria which sour milk, are killed by pasteurization, and the deleterious effects which result from the high temperatures necessary for sterilization are also avoided.

Decay. The process commonly known as decay is very largely due to fermentation or to digestion by bacteria and other saprophytic fungi that live and feed on the organic material which exhibits the appearance and phenomena of decay. The bacteria which cause decay are saprophytes, and like the yeasts they secrete digestive and energy-forming ferments which attack the cell walls and other lifeless parts of animal and plant remains upon which they live. Parts of these organic remains are thus fermented and digested, with the result that the entire form and structure of the part attacked is often destroyed and crumbles away. Accompanying this fermentation and digestion of organic matter, gases, such as ammonia and sulphureted hydrogen, are produced. In the case of the flesh of animals or of material containing a large percentage of nitrogenous compounds the gases liberated give rise to odors which we associate with putrefaction. Nitrates and sulphates are also formed which are carried back into the soil, and these compounds ultimately serve as raw food elements absorbed by the roots of green plants. The decay of leaves and tree trunks in a forest, already mentioned, is a good instance of decay produced by saprophytic bacteria and other fungi, and similar activities of such bacteria in liquids are familiar phenomena. The souring of milk is caused by a 
ferment secreted by a saprophytic bacterium which causes the sugar of milk to be transformed into lactic acid.

Milk sugar + lactic ferment = lactic acid + lactic ferment.

Similarly, cider is changed into vinegar by certain ferments secreted by bacteria. This process is brought about by the oxidation of alcohol in the cider to form the acetic acid of vinegar.

Alcohol + oxygen + acetic ferment

$$
=\text { water }+ \text { acetic acid }+ \text { acetic ferment. }
$$

Many other familiar instances might be given in which saprophytic bacteria cause profound changes in organic bodies by the secretion of ferments which enable the bacteria themselves to digest food or produce energy for their own life processes.

Disease. Certain parasitic bacteria, commonly designated as disease germs, cause diseases in animals and plants by the secretion of poisonous substances called toxins. The toxins are products manufactured, like ferments, by the protoplasm of the bacterial cells. These toxins then diffuse out into the blood and into the tissue cells of the organism attacked by the bacteria. When thus liberated in the system of another organism, the toxins cause the various symptoms characteristic of particular bacterial diseases. The toxins resemble the ferments, or enzymes, in being cell secretions; but they do not cause fermentations or digestion, and they have no known function for the bacterial cells secreting them. Similar poisonous toxins are secreted by snakes, by the castor bean, and by poisonous mushrooms. The toxins are not, therefore, confined to bacteria, although the bacterial toxins are the most important on account of the diseases which they cause in man, other animals, and plants. A large number of plant diseases are now known to be due to bacteria.

Antitoxins are secretions of the cells of the organism attacked by toxic bacteria, which tend to neutralize the toxins secreted by the bacterial cells. Diphtheria, for instance, is produced by bacteria growing on the lining membrane of the throat. The diphtheric bacteria do not enter the body of the diseased organisms, but secrete the diphtheric toxin, which is circulated by 
the blood and causes the symptoms of the disease in man and animals. To counteract the effect of the toxin the body cells of the diseased person secrete antitoxins, which, if secreted in sufficient amount, can neutralize the effects of the diphtheric toxin and thus arrest or stop the progress of the disease. Other

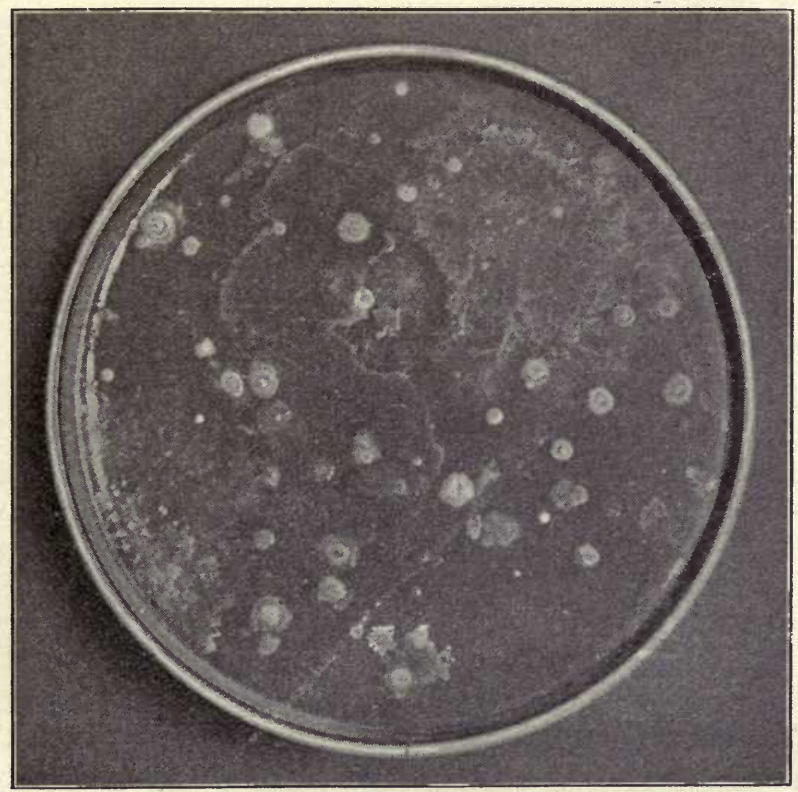

Fig. 138. Bacterial colonies growing on a gelatin culture

A sterilized plate of gelatin exposed for five minutes in the hallway of a schoolroom, and then closed for five days, developed the above colonies. This illustrates the dissemination of bacteria in the air and the danger of increasing the normal number by stirring up the dust on the floor of a room. From Bergen and Caldwell's "Introduction to Botany"

antitoxins produced in animals may, as in the case of diphtheria, be injected into the blood of the diseased person and thus aid in checking the progress of the disease. Antitoxin treatment is especially helpful in diphtheria and tetanus, or lockjaw.

The ptomaines are complex chemical bodies which develop in various organic substances, including canned foods, ice cream, etc. They are sometimes poisonous and sometimes harmless, and must not be confused with the toxins, which are definite poisonous 
secretions giving rise to disease. The ptomaines are regarded by some as bacterial secretions and by others as by-products of the decomposition caused in organic material by various saprophytic bacteria. Ptomaine poisoning is more likely to occur in warm weather, since conditions are then more favorable to general bacterial activity.

\section{SUMMARY}

From the above discussion it has been found not only that bacteria have the same general cell structure as other organisms but that their physiological activities and products closely resemble those of the yeasts and other saprophytic and parasitic fungi to which they are most closely related. The decay and putrefaction caused by bacteria is indirectly a result of certain digestive and fermentative processes which belong in the same eategory as similar nutritive processes occurring in yeasts and other fungi. Even the disease-producing power of bacteria is due to products of their cell protoplasts, which are not peculiar to the bacterial cells alone. The striking effects of bacteria on other organisms and upon organic material are due, therefore, to their great numbers and to the immense scale upon which they act, rather than to any marked peculiarity either in their organization or in their physiological processes.

\section{MOLDS}

Everyone is familiar with the appearance of molds on bread, jelly, and other household products. The white, fluffy character of the molds is due to the innumerable colorless filaments which comprise the plant body. These filaments are individually called hypho, while the entire white mass of filaments constituting a mold colony is called a mycelium. The hyphæ of the molds, and other fungi as well, resemble very closely the filaments of green algæ like Vaucheria or Spirogyra, except that they lack chloroplastids and green chlorophyll. The mycelium which is formed by them usually occurs both on the surface and within the substances upon which they grow. The term aërial hyphoe or aërial mycelium is applied to the visible surface filaments of a mold, while those which grow within a substance are called submerged hyphoe or submerged mycelium (Figs. 139 and 140). 
This differentiation of the plant body of a mold into an aërial and a submerged portion has an important physiological bearing on the life of the plant. 'The aërial hyphæ give rise to the spores, which are thus in a position to be readily disseminated in the air, while the submerged hyphæ ramify through the nutrient

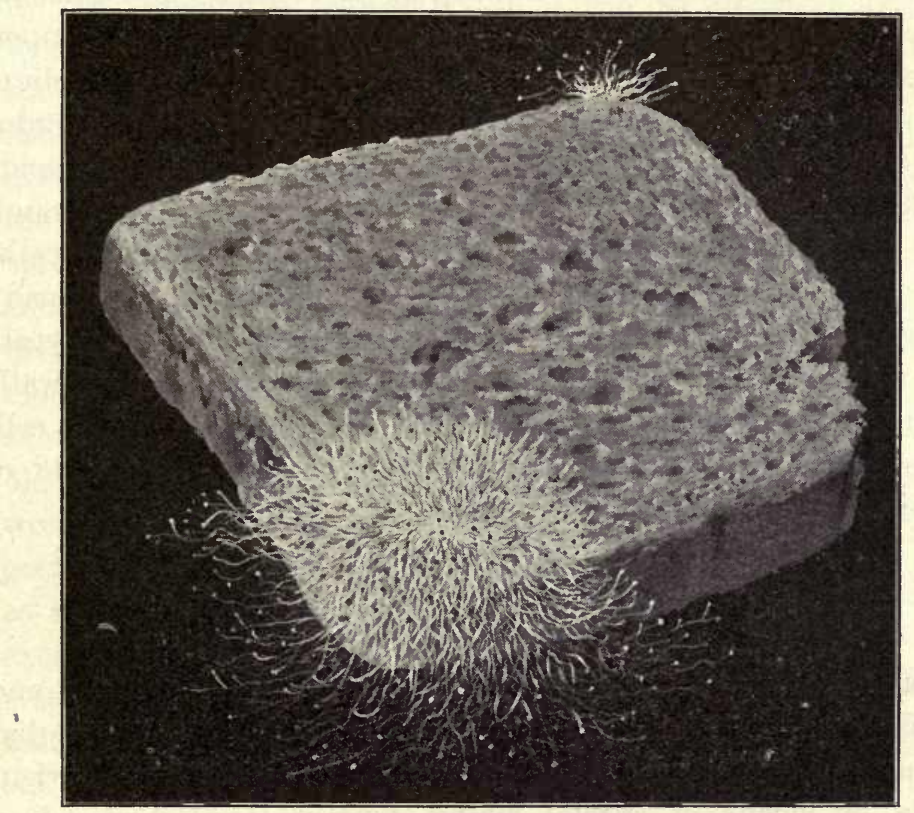

Fig. 139. The habit of the black mold (Rhizopus) growing on bread

The black heads are the sporangia growing from the aërial mycelium of the mold. After Conn

medium on which the mold is growing, and both digest and absorb food for the entire fungus plant. The submerged hyphæ thus serve the double function of digesting and absorbing foods. We see, therefore, that even in these very simple plants there is a division of labor which amply meets their demands for food and the need for wide distribution. These facts will become clearer by the study of two or three common types of molds selected from two different groups of fungi. The black molds, represented by Rhizopus, belong to the lowest group of algal 
fungi, while the blue-green molds belong to the sac fungi. They are, however, conveniently studied together on account of the similarity in their general structure and mode of life.

-The black molds are readily distinguished by the naked eye on account of the dark spore cases, or sporangia, which are borne by the older portions of the aërial mycelium. If examined closely, these sporangia look like black spheres supported upon minute stalks, or hyphæ, springing from the white aërial mycelium of the mold. The younger sporangia are white, becoming gradually darker with age. Another characteristic of the black molds is the peculiar structure of the hyphal filaments, which, though long and frequently branched, are yet composed of single cells, like Vaucheria, without partitions or septa but with many nuclei. They have been likened to a greatly elongated yeast cell which with its growth has failed to form new cell walls to inclose the repeatedly dividing nuclei of the growing cell. A convenient species for study is the common black mold of bread, known as Rhizopus nigricans.

\section{RhIzopus nigricans (BLACK Mold)}

Habit. Rhizopus has the same branched multinucleate and unicellular hyphæ as the rest of the black molds. It is peculiar in that it spreads over the surface of bread or other nutrient media by means of special hyphæ termed runners or stolons. The mold spreads by these stolons in much the same manner as strawberry plants spread by runners growing out from the mother plants. The stolons are hyphæ which grow out radially from centers where the mycelium is already established. At certain intervals these hyphal stolons send out rootlike submerged hyphal branches, which penetrate the nutrient medium on which it grows and anchor the aërial mycelium. The sporangia and spores are borne on erect aërial hyphæ which spring from the points on the stolons where the rootlike hyphæ grow out. The sporangia and spores are thus borne at the most favorable points for receiving food from the submerged hyphæ, as these digest and absorb the starch and other nutrient substances of the bread. 
Nutrition. Rhizopus is able to grow not only upon bread but upon a great variety of organic material if kept in moist places. From some substances it is undoubtedly able to absorb foods which are already in solution, while in other cases, as on breads, much of the food material is insoluble and must first be digested by the submerged hyphæ before it can be absorbed and used by the cells of the mold mycelium or by the sporangia during spore formation (Fig. 140). In order to digest the food the submerged hyphæ secrete digestive ferments similar to those already discussed under yeasts. These digestive ferments diffuse out of the hyphæ and convert the starches, fats, and proteins of the bread on which the mold is growing into soluble and diffusible foods. In other words, the mold digests its food much as higher plants do, except that the digestion in the mold takes place entirely outside of the body

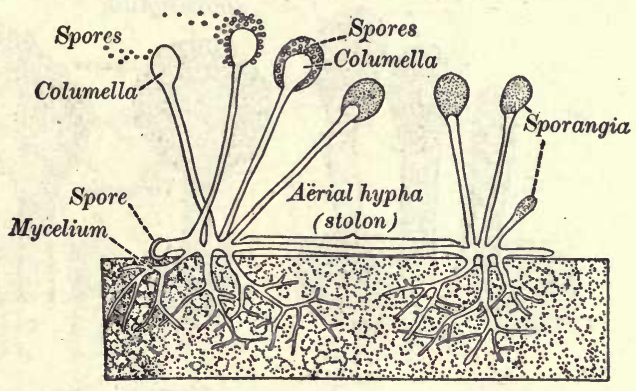

FIG. 140. A drawing illustrating the growth of Rhizopus on and within a piece of bread

The absorbing and feeding mycelium, composed of branched hyphæ, is shown within the bread; also a stolon and two groups of sporangia cells of the plant and within the nutrient medium immediately surrounding its hyphæ. Some of the black molds, in addition to the digestive enzymes, are able to secrete enzymes which bring about fermentations in sugar solutions exactly like those of the yeast.

Asexual reproduction. The hyphæ which bear the sporangia and spores in Rhizopus arise at the point of origin of the rootlike outgrowths which spring from the stolons, where they first grow out as short, erect branches of the mycelium (Fig. 141). These aërial hyphæ soon begin to swell at the ends, producing a light-colored spherical enlargement which is the beginning of the future sporangium. The entire structure, including the erect hypha and its swollen end, is now called a sporangiophore, 
or sporangium bearer. A cell wall next appears, separating the young sporangium from the hypha which bears it, thus forming a greatly swollen terminal and spherical sporangial cell which is filled with dense cytoplasm and many nuclei. This sporangial cell now expands with great rapidity, and with its expansion the wall separating it from its hyphal stalk grows in surface area and assumes a convex form, protruding into the growing sporangium until it comes to occupy fully one half or two thirds of the sporangial cavity (b), when it is called the columella. The mold, if
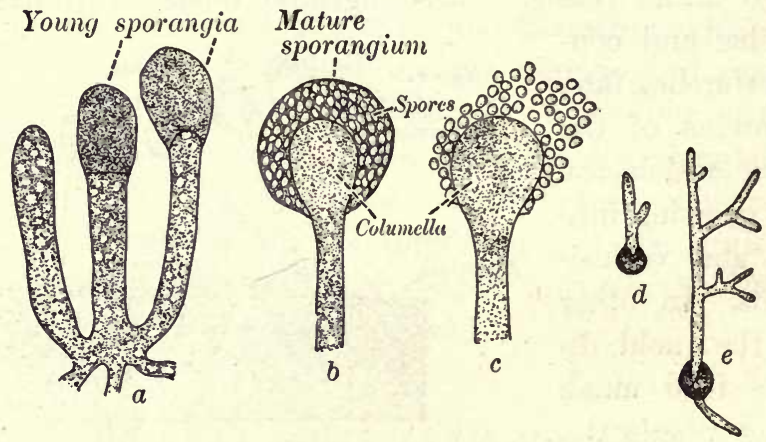

Fig. 141. Development of the sporangia and spores in Rhizopus

$a$, young sporangia occurring as terminal enlargements of aërial hyphæ; $b$, older sporangium with columella and spores differentiated; $c$, spores being shed by rupture of sporangium wall; $d$, early stage of spore germination; $e$, later stage, with branched hyphal filament

observed at this stage with a hand lens, appears to be dotted with round white or gray balls, which are the young sporangia. Meanwhile internal cell processes which are to result in the formation of the spores have been going on in the cytoplasm of the sporangium. As the spores mature they secrete a black pigment, so that the sporangia become darker with age. Finally the sporangium cavity is filled with innumerable dark spores, and the plant now presents the familiar appearance of the black molds, owing to the large number of black sporangial heads which cover its surface. When the sporangia are ripe, the sporangium wall ruptures by drying, and the light spores are widely scattered by wind in the open and by air currents in dwellings. 
The germination of the spores takes place as follows: When they come to rest on a proper nourishing medium, the spores absorb water, the outer coat ruptures, and the delicate inner coat expands into a tubular hypha, into which the protoplasm of the spore flows (Fig. 141, $d$ and $e$ ). As the hypha elongates it absorbs new nutriment, by means of which it grows and branches to form a new plant body, or mycelium. Usually several spores germinate in close proximity, and the hyphæ produced by the different spores commingle to make one fluffy, white mycelium characteristic of the black molds.

Sexual reproduction. Under special conditions two hyphæ, belonging to separate mycelia of different kinds, or strains, may conjugate and produce a zygote. Each hypha sends out a fertile hyphal branch, or bud, called a suspensor. The two branches grow toward each other in much the same way as the

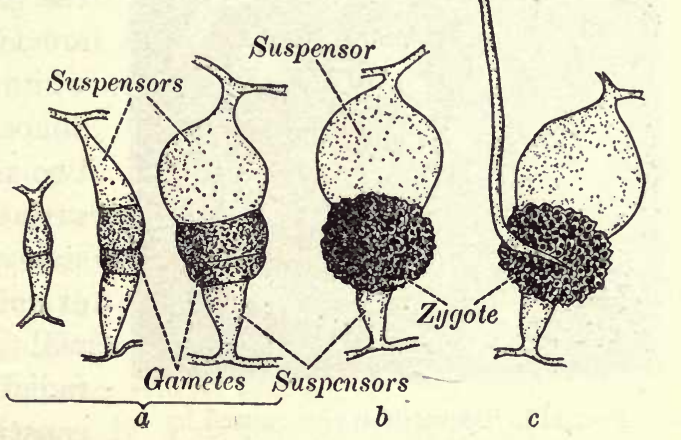

Fig. 142. Sexual reproduction in Rhizopus

$a$, formation of the suspensors and gametangia; $b$, formation of the zygote; $c$, germination of the zygote to form a new sporangium conjugating tubeș do in Spirogyra, and each suspensor cell then cuts off at its apex a cell which corresponds to a gametangium (Fig. 142). The two end cells, or gametangia, of the approaching suspensors meet, and the protoplasts (gametes) unite after the solution and disappearance of the cell walls of the two gametangia at the point of contact. The zygote cell thus formed enlarges and forms the usual heavy protective walls of a zygote. After a period of rest this zygote may germinate and produce a new mycelium (c), from which sporangia and asexual spores will be formed as described above. Although the sexual reproduction of Rhizopus and Spirogyra are similar, the two plants have no near relationship. 


\section{Penicillium (Blue Mold)}

Habit. The common blue-green molds are found on decaying fruits, vegetables, and foods of various kinds. They differ from the black molds in that their hyphæ are subdivided into many cells, each cell of which is provided with a single nucleus. They also produce asexual spores by the transverse division of special spore-bearing hyphæ and not by cell division within a

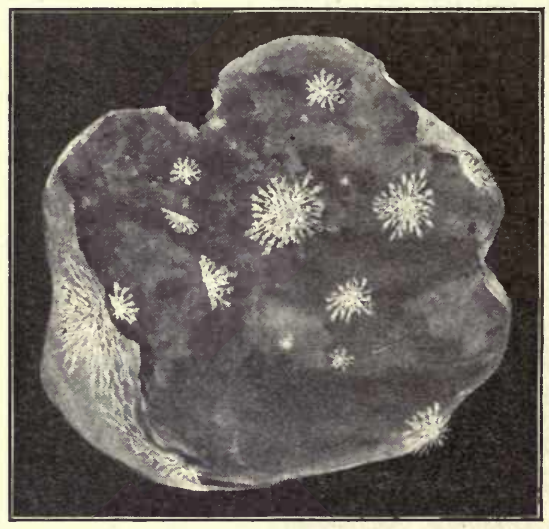

Fig. 143. Decay of apples caused by

The mycelium of the mold penetrates the apple, as in the case of Rhizopus on bread (Fig. 140), and breaks down its substance, thus causing decay. After Conn mold growth

sporangium, as in Rhizopus.

When fruits or other nutrient media suitable for the growth of Penicillium are left in a moist place, white patches of mold are almost certain to appear in two or three days on the surface of the exposed substance (Fig. 143). Careful examination of these areas will reveal more or less radiate patches of hyphæ constituting the young mold colonies of Penicillium. This radiate appearance will be found to be due to the fact that the new hyphæ which make up the white mycelium of the mold are growing out centrifugally from the point where the mold spores started to germinate. This centrifugal growth continues until neighboring patches unite into one extensive mycelium, thus obscuring the method of origin.

The patches of inycelium described above start from spores which have been deposited from the air on any given nutrient medium. These spores germinate, like those of Mucor already described, by the rupture of the outer coat of the spore and the protrusion of the inner coat to form a hyphal filament. If spores of Penicillium are sown on prune juice or on some other 
favorable medium and are then placed in a suitable temperature $\left(15^{\circ}-30^{\circ} \mathrm{C}\right.$.) for twenty-four hours, all stages in the germination process. can be observed with a sufficient magnification. The elongation of the inner coat to form a hypha is accompanied by nuclear and cell division, so that the resulting hyphæ and mycelium are multicellular. The individual hyphæ have a delicate cell wall with highly granular vacuolate cytoplasm, and repeatedly give off branches, ultimately forming the patches of mold already described. In nature and in the artificial sowings of spores more than one spore usually starts a mycelium at a given point. The centrifugal method of growth which follows is advantageous in bringing the mycelium in contact with more of the nutrient material and in forming a larger surface for the formation of spores. As growth proceeds submerged hyphæ grow down into the fruit or other nutritive medium and perform the functions of digesting and absorbing food for the entire plant.

Asexual reproduction. If patches of Penicillium are examined, it will be seen that they gradually change their color from pure white to sage green. This change in

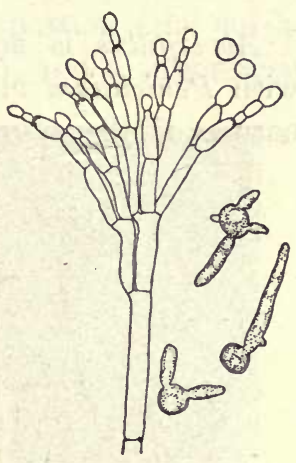

Fig. 144. The method of producing spores in Penicillium

Consult the text for a discussion of the sporeforming process. At the right are germinating spores. After Thom color usually begins in the older central portion of the circular patches of mycelium and spreads from the center toward the circumference of any given mold colony. It is due to the formation of innumerable spores, which, taken in the mass, are green in color. The spores are formed upon erect aërial hyphæ, which branch repeatedly at the end, forming a brushlike or treelike growth of short hyphal branches (Fig. 144). Each branch then begins to constrict below the apex just as though a thread had been tied around the hypha at this point and then gradually tightened. This process is repeated until each hyphal branch of the main spore-bearing hypha is converted into a row of spores. The older spores at the ends of 
the branches break off and are disseminated by air currents, thus scattering the mold spores widely. The large number of spores produced, and the great resistance of the spores to extremes of temperature and moisture, account in part for the wide distribution of Penicillium in nature.

\section{ASPERGILLUS}

Aspergillus is another blue-green mold which often occurs with Penicillium in cultures, and differs from it largely in the nature of the spore-bearing hyphæ (Fig. 145). These hyphæ

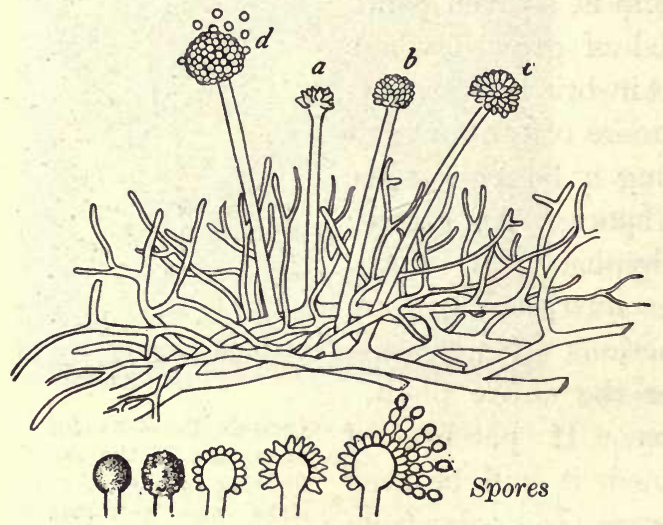

Frg. 145. A colony of Aspergillus, showing mycelium and spore clusters

The lower figures show in detail the method of spore formation. After Conn in Aspergillus swell at the end into a large, spherical cell which looks like a young sporangium of Rhizopus. The swollen end cell then buds out into innumerable short, radiating hyphæ, which together form a compact head. Each radiating hypha then constricts to form a chain of spores, exactly as in Penicillium. In other re-

spects Aspergillus is scarcely distinguishable from Penicillium and has essentially the same general appearance and biologic history.

Both Penicillium and Aspergillus produce gametes which fertilize and produce spore fruits. The sexual process in the molds and in many other fungi seems, however, to have become quite subordinate to the asexual spore process, upon which these fungi depend almost entirely for their perpetuation and wide distribution. 


\section{MUSHROOMS AND THEIR ALLIES}

\section{Mushrooms}

The mushrooms belong to the higher fungi and include among their allies the puffballs, the bracket fungi, and the rusts and smuts of cereal grains.

Habit. The plant body of a mushroom is divided, like that of the molds already studied, into an aërial portion (the mushroom proper), which bears spores (Fig. 146), and a submerged

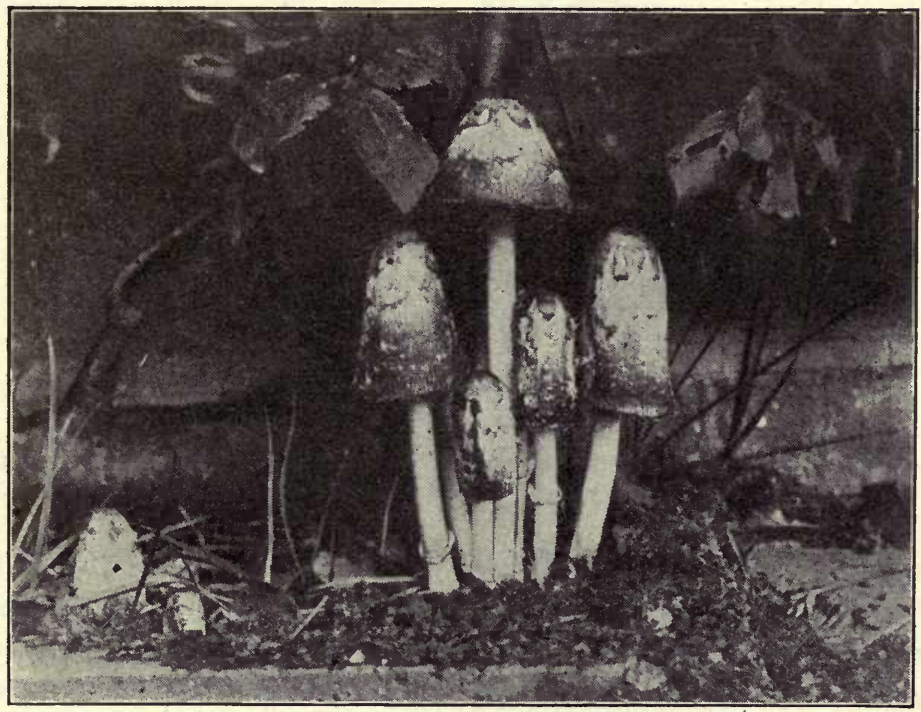

Fig. 146. Habit of the "shaggy mane" mushroom, Coprinus comatus After Buller

mycelium, which digests and absorbs food from the organic matter in the soil. The mushrooms are therefore saprophytes and grow best around old stumps or in soil which is rich in decaying vegetable remains. The mycelium, often called spawn, traverses this soil in the form of long white threads made up of strands of mycelial fibers closely interwoven. This spawn may live for several years, and produces each season a new crop of mushrooms. The mushroom plant above soil is therefore a 
kind of fruit body, which expands, produces spores, and dies down again in a few days, while the real lasting and perennial portion of the plant is the feeding submerged mycelium within the soil.

The spore-bearing mushroom fruit is definitely adapted to the production and dispersal of large numbers of asexual spores. Its parts are the stipe, or stalk, the pileus, or umbrella, and the lamelloe, or gills. The stipe bears the pileus at its apex and serves to lift it into the air when the spores are ripened and ready to be shed. The pileus bears the lamellæ, or gills, on

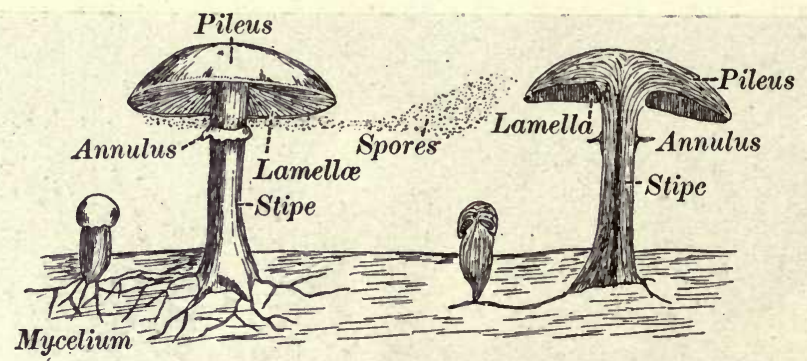

Fig. 147. Diagrammatic figures of young and mature mushrooms growing in the soil

Note the soil mycelium similar to that of Rhizopus in bread (Fig.140). Adapted from Buller's "Researches on Fungi"

its under surface as radiating plates which extend from the stipe to the margin of the pileus and greatly increase the sporebearing surface of the mushroom fruit, since the spores are borne over the entire surface of each lamella. Buller estimated that a single lamella of Coprinus comatus produced about $24,480,000$ spores and that all of the lamellæ of this mushroom bore approximately $5,240,000,000$ spores.

When the mushroom is young (Fig. 147), the pileus is bent down like a folded umbrella, thus protecting the young lamellæ and spores during the early stages of their development. Some mushrooms have a further protection for the lamellæ and spores in the form of a delicate veil composed of hyphæ which stretch from the edge of the pileus to the young stipe, so that the lamellæ are inclosed in a chamber away from the outside air. As the 
mushroom matures, the stipe elongates and the pileus expands, so that the lamellæ are lifted into the air and properly disposed for the dissemination of the spores. At the same time the veil is ruptured, and a remnant of it clings to the stipe as a ring of tissue, called the annulus, marking the former junction of the
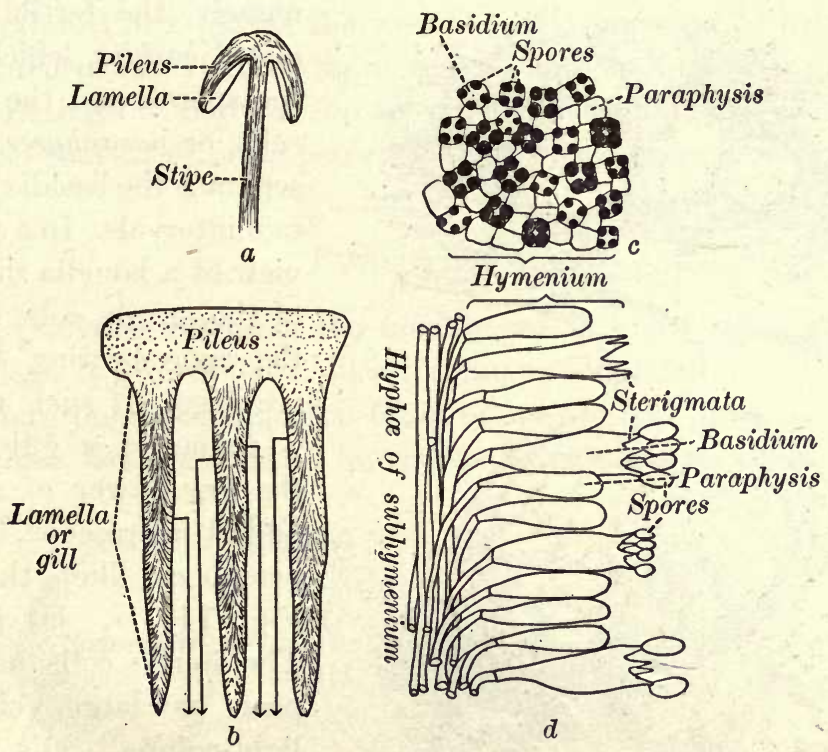

Frg. 148. Spore formation in a mushroom, Coprinus

$a$, vertical section of the mushroom fruit; $b$, vertical section of three lamellæ, or gills (the arrows indicate the path of the spores when shot off from the sterigmata); $c, d$, portions of the hymenium showing spores on the basidia ; $c$, surface view of the hymenium; $d$, sectional view. Further discussion in the text. $b, c, d$, redrawn from Buller's "Researches on Fungi"

veil with the stipe. The output and dissemination of spores is also greatly facilitated by the structure of the lamellæ and by the method of producing and freeing the spores by the specialized spore-producing cells, or basidia.

Asexual reproduction. The lamellæ are formed by hyphæ which grow downward from the pileus and unite to form the various layers of the gills.

The central portion of a lamella is made up of vertically arranged hyphæ which unite by cross branches to form the 
trama. The hyphæ of this central trama turn outward toward the surface of the lamella and end in large, club-shaped cells which form the hymenium, or spore-bearing layer (Fig. 148, $d$ ). This hymenium covers the entire surface of the lamella like an epidermis, and is composed of two kinds of club-shaped cells,

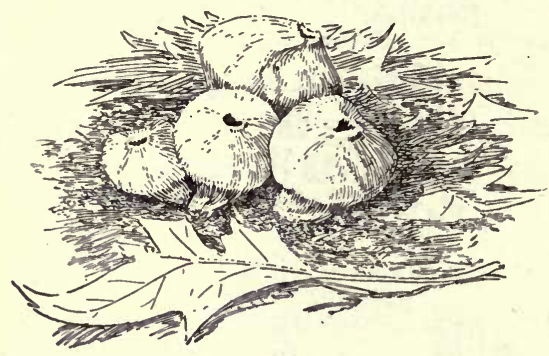
namely, the fertile cells; called basidia, which form the spores, and the sterile cells, or paraphyses, which separate the basidia at certain intervals. In a surface view of a lamella the ends of the sterile cells and of the spore-bearing basidia are seen. If such a view is taken of a gill under the low power of a compound microscope, an appearance like that in Fig. 148, $c$, is shown. The sterile cells now appear as large, circular, light-colored cells, while the spores borne by the basidia are seen in groups of four small, dark bodies.

Fig. 149. Two species of puffballs, the upper one shedding spores

This view also gives one

a good idea of the continuous epidermislike character of the hymenium. When the hymenium begins to bear spores, each basidium buds out at its large, free end into four slender protrusions of the cell wall, called sterigmata, and each sterigma then swells up at the end into a spherical spore cell, into which cytoplasm and a nucleus flow from the main body of the basidium. The spores change in color as they mature, and cause a change in the color of the gill from white to black, brown, or pink in many species of mushrooms. When they are ripe they are shot off from the sterigmata 
and fall vertically in the space between the two adjacent gills. Buller estimates the spore discharge in Coprinus comatus, the common shaggy Coprinus, to aggregate one hundred million spores per hour during the period of active spore discharge, so that if any considerable proportion of these spores germinated and produced new plants, the world would very soon be overrun with mushrooms. Most of these reproductive bodies of the mushroom fail to grow, however, on account of an unfavorable environment, so that there is no perceptible increase of the species.

\section{Puffballs}

The puffballs resemble the mushrooms in being saprophytes. The plant is also divided, like the mushrooms and molds, into an underground feeding mycelium, or spawn, and an aërial portion which bears spores. The spawn is identical in appearance

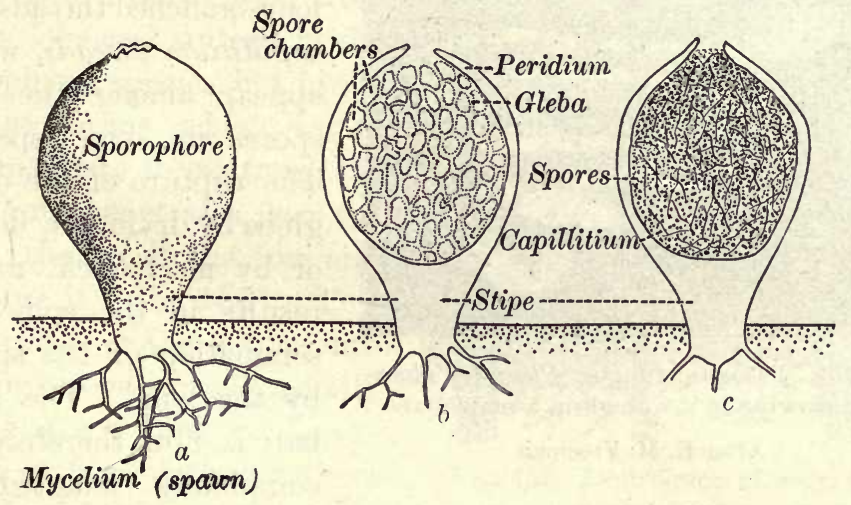

Fig. 150. Drawing showing the external and internal structures of a puffball $a$, external view with mycelium in the soil; $b$, median long section showing gleba and peridium; $c$, ripe puffball, in which the gleba is transformed into spores and capillitium

with that of the mushroom, but the aërial fruit body is the subspherical puffball seen in nature (Fig. 150): The hyphæ of which the puffball is composed differentiate very early into one or more dense outer layers, called the peridia (singular, peridium), and an inner loose mass of hyphæ, called the gleba. This inner gleba 
then forms spore cavities which are lined with a continuous hymenial layer like that which covers the outer exposed surface of a mushroom lamella. When the spores are ripe, the hymenium and the hyphæ of the trama, on which the hymenia of the several spore cavities are borne, break down, and the spores become free

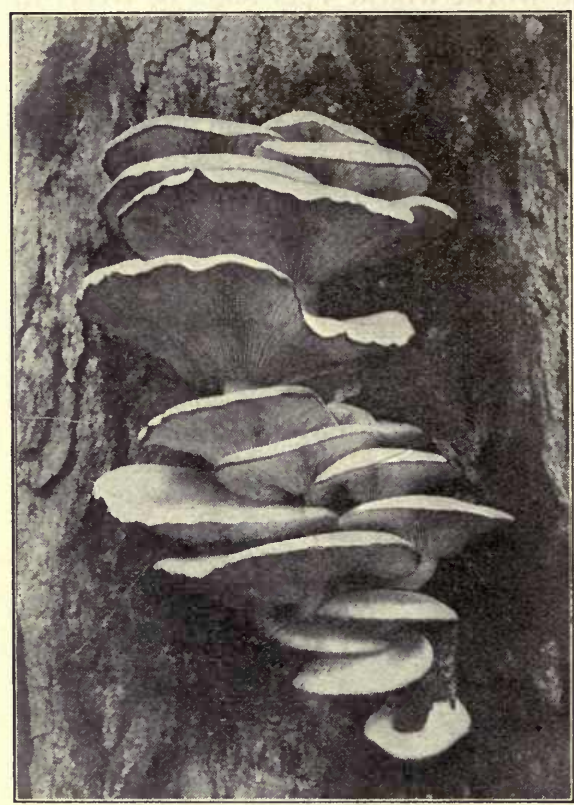

Fig. 151. Parasitic fungus, Pleurotus ulmarius, growing in a wound on a maple tree After E. M. Freeman within the peridium, or outer covering. The drying of the entire disorganized portion of the gleba and the growth of the spores result in the formation of a large central spore cavity filled with ripe spores. Some of the hyphæ of the gleba may also thicken to form long-branched threads, the capillitium threads, which appear among the ripe spores in some species. The rupture of the outer gleba by drying, by decay, or by mechanical means results in the wide dissemination of the spores by the wind. The puffball is not, therefore, as completely adapted to spore dissemination as the mushroom, in which the elongated stipe and the exposed lamellæ facilitate spore scattering.

\section{Bracket FungI}

The bracket fungi (Fig. 151) are also closely related to the mushrooms and puffballs on account of their method of forming spores by means of basidia arranged in the form of hymenial layers. These hymenial layers, in some species, cover either 
smooth or greatly roughened surfaces, but in the more common species they line pores on the under side of the shelflike fruit body. Since these pores open out on the under surface of the fungus, the spores are readily disseminated, as in the mushroom, by being shot off from the basidia into the open space of the pores, from which they fall downward into the open air.

The bracket fungi are often very hard and woody, but sections cut from the plant body show that it is composed entirely of thickened hyphæ. The feeding mycelium penetrates and absorbs food from rotten logs and stumps in the saprophytic species, but in the parasitic forms, which are so destructive to forest trees, the mycelium penetrates into the living tissues of the tree and destroys it (Fig. 152). This penetration of the hyphæ into the hard wood of trees usually begins at a wound on the exposed surface of the trunk or branches, and its entrance into the tree is effected by the secretion of cellulose and wood ferments, which digest a passage for the hyhæ. Once within

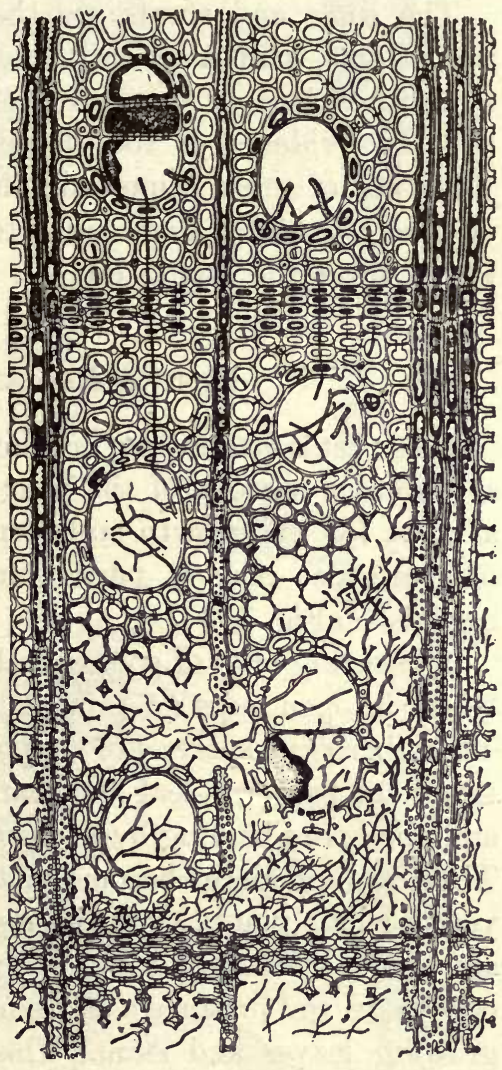

Fig. 152. Destruction of wood by fungus hyphæ

The figure shows the mycelium of Polyporus squamosus penetrating and breaking down the wood-cell walls by digestion. From Duggar's "Plant Diseases." After Buller the tree, these hyphæ branch within its tissues and not only break down the wood-cell walls but attack the living cells of the tree and their stored food. The bracket fungi thus destroy living forest trees and logs, railroad ties, and lumber. 


\section{RUSTS AND SMUTS}

The rusts and smuts are the most distant relatives of the mushrooms, puffballs, and bracket fungi. The relationship is established largely through their method of producing spores, but the evidence is too detailed for our present discussion. They are of great importance in agriculture on account of the destruction which they cause to the various species of cereals which form the staple crops.

\section{Rusts (PUCCINIA Graminis)}

This rust is heteroeious, producing four different kinds of spores at different seasons of the year and in using two plant species as hosts for the growth and formation of its spores. Each kind of spore also plays a particular rôle in the life history of the organism (Fig. 153).

The spring crop of spores, called ceciospores or acidiospores, are borne in open cups $(F)$ on the leaves of the common barberry. These barberry spores are supported by a mycelium which penetrates the barberry leaf as a parasite and absorbs food for itself and the spore-bearing hyphæ produced within the barberry cups. These spring spores become mature at about the time when the young wheat plants are springing up in the fields, and when they are blown by the wind and fall upon a young wheat plant they germinate and produce a dense mycelium within the tissues of its growing leaves and stem. This inner parasitic mycelium then forms red-rust spores, in groups called sori $(B)$, at certain points along the surface of the leaves and the stem. These masses of spores break through the epidermis and form the long lines of spores (A) familiarly known as red rust.

These summer spores, called urediniospores or uredospores, are single-celled and are borne on a short stalk. They are quickly disseminated, and since they germinate at once under favorable conditions, they serve to spread the rust very widely in the fields of grain in early summer. In warm regions the red-rust spores often survive the winter and start the rust in the spring. 

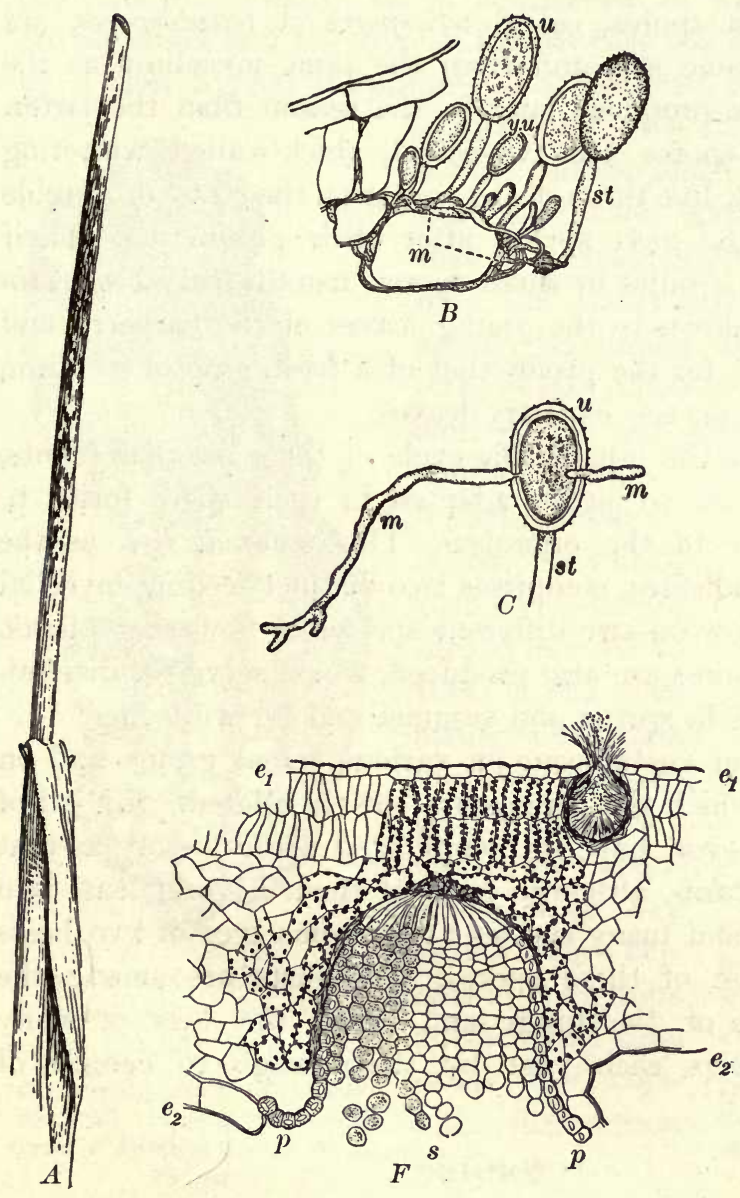

Fig. 153. Wheat rust (Puccinia graminis)
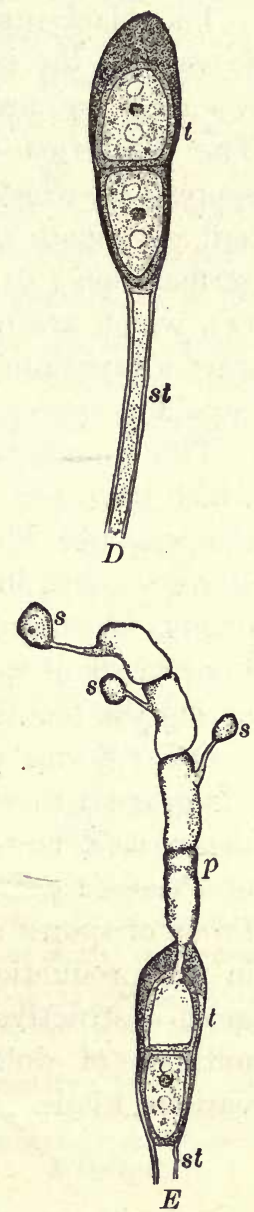

$A$, portion of stem of wheat with rust spores in groups, or sori; $B$, sectional view of a sorus, with young uredospores $(y u)$ and mature uredospores $(u)$, stalks $(s t)$, and mycelium $(m)$ within the stem tissues; $C$, red-rust spore (uredospore) with stalk (st) sending out infection hyphæ $(m) ; D$, two-celled black-rust spore (teleutospore, $t$ ) with greatly thickened cell wall and stalk $(s t) ; E$, germination of the upper spore cell of the teleutospore $(t)$ to form the promycelium $(p)$, bearing disseminating spores (sporidia, $s) ; F$, section of barberry leaf showing the recidium cup with spores $(s)$, the wall of the cup, or peridium $(p)$, and the upper and lower epidermis of the leaf ( $e_{1}$ and $\left.e_{2}\right)$. Adapted from Duggar's "Fungous Diseases of Plants" 
The black-rust spores, called teliospores or teleutospores, are formed in the same sori and from the same mycelium as the red rust, but are produced later in the season than the latter. The black-rust spores are two-celled, thick-walled wintering spores $(D)$ which live through the winter on the straw or stubble and germinate the next spring after their production. Their germination $(E)$ results in small spores, usually called sporidia $(s)$, which are blown to the young leaves of the barberry and start a mycelium for the production of a fresh crop of æcidium cups and spores on the barberry leaves.

This completes the remarkable cycle of these peculiar plants, which have become so highly adapted in their spore forms to the seasonal life of the organism. This seasonal life, as the above account indicates, comprises two distinct feeding mycelial plants, which grow on two different species of flowering plants. Four kinds of spores are also produced, which serve for disseminating the plants in spring and summer and for wintering.

Other forms of rusts occur on various cereal grains and on the grasses, to which the cereals are closely rélated. Not all of these rusts, however, have so complicated a life history as that of Puccinia graminis, although most of them have at least two forms of spores and many species avail themselves of two hosts in the production of these spores. The rusts are among the most destructive of the fungi and cause the loss of many millions of dollars each year by the damage to cereals of various kinds.

\section{Smuts}

The most familiar smut is that of Indian corn (Ustilago zeae) (Fig. 154), which causes the distortion of the kernels of corn on the cob and produces the large black masses of smut spores seen in fields of corn in the autumn. If the smutted ears of corn are examined early in the season, it will be found that the fungus gradually replaces the kernels as it absorbs the food stored in them. Later the infected kernels grow to many times the size of the original kernels and produce great masses of spores in cavities which resemble somewhat those already described in 
the puffball. Finally all of the tissues of the original kernel are absorbed and the hyphr die, leaving the spores surrounded by a thin hyphal membrane. The rupture of this membrane

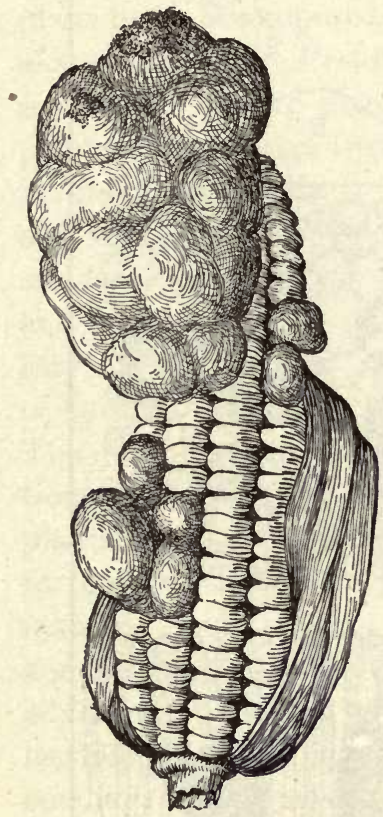

Fig. 154. Kernels of corn infected with corn smut (Ustilago zeae)

Observe the outline of the kernels still visible in the upper, greatly swollen mass. After Duggar allows the spores to escape and to be widely disseminated in the fields. Like the black spores of the wheat rust these smut spores carry the plant over the winter and germinate in the spring, producing the smaller spores, or sporidia, which are blown to young corn plants and start the fungus for another season.

Other smuts on onions, wheat, oats, etc. - have a history similar to that of the corn smut. The smuts are very destructive where they occur in abundance, since they attack the kernels of the grains affected and thus cause the complete destruction of the seed.

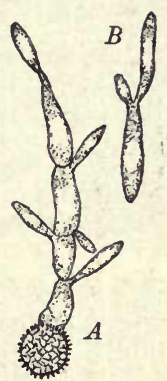

FiG. 155. Spore germination in the spores of corn smut(Ustilago zeae)

Note the conidia, or disseminating and infecting spores, being produced from the sides of the single germ tube, or hypha, springing from the spore. After Brefeld

\section{LICHENS}

Structure and habit. Lichens occur on the bark of trees, on old fences, and even on earth and stones in certain localities. They are grayish green in color and may occur as flat, leaflike expansions (foliaceous lichens) (Fig. 156), as powdery crusts (crustaceous lichens), or as shrubby, erect outgrowths (fruticose lichens). The fruticose lichens often form conspicuous coverings 


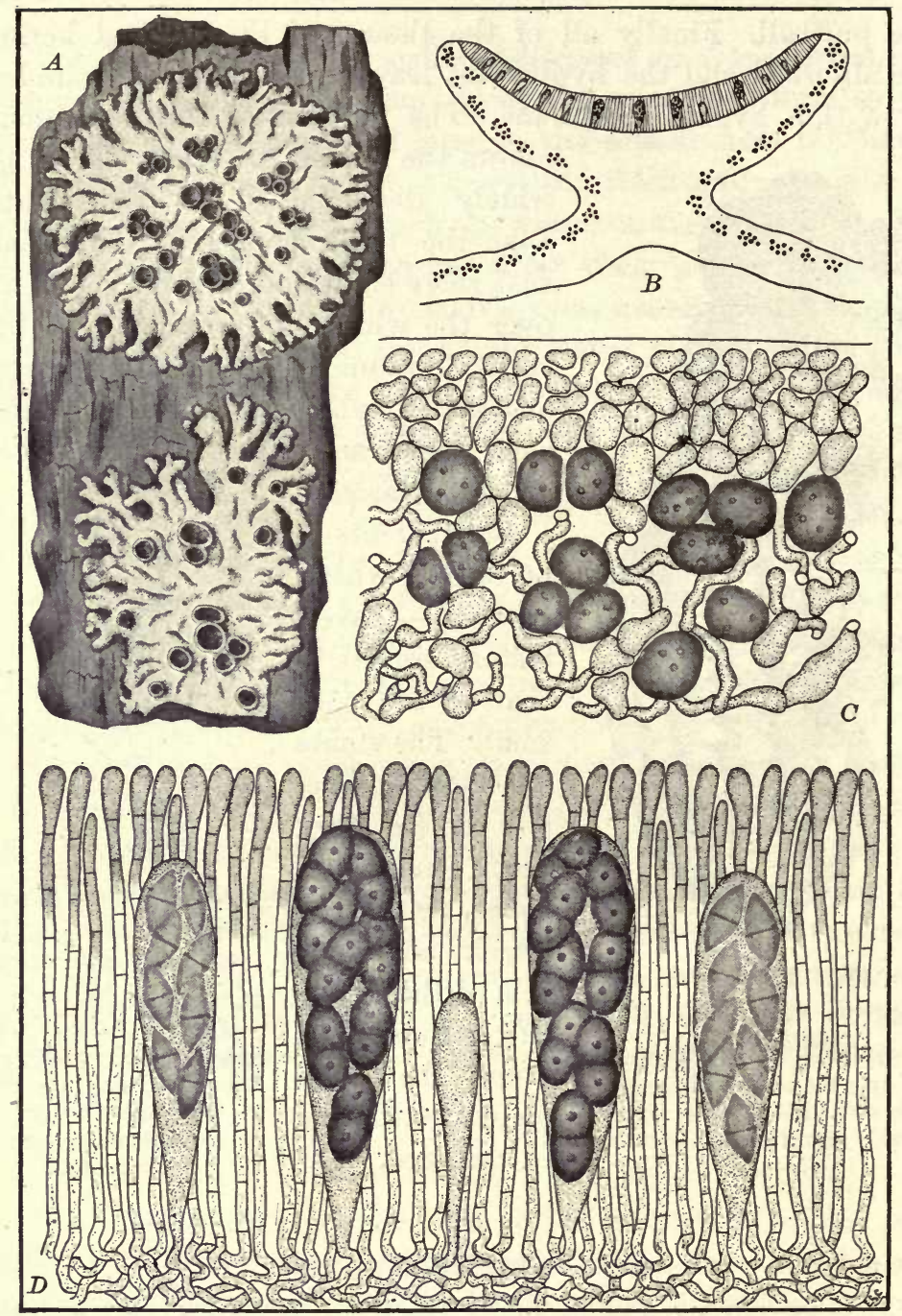

Fig. 156. Habit and structure of a common tree lichen, Physcia stellaris $A$, habit of plants bearing reproductive cups, or apothecia ; $B$, section of a reproductive cup, showing the dark spore-bearing layer (hymenium) lining the cup; $C$, structure of upper portion of plant body, including the green algal cells; $D$, portion of the hymenial layer, greatly magnified, showing spore sacs (asci), with dark-colored spores, and the lighter paraphyses. From Bergen and Davis's "Principles of Botany" 
of branches in warm, moist climates, where they are popularly known as gray moss, since they resemble mosses very closely.

The grayish-green color of lichens is due to the fact that the plant body is composed of two distinct plants: namely, a green alga resembling Protococcus and a colorless fungus like the molds. These two plants are associated as partners in the same plant body, the alga furnishing the sugar made by photosynthesis in its green cells, and the fungus absorbing the water and soil salts necessary for the growth of each partner. The physical relations of the two plants are such as to enable them to maintain their partnership to the best advantage. The flat, leaflike body of a common foliaceous lichen is composed largely of moldlike fungus hyphæ, not unlike a very dense

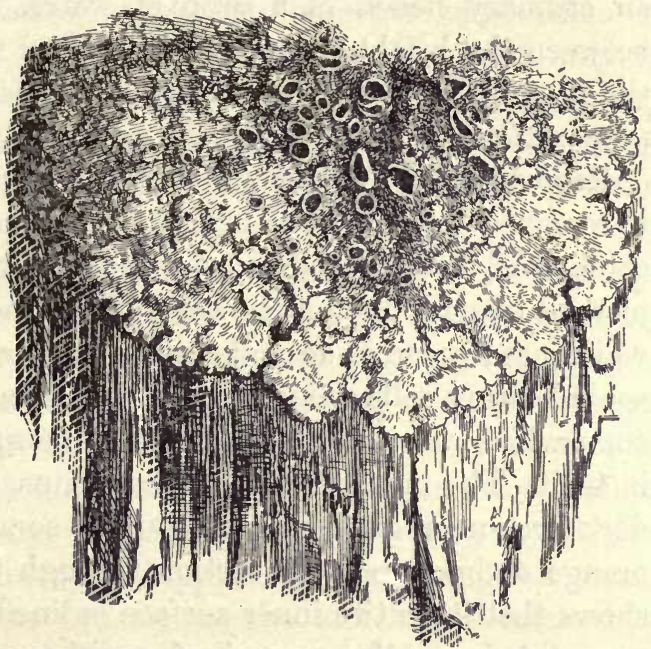

Fig. 157. A common lichen, Parmelia, on the bark of a hickory tree

The spore-bearing cups are clearly shown

and regularly formed mycelium. The hyphæ on the upper and lower surfaces of the flat expansion are short and closely adherent (Fig. 156, C), thus forming the protective outer layers termed respectively the upper and lower cortex. Between these two layers the hyphæ form a looser structure termed the medulla, or central part of the lichen body. The algæe are commonly distributed in a layer just beneath the upper cortex, where they are most advantageously exposed to light. The fungus hyphæ either penetrate the algal cells by means of short branches which absorb food from the vacuole, or they adhere closely to the algæ and absorb nutriment through their cell wall. 
The relation of the fungus to the algæ is therefore that of a parasite on a green plant, while the algæ probably derive some benefit from the fungus in raw food materials and protection. Such a relationship is called symbiosis, which means a partnership with mutual advantage to each partner. This physical relation of the algæ and the fungus of the lichen body is easily demonstrated by making sections or by teasing out small portions of an ordinary lichen in a drop of water. The striking contrast between the bright-green algal cells and the colorless hyphæ of the fungus enables one to determine without difficulty in such preparations the relation and the nature of the two plant partners.

Reproduction. Almost any part of the plant body of a lichen, if removed from the mother plant and placed under favorable conditions, can reproduce the plant vegetatively. The usual method of reproduction, however, is by means of asexual spores, which are formed in special club-shaped cells termed asci. These spore-bearing cells occur in great numbers in the so-called lichen cups, which are easily seen covering the upper surface of lichens in fruit. The inner surface of these cups, or apothecia, is usually dark brown or gray in color, but in some instances it may be orange or brick red. A section through the cup (Fig. 156, B) shows that its entire inner surface is lined with the club-shaped spore-bearing cells, or asci, alternating with greatly elongated sterile hyphæ called paraphyses. Each club-shaped spore-bearing cell contains from two to eight spores, which are expelled with some force from the ascus when they are ripe. These spores germinate like mold spores, and if the hyphæ thus formed come in contact with the proper algæ, they attach themselves to the algal cells and gradually form a new lichen plant. It is thus seen that the production of a new lichen is entirely dependent upon spore reproduction by the fungus. In this manner lichens arise in nature wherever rough, moist surfaces offer the proper conditions for their growth. On account of their great hardiness and their ability to make their own food the lichens are among the most widely distributed plants in nature. 


\section{FUNGI AND PLANT DISEASE}

Nature and importance. The diseases of plants caused by fungi are scarcely less important than the diseases of animals and man, on account of their intimate relation to the world's food and lumber supply. These facts were abundantly emphasized to the public during the Great War, when government and state experts demonstrated the immense importance of fungous pests to our national food supply. We have only to recall the damage done by such fungi as the grain rusts and smuts, the potato rot, the apple scab, the grape mildews, and the tree-killing fungi to realize the national importance of fungi and fungous diseases.

Some plant diseases are produced by bacteria, as in man and animals, but the greater number are caused by filamentary fungi similar to the molds, rusts, and smuts studied in previous pages.

Spread of plant diseases. The rapid spread of plant diseases is due in large measure to the very unusual number of asexual spores produced by the fungi, of which we have had illustrations in the molds, smuts, and mushrooms. Indeed, many fungi, like the mushrooms and smuts, have apparently abandoned the sexual method of reproduction entirely for the more rapid method of producing abundant asexual spores.

These spores are light and so, like the bacteria, are constantly blown about and earried long distances by air currents. As a consequence some fungous diseases spread with startling rapidity, resulting in widespread injury.

The recent spread of the disease known as chestnut blight, caused by the fungus Endothia parasitica, is a well-known illustration of the rapid spread of such a destructive disease by means of spores. The chestnut blight is supposed to have been imported from China and to have spread from New York City, as a center, about 1904. It has now almost wholly destroyed the native chestnut trees over large areas in the forests and cities of the eastern United States. The pine-tree blister rust has had a similar history and threatens to destroy many thousand feet of valuable pine timber unless its ravages can be checked. 
The dissemination of fungi by spores lodged on seeds or by means of mycelium within the seeds is another method of spreading fungous diseases. The rusts and smuts are often thus disseminated by spores lodged on the kernels of the grain when the seeds are sown. These spores then germinate with the seeds and attack the young seedlings while their tissues are tender and allow the fungus hyphæ. to penetrate them.

Beans are infested with a fungous disease known as anthracnose, which often does great damage to the crop. The mycelium of the fungus causing the disease grows into the ovule during its active vegetative life and hibernates there during the resting period of the seed. The next spring, when the beans are planted, these hibernating hyphæ also grow and form a mycelium throughout the tissues of the bean plant. If conditions are right for vigorous fungus growth, the mycelium attacks the pods and developing seeds and destroys the crop.

In this manner a dangerous disease may readily be spread through the exchange or sale of seed, and the infection of a previous year may cause the real damage to the crop in the year following infection. Local spreading of fungous diseases is often caused in forests by certain fungi which attack the roots of trees. These fungi form long stolons, composed of fungus hyphæ, which grow for considerable distances and penetrate the roots of adjacent trees, infecting them with the disease.

In addition, dissemination of fungi is effected by water, as in the water molds (Saprolegnia), by animals (including insects and fish), and by man in the shipping and transportation of plants and plant parts from state to state and from one country to another. The spread of disease by this method has been greatly increased in recent years by the shipping of seeds and entire plants from state to state and from European countries into the United States.

Infection. Plants have few openings into the inner tissues of the body, corresponding to the nose, mouth, and ears of man and other animals, through which germs can enter and cause disease. Infection in plants, therefore, is more likely to take place through wounds or through the stomata. 
Infection of forest trees (Fig. 151), for instance, usually takes place at points where wounds have been made by pruning or where the bark has been injured by animals. In a similar manner the infection of fruits is usually caused by bruising, which breaks the epidermis and thus offers an opening to fungi, whose spores germinate in the wound. It is for this reason that shippers of apples and other fruits exercise the greatest care in preparing the fruit for shipment. This is done by drying and rubbing the surface of the fruit, by discarding infected specimens, and by wrapping each fruit in paper to prevent contact and infection from adjacent specimens. Many of these fruits are infected and rotted in part by molds like Penicillium (Fig. 143), the spores of which are on the skin of the fruit when it is packed. In other instances fungi infect host plants by boring through epidermal walls through the agency of ferments, which destroy the tissues ahead of the entering germ tube. Infection by a filamentary fungus is often accompanied by infections on the part of bacteria also, which complete the processes of decay inaugurated by the higher fungous parasite.

Invasion and disease production. The fungus hyphæ, when they have once penetrated into the interior tissues of a plant, spread and destroy the tissues by the growth of a mycelium, exactly as in the case of mold on bread (Fig. 140) or like a tree parasite (Fig. 152). The invasion and breaking down of the tissues is effected through the secretion of ferments by the hyphæ of the invading mycelium, just as the hyphæ of Rhizopus digest the starch in a slice of bread by the secretion of digestive ferments. In this manner the mycelium of a parasitic fungus may penetrate the hardest wood of trees, and then dissolve out the wall substance and the stored food by a digestive process which results in decay and death. Smuts and similar fungi, which start to grow with the seed, keep pace in the above manner with the growth of the seedling, following along the paths of the least resistant tissues to places where the spores are to be formed.

This invading of the plant body, and the production of disease, is, however, a much slower process in plants than the distribution 
of bacteria and their poisonous toxins by means of the blood stream in animals. As a consequence, the period of disease production is greatly prolonged in plants as compared with animals, and may continue in perennials, like trees, for many years without causing death.

The exact manner in which fungi destroy plant structures and materials which are commercially important to man varies with the fungus and the host. In the case of forest trees the damage comes from the breaking down of the tissues of the tree, so that the wood is injured, as well as from the checking of the healthy growth and development of the tree. In herbaceous plants the fungous disease usually weakens the plant to such an extent that its productiveness is greatly lessened, or it may destroy the plant entirely. In other cases, as in the attack of grain by smuts, the spores are formed in such numbers in the ovules that the grain itself is almost entirely destroyed. In soft plant structures, like fruits and potatoes, the mold or other parasite simply starts the process of decay, which is then carried on by bacteria until the entire structure is destroyed.

Remedies. The remedy for the great losses in food, lumber, and nursery stock, which result from fungous diseases, lies in a better knowledge of the nature of these diseases and in a more strict oversight, on the part of individuals and of state and national governments, of their control and dissemination. The United States government now employs expert plant pathologists, who study the origin and spread of plant diseases throughout the country. They are also coöperating with state governments, through the state experiment stations, in an endeavor to check the progress of dangerous fungous pests. Expert pathologists are also maintained by the United States government to inspect imported seeds and plants, so as to prevent the introduction of plant diseases from foreign countries. Entire shipments of fruits, vegetables, and nursery stock are often condemned by these government officials and denied entrance into our ports. Much insight is also being gained into the various methods of controlling various diseases by sterilizing seeds before planting and by the destruction of certain fungous hosts which harbor dangerous 
parasites. The order of the government to destroy the barberry bushes during the late war is an instance in point. These barberries were known to be infected by one phase of the wheat rust, which winters on wheat straw or stubble. By destroying the barberry the government hoped to eradicate this dangerous pest. Recent orders of the government, looking toward the control of shipments of bulbs and nursery stock from Holland and

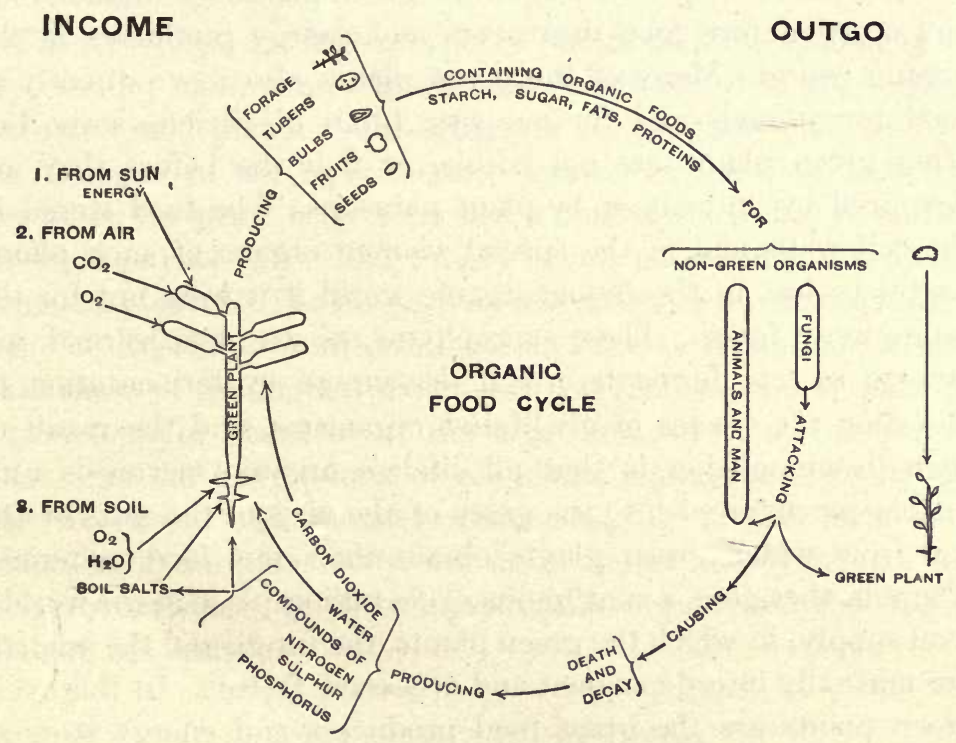

Fig. 158. The organic food cycle

other European countries, are interesting indications of the awakening of the country to the great danger and commercial importance of fungous diseases to our ornamental and food plants.

\section{THE ORGANIC FOOD CYCLE}

From what has been learned concerning the life of the fungi the student is prepared to understand more clearly the relative position of the fungi and the green plants as regards the food supply of all organic life (Fig. 158). We have seen that the 
green plants, on account of their chloroplasts, are able to convert the simple elements of the air and the soil into organic food. For this purpose they are able to draw on the energy of the sun through the agency of their chlorophyll pigment. Such plants are thus able to secure an unlimited supply of outside energy, which makes them the great food makers and accurnulators for the rest of organic nature. The colorless fungi and the animals are dependent upon the green plants for organic food and are therefore food destroyers and energy producers in the organic world. Many of the green plants also serve directly as food for animals and for parasitic fungi of various sorts, but other green plants are not edible, or they die before they are devoured by animals or by plant parasites. The food stored in the cell walls and in the special storage organs of such plants would be lost to the living organic world if it were not for the saprophytic fungi. 'These saprophytes, as we have learned, are able to secrete ferments which decompose by fermentation or digestion the tissues of all lifeless organisms, and the result of such decomposition is that all lifeless organic matter is ultimately reconverted into the gases of the air and the salts of the soil from which green plants obtain their raw food materials. 'There is, therefore, a continuous cycle taking place in the world's food supply, in which the green plants, the fungi, and the animals are mutually interdependent and necessary factors. In this cycle green plants are the great food producers and energy storers; animals and parasitic fungi are food users and energy producers; and the saprophytic fungi are the great scavengers and reconverters of lifeless organic matter into new compounds, which can be used over again by the food-building green plants. It is difficult to see how any one of the members of this triple alliance of organic forms could exist for long without the presence of the other two members of the organic world. When we take this larger view of nature, therefore, we see that the fungi are, on the whole, useful plants. 


\section{CHAPTER XIV}

\section{BRYOPHYTES (LIVERWORTS AND MOSSES)}

The Bryophyta are higher types of plants than the Thallophyta, approaching more nearly to the conditions found in the higher spore and seed plants in the structure of the plant body and in the character of the reproductive organs. In the liverworts, or Hepaticae, the plant body is termed a thallus, since they resemble the thallophytes (algæ and fungi) in not possessing true roots, stems, and leaves. The mosses (mивci) are more highly organized than the liverworts and have stem and leaves resembling somewhat those of the higher plants, but this leafy-stemmed plant is a gametophyte plant, as in the algx, instead of a sporophyte plant, like the leafy plants of all plants above the bryophytes. The liverworts and mosses, while differing in the form and structure of the plant body, are nevertheless closely related by the great similarity of their reproductive organs and the stages of their life histories.

\section{HEPATICAE (LIVERWORTS)}

The simplest members of the Hepaticae are amphibious plants, which occupy wet banks and overhanging rocks on the borders of streams and lakes. They are named Hepaticae from their fancied resemblance to the lobes of the human liver. As might be expected, these simplest land plants exhibit transition stages between the aquatic algæ and the highest spore plants (represented by the mosses and ferns). The simplest forms are mere ribbons of green cells, resembling in their structure the leaf of Elodea or of a moss. They are attached to the mud or wet rocks over which they grow by fine, hairlike roots called rhizoids, which closely resemble the root hairs of the higher plants. 
Among the higher liverworts a more bulky plant body enables these plants to live in less moist situations than the lower types and to approximate more nearly in their form and structure to true land plants. These higher forms closely resemble in structure a leaf of the higher plants and are attached, like the simpler species, by means of rhizoid roots.

The production of new plants and the wider distribution of the species take place by means of nonmotile spores borne in special spore cases, or sporangia, which are new structures in the life history of plants thus far studied.

\section{RICCIOCARPUS}

Habit and habitat. Ricciocarpus (Fig. 159) is a hydrophytic liverwort which floats on the surface of ponds and lakes or grows on mud along the shore where the water has receded.

The general structure of the plant body is similar to a green leaf in its organization, with green chlorophyll tissue on its upper exposed surface adapted for photosynthesis. The lower surface is furnished with rhizoids, and with platelike structures in the form of scale leaves, which serve in part to keep the plant upright on the surface of the water. In the case of plants growing on mud the rhizoids function as roots in the absorption of water and soil salts.

Gametophyte. The plant body of Ricciocarpus is the gametophyte, which bears the reproductive organs in furrows on the upper surface, back of the growing points. The reproductive organs, which we have called gametangia in the algæ, are here more highly organized and hence have other names applied to them. The male reproductive organ is called the antheridium and is composed of an outer layer of cells, called wall cells, which inclose and protect the inner mass of mother cells of the sperms, or male gametes (Fig. 159,g). The protoplasts of these mother cells differentiate to form the male gametes, and when the gametes are fully formed, the walls separating the mother cells are absorbed and the gametes are liberated by the rupture of the antheridium at its apex. 
The female reproductive organ is also more highly organized than similar organs in the algæ and is called an archegonium (Fig. 159,,$d$ ). This archegonium is flask-shaped, with an elongated neck and an enlarged venter. The outer cells of the neck and venter form a protective wall layer like that of the antheridium, inclosing a central column of cells more immediately

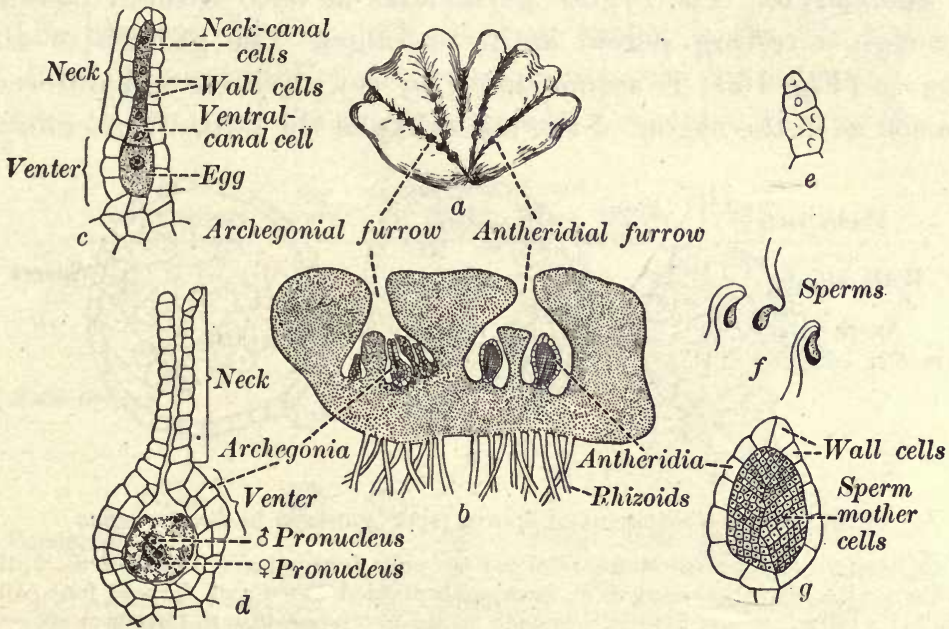

Fig. 159. Habit and sexual reproductive organs of Ricciocarpus

$a$, habit of a single plant of Ricciocarpus; $b$, section of the plant represented in $a$ cut through two reproductive furrows containing archegonia and antheridia; $c$, section of young archegonium; $d$, section of archegonium with fertilized egg cell containing male $\left(\sigma^{*}\right)$ and female $(q)$ pronuclei ; $e$, young antheridium; $f$, sperms with flagella; $g$, section of mature antheridium

concerned with fertilization and the growth of an embryo. This central column of cells includes the neck-canal cells, the ventralcanal cell, and the female gamete.

Fertilization takes place when the plants are wet with rain, dew, or spray, since this is the proper condition for liberating the gametes and for the locomotion of the motile male gametes. When the female gamete is ready for fertilization, the neck-canal and ventral-canal cells disorganize and form a mucilaginous substance which absorbs water, ruptures the archegonium at its apex, and exudes in the form of a viscid drop in which the 
liberated male gametes become entangled. The male.gametes then swim down the canal of the neck, made by the disorganization of the canal cells, and one successful gamete enters the egg and fertilizes it. The unsuccessful gametes, as in the algæ and fungi, are unable to penetrate the fertilized egg cell and die in the neck canal or the venter.

Sporophyte. 'The zygote germinates at once without passing through a resting period as in the algæ. The growth of the zygote (Fig. 160) is accompanied by cell division and differentiation as in the zygote of the mandrake or the bean. The embryo
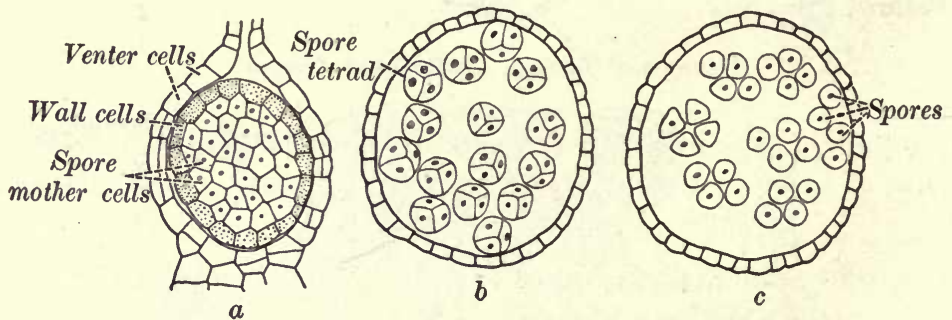

Fig. 160. Development of spores (sporogenesis) in Ricciocarpus

$a$, early spore mother-cell stage, with mother cells forming a cellular tissue; $b$, the mother cells, after becoming free, have each divided twice and formed four cells, called a tetrad; $c$, the four cells of each tetrad in $b$ separating to form four spores

which results is, however, very simple in Ricciocarpus as compared with the embryos of the higher plants just referred to. It is composed of an outer layer of wall cells which incloses and protects a mass of cells which ultimately become the mother cells of spores. With the enlargement of the embryo the spore mother cells become free from each other, and each mother cell divides twice to form groups of cells in fours, called tetrads. Each cell of a tetrad gives rise to a spore. By this process the embryo is converted into a spore case, or sporangium, filled with spores. The rupture of the wall of this spore case liberates the spores, which then germinate at once to form new plants in a proper habitat.

Such a plant body as that described above, which results from cell division and differentiation of the zygote, is called a sporophyte, since its main function is the bearing of spores. 
We shall learn, as indicated above, that this simple sporophyte of the liverwort is the forerunner of the leafy green plant body of the ferns and the seed plants like the mandrake and the bean.

The sporophyte of Ricciocarpus is therefore a simple kind of sporophyte plant, produced from the zygote, which grows as a parasite on the mother plant, or gametophyte, and is devoted wholly to the production of spores.

Life history. In Fig. 161 the main stages in the life history of Ricciocarpus and Vaucheria are represented by diagrammatic figures to bring out the essential points of contrast between them.

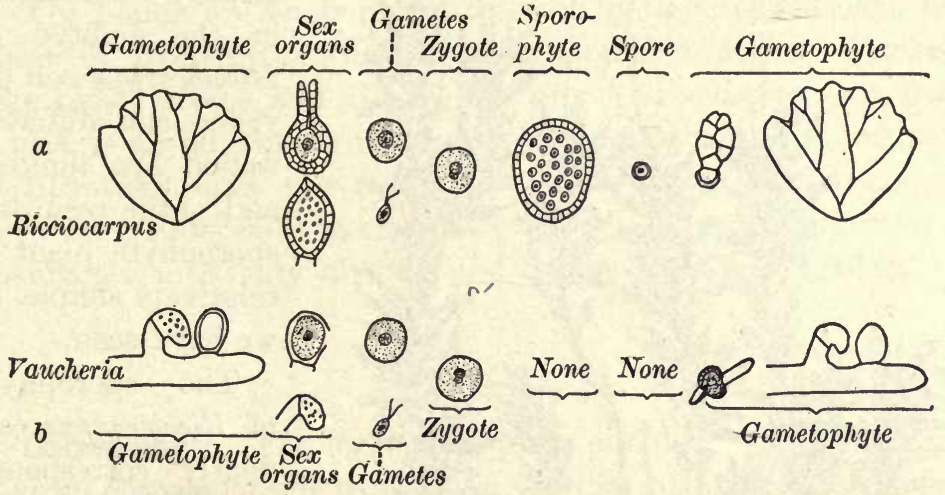

FIG. 161. Life history of Ricciocarpus and of Vaucheria, represented graphically $a$, stages in the life history of Ricciocarpus; $b$, stages in the life history of Vaucheria

In the liverwort the gametophyte is bulkier and more highly differentiated than in the alga, and is thus better able to meet the aquatic or amphibious conditions under which it has to live.

The reproductive organs, instead of being, as in the alga, single-celled structures adapted to form the gametes, are complex cellular organs, with an outer protective cell layer inclosing the more delicate cells which form the gametes and aid in fertilization.

The zygote is formed in both the liverwort and the alga by the union of the male and female gametes in fertilization, and consists in each case of a single cell with a protective cell wall, cytoplasm, and nucleus, like the zygote of Spirogyra. 
In the alga this cell usually passes through a resting period, after which it germinates to form a new filamentary plant,

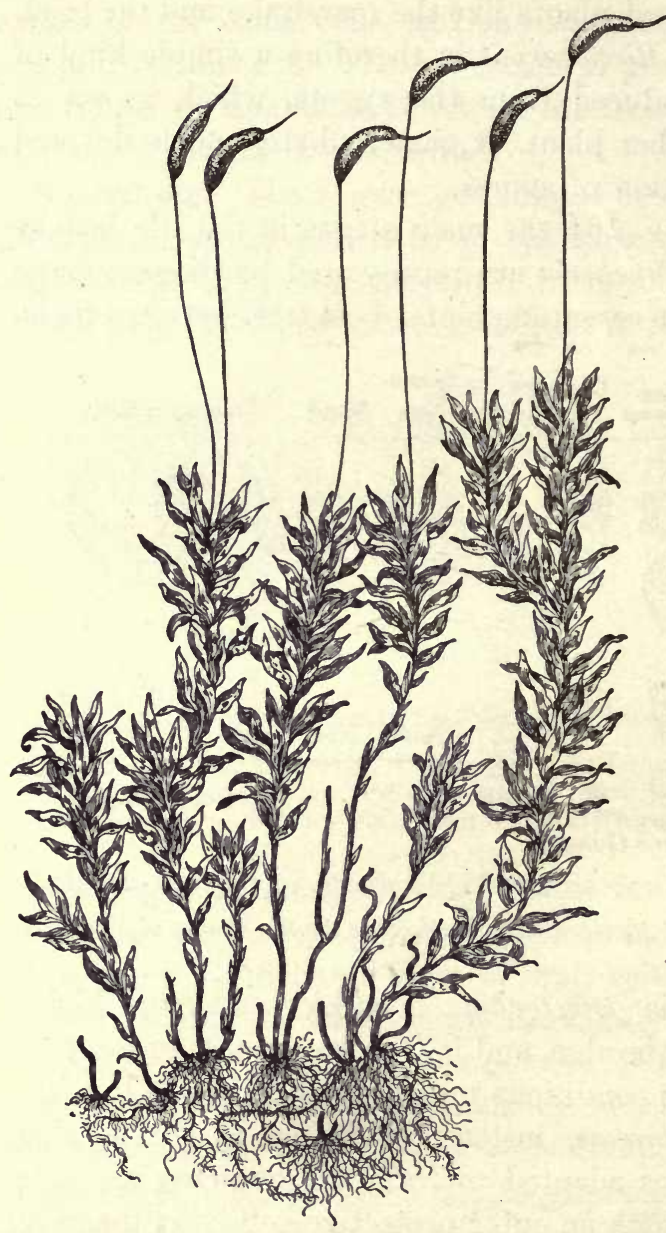

Fig. 162. Habit of a moss, showing the leafy gametophytes and sporophytes. After Sachs or gametophyte.

In Ricciocarpus the zygote becomes an embryo plantlet, or sporophyte, through cell division and cell differentiation, as in the higher plants already studied, but in the embryo of Ricciocarpus cell division and differentiation are limited, and so the resulting sporophyte plant is relatively simple, as we have seen.

The sporophyte of Ricciocarpus evidently corresponds in the life history to the embryo and the adult plant of the bean or the mandrake, since they are all derived by similar processes from an initial fertilized egg cell, or zygote. Plants like Ricciocarpus and the mandrake, which have a sporophyte plant alternating in the life history with the gametophyte plant, are said by botanists to have an alternation of generations in the life cycle. 


\section{MUSCI (MOSSES)}

Habit. The mosses are leafy-stemmed plants and are hence much more highly organized than the liverworts, which are their nearest relatives. The stem of such a moss as Funaria is a delicate structure with the leaves arranged spirally upon it. The leaves are also very simple, being composed of a single layer of chlorophyll-bearing cells, except in the central conducting strand, corresponding to a midrib, where the cells are elongated and are two or more layers thick.

The plants are anchored in the soil by delicate rhizoids, like those of liverworts, which serve a similar function. Funaria, like most mosses and liverworts, grows in clusters, - a habit which is an advantage in conserving moisture and in insuring fertilization, since the male and female organs are borne on separate plants and the clustering habit is necessary in order to insure the proximity of male and female organs (Fig.163).

\section{FUNARIA}

Gametophyte. The leafy moss plant just described is the gametophyte corresponding to the flat, leaflike plant body of Ricciocarpus and the other liverworts. In Funaria the reproductive organs (archegonia and antheridia) are borne in terminal reproductive buds.

The antheridia are borne in the form of open disks (Fig. 163, c), which are easily recognized by the brick-red color of the wall cells.

The archegonia (Fig. 163,b) are produced in closed buds, which are not easily distinguished from vegetative buds (Fig. 163,a).

The antheridia and archegonia are almost identical in structure and function with those already described in Ricciocarpus, so that no additional description of them is necessary. The antheridia (Fig. 163, $d, e$ ) are accompanied by multicellular hairs, called paraphyses, with enlarged terminal cells.

Fertilization takes place, as in the liverworts, when the plants are wet with rain or dew, which facilitates the opening of the sex organs and the migration of the motile male gametes. 
The male gametes swarm down the opened neck canal of the archegonium as in Ricciocarpus, and the egg cell is fertilized by one successful male gamete. 'The zygote which results, like that of Ricciocarpus, forms an outer cell wall and begins to germinate without passing through a resting period.

Sporophyte. The embryo (Fig. 164, a) which results from the early divisions of the zygote cell resembles somewhat the young embryo sporophyte of Ricciocarpus. Elongation of this cellular embryo then begins, resulting in a rodlike structure which carries
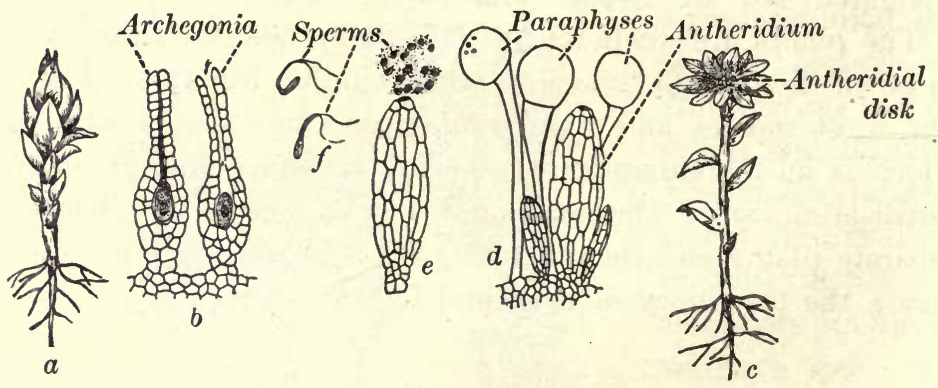

Fig. 163. Male and female plants and sex organs of a diœcious moss, Funaria $a$, female plant with reproductive bud; $b$, archegonia (left, young archegonium with egg and canal cells; right, older archegonium with egg in the venter); $c$, male plant with group of antheridia forming antheridial disk at apex of plant; $d$, group of antheridia and paraphyses; $e$, mature antheridium expelling sperms; $f$, sperms with flagella

the old archegonium wall upon its apex like a cap, the wall cells having separated from the plant at the base of the venter (Fig. 164, $c$ and $d$ ). This rodlike cellular zygote grows not only upward but downward as well, so that it bores with its basal end into the tissues of the leafy moss plant, from which it now draws its food and water like a parasite. After it has become established in the leafy gametophyte plant, the rod begins to enlarge in its upper part to form the rudimentary spore case known as the moss capsule, while its lower part is converted into a strong, flexible rod called the seta. This spore case, or capsule, although at first composed of simple, undifferentiated cells, soon becomes differentiated into a complex cellular organ for the production of spores. In many mosses the outer cell layers of the 
capsule beneath the epidermis are green and are therefore able to carry on some photosynthesis, but the greater part of the food required for the development of the spores within the capsule is derived from the mother plant through the seta.

When the spores are ripened, the inner tissues of the capsule break down and the spores lie free within its central cavity.

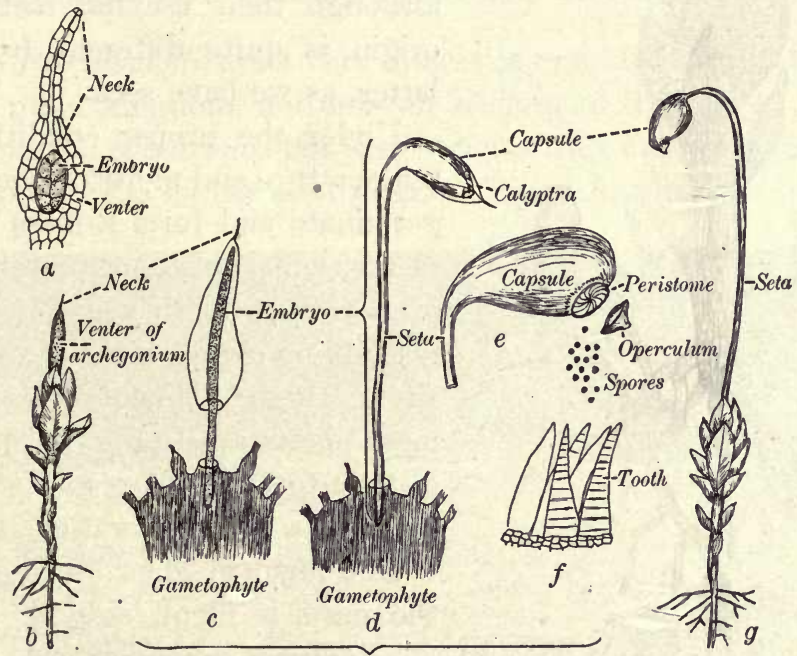

Fig. 164. Stages in the development of the sporophyte in Funaria

$a$, archegonium containing the cellular embryo of a young sporophyte; $b$, moss plant with rodlike embryo still in the expanded archegonium; $c$, rod stage of the sporophyte with the old archegonium wall, neck, and venter; $d$, mature sporophyte with parts differentiated ; $e$, parts of the capsule; $f$, portion of the peristome; $g$, gametophyte and sporophyte in proper relation

The mature capsule at this time consists of the following structures, represented in Fig. 164, $e$ and $f$. A lid, or operculum, is released at the apex of the capsule, thus exposing the light spores to the air. When the lid is removed, a circle of hairlike teeth, the peristome; is seen to guard the mouth of the capsule. These teeth have the power of movement and tend to close over the mouth of the capsule in wet weather and to open out in dry weather, when the light spores are more easily scattered by the wind. In many mosses the teeth open by chinks in dry weather only, so that the spores are sifted out, like salt out of a salt shaker, 
when the elastic seta allows the capsule to be swayed by the wind. We see, therefore, how admirably the moss capsule is organized to form and disseminate the spores which give rise to new moss

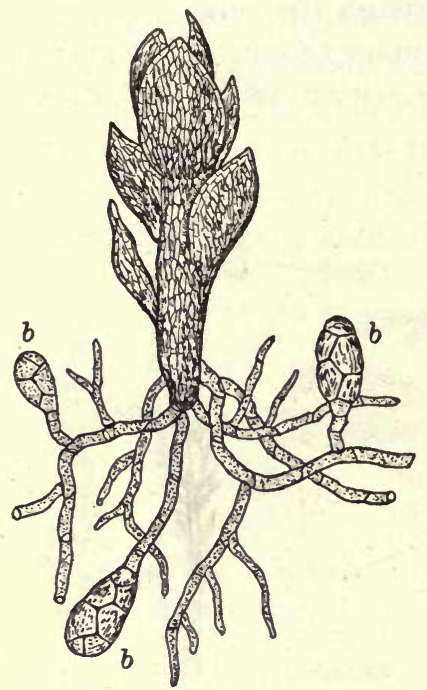

Fig. 165. Protonema and young moss plants

Young plant of a moss attached to a protonema with reproductive buds $(b)$ plants. These spores are highly protected cells, not unlike the $\mathrm{zy}$ gotes of Spirogyra in structure, although their asexual method of origin is quite different from the latter, as we have seen.

Under the proper conditions of temperature and moisture the spores germinate and form a mass of filaments, which often coat wet banks or flowerpots with a green coating resembling closely some of the algx. From these filaments new leafy moss plants arise (Fig. 165), which in turn form gametes and start the cycle of a new generation.

Life history. The life history of the moss is identical with that of Ricciocarpus except in the greater complexity of the gametophyte and sporophyte plants and in the details of spore germination. In both cases the gametophyte and sporophyte alternate in regular sequence in the life history, the gametophyte plant doing the work of photosynthesis and the bearing of the reproductive organs, while the sporophyte lives parasitically on the gametophyte and produces the spores. 


\section{CHAPTER XV}

\section{PTERIDOPHYTES (FERNS, EQUISETA, AND CLUB MOSSES)}

The Pteridophyta are a very interesting and important division of the plant kingdom, not only on account of their great beauty and common occurrence but also because they are the undoubted ancestors of the higher seed plants. These fernlike ancestors of

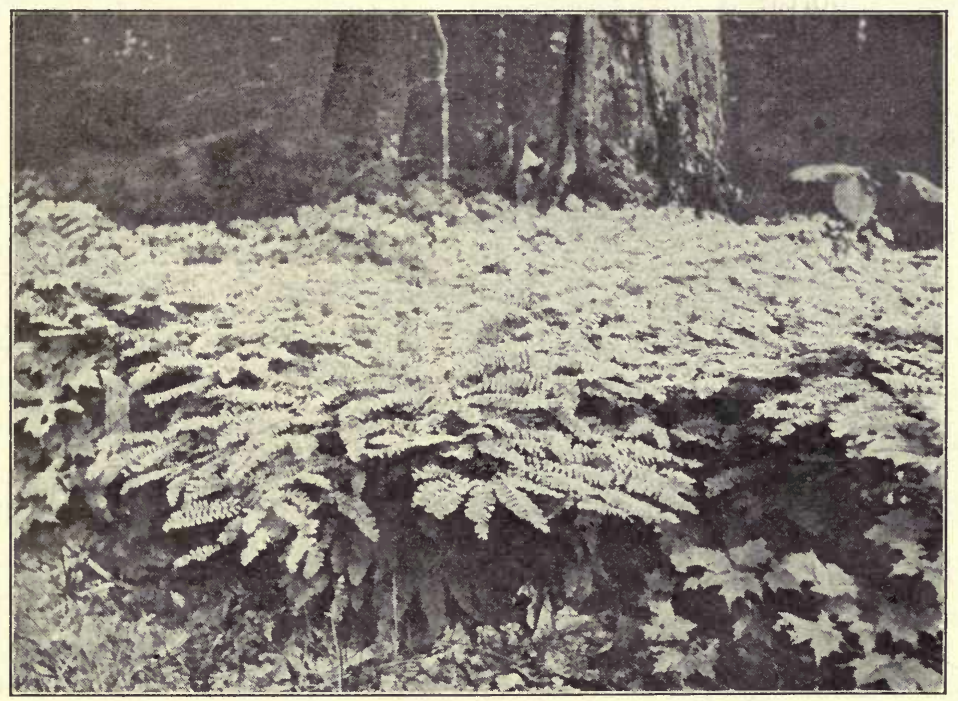

FIG. 166. Maidenhair ferns (Adiantum) growing in a natural habitat in the forest

the pines and their allies, as well as of the flowering plants (like the beans, mandrake, and clovers), date back to the coal period.

The special advances made by the Pteridophyta over the plants below them relate to the much greater importance of the sporophyte as compared with the gametophyte in the life histories of all its members, and to the differentiation of the 
spores into two distinct kinds, which has been effected by some of the higher living species and fossil species of the coal age.

These special features of their life histories indicate clearly the important part played by the early ancestors of modern ferns in the origin of the living seed plants. These important features in the evolution of the plant body and reproduction of the Pteridophyta will be more clearly understood after con-

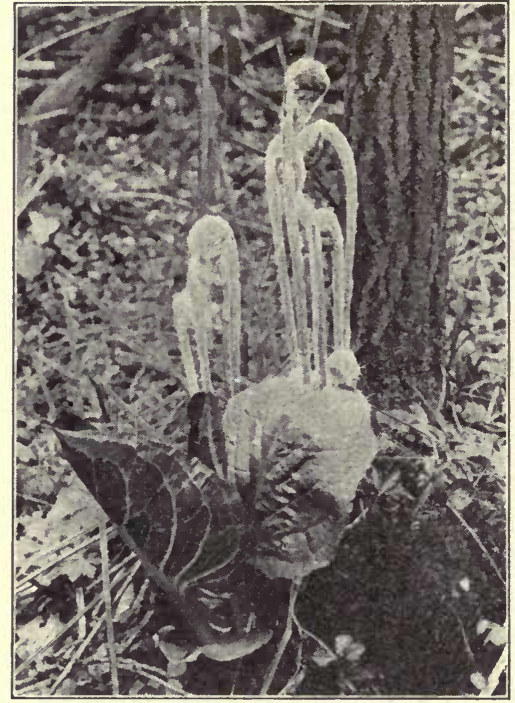

Fig. 167. Coiled tips (circinate vernation) of a fern, Osmunda claytoniana sidering the life history of type species selected from the three great orders of living Pteridophyta, namely, the true ferns, the lycopods, and the horsetails.

\section{FILICALES (FERNS)}

Habitat and habit. The ferns are quite unlike the mosses and liverworts in their structure and mode of life, since they are true land plants with an organization similar to that of the higher seed plants. The great majority of ferns inhabit the floor of forests, where the light is dimmed by overhanging foliage and the soil is moist and rich with decaying humus. A few forms only are adapted to xerophytic conditions, and fewer still are hydrophytes. Most ferns are therefore typical medium land plants, or mesophytes, adapted to the conditions of light, soil, and air found on the forest floor (Fig. 166). The leaves are large and divided to form the characteristic compound leaf seen in most ferns. The leaf blades are thin, and the mesophyll cells possess an abundance of chlorophyll, which is favorable to the manufacture of starch and sugar in a dim and moist atmosphere; the stem in most instances is an underground rhizome 
like that already described for some herbaceous seed plants, and the young leaves spring from the apex of the stem and uncoil in the peculiar circinate manner shown in Fig. 167. They are often protected in the early stages of their development by the abundant scales, or ramenta (singular, ramentum), which are so characteristic of ferms. The roots (Fig. 168)are true roots, like those of seed plants, and not hairlike rhizoids like those of the moss group.

The cell structure and the tissues of the stem, roots, and leaves of ferns are also very similar to those of the higher plants. Indeed, ferns are the first of the vascular plants which possess

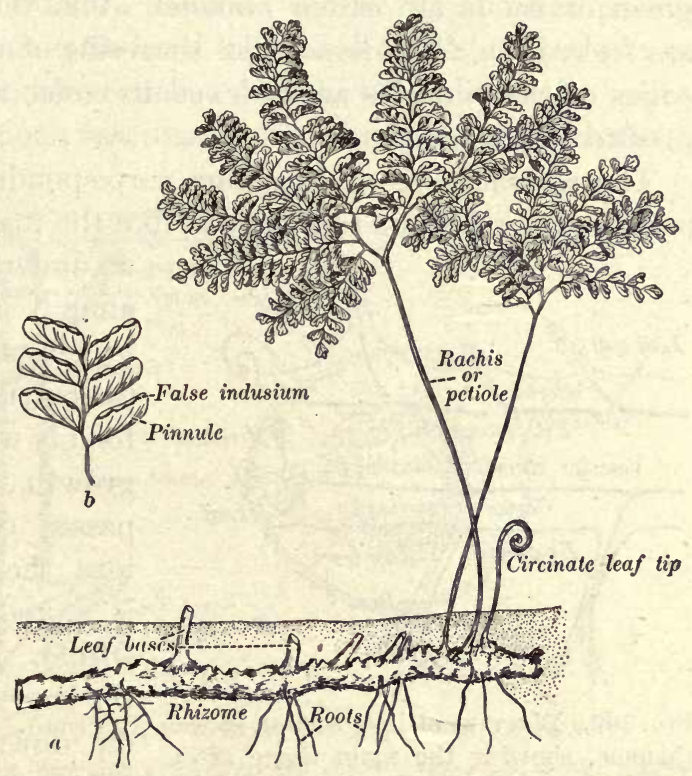

Fig. 168. Habit and parts of a single plant of Adiantum $a$, the entire plant; $b$, portion of a pinna

a true vascular conducting system composed of phloëm and xylem. For these various reasons they are now regarded as the ancestors of higher seed plants.

\section{SPOROPHYTE}

\section{ADIANTUM (MAIDENHAIR FERN)}

Habit. The maidenhair fern is like the other members of the Filicales, mentioned above, in having an underground stem, or rhizome (Fig. 168), which is thickly covered with ramenta. These scales are particularly abundant at the growing point and on the young leaves, and serve as a protective covering. 
The young leaves unfold in the spring in the circinate manner represented in Fig. 168 and finally develop a dark, shining, elastic leafstalk, which forks repeatedly to form the compound leaf. The mature leaf is pinnate (like a feather), and the ultimate green divisions are called pinnules. The veins of the pinnules are forked, or dichotomous, like the veins of other ferns, and the edges of the pinnules are reflexed to cover the sporangia which produce the spores.

The seasonal life of Adiantum corresponds exactly to that of perennial herbaceous seed plants, like the mandrake, which have

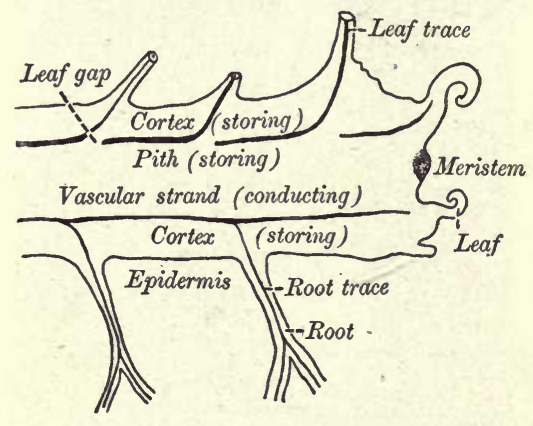

Fig. 169. Diagram of long section of the rhizome, showing the main tissue areas and their principal functions an underground rhizome and annual aërial parts. The leaves manufacture food by photosynthesis; some of the food is used for the season's growth, while the excess passes down the leaf and into the rhizome, where it is stored for use the next spring. When frost comes, the leaves of the maidenhair die down and the rhizome hibernates and carries the plant over the winter. With the advent of spring the reserve food is digested, the young leaves begin to uncoil, and another active period in the life of the plant is begun. In order to carry on these various functions the tissues of the fern stem and leaf are more highly differentiated than those of any plants yet studied except the seed plants.

Structure. The general arrangement of the main tissues of the rhizome of Adiantum is diagrammatically represented in Fig. 169. In this figure it may be noted that the tissues of the fern stem are the same in kind and general arrangement as that already studied in herbaceous dicotyledons. The outside of the stem is protected by an epidermis and by the thick-walled cells of the outer cortex, which constitute a kind of exoskeleton for the entire stem. The inner portions of the cortex and pith are 
composed of storage parenchyma, in which the reserve food is stored for the spring growth. The vascular system is composed of a phloëm and xylem cylinder which runs lengthwise of the stem. From this vascular cylinder strands called leaf traces pass out through the cortex into the leaves. Where a leaf trace passes off from the vascular cylinder a break called a leaf gap occurs (Fig. 169). The roots also connect with the central vascular cylinder, so that a continuous vascular system exists in the maidenhair

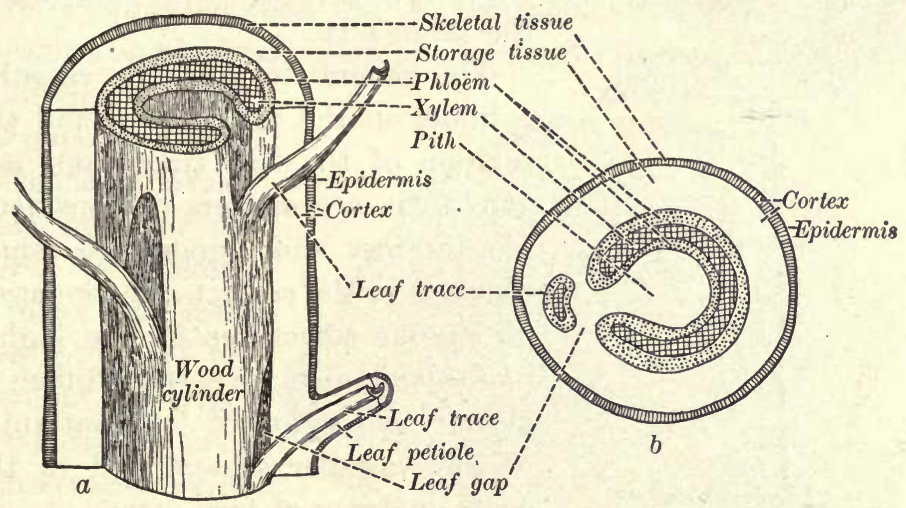

FIg.170. Gross anatomy of a portion of the rhizome of Adiantum

$a$ shows the relation of the vascular system to the other tissues and to the leaves (note the breaks or leaf gaps in the vascular cylinder, caused by the exit of the leaftrace bundle; the cortex has been removed from one side to expose the wood cylinder, leaf gaps, and leaf traces in surface view); $b$, transverse section of the rhizome cut in the region of a leaf gap and a leaf trace

and other ferns as in the higher plants. The arrangement of the tissues in a transverse and a longitudinal section of the rhizome is shown in Fig. 170. Fig. 170, $b$, shows the concentric arrangement of phloëm and xylem which is characteristic of fern stems. In this arrangement the phloëm completely incloses the xylem, flanking it on the outside next to the cortex and on the inside next to the pith. In the seed plants, including the alder and Salvia (Fig. 56), it will be remembered that the arrangement was collateral; that is, with the xylem on the inside of the vascular cylinder next to the pith, and with the phloëm on the outside next to the cortex. The concentric arrangement of phloëm and 
xylem in the ferns is a very ancient one in vascular plants and gave rise to the collateral arrangement by a gradual process of evolution, the discussion of which is not within the scope of an. elementary textbook: 'The cambium is entirely lacking in the stem of the maidenhair and in the stems of most living ferns,

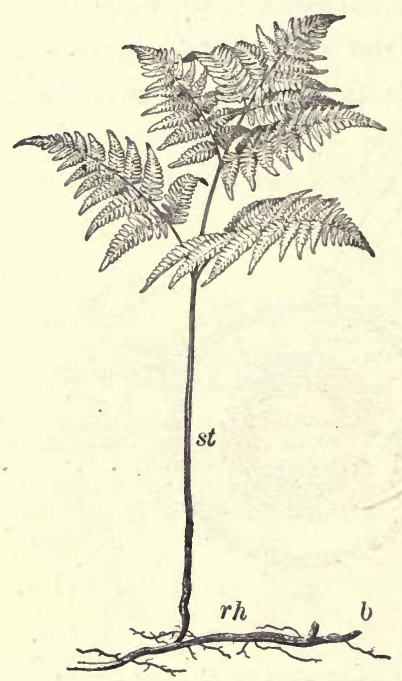

Fıg. 171. Plant of Pteris aquilina (the eagle fern), showing rhizome and leaf

The rootstock $(r h)$ is horizontal and grows underground; upon it are the buds $(b)$ and the upright leafstalk (st) so that no secondary phloëm, xylem, or wood rays are developed. Storage is therefore largely limited, in all living Filicales, to the wide cortex and the large pith.

The general form of the vascular cylinder in the maidenhair, and the relation of the leaf traces and leaf gaps to it, are matters of considerable interest and importance, since they aid in the correct interpretation of similar structures in the higher plants and also give the student a clearer conception of the continuity of the vascular system and of the gross anatomy of fern stems.

Fig. $170, a$, shows a portion of the rhizome greatly magnified, in which the outer cortex has been removed from one side so as to expose the central vascular cylinder composed of xylem and phloëm. By an inspection of this figure it may be seen that a portion of the phloëm and xylem ring of the central cylinder passes out to form the vascular bundle of each leaf petiole. This vascular bundle, or leaf trace, thus connects the phloëm and xylem of the main vascular ring with the leaf veins. The figure shows also that the entire vascular cylinder of the fern rhizome is not a completely closed cylindrical tube, but is broken at intervals by leaf gaps. In fern rhizomes where the leaves are large and close together the gaps are more frequent, and the vascular cylinder comes to resemble a 
latticework or meshwork with vascular strands composed of phloëm and xylem surrounding the large and frequent leaf gaps.

In Fig. 170, $b$, the relation of the leaf trace and the leaf gap is shown as it would appear in a transverse section.

\section{STRUCTURE OF PTERIS (BRACKEN FERN)}

In the bracken fern (Pteris aquilina) the vascular system is more complicated than that of the maidenhair fern. Instead of a nearly complete vascular cylinder of phloëm and xylem, broken by infrequent short leaf gaps, as in the maidenhair, the vascular cylinder of Pteris is broken by many long leaf gaps into a set of vascular strands, or bundles, which form a coarse meshwork, with infrequent union of the strands composing the mesh. This meshwork, when cut across, forms the outer circle of

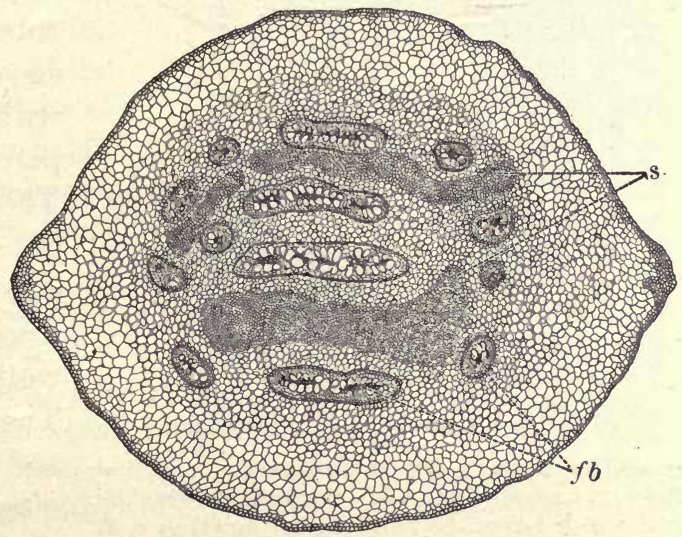

Fig. 172. Section of the rhizome of Pteris aquilina $s$, internal skeletal tissue composed of thick-walled fibers ; $f b$, vascular strands of the meshwork comprising the vascular cylinder of Pteris vascular buindles seen in Fig. 172. The two inner bundles, flanked on either side by dark masses of skeletal supporting tissues, are formed by a budding process from the outer bundle cylinder in the early stages of the development of the stem. In Fig. 173, $a$, the section of the young stem of Pteris is seen to correspond almost exactly with that of Adiantum. In $b, c$, and $d$ the budding off of a central bundle and the division of the single bud to form the two central bundles of the adult stem are diagrammatically shown. The origin of the two dark masses of skeletal tissue from 
a single strand, and the conversion of the solid vascular bundles by an increasing number of leaf gaps, is also shown in the figures. At the left in $c$ and $d$ a root is being given off.

The minute structure of the tissues of ferns is strikingly shown in the large-celled tissues of Pteris. Each vascular bundle is concentric, as in the maidenhair, with a central xylem
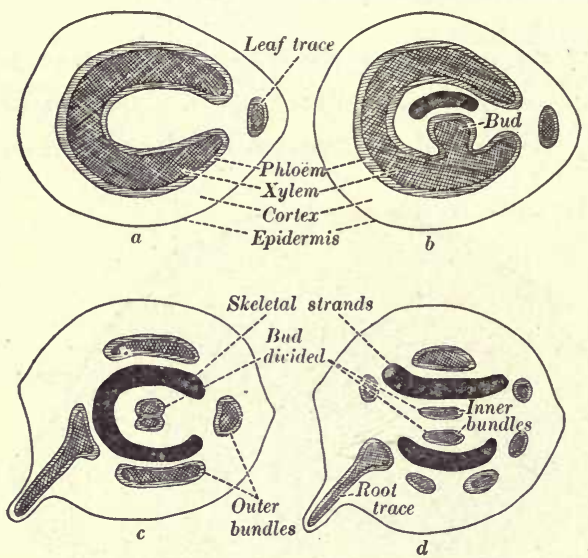

FIG. 173. Diagram illustrating the development of the stem tissues in Pteris aquilina

$a$, section through a young rhizome; $b$, section through an older portion of a rhizome, showing the bud which forms the two internal bundles; $c, d$, older stages in the development of the internal bundles and the skeletal tissue. After Jeffrey. Redrawn from Jeffrey's "Anatomy of Woody Plants" mass surrounded by a sheath of phloëm.

In long sections the phloëm is seen to be composed of large sieve tubes having numerous sieve plates, together with considerable phloëm parenchyma (Fig. 174). The xylem is made up of single-celled waterconducting elements, or tracheids, which have the wall markings of ducts. These tracheids in Pteris and some other ferns often unite end to end to form primitive water ducts. Such a primitive duct, with a ladderlike (scalariform) arrangement of the thick and thin places of the cell wall, is shown in Fig. 174. A large sieve tube, with innumerable sieve plates of varying form and size, is also shown. Both the duct and the sieve tube are flanked by living storage parenchyma cells. The tissues of the cortex and epidermis are similar in arrangement and structure to those of the maidenhair. The cell walls of the epidermis and skeletal tissues are greatly thickened, and the cells of the latter are elongated and are fibrous in their nature. These two tissues, together with the inner skeletal strands, form an admirable protective and strengthening exoskeleton for the fern rhizome. 


\section{SUMMARY}

As regards their vegetative structures the ferns may be characterized as follows :

1. They are the simplest vascular plants and have become adapted to a moist mesophytic environment by the formation of large, thin, dark-green leaves.

2. They are really herbaceous perennials, with a seasonal life quite comparable to that of many seed plants with a perennial underground stem. The tree ferns of the present day and those of the coal period are exceptions to this general herbaceous habit.

3. A few ferns are adapted to dry conditions, and some are hydrophytes.

4. The anatomy of the fern rhizome, or stem, has the same general tissue arrangements as the herbaceous stems of the higher flowering plants, with a large pith, a wide cortex, and a narrow wood ring. The distinctive features are the conspicuous leaf gaps (which often trans-

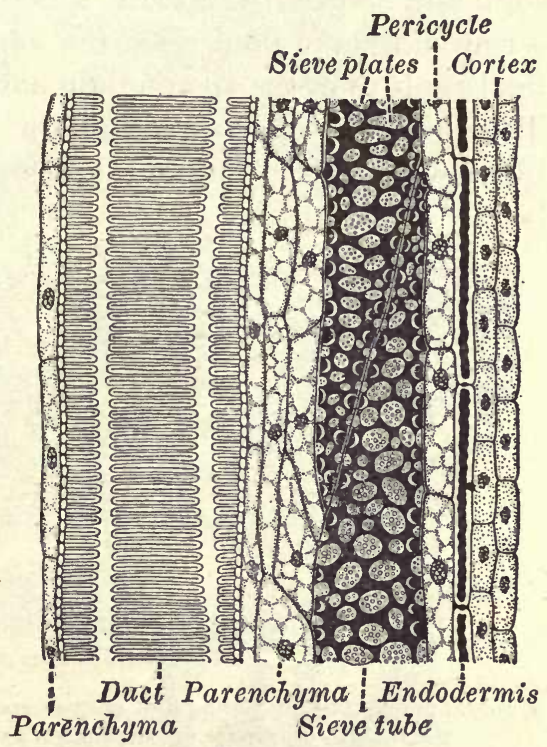

Fig. 174. Vascular tissues of the rhizome of Pteris in long section

After Jeffrey. Redrawn from Jeffrey's "Anatomy of Woody Plants" form the vascular cylinder into a meshwork instead of an unbroken cylinder) and the concentric arrangement of phloëm and xylem. There is no cambium layer and consequently no increase in diameter by the production of annual rings of phloëm and xylem. The water-conducting elements are more primitive in type than those of the higher seed plants studied earlier in the course.

\section{ASEXUAL REPRODUCTION}

The leaves of ferns bear the asexual spores in definite organs, called sporangia, located on the lower side of the leaves along the veins. These sporangia are borne in groups called sori 
(Fig. 176, $A, B, C$ ) and are covered in the maidenhair by the reflexed margins of the pinnules (Fig. 168, b). Each sporangium (Fig. 175) is composed of a stalk and an enlarged spore case, or sporangium proper. This spore case is composed of an outer layer of cells and an inner mass of cells which ultimately form the spores. A mature sporangium is also furnished with a ring of thick-walled cells, the annulus, which brings about the final rupture of the sporangium and the scattering of the spores. This process is effected as follows: 'The cells of the annulus are filled with water during the early stages in the development

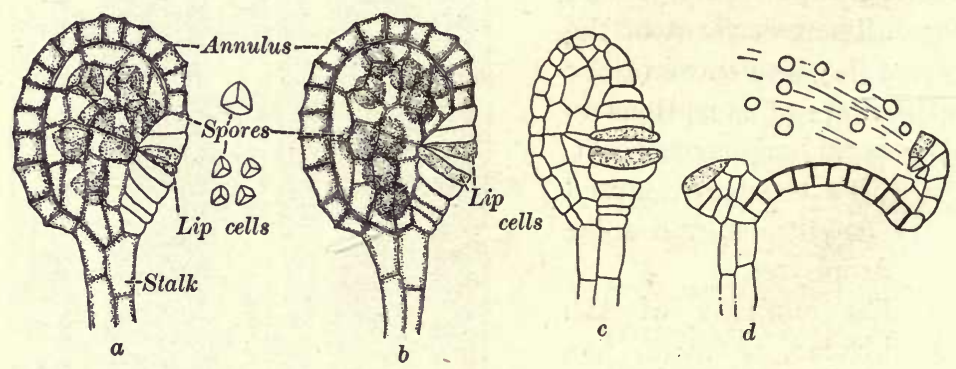

Fig. 175. Sporangia of a fern, showing the development and dissemination of spores

$a$, sporangia containing young and mature spores; $b, c$, opening of the sporangia at the lip cells; $d$, spore dissemination by the movement of the annulus

of the sporangium. As the sporangium matures and the spores ripen, this water begins to evaporate, and the thin outer walls of the annulus cells are pulled in and become U-shaped. They are supposed to be pulled in by the cohesive strength of the diminishing water in their cell cavities. As the outer walls become concave the entire annulus bends backward and ruptures the wall of the sporangium at its weakest point, called the stoma. Finally, when the water is nearly withdrawn from the cells, its tension breaks, and their overstretched, thick inner and radial walls snap the annulus back to its original position (Fig. 175, d). This snap throws the spores so that they can be disseminated by wind and air currents. These spores are highly protected cells which have the power of withstanding drought and other 

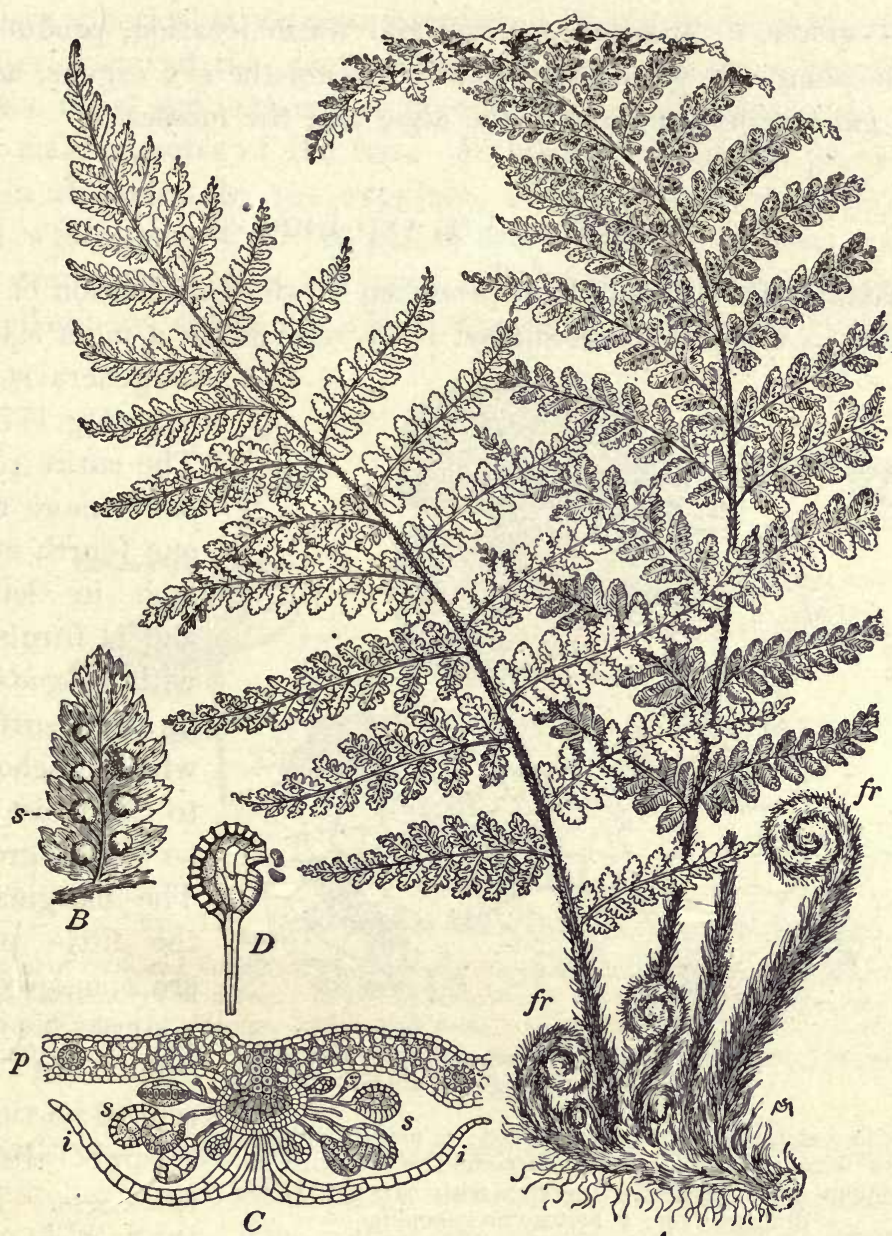
The spores, in a suitably moist and warm location, produce a little plant, the gametophyte, which bears the sex organs, as in the gametophyte plants of the algæ and the mosses.

\section{GAMetophyte AND Embrío}

Habit. The gametophyte produced by the germination of the spores is a green, heart-shaped body resembling a moss leaf in

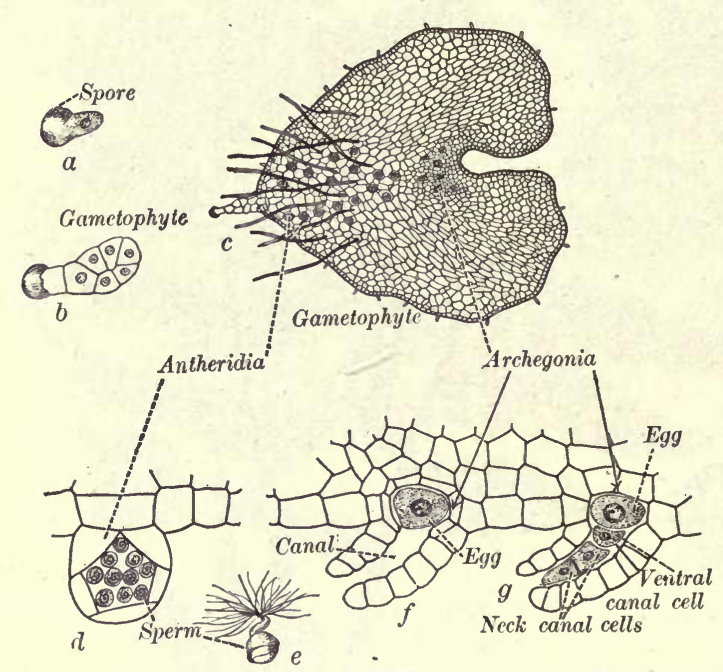

F1G. 177. Spore germination, gametophyte, and sex organs in ferns

$a, b$, two stages in spore germination; $c$, mature gametophyte with antheridia and archegonia; $d$, mature antheridium with sperms; $e$, sperms with cilia; $f$, young archegonium; $g$, mature archegonium its general structure (Fig. 177, c). The entire plant is not more than one fourth of an inch in length and is furnished with rhizoids on its under surface, which anchor it to the moist soil on which it grows. The margins of the little plant are composed of a single layer of cells, but the central portion is several cells in thickness. This thick portion is called the cushion and bears the reproductive organs and the rhizoids. It is thus equipped like the moss for carrying on its own nutrition and for producing the sex organs necessary for sexual reproduction.

Sexual reproduction. The reproductive organs (Fig. 177) resemble those of the liverwort and moss so closely that it will not be necessary to describe them in detail. The archegonia are borne at the anterior end of the cushion, while the antheridia are more 
or less spherical structures distributed among the rhizoids at the posterior end of the plant. This position of the sex organs next to the moist soil is therefore favorable to fertilization by the motile male gametes of the fern. Fertilization is always preceded, as in the moss, by the expulsion of the male gametes during wet weather or when the plants are covered with dew, and by the transformation of the canal cells of the archegonium into a mucilaginous substance which attracts the sperms to the eggs.

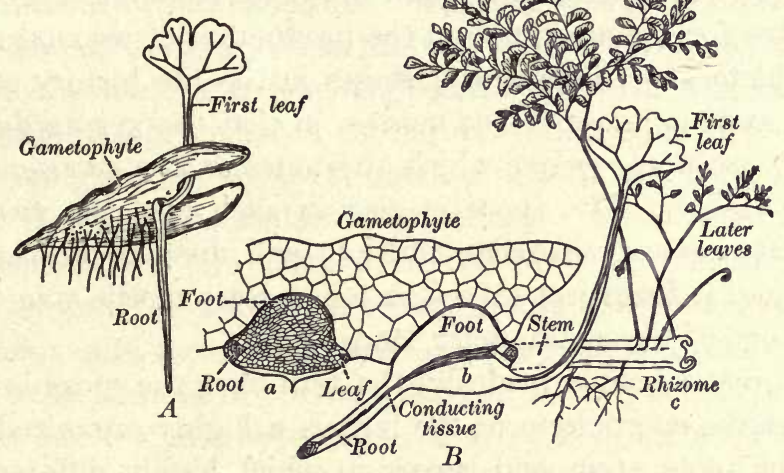

Fig. 178. Gametophyte and embryo of Adiantum

$A$, gametophyte and young attached sporophyte of Adiantum; $B$, section of gametophyte and embryo of a fern: $a$, young embryo; $b$, more mature embryo with first leaf; $c$, permanent rhizome, leaves, and roots, which grow from the stem portion of the embryo in $b$. The embryonic root, foot, and leaf of the embryo in $b$ disappear when the plant in $c$ is fully established

Embryo. After fertilization, while still in the venter of the archegonium, the zygote germinates to form the embryo (Fig. 178, B, a). The young sporophyte, quite unlike that of the moss, very early differentiates into a primary root, stem, and cotyledon, which indicates that it is to become a real leafy plant with the usual organs of the higher plants (Fig. $178 \mathrm{~B}, \mathrm{~b}$ ). In addition to these primary organs the embryo spore plant develops a mass of cells called the foot, which remains in contact with the gametophyte plant and absorbs nourishment from it for the growing embryo until the latter is self supporting (c) and the gametophyte dies. 
The adult fern plant, which was described above, is produced entirely from the stem portion of the embryo sporophyte. The relation of the adult plant to the embryo is diagrammatically illustrated in Fig. 178, $B, b, c$, in which the embryonic stem is represented as having elongated into the horizontal leaf-androot-bearing stem of the adult fern. In the figure the primary structures - root, foot, and first leaf - are unshaded, while the perennial adult fern plant is left shaded. This perennial fern plant, therefore, corresponds to the moss capsule in the life cycle of the two organisms, since both originate from the zygote and both have for a main function the production of asexual spores.

Life history. The essential stages in the life history of ferns are the same as those of the mosses, in that the zygote develops in each case a sporophyte which alternates with a gametophyte in the life history. The spore is also in each case the means by which the mosses and ferns multiply and disseminate their offspring, and it furnishes, therefore, a necessary motile stage in the life history of these stationary land plants.

The great advance made by the fern over the moss is in the fact that the sporophyte in the fern is a highly organized plant with true roots, stem, and leaves, in which highly differentiated tissue systems provide amply for food making, food conduction, and food storage. The fern sporophyte is therefore an independent food-making and spore-producing plant, quite different from the leafless and rootless parasitic sporophyte of the moss. The gametophyte, on the contrary, has become greatly reduced in the fern and has for its sole functions the production of gametes and the temporary support of the embryo sporophyte (Fig. 178, A). The gametophyte is therefore short-lived and usually dies at the close of a single season's growth. We shall see that this reduction of the gametophyte to a comparatively simple plant structure marks the beginning of the almost complete disappearance of this phase of the life history in the highest seed plants. Since the ferns are the undoubted ancestors of the early seed plants of the coal period, the facts of their life history are of the utmost interest and importance to a proper understanding of the conditions which we are to discuss later in those plants. 
Chromosome reduction. Reduction of the chromosomes takes place in ferns in the first divisions of the mother cells, so that the spores have one half as many chromosomes in their nuclei as the nuclei of the mother cells (consult Fig. $43, b$ ). The nuclear division which follows to form cells of the gametophyte and the gametes is of the ordinary vegetative type (Fig. 43, a), in which each daughter nucleus is supplied with the same number of chromosomes as the nucleus from which it arose. As a result all cells of the gametophyte, including the gametes, have the reduced number of chromosomes. When fertilization takes place, the nucleus of the zygote has the two sets of chromosomes supplied by the male and female gametes (consult Fig. 44), and this double number persists throughout all cellular divisions in the sporophyte plant until the mother cells of the spores divide to form the first two cells of the tetrads. The two alternating generations in the life history of the ferns are therefore distinct as regards the number of chromosomes in the nuclei of their constituent cells, the gametophyte and the gametes always having one half the number of chromosomes of the zygote, the embryo, and the adult sporophyte, as in the higher plants (Fig. 44). The chromosome changes in the life history, as noted above, always take place in the first division of a spore mother cell to form tetrads and in the formation of the double cell, or zygote, which results from the union of the gametes. The great differences in the form and structure of the two alternating generations in the fern are therefore paralleled by a striking difference in the chromosome number in each generation.

\section{LIFE HISTORIES OF THE FERN ALLIES (EQUISETALES} AND LYCOPODIALES)

The spore plants of the Equisetales and Lycopodiales differ so much from the true ferns in external appearance and structure that they would never be recognized as fern allies by the ordinary observer. This alliance was first recognized by botanists after Hofmeister, in 1851, had worked out the life histories of some of the higher spore and seed plants and had established 


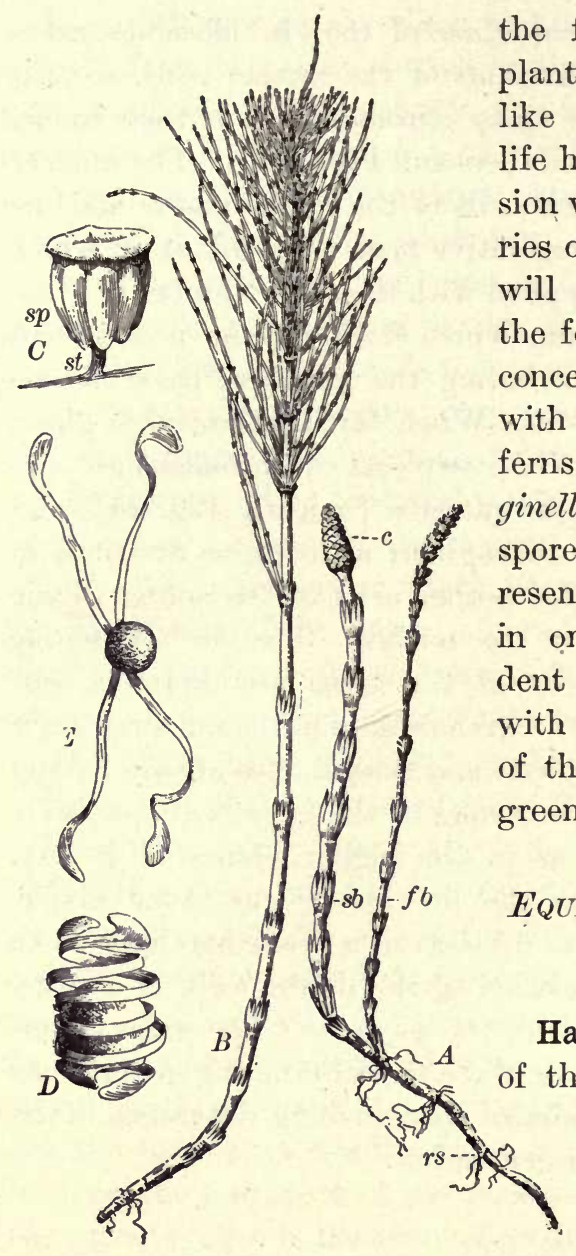

FIg. 179. Equisetum arvense

$A$, plant in early spring, with aërial branch $(s b)$ bearing strobili $(c)$ and a leafy branch $(f b) ; B$, a green summer shoot; $C$, sporangiophore with stalk $(s t)$ and sporangia $(s p) ; D$, spore with expanded elaters ; $E$, spore with contracted elaters

the fact that all higher green plants had similar stages and a sequence of stages in their e histories. In the brief discuswhich follows, the life histoof the equiseta and lycopods be compared with that of ern, in order to extend the conceptions that have been gained with regard to the life history of ferns and of the fern allies. Selaginella, with its two kinds of pores, will be taken as a representative of the Lycopodiales, in order to introduce the student to the problems connected ith the origin of the seed and f the seed habit in the highest reen plants.

Equisetales, oR Horsetails SPOROPHYTE

Habit. The sporophyte plants the equiseta differ in important particulars from those of the ferns on account of their xerophytic form and structure (Fig. 179). Some of the modern equiseta, like a few of the ferns, inhabit wet marshes and ponds, but they all retain the xerophytic structure and appearance which is derived from their treelike ancestors of the coal period. The xerophytic structures of the equiseta are therefore genetic and not adaptive characteristics. 
These xerophytic characteristics are apparent in the minute, scalelike leaves, the highly protected epidermis of the stems, and the poorly developed vascular system in the horsetails, all of which are in strong contrast to the large leaves and highly organized vascular system of their mesophytic allies the true ferns. The plant body in the equiseta is composed of an underground perennial stem, or rhizome, which serves the function of storing food and of hibernation when the aërial parts of the plant are destroyed by cold or drought. This perennial rhizome gives rise to annual green shoots, which are simple in some species and highly branched in others. These aërial stems, since they produce distinct chlorophyll-bearing tissue, serve the double function of making starch by photosynthesis and of bearing asexual spores. The plant body thus manifests the same physiological division of labor as we have already noted in the ferns, but the work of photosynthesis and spore bearing is performed in the equiseta by the annual shoots, instead of by the leaves as in the ferns.

Asexual reproduction. The spores are borne in strobili, or cones, which terminate either the regular green shoots or special reproductive branches as in Equisetum arvense (Fig. 179). The reproductive cones $(c)$ consist of a number of shield-shaped sporangiophores $(C)$, each of which bears several sporangia, shaped like the finger of a glove. When the spores are ripe, the axis of the cone, or strobilus, lengthens, thus separating the sporangia, which then open along a seamlike layer of cells on one side and liberate the spores. Each spore $(E)$ is furnished with four appendages, called elaters, which open out in dry weather and close up around the spore in a moist atmosphere. When spread out, the elaters assist in the distribution of the spores by air currents. The spores germinate much as in the ferns and produce a gametophyte plant, which bears the gametes. The peculiarity of the spores of Equisetum in this regard is that some spores produce gametophytes which bear antheridia only, whi'e others produce only female gametophytes. The spores are alike in form and are therefore said to be homosporous; but they are evidently differentiated physiologically, since they produce either a 
male or a female plant. This physiological differentiation of spores in Equisetum is very important, since it indicates how those spore differences probably originated which gave rise to the so-called heterosporous plants, of which we shall learn in our later studies.

\section{GAMETOPHYTE AND EMBRYO}

The male and female gametophytes of the equiseta resemble those of the ferns, except that they are more highly branched (Fig. 180). The male plants are small and bear the male sex organs at the ends of the lobes or on the margins of the

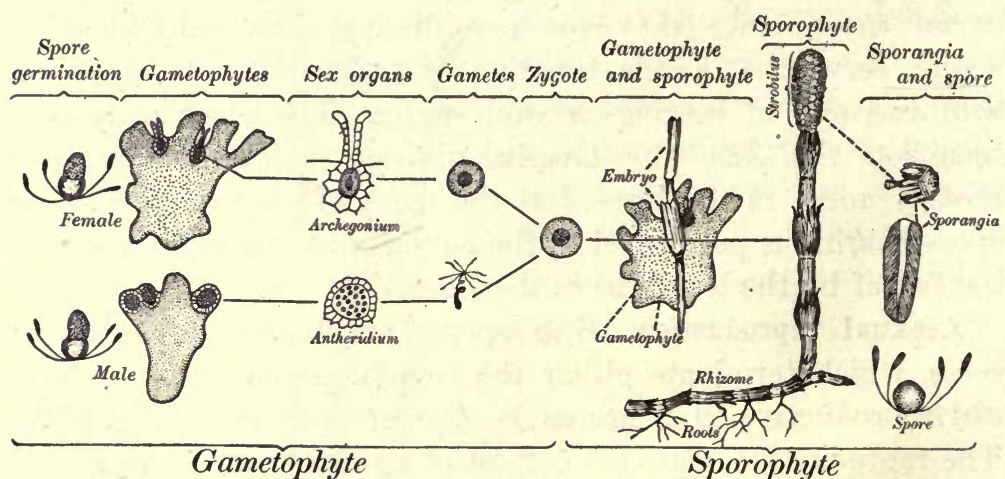

FIG. 180. Diagram showing life history of Equisetum

gametophyte. The female plants are larger than the males and bear the female gametangia on a thickened, cushionlike portion of the gametophyte beneath green, lobelike extensions of its upper surface. The male and female sex organs (antheridia and archegonia) and the gametes indicate by their structure and form their close relation to similar reproductive cells and organs of the ferns. Fertilization takes place by means of motile male gametes, which are liberated in films of water formed by rain or dew. Fertilization of the female gamete is followed by the development of a parasitic embryo sporophyte, as in the ferns.

Embryo. The general form and structure of the embryo sporophyte in the equiseta is determined by the kind of adult sporophyte into which it is destined to develop. We find as a 
consequence that the equisetum embryo, on account of the prominence of the stem in the adult plant, forms as its prominent organs a rudimentary stem, root, and sucking foot, instead of a cotyledon, root, and foot, as in the ferns. This embrya remains parasitic on the green gametophyte for a time, but ultimately gives rise to the adult sporophyte already described.

Life history. The life history of Equisetum (Fig. 180) will be found upon inspection to present the same sequence of stages as that of the fern, together with a close resemblance between the two forms as regards the structure of their reproductive organs and cells. These facts, as stated above, establish the close alliance of the equiseta and the ferns in spite of the great dissimilarity in the form and structure of the adult sporophytes.

\section{Lycopodiales, or Club Mosses}

\section{SPOROPHYTE}

Habit. In the Lycopodiales the sporophytes are often mistaken for mosses on account of their small leaves and their habit of growing in clusters or mats on the forest floor of temperate and tropical regions. Their structure and life history, however, indicate clearly that they are near relatives of the ferns and equiseta described above. The Lycopodiales are divided into two closely related families, the lycopods and selaginellas, on account of an important difference in the method of producing their asexual spores. The lycopods, commonly known as ground pines (Fig. 181), bear but one kind of spores, like the ferns and equiseta, but the selaginellas (Fig. 182) produce two kinds of spores in different sporangia on the same plant. In other respects the sporophytes of the two families are quite similar, with their horizontal branching stems creeping over the surface of the ground and with the erect spore-producing shoots (strobili) furnished with the small green leaves characteristic of the order. The entire plant body is anchored to the ground by naked branches sent out from the horizontal stem; these grow downward and take root in the soil much like the runners of a strawberry plant. 
Asexual reproduction. The erect branches terminate in the reproductive strobili, or cones, formed of modified leaves, which

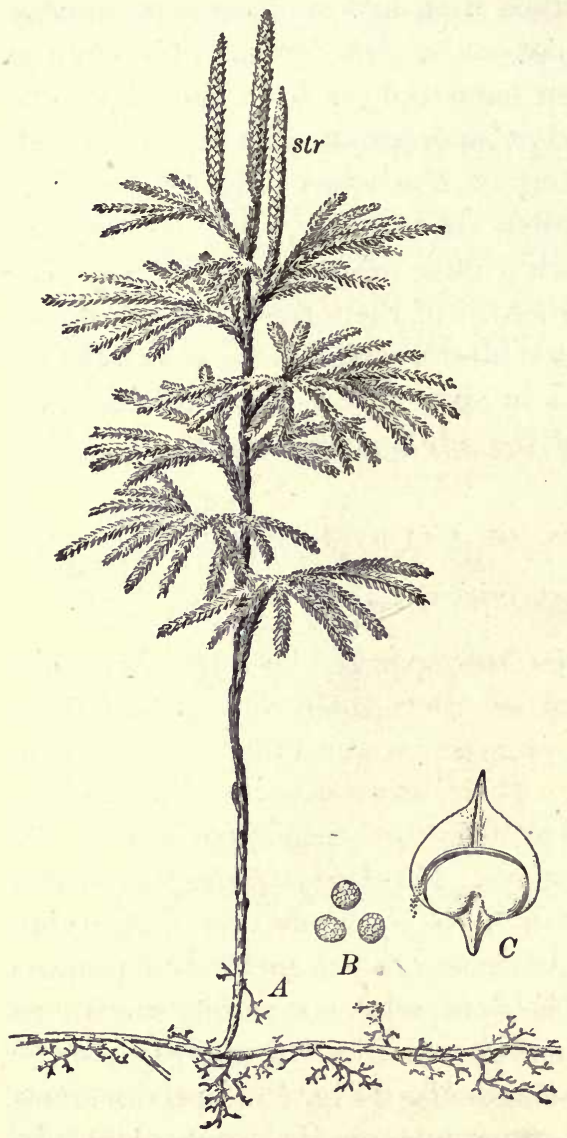

Fig. 181. The habit of a lycopod (Lycopodium)

$A$, plant with horizontal rhizome and erect, leafy spore-bearing stem; str, strobili; $B$, spores; $C$, sporophyll and kidney-shaped sporangium. From Bergen and Caldwell's "Practical Botany" bear the sporangia and spores. These very highly modified leaves are called sporophylls, which means leaves that bear spores. The sporophylls and sporangia of the lycopods and selaginellas are very similar except that in Selaginella there are two kinds of sporangia and two kinds of spores. The selaginellas are therefore said to be heterosporous, or with different spores, to distinguish them from the common ferns, equiseta, and lycopods, which are homosporous, that is, with one kind of spores. It should be stated also that some of the true ferns now living are heterosporous, and that many of the fossil Pteridophyta of the coal period bore two kinds of spores. Since the heterosporous condition is of the greatest importance for an adequate understanding of the life history of seed plants, the following description of the sporangia and spores of Selaginella is given to explain the nature of heterospory in a common tropical species of the Lycopodiales. 
Heterospory. In the fertile shoots of Selaginella the small spores, called microspores, are borne in microsporangia at the apex of the reproductive strobili, while the large spores, called megaspores, are produced in large megasporangia at the base of the strobili (Fig. 182). The microspores, like the spores of the equiseta and the homosporous lycopods, are produced in large numbers in each microsporangium, but the large megaspores are reduced to from two to four spores in each of the megasporangia (Fig. 183). This discrepancy in size is closely related to the function of the two kinds of spores in the rôle which each plays in reproduction. "The diameter of the megaspore is usually about ten times that of the microspores, which is equivalent to a proportion of $1000: 1$ in bulk." The larger megaspores produce-female gametophytes and on account of their size and weight are not easily scattered by the wind when shed from

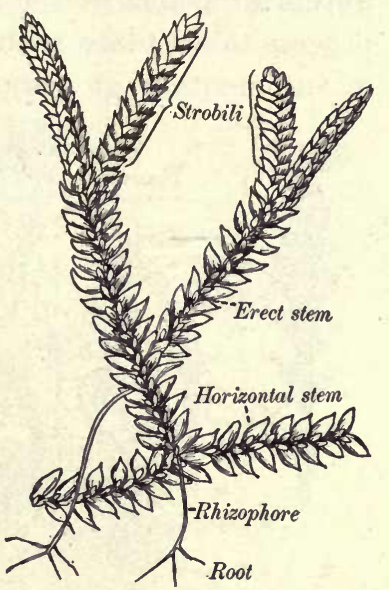

Fig. 182. Habit of Selaginella with strobili the sporangium. The small microspores, on the contrary, are light and easily scattered, like the pollen grains which we have already studied in the higher plants. Like the pollen, therefore, the microspores are the mobile spores of Selaginella, which can be borne to the heavy megaspores at the proper time for reproductive purposes. Since, however, this association of megaspores and microspores is necessarily a matter of chance, it follows that the microspores must be produced in great numbers to insure fertilization. Although the differentiation of spores in Selaginella and in other heterosporous Pteridophyta must be regarded as the first step toward the evolution of seeds, it is, nevertheless, in some respects a wasteful arrangement and not without its difficulties in insuring a meeting of the male and female gametes. In the higher seed plants this difficulty is overcome in part by the production of the stigma as a receptive apparatus for the pollen. 


\section{GAMETOPHYTES AND EMBRYO}

The spores of Selaginella begin the process of germination while they are still retained in the sporangium and are being nourished by the mother sporophyte. The germination process differs from that of the ferns and equiseta also in that the entire process takes place within the spore coat and results, therefore, in rudimentary gametophytes, which are permanently retained

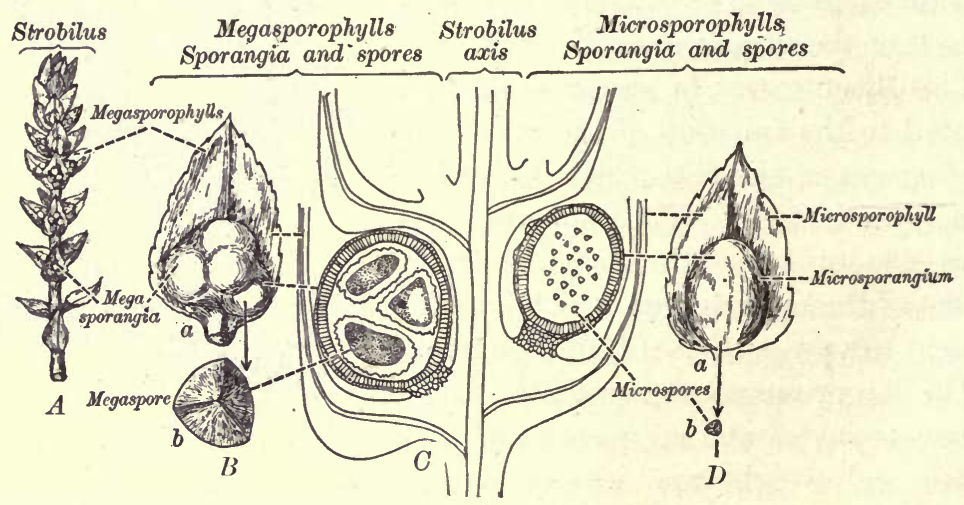

Fig. 183. Spore production in Selaginella

$A$, strobilus with megasporophylls and megasporangia; $B$, megasporophyll with sporangium and spores; $C$, median long section of a strobilus; $D$, microsporophyll with sporangia and spores

in the male and female spores. The female gametophyte is ultimately exposed to the air by the rupture of the large megaspore along three lines of weakness in the spore coat, which thus exposes the gametophyte and the archegonia in preparation for the fertilization process (Fig. 184, $a, b$ ). The germinated female spores may still be retained in the sporangium or they may be shed upon the earth, where the archegonia and gametes go through the same preparatory stages for receiving the male gametes as we have already described in the mosses and ferns.

Meanwhile the rudimentary male gametophytes are developed inside of the microspores; each of these consists essentially of a small cell representing the male gametophyte, and of an antheridium composed of wall cells and of several sperm mother cells 
which finally form free male gametes within the microspore (Fig. 184, c). These male gametes are then liberated by the rupture of the spore coat and fertilize the female gametes. In some species of Selaginella fertilization and the early development of the embryo take place within the female sporangium, which has previously opened and admitted the germinated microspores. In
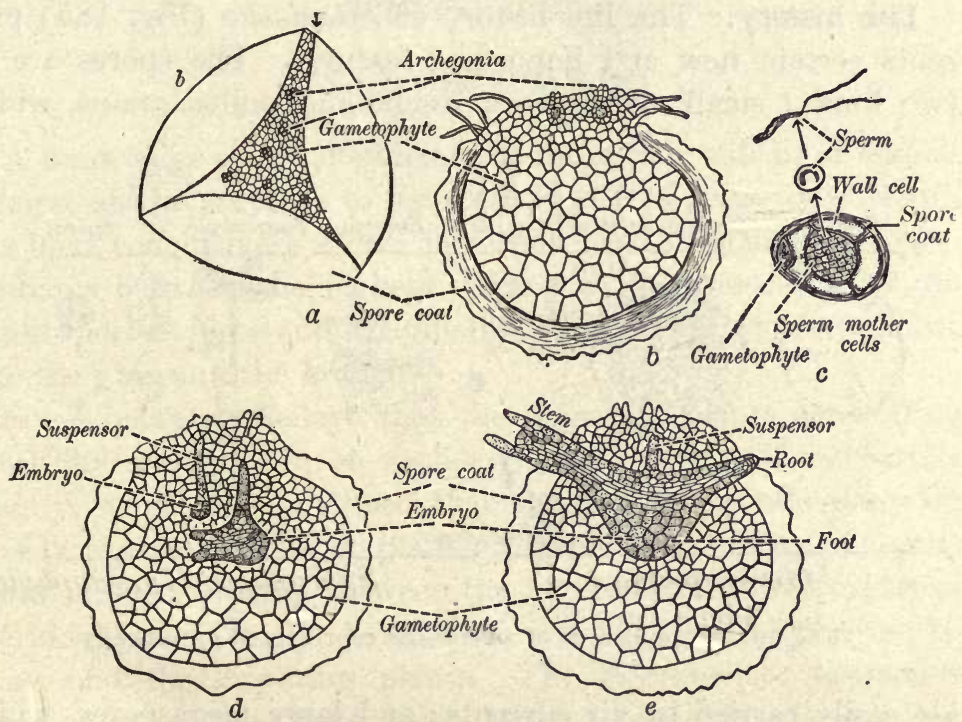

Frg. 184. Gametophytes, sex organs, and embryo of Selaginella

$a$, megaspore opening to expose the inclosed gametophyte and archegonia preparatory to fertilization; $b$, sectional view of a megaspore and gametophyte; $c$, section of a microspore, gametophyte, and sperm mother cells, with sperms above; $d, e$, three stages in the development of the sporophyte of Selaginella; $e$, young and differentiated sporophyte, with elongating root and stem

other instances fertilization may take place on the soil, where the female spores complete the germination process. The process of fertilization, as in all other instances among the higher plants, produces an embryo sporophyte which ultimately grows into an adult spore plant of Selaginella.

Embryo. The young embryo (Fig. 184, d) is early differentiated into the embryo proper and the suspensor, which serves to force the embryo down into the nutritive tissue of the gametophyte. The embryo then develops two cotyledons and a rudimentary 
stem, root, and foot surrounded by food material previously stored in the gametophyte within the spore. The further growth of the embryo (e) is accomplished by the elongation of the young stem (or hypocotyl) and the root, which bore their way out of the gametophyte and become adjusted to soil and air in response to gravity and light.

Life history. The life history of Selaginella (Fig. 185) presents certain new and important features. The spores are of two kinds: small microspores, resembling pollen grains, which

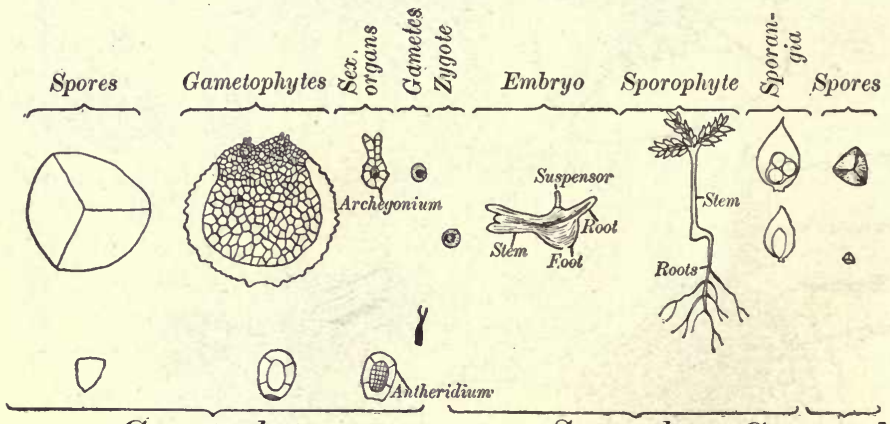

Gametophyte

Sporophyte Gametophyte

FIG. 185. Life history of Selaginella represented graphically

are easily carried by air currents; and large megaspores, which develop a nutritive gametophyte and female gametes. Spore germination takes place while the spores are still in the sporangia, and consequently the gametophyte plants thus formed within the spore coats are nourished by and are parasitic upon the mother sporophyte. The male gametophytes are reduced to gamete-producing structures and are composed of a singlecelled plant body and an antheridium bearing motile male gametes. The female gametophytes are richly stored with food which supports the embryo and young sporophyte until the latter becomes adjusted to its environment and is self-supporting. The heterosporous selaginellas thus approach the conditions found in the higher seed plants, in that they bear two kinds of spores, one of which, the megaspore, is stored with food reserve derived from the mother plant. 


\section{CHAPTER XVI}

\section{GYMNOSPERMS}

\section{CYCADALES (CYCADS)}

A knowledge of the life history of the cycads will be of special interest and importance to the student at this point, since botanists have conclusively shown in recent years that they are intermediates between the highest spore plants, represented by the heterosporous ferms and selaginellas, and the higher seed plants, like the pine and the bean.

Some botanists believe that the flowering plants arose from cycadlike ancestors in an earlier geologic period of the earth's history, while all are agreed that the modern cycads resemble closely a. great plexus of plant groups which in Carboniferous times bridged the gap between the highest living heterosporous pteridophytes (namely, Selaginella) and such seed plants as the pines and the flowering plants. The intermediate characteristics of the cycads have been shown to involve not only the reproductive features in their life history but also their general habit and vegetative structure.

\section{ZAMIA}

\section{SPOROPHYTE}

In Zamia (Fig. 187) the leaves resemble those of ferns in size and form, and the tuberous stem is covered with the scalelike bases of former leaves, as in many tree ferns. The internal anatomy of the above organs, as well as their external form, indicates also a fernlike ancestry. The male and female strobili are borne on separate plants and resemble in form and size the cones of pines. The microsporophylls are much larger, however, than 
the similar structures in the pine, and the numerous sporangia are usually grouped in sori, like the ferns (Fig. 187, C, a). The microspores resemble those of Selaginella and are called pollen grains. The megasporophylls are shield-shaped and bear two megaspo-

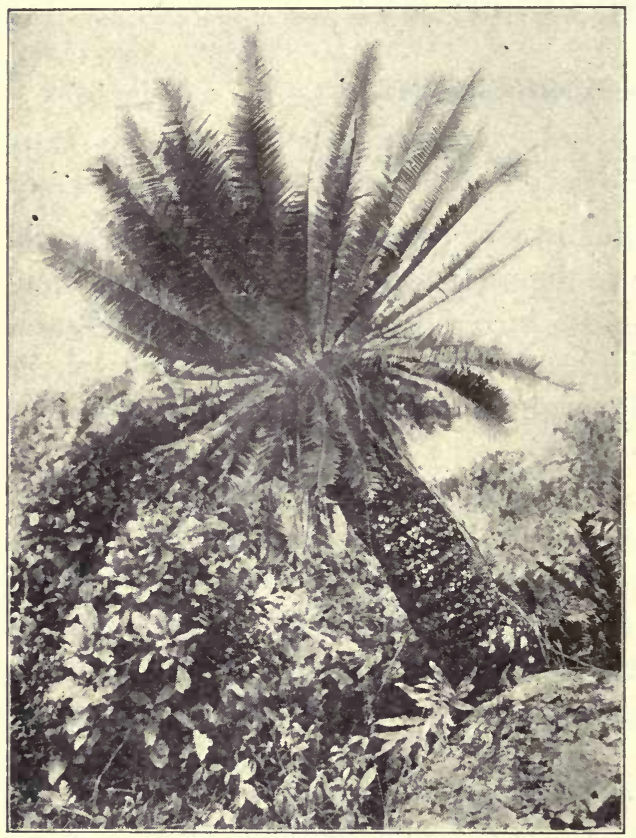

Fig. 186. The habit of a cycad, Dioön edule, growing in Mexico

The trank of this specimen was 15 meters (nearly 50 feet) high, and the age was estimated to be over nine hundred years. After Chamberlain rangia on each sporophyll (Fig. 187, $B, a$ ), which exhibit many characteristics of the early types of ovules. and seeds found in the fossil plants of the coal period. Each megasporangium consists of a mass of cellular tissue enveloped, as in the higher seed plants, by an integument (Fig. 187, B), which forms a long micropyle leading to the sporangial tissue. A single megaspore is ultimately developed from a mother cell which is deeply buried within each young megasporangium, as in Fig. 187, $B$, a.

\section{GAMETOPHYTES, EMBRYO, AND SEED}

The microspores and megaspores both form gametophytes within the spores by germination, as in Selaginella, and the female gametophyte and archegonia are permanently retained within the sporangium. The megasporangium is also furnished with an integument, which marks a new departure in the plants studied thus far in our consideration of the great plant groups. 
The female gametophyte (Fig. 187, B) is formed within the megaspore, which enlarges as the gametophyte grows, until it comes to occupy most of the space within the megaspore wall, only a remnant of the sporangial tissue being left at the micropylar end of the sporangium. At the micropylar end of the gametophyte from three to five archegonia are formed. The male gametophyte at first consists of a single gametophyte cell and of

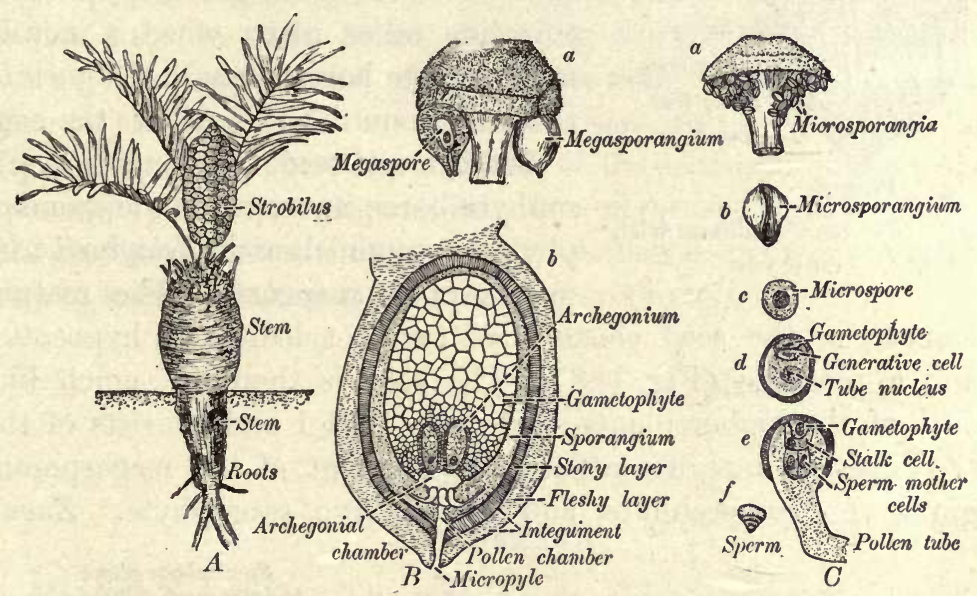

F1G. 187. Habit and reproductive structures of a Florida cycad, Zamia

$A$, plant of Zamia bearing a female strobilus ; $B, a$, megasporophyll, sporangia, ard spores; $b$, megasporangium (ovule) with gametophyte, archegonia, pollen grains, and pollen tubes; $C, a$, microsporophyll and sporangia; $d, e$, two stages in the

germination of the microspore and in the production of the male gametophyte

an antheridial cell, called the generative cell (Fig. 187, $C, d$ ). The generative cell then divides and there are ultimately formed a stalk cell and two sperm mother cells, each of which then develops a motile sperm (Fig. 187, C, e, f). Potlination takes place when the sporophylls of the female strobili separate and the microspores are borne to them by air currents. The microspores are drawn into the micropyle by a secretion which contracts with drying and carries the spores into a chamber (called the pollen chamber) developed in the sporangial tissue at the base of the micropyle (Fig. 187, B). The pollen tubes developed by the 
microspores bore into this sporangial tissue and absorb nourishment for the developing gametes. When the eggs are ripe, the sporangial tissue between the pollen chamber and the female

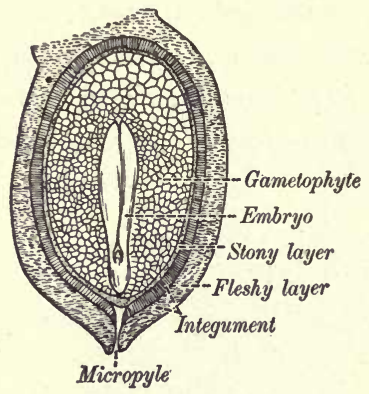

FIG. 188. Seed of Zamia with embryo gametophyte breaks down and the motile male gametes are cast out into a depression in the gametophyte, called the archegonial chamber, into which the necks of the archegonia open. Fertilization takes place when a motile male gamete bores through the neck of the archegonium and fertilizes the egg.

Embryo and seed. The embryo sporophyte bores its way into the gametophyte tissue, much as in Selaginella, by means of a suspensor. The mature embryo in the seed consists of two cotyledons, a hypocotyl, and a plumule (Fig. 188). The seed is therefore much like that of the higher plants studied in Part I and consists of the seed coat, or integument, a mere remnant of the megasporangium, the gametophyte, and the embryo sporophyte. Zamia

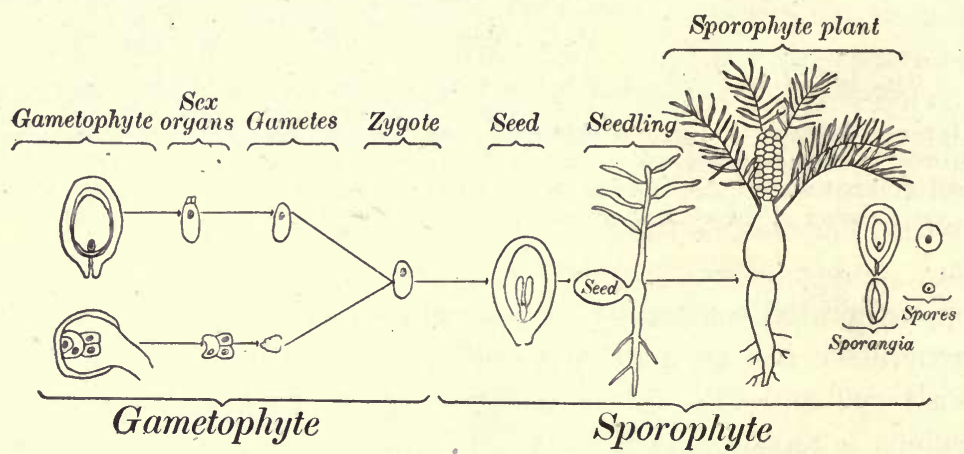

Fig. 189. Life history of Zamia represented graphically

is thus the first plant among the plant groups now under consideration which forms a true seed. Germination of the seed results in a seedling sporophyte which develops into the mature Zamia plant, similar in habit to Dioön (Fig. 186). 
Life history. It will be seen from the above brief account of the eycad that it has the same general stages in its life history as Selaginella (Fig. 185). The new features are concerned principally with the formation of a pollen tube, the changed relations of the megasporangium and megaspore, and the formation of seeds. The retention of the megaspore in the sporangium, which remains longer on the mother plant than in Selaginella, is accompanied in the cycad by pollination and the formation of a pollen tube to serve as an anchoring and absorbing structure during the development of the motile male gametes. The permanent retention of the megaspore has also resulted in the formation of a true seed composed of the megasporangium, the gametophyte, the megaspore, and the embryo sporophyte. When the seed germinates, the sporophyte resumes its growth and gives rise to a new adult cycad plant, or sporophyte.

\section{CONIFERALES}

\section{The Spruce (PICEA)}

\section{SPOROPHYTE}

Habitat and habit. The spruce tree, which is the spore-bearing plant, or sporophyte, has the same general form and mode of growth as the pine tree described earlier in the text. Like the pine the spruce is an erect tree type with an excurrent trunk and pyramidal crown, which results from its mode of growth and the spiral arrangement of its branches.

The spruce differs from the pine in that its needlelike leaves are borne directly and singly on the main shoot instead of in pairs or clusters on the end of minute dwarf shoots. Like the pines and their allies the spruces also inhabit mainly northern or mountainous regions and are typically xerophytic in habit and structure, although they adapt themselves readily to cultivation and to mesophytic conditions. In stem structure the spruces are intermediate between the pteridophytes and the woody-stemmed flowering plants, as the following account of the structure of the spruce will indicate. 
Structure. The broad outlines of structure in the spruce stem are identical with those of the woody stems of the alder, ash, and other trees and shrubs belonging to the flowering plants already

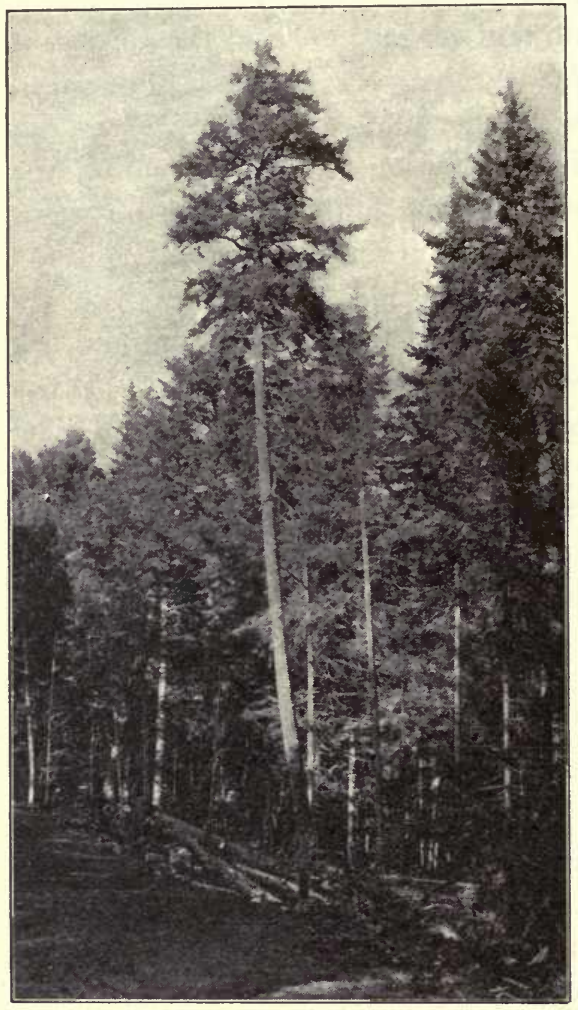

FIG. 190. Virgin forest of red spruce (Picea rubra) in the Adirondack Mountains

Photograph furnished by the United States Division of Forestry. studied (Figs. 191 and 192). The bark is composed of thick layers of cork, which scale off in flakes on the main trunk and the older branches of the tree. This corky bark, like that of other trees, is formed by a special cork cambium which arises in a layer of cortical cells beneath the epidermis during the first year's growth of the main stem or its branches. The epidermis is soon cut off from its water supply by this new growth of a corky bark, and is then sloughed off.

The cortex forms a wide zone of storage tissue in the young stem, but is destroyed later by being crushed between the corky bark and the expanding central cylinder of phloëm and xylem. A distinctive feature of the cortex in the spruces and pines is the formation of the large resin canals, which contain the resin common in coniferous trees. The central cylinder presents the same general features as that of other trees. The cambium is flanked on either side by the phloëm and xylem rings, and the annual wood rings are also sharply marked by the difference in structure of the spring and summer wood. 


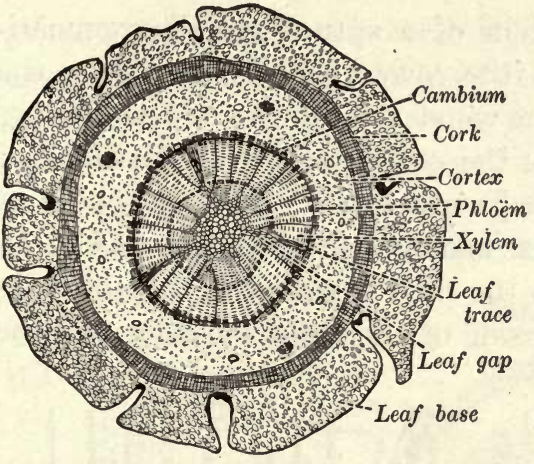

Fig. 191. Cross section of spruce stem two years old

The xylem is distinctive in Picea and the other Coniferales (including the common pines, hemlocks, and cedars) by being made up almost exclusively of single-celled water-conducting elements called tracheids (Fig. 193). These single-celled tracheids resemble those of the ferns and do the work of the long vessels, or ducts, in woody and herbaceous stems of the flowering plants. The tracheids of Coniferales are furnished with peculiar bordered pits representing thin places in the cell wall, which later become partially roofed over by the extension of the adjacent thicker portious of the tracheid wall. The wood rays have also certain distinctive features in the spruce and its allies, but their general structure and function is the same as that of the rays of the higher flowering plants. The pith is small and is more or less irregular in outline on account of the leaf gaps, which, as in the ferns, cause breaks, or gaps, in the otherwise solid vascular cylinder of a young stem.

The general relation of the leaf traces and leaf gaps to the vascular

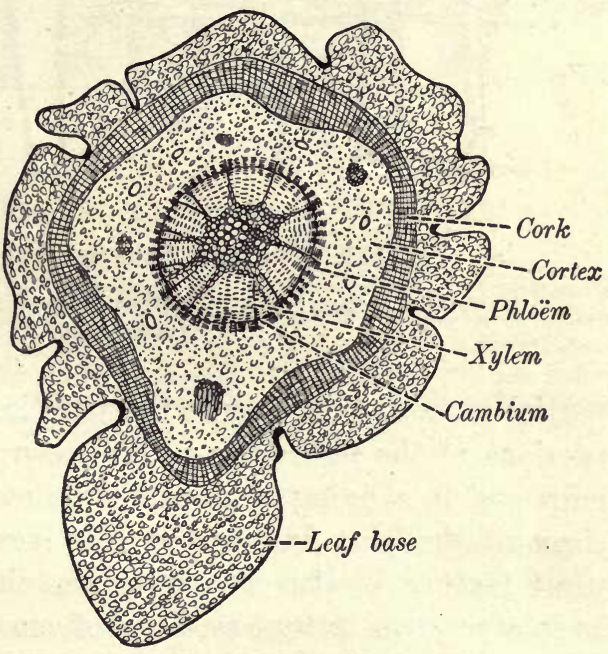

Fig. 192. Cross section of spruce twig of the first year

The radiate form of the pith is due to the numerous leaf gaps at the bases of leaf traces, or vascular bundles, going out to the leaves. The irregularities in the outer cortex are due to the effect of leaf bases 
cylinder in a two-year-old stem of a spruce are diagrammatically shown in Fig. 194. In the lower part of the figure the cortex and one annual ring of wood are represented as having been removed so as to expose the outer surface of the wood of the first year. On this exposed surface the leaf gaps appear as in the vascular cylinder of the maidenhair fern (Fig. 170). In the upper portion of Fig. 194 the pith is removed and the long leaf gaps are visible on the inside of the wood cylinder. In the

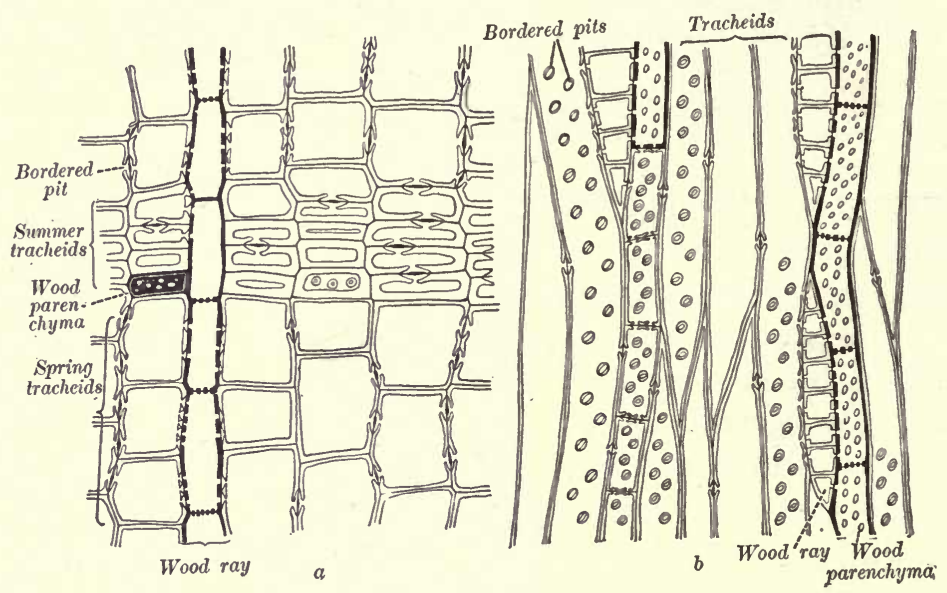

FIG. 193. Structure of the wood of the spruce (Picea)

$a$, transverse section; $b$, long section. Copied from Jeffrey's "Anatomy of Woody Plants"

partial cross-section view at the junction of the upper and lower portions of the figure the pith is seen to continue into the leaf gaps and in a living stem would be continuous with the cortex through the gap during the first year's growth. This structural feature is also shown in the first annual ring of wood as it appears in actual sections of one- and two-year-old stems of the spruce (Figs. 191 and 192). The young wood cylinder of the spruce during the first year is therefore similar to that of a fern like the maidenhair in having leaf gaps where portions of the wood and phloëm cylinder pass out to form a leaf trace. This persistence of a fern characteristic in the stem structure of 
a seed plant indicates, according to the teachings of modern anatomy, that the spruces have been derived from plants with a fern ancestry. In older spruce stems the leaf gaps are covered over by the later-formed annual rings of wood, but they are still evident as radial projections of the pith. The leaf traces are, however, persistent throughout the life of the evergreen leaves and may often be seen to connect with the leaf gaps, as in Fig. 194. These leaf traces serve to connect the phloëm and xylem of the vascular cylinder of the branches with the green tissues of the needle leaves. The summary on the following page gives the important points of similarity between the anatomy of the stem in the maidenhair fern and in the spruce, and also the general advances in structure made by the Coniferales as compared with the pteridophytes.

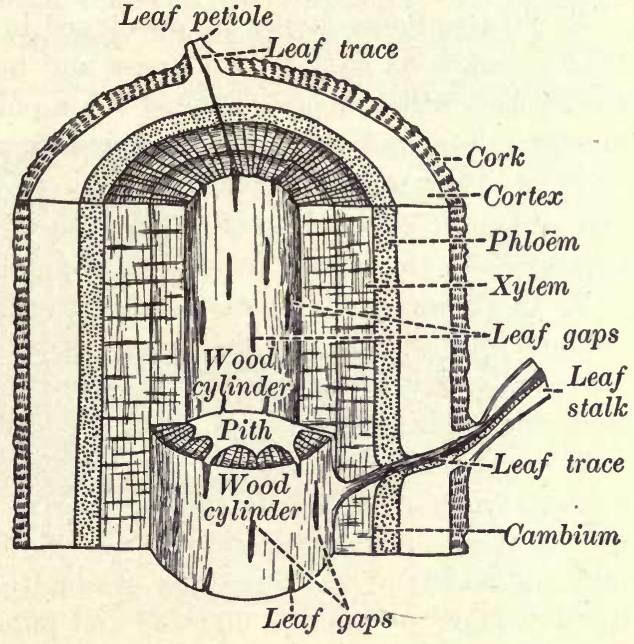

Fig. 194. Gross anatomy of the stem of a spruce branch two years old

The surface of the wood cylinder is exposed in the lower half of the figure by the removal of the cortex. The inner portion of the wood cylinder is shown above by the removal of the pith. Compare with the similar figure of the fern rhizome (Fig. 170). Note.the breaks, or leaf gaps, in the wood cylinder, as in the fern

The leaves of the spruce are strictly xerophytic in structure, as is shown by their small size and by the thick-walled outer layers of cells, which include both the epidermis and one or more layers of cells beneath it. Under this hard outer covering of cells the green mesophyll forms a wide, cortexlike layer containing chloroplastids. The central cylinder of the leaf is occupied at the base by two bundles which join into one in its upper portion. This vascular system of the leaf, as already indicated, is a continuation of the leaf trace connecting 
the living mesophyll cells of the leaf with the water-conducting tissue of the main stem and its lateral branches.

The root of the spruce and its relatives does not present any new features that need be discussed in an elementary textbook.

\section{SUMMARY}

1. Two cambium layers are developed in the spruce, which enable it to increase its stem in thickness and to form a protective outer cork jacket which insures against too rapid changes in temperature, loss of water, and the attacks of insects and fungi. In ferns this cork jacket is unnecessary, since the stem is usually underground and the outer skeletal layer, once formed, is in no danger of being destroyed by the annual growth of the stem in diameter.

2. The growth of the cambium forms a wide wood and phloëm cylinder for conducting the larger quantities of foods and water made necessary by the greatly increased leaf exposure of the spruce trees and their allies.

3. Food storage is provided for in the wood rays of the central cylinder instead of in the pith and cortex, as in the ferns. This provision was made necessary in the higher plants when the wide pith and cortex of the ferns was gradually eliminated as a result of the secondary production of wood and phloëm by the cambium.

4. The leaf traces and gaps are present in the spruce, but they become buried by the secondary products of the cambium. Their presence in the spruce stem is an indication of the relationship of two groups of plants which in other respects are widely separated.

Asexual reproduction. The spruces are moncecious, bearing both staminate and ovulate strobili on the same tree. Each strobilus is a modified shoot, like the strobili of the lycopods and cycads, with a central axis and lateral sporophylls arranged in a spiral form. The staminate strobili terminate lateral shoots at the ends of the main branches (Fig. 195, a), where they live through the winter in the bud stage and first make their appearance, in temperate climates, early in May. The microsporophylls are scalelike, and each microsporophyll bears two microsporangia on its lower abaxial surface (Fig. 197, e,f). A microsporophyll with its two sporangia is commonly called a stamen, 
as in the mandrake, although it resembles the sporophylls and sporangia of the lycopods and cycads quite as closely as it does the stamens of ordinary flowering plants. Each microsporangium produces a large number of microspores by tetrad division of microspore mother cells, exactly as in the ferns, Selaginella, and cycads, so that the spore-forming processes in the spruce microsporangia are identical with those of the sporangia of the lower vascular plants already studiect The microspores of the spruce are therefore true spores, exactly comparable to the microspores

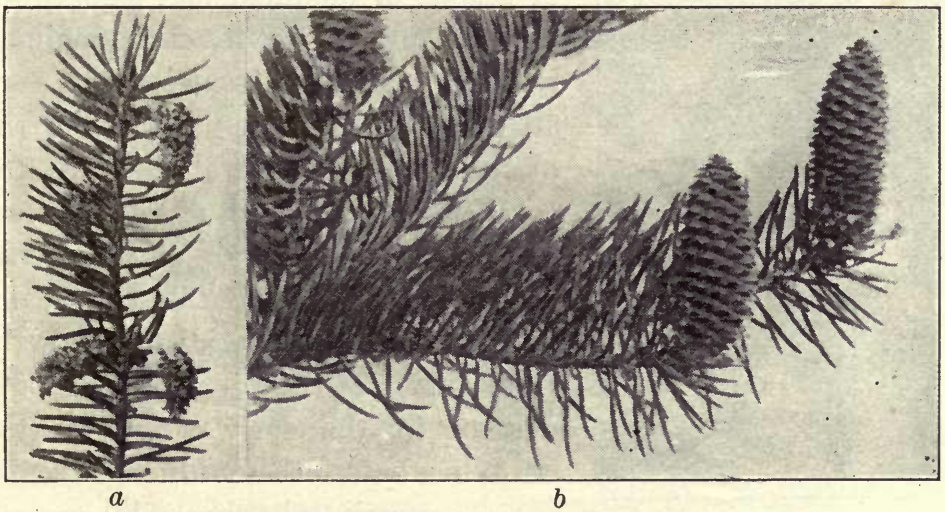

Fig. 195. Spruce twigs with staminate and ovulate strobili in May $a$, male strobili ; $b$, female strobili. Note the erect position of the female cones ready to receive pollen

of Selaginella. Each microspore, or pollen grain, when mature, is furnished with two expanded sacs, or wings (Fig. 198, c), formed by the inflation of the outer coat of the microspore. When the microspores are ripe, the microsporangia split down the center of each sporangium, or anther sac, and the light-winged spores are widely scattered, thus effecting pollination.

The ovulate strobili grow at the ends of last year's twigs, where they remain in the bud stage, like the staminate strobili, through the first winter. They make their appearance, in temperate regions, from the first to the fifteenth of May, occurring as beautiful red erect strobili (Fig. 195, b). They retain this erect position for two or three weeks, until pollination is effected 
by the microspores' falling into the space between the open megasporophylls and the axis of the strobilus. After pollination the megasporophylls close by excessive growth on the abaxial surfaces, and the cones gradually change their position, owing to carpotropic movements, finally assuming the pendulous position shown in Fig. 196. These ovulate strobili of the spruce are

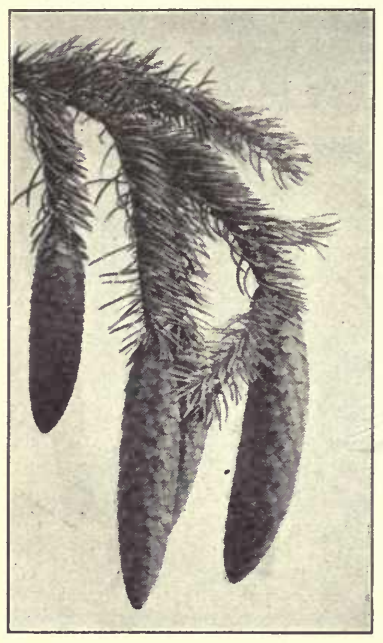

FIG. 196. Spruce cones in June after pollination

These cones were photographed about a month later than those represented in Fig. 195. Note the change in size and position of the cones at the time of pollination and during seed formation more complex in structure than the staminate strobili, since each ovulate strobilus bears on its axis two kinds of scales, or modified leaf structures, instead of one, as in the staminate strobili. The large scales which constitute the conspicuous part of the mature cone, or strobilus, are the ovuliferous scales, each of which bears two megasporangia, or ovules, at its base. These large ovuliferous scales really arise as adaxial outgrowths from very small scales which are only evident in the early stages of the strobilus, before the ovuliferous scales have outstripped them in growth (Fig. 197, c). The large ovuliferous scales probably represent two sporophylls of a reproductive branch, which grew in the axils of leaves corresponding to the minute scales of the young cones. For our purposes we may properly term the ovuliferous scales megasporophylls, and consider the ovulate strobilus a compound strobilus with both bracts and sporophylls, instead of a simple strobilus, like that of the staminate cones.

The-megasporangia, or ovules, of the spruce are similar to those of the cycad, with a single integument surrounding the sporangium tissue proper. Each megasporangium produces a single large megaspore (Fig. 197, b), which finally occupies a considerable portion of the sporangium. The history of its 
development shows that each megaspore is produced by a single mother cell, which lies deeply buried in the tissue of the young sporangium. This mother cell divides by tetrad division, and one of the cells of the tetrad forms the single successful megaspore. The ovules of the spruce, like those of the cycad, are therefore true megasporangia, in which a single megaspore, produced by the usual processes of spore formation, is formed and permanently

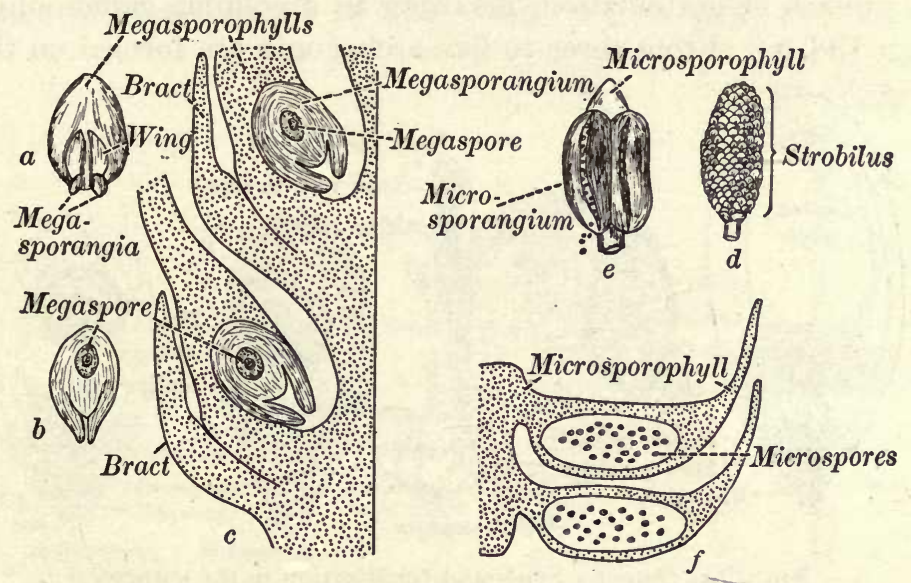

Fig. 197. Megasporangia and microsporangia with spores

$a$, megasporophyll (adaxial view) with two ovules (megasporangia); $b$, megasporangium in median section, showing the single megaspore; $c$, portion of a strobilus in section, showing megasporophylls, bracts, megasporangia, and spores; $d$, male strobilus; $e$, microsporophylls and sporangia; $f$, microsporophylls, spores, and sporangia (sectional view)

retained within the sporangium, instead of being shed, as in Selaginella. As the megaspore enlarges, it germinates and produces a true cellular gametophyte (Fig. 198,a). After fertilization the megasporangium becomes the seed, furnished with a hard seed coat, or integument, and an embryo spore plant produced by the fertilized egg.

\section{GAMETOPHYTES AND EMBRYO}

The male gametophyte in the spruce is similar to that of the cycads and is formed within the microspore (Fig. 198, d) as a result of germination. It consists at first of two cells (the 
gametophyte proper) and of an antheridial cell called the generative cell. The generative cell then divides to form two cells, a stalk cell and a body cell. When the pollen tube forms, the stalk cell disorganizes and frees the body cell, which then divides in the tube to form two male cells (Fig. 198,e). These nonmotile male cells correspond to the motile sperms of the cycads and ferns.

The female gametophyte is formed within the megaspore by the process of germination, resulting in a cellular gametophyte (Fig. 198,a). From three to five archegonia are formed on this

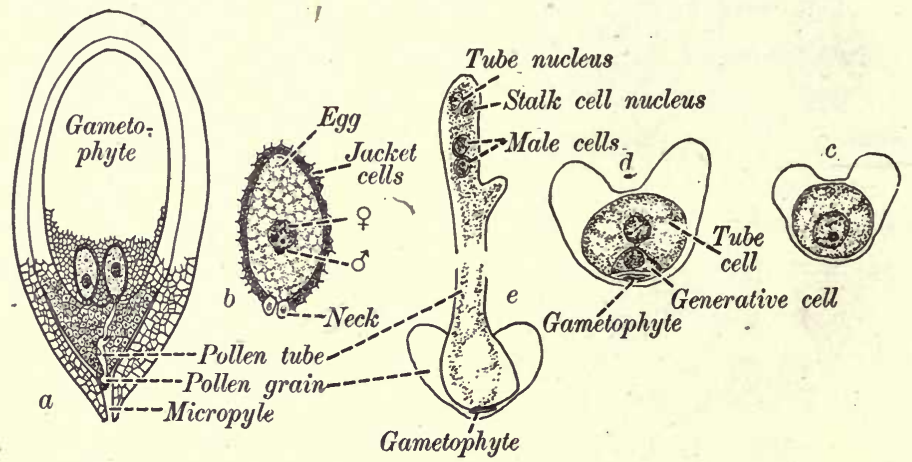

Fig. 198. Gametophytes and fertilization in the spruce

$a$, ovule at the time of fertilization; $b$, archegonium with a fertilized egg and male $\left(\sigma^{\circ}\right)$ and female $(q)$ pronuclei ; $c, d$, microspores before and after germination to form the gametophyte; $e$, pollen tube and male cells, or sperms

gametophyte at its micropylar end, each archegonium (b) being composed of a large egg cell, a layer of cells called the jacket cells, and the neck cells. Pollination is effected by means of the wind when the young female cones are erect on the ends of the branches. The cone scales are then,open (Fig. 195, b), and the pollen sifts down between them and comes to rest in contact with the micropyles of the ovules. A sticky secretion is exuded by the micropyle, as in the cycads, which draws the microspore into the micropyle until it rests on the surface of the megasporangium. No distinct pollen chamber is formed in the spruce, like that in the.cycad megasporangium. The pollen tube begins to grow down into the megasporangium early in May, soon after pollination, and reaches the archegonia late 
in June (Fig. 198, a). Just before fertilization the end of the pollen tube penetrates the neck of an archegonium and then ruptures, liberating the male cells in contact with the egg. The union of one of these male cells with the egg cell completes the process of fertilization and initiates the formation of the embryo.

Embryo. As soon as fertilization has taken place, the conjugate nucleus, formed by the union of the male and female

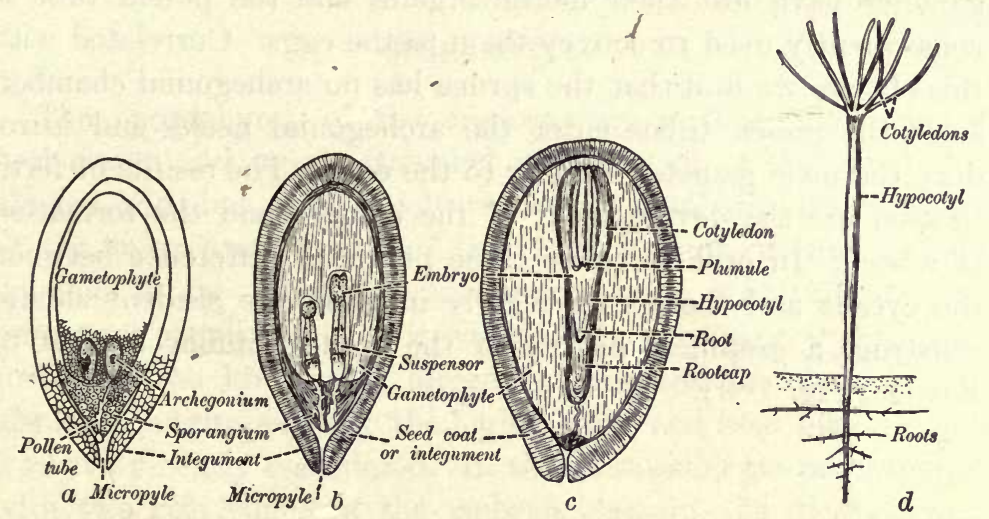

FIG: 199. The ovule, seed, and seedling of the spruce

$a$, ovule at the time of fertilization; $b$, two embryos developing as a result of fertilization; $c$, seed developed from $a$ with only one embryo; $d$, young seedling developed from a seed by germination and growth

pronuclei, divides to form eight nuclei, which then pass to the bottom of the egg. Around these nuclei eight cells are ultimately formed, which constitute the beginning of the proembryo. This proembryo soon differentiates into a suspensor, composed of four greatly elongated cells, and the embryonic - cells which are to form the embryo proper (Fig. 199, $b$ ). The embryonic cells finally produce the embryo within the seed. This embryo is composed of the hypocotyl, or stem, the root, and numerous first leaves, or cotyledons, surrounding the terminal plumule, or bud. These structures are shown more plainly in Fig. 199, $d$, which represents a seedling sporophyte of the spruce produced by the germination of the seed. The seed 
is thus composed of the seed coat, or integument (which forms a part of the mother sporophyte plant), of the gametophyte, and of the young sporophyte, or embryo, which represents a new sporophyte generation (Fig. 199,c).

Life history. The life history of the spruce is similar in all essential respects to that of the cycads, represented by Zamia. In both instances the megaspore is permanently retained in the megasporangium. In the spruce and its relatives the male gametes have lost their motile organs, and the pollen tube is consequently used to convey them to the eggs. Correlated with this change we find that the spruce has no archegonial chamber, since the pollen tubes enter the archegonial necks and introduce the male gametes directly to the eggs. The results of fertilization are the development of the embryo and the formation of a seed. In order to have these points of difference between the cycads and the spruce clearly in mind the student should construct a graphical history of the spruce similar to that of Zamia (Fig. 189). 


\section{CHAPTER XVII}

\section{ANGIOSPERMS \\ DICOTYLEDONS}

\section{SPOROPHYTES}

The sporophytes of the angiosperms include the common herbaceous and woody-stemmed plants, such as the mandrake, clovers, and elms, with which we became familiar in the first part of the text. On account of the large amount of time already devoted to the vegetative and reproductive structures of this important group of plants it will only be necessary at this point to review the knowledge already gained and to relate the life history of angiosperms to the higher spore and seed plants which we have recently considered. In this discussion the angiosperms with two cotyledons in the embryo, namely, the dicotyledons, have been chosen to represent the group, while the monocotyledons will be reserved for a separate and special treatment.

Structure. In connection with the following brief summary of the important advances in anatomy made by the dicotyledons the student should consult the figures and review the text relating to the structure of woody and herbaceous stems in Part I, and also the structure of Adiantum and the spruce in Part II.

The advances in structure relate mainly to the stem tissues, since the leaves of dicotyledons are not much more highly organized than those of ferns and cycads.

The general arrangement of the stem tissues in the woody types of dicotyledons is very similar to that of the spruce, and the advances made by the spruce in this respect, as compared with the pteridophytes, apply to the trees and shrubs among dicotyledons. 
The herbaceous dicotyledons resemble the herbaceous ferns in having a wide storing cortex, a large pith, and a narrow vascular cylinder. They differ from the ferns and are like the woody dicotyledons in the collateral structure of the phloëm and xylem and in the nature of the tissue elements, which are essentially the same in all dicotyledonous stems.

The storage system of cells in the dicotyledons, particularly in trees and shrubs, is for the first time amply provided for by large and small wood rays and by wood parenchyma abundantly distributed throughout the primary and secondary wood. In living pteridophytes the wood rays are lacking, and gymnosperms have neither the rays nor the wood parenchyma so largely developed as in the woody dicotyledons. (Compare the figures illustrating the anatomy of Adiantum, alder, and spruce.) This highly developed storage system of the woody dicotyledons compensates for the small size of the pith and cortex, which serve the storage function for a short time only in these plants, since the death of the cortex and pith in old dicotyledonous stems relegates the entire storage function to the wood rays and the parenchyma of the vascular cylinder.

The conducting cells are the familiar ducts which constitute long tubes for the rapid transfer of water necessitated by the immense leafage of the broad-leaved dicotyledons.

The average length of the tracheids which compose the waterconducting elements of gymnosperms is from two to four millimeters, while that of the ducts in dicotyledons ranges from a iew centimeters to several feet in length. The ducts, therefore, offer much less resistance to the rapid flow of water up the tree trunk in a dicotyledon than the tracheids do in a spruce or other gymnosperm (compare Figs. 53 and 193).

The great differentiation in kind and arrangement of tissues in the stems of dicotyledons is also a distinctive feature in these plants, since ducts of various kinds and sizes, strengthening fibers and tracheids, wood rays and storage parenchyma, are all adapted, in them as in no other plants, to the proper performance of their respective functions. This elaborate differentiation of tissues culminates in the woody-stemmed trees and shrubs. 
The flower. The flower in the angiosperms presents some new and distinctive features which are common to both dicotyledons and monocotyledons. The important advances made by the flower, as compared with the strobilus of plants below the angiosperms, relate to the development of a showy perianth and of a closed megasporophyll, or pistil, and to certain modifications in the relation and number of sporophylls borne on the floral axis, or receptacle. These new features can be most easily presented

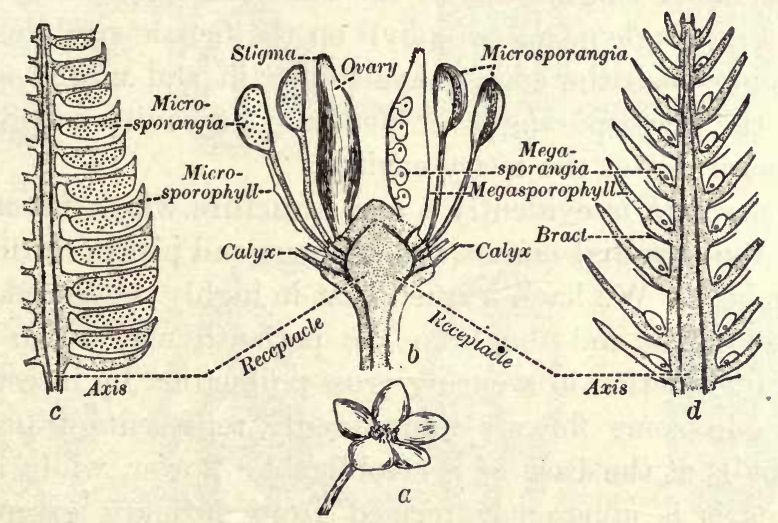

Fig. 200. Diagram designed to illustrate the corresponding parts of the spruce strobili and the flower of the marsh marigold (Caltha palustris)

$a$, flower of the marigold; $b$, section of the flower; $c$, median long section of the staminate strobilus of the spruce; $d$, similar section of the ovulate strobilus

by instituting a comparison between a simple flower like that of the marsh marigold (Caltha palustris) and the strobili of a gymnosperm like the spruce (Fig. 200). In the marigold flower the perianth and the numerous stamens and pistils are arranged in a spiral form on a dome-shaped receptacle like the sporophylls on the axis of a spruce cone. Such flowers with spirally arranged parts evidently correspond more nearly to the strobilus of the plants below them than the cyclic flowers of the mandrake and locust, in which the separate sets of floral organs are arranged in cycles on a flattened receptacle. If a median longitudinal section of a marigold flower is compared with similar sections of the male and female strobili of the spruce, the 
corresponding parts of the flower of the angiosperms and the strobili of the gymnosperms are at once made apparent.

The receptacle of the flower evidently corresponds to the axis of a strobilus, although it is greatly shortened and somewhat flattened at its apex. The stamens correspond to the microsporophylls and microsporangia of the spruce strobilus, the filament representing a highly modified, slender microsporophyll, and the anther sacs representing microsporangia borne at the apex of the sporophyll. A single pistil of the marsh marigold flower corresponds to a single megasporophyll on the female strobilus of the spruce, in which the edges have folded in and united so as to inclose the megasporangia, or ovules, in a cavity called in the angiosperm flower the ovary cavity.

The perianth is evidently a new structure which functions to protect the essential organs, the stamens and pistils, during their development. We have learned that in highly organized flowers like the locust and the bean the perianth may also serve an important function in securing cross-pollination by insects. The perianth in some flowers undoubtedly represents transformed sporophylls at the base of a strobiluslike flower, while in other instances it is apparently formed from ordinary green leaves below the sporophylls. We may conclude, therefore, that the angiosperm flower, represented by the flower of the marsh marigold, is a highly modified strobilus, in which many changes have taken place during its long course of evolution, including the shortening of the axis, or receptacle, and the transformation of simple sporophylls and sporangia into stamens and pistils and of certain sporophylls, or green leaves, into the parts of the perianth, namely, the calyx and corolla.

The evolution of the sporophylls and sporangia of the angiosperm flower will be more fully understood if a further comparison is made between these structures and the corresponding structures in the other vascular plants already studied. In Fig. 201 the microsporophylls and sporangia of Selaginella, spruce, and marsh marigold are compared with a portion of the sporophyll, or leaf, of Adiantum. From this figure it will be seen that the sporophyll has become gradually reduced in size from 
the fern to the angiosperm, until it has reached its limit in the slender filament of the angiosperm stamen. With this gradual reduction in size its original chlorophyll tissue has been lost, together with its power of making starch, so that the microsporophyll now serves a single function, namely, that of producing
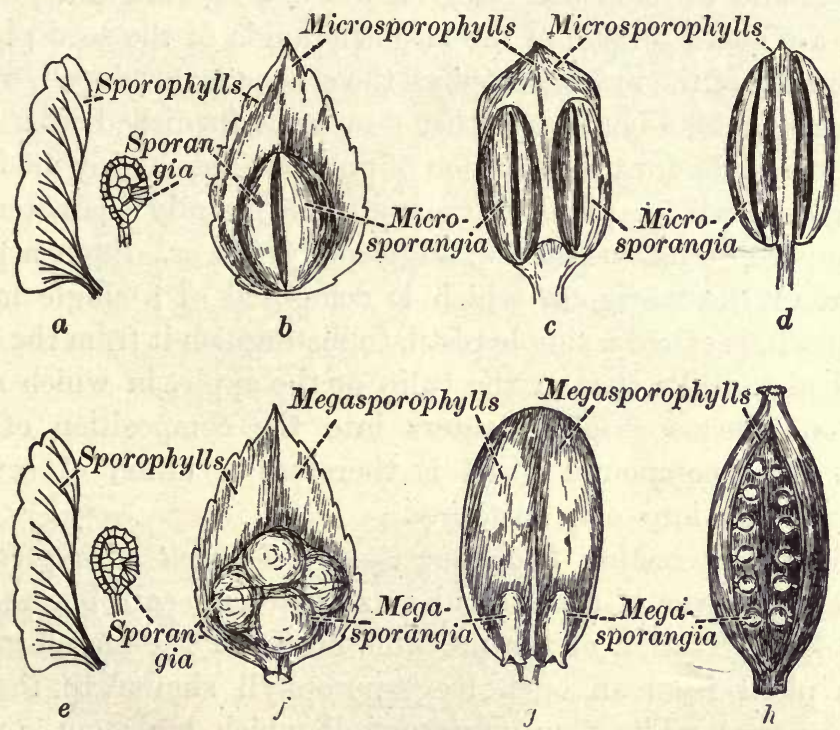

Fig. 201. Diagram illustrating the homologous, or corresponding, parts of the sporophylls and sporangia of the higher spore and seed plants

$a$, pinnule (sporophyll) and sporangium of the maidenhair fern; $b$, microsporophyll and microsporangium of Selaginella; $c, d$, corresponding parts of the anthers of the spruce and of the marsh marigold; $e$, pinnule (sporophyll) and sporangium of the maidenhair fern; $f$, megasporophyll and megasporangium of Selaginella; $g$, ovuliferous scale and ovules of the spruce; $h$, ovary and ovules of the marsh marigold

microspores, instead of the double function of spore production and photosynthesis, as in the ferns. The sporangia have also become transformed from the simple, distinct sporangia of the ferns into the four united microsporangia of the angiosperms, borne on a single sporophyll.

The pistil, which may be composed of a single megasporophyll, as in the marsh marigold, or of several megasporophylls, as in the lily or the apple, represents a still greater transformation in sporophyll 
structure than that outlined above for the microsporophyll. A single pistil of the marigold evidently corresponds to one megasporophyll of the spruce, with its edges turned in and united to form the ovary cavity. At the point of union of the edges of the sporophyll, which form the placenta, the megasporangia, or ovules, bud out and develop the integuments and sporangium proper, which are characteristic of the megasporangia of the seed plants. At the apex of the megasporophyll the stigma is developed, which in the marigold, as in many other flowers, is furnished with hairlike outgrowths for the retention of pollen. This highly modified megasporophyll, or pistil, is the most universally characteristic and important feature of the angiosperm flower. Such a pistil as that of the marigold, which is composed of a single megasporophyll, is called a simple pistil, to distinguish it from the compound pistils like that of the tulip or the apple, in which more than one megasporophyll enters into the composition of the ovary. The compound pistil is therefore a union of several simple pistils into one structure.

It is an interesting fact that the development of a pistil in the young flower of a marigold or a buttercup corroborates the above interpretation of the probable origin of the closed angiosperm pistil from an open megasporophyll similar to that of gymnosperms. The young sporophyll which buds out on the receptacle of a developing flower of a marigold or a buttercup is at first an open sporophyll resembling a rudimentary spruce sporophyll but with a concavity toward the axis of the flower. 'The edges of this concave sporophyll gradually approach each other by growth and finally unite to form the ovary cavity. Meanwhile the megasporangia, or ovules, bud out upon the uniting edges where the placenta is to be formed, and the stigma develops at the apex of the leaflike sporophyll. By further growth the mature closed pistil of the marigold is formed.

Asexual reproduction. The anther of the angiosperms is usually composed of four lobes, visible from the outside, which represent four microsporangia. These four microsporangia are shown in Fig. 202, A, as they appear in a transverse section of a mature anther of a lily. In a younger anther than that 
represented in the figure the pollen grains would be replaced by mother cells, which form the pollen, or microspores, by tetrad division, as in ferns, cycads, and spruces. The mother cell first
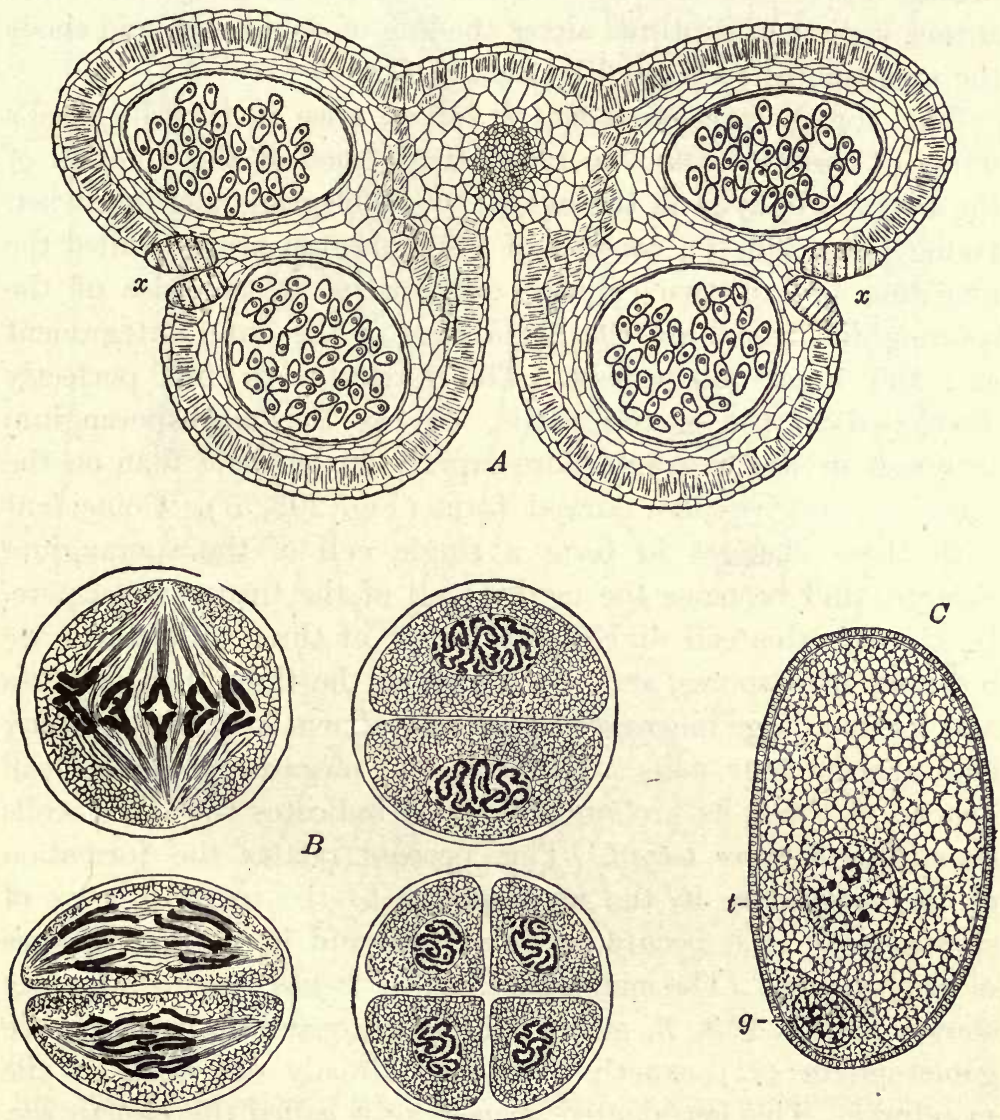

Fig. 202. Anther and pollen formation in a lily

$A$, mature anther with four microsporangia containing pollen grains; $B$, the process of forming microspores by tetrad division; $C$, gametophyte $(g)$ and tube nucleus $(t)$

in a germinated microspore. From Bergen and Davis's "Principles of Botany"

divides into two cells $(B)$, with a reduction in the number of chromosomes (consult Fig. 43, b). These two cells then divide again and form the four cells of the tetrad. Each cell of the 
tetrad then develops into a microspore, or pollen grain. When the pollen grains are ready to be shed, the cellular partition separating the two microsporangia on each side of the anther breaks down, and the two anther sacs are thus formed. The' anther wall then ruptures along the line of dehiscence and sheds the microspores (consult Fig. 202, $A, x$ ).

The young megasporangia, or ovules, arise in the shepherd's purse (Capsella) from two placentæ, formed at the junction of the two sporophylls in the ovary. Each megasporangium, when young (Fig. 203, A), consists of a sporangium proper, called the nucellus, and of two cellular outgrowths at the base of the sporangium, which are the beginnings of the outer integument and the inner integument. The funiculus is not perfectly developed in the young ovule, but as the megasporangium increases in size it grows more rapidly on one side than on the other, which gives it a curved form (Fig. 203, B). Coincident with these changes in form a single cell of the sporangium enlarges and becomes the mother cell of the future megaspore. In Capsella this cell divides into a row of three cells which are potential megaspores, and the lowest of the three then enlarges and forms a large megaspore, such as is shown in $B$, es. In many angiosperms four cells arise from the megaspore mother cell instead of three, as in Capsella, which indicates that these cells constitute a spore tetrad. This process relates the formation of the megaspore in the angiosperms to the usual process of sporogenesis as it occurs in Selaginella and in the microspores of angiosperms. The megaspore, when it has reached the size shown in Fig. 203, $B$, germinates at once and forms a female gametophyte $(C)$ exactly like that already described in the mandrake. This germinated megaspore is called the embryo sac.

\section{GAMETOPHYTES AND FERTILIZATION}

The female gametophyte of Capsella (Fig. 203, C) corresponds exactly to that of the mandrake (Fig. 86, b) and the iris (Fig. 204). It consists of the egg cell, or female gamete, the synergidæ (which are closely associated with the egg), the polar 


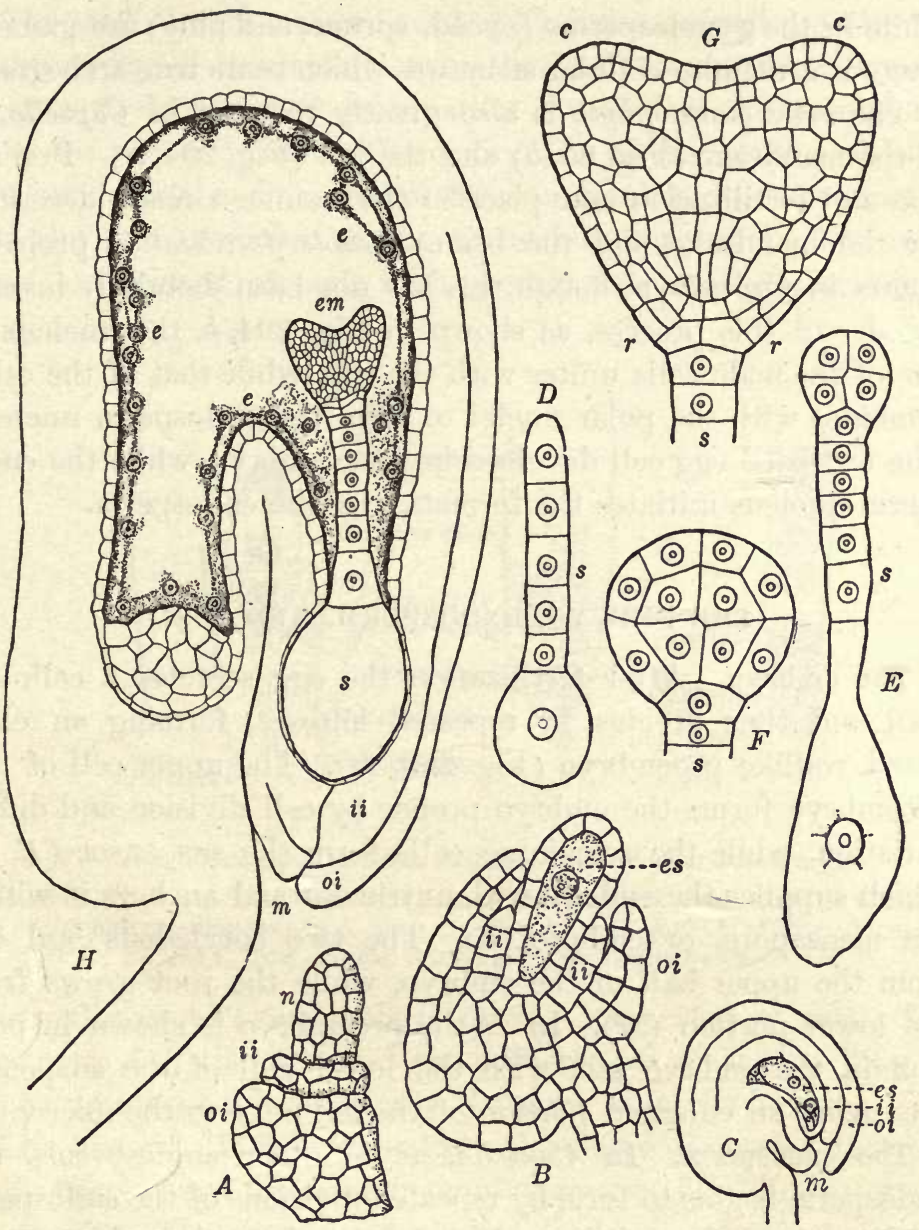

Frg. 203. Development of the ovule and embryo of shepherd's purse (Capsellc) $A-C$, stages in the development of the ovule; $n$, nucellus of megasporangium; $i i$ and $o i$, inner and outer integuments; $m$, micropyle; es, embryo sac; $H$, mature ovule (megasporangium) with embryo sac, embryo $(\mathrm{em})$, and endosperm nuclei $(e) ; D-G$, stages in the development of the embryo ; $s$, suspensor; $r$, root; $c$, cotyledons

nuclei, and the antipodal cells. The student will note at once the great difference between this reduced gametophyte of the angiosperms and that of the cycads and spruce. In the angiosperm the female gametophyte consists of six cells and two nuclei, 
while in the gymnosperms (cycad, spruce, and pine) the gametophyte is a definite cellular structure which bears true archegonia.

The male gametophyte is also greatly reduced in Capsella, as in the mandrake (Fig. 85, b) and the iris (Fig. 204,a). Pollination and fertilization take place in the manner already described for the mandrake and the bean. Double fertilization probably occurs in Capsella, although this has not been definitely investigated. In this process, as shown in Fig. 204, $b$, the nucleus of one of the male cells unites with the egg, while that of the other combines with the polar nuclei to form the endosperm nucleus. The fertilized egg cell develops into the embryo, while the endosperm nucleus initiates the formation of the endosperm.

\section{THE EMBRYO, ENDOSPERM, AND SEED}

The embryo. After fertilization the egg secretes a cellulose wall and then divides by repeated mitoses, forming an elongated, rodlike proembryo (Fig. 203, D). The upper cell of this proembryo forms the embryo proper by cell division and differentiation, while the remaining cells form the suspensor $(E, F)$ which supplies the embryo with nutriment and anchors it within the megaspore, or embryo sac. The two cotyledons bud out from the upper half of the embryo, while the root grows from the lower portion $(G)$. In $I$ the proembryo is shown in position in the embryo sac, with the lower cell of the suspensor occupying an enlarged portion of the sac next to the micropyle.

The endosperm. In Capsella, as in other angiosperms, the endosperm begins to form by repeated divisions of the endosperm nucleus, formed, as indicated above, by the union of one male nucleus and the two polar nuclei.

The numerous endosperm nuclei thus formed accumulate in the peripheral layer of cytoplasm which surrounds the large central vacuole of the embryo sac, where they form a layer of free nuclei (Fig. 203, H, e). These free nuclei never form a permanent cellular endosperm in Capsella, since they are gradually absorbed by the growing embryo, which in a later stage of its development fills the embryo sac. 
The seed. In the ripened seed of Capsella the embryo fills the entire cavity of the embryo sac; the food reserve necessary for its growth during seed germination is stored in the cotyledons, as in the pea and bean. The embryo, as in the latter seeds, has two cotyledons, a plumule (or first terminal bud), a hypocotyl, and the root meristem at the tip of the hypocotyl. The seed coats are formed of cells with greatly thickened walls, which

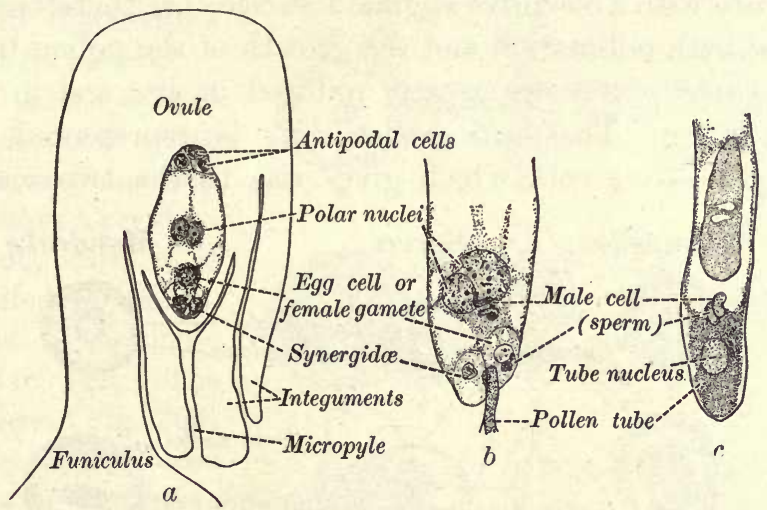

Fig. 204. Ovule, pollen tube, and fertilization in Iris and a lily

$a$, ovule of Iris with embryo sac and female gametophyte at the time of fertilization; $b$, double fertilization in a lily; $c$, pollen tube of Iris. $b$, after Gü̈nguard; $a$ and $c$, from original drawings by M. Louise Sawyer

effectually protect the embryo during its period of rest. The germination of the seed and the adjustments of the seedling to the environment are essentially the same as in the bean.

Life history. It is evident from the above discussion that the angiosperms have the same general stages in their life history as the spruce. The new features relate to details of structure and reproduction already discussed and hence need only a brief treatment in the form of a summary at this point.

The flower of the angiosperms is a highly modified strobilus in which the microsporophylls have been transformed into stamens and the megasporophylls into one or many pistils. The closed pistil, with the stigma differentiated for the reception of pollen, is the most distinctive feature of the angiosperms, although the perianth, when developed, is an important characteristic. The 
perianth is usually composed of modified sporophylls, but in some flowers the leaves immediately below the sporophylls have been transformed into sepals and petals.

Pollination devices are more highly developed in the angiosperms than in any of the gymnosperms, and the pollen tube traverses the tissues of the style, stigma, and ovary cavity before coming in contact with the micropyle. The development of a closed pistil with a receptive stigmatic surface has therefore greatly modified both pollination and the growth of the pollen tube.

The gametophytes are greatly reduced in size and in cellular differentiation. The male gametophyte is represented by the single generative cell, which gives rise to the two male cells

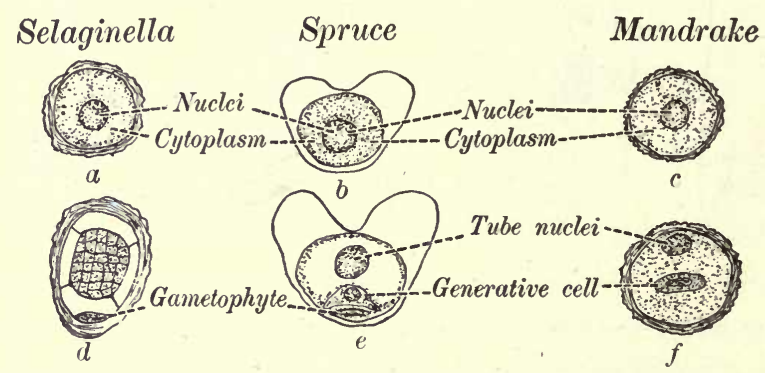

Fig. 205. Diagram illustrating the homologous, or corresponding, structures of microspores and male gametophytes in Selaginella, spruce, and mandrake

$a-c$, microspores; $d-f$, germinated microspores and gametophytes

within the germinated pollen grain, or microspore. The female gametophyte is reduced to the egg apparatus, polar nuclei, and antipodals in the embryo sac. The embryo develops from the fertilized egg cell within the embryo sac and passes into a resting stage within the seed. The full development of the sporophyte begins with seed germination, when the embryo resumes its growth, fed by the endosperm stored up around the embryo or within its cotyledons.

\section{SUMMARY AND COMPARISONS}

A brief summary and comparison of the heterosporous plants from Selaginella to the angiosperm will suffice to indicate the striking advances made by the highest spore-bearing and seed plants in 
the evolution of the seed and the seed habit. The more important of these changes are represented graphically in Figs. 205 and 206. In these figures it may be seen that the microspores have remained quite similar in form, size, and structure, since their function has not changed. In the angiosperm, as in Selaginella, the microspores still serve the function of bearing the male gametes to the female gametes, and are hence small, light, and highly protected cells, produced in great numbers by the microsporangia to guard against waste in distribution by wind or insects.

The male gametophyte has been gradually reduced, as was explained in a previous paragraph, until it is represented in the angiosperm by a single generative cell. The megaspores (Fig. 206) have become reduced to a single megaspore in each megasporangium, both in the gymnosperms, represented by the spruce, and in the angiosperms, represented by the mandrake. The important advance made by the latter groups of plants over Selaginella and
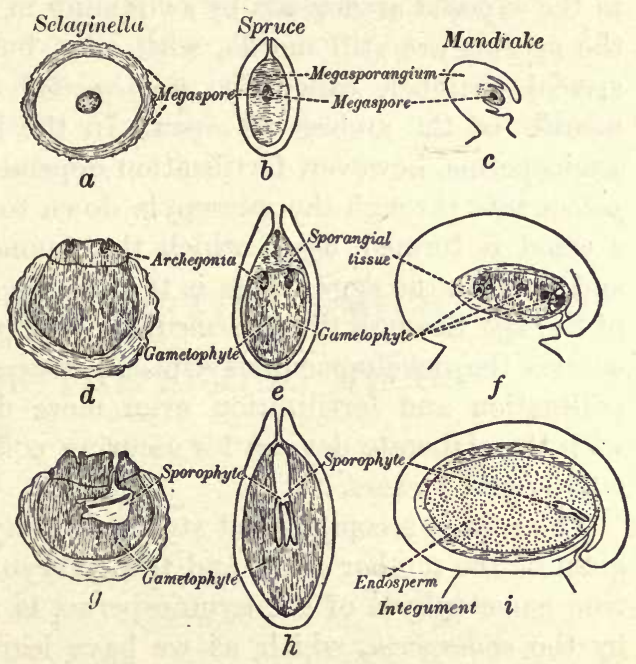

Fig. 206. Diagram showing the homologous, or corresponding, parts of megaspores, megasporangia, gametophytes, and sporophytes of Selaginella, spruce, and mandrake

$a-c$, megaspores; $d-f$, gametophytes; $g-i$, gametophytes and sporophytes. $h$ and $i$ are seeds similar heterosporous Pteridophyta is that of retaining the megaspore permanently in the sporangium. The megasporangium thus becomes indehiscent and is shed from the mother plant and disseminated with the contained megaspore and embryo. The female gametophytes remain cellular structures produced by the germination of the megaspore in both Selaginella and the spruce. In Selaginella, however, the gametophyte and archegonia are still exposed by the opening of the megasporangium and spore. In the indehiscent megasporangium of the spruce the cellular gametophyte is retained permanently in the spore within the megasporangium, but 
it still bears definite archegonia with neck cells and a ventral-canal cell nucleus inherited from its fernlike ancestors of the coal period.

In the angiosperm no cellular gametophyte is formed before fertilization, and the gametophyte is reduced to the egg apparatus, polar nuclei, and antipodals. Pollination and fertilization methods are also greatly modified, in both the higher gymnosperms and angiosperms, by the permanent inclosure of the gametophyte in the megasporangium. In Selaginella the motile sperms reach the eggs in the exposed archegonia by swimming in films of water. In cycads the sperms are still motile, with cilia, but they are liberated in a special chamber, called the archegonial chamber, into which the mouths of the archegonia open. In the higher gymnosperms and angiosperms, however, fertilization depends upon the growth of the pollen tube through the micropyle down to the egg; by this growth a canal is formed, down which the nonmotile male gametes reach and fertilize the eggs. This is true siphonogamy, or the fertilization of the egg through the intermediary of a pollen tube. In the angiosperms the development of a pistil, with stigma and style, renders pollination and fertilization even more difficult and is correlated with the elaborate devices for securing pollination observed in many angiosperm flowers.

The seed is a complicated structure composed of the megasporangium of the mother plant and the embryo. In the angiosperms the true gametophyte of the gymnosperms is replaced in many species by the endosperm, which, as we have learned, is a nutritive tissue formed as a result of fertilization. In other respects the seeds of angiosperms and gymnosperms are quite similar in structure. The most striking advances and changes leading to the evolution of seeds are, therefore, the reduction of both male and female gametophytes, the reduction in the number of megaspores produced by the megasporangia, the retention of the megaspores and female gametophytes permanently within the sporangia, and the changes in methods of pollination and fertilization correlated with this retention. 
PART III. REPRESENTATIVE FAMILIES AND SPECIES OF THE SPRING FLORA 



\section{CHAPTER XVIII}

\section{DESCRIPTIVE TERMS}

For the study of plants in the field the student will need certain descriptive terms which have not been given in the preceding pages. In the following brief discussion, therefore, we shall define the more important descriptive terms which the student will need in studying the trees, shrubs, and herbaceous plants of the spring flora.

\section{VEGETATIVE PARTS OF PLANTS}

Habitat. The term habitat is used to indicate the nature of the environment in which individual plants or plant groups live. The most common classification of habitats is that already described, based upon the conditions of moisture, soil, and light which constitute the environment. Habitats may therefore be designated as mesophytic, xerophytic, hydrophytic, and tropophytic, according as the plants inhabiting these areas are mesophytes, xerophytes, hydrophytes, or tropophytes in habit.

Habit. The term habit includes the form and general appearance of plants, based upon stem, branch, and leaf characters. Thus, trees like the pines and spruces, with a main excurrent trunk, are said to be erect in habit as compared with trees like the elm, apple, and oak, in which the equal growth of several branches produces a spreading habit. Plants are also said to be caulescent when possessed of a definite aërial stem, to distinguish them from plants like dandelions and strawberries, which are designated as acaulescent, or without a visible aërial stem.

Stems. Stems are either aërial, growing aboveground, or subterranean, growing largely or wholly underground. The main types of underground stems may be defined as follows:

Rhizomes, like those of ferns or Solomon's seal (Fig. 207), are horizontal underground stems furnished with buds and scalelike 
leaves which serve both for storage and for the support of aërial parts growing from them. On account of their underground habit they often become highly modified in both form and structure.

Runners and stolons are horizontal stems much like rhizomes except that they run over the surface of the ground, in which they frequently take root and give rise to new plants, as in the strawberry (Fig. 76). Prostrate stems are like runners except

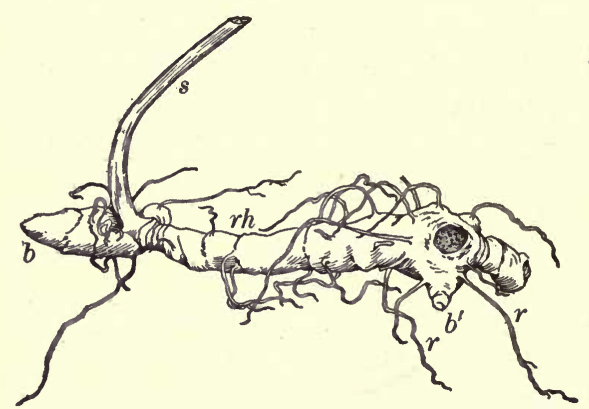

Fig. 207. Rootstock of Solomon's seal

$b, b$, buds; $r$, roots; $s$, flowering stem that they rarely take root in the soil over which they trail.

Bulbs like the onion and tulip (Fig. 79) are short, erect stems which bear scalelike leaves filled with reserve foods surrounding a terminal bud.

Corms are short, erect, fleshy stems (Fig. 80) with inconspicuous scale leaves. They usually have a prominent terminal bud and less conspicuous lateral buds in the axils of the scale leaves.

Tubers, like the potato (Fig. 78), are essentially greatly shortened rhizomes with scale leaves and lateral buds. They are usually filled with stored reserve food.

Leaves. Fig. 208 illustrates certain characters of leaves, pertaining to their form, margin, leaf tips, and venation, which are of importance in characterizing and identifying plants. The figures are self-explanatory, since the proper terms descriptive of the leaves are used in connection with the figures. The terms pinnate, palmate, and parallel, used in connection with the leaf shapes, indicate the type of venation characteristic of each leaf. Parallel venation is characteristic of such leaves as those of the linear type, found in grasses and members of the lily family, where the secondary veins run lengthwise of the leaf parallel to the midvein. Pinnate and palmate venation are found in the broad-leaved herbs and trees which belong to the dicotyledons. 


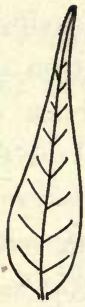

$a$

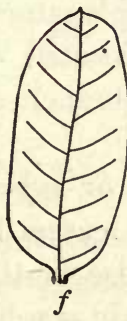

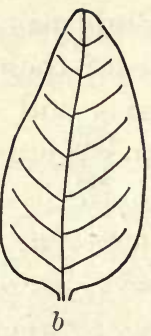
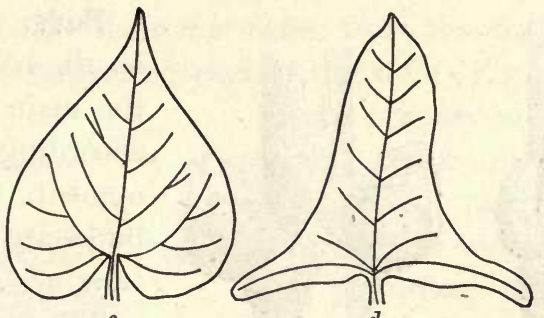

$c$

$d$
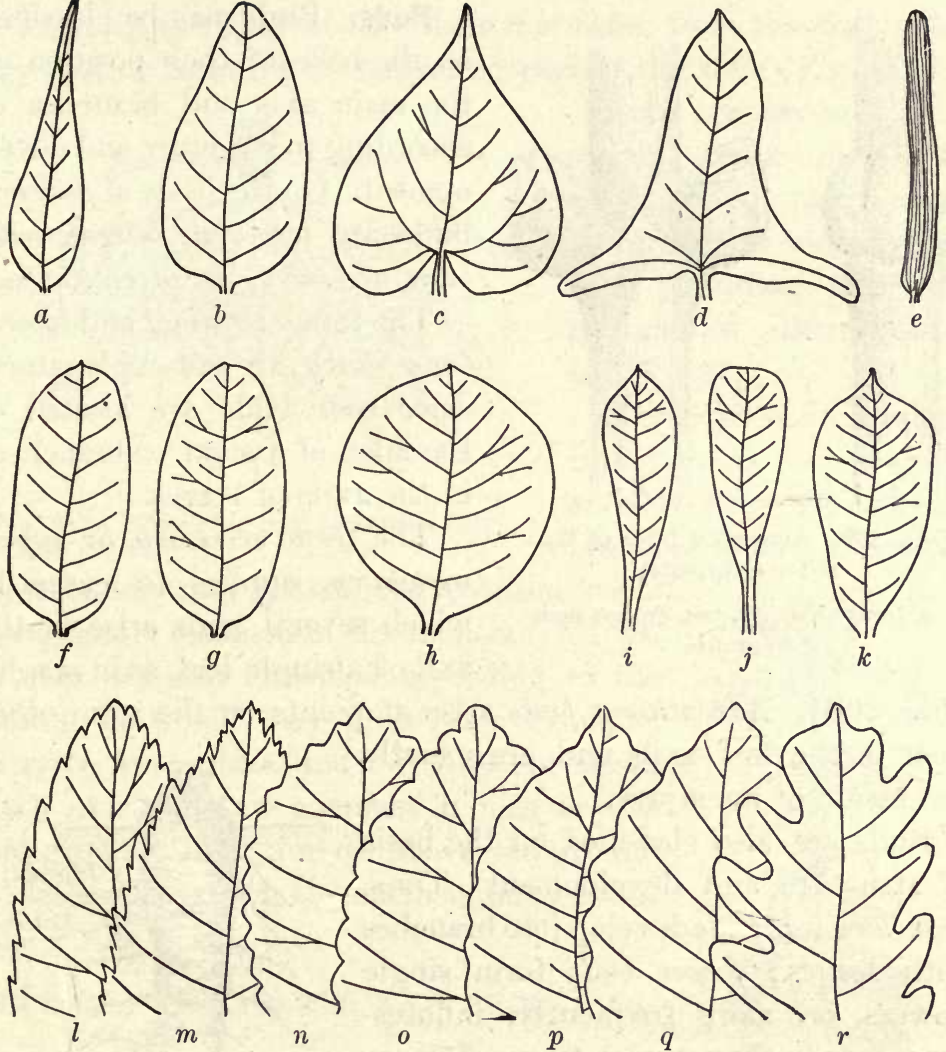

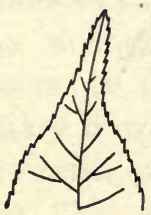

$s$

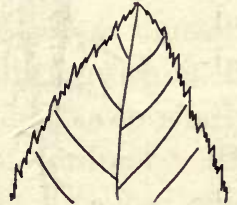

$t$

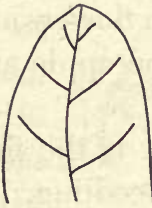

$u$

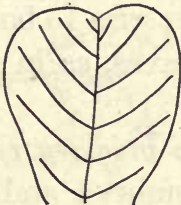

$v$

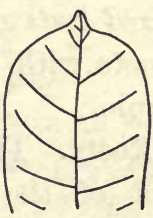

$w$

Fig. 208. Diagrams illustrating differences in the form, venation, margin, and apex of leaves

$a-k$, form and venation of leaves : $a$, lanceolate pinnate ; $b$, ovate pinnate ; $c$, heartshaped palmate; $d$, halberd-shaped palmate; $e$, linear parallel; $f$, oblong pinnate; $g$, oval pinnate; $h$, orbicular pinnate; $i$, oblanceolate pinnate; $j$, spatulate pinnate; $k$, obovate pinnate. $l-r$, different kinds of leaf margins $: l$, serrate; $m$, double serrate; $n$, dentate ; $o$, crenate; $p$, undulate; $q$, sinuate ; $r$, lobed. $s-w$, different kinds of leaf apexes: $s$, acuminate; $t$, acute; $u$, obtuse ; $v$, emarginate ; $w$, mucronate 


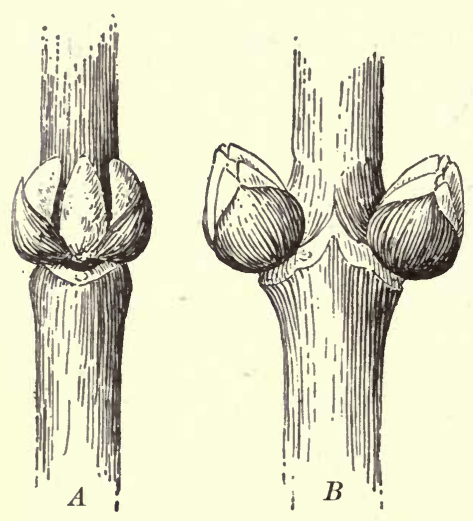

Fig. 209. Accessory buds of box elder (Negundo)

$A$, front view ; $B$, two groups seen in profile

Buds. Buds may be classified on the basis of their position on the main axis and branches or according to structure and development. On the basis of position, buds are terminal, lateral, axillary, accessory, or adventitious.

The terms terminal and lateral (or axillary) are self-explanatory, since such buds are located at the apex of a stem or branch or in the axils of leaves.

The term accessory, or supernumerary, applies to cases in which several buds arise in the axil of a single leaf, as in maples (Fig. 209). Adventitious buds arise at points on the stem other than in the leaf axils and are usually the result of an injury.

Buds are also classified on the basis of structure and development. Thus, branch or leaf buds develop into branches with leaves; flower buds form single flowers, or, more frequently, inflorescences, as in the common types of trees; mixed buds give rise to both flowers and shoots with leaves, as in the apple and maple.

Roots. The primary root is the first root of the embryo and seedling. In most dicotyledons the primary root forms a taproot or a single main root, with small lateral roots, as in the beet, carrot, and dandelion (Fig. 210). The primary root may be permanent or tem-

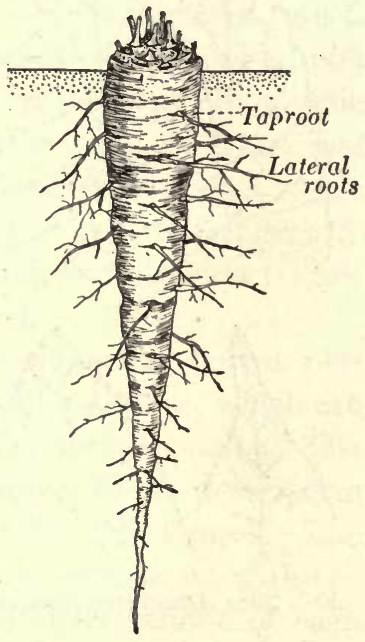

Frg. 210. Fleshy taproot of the carrot porary, being replaced in the latter instance by secondary roots which grow out from the lower part of the stem. 
Secondary root systems take their rise either from the main primary root or from the underground part of the stem (Fig. 211).

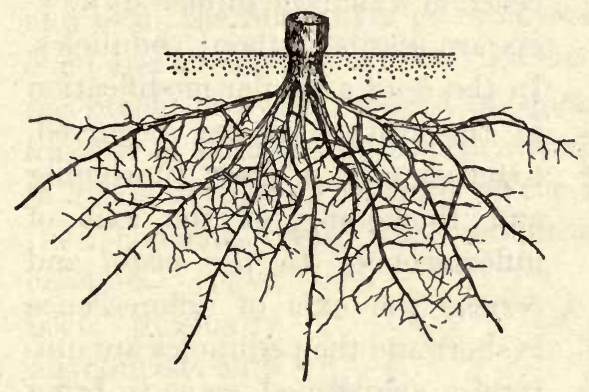

Fig. 211. Branching root system of corn

Both primary and secondary roots may be either fleshy or fibrous in form, and surface or deep, according to their method of distribution in the soil.

Adventitious roots arise from stems or leaves and are thus not lateral branches from a primary or a secondary root system. Adventitious roots are especially common in the monocotyledons.

Roots are also classified according to the medium in which they grow; for example, soil roots, water roots, and air roots (or aërial roots). Air roots are common in corn as prop roots, growing from a node above the ground and later penetrating the earth as brace roots.

\section{REPRODUCTIVE PARTS AND PROCESSES}

Inflorescence. The inflorescence has already

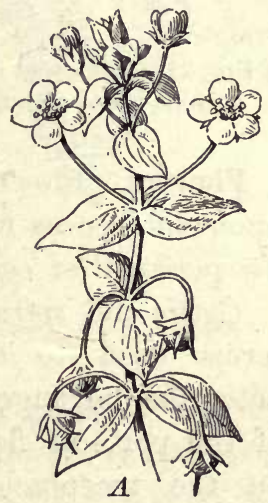
been defined as a cluster of flowers, to distinguish it from solitary flowers borne singly in the axils of ordinary leaves (Fig. 212, A). Some of the main types of inflorescence are indicated in Fig. 213. In this figure the flowers are indicated by circles at the ends of the stems, or peduncles, which arise

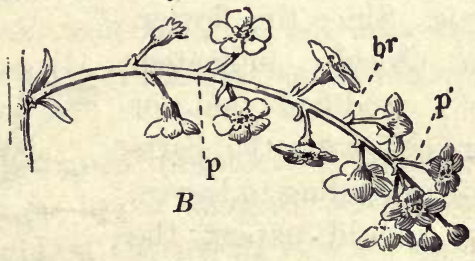

Fig. 212. Solitary flowers and cluster $A$, axillary and solitary flowers of the pimpernel; $B$, raceme of red currant; $p$, axis of inflorescence; $p^{\prime}$, peduncle; $b r$, bract from the axils of bracts. All of the main types of inflorescence can be derived from the raceme if we suppose certain modifications to 
have arisen in the raceme type during the development of other kinds of inflorescence. Thus, the spike is a racemelike inflores-
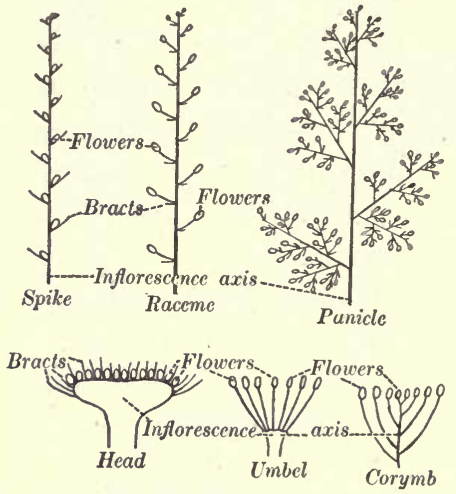

Fig. 213. Diagram illustrating various types of inflorescence cence in which the numerous flowers are sessile, without peduncles. In the head a similar modification in the peduncles has occurred, together with a great shortening and thickening of the axis of inflorescence. In the umbel and corymb the axis of inflorescence is short and the peduncles are uniformly lengthened, so as to bring the flowers at the same level, making a flat-topped inflorescence. Other types of inflorescence represent similar modifications from the simple racemose type.

Flowers. Flowers have various terms applied to them, based upon differences in form, structure, the presence or absence of the perianth or essential organs, and their spiral or cyclic plan.

Cyclic and spiral are terms used to designate the arrangement of the parts of flowers on the receptacle, or axis. Since the flower, as already indicated, is a modified bud, or branch, with the parts corresponding to leaves, we should expect the same cyclic or spiral arrangements of its floral parts on the receptacle
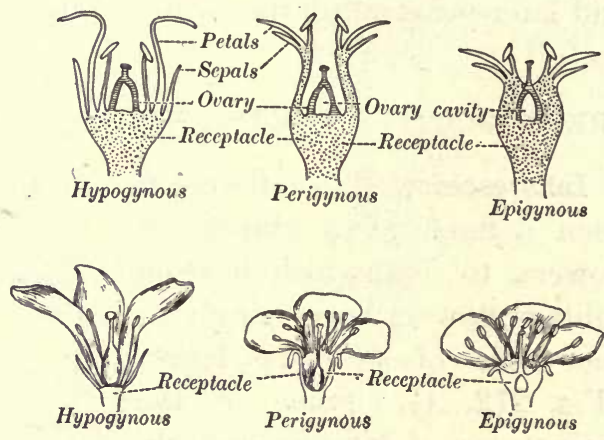

Fig. 214. Hypogynous, perigynous, and epìgynous flowers

Diagrams above; median sections of flower below as we have already found in the leaves on the stem axis. In our discussion of the flower of the marigold it was indicated that such flowers, with spirally arranged parts, represented the 
simplest floral types, comparable to the strobilus of some of the lower orders of plants. In the cyclic flowers, like the mandrake and bean, the floral axis, or receptacle, has become greatly shortened and broadened, so that the floral parts are arranged on the receptacle in cycles instead of spirals as in the marigold. The number of floral parts in each whorl is correspondingly reduced in cyclic flowers on account of the shortening and flattening of the receptacle. With these modifications in the receptacle other changes have been gradually introduced with the evolution of flowers, giving rise to what are known as perigynous and epigynous flowers, as distinguished from the more simply arranged hypogynous flowers (Fig. 214).

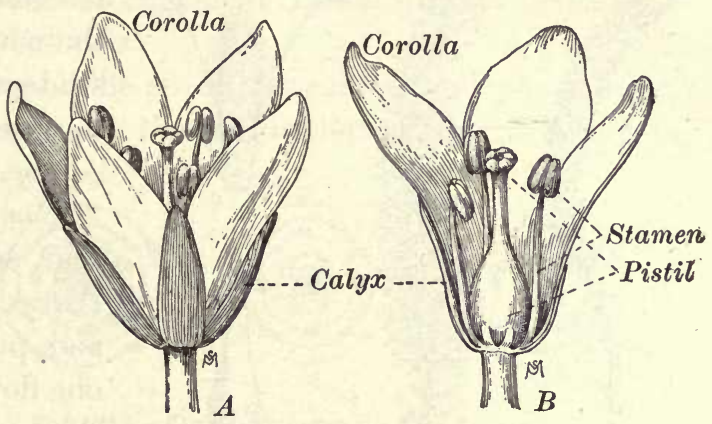

Fig. 215. Complete flower of the alpine azalea (Loiseleuria)

$A$, exterior view ; $B$, sectional view. After H. Müller

Hypogynous flowers have the parts of the perianth and the two sets of essential organs (stamens and pistils) arranged separately on the receptacle. The spiral flowers of the marigold and the cyclic flowers of the mandrake and bean are common illustrations of hypogynous flowers with cyclic and spiral arrangements of their floral parts.

Perigynous flowers have a cup-shaped receptacle, which bears the perianth and the stamens on its upper margin. These parts thus appear to surround and inclose the pistil, which remains free in the center of the flower. Flowers of the cherry and of the common wild rose are familiar illustrations of perigynous flowers.

Epigynous flowers are common in the apple and in many flowers of the Compositoe, to which the yarrow and dandelion belong. In such flowers the receptacle is cup-shaped, as in perigynous flowers, but yet forms a part of the ovary cavity, which 
is roofed over by the sporophyll or sporophylls of the pistil proper. The remaining parts of the epigynous flower seem to arise from the upper margin of the ovary, surrounding the style and stigma.

Flowers are also variously classified on the basis of the presence or absence of certain floral parts and of the form of floral parts, and also on the basis of certain arrangements for insuring

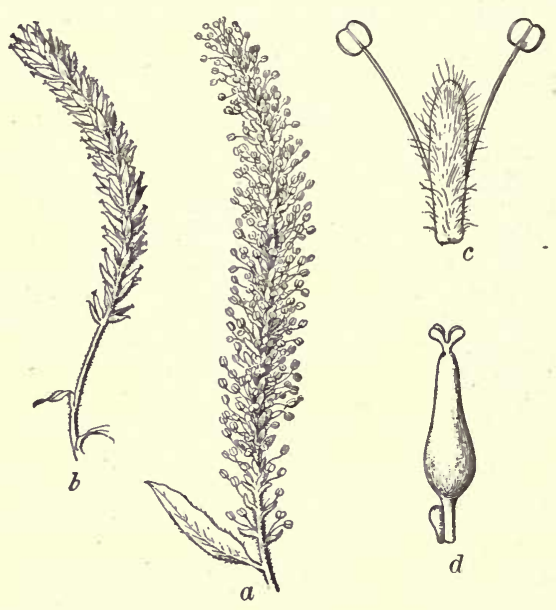

FIg. 216. Catkins and diœcious flowers of a willow (Salix alba)

$a$, staminate catkin ; $b$, pistillate catkin ; $c$, staminate flower; $d$, pistillate flower. From Bergen and Caldwell's "Practical Botany"

of essential organs and are thus either wholly staminate or wholly pistillate. Plants which bear imperfect flowers are said to be either monocious or dicecious, according as they bear imperfect flowers of both kinds on the same or on different plants. Thus, the willows (Fig. 216) are imperfect and diøecious, since they have only staminate flowers on one tree and only pistillate flowers on another tree of the same species. The oak, on the contrary, is imperfect and monœecious, with both staminate and pistillate flowers on the same tree (Fig. 238).

Regular flowers have all of the parts of one set of organs alike in form, as in the azalea, while irregular flowers have the 
parts of one or more sets of organs irregular in form, as in the flowers of the pea, bean, and locust (Fig. 89).

Floral plan. In a floral plan (Fig. 217) the parts of the flower are represented in transverse section as though reduced to a common plane. In dicotyledons the parts are usually in fours or fives, while in monocotyledons they are on the plan of three. It will be noticed also that the parts of each set of floral organs alternate with those adjacent to them, like the leaves on a leafy shoot. This alternate arrangement is another evidence that - flowers are modified shoots.

Pollination features. The special structural and physiological phenomena concerned with the pollination of flowers relate for
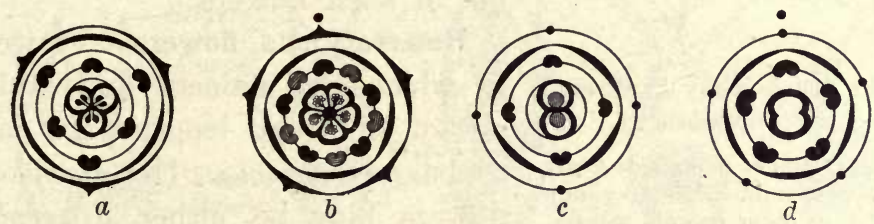

Fig. 217. Floral diagrams

$a$, lily family; $b$, heath family; $c$, madder family; $d$, composite family. The dot above $b$ and $d$ indicate the stem axis; the sepals are represented with midribs; the

lighter stamens in $b$ represent an alternate whorl of stamens. After Sachs

the most part to devices for securing cross-pollination and close-pollination. The more important of these structural and physiological phenomena are the following:

Anemophilous and entomophilous. The principal agents by which flowers are close-pollinated or cross-pollinated are the wind and insects. Flowers in which the pollen is carried to the stigma by the wind, as in corn, poplar, and oaks, are said to be anemophilous, or " wind-loving," while insect-pollinated flowers, like the locust and bean, are said to be entomophilous, or "insectloving." Anemophilous flowers are usually characterized by inconspicuous color, abundance of light pollen, and lack of odor.

Dichogamy. In many perfect flowers the stamens and stigma mature at different dates in the same flower, - a condition defined by the term dichogamy (Fig. 218). If the stamens ripen earlier than the stigmas in such flowers, the flowers are said to 
be protandrous, while flowers in which the stigmas ripen before the anthers are said to be protogynous. It is evident that protan-

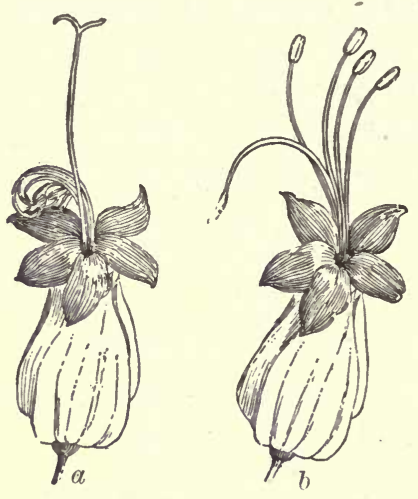

Fig. 218. Dichogamy in flowers of Clerodendron

$a$, the pistil is bent to one side away from the ripe stamens in the young flower; $b$, in older flowers the stamens wither and the stigmas are exposed for the reception of pollen drous and protogynous flowers are necessarily either close-pollinating or cross-pollinating. Homogamous flowers are flowers in which the stamens and pistils ripen together, thus making self-pollination possible.

It is evident that where flowers are imperfect either close-pollination or cross-pollination is insured, since self-pollination would be impossible in such flowers.

Heterostylous flowers. Flowers in which the stamens and styles are of different lengths are said to be heterostylous. Heterostylous flowers may be either dimorphic (Fig. 219) (with two lengths of stamens and pistils) or trimorphic (with three lengths of stamens and pistils). In either case each set of stamens matches one length of pistils, so that insects carry pollen from the anthers of one flower to stigmas of the same height in other flowers on the same or on a different plant, thus effecting either a close-pollination or a cross-pollination.

Odor, nectar, color, and movements. In flowers adapted to insect pollination, or

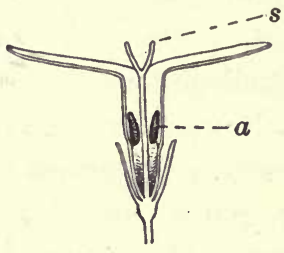

$A$

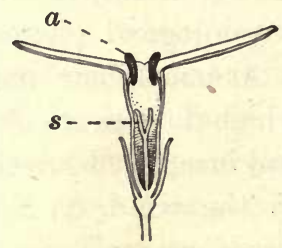

$B$

FIG. 219. Dimorphic stamens and pistils in bluets $A$, form with long style; $B$, form with short style; $a$, anthers; $s$, stamens entomophily, the insects are undoubtedly attracted to many flowers by their odor, nectar, or color, a fact which insures close-pollination and cross-pollination. The odor is due to the 
secretion of volatile oils by the petals or other floral parts, while the nectar is secreted by nectar glands, usually located at the base of the pistils on the receptacle. In addition many flowers possess the power of movement in the stamens and pistils by which the anthers and stigmas are either separated or approximated when ripe, thus insuring either close-pollination, cross-pollination, or self-pollination.

Pistils, seeds, and fruits. Pistils are either simple or compound, according as they are composed of one or more sporophylls, or carpels. Simple pistils are composed of one carpel,

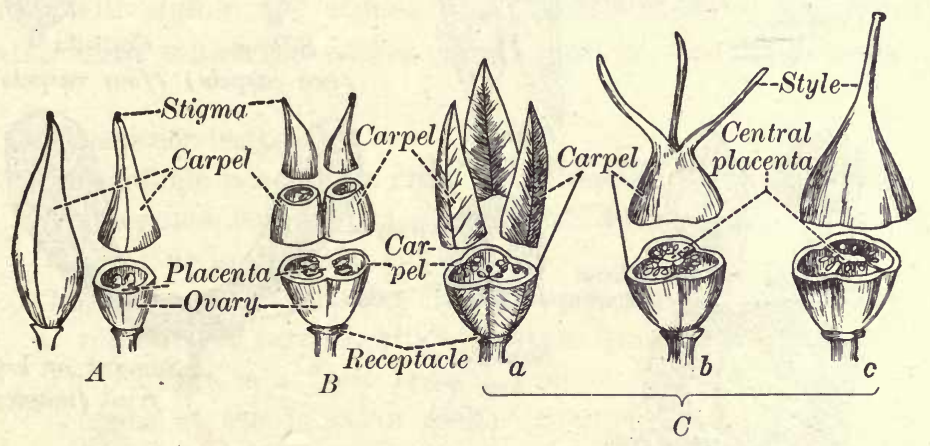

Fig. 220. Simple and compound pistils

$A$, simple pistil with one carpel; $B$, compound pistil with two carpels and central placenta; $C$, compound pistils ( $a$, with parietal placentæ; $b$, $c$, with central placenta)

or megasporophyll, as in the mandrake, bean, and locust (Fig. 220, A). Compound pistils are composed of two or more carpels, or sporophylls, so united as to inclose one or more seed cavities, or locules (B). The placentoe, or lines of attachment of the ovules, may be either central or parietal $(C)$.

Ovules are of three main types, according to their form and the relation of the ovule proper to the funiculus. Orthotropous, or straight, ovules grow straight, without curvature, from the funiculus, or stalk. Campylotropous ovules are curved, owing to the greater growth of one side of the ovule during its development, as in Capsella (Fig. 203, C). Anatropous ovules are the most common type, in which the ovule becomes completely inverted during its early development and adheres to 
the funiculus throughout its entire length. The ridgelike junction of the ovule and the funiculus is called the raphe.

Fruits are usually formed as a result of fertilization, and consist of the ripened ovary or of the ovary and the receptacle,

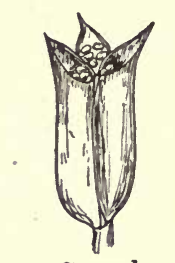

Capsule (three carpels)
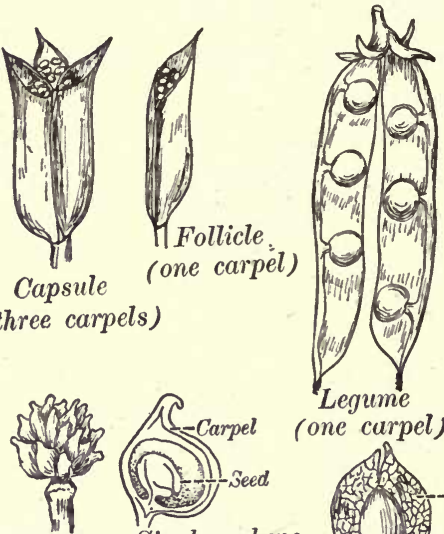

Achenes Single achene
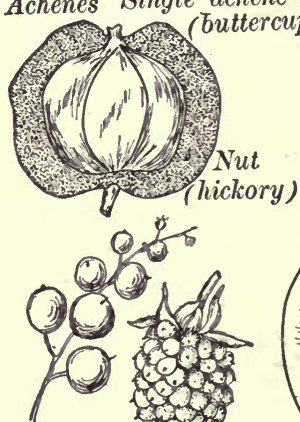

Berry

(Smilacina) Aggregate

(mulberry)

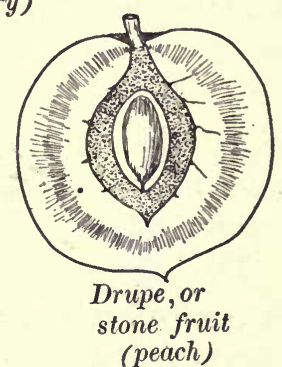

Samara, or

key fruit (elm)
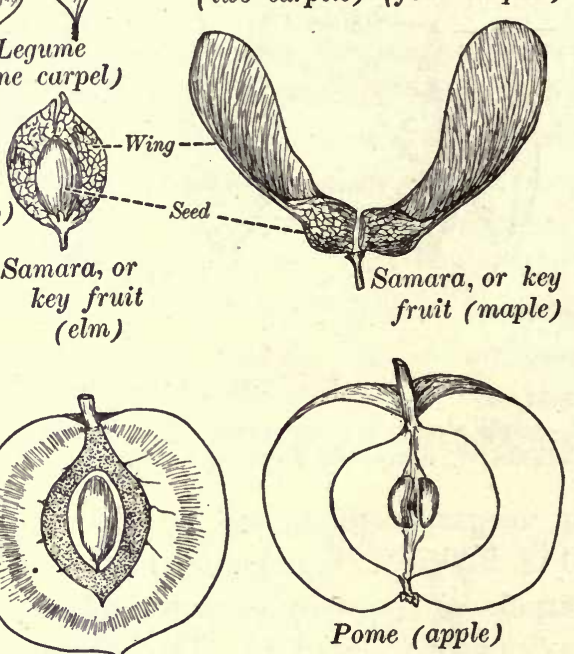

Pome (apple)

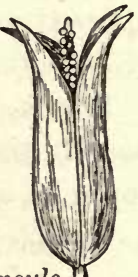

Capsule (two carpels) (four carpels)

Fig. 221. Different kinds of fruits

Upper row, dry dehiscent fruits ; middle row, dry indehiscent fruits; lower row, fleshy fruits

as in the pome, drupe, and aggregate fruits (Fig. 221). They are variously classified on the basis of their form and structure. In the following classification the terms dry and fleshy indicate whether the ovary (or the ovary and receptacle combined) 
becomes dry and hard in ripening, like the fruit of the pea and buttercup, or soft and fleshy, as in the apple, pear, etc.

Dehiscent fruits, upon ripening, split open along the junction of the carpel or carpels, namely, along the placenta (septicidal dehiscence) or along the back of the carpels between the placental junction line (loculicidal dehiscence).

Indehiscent fruits remain closed, as in the cereal grains and strawberry, where the entire ovary and inclosed ovule is shed and disseminated together.

The following classification and the accompanying illustrations will enable the student to classify most of the common fruits with which he comes in contact in the field.

\section{Dry dehiscent fruits :}

The follicle is a simple fruit which dehisces along one side.

The legume, or pod, is a simple fruit which dehisces along two sides, as in the bean and pea.

The silique is a fruit, like that of mustard, composed of two sporophylls, or carpels, which separate from the central partition.

The capsule is a fruit formed from a compound ovary which opens at the junction of the sporophylls or between these junction points.

\section{Dry indehiscent fruits :}

The achene is a simple dry fruit in which the single seed is free from the ovary wall, as in the buttercup.

The caryopsis, or grain, is the fruit of the grasses and cereals in which the ovary wall adheres to the seed.

The nut is a fruit in which the ovary wall becomes the indurated resistant wall of the fruit.

The samara, or key fruit, like that of the maple and ash, is a fruit furnished with a winglike outgrowth of the ovary wall.

Fleshy fruits, simple or compound :

In the berry the ovary wall becomes fleshy and incloses one or more seeds.

In the pome the ovary wall is fleshy but with an indurated central part inclosing the seeds, as in the core of the apple.

Drupes are stone fruits, like the cherry and plum.

Aggregate fruits, like the blackberry, have several simple stone fruits aggregated or massed together on one receptacle. 


\title{
CHAPTER XIX \\ TREES, SHRUBS, AND FORESTS
}

\author{
IMPORTANCE AND USE
}

Ornament and protection. The ornamental and protective function of trees is so well known that very little can be said to emphasize this aspect of their importance to man. The shade trees of our cities and towns, the great beauty of trees and shrubs on private lawns and in public parks, the picturesqueness of the mountains and of the open country with wooded hills and streams, all attest to the value of trees and shrubs and to the need of an adequate knowledge of their habits and uses.

These facts are more evident if one travels from the wooded regions of the East or the Far West across the Western prairies, where little protection is offered against wind, sun, and storm except where early settlers have established windbreaks by setting out trees or where streams are bordered by protective stands of timber. While isolated trees, or trees in small groups, are thus contributory to man's pleasure and comfort, it is to trees aggregated in forests that one must turn in order to understand the great importance of tree life to the industrial life of men and to the progress of civilization.

The national forests. The national forests of the United States formerly occupied an area of $850,000,000$ acres, which has been reduced at the present time to about $545,000,000$ acres. This vast forest domain includes the northern coniferous forests of the Great Lakes and the mixed coniferous and hardwood forests of the New England States; the great southern forests, composed largely of pines; the central, sparsely covered forest of hard woods; and the Rocky Mountain and Coast Range forests of the extreme western states (Fig. 223). 
The great value of trees in this forest domain is enhanced by the fact that a large part of it is in the mountains and in regions like the pine barrens of the Southern states, where the land is not of value for agriculture on account of the unproductiveness of the soil in those regions. The trees thus render an otherwise unfruitful region productive, and serve at the same time as a protection against floods, erosion, and drought by their control of rainfall and other climatic factors.

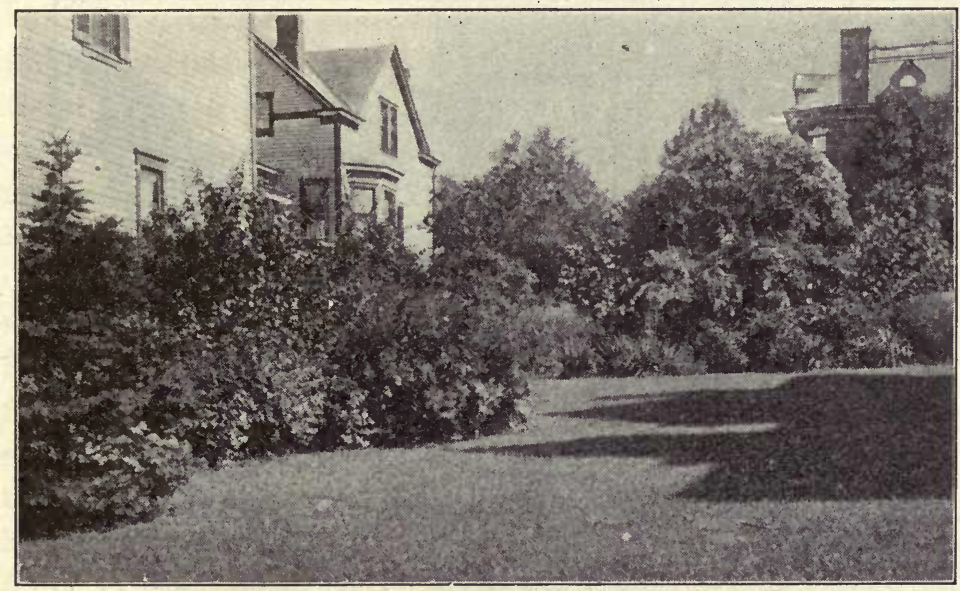

Fig. 222. The ornamental funetion of trees and shrubs

Photograph furnished by the United States Forest Service

Climate and water supply. The factors of climate which are controlled in any ineasure by forests are concerned largely with temperature, air movements, and water control. The effect on temperature is one which is felt only in the immediate vicinity of the forests themselves, and not over the country at large. It is a well-known fact that the leaves of trees in a forest absorb a large part of the heat which falls upon them, and that they utilize this heat in warming the leaves, in making sugar and starch, and in the evaporation of water vapor. The rich covering of humus on the forest floor also absorbs heat and protects the soil beneath from absorbing and radiating it as the soil in naked exposed regions would. As a consequence 
the forest has a general cooling effect on the air in its vicinity and so protects the soil from drying up. Air movements in the form of winds and storms are also restricted by forests, which therefore serve as effective windbreaks and at the same time affect the temperature of a region in both winter and summer. The early settlers in the West soon learned this advantage of trees as windbreaks and planted cottonwoods and other quickgrowing trees on the north and west sides of their holdings.

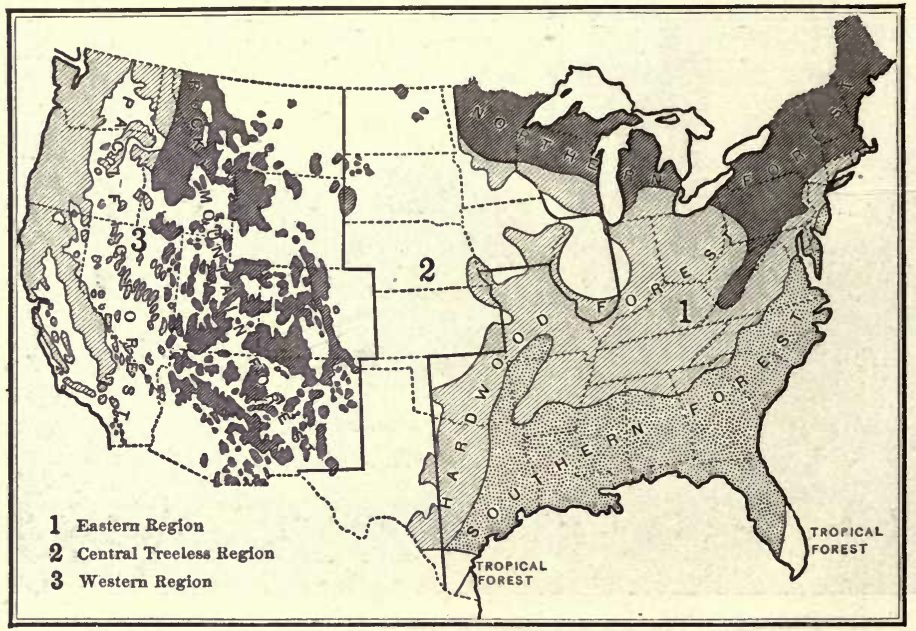

FIG. 223. General map of the forest areas of the United States

In addition to the effect on the relative humidity and temperature of a region the forests have an important function in the control of water falling in the form of rain or snow.

Forest control of rainfall and floods. Rainfall is supposed by some to be increased by the presence of great forests, and the investigations of European foresters would seem to bear out this assumption. Other data, however, gathered with equal care by experienced scientific foresters, yield opposite results, and it is doubtful whether the forests have any marked effect on precipitation.

Floods and erosion, or the wearing away of soil by water, are so largely controlled by forests that this control is now 
reckoned among the most beneficent and important effects of the forest cover. When rain falls over a dense forest, from one tenth to one fourth of it is caught by the crowns of the trees, while the forest floor of humus soil and roots holds the remainder. It is estimated that a forest floor " can hold for a while a rainfall of five inches." This water is then gradually evaporated or is slowly drained off into streams, lakes, and the sources of springs. Mountain. streams, which irrigate fertile valleys, are thus fed and sustained at their source. In like manner the supply of water for the great irrigation systems of the West, and for the water supplies of large cities like Denver, Los Angeles, and San Francisco, comes from mountain springs, lakes, and streams, which are protected at their source by forests.

Rain which falls upon unforested soil has a very different effect from that

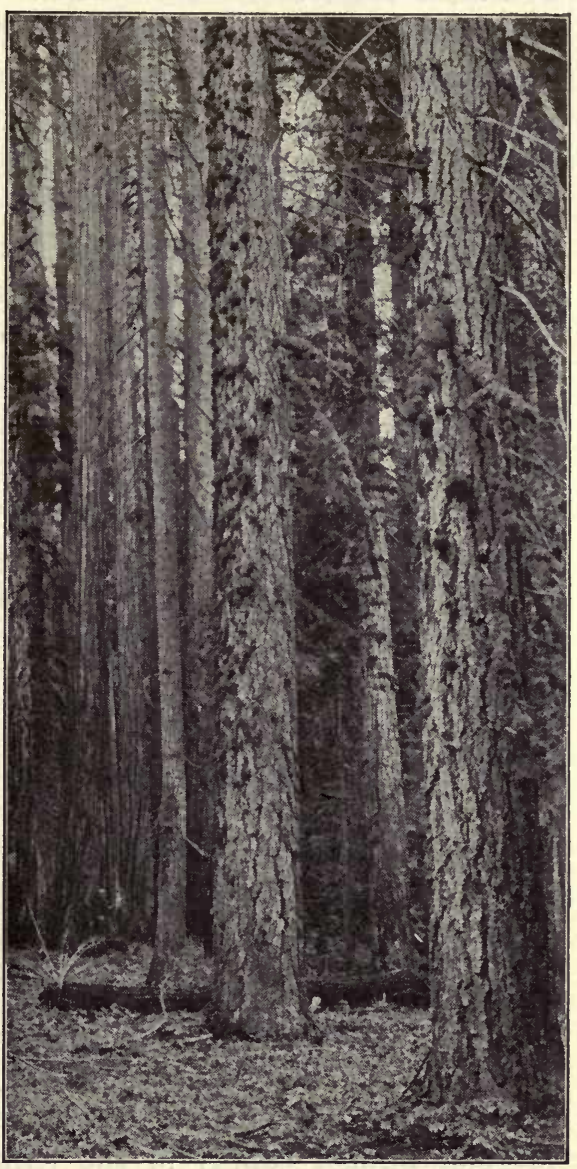

FIG. 224. Pacific-coast forest of Douglas fir and western red cedar, Tacoma, Washington

Photograph furnished by the United States Forest Service outlined above, especially in mountainous and hilly regions, such as those occupied by most of our national forests. In a region denuded of forests the rain falls directly upon the soil, 
which is apt to be beaten into a hard surface layer or, if soft and porous, to become quickly saturated and give way. The result is almost certain to be a disastrous flood and often immense damage caused by greatly swollen streams. In the Adirondacks and in California great damage has already been done in this way where the forests have been wholly or partially cut off or injured by grazing. It is estimated that " upward of two hundred square miles in the United States is annually laid waste by erosion" and that much of this great waste could be prevented by protecting or replanting the forests in the eroded regions. When swollen mountain streams reach the valleys at the foot of the mountains, they flood them and at the same time deposit sand, gravel, and even large bowlders on once fertile and productive soil. Not only must the cutting of forests on mountain slopes be carefully regulated, therefore, but denuded areas need to be systematically reforested by governments, either state or national, which possess resources adequate for such great tasks.

Forest products. A great variety of forest products are derived from the national forests, including turpentine, tar, formaldehyde, and rosin, in addition to the more important wood pulp, timber, and lumber supplies. The lumber and timber are used for various purposes in the industries and the home. These uses include firewood, lumber for construction and building, cooperage, and veneers, and timber for the making of excelsior and wood pulp, railroad ties, and telephone and telegraph poles. For these various purposes it is estimated that "we take from our forests yearly, including waste in logging and manufacture, more than 22,000,000,000 cubic feet of wood, valued at $\$ 1,375,000,000$." It is also estimated that almost half of the original lumber supply of the United States has already been used and that "the present rate of cutting for all purposes exceeds the annual growth of the forests."

The remedy. The obvious remedy for this condition is the scientific control of timber cutting and the replanting of the forests, already in process of depletion, by the state and national governments. It is therefore of the greatest importance that the United States government has adopted the policy of caring for 
and extending its control over an ever-increasing forest area. The extent to which this policy is being carried out by our national government is indicated by the following data. "On June 30, 1917, there were 147 national forests with a total of $155,166,619$ acres," yielding an annual income of $\$ 3,500,000$. On the above date the government employed in this work between three and four thousand men, including forest supervisors and rangers, lumbermen, scalers, planters, and clerks. These various officers are distributed to the different national forests in the proportion necessitated by the labor to be performed. They have a great variety of work, including the prevention and control of forest fires, the scientific cutting and marketing of timber, the control of grazing privileges, and the replanting of depleted forests. This mere enumeration of the extent, use, and control of the national forests of the United States is all that can be attempted in an elementary textbook of botany, but every student should acquaint himself with this great industry of our national government, which means so much to the present and future prosperity of our country.

\section{REPRESENTATIVE GROUPS OF FOREST TREES}

The trees which comprise the forests of the United States belong to the gymnosperms, or naked-seeded plants, represented by the pines and the spruces, and to the angiosperms with a closed pistil, represented by the elm, oak, and maple. In the following species, selected from these two great tree groups, both the economic and the biological features will be considered as concrete illustrations of the importance and interest attached to forest and ornamental trees.

\section{GYMNOSPERMS (EVERGREENS)}

\section{The Spruces (PICEA)}

Habitat and habit. The spruces form an important part of the great coniferous (cone-bearing) and mixed forests of the northeastern portion of the United States, the Appalachian region, the 
Rockies, and the North Pacific coast. The red, white, and black spruces are found mainly in the northeastern and Appalachian forests ; the Engelmann spruce has its home in the Rocky Mountains; while the Sitka spruce, so important in the construction of aëroplanes in the World War, is found exclusively on the

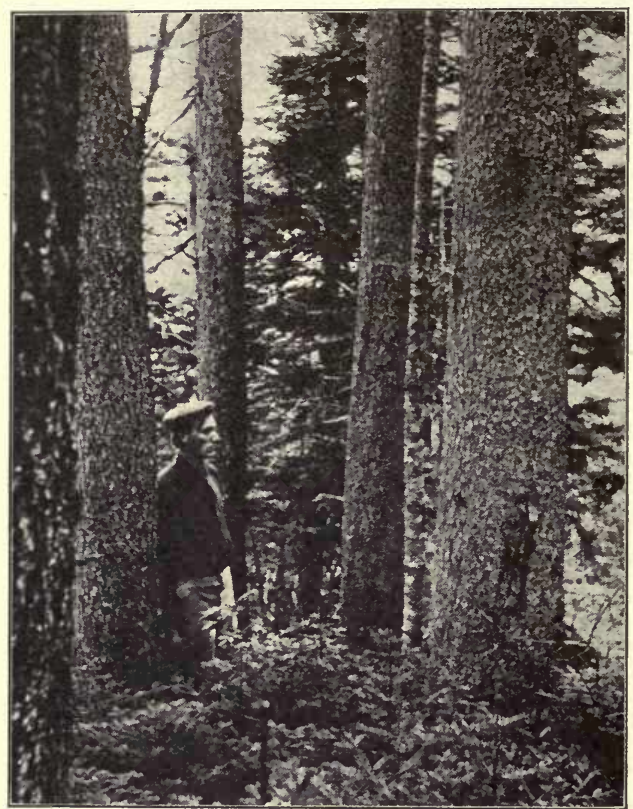

Fig. 225. Virgin stand of red spruce with reproduction of tolerant spruce and fir in the White Mountains, New Hampshire

Photograph by the United States Forest Service Pacific coast. The habitat of the spruces is thus confined largely to well-drained uplands or to mountain slopes. Like many other plants, spruces are often found to be occupying situations to which they are not perfectly adapted, including marshes and swamps, on account of the lack of competition in these habitats with the more highly organized hard woods, such as the oak and maple.

Tolerance. An important factor in the distribution and success of the spruces is due to the light requirement of the different species, especially in the younger stages of growth. They belong to the so-called tolerant trees, which have the power to grow, while young, in the shade of other trees (Fig. 225). "Having once gained a foothold in a selection forest, the young spruce grips life tenaciously, struggles along for many years under the shade of the forest, and gradually forces its way upward as natural thinning reduces the number of its overtopping competitors." Balsam is often found 
with spruce on the forest floor (Fig. 226), since it too is a tolerant species, growing in the shade of the other forest trees. Balsam is, however, the stronger competitor of the two in such situations, on account of its more plentiful seeds and rapid growth.

Seed production. The distribution of forest trees, and their power to reproduce a forest once destroyed, is determined largely by the number of seeds produced and by the viability of the seeds, or their power to germinate and grow under the conditions presented in a given habitat. Spruces, like other cone-bearing gymnosperms, begin to produce large quantities of winged seeds when the trees have reached the proper age. The seed-producing stage has been found to vary in the spruce with the conditions under which it lives. In the forest it begins

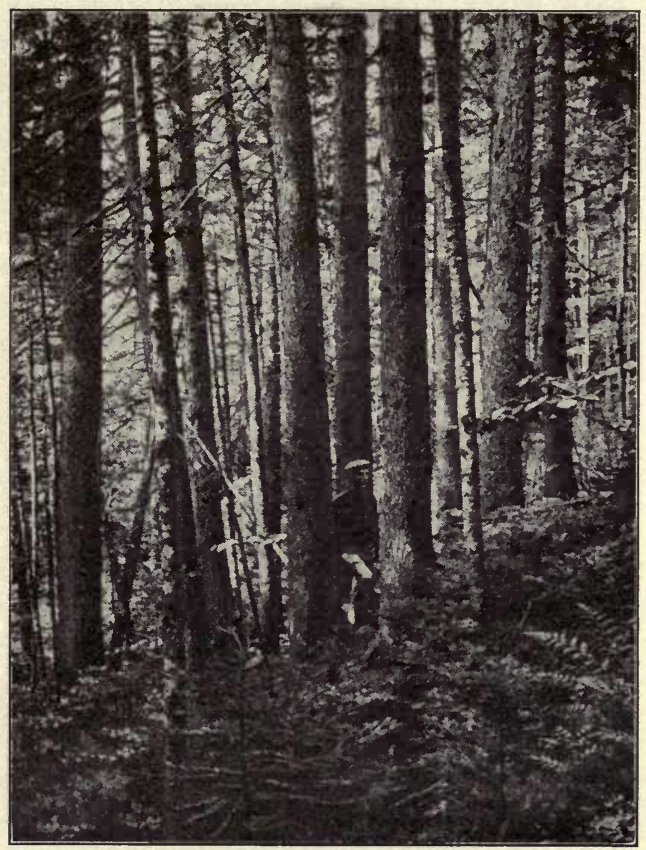

Fig. 226. Balsam, a tolerant tree, growing beneath a virgin stand of red spruce in the White Mountains Photograph by the United States Forest Service to bear when the crown succeeds in reaching the light, which may be at the age of twenty or thirty years or may be delayed until the tree is one hundred years old. "In the open, and under favorable soil conditions, seed production begins as early as the fifteenth or twentieth year, and heavy crops follow by the thirtieth or thirty-fifth year." The seeds mature in late September and germinate in the same fall or the next spring, producing in good soil a new stand of spruce. 
Maintaining the supply. Maintaining the supply of spruce for wood-pulp production and other commercial purposes is closely connected with the amount and nature of the seed production, since spruce forests are recreated or regenerated largely by means of seeds. In the case of hardwood trees, to be discussed below, vegetative reproduction by means of sprouts from the stumps is often used in the regeneration of a forest destroyed

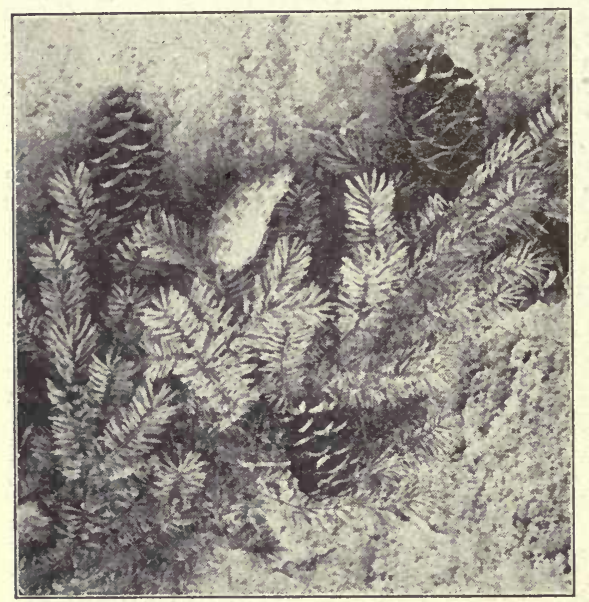

Fig. 227. Ripe cones of big-cone spruce in the Cleveland National Forest, California

Photograph by the United States Forest Service

by cutting or by other agencies. In the case of most of the coniferous trees the sprout method is not possible, since, with few exceptions, these trees do not reproduce vegetatively in this manner. The production of new stands of spruce by means of seeds may be either by the natural method, where the growth of seedlings occurs in a spruce or a mixed forest (Fig. 229), or by artificial sowing of seed. Where seedlings are to be grown by the natural method, care must be taken to eut out enough of the standing timber to facilitate the growth of the spruce at each stage of its development. In time, most or all of the larger trees of such an area will need to be cut, to allow the new spruce forest to develop normally, with plenty of soil space and light exposure. In other instances clear spaces are cut in the forest, with bordering mother spruce trees, from which the seed will be distributed and sown naturally over the cleared ground. In such cases the surrounding trees, if the clearing is not too large, protect the ground from drying and furnish partial protection to the growing seedlings. These sheltering trees must be allowed to stand until the young growth can bear direct exposure. 
Seeding with spruce seeds may also be done artificially by scattering seeds on soil denuded of forest trees or by sowing the seeds in prepared seed beds (Figs. 230 and 231). In this case the seedlings, when they have reached the desired age, must be transplanted to the forest area where the new forest is to be grown. This method is, on the whole, the best and will probably be more largely employed in the future than in the past by the state and national governments. Valuable species for this purpose are the white spruce (Picea canadensis) and the Norway spruce (Picea abies), while the red spruce (Picea rubra) is more difficult to manage on account of its slow growth in early life.

Commercial importance. The wood of the spruce, like that of the pines and of the other cone-bearing trees, belongs to the class called softwood,

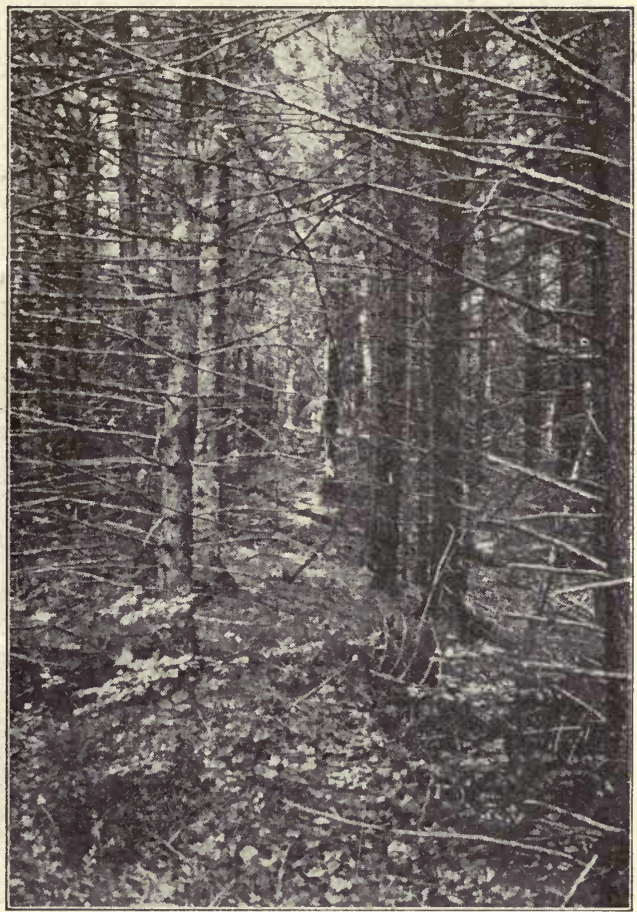

Fig. 228. Reproduction of spruce (second growth) in New Hampshire

Photograph by the United States Forest Service to distinguish it from that of the broad-leaved hardwood trees like the oak, maple, hickory, and poplar. The term is a purely conventional one, since many kinds of soft woods are harder and more durable than some of the so-called hard woods, like poplar, basswood, and willow. The real characteristic of spruce and other coniferous woods which gives them their value and distinguishes them from the wood of broad-leaved species is the 
character of the wood elements which make up the bulk of the wood. The student will recall that in spruce wood (Fig. 193) the water-carrying elements were the one-celled, thick-walled tracheids instead of the wide ducts of the alder and other hard woods. The small diameter of these tracheids, their thick walls, and their uniform size throughout the tree trunk make the even, fine-grained wood of the spruces and other conifers, like the white pine, extremely valuable in the industries.

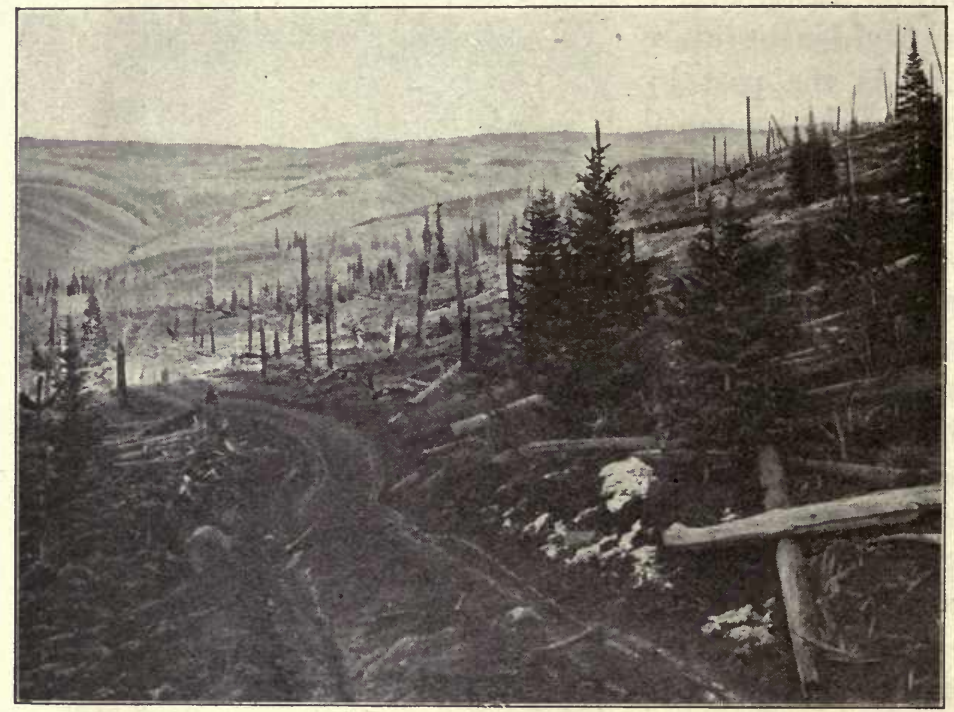

Fig. 229. Regrowth of aspen and spruce on a burned area in the San Francisco Mountains, Arizona

Photograph furnished by the United States Forest Service

The long, fibrous character of these tracheids and their close union with each other also contribute to their great value in the wood-pulp industry and in the making of aëroplanes from the now famous Sitka spruce. "Spruce is an aristocrat among woods. Its outstanding characteristics are combined elasticity and the ability to withstand sudden strain and shock."

The most extensive use of spruce at the present time is in the making of wood pulp for newspaper stock. Something 


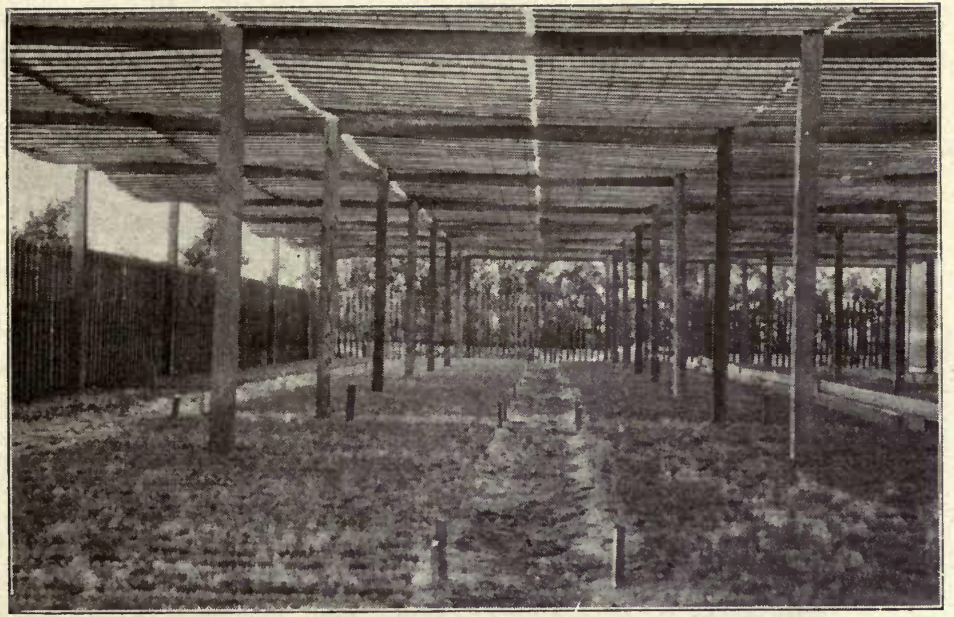

FIg. 230. State nursery at Saranac, Adirondack Mountains, New York

Two-year-old seedlings of yellow pine in the foreground. Photograph furnished by the United States Forest Service

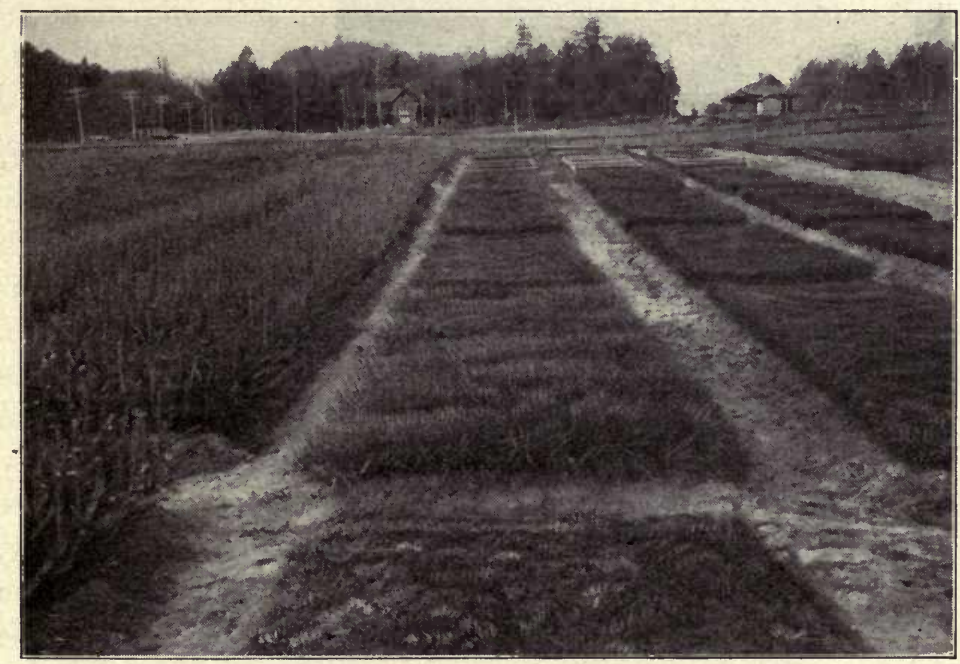

Fig. 231. Nursery of Austrian and yellow pine in the Kansas National Forest Austrian pine on the right, yellow pine on the left. Photograph furnished by the United States Forest Service 
over four million cords of wood is used for this purpose annually, of which about 60 per cent has been spruce wood. Red spruce has been the principal contributor to this great enterprise, but other woods are now being used on account of the depletion of the American and Canadian forests in the trees of this species. Spruce wood is also widely used in slack cooperage and in building and interior finishing. 'The great value of

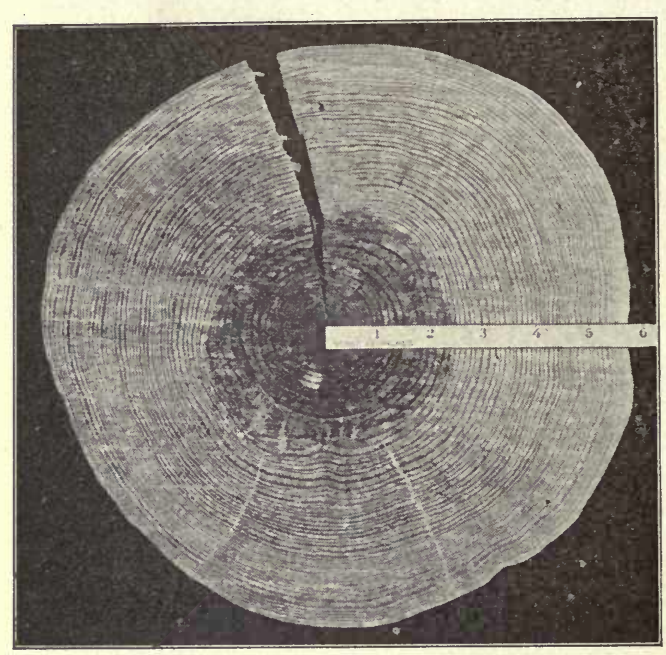

Fig.232. Transverse section of tree trunk of long-leaf pine, showing annual rings, heartwood, and sapwood Photograph furnished by the United States Forest Service spruce wood in the industries has stimulated the government to investigate new methods for its preservation and regeneration in the forests, which will undoubtedly result in preserving these valuable trees to future generations.

The Pines (PINUs)

Habitat and habit. The pines originally occurred in both mixed and pure forests in the northern, southern, and western national forests. The great stand of pure pine in the northern forests in the Great Lakes region has been almost wholly depleted, however, so that the southern forest of long-leaf and short-leaf pine, loblolly pine, and cypress is one of the principal sources of pine lumber to-day. This is also the great seat of the turpentine industry, which has exacted a. heavy toll on southern pines under the old wasteful system of tapping the trees for turpentine. The Western Rocky Mountain and Pacific Coast forests also supply pine lumber in large quantities from the western yellow, lodgepole, sugar, and white pines, which find their natural home in 
oaks, poplars, hickories, ash, willows, and other well-known species of broad-leaved trees. This hardwood forest of the central region differs from that of the coniferous forests in that it is not so continuous, being composed of smaller local forest stands or of groups of trees on farms, known as the farm wood lot.

Reproduction. The hard woods belong to the angiosperms, or true flowering plants, and are hence sharply distinguished from the cone-bearing gymnosperms. The flowers of the fruit bearers, such as the apples and plums, have already been discussed and are familiar features of these trees in the spring on account of their great beauty and fragrance. Many of the shade and timber trees, however, reproduce by means of very simple flowers which are rarely known to anyone except the student of botany. In some cases, as in the oaks (Fig. 238), these simple flowers are thought by many botanists to indicate a very early ancestry, even antedating that of herbaceous species, while others regard the simplicity of the flowers as

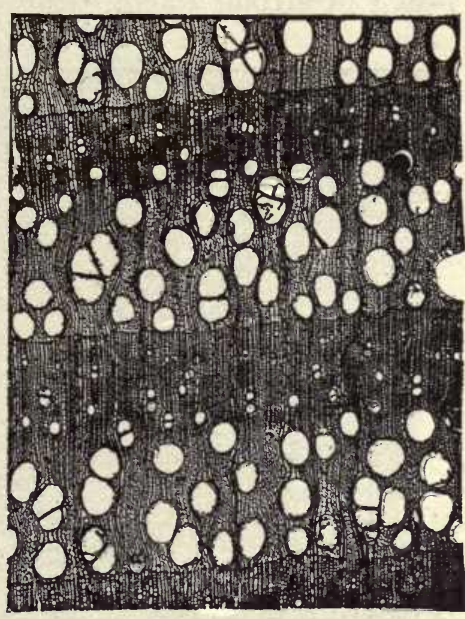

Fig. 234. Transverse section of the wood of sassafras, showing its ringporous character

Photomicrograph by R. B. Hough indicative of a reduced condition. In most of these cases pollination is anemophilous (by the wind) and the trees are either monœcious or diœcious.

Many trees produce winged fruits, which greatly facilitate their dissemination, as in the poplars, which so frequently reforest burned-over areas on mountain slopes. Other species, like the oaks and hickories, produce heavier nut fruits, which are not easily distributed and hence limit the range of these species.

Commercial importance. The commercial importance of the hardwood trees is determined by the character of the fruit and the wood. In the wild state the wood is the most important 
commercial product of these trees, and there is much greater structural variation in the hard woods than in the soft-wooded gymnosperms on account of the ducts and fibers in hard wood. The hard woods are all characterized by the possession of large water ducts, which render the wood more porous and make it less uniform in texture than is the case in spruce and pine. Between the pores, as we have already learned, the wood is composed of strong strengthening fibers and living cells, which are
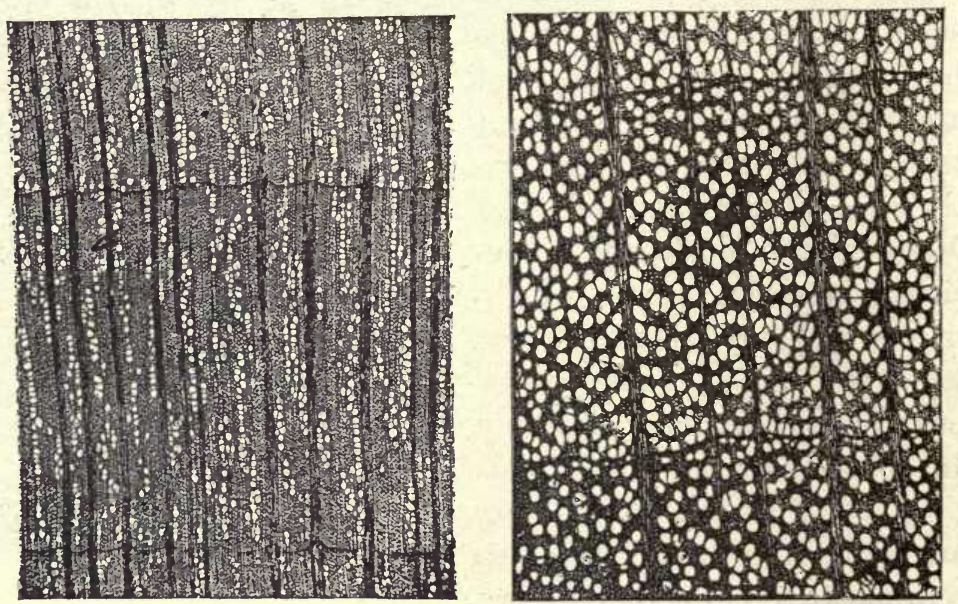

Fig. 235. Diffuse-porous woods of the sycamore and holly

Photomicrograph by R. B. Hough

usually more abundant in the summer wood than in the spring wood (Figs. 47 and 55). The large number of species of hardwood trees and the great variety in the character of the wood make this group of importance in supplying lumber and timbers for almost every commercial purpose. Two varieties of hard wood are recognized in the industries, namely, ring-porous wood and diffuseporous wood. Ring-porous wood, like that of the sassafras (Fig. 234), has the spring ducts in the early spring wood, while in diffuse-porous woods (Fig. 235) the ducts, or pores, are scattered throughout the entire wood ring. In ring-porous woods the annual rings are more distinct than in diffuse-porous woods. 


\section{THE WHITE OAK (QUERCUS ALBA)}

Habitat and habit. The white oak may be taken as a typical example of a hardwood forest tree as compared with the softwood coniferous trees represented by the spruces and pines. It

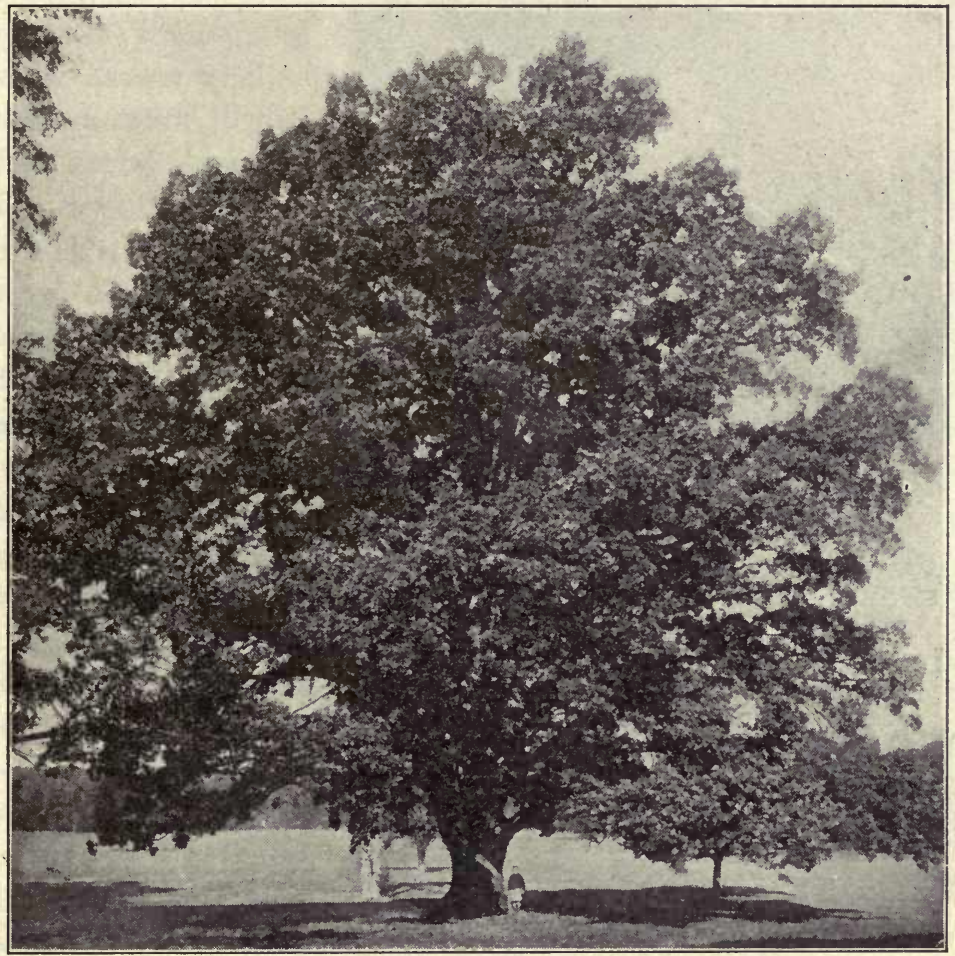

Frg. 236. Spreading habit of the white oak (Quercus alba)

Photograph furnished by the United States Forest Service

forms an important constituent of the great central hardwood forest, where it attains its best development " on the western slopes of the Allegheny mountains and in the central Mississippi and lower Ohio basins." While the white oak can maintain itself on almost any soil, the above habitat indicates its preference for the rich, loamy soils on gentle slopes, bottom lands, and coves. 
It rarely occurs alone, but rather in mixed forests with other oaks, chestnuts, basswoods, and tulip trees. The white oak is comparatively tolerant in early life, growing under the shade of other trees if the canopy is not too low or too dense. In such

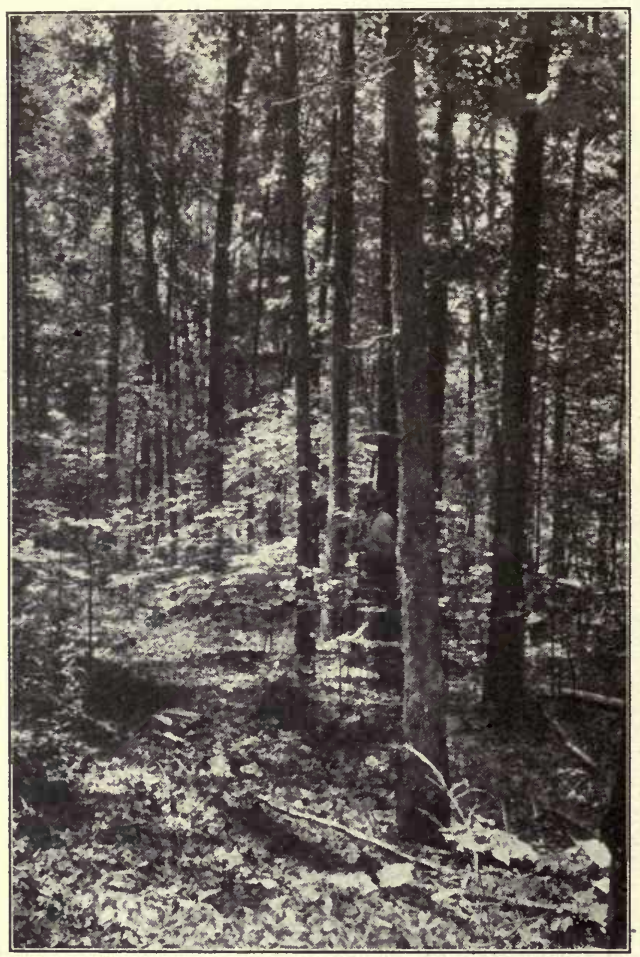

FIG. 237. Stand of young white oak timber on bottom land of a small creek

Photograph furnished by United States Forest Service situations it grows very slowly for a long period (as many as fifty years), when it will take on new life and grow with great rapidity if the trees which shade it are logged so as to expose it to the sunlight. When fullgrown it is of the spreading type, and its top forms a broad dome with numerous branches (Fig. 236). It usually reaches a height of from 60 to 100 feet, with a diameter of from 2 to 4 feet, although it may grow to a height of 150 feet, with a diameter of from 6 to 8 feet. The bark on the young twigs and branches is greenish gray, but on the mature trunk it varies from pale gray to white, with shallow fissures and flaky scales. The leaves are deeply lobed, with the rounded lobes characteristic of the white, as distinct from the black, oaks. The buds in the wintering shoots are short and blunt, reddish gray in color, and sometimes covered with a distinct bloom. The white oak is easily identified by the above characteristics. 
Reproduction. The white oak is monoecious, with the imperfect flowers borne on the young shoots and the twigs of the previous season (Fig. 238). The male flowers occur in loose spikes or catkins, and the female flowers in few-flowered spikes in the axils of leaves above the clusters of male catkins. Each male flower is composed of a group of from 5 to 8 stamens in an inconspicuous perianth with from 5 to 9 lobes. Each female flower is composed of a single pistil with a rudimentary perianth. The two or three pistillate flowers which occur in a single spike are enveloped in a cup formed of coherent bracts, which ultimately develop into the cupule, or cup, of the fruit.

Pollination is by the wind (anemophilous). For this the white oak is adapted by its abundant light pollen grains produced in pendent catkins, which are easily swayed by air currents.

'The fruit is a nut, or acorn, resting in the cupule, or cup, formed, as indicated above, by bracts which surround each spike of female flowers. In

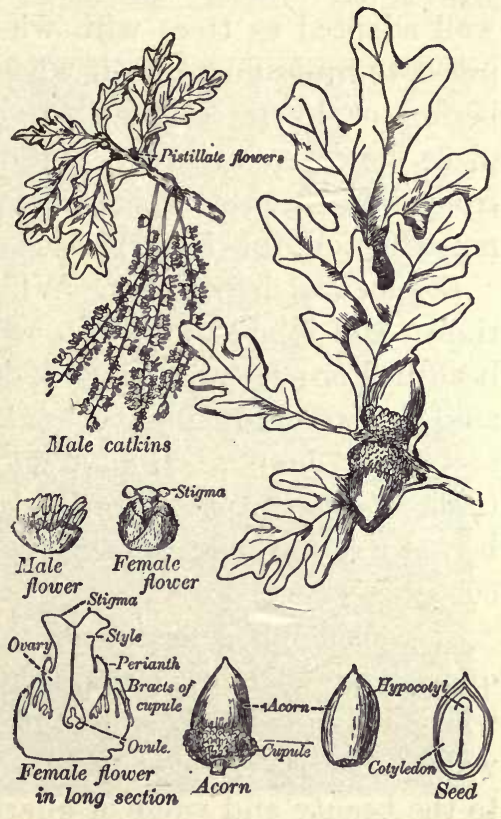

Frg. 238. Drawing of the inflorescence, flowers, and fruit of the white oak the white oak the acorn matures each fall, unlike the black-oak series, in which the acorn ripens the second season after pollination. The acorn germinates in the fall, sending a long taproot deep down into the soil. The plumule and root are liberated from the seed by the lengthening of the petioles. The age at which the white oak forms its seeds varies with the conditions - under which it is growing. Where trees grow in the open in rich, well-watered soil, seed production may occur in the eighteenth or twentieth year; in open woods it occurs at the age of forty 
years; but "under normal forest conditions acorns are not produced, as a rule, before the seventieth or eightieth year." Moreover, a large proportion of the seed is destroyed by worms, rodents, and other animals having access to the forests or wood lots where oak trees are found. The heavy acorns are distributed mainly by gravity or by squirrels which bury them and never dig them up again. The white oak, therefore, is not so well adapted as trees with winged seeds, like the spruces and pines, to disseminate itself where it has been destroyed by lumbering or by forest fires. Vegetative reproduction often takes place, however, in such situations by the outgrowth of sprouts from the stumps, which maintain this power of vegetative reproduction for long periods.

Commercial importance. White oaks are chiefly valuable for timber and lumber, although, where they are native, they form a beautiful ornamental tree with dense shadle. The wood (Figs. 47 and 55) is very dense and hard, with great resistance to disease and decay, both when alive and when used for railroad ties or timber in construction work. Its main uses are for sawed lumber, switch ties, and timbers for ships and bridges and for tight cooperage.

A considerable portion of the lumber from the best logs is quarter-sawed and then shipped to furniture and cabinet factories, where it is used as a substitute for other woods, such as black walnut, cherry, and mahogany. The broad wood rays add greatly to the beauty and value of quarter-sawed oak (Fig. 50). Tightcooperage staves were early made from white oak for the preservation and shipping of alcohol, wines, and liquors, as well as for oil, molasses, and other fluid or semifluid substances which must be confined in tight receptacles. The compact, hard wood of the white oak is admirably fitted for such purposes. In shipbuilding and in making piles for bridges white oak is also very valuable, for the reason that it does not readily deteriorate in situations where it is subjected to alternate dry and moist conditions.

Like most of the valuable forest trees white oak is rapidly. diminishing in amount and quality, so that its propagation is an important forestry problem. 


\section{The Willows (SALICACEAE)}

Habitat and habit. Most of the willows are hydrophytic in their habitat, growing along the borders of lakes and streams, in swamps, or in moist soils (Fig. 239). A few species are adapted to mesophytic conditions and are capable of cultivation in comparatively dry soils. In habit the majority are shrubs,

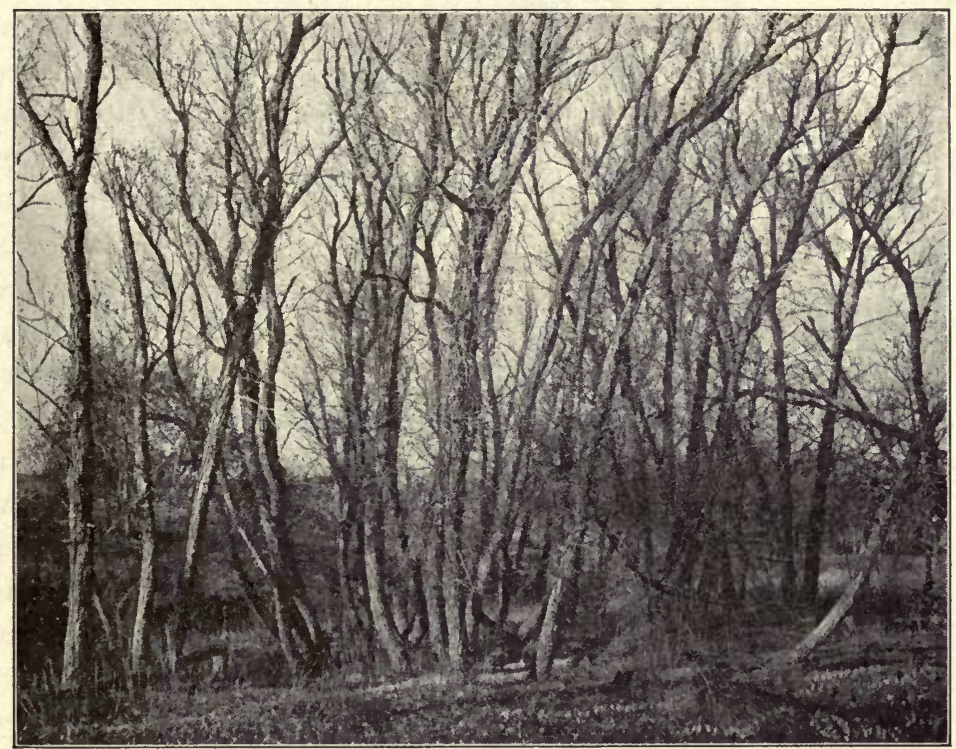

Fig. 239. Natural habitat and habit of willows along a creek

Photograph furnished by the United States Forest Service

while a few forms grow to be large-sized, spreading trees from 50 to 100 feet in height, attaining to a diameter of two or three feet.

The bark of the young twigs (Fig. 240) is smooth or hairy, while that on the main trunk and the older branches is flaky. The leaves are long and narrow, with an entire or slightly notched margin and with a short petiole bearing stipules, or appendages, at its base, where it joins the main stem. 
Reproduction. The flowers of the willows occur in the form of catkins (Fig. 240), which are a modified form of a spike in which the bracts are represented by conspicuous scales. The catkins of the pussy willow (Salix discolor) are thick and oval and have received the name pussy on account of the silky hairs that clothe.

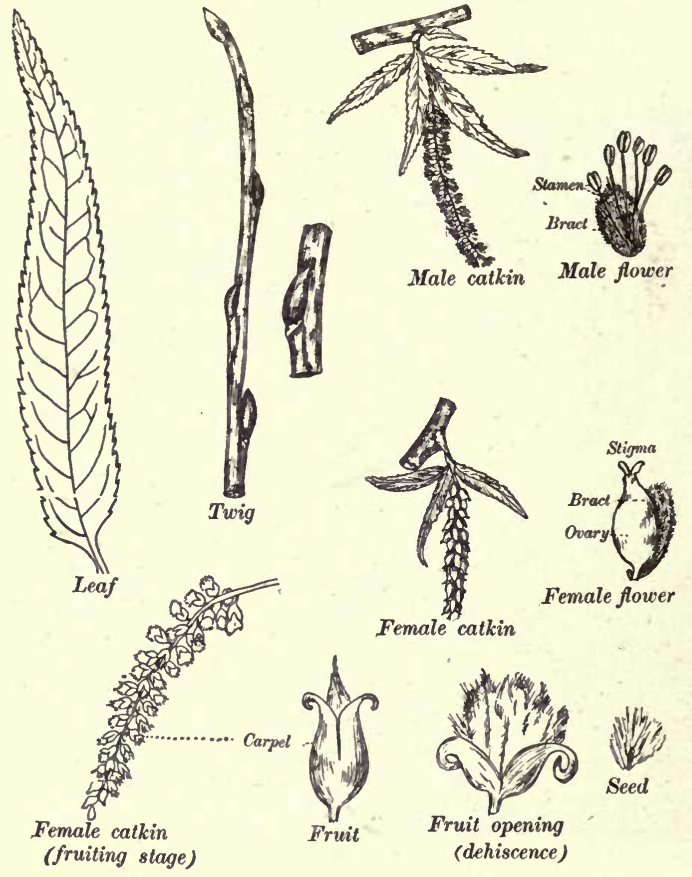

FIG. 240. Inflorescence, flowers, and fruit of willow the young catkins in early spring. Since these catkins are borne on separate trees, the willows are dicecious and are entomophilous, or cross-pollinated by the insects which visit the flowers for the nectar secreted by nectar glands at the base of the flowers. The pollen is abundant and sticky, so that frequent pollination is assured although the flowers occur early in the spring.

The individual flowers are very simple, the females consisting of a simple compound ovary composed of two sporophylls subtended by a hairy bract. The male flowers bear two or more stamens, according to the species, in the axil of a hairy bract (Fig. 240).

The fruit is a capsule, or pod, and the seeds ripen early in the spring, after fertilization. The two sporophylls comprising the fruit separate so as to liberate the seeds when they are ripe. The long, silky hairs of the seeds facilitate their distribution by the wind, or by water currents. 
The poplars, which are closely related to the willows, have a similar natural habitat, but they grow to a greater size and are of more value for timber or as ornamental trees than the willows. The reproductive organs of willows and poplars are similar in that the simple flowers of the poplars are borne in catkins, as in the willows (Fig. 241). The individual flowers of the poplar, however, differ in detail from those of the willow. The stamens in the poplar are borne in considerable numbers on a bract with

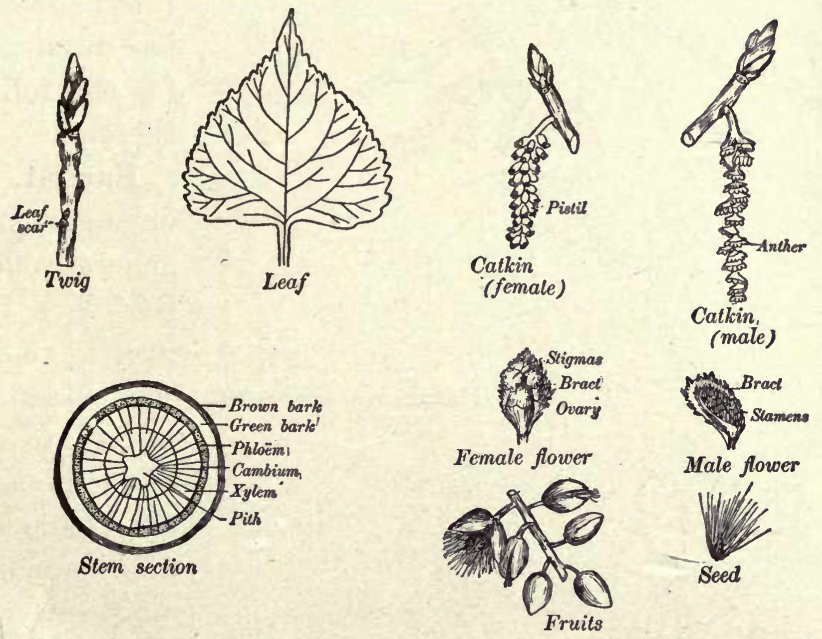

Fig. 241. Vegetative and reproductive parts of a poplar

a hairy or roughened margin. The pistil resembles more nearly that of the willow, but is characterized by a larger and muchlobed stigmatic surface. Each pistillate flower is in the axil of a bract like that which bears the stamens. The seeds in the cottonwood (Populus deltoides) are well known for their cottony covering of long, silky hairs. These hairs are outgrowths of the seed coat, and when the fruit opens they expand and the seeds can then be blown about by air currents. Pollination in the poplars, unlike the willows, is effected by the wind (that is, they are anemophilous), and the pollen, as in all wind-pollinated plants, is produced in great profusion by the conspicuous pendent male catkins, from which the pollen is easily scattered. 


\section{Aceraceae (Maple Family)}

\section{THE SUGAR MAPLE (ACER SACCHARUM)}

The maple family includes about seventy species, among which are the common soft, or silver, maple, the ash-leaved maples, or box elders, and the various kinds of hard maples, of which

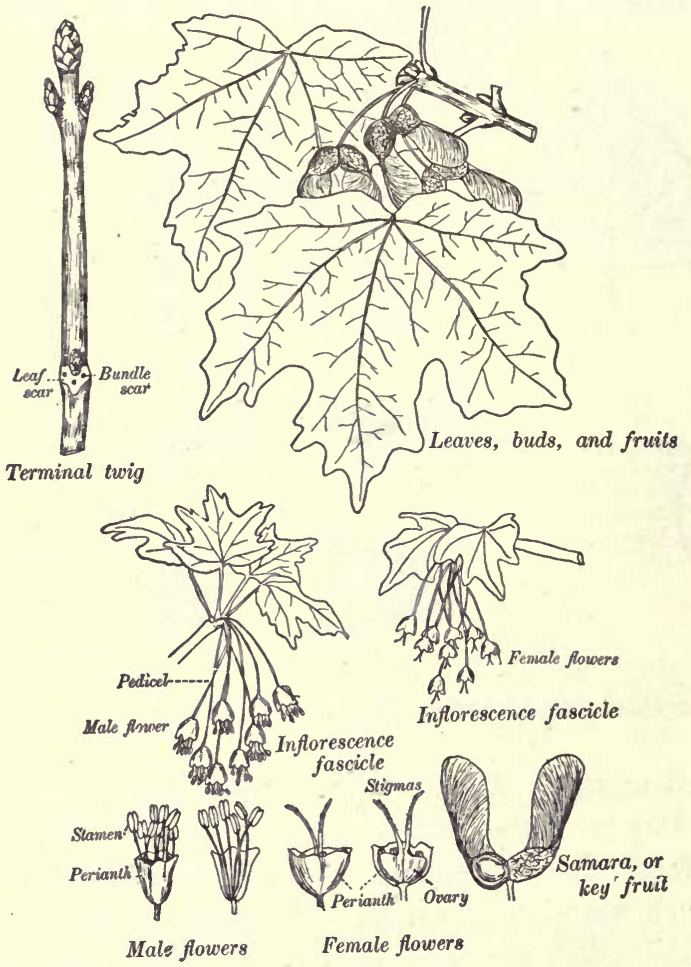

FIG. 242. Flowers, fruits, and seeds of the sugar maple (Acer saccharum) the sugar maple (Acer saccharum) has been chosen for the following sketch.

Habitat. Rock, or sugar, maples generally flourish best in a fertile, loamy soil, but can adapt themselves to other kinds of soils if they have a medium amount of moisture. This tree is distinctive in habit because of its symmetrical, broad, round top, so characteristic of old trees. The bark on older trees is deeply furrowed and is

divided into broad plates or large flakes. The leaves are broad, thin, and deep green, with the margin divided into five points, or lobes (Fig. 242).

Reproduction. The flowers in the sugar maple are borne in corymblike clusters with long, hairlike pedicels. Some of the 
flowers are perfect, while others are imperfect. The trees are therefore either diøecious, with male flowers on one tree and female flowers on another, or polygamo-monœecious, that is, with some imperfect flowers of both sexes on the same tree.

The individual flowers (Fig. 242) have a five-lobed perianth inclosing both sets of essential organs in the perfect flowers or one set in the imperfect flowers. The pistillate flowers usually

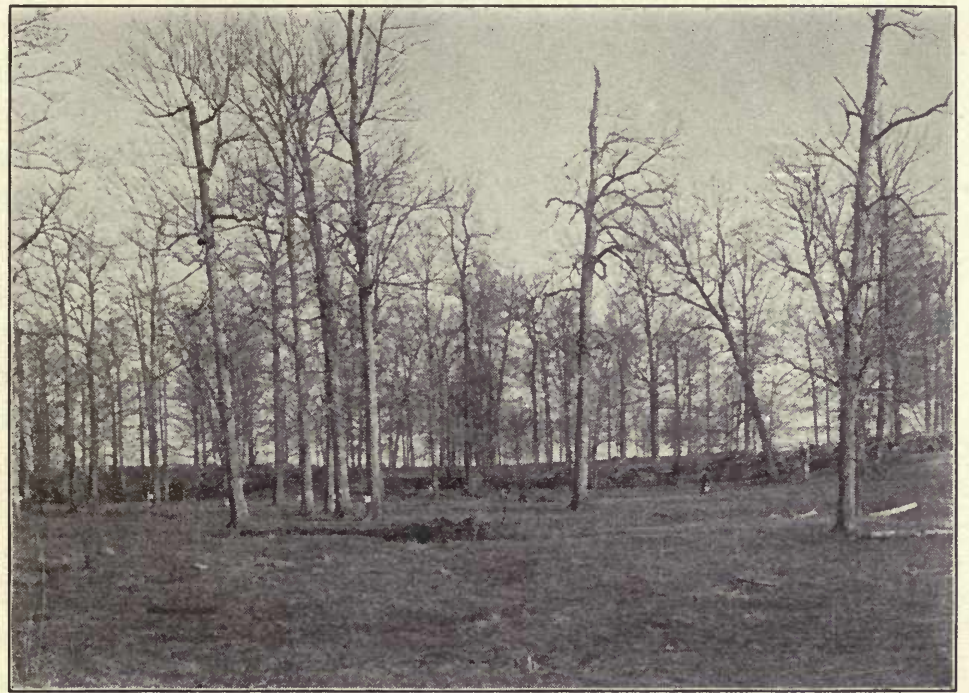

Fig. 243. A grove of sugar maples which have been tapped for over eighty years

From Bergen and Caldwell's "Introduction to Botany"

contain rudimentary stamens. Pollination is entomophilous, and the fruits are of the samara type, the wings being formed by an outgrowth from the ovary wall.

Distribution and importance. Sugar maple is distributed over the entire eastern part of the United States, occurring in the northern and central hardwood forests and as a shade and ornamental tree. The wood is diffuse porous, with the ducts scattered throughout the annual rings and separated by dense masses of wood fibers. It is preëminently a manufacturer's wood, the demand being that of a planing-mill product. These products 
include flooring, ceiling, and molding and door lumber. In addition the wood is used for a great variety of purposes, - for agricultural implements, musical instruments, tools, and excelsior. Curly and bird's-eye maple (Fig. 49) are of special value for interior finish and decorative effects. The curly effect is due

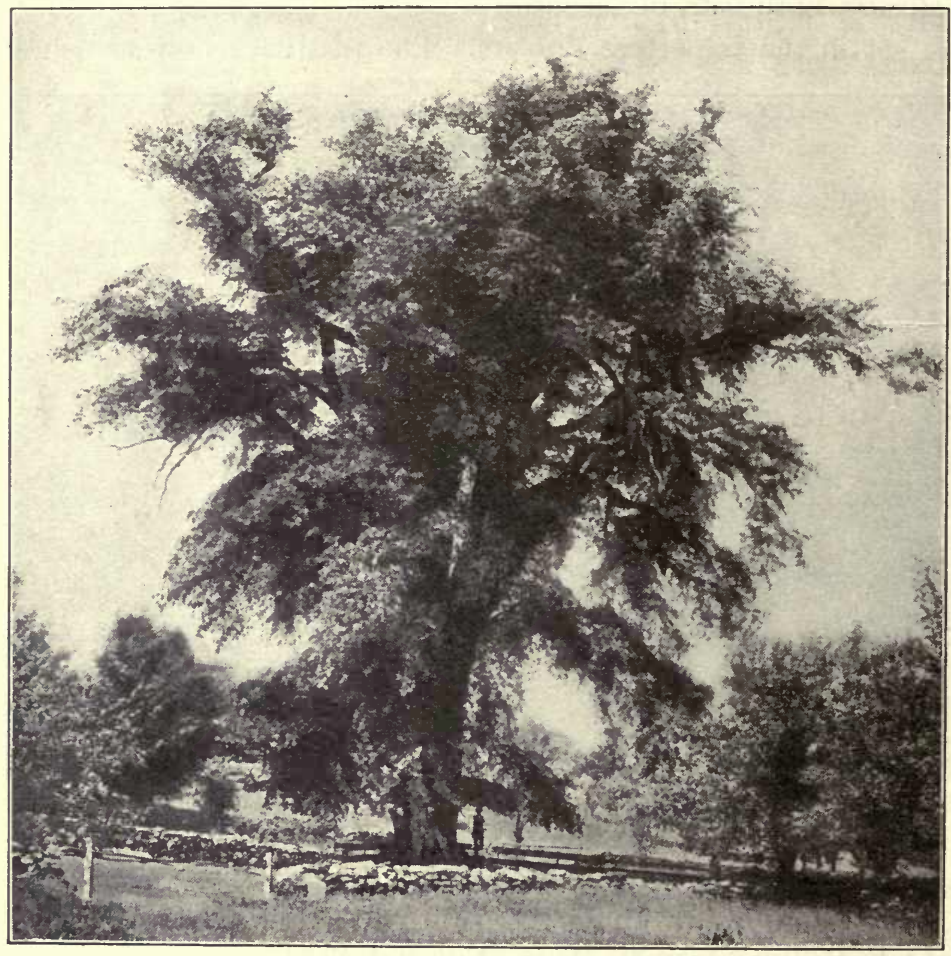

FIg. 244. Habit of the American elm (Ulmus americana) in summer Photograph furnished by the United States Forest Service

to the fact that the grain is wavy, instead of straight as in most trees. Bird's-eye effects may be due to the distortion of the annual wood rings by latent buds, unsuccessful branches, or injuries produced by wood borers. The maple-sugar industry is also one of considerable importance in the east and in the states of Michigan, Wisconsin, and New York. For this purpose the sap of the black maple and that of the sugar maple are the most valuable. 


\section{ULMACEAE (ELM FAMHL) \\ THE WHITE ELM (ULMUS AMERICANA)}

Habitat and habit. The white elm, with its near relatives the slippery elm (Ulmus fulva) and the cork elm (Ulmus racemosa), occupies a wide area in the United States from the Rocky Mountains to the eastern seaboard. In these regions it does not form pure forest stands like many other forest trees, but occurs with such moistureloving trees as the cottonwoods, silver maples, and sycamores along streams, in river bottoms, in farm wood-lots, and on lawns where there is good, well-drained soil. It rarely occurs among oaks, pines, and hickories, which grow in drier soils.

The elm should also be classed as an intolerant tree, since it needs plenty of light for its best de-

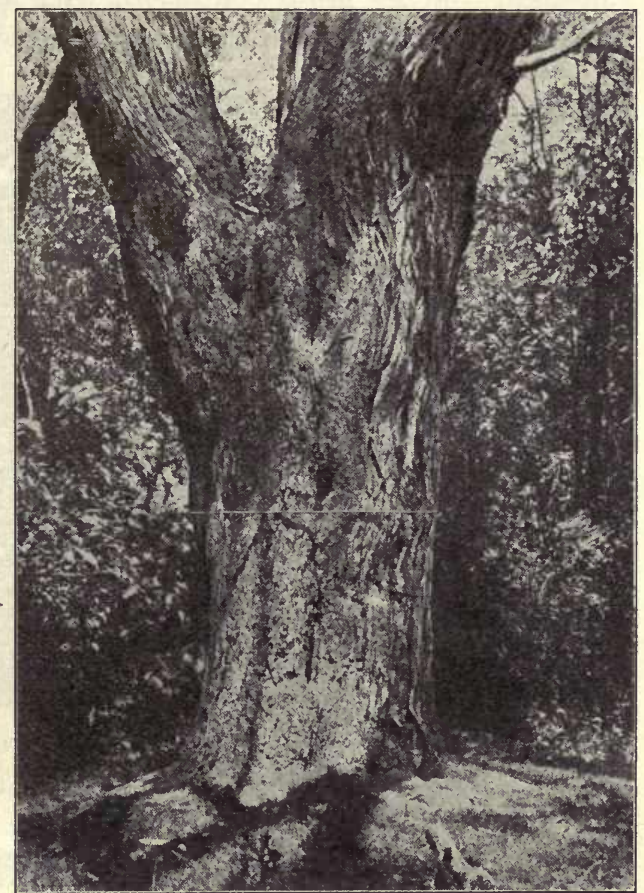

Fig. 245. Tree trunk and bark of the white elm Photograph furnished by United States Forest Service velopment. The graceful habit of the elm makes it one of the most valuable of trees for ornamental purposes on private lawns and as a shade tree in public parks and city streets.

Reproduction. The flowers of the elms occur in dense, racemelike clusters, or fascicles, which open in early spring before the leaves are out (Fig. 247). The flowers on different parts of 
the tree are of various sorts, some being perfect, with both pistils and stamens in the same flower, while others are imperfect, with either stamens or pistils in a single flower. In the latter instances both sets of organs are usually present, but one set (either pistils or stamens) is abortive and hence useless in reproduction. These abortive organs furnish an interesting example of the persistence of structures which were once useful but have ceased to function. Such a floral condition, with a mixture of perfect and imperfect flowers on the same tree, is designated as polygamous.

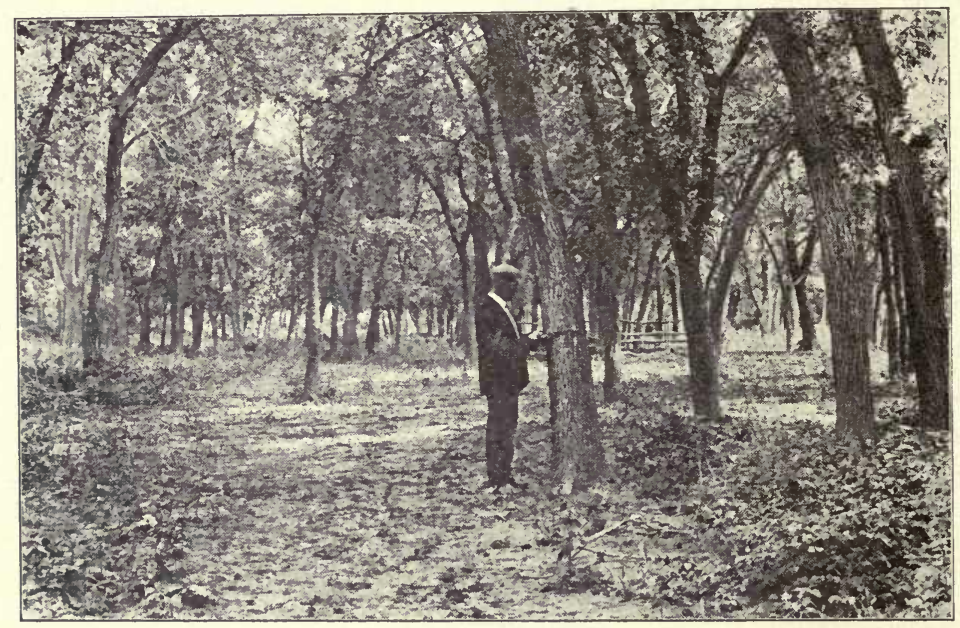

Fig. 246. Natural habitat of elms on a creek bottom

Photograph furnished by the United States Forest Service

Pollination is necessarily either self-pollination or crosspollination and is effected by wind or by insects. The fruit is a winged samara, or key fruit, the wings being an outgrowth of the ovary wall which takes place as a secondary result of fertilization along with the formation of the embryo and seed. This winged fruit aids materially in the dissemination of the elms.

Commercial importance. In addition to its value as a shade and ornamental tree the elm is of considerable importance in the production of lumber for certain purposes. The wood is of the ring-porous type, with a single circle of large spring ducts in 
each annual ring. The remainder of the ring is composed of alternating bands of small ducts and bands of fibers. The wood rays and wood parenchyma are inconspicuous. The value of elm for commercial purposes is due to its strength and to the qualities which enable it to withstand bending and shock better than many other kinds of woods. Consequently its greatest use is in industries such as slack cooperage and the making of baskets, boxes, crates, and vehicles. In the making of hoops and staves for cooperage, as well as of rims or bands in baskets and crates, its bending quality and its strength are of particular value.
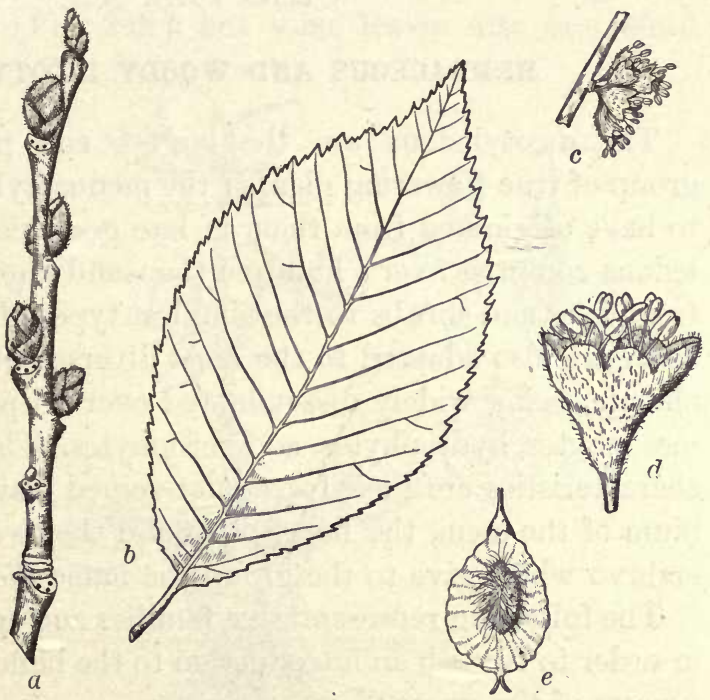

Fig. 247. Vegetative and reproductive structures of the red, or slippery, elm (Ulmus fulva)

In vehicles it is used chiefly in body work. Elm

wood is also used for a great variety of industries, such as the making of chairs, musical instruments, agricultural tools, etc.

The principal sources of elm wood are the states bordering on the Great Lakes (particularly Michigan and Wisconsin) and those in the southern Mississippi region (that is, Arkansas, Mississippi, and Louisiana). Like most valuable woods, however, it is being rapidly depleted and must either be replanted or replaced by other woods of a similar character. 


\section{CHAPTER XX}

\section{HERBACEOUS AND WOODY DICOTYLEDONS}

The dicotyledons are the largest and probably the oldest group of true flowering plants; the monocotyledons are supposed to have originated from them in late geologic times. The dicotyledons comprise over a hundred thousand known species, ranging from trees and shrubs to the simplest types of herbaceous plants. They are also adapted to the most diverse conditions of soil and climate, being widely disseminated orer all parts of the earth as mesophytes, hydrophytes, and xerophytes. Their chief distinctive characteristics are their broad, net-veined leaves, the active cambium of the stem, the floral plan, and the two cotyledons of the embryo which give to the group the name dicotyledons.

The following representative families and species are discussed in order to furnish an introduction to the biological and economic aspects of the group.

\section{RANUNCULACEAE (BUtTercup Family)}

The Ranunculaceae include many of our commonest and most typical plants of the spring flora. Among these the buttercups, the marsh marigold, the hepaticas, and the wood anemones are early harbingers of spring, which represent at the same time the simpler types of plants in the family, with regular spiral and hypogynous flowers. Among the more highly modified and showy flowers of the family the columbines, larkspurs, and clematis represent both wild and cultivated species of great beauty and usefulness as ornamental plants. Most of the species are stemless herbs with simple leaves, which produce their flowers and fruits early in the spring. 


\section{CALTHA (MARSH MARIGOLD) AND RANUNCULUS (BUTTERCUP)}

Habitat and habit. Caltha palustris, the marsh marigold, differs from the majority of the buttercups in being a hydrophyte, inhabiting marshes and swamps along the margins of streams and lakes. The heart-shaped, rounded leaves are mostly radical, or root, leaves (Fig. 248), but some leaves also are found on the flowering stems, which grow up annually and produce clusters of bright yellow flowers. The buttercups are quite diversified in both habitat and habit, being represented by true mesophytes and hydrophytes as well as by caulescent and acaulescent species.

Reproduction. In both Caltha and the true buttercups the flowers are simple and

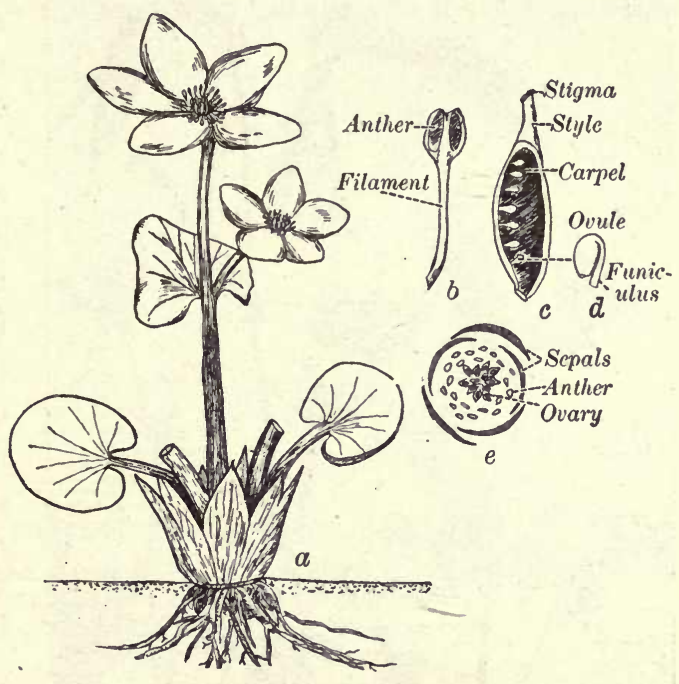

Fig. 248. Habit and reproductive organs of the marsh marigold (Caltha palustris)

$a$, the plant ; $b$, stamen; $c$, long section of a pistil, showing the ovules on the placenta; $d$, ovule; $e$, floral diagram regular, and are thus representative of the simplest types of floral structure among the plants of the Ranunculus family. The account already given of the flower of Caltha indicates its close alliance with the spiral, hypogynous flowers whose structure is similar to that of a strobilus (Fig. 200).

Cross-pollination is provided for in both Caltha and the buttercups, although close-pollination and self-pollination are possible in most cases. The following account of the adaptation of the flowers to pollination is applicable to both Caltha and the common buttercups. 
In the young flowers the stamens are massed closely around the pistils, with the anthers just below the stigmatic surfaces. In the older flowers the outer stamens ripen first, and since the

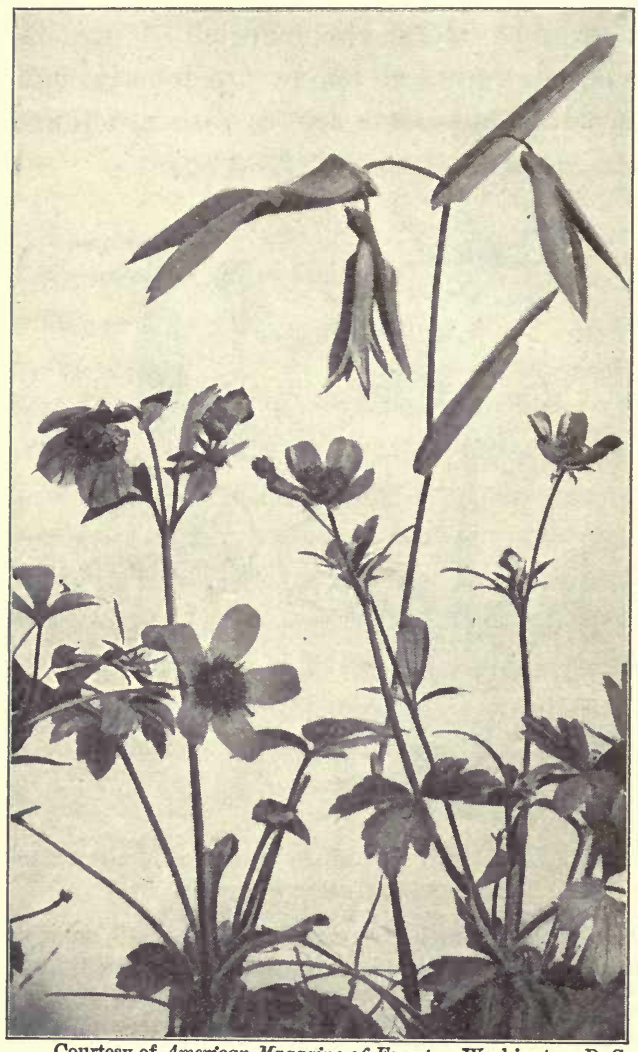

Courtesy of American Magazine of Forestry, Washington, D. C.

Fig. 249. Habit of the common buttercup

Photograph by Dr. R. W. Shufeldt anthers are extrorse (that is, on the outer abaxial surface of the filament), the pollen as it is shed is not likely to reach the stigmas without insect aid. The dehiscence of the remaining anthers, after the first, is centripetal (that is, from the outside of the flower toward the centrally located pistils), which further insures against selfpollination. Insect visitors are attracted by the bright yellow color of the petals and by the abundant nectar, which is secreted in two shallow depressions on either side of each ovary. When they alight upon the center of the flower with pollen on the abdomen derived from other flowers, they effect either close-pollination or cross-pollination, according to the source of the pollen. Insects which pass from the outer border of a flower across the center are likely to effect self-pollination. The fruit is a many-seeded follicle which splits down the placental suture and liberates the seeds. 


\section{AQUILEGIA (COLUMBINE)}

In the columbines the flowers are very highly modified and irregular, the five petals being developed into long spurs with nectaries at the tips. The flowers are pendulous, and the nectar secreted in the end of the spur is thus protected from rain. They are pollinated by bumblebees, which possess a proboscis long enough to penetrate to the nectar at the base of the spur, which may be from 15 to $20 \mathrm{~mm}$. in length (Fig. 250, a).

The following account of the pollination of Aquilegia vulgaris, taken from Knuth's Handbook, will serve to indicate the details in other species. "Bumblebees, which possess a probos-

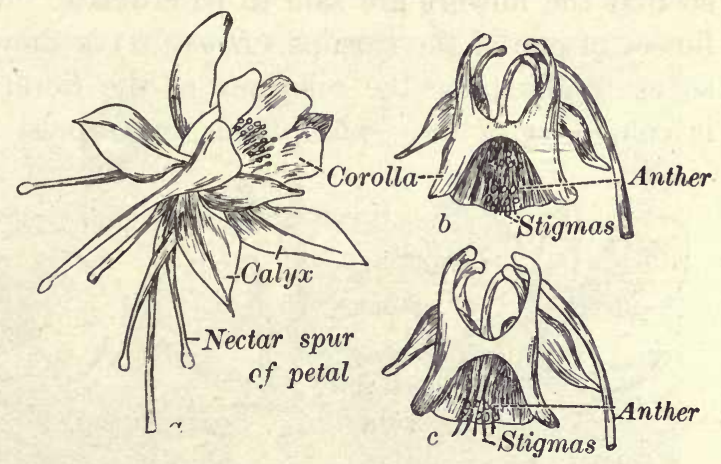

Fig. 250. Flowers of the columbine (Aquilegia)

$a$, single flower; $b$, young flower with anthers surrounding the stigmas; $c$, older flower with pollen shed and stigmas protruding. From Knuth's "Handbook of Flower Pollination"

cis long enough to reach the nectar in the legitimate way, cling to the flowers from below, holding on to the base of the spur with the forelegs and pushing the head into the spur. In so doing the underside of the abdomen comes in contact, in the young flowers, with the pollen-covered anthers which surround the pistil (Fig. 250, $b$ ). In older flowers (c) the same region of the body touches the somewhat spreading stigmas which project from among the stamens, so that cross-pollination is necessarily effected. Should insect visits fail, automatic self-pollination readily takes place, for the carpels (pistils) grow down in the middle of the stamens, and by elongation of the style the stigmas ultimately reach a lower level than the anthers." Other members of the Ranunculaceae are also highly modified for securing cross-pollination by insects. 


\section{CRUCIFERAE (MUSTARD FAMILY)}

The Cruciferae include some of the most important cultivated commercial varieties of plants, such as the cabbage, turnip, radish, and cress. The most distinctive features of the family are the acrid juice and the peculiar structure of the flowers and fruit.

Reproduction. The parts of the flowers are in sets of four members each, the petals being so related as to form a cross, so that the flowers are said to be cruciate. In Fig. 251, $a$, the flower of one of the turnips (Brassica) is shown in long section, so as to illustrate the relations of the floral parts. The pistil is composed of two sporophylls, or carpels, and the cavity of
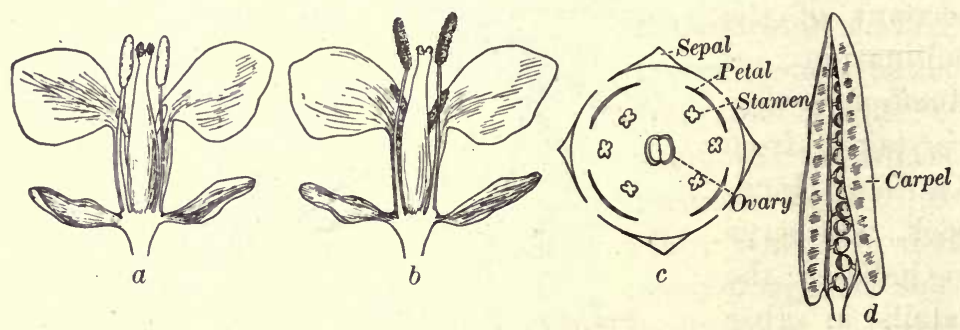

Fig. 251. Flowers and fruit of a cruciferous plant (Brassica) $a$, young flower with unripe anthers; $b$, older flower, anthers shedding pollen; $c$, floral plan; $d$, fruit (silique)

the ovary is separated by a false partition, or septum, which runs between the two placental ridges (see the section of the ovary in the ground plan of a cruciferous flower, Fig. 251, c).

The stamens are six in number, two short and four long, the four long stamens representing two stamens which branch at the base. Cross-pollination may be effected through the agency of insects, or self-pollination from the anthers of the long stamens. When the flowers are young (Fig. 251, a), the anthers of the long stamens are often separated from the stigma by the movements of the filaments. In more mature flowers $(b)$ the anthers move toward the stigma, so that self-pollination is effected. The fruit (d) is a pod, called a silique. When ripe the two halves of the pod separate from the septum, or partition, to which the seeds cling, and the seeds are then disseminated. 
Commercial varieties. The commercial varieties of the Cruciferce are of the greatest importance economically and are also of special interest to the student of variation and development in cultivated plants, since some very remarkable transformations have been effected in them by culture. Thus, in the cabbage the stem has become greatly shortened and the normal leaves transformed into the overlapping, fleshy leayes of the cabbage head. In Brussels sprouts the stem has retained its normal length,

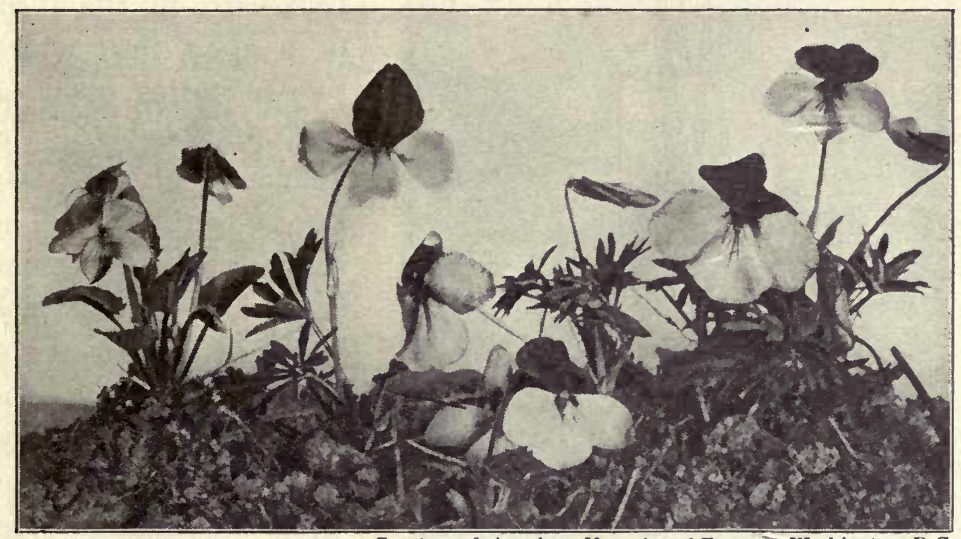

Courtesy of American Magazine of Forestry, Washington, D.C.

Frg. 252. Habit of violets with flowers

Photograph by Dr. R. W. Shufeldt

but the lateral buds have been enlarged until they look like small cabbage heads. In turnips and radishes the main taproot has become highly modified into the fleshy roots of the commercial varieties. In the transformation of the cabbages, cauliflower, Brussels sprouts, etc. mutation is thought to have occurred, thus giving rise to some of the striking variations in these plants which have been accentuated by. cultivation.

\section{Violaceae (Violet Family)}

The Violaceae are of special interest on account of their great beauty, both in the wild state and under cultivation. The cultivated pansy is a hybrid of several wild species of violets. 
Biologically considerable interest attaches to these beautiful plants on account of the unusual devices which result in crosspollination by insects. Fig. 252 illustrates the usual habit of the violet plants and the highly modified floral structure. In Fig. 253 a sectional view of the flower of Viola pinnata is also shown, which will enable the student to understand more fully the wonderful mechanism for securing pollination and fertilization in this species. The lower petal of the flower is projected

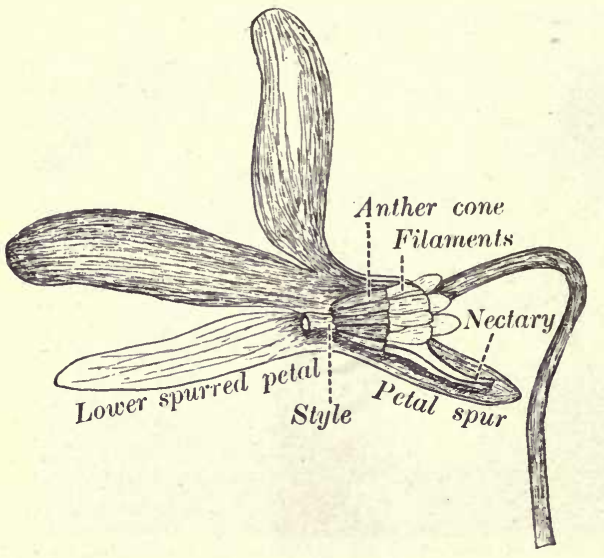

Frg. 253. Structure of the flower of a violet with reference to pollination

Adapted from Knuth's " Handbook of Flower Pollination" backward in the form of a spur, into which two nectaries grow as continuations of the filaments of the two lowest anthers. The stamens form a cone around the style, which is closed except on the lower side between the two lowest anthers. The style projects beyond the stamen cone, bearing the stigma at its end. When an insect probes down the cavity of the spur for nectar from the nectaries, he spreads the lower anthers slightly, which allows pollen, already dehisced into the anther cone, to sift out upon his proboscis. This pollen cannot reach the stigmatic surface of the same flower upon the withdrawal of the proboscis, on account of its position and structure. When, however, the next flower is visited for nectar, the stigma is almost certain to be dusted with foreign pollen gained from a previously visited flower, and so to be either close-pollinated or cross-pollinated. The fruit is a pod which opens by three valves, and the seeds are often thrown to considerable distances by a special mechanism. The two walls of each valve contract as they become dry, and squeeze the hard seeds, which are thus thrown from the mother plant. 


\section{Rosaceae (Rose Family)}

The rose family comprises over 14,000 species, including an unusually large number of ornamental, food-producing, and fruitproducing varieties of great value and importance to man. The family is also noted for the great variation in its species as regards habit, since it includes all types of plants from acaulescent herbs, like the potentillas and strawberries, to shrubs and trees, like the roses proper, the spireas, and the apples, pears, and plums.

In the treatment of the following representative species emphasis will be placed largely upon reproduction and fruit formation, since this is the distinctive feature of the rose family which is of the greatest interest and importance.

\section{FRAGARIA (STRAW- BERRY)}

\section{Habit and history.} The cultivated strawberry is a direct descendant of one of the

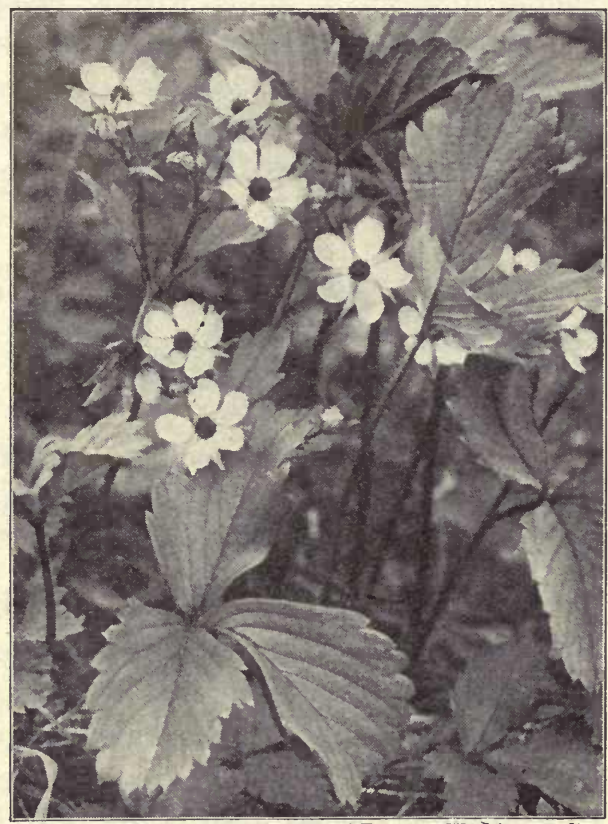

Courtesy of American Magazine of Forestry, Washington, D.C.

Fig. 254. Habit and flowers of the American strawberry

Photograph by Dr. R. W. Shufeldt

wild strawberries, and its large size and greatly improved fruit is due to cultivation and breeding by man during the last fifty years. Professor L. H. Bailey, a recognized authority on the evolution of our native fruits, ascribes the origin of our present varieties of cultivated strawberries to the improvement of a wild species from Chile, known as the Chilean strawberry (Fragaria 
chiloensis). Like most of the other fruits of this order the strawberry is therefore a product of the breeding and improvement of wild plants by the methods of hybridizing and breeding recorded in earlier chapters. Vegetative reproduction by means of runners has already been explained in Part I and is there illustrated, together with the acaulescent habit of these plants, in Fig. 76 .

Flowers and fruit. The flowers in the strawberry are quite similar to those of Caltha, with an elongated receptacle on which the numerous stamens and the cluster of simple pistils are arranged spirally (Fig. 255, a, b). The flowers are usually perfect, but some commercial varieties have only pistillate flowers, while others have pistils with few stamens (Fig. 255,e,f). In
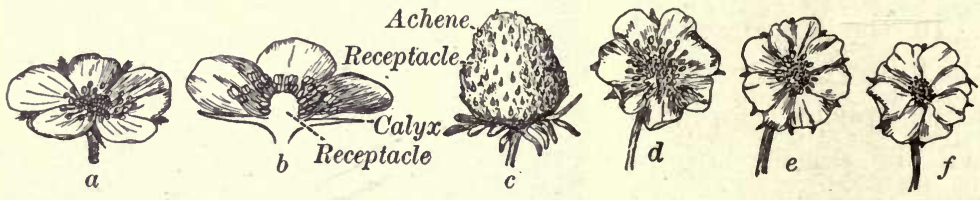

Fig. 255. Flowers and fruit of the strawberry (Fragaria)

$a, b$, surface and sectional views of a perfect flower; $c$, fruit; $d$, perfect flower; $e, f$, imperfect flowers ( $e$, pistillate flower; $f$, staminate flower)

planting such varieties care must be taken to have both pistillate and pollen-bearing individuals in the same or in alternate rows.

Cross-pollination is effected by insects, since the perfect flowers are protogynous, the stigmas ripening before the stamens. Pollination and fertilization in the strawberry, as in so many other fruit-forming species, results not only in the development of the seeds but also in great changes in the receptacle, by which it becomes greatly enlarged and fleshy to form the fruit. The seeds remain inclosed in the ovary wall, which dries and forms a true achene.

The fruit is therefore composed largely of fleshy receptacle, with the hard achenes scattered over its surface and commonly designated as seeds by those who eat the ripened fruit. The dissemination of the wild strawberry is undoubtedly facilitated in nature by animals that eat the fruit and eject the dried achenes and seeds with their excrements. 


\section{RUBUS (RASPBERRIES AND BLACKBERRIES)}

Habit and history. The raspberries and blackberries now under cultivation had the same general origin and history as that of the cultivated strawberry in that they have been derived in almost every instance from wild ancestors. Thus, the common black-cap raspberries seem to have originated from chance seedlings, possibly mutations, first discovered in Ohio in 1832 by Nicholas Longworth. The red raspberries probably had a similar origin from wild. ancestors in Canada and the northeastern portions of the
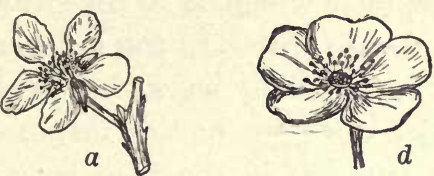
United States. The cultivated blackberries have evidently been developed from the wild species of our own country and came gradually into use by the early settlers.

Both raspberries and blackberries are prickly shrubs with simple or compound leaves and with aërial stems, called canes, which produce leaves and buds for the first year only. From these first-year

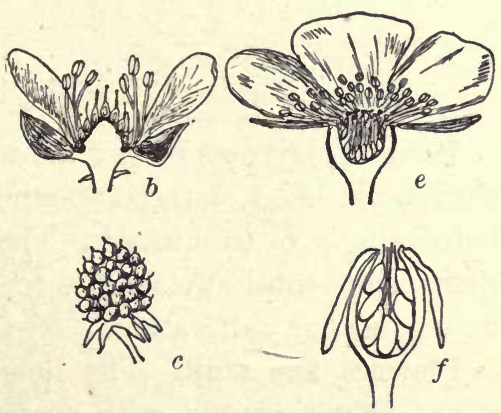

Fig. 256. Flower and fruit of the blackberry and the rose $a, b, c$, blackberry; $d, e, f$, rose canes lateral buds develop the flowers and fruit of the second season. After the formation of the fruit the canes die and are replaced in many species by sucker shoots which spring up from the roots. In the black raspberry vegetative reproduction takes place by means of branches which bend over and take root, thus giving rise to new plants (Fig. 75).

Flowers and fruit. The flowers are either axillary and solitary or are borne in clusters. They resemble the flowers of the strawberry in being perfect and perigynous, with an enlarged receptacle (Fig. 256, b). There are five petals, many separate pistils crowded on the receptacle, and numerous stamens. 
Close-pollination and cross-pollination are effected by insects, which visit the flowers for the nectar secreted by a ringlike nectary at the base of the stamens.

The fruit is an aggregate of small stone fruits, or clrupes, in both the raspberry and the blackberry, the separate drupes arising from the ovary wall, which forms, in ripening, an outer fleshy wall and an inner stony part inclosing the seed. In the blackberry (Fig. 256, c) these stone fruits are aggregated on a fleshy receptacle which, as in the strawberry, forms a part of the ripened aggregate fruit. In the raspberry the separate stone fruits unite by interlocking hairs, but separate easily from the receptacle, which does not in this instance enter into the edible portion of the fruit, as in the blackberry and the strawberry.

\section{ROSA (ROSES)}

Habit. The roses proper are woody perennials, often becoming shrubby in habit, with compound leaves and conspicuous flowers borne singly or in clusters. The plants are principally noted for their ornamental effects, due to the great size and beauty of the flowers under cultivation.

Flowers and fruit. The flowers of the wild and cultivated roses are perigynous, with an urn-shaped receptacle which bears the conspicuous petals, calyx tubes, and stamens on the rim of the cup (Fig. 256,e). The pistils are numerous, springing from the base of the receptacle and inclosed by it.

Pollination. The stigmas protrude beyond the urn-shaped receptacle in the open flower, and the stamens bend outward, facilitating cross-pollination, although self-pollination is not precluded. In the development of the fruit, or rose hip (Fig. $256, f$ ), both the pistils and the receptacle have a part. The pistils develop into hard, bony, achenelike fruits inclosed by the receptacle, which becomes fleshy and bright red or scarlet at maturity. The stamens and corolla disappear during fruit formation, but the calyx lobes adorn the upper border of the rose hip after it is ripe. The seeds are scattered when the fruit decays, or they may be disseminated by animals if the fruit is eaten. 


\section{PRUNUS (CHERRY)}

Reproduction. The flowers of the cherry are borne in lateral buds on dwarfed shoots called spur shoots. The flowers are perigynous (Fig. 257, $f$, and 259,b), as in the genus Rosa, but there is only one ovary in the urn-shaped receptacle of each flower.
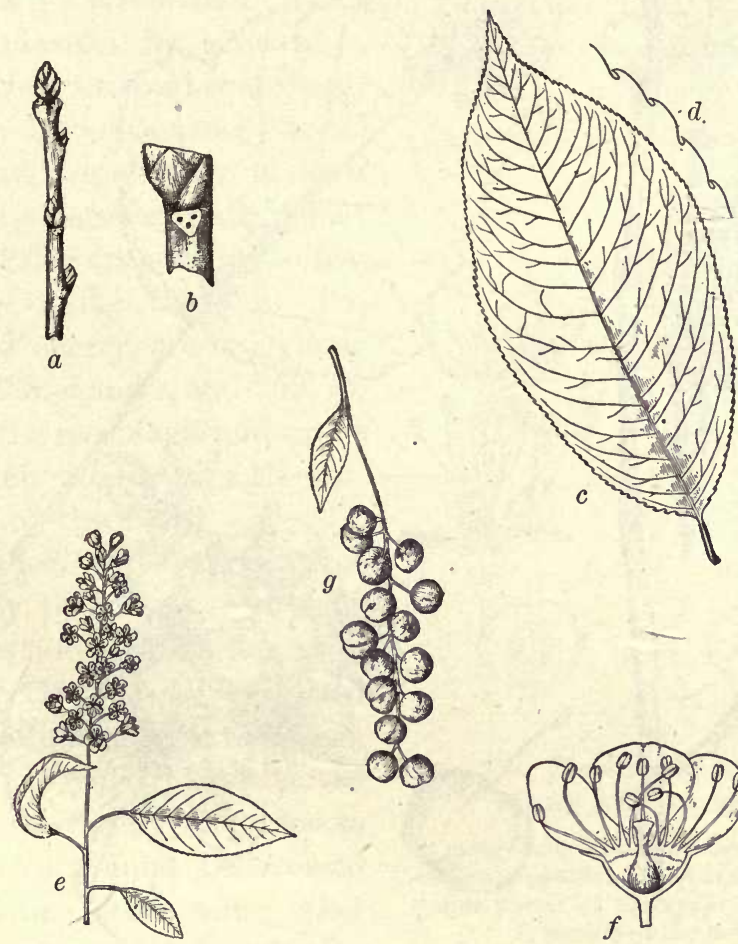

Fig. 257. Black cherry (Prunus serotina)

$a$, winter twig; $b$, portion of the same enlarged; $c, d$, a leaf and the leaf margin; $e$, racemose inflorescence; $f$, perigynous flower in section; $g$, fruit cluster. From "Michigan Trees." Photograph furnished by Dr. Charles H. Otis

In the ripening of the fruit the pistil forms the entire fruit, the inner portion of the ovary wall forming the stone and the outer portion the fleshy part of the fruit. The receptacle and the floral parts formed upon its upper margin disappear with the development of the fruit (Fig. 257, $g$, and 259,c). 


\section{MALUS (APPLES AND CRABS)}

The cultivated apples and crabs (Fig. 258) are known to have had a common origin from wild ancestors resembling the wild crabs of to-day. In many instances these wild ancestors are of

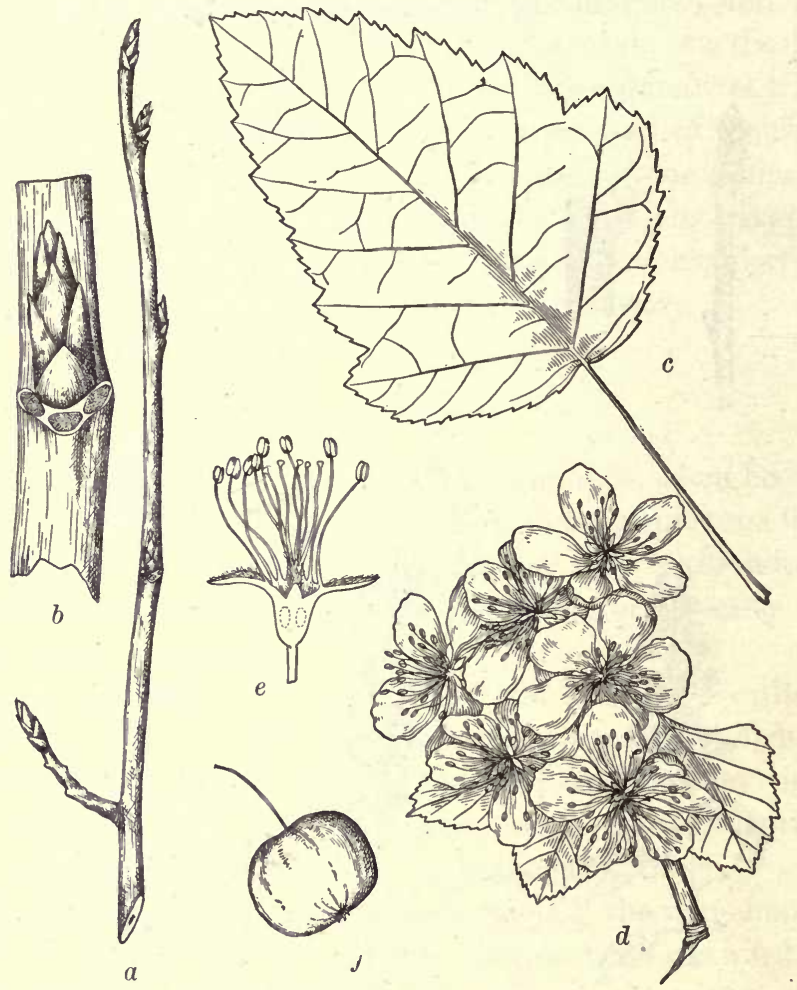

Fig. 258. American crab (Pymus coronaria)

$a$, winter twigs ; $b$, portion of twig enlarged showing leaf scar and bud; $d$, inflorescence; $e$, flower in long section, with petals removed, showing its epigynous character; $f$, fruit (pome). Photograph furnished by Dr. Charles H. Otis

American origin, but in other instances, particularly in the case of apples, the early ancestors are of European origin, and the cultivated varieties therefore originated on foreign soil. The plants are in all instances trees of a diffuse habit of growth and are of no particular value except for the production of fruit. 
Flowers and fruit. The flowers in most common apples are borne in clusters, on dwarf or spur shoots, which occur as lateral shoots on the more rapidly growing leafy branches of the tree. The buds which bear the flowers are mixed buds, bearing terminal flower clusters and a few leaves at the base of the year's growth. Since the spur shoot is usually terminated by a flower cluster, its growth in length from this bud is stopped, but may be continued by a lateral bud, which may in turn produce a terminal mixed bud and bear flowers and fruit. The flowers, unlike those of the rose and cherry, are epigynous (Figs. 258, $d$ and $e$, and 259, $e$ ), so that the receptacle and ovary are closely adherent in the formation of the fruit.

Cross-pollination takes place normally by insects, but some varieties are self-sterile; that is, they fail to be fertilized when pollinated with their own pollen. In such instances the self-sterile variety, in order to set its fruit, must be crossed with pollen from some other variety with which it is fertile.
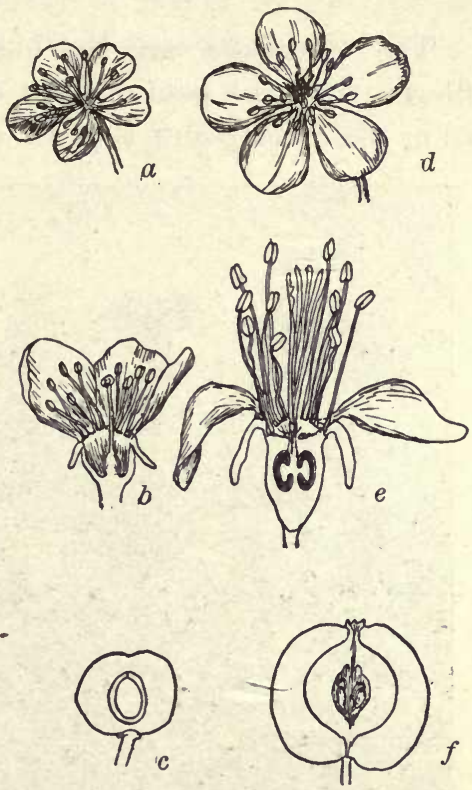

Fig. 259. Flower and fruit of the cherry and the apple

$b$, perigynous flower of the cherry, in section; $c$, section of the drupe; $e$, epigynous flower of the apple, in section; $f$, section of the pome

In the development of the fruit the ovary wall forms the membranous core and a portion of the flesh immediately surrounding it. The outer and greater portion of the fleshy part of the apple is, however, developed from the receptacle.

In the apple and many other members of the rose family the seeds are disseminated mainly by animals, especially birds, which eat the fruits and the seeds. The hard seeds resist the action of the digestive juices and are cast out with animal excrements, 
often at great distances from the mother plant. Marked exceptions to the above rule occur in Geum triflorum, which has hairy pistils which elongate and distribute the seeds by the wind.

\section{Compositae (Composite Family)}

The Compositae are the highest type of the Sympetalae, or flowers with the petals of the corolla united instead of separate as in the other plants thus far studied. They are represented by

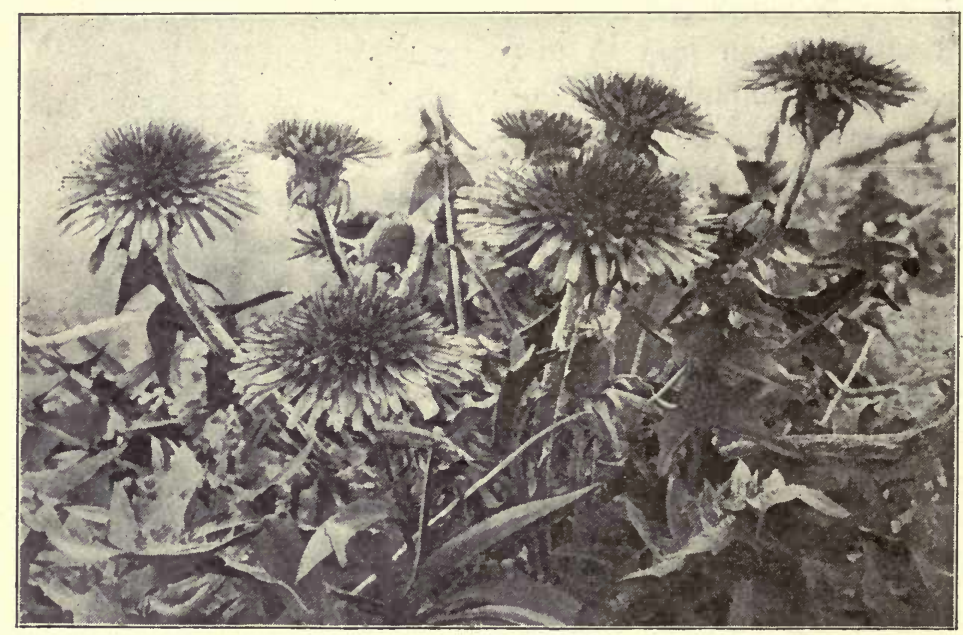

Courtesy of American Magazine of Forestry, Washington, D.C.

FIg. 260. Habit and flowers of the dandelion (Taraxacum)

Photograph by Dr. R. W. Shufeldt

such common species as the dandelions, asters, sunflowers, marigolds, and chrysanthemums. Very few of the species of Compositae, however, are represented in the spring flora, and hence the following discussion will be limited to a single species.

\section{ACHILLEA MILLEFOLIUM (YARROW)}

The yarrow is a perennial herbaceous plant with leaves dissected into narrow segments and with terminal flower clusters in the form of compound corymbs. It is common in spring by 
roadsides and in waste places and is a convenient type for study. The reproductive structures of the Compositae are so similar that when one type is mastered the variations from this type are easily understood.

Inflorescence. The flowers of the yarrow are borne in dense heads, each surrounded by a cluster of bracts, which together form the involucre (Fig. 261, A). Each separate head of flowers
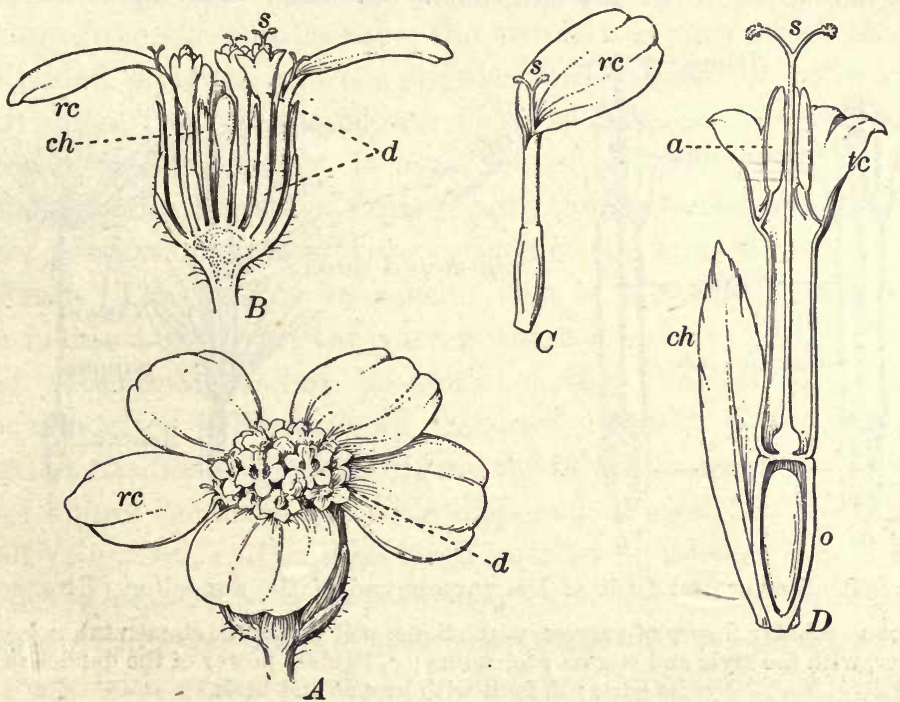

Fig. 261. Inflorescence and flowers of yarrow (Achillea)

$A$, single head, or inflorescence; $B$, section of the same; $C$, ray flower; $D$, disk flower; $a$, anther; $c h$, bract; $d$, disk flower; $o$, ovary; $r c$, corolla of ray flower; $s ;$ stigma ; $t c$, corolla of tubular flower

thus represents an inflorescence with a broad, flat receptacle on which the small flowers are closely crowded. The outer flowers bear an outgrowth of the corolla which is strap-shaped, while in the central flowers the corollas are tubular. The outer strapshaped flowers are the ray flowers of the head and serve to render it more conspicuous.

Flowers. The flowers are all epigynous, with the floral parts borne above and upon the ovary, which is composed of two sporophylls, or carpels. The perianth is composed of a corolla the 
parts of which are united to form a tube. The calyx is absent in the yarrow, but in many other Compositae it is split into a large number of fine hairs which together are called the pappus.

The essential organs comprise five stamens and a single compound pistil composed of two sporophylls, or carpels. The ovary bears a single seed and forms an achene in fruit. The style splits at the apex to form two lobes, the inner faces of which are roughened to form the stigmatic surfaces. The apex of each

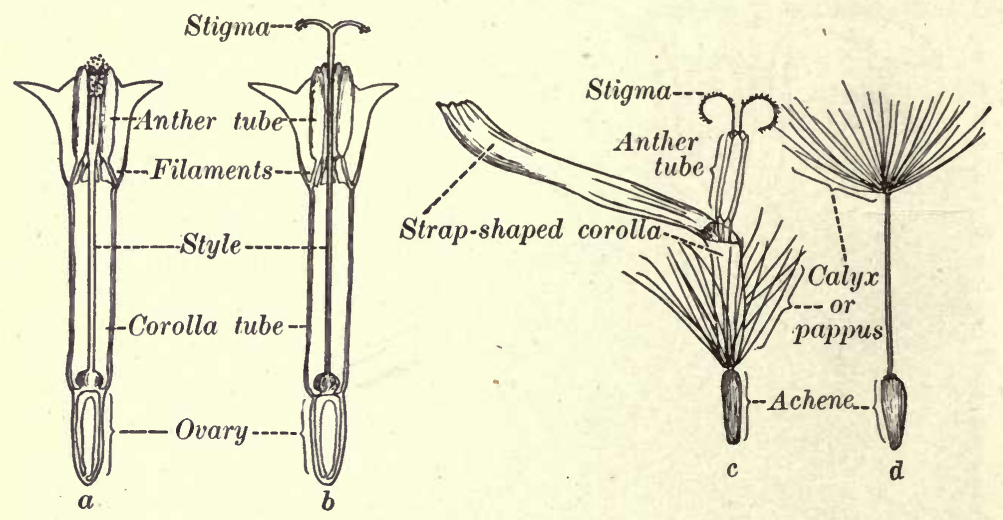

FIg. 262. Flower and fruit of the yarrow and of the dandelion (Taraxacum) $a$, young tubular flower of yarrow, with stigma still within the stamen tube; $b$, older flower, with the style and stigma protruding; $c$, ligulate flower of the dandelion and its parts; $d$, fruit with pappus and beak

stylar lobe is also furnished with a brush of hairs which together constitute the stylar brush. The stamens are attached to the corolla by slender filaments, and the anthers are united to form a tube around the style.

Pollination. The umbellate inflorescences of the yarrow form a continuous flat-topped floral mass which is rendered conspicuous by the white or pink ray flowers. Since there may be as many as one hundred heads in the entire inflorescence, with from fifteen to twenty flowers in each head, a visiting insect is certain to come in contact with a large number of separate flowers, all of which are especially adapted to secure cross-pollination. The mechanism of cross-pollination is concerned with the structure 
of the flower already described and with the relation of the anther tube and the stigmatic surfaces in young and old flowers. The flowers are protandrous, and in young flowers the pollen is shed while the style is short and the stigmatic surfaces closely approximated (Fig. 262,a). As the flower matures the style elongates and the stylar brush pushes the pollen out of the anther tube, where it may come in contact with an insect's body passing over the flower. When the end of the style and the stylar brush emerge from the corolla tube, the two halves into which the tip is divided separate, and the stigmas are exposed for pollination with pollen from another flower (Fig. 261, D, and Fig. 262, $b, c$ ). In case cross-pollination is not effected self-pollination may be brought about by pollen falling from the stylar brush upon the outer portion of the stigmatic surface in the same flower.

Fruit. The fruit is an achene, that is, a ripened ovary with one inclosed seed. In the yarrow there is no special device for seed dissemination, but in many of the Compositae, such as the dandelion (Taraxacum), Spanish needles (Bidens), and thistle (Carduus), special outgrowths in the form of the pappus or of spines facilitate the wide dispersal of seeds by animals or wind (Fig. 262, $c, d$ ). The large number of flowers that are so perfectly adapted to cross-pollination, and the special devices for disseminating the seeds, enable the Compositae to spread and to increase with extreme rapidity wherever opportunity is offered.

\section{DICOTYLEDONS AND MONOCOTYLEDONS}

The angiosperms which we have studied thus far have all belonged to the dicotyledons, or plants like the bean, mandrake, and marigold, which have two cotyledons in the embryo. The monocotyledons have but one cotyledon in the embryo, - a condition which is supposed to have arisen by the loss of one cotyledon in the ancestral dicotyledons. The close relationship of the two groups is further emphasized by the great similarity - between them in the structure of the flower and in the gametophyte plants resulting from the germination of the spores, and also by the occurrence of species with intermediate characters. 
The original distinction between the monocotyledons and the dicotyledons was based upon the number of cotyledons in the embryo, as the names signify, since the monocotyledons were known to have embryos with but one cotyledon, while the

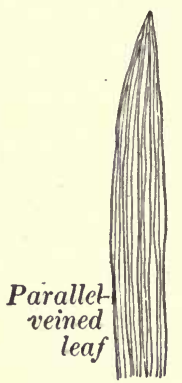

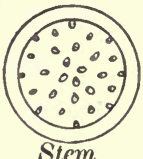

Stem

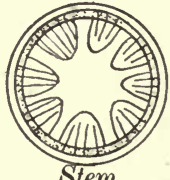

Stem

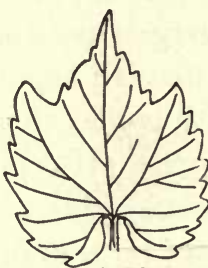

(vascular cylinder) Netted-veined leaf

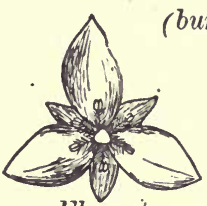

Flower'

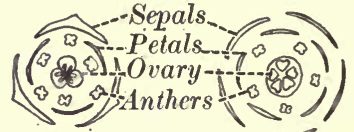

Floral plan, of three
Floral plan
of five

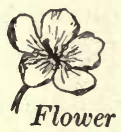

Flower

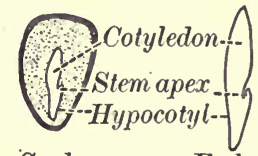

Seed

Embryo

(monocotyledon)
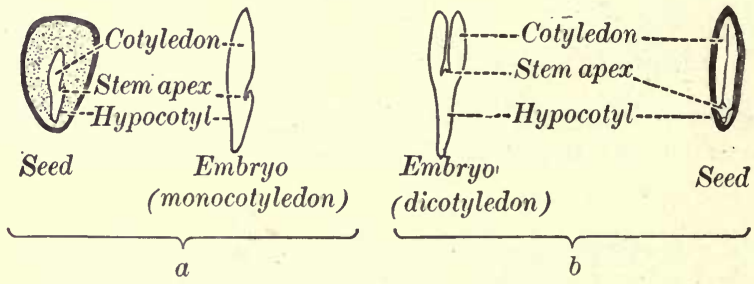

Embryo'

(dicotyledon)

Seed

Fig. 263. Comparison of monocotyledons and dicotyledons

$a$, vegetative and reproductive structures of a monocotyledon; $b$, similar structures of a dicotyledon

embryos of dicotyledons had two cotyledons, as in the common beans and peas. The mature plants of the two groups also manifest quite marked distinctions, which apply to the form and venation of the leaves, the structure of the stem, and the numerical plan of the flowers (Fig. 263).

The leaves of monocotyledons are usually linear with parallel venation, like the leaves of lilies and grasses. The venation is 
also designated as closed venation, since the veins do not terminate in the margin of the leaves, which therefore remain smooth. In dicotyledons the leaves are usually netted-veined, and the veins end free in a rough margin.

The stems of monocotyledons also have scattered vascular bundles without a cambium layer, while those of dicotyledons form a cylinder in which the cambium adds new tissue to the phloëm and xylem layers annually.

The flowers of the monocotyledons are habitually on the plan of three, while dicotyledonous flowers are more frequently on the plan of four or five parts in a whorl for each set of floral organs. These distinctions between dicotyledons and monocotyledons are graphically illustrated in Fig. 263 and are concisely stated in the summary below.

\section{SUMMARY}

The embryo of monocotyledons has but one cotyledon and a lateral stem tip, while dicotyledons have two cotyledons and a central stem tip.

The monocotyledons usually have leaves with parallel-veins, and the veinlets do not end free in the margin of the leaf, as in dicotyledons, in which netted venation prevails.

In the stems of monocotyledons the vascular bundles are scattered. In dicotyledons the xylem and phloëm form a cylinder inclosing the central pith.

The flowers of monocotyledons are in threes, but those of dicotyledons are in fours or fives. 


\section{CHAPTER XXI}

\section{MONOCOTYLEDONS}

Habitat and habit. The monocotyledons include over forty known families and about twenty-five thousand species of plants, the greater number of which are water-loving, being either true hydrophytes, like the pondweeds (Pontederia and Potamogeton), or semiaquatics, like the marsh grasses and sedges. A few forms, such as the yuccas and desert grasses, are xerophytic, while a large number should be classed as tropophytes, adapted to alternating seasons of moisture and drought. Among these plants are many of the wild and cultivated species in which the underground stem takes the form of a rhizome, bulb, or corm, which enables the plant to live securely underground during an inclement season. The favorable season with such plants is used for the growth of aërial stems bearing leaves which make food, and for the production of flowers, fruit, and seeds. The majority of these species have the characters already ascribed to monocotyledons, namely, narrow, parallel-veined leaves, aërial stems with scattered bundles, and flowers on the plan of threes.

Reproduction and seasonal life. The monocotyledons with underground stems reproduce sexually by means of flowers and vegetatively by means of corms, bulbs, runners, or tubers formed as offshoots from the mother plants. Vegetative reproduction facilitates local increase in the immediate vicinity of the mother plant, while seeds, formed as a result of sexual reproduction, facilitate the distribution of the species over wide areas. The seasonal life is therefore much the same as that of the white sweet clover and of the perennials outlined in the summary of the seasonal life of plants in Part I.

The commercial importance of the monocotyledons is due to the great beauty of many species, such as the lilies, tulips, and hyacinths, and to the value of other species for food and forage. 


\section{COMMELINACEAE (SPIDERWORT FAMILY)}

\section{TRADESCANTIA (SPIDERWORT)}

Habitat and habit. The spiderworts are among the commonest wild and cultivated plants of the spring and summer flora. They usually inhabit gravelly, sandy, or alluvial soils in woods or along railroads and river banks. In Tradescantia virginiana (Fig. 265) the stem is both aërial and subterranean. The aërial stem bears the long, parallelveined leaves and blue flowers characteristic of the species. The underground stem is tuberous and gives rise to one or more lateral flowering shoots by means of lateral buds. It also enables the plant to live over the late summer, autumn, and winter, furmished with buds and reserve
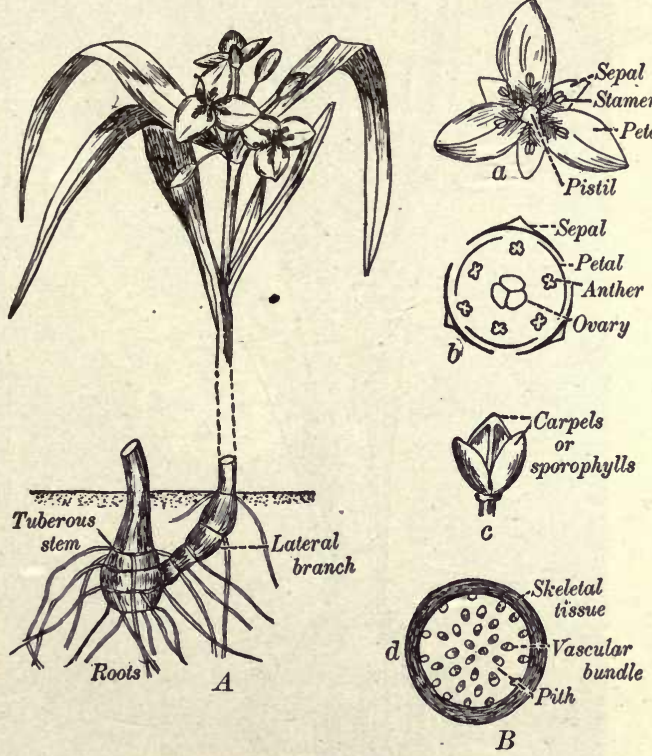

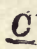

Frg. 264. Habit and reproduction of the common spiderwort (Tradescantia)

$A$, plant in flower; $B$, reproductive structures and stem section ( $a$, front view of flower; $b$, ground plan; $c$, dehiscent fruit; $d$, section of stem); $C$, leaf and parallel venation

food for the early spring growth. Tradescantia is thus a semixerophyte or semitropophyte, like many of the cultivated bulbous monocotyledons.

Reproduction. The blue or purple flowers are ephemeral, that is, lasting for a day only, and have their parts arranged in threes (Fig. 264, B) like other typical monocotyledons. 
Pollination is by means of insects, which visit the flowers in great numbers and pollinate the stigma, which protrudes beyond the anthers and hairy filaments. The fruit is dry and opens by

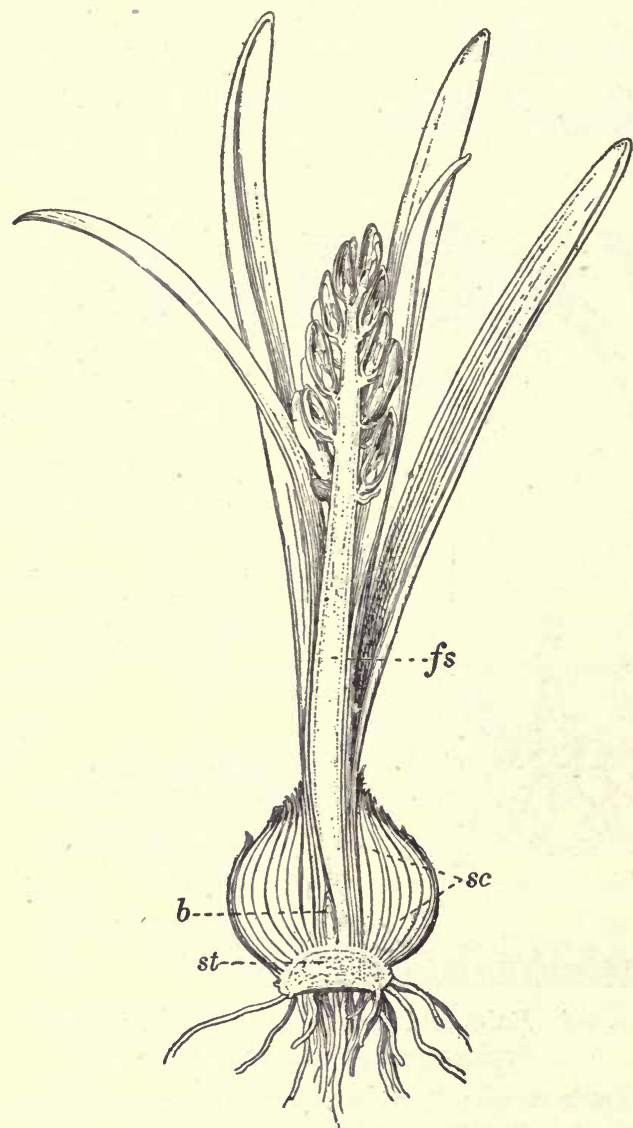

three valves, representing the three carpels of the ovary, and liberates the smooth black seeds for dissemination. Thus the plant reproduces in two ways, - sexually by means of flowers, fruits, and seeds, and vegetatively by means of tubers with lateral buds, which very often produce underground branches of considerable length.

Fig. 265. Lengthwise section of hyacinth plant $s t$, stem; $b$, young bulb for next year's growth; $s c$, bulb scales; $f s$, flower stalk. Reduced

\section{ILILIACEAE (LILY FAMILY)}

The Litiaceae are of principal interest on account of the large number of ornamental plants included among them, although food plants, such as the onions and asparagus, are also members of this important family. The ornamental plants include the common cultivated lilies, the spring tulips, hyacinths, narcissus, and lily of the valley. Among the wild species of greatest beauty are the dogtooth violet (Erythronium), the bellwort (Uvularia), 
Trillium, false Solomon's seal (Smilacina), and Ornithogalum (Fig. 266). Most of these plants spring from bulbs, tubers, or rhizomes, and so are adapted to a tropophytic existence and a

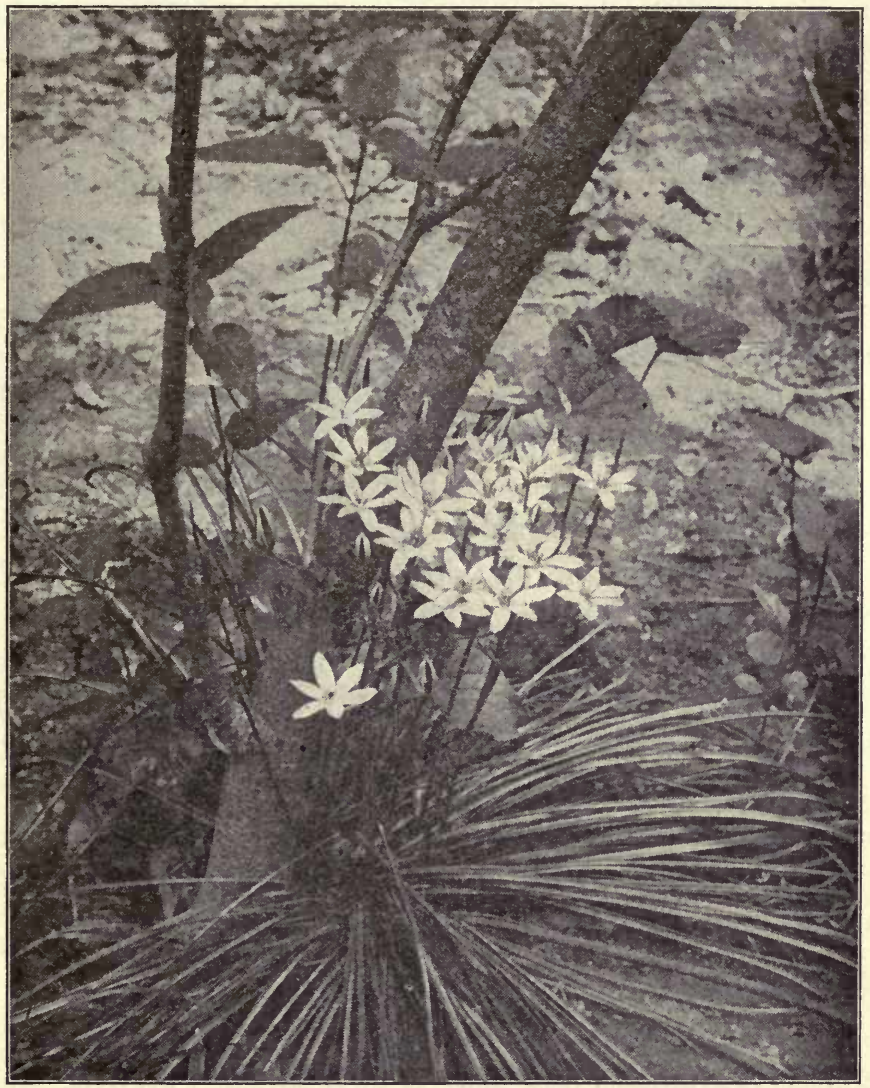

Courtesy of the American Magazine of Forestry, Washington, D. C.

Fig. 266. Ornithogalum, star of Bethlehem

Natural habitat and habit of a flower in bloom. Photograph by Dr. R. W. Shufeldt

corresponding seasonal life. Many of the cultivated species originated in arid regions, where the short rainy season is followed by a long dry period, as in California or the Mediterranean region. In such habitats the underground stem enables the plant to live securely during the dry season, while the great store of food in 
the bulbs, tubers, or rhizomes facilitates the rapid growth of aërial shoots and flowers during the short rainy season. In temperate regions the wild species often inhabit hillsides or banks where dry conditions are imposed during the summer months.

Commercial importance. This habit of producing underground stems facilitates the cultivation of the Liliaceae for commercial purposes, since the bulbs, corms, and rootstocks thus produced allow of shipments and long storage, which would otherwise be impossible. Tulips and hyacinths, for example, are grown in Holland for shipment to this country. The bulbs arise as lateral buds which produce the young bulbs or offsets in considerable numbers each year from the mother bulbs (Fig. 79). Ten or twelve daughter bulbs are produced from one mother tulip bulb in a season, and as many as twenty or more from a hyacinth bulb. These offsets are grown for from three to five years, then dried and shipped to various parts of the world for the growth of flowers for ornamental and decorative purposes. The biological and commercial history of the narcissus, lilies, onions, and other ornamental and food-producing varieties of the lily family is very similar to that briefly sketched for the tulip and the hyacinth. Most of these species have been greatly improved, as regards variation in color and size, by hybridization; but the perpetuation of such variation must be secured by vegetative reproduction, as we learned earlier in the study of reproduction, hybridization, and breeding.

\section{SMILACINA AND ERYTHRONIUM}

Habitat and habit. The species of Smilacina usually inhabit wooded slopes in the shade of trees, where a considerable amount of humus is present in the soil. They represent those species of the Liliaceae which have both an underground and an aërial stem, with the usual differentiation in function between the two kinds of stems. The aërial stem bears the leaves and the cluster of flowers of the summer season, while the underground stem serves for food storage, conduction, and the formation of buds for the next season's growth (Fig. 267,A). During dry periods, and in 

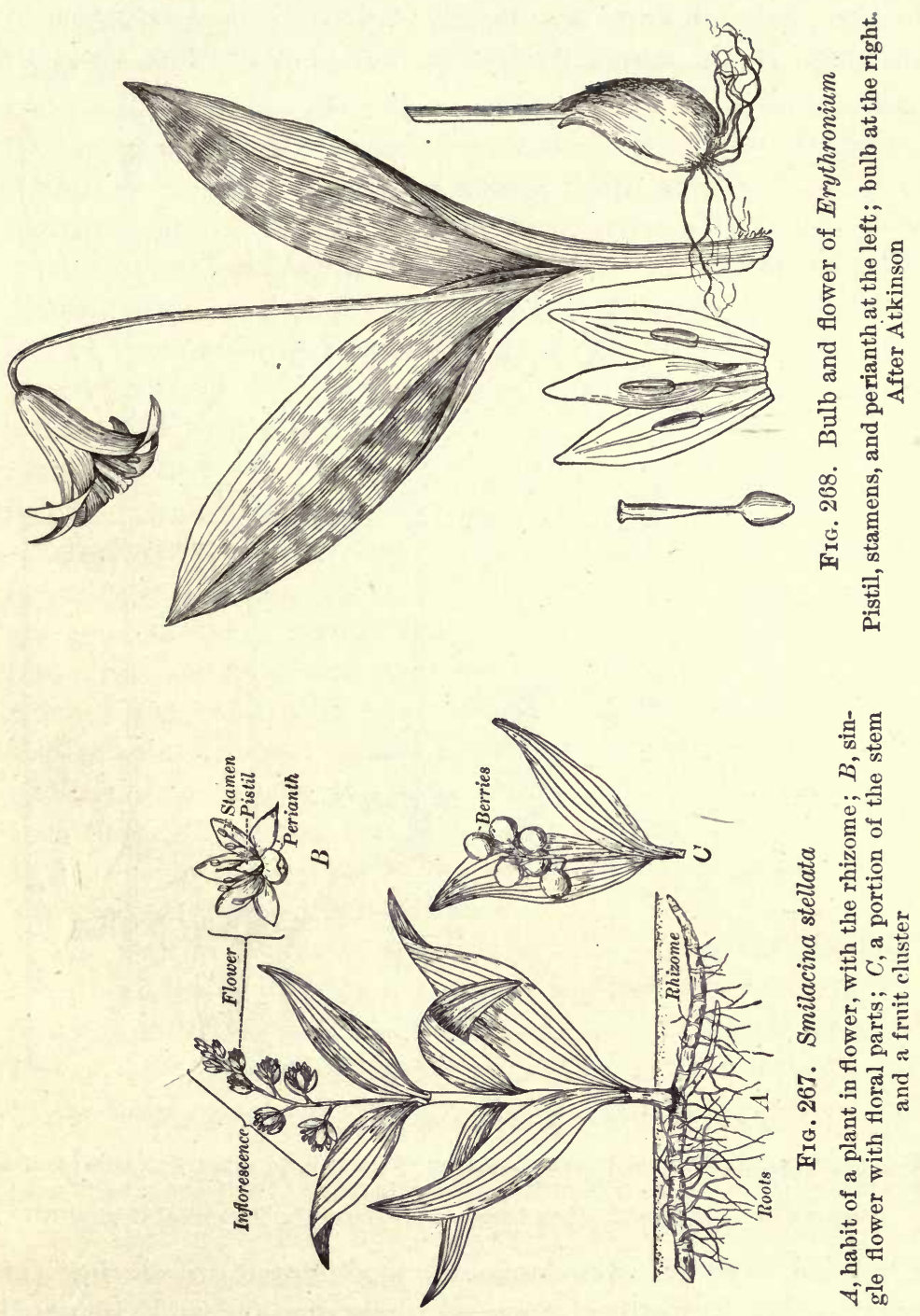
the winter months, the underground rhizome enables these plants to live protected from any danger of drought or destruction by freezing. In the spring the young buds and growing roots are

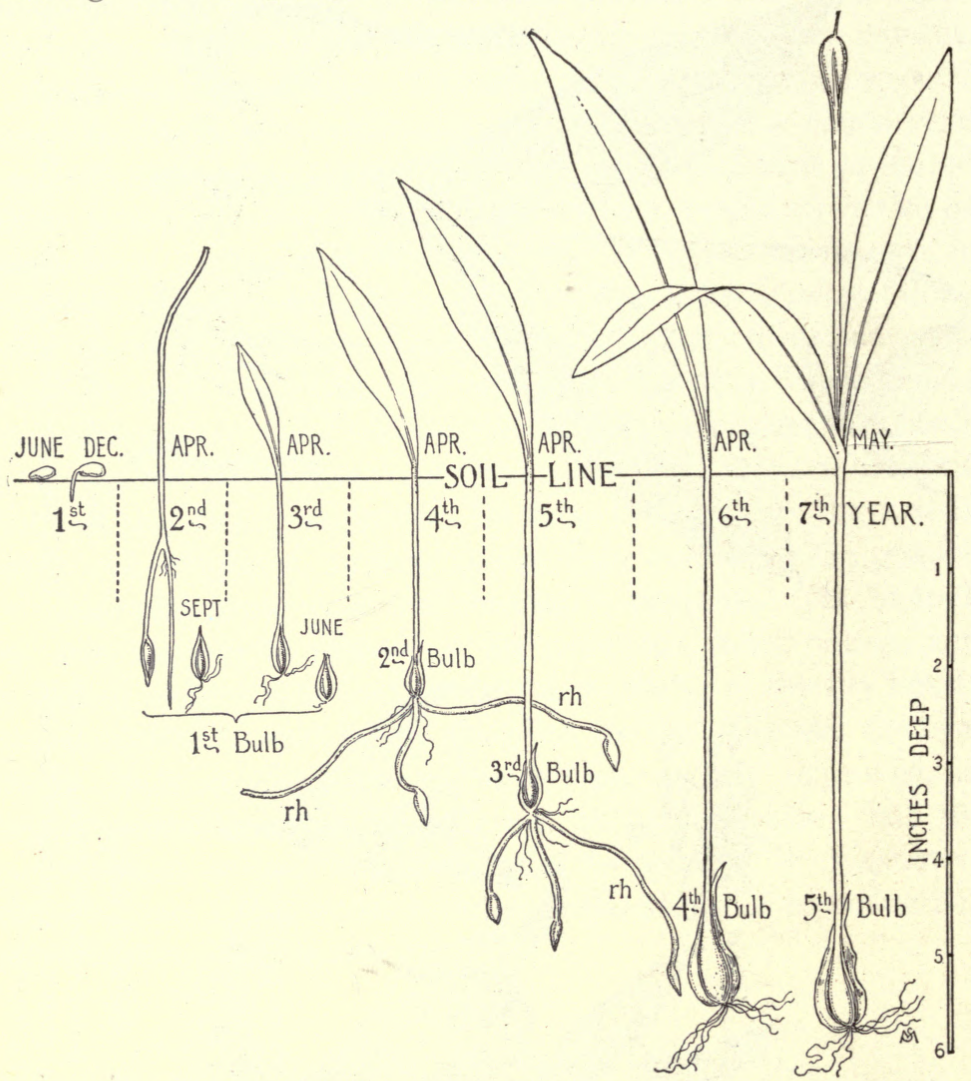

Fig. 269. Stages in the development of Erythronium

First year, germinating seed and seedling; second and third years, first bulb; fourth and fifth years, new bulbs being formed deeper in the soil; sixth and seventh years,

larger bulbs and plants. From Bergen and Caldwell's "Practical Botany"

furnished with an abundance of food stored up during the previous season in the rhizome and now digested and circulated for use in spring growth.

Erythronium (Fig. 268) occupies much the same habitat as Smilacina and has the same general habit and seasonal life. The 
underground stem is in the form of a solid, scaly bulb from which two characteristically spotted leaves and a single flower scape grow each spring. The most distinctive feature of Erythronium is the peculiar method by which the bulbs of each season become more deeply buried in the soil. The new bulbs are formed at the ends of runners arising from buds in the axils of the scale leaves. The runners of each season (Fig. 269) grow downward and so bury each new daughter bulb a little deeper in the soil than the mother bulb. Six or seven years are necessary for the production of a bulb strong enough to bear flowers. The seasonal history of Erythronium and Smilacina is therefore not unlike that of the tulip and hyacinth or of a perennial woody plant in which a long period of vegetative activity is necessary before reproduction by flowers and fruit is possible.

Reproduction. The flowers in Smilacina and Erythronium differ from those of Tradescantia in that both whorls of the perianth are colored like a corolla and are therefore not differentiated into a distinct calyx and corolla. This feature is also characteristic of the cultivated lilies, tulips, and hyacinths, to which Smilacina and Erythronium are closely related. Cross-pollination is effected by insects, since the stamens, when ripe, are shorter than the pistil, so that the stigma protrudes beyond the anthers. It is thus in a position to receive pollen from insects which have recently visited other flowers.

The fruit in Smilacina (Fig. 267,C) is a berry, and the seeds are disseminated when the berry disintegrates, or they may be distributed by animals which eat the fruit. In Erythronium the fruit is a capsule which splits into three valves corresponding to the three carpels of the ovary, thus disseminating the seeds.

\section{Iridaceae and Araceae (Iris and Arum Families)}

The species of these two families are of particular interest on account of the special adaptations of the flowers for securing cross-pollination by insects. Their vegetative characteristics are also of biological importance as indicating the characteristic habit of the aërial and underground parts of the monocotyledons already emphasized in Tradescantia and Liliaceae. 


\section{IRIDACEAE (IRIS FAMULY) \\ IRIS VERSICOLOR (COMMON BLUE FLAG)}

Habitat and habit. The common wild blue flag of the spring flora inhabits swampy land on the borders of streams and lakes

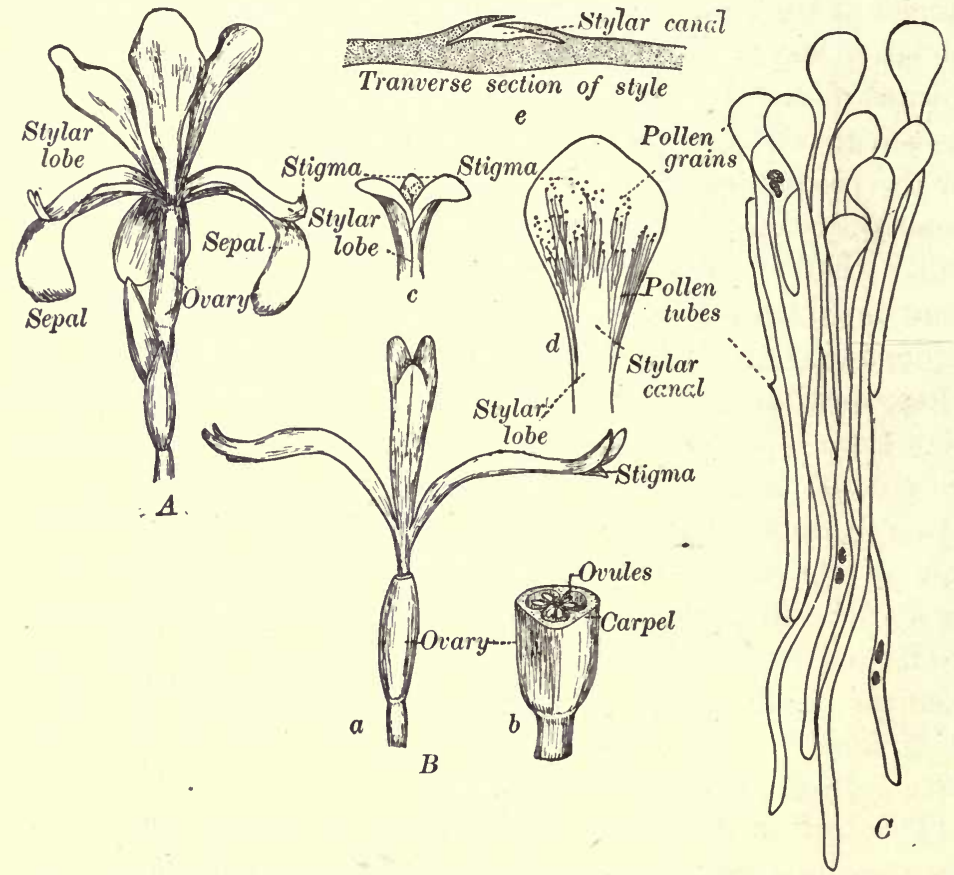

Fig. 270. Flower and reproductive structures of the iris (Iris versicolor)

$A$, flower and its parts; $B$, ovary, style, and stigma; $C$, pollen tubes

and is thus adapted to a hydrophytic habitat. Like many plants of such habitats, however, its habit, represented by the form and structure of its leaves and underground stem, is that of a xerophyte. This is probably due to the large percentage of organic matter, including organic acids, in the water of the soil which surrounds the roots of these plants in the marshes and swamps where they live.

The short aërial stem bears characteristic monocotyledonous leaves and highly organized flowers. 
The cultivated forms of Iris have a similar habit and also a similar floral structure, which has become highly modified by hybridization and selection with a view to increasing the size, color, and beauty of the flowers.

Reproduction. The flower (Fig. 270, A) is epigynous, with the floral parts arranged in sets of three each. The outer lobes of the perianth are large and highly colored, while the inner ones are smaller and less conspicuous. The three stamens spring from the base of the outer perianth lobes, and each stamen is beneath a branch of the three-lobed style. The stigmas are on the upper surface of a flap which grows out from each stylar lobe. When an insect visits the flower for nectar, it comes to rest upon one of the outer lobes of the perianth and then crawls down into the flower to probe for nectar at the base of the perianth. In so doing it dusts pollen onto its back, which cannot reach the stigmatic surface as it crawls out, but is in a position to pollinate a stigna of the next flower visited. The pollen germinates quickly on the stigma of Iris, and.the pollen tubes follow down the stylar canal in the center of the style to the ovules, where fertilization takes place. The flower is thus admirably adapted to cross-pollination by insects.

\section{ARACEAE (ARUM FAMILY) \\ ARISAEMA (JACK-IN-THE-PULPIT)}

Habitat and habit. The common jack-in-the-pulpit (Arisaema triphyllum) is one of the most familiar representatives of the Arum family in the spring flora. It grows naturally in moist humus soil in the shade of trees and is thus mesophytic in habit and habitat.

The aërial stem bears one or two leaves and peculiar greenish flowers. The underground stem is a turnip-shaped corm which bears the annual aërial shoot from a terminal bud.

The seasonal history of the jack is an interesting one and will serve as another illustration of the peculiar adaptations of some of the monocotyledons with underground stems for perpetuating themselves by vegetative reproduction. The new roots arise each year from the upper part of the corm (Fig. 271, $A$ ), while 
the nourishment is stored in the base of the corm for the early growth of the bud which produces the annual aërial stem. The new corm is therefore formed above the old corm each season, and the tissues of the latter disappear as the new corm is formed above on its remains. Large corms also produce lateral

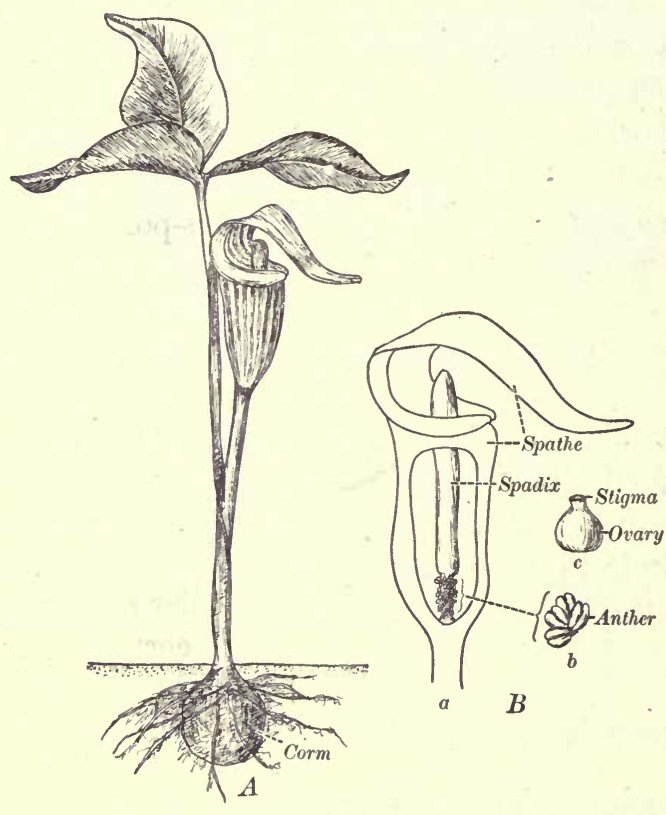

Fig. 271. Habit and flower of jack-in-the-pulpit (Arisaema)

$A$, plant with flowers and corm ; $B$ ( $a$, spathe, spadix, and staminate flowers; $b$, cluster of stamens; $c$, pistil). Copied

from Curtis's "Nature and Development of Plants" buds, similar to those of the gladiolus (Fig. 80), which give rise to a circular cluster of corms around the mother corm.

\section{Sexual reproduc-} tion. The flowers are borne on a fleshy axis called the spadix, which is included in a bractlike structure called the spathe (Fig. 271, $B, a$ ). The entire structure is often mistaken for a flower, although it is really an inflorescence.

Cross-pollination is assured, since male and female flowers are usually borne on separate plants. The male plants are also smaller, as a rule, than the female plants, which is -an advantage, since the female plants must produce seed and fruit and so need the great store of reserve food contained in the larger corms. The production of pistillate flowers from the larger corms is supposed to be connected with the abundant food supply. The fruit is composed of a cluster of beautiful red berries borne on the lower, fleshy portion of the spadix. 


\section{ORCHIDACEAE (ÖRCHID FAMILY)}

\section{CYPRIPEDIUM (LADY'S-SLIPPER, OR MOCCASIN FLOWER)}

Habitat and habit. The flowers of the lady's-slipper belong to the orchid family (the flowers of which are famous for their great
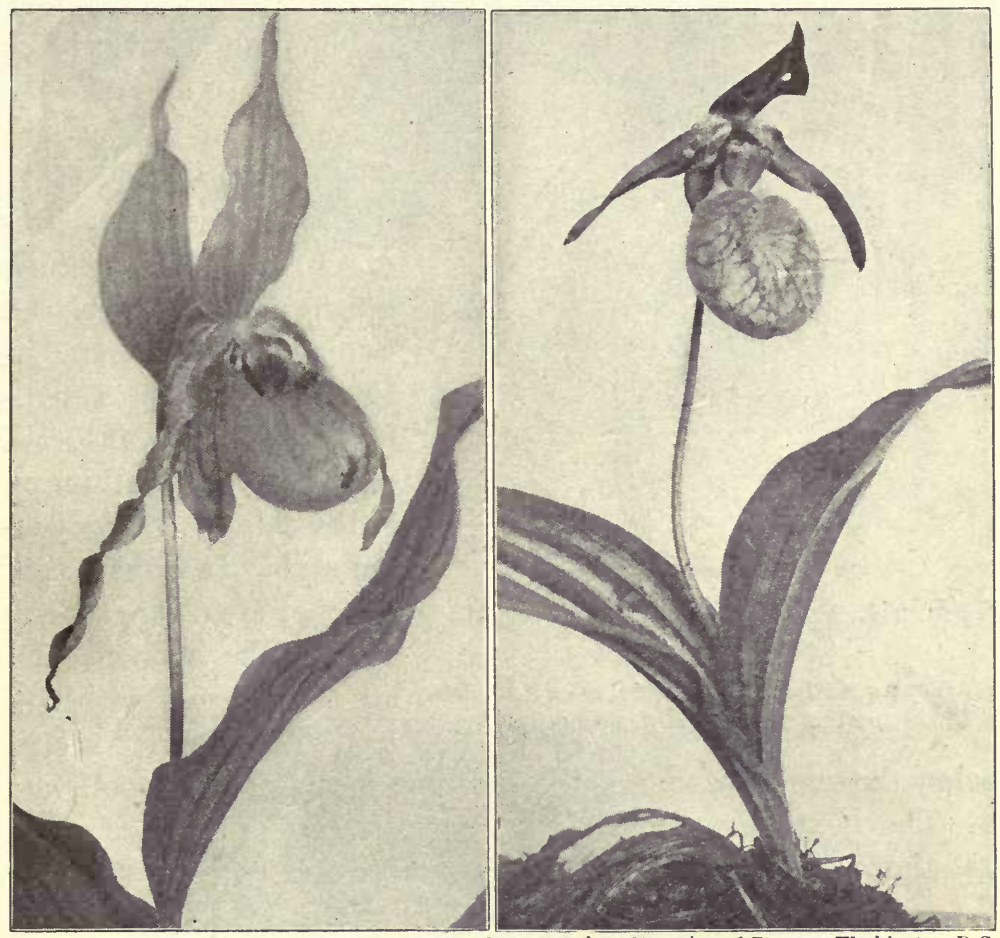

Courtesy of the American Maguzine of Forestry, Washington, D.C.

Fig. 272. The yellow lady's-slipper

(Cypripedium pubescens)

Photograph by Dr. R. W. Shufeldt
Fig. 273. The pink lady's-slipper

(Cypripedium acaule)

Photograph by Dr. R. W. Shufeldt

beauty as conservatory plants) and are biologically interesting on account of their wonderful mechanisms for securing crosspollination by means of insects. The plants inhabit moist, shady woods with soil containing plenty of humus, or, in the case of some species, low, marshy regions along streams, ponds, and lakes. Their habit is sufficiently illustrated in the text figures 
of the common yellow lady's-slipper (Cypripedium pubescens) (Fig. 272) and the pink species (Cypripedium acaule) (Fig. 273).

Reproduction. The flowers of the lady's-slipper are simpler than those of the true orchids, but are nevertheless very highly modified in such a manner as to prevent self-pollination. One of the petals is developed into a saclike structure (the labellum,

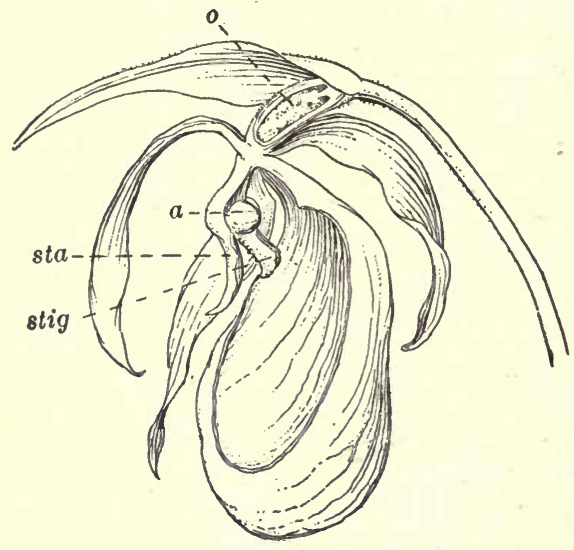

Fig. 274. Vertical section of a flower of Cypripedium acaule

$o$, ovary ; $a$, anther of a perfect stamen ; sta, imperfect stamen : stig, stigma or lip) observed in the figures, with a narrow entrance above for insects. The style is highly modified and projects into this opening to the labellum, bearing the stigma and two stamens on its underside (Fig. 274). Insects make their way into the cavity of the labellum on either side of the projecting style. In order to get out they are obliged to rub against the stamens and are thus dusted with pollen. The stigma is situated below the stamens, so that the outgoing insect does not pollinate it. The next flower visited, however, is certain to receive on its stigma the pollen from the flower previously visited.

\section{Alismaceae And Pontedertaceae}

\section{SAGITTARIA AND PONTEDERIA}

Habitat and habit. Sagittaria (arrowhead) and Pontederia (pickerel weed) are good examples of hydrophytic monocotyledons which inhabit shallow water on the margins of lakes or streams. In Sagittaria the flowering stem and leaves arise from stolons which are buried in the mud at the bottom of the lake or stream. In Pontederia the underground stems are rhizomes which give rise to a flower-bearing shoot and long-petioled leaves. 

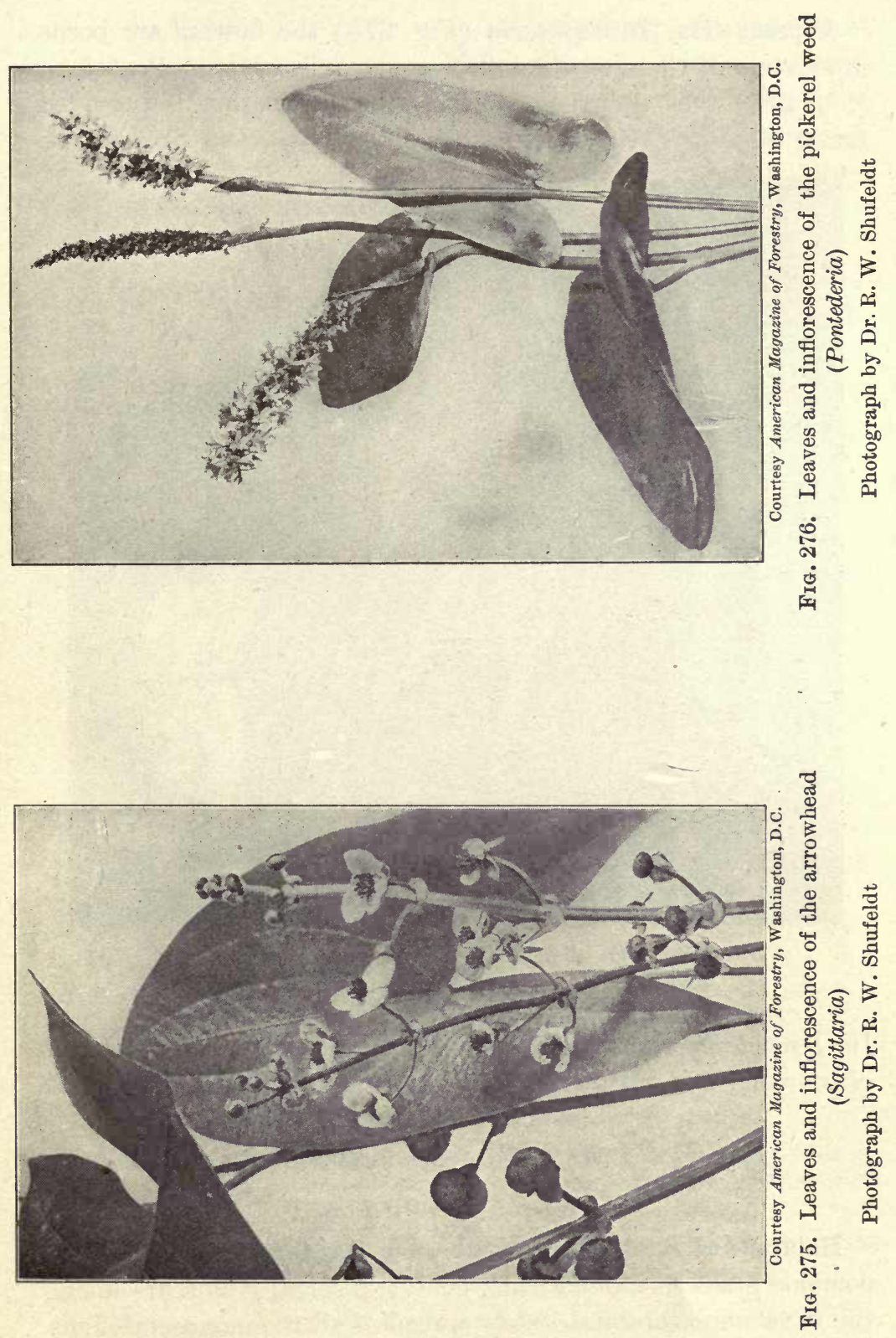
Reproduction. In S'agittaria (Fig. 275) the flowers are borne in clusters of three on the inflorescence axis, while in Pontederia (Fig. 276) they occur in spikes. Cross-pollination is provided for in Sagittaria, since plants are either moncecious or dioecious.

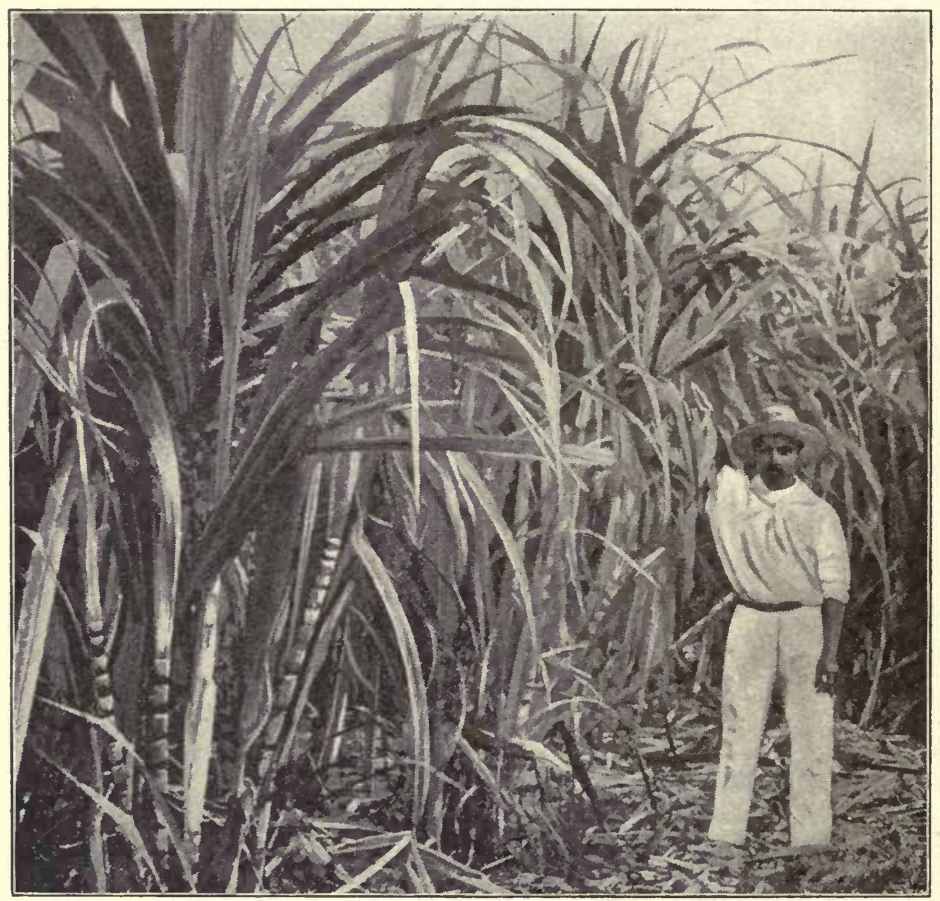

FIG. 277. A field of sugar cane at Vera Cruz After Freeman and Chandler

In Pontederia the flowers are trimorphic, with three lengths of stamens and pistils, which also insures eross-pollination.

\section{Gramineat (Grasses ANd Sedges) FORAGE AND FOOD PLANTS}

Habitat and habit. The members of this family, including the common grasses, bamboo, sugar cane, and cereal grains, are among the most important and widely spread of all the monocotyledons. The grasses proper are social plants, forming vast associations 


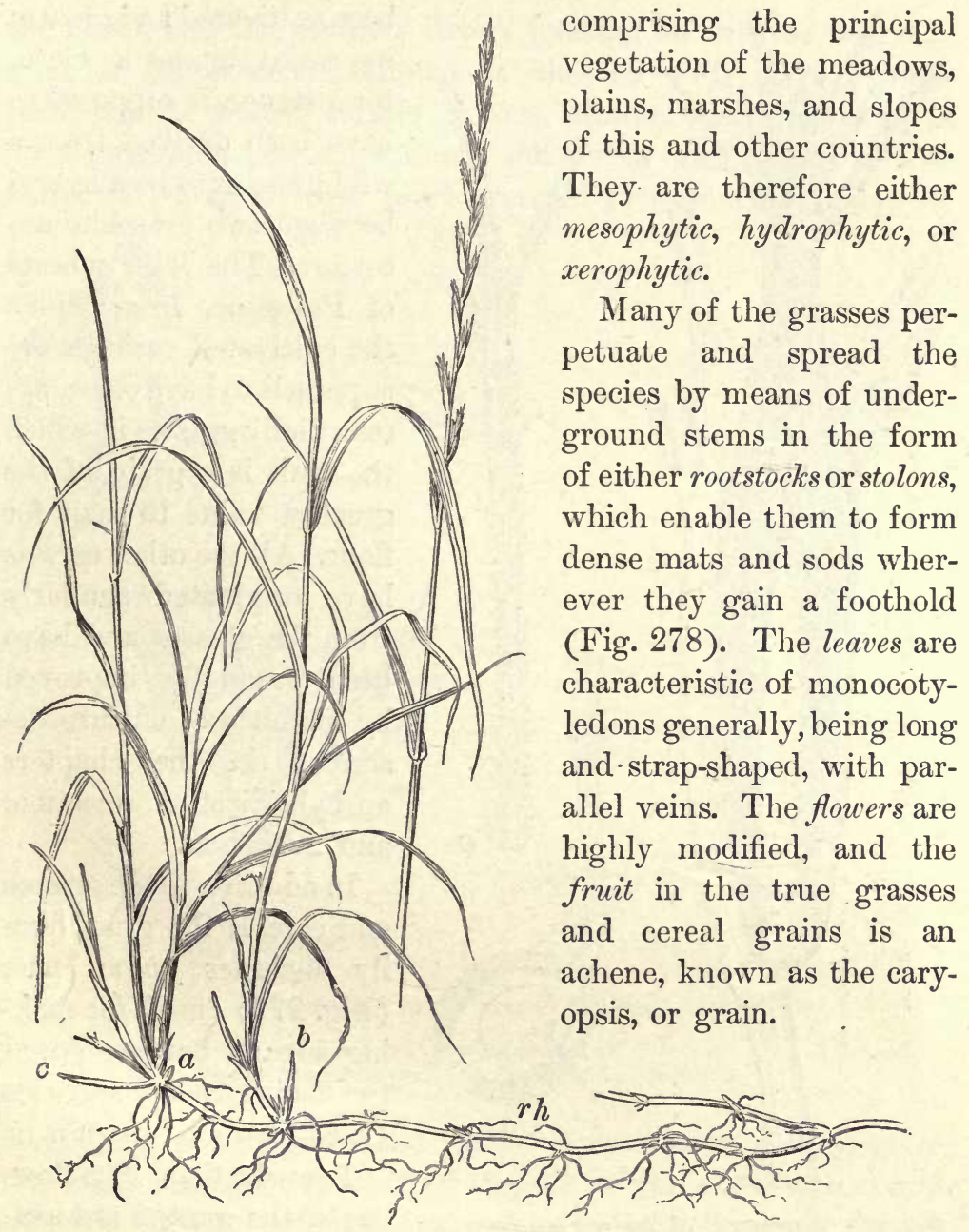

Fig. 278. Habit of the couch grass, a weed pest

Note that the aerrial stems spring from nodes of the underground rhizome. When the rhizome is cut in pieces, each node can reproduce a new plant and so spread the weed

Economic importance. The true grasses are of the greatest importance in furnishing pasturage and hay for animals and in providing a good turf for lawns and meadows. The cereal grains, including corn, wheat, oats, and rye, are all grasses which have 


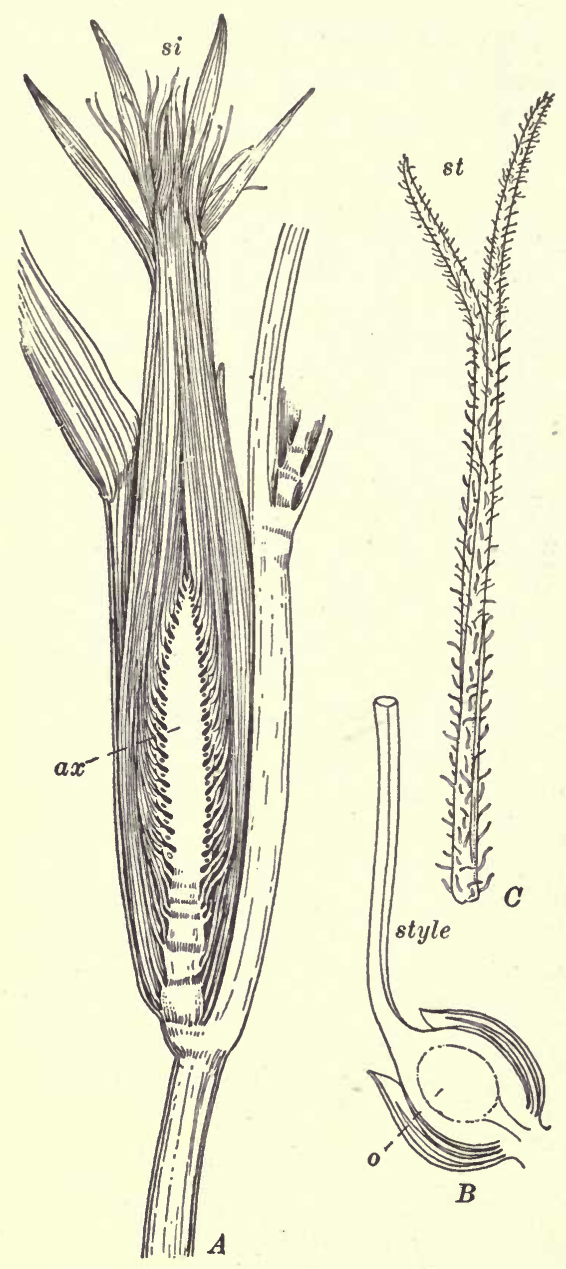

Fig. 279. Structure of an ear of corn, or pistillate inflorescence

$A$, section of a young ear, showing the cob, or axis of inforescence $(a x)$, and the silk, or style, and stigmas $(s i) ; B$, ovary, showing ovule $(o)$, and style; $C$, upper portion of style (silk) and stigmas, enlarged been cultivated and greatly improved by man. Corn, for instance, is supposed to have been derived from a wild grass or to be a hybrid between two grasslike ancestors. The wild wheats of Palestine, from which the cultivated varieties are supposed to have come, are essentially grasses in which the fruit is a grain of the greatest value to man for flour. All the other cereals have originated similarly from the grasses and have been gradually improved by methods of culture described in the chapters on hybridization, selection, and evolution.

In addition to the grasses and cereals the grass family includes sugar cane (Fig. 277) (used for making sugar), bamboo (used for fishing rods), and rice (used as a food plant).

Reproduction. The flowers of the grasses are usually very highly modified, and their relation to the flowers of the monocotyledons thus far studied is difficult to determine. In the corn plant (Fig. 102) the flowers are borne separately, the staminate flowers forming a compound inflorescence, known as 
the tassel, and the pistillate flowers forming an entirely different kind of inflorescence, known as the ear (Fig. 279). Crosspollination is almost certain in corn, although self-pollination is not impossible where the stamens and pistils ripen together. The anthers on a single corn plant are said to produce as many as
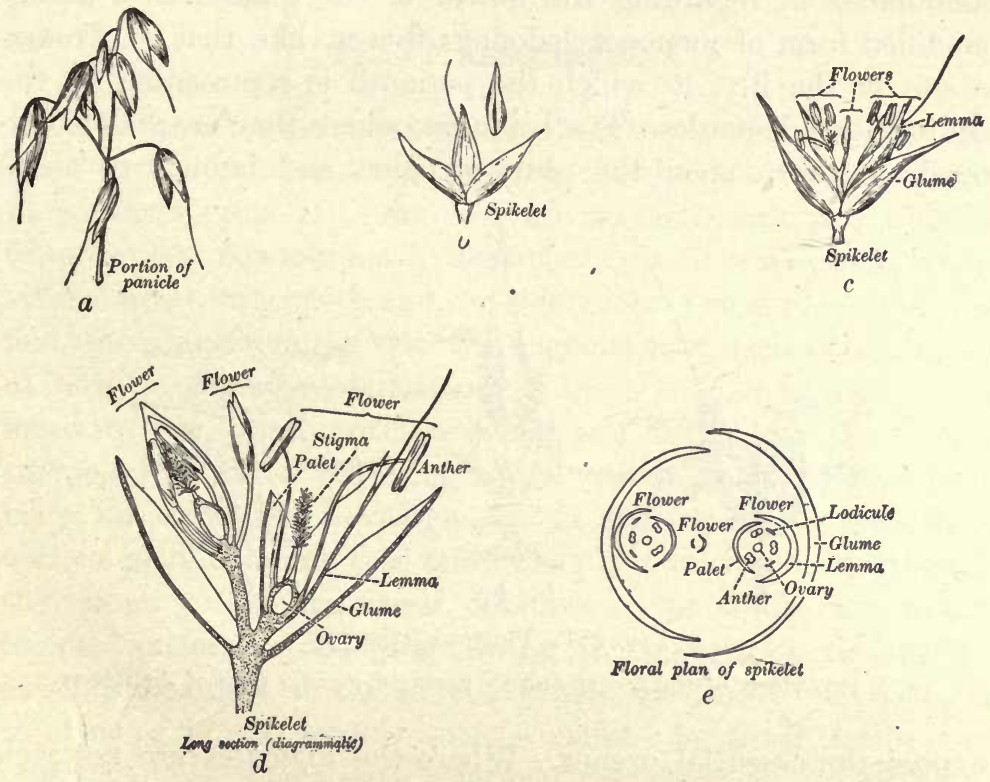

Fig. 280. Inflorescence and flower of the oat (Avena sativa)

$a$, portion of an oat panicle, or inflorescence; $b, c, d$, different views of the oat spikelet and its parts; $e$, floral plan of spikelet

$50,000,000$ pollen grains, so that complete wind pollination of the many stigmas constituting the silk of the ear is practically assured. Where pollination does not occur, the seeds do not develop.

The flowers of the true grasses and cereal grains are usually borne in dense spikes, like those of wheat and timothy, or in compound clusters made up of separate spikelets, like the panicle of oats (Fig. 280,a). The spike, like the panicle, is composed of several spikelets, each spikelet containing one or more flowers (Fig. 280, b-e). The flower proper (Fig. 281) is greatly reduced, 
being composed of a pistil with two plumose stigmas, three stamens, and two small rudimentary organs at the base of the pistil, called lodicules. Each flower is usually surrounded by two pairs of bracts. The outer bracts are called glumes; the inner bracts are called the palet and the lemma. Botanists are almost unanimous in regarding the flower of the grasses as a highly modified form of monocotyledonous flower, like that of Tradescantia or the lily, in which the perianth is represented by the rudimentary lodicules. The lodicules, where they are well developed, serve to open the glumes, palet, and lemma, so as to

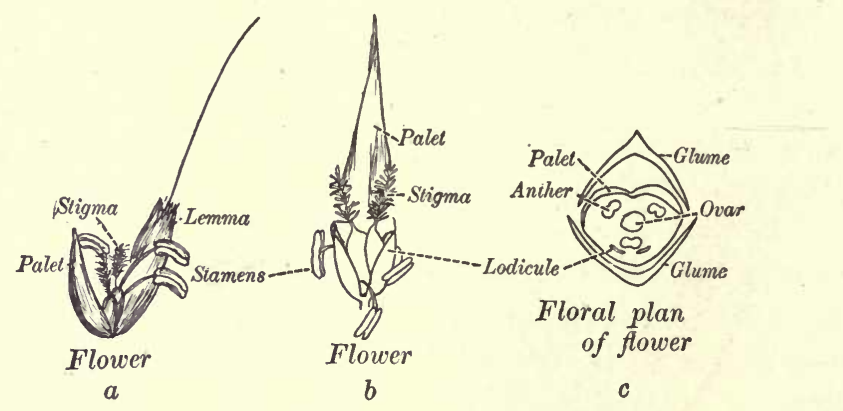

Fig. 281. Flower of the oat

$a, b$, two views of the flower and its parts; $c$, ground plan of the flower

expose the essential organs. Where the lodicules are not well developed, the flowers remain closed. Cross-pollination, where this occurs, is usually effected by the wind, the anthers being attached to the filaments at the middle, so as to be readily swayed to and fro for the scattering of pollen. In many of the cereal grains, like wheat and oats, the opening of the flowers and the act of pollination occur early in the day and are often completed in a relatively short time.

The fruit is the caryopsis, or grain, which is formed by a union of the ovary wall with the seed coat, making a dry indehiscent fruit. 


\section{CHAPTER XXII}

\section{PLANT ASSOCIATIONS}

In the previous chapters plants have been considered as separate individuals or as groups of closely related individuals known as species. In nature, however, each plant is a member of some plant community, living together with other plants which vitally affect its growth and development. The student will also find upon investigation that the vegetation of each region is not uniform in character, but is made up of smaller units, such as meadow, pond, and cultivated field and lawn, each of which is composed of plants requiring similar conditions of soil and climate for their best development. It will be found that these various plant communities, known as plant associations, although they seem to the uncritical observer to be stable, are nevertheless constantly changing, owing to competition among the existing members, to the advent of new species by migration, or to changes in the earth's surface caused by fires, floods, and other agencies, which have a far-reaching effect on vegetation.

Kinds of plant associations. The most general classification of plant associations is that mentioned in the first part of the text, namely, the mesophytic (Fig. 282), hydrophytic (Fig. 286), and xerophytic (Fig. 71) plant associations, based upon the available water in the soil. To this general classification a fourth should be added, to include the halophytic plant associations, which inhabit salt marshes and ponds where the amount of various salts in the water is excessive. These larger associations are usually subdivided into smaller ones, which reflect more accurately the particular environmental conditions prevailing in particular habitats.

Thus, the hydrophytic association comprises smaller pond, lake, stream, and swamp associations, which differ either in the kinds 
of species composing them or in the proportion existing between the constituent species of each minor association. In a similar manner, the xerophytic associations include lesser desert, dune, cliff, bog, and saline plant associations, while mesophytic plants form distinct associations in the form of forests, meadows, prairies, and cultivated fields. These large and small plant associations

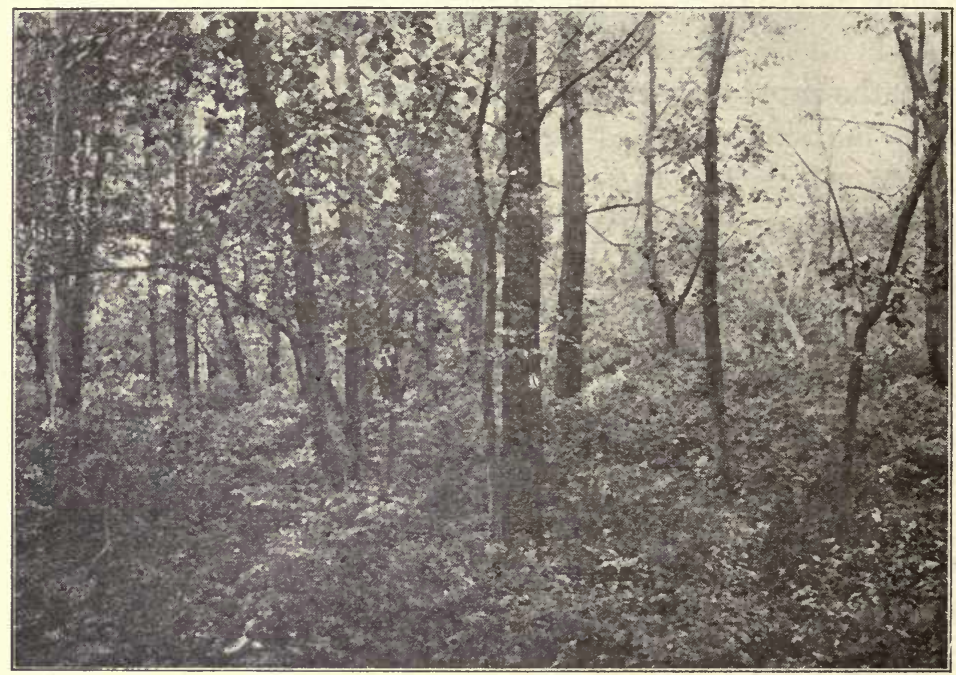

FIG. 282. A new mesophytic forest association (twenty-five years old) of honey locust, white elm, and black walnut

Photograph furnished by the United States Forest Service

form the units which comprise the vegetation of local areas and, finally, of the entire land surface of the globe occupied by plants.

Origin of new associations. In order to understand the important phenomena connected with the origin and development of plant associations it will be necessary to consider certain dynamic aspects of plant life, including the migration of plants from one locality to another, the invasion and occupation of new territory by such migrants, and the replacing of one plant population by another in new regions.

It is evident that the migration of plants by means of mobile seeds, fruits, or other reproductive parts must be a potent factor 
in the formation of new plant communities in denuded areas or in repopulating old ones where space still remains for the introduction of new individuals or species. It is clear also that the rapidity with which any given plant will invade such areas will depend in part upon the nature of the device which it possesses for seed dissemination. The student is already familiar with some

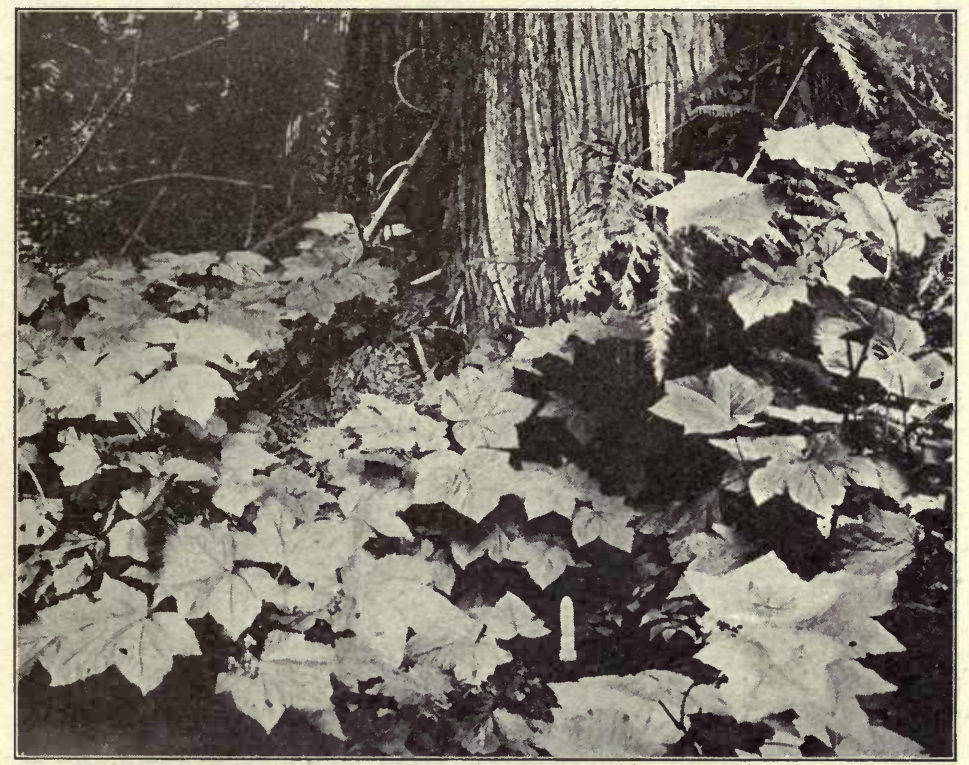

Fig. 283. Association of plants in a forest, - blackberries forming a layer associated with cedar (Thuja plicata), Cedar Mountain, Idaho

After Clements

of these devices in the plants previously studied in the field and laboratory. Thus, the wide distribution of willows and poplars along the borders of streams and on the shores of lakes or ponds is due to the long, silky hairs on the seeds, which enable them to migrate by means of air currents. The rapid migration and wide distribution of such composites as the dandelion and yarrow is due to the very effective parachute of hairs which serves as a flying apparatus for the fruits of these plants. In the case of fruits with wings, like the maples and pines, or of the heavy 
fruits, like the nuts of the hickory and oak, the devices are less effective and serve for local rather than for wide seed distribution. The seeds of edible fruits, like berries, apples, and cherries, are also very widely disseminated by birds and other animals, which cast the seeds with their excrements in regions far removed from the home of the mother plant. Almost innumerable

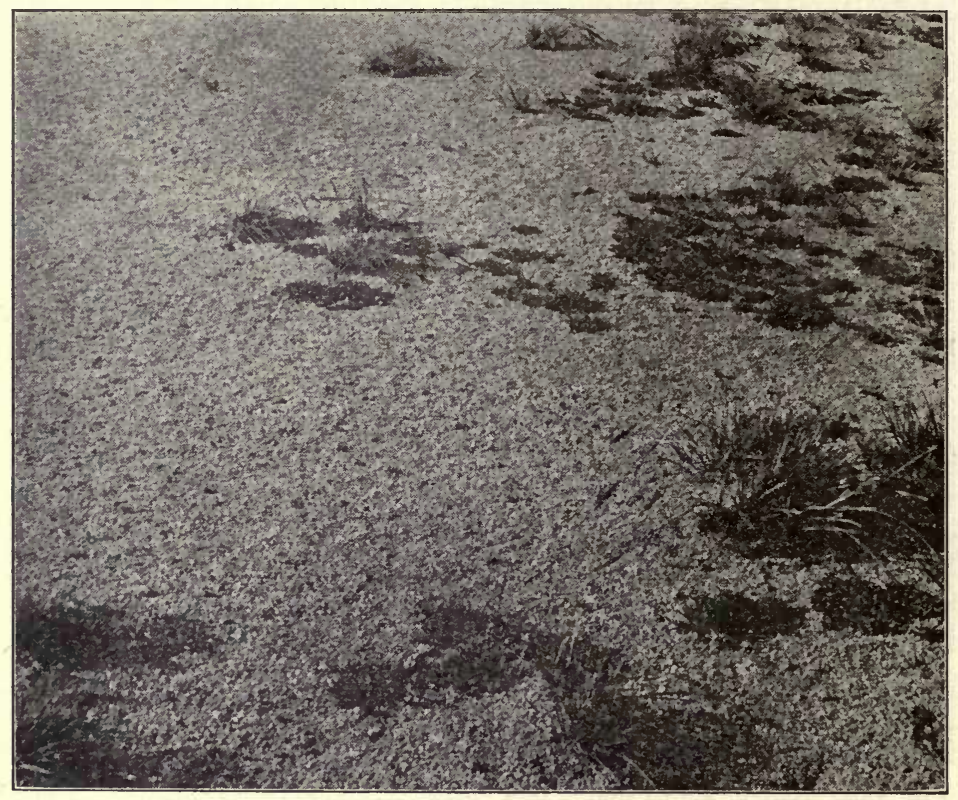

Fig. 284. Invasion of a grass, Agropyron, into bare sand by groups, Mount Garfield, Pikes Peak, Colorado

After Clements

examples might be added of other devices by which the mobile seeds, fruits, and other reproductive parts of plants migrate and invade new regions.

The immediate effect of such migrations and invasions (Figs. 284 and 285) in the formation of new plant associations is most easily observed where tracts of land occur which are devoid of vegetation. Such land surfaces may exist in gardens, lawns, and fields, or they may be the result of fire, flood, or other destructive 
agencies. In all such cases of denuded land areas the first invaders have a free field, without competition on the part of other plants, which is a very important factor in the success of invaders into old plant communities. The main restrictions on the occupation of these naked surfaces are those imposed by the nature of the invading plants themselves and by the environmental conditions obtaining in the invaded area. Thus, strictly water-loving

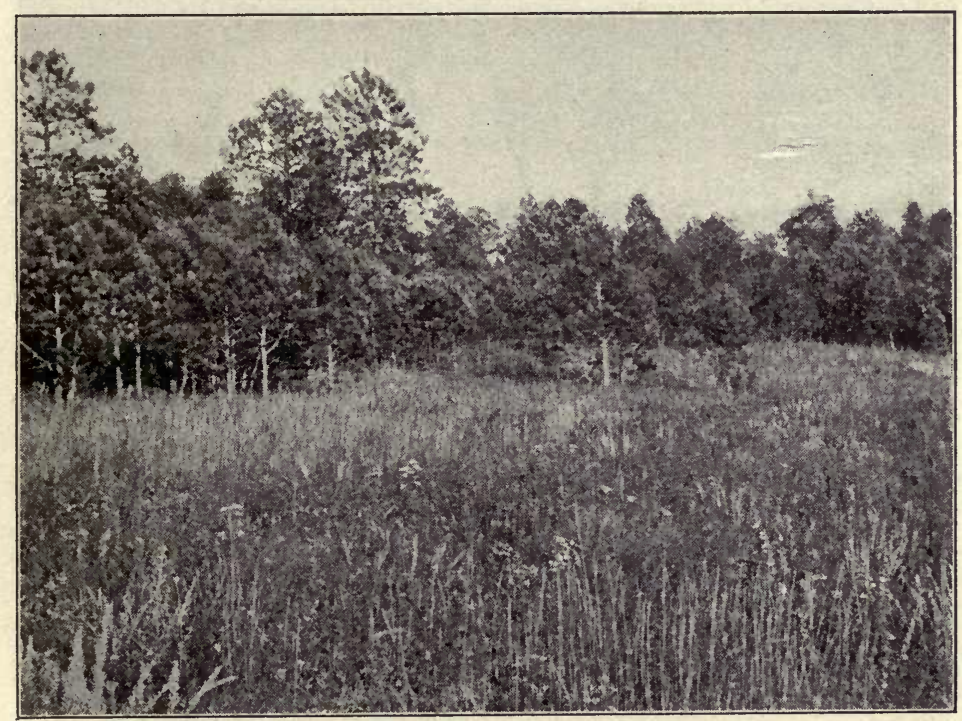

Fig. 285. Invasion of Pinus ponderosa into plains grassland, Black Forest, Colorado

After Clements

plants will not thrive on an upland tract, and plants accustomed to medium, or mesophytic, conditions will not grow or thrive in a desert soil. In other words, the invading species must be more or less closely adapted to the soil and climate of the invaded region in order to survive and become a permanent member of a new plant community or association. The first invaders are usually annuals or biennials furnished with mobile seeds, fruits, or vegetative parts. These are in turn succeeded by hardy perennials like the grasses, which may establish a permanent plant 
association. In other cases, as we shall learn later, the herbaceous plant association may be replaced in time by a shrub or a forest plant association. Many plants, also, when once established in a given locality spread by vegetative means and gradually drive out other competitors. This is notably true of plants with runners, stolons, rhizomes, and other underground parts, already discussed under vegetative reproduction in the first part of the

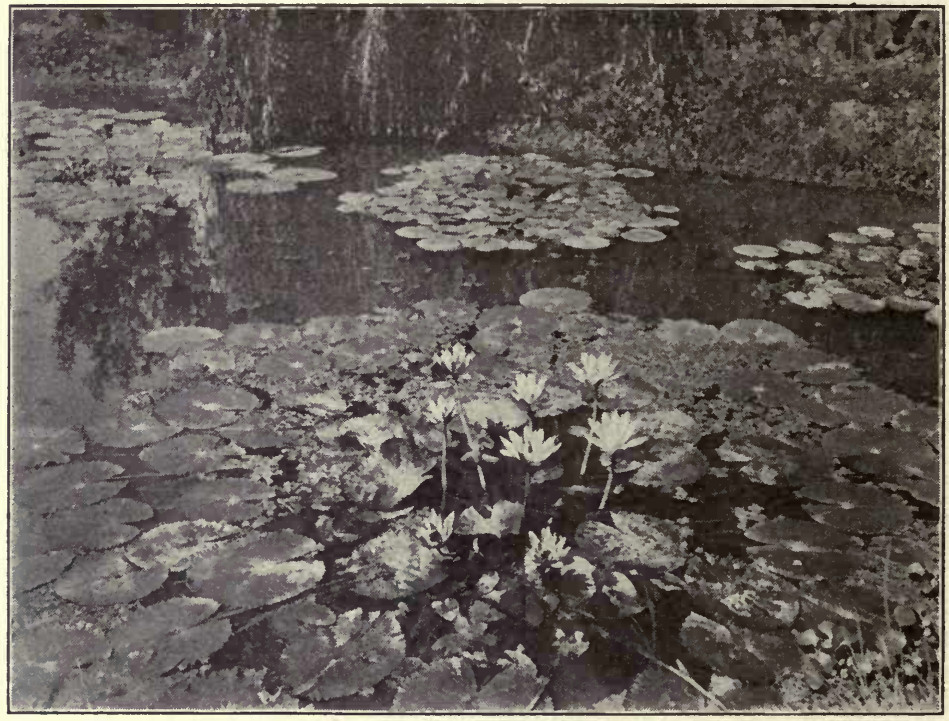

Fig. 286. The pond lily, an aquatic with floating leaves and submerged stems

text. In the end a new plant association will be formed, composed of plants adapted to the conditions of soil and climate which obtain in the given region. The above sketch of the main factors involved in the making of a new plant association on a denuded tract is probably a fairly accurate picture of the manner in which the existing plant associations which constitute the earth's vegetation have arisen.

Succession. The term succession involves the idea of replac-. ing the plants comprising a given association by a new plant population which invades and finally occupies the ground formerly held by the old association. This conception can be most 
easily explained by a concrete illustration of succession often exhibited in the history of a pond or lake which becomes gradually filled with soil and decaying vegetation. The plants comprising the first association in such a habitat are wholly hydrophytic and consist of free masses of algæ and of shore plants such as flags (Iris), bulrushes (Scirpus), and arrowhead

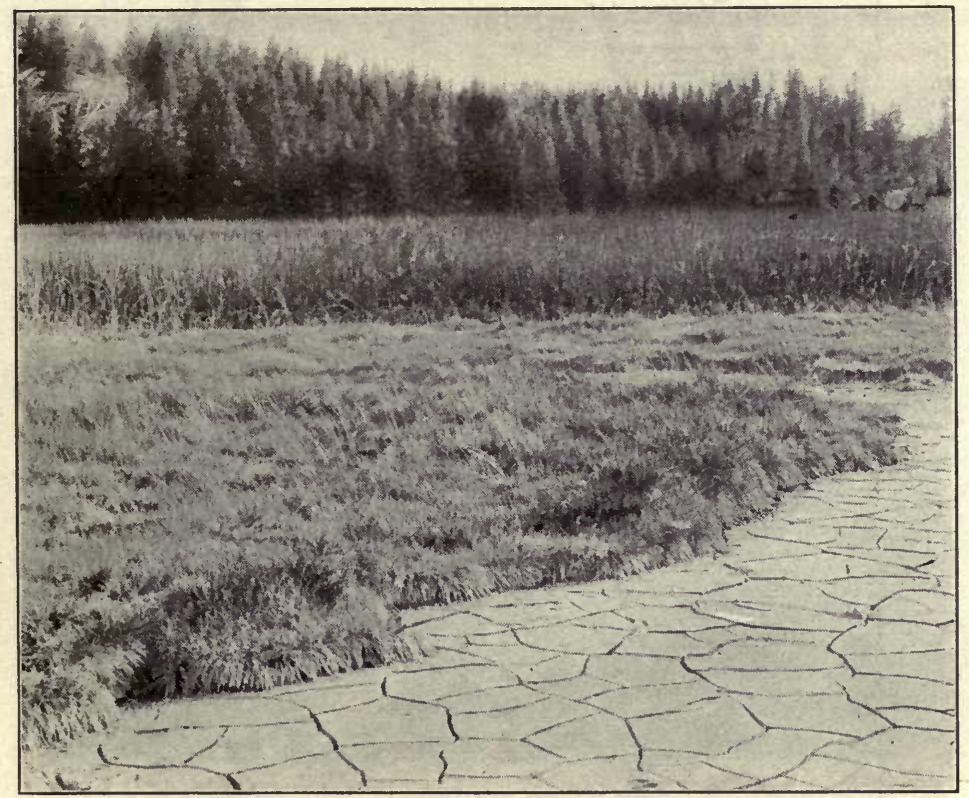

Fig. 287. Zonation of grass (Deschampsia), bulrushes (Scirpus), and pines (Pinus ponderosa)

After Clements

(Sagittaria). As the pond or lake becomes shallower by the accumulation of vegetable remains and the inwash of soil the shore plants encroach more and more upon the water area, followed by grasses and sedges which convert the old shore line into a marsh or bog. This marsh or bog may form a permanent plant association for a considerable period or it may be converted by willows, alders, and similar shrubs into a thicket, and then by poplars, ash, maples, and oaks into a typical mesophytic forest. 


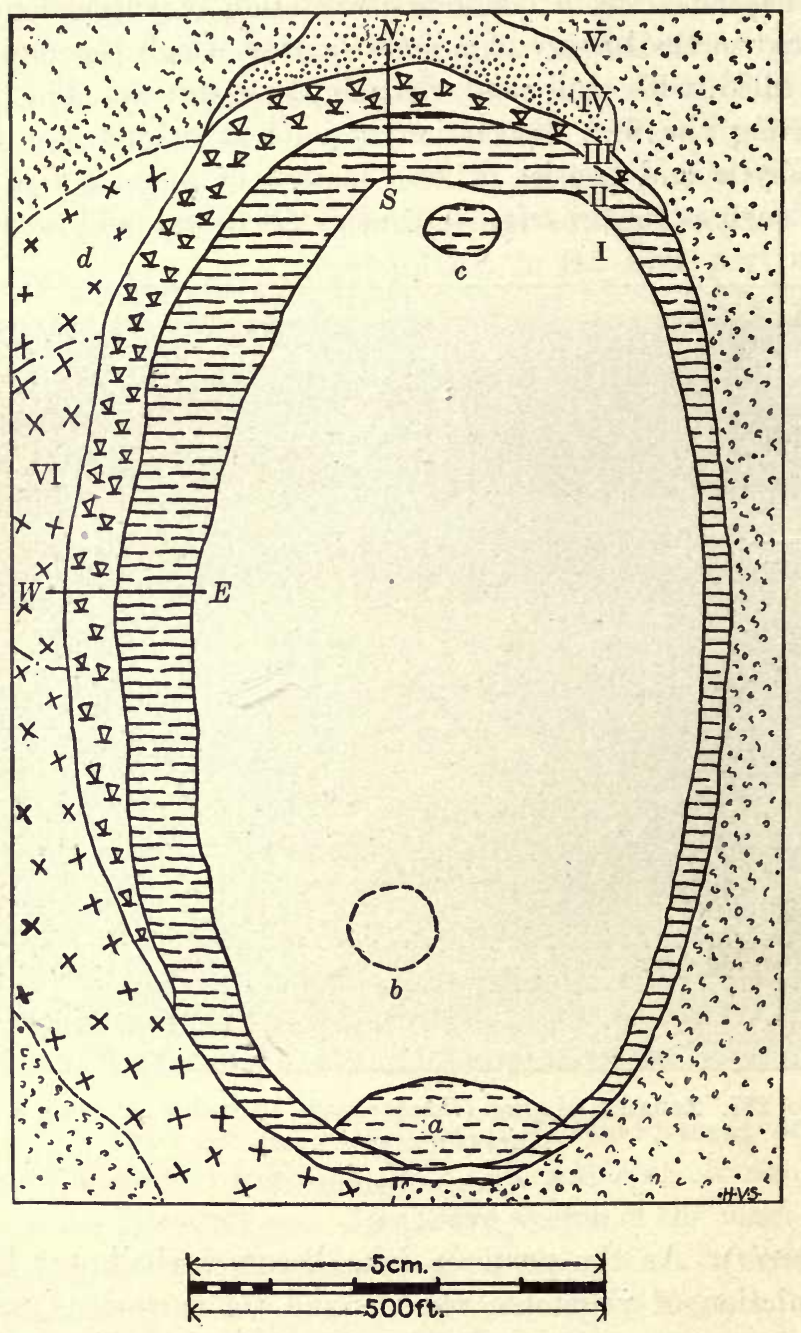

FIG. 288. Diagram illustrating zonation and succession around a pond $I$, pond ( $a$, floating pipewort (Eriocaulon) ; b, deepest portion of pond; $c$, rushes (Juncus) forming an association); II, bog zone ; III, swamp thicket zone; $I V$, sand pit and incomplete xerophytic zone; $V$, dry meadow zone; VI, dry woodland zone ( $d$, birch woodland). From Bergen and Davis's "Principles of Botany" 
The length of time required for one type of association to succeed and supplant another in such an instance as that just described, as well as the composition and nature of the plant populations which follow one another in any given succession, will of course vary in different cases, but the general facts regarding succession will hold for all similar habitats. Another familiar illustration of plant succession is often observed in forests where fires destroy the trees over wide tracts. The first invaders are

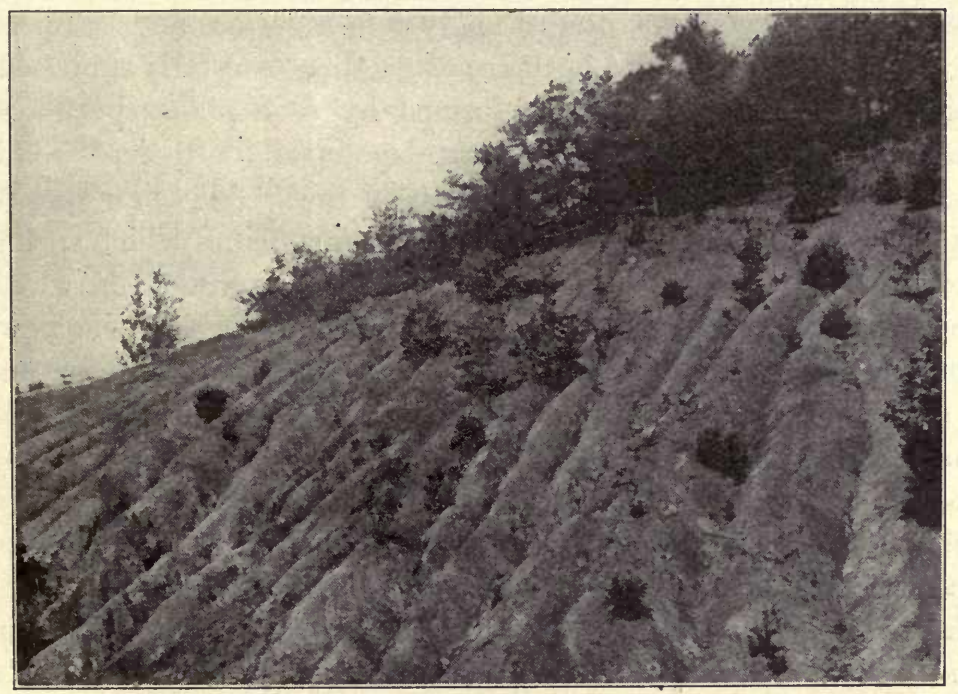

FIG. 289. A hillside once forested, but now bare and eroded Photograph by United States Forest Service

here, as in most other instances, herbaceous plants, including the fireweeds (Epilobium and Lrechtites), which are able to live and thrive in the burned-over area. The herbaceous plants are in time succeeded by aspens and conifers in some regions, or by forests of deciduous hardwood trees in others. Similar processes may be observed along the shores of most lakes and streams where new land is formed by changing water levels or in cultivated fields and gardens where land is plowed and allowed to lie fallow long enough for the first occupants to be routed by later competitors. 
The arrangement of plants in regular zones, termed zonation (Figs. 287 and 288), in regions where one association is being replaced by another, is often very evident, particularly on the borders of streams, ponds, and lakes. In some such instances the zones are very distinctly marked, while in others they merge gradually into one another, creating tension lines of great competition where two kinds of vegetation struggle for supremacy.

General instability of vegetation. The changes noted above in new plant associations, due to migration, invasion, and competition, are far more general in their nature than is usually supposed. The great numbers of seeds formed by each plant species in nature, and the admirable devices developed for their dissemination, result in a wide annual sowing of the seeds of new species in every old plant association. If the young plants which spring from these seeds are better adapted to the environment in which they chance to spring up than the existing species, the newcomers will gradually drive out the older inhabitants and become a new element in the population of the old association. Familiar instances of such changes are seen in the invasion of a lawn by dandelions, and of roadsides and cultivated fields by weeds. Old associations are thus constantly changing in the kinds of species constituting them. Another potent cause for far-reaching changes in existing vegetation is the gradual change going on in the surface features of the earth, caused by elevations and subsidences of the earth's crust and by water erosion. Geologists tell us that the continents are slowly but surely being leveled off by the wearing down of the hills and by the deposit of the eroded soil in the valleys by water action (Fig. 289). By these processes hills and slopes are denuded, new and barren cliffs are formed, and existing plant populations are covered and destroyed in the valleys and on flood plains. These constant readjustments initiate new plant associations, which form the first of a series of plant populations, followed by successions such as those described above. What is known as vegetation is therefore in constant flux and change, although the individual plants which comprise it are themselves immobile. 


\section{SUMMARY}

The vegetation of the earth's surface is not homogeneous but is composed of units of varying composition which are termed associations. These units of vegetation have apparently originated, as new associations arise to-day, in regions where the land is partially or wholly deprived of its vegetation by natural or artificial means. Some of the main factors concerned in the origin and development of a new plant association are the migration of plants by means of reproductive parts, the adaptation of plants of different kinds to different environmental conditions, and competition between the individuals of a growing plant population. Plant associations have also been found to be constantly changing, owing to competition and to changes in habitats due to natural and artificial causes. The general instability of existing vegetation is the natural outcome of these various factors, which are in continual operation over large areas of the earth's surface. 



\section{INDEX}

(References to illustrations are indicated by asterisks accompanying page numbers)

Absorption, mechanism of, 137

Absorption by roots, 139

Acaulescent, 353

Accessory buds, 356

Acer saccharum, 88*, 390*, 391*

Aceraceae, 390, 393

Achene, 364, 365, 412*

Achillea, flower of, 411*, 412*

Achillea (yarrow), 411*

Acorn, 385*

Acuminate leaf apex, 355*

Acute leaf apex, 355*

Adiantum, embryo of, 309*

Adiantum, gametophyte of, 309*

Adiantum (maidenhair fern), 297*, 299*, 300*, 301*

Adjustments, summary of, 44

Adjustments of American elm, 43*

Adjustments of bean leaves, 28*, 29*

Adjustments of caladium, 38*

Adjustments of clover, 39*, 40*

Adjustments of common plants, 37

Adjustments of corn roots, 32*, 33*, 34*

Adjustments of dandelion, 41*

Adjustments to environment, 23-45

Adjustments of garden pea, 24*

Adjustments of nasturtium, 26*

Adjustments of plant body, 14

Adventitious buds, 356*

Adventitions roots, 357

Aciospores (æcidiospores), 274, 275*

Aërial roots, 357

Aërobic, 249

Aggregate fruit, 364*, 365, 405*

Air spaces of leaf, 115

Alder, stem structure of, $91 *, 92 *, 93 *$

Alfalfa, roots of, 112

Algæ, 219-241

Algæ, life histories of, 228, 229

Alismaceae, 428-430

Alnus, stem structure of $92 *, 93 *$

Alnus mollis, stem sections of, $91 *$

Alternation of generations, 292*, 311* A manita muscaria, 267

Ambrosia, 19
American crab, 408*

Anaërobic, 249

Anaphase, 75*, 77

Anatomy, 213

Anatropous ovule, 363

Anatropous ovule of Iris, 347*

Anemophilous, 361

Angiosperms, 337

Angiosperms, trees of, 380

Animate environment, 7

Annual bean plant, physiology of, 127*

Annual bean plant, seasonal history of, 126-130, 129*

Annual bean plant, summary, 130

Annual ring, 85*

Annulus of fern sporangium, 306*

Annulus of mushroom, 268*, 269

Anther, 162*

Anther, structure of, $343 *$

Anther and sporophylls, 341*

Antheridium, 288, 289

Antheridium of Ricciocarpus, 231*

Anthracnose, 282

Antipodals, 164, 165*

Antitoxins, 256

Apogeotropic, 20*, 30

Apophototropic, 30

A potropic, 30

Apple, flower and fruit of, 409

Apple tree, body plan of, 15*, 16*

Aquilegia (columbine), 399*

Araceae, 425, 426*

Archegonium, 289*

Archegonium of Ricciocarpus, 289*

Arisciema, 426*

Ascent of water, 144, 146

Ascent of water, path of, 141*

Asci of lichen, 278*, 280

Asclepias, leaf structure of, 113*, 115*

Ascophyllum, habit of, 236*

Aspergillus, 266*

Aspidium, fern, 307*

Assimilation, 125

Assimilation of bean plant, 129

Associations, origin of, 436

Associations, plant, 435,445 
Avena sativa, flower of, 433*, 434*

Axil of leaf, $17 *$

Axillary branch, 17

Axillary bud, 356

Axis of bud, 68*

Axis of strobili, 339*

Azalea, flower of, 359*

Bacteria, 250, 258

Bacteria, cell division in, 252*

Bacteria, colonies of, 257*

Bacteria, forms of, 251*

Bacteria, spore formation in, 253*

Bacteria, spore germination in, 254*

Bark, 84, 85*, 86*, 90, 101*

Bark, formation of, 102

Bark in Salvia, 105

Basidia of mushroom, 269*, 270

Bean, growth of, $67 *$

Bean, sensitive roots of, 34,36

Bean plant, seasonal life of, 126, 127*, 129*

Bean pulvini, 15*, 16*

Bean roots, sensitiveness of, 36

Bean seedlings, 4

Beer making, 250

Ber̂ry, 364*, 365, 421, 422

Bidens (Spanish needles), 413

Biennial plant, seasonal life of,130,131*

Biology, historical sketch of, 212

Biology, plant, 3

Biology, subdivisions of, 216

Bird's-eye maple, 88*, 90, 392

Black cherry, $407 *$

Black raspberry, vegetative reproduction of, 155*

Blackberry, flower and fruit of, 405*

Blackberry association, 437*

Blackberry hybrids, 183

Body plan, summary of, 23*

Body plan of apple, 15*

Body plan of buckwheat, 17

Body plan of elm, 21*, 22*

Body plan of herbaceous plants, 18*

Body plan of lilac, 15*

Body plan of pine, 20*

Body plan of plants, 15*, 16*, 17*

Bordered pits, 327, 328

Botany, historical sketch of, 212

Bracken fern, anatomy of, 303*, 304*, 305*

Bracket fungi, 272*, 273

Brassica, flowers of, $400 *$

Bread making, 249

Breeding, plant and animal, 216

Brown, Robert, 55

Brussels sprouts, 401
Bryophyta, 287-297

Buckwheat, body plan of, 17*

Buckwheat, growth of, 60*

Bud, lateral, 15*

Bud growth, 68* 70*,71*

Bud scales, 68*

Bud structure, 68*, 70*

Budding, 245

Bud-scale scars, 69*

Buds, accessory, 356*

Buds, adventitious, 356

Buds, flower, 356

Buds, kinds of, 356

Buds, lateral, 356

Buds, leaf, 356

Búds, mixed, 356

Buds, supernumerary, 356

Buds, terminal, 356

Bulb, hyacintl, 418*

Bulb, tulip, 157*

Bulbs, 354

Bulbs, culture of, 420

Bulbs, development of, 422*

Bundle scars, 84*

Burbank and plant breeding, 182, 183

Buttercup, 398*

Cabbage, 401

Cacti, ornamental, 148*

Caladium, adjustments of, 37, 38*

Caltha palustris, 339*, 397*

Calyptra of moss, 295*

Calyx, 161, 169*

Cambium in alder, 92*, 93*

Cambium in lilac, 84,85

Cambium in oak, 86

Cambium of roots, 111

Cambium in Salvia, 106*

Cambium layer, 95, 96*, 98

Camptosorus, vegetative reproduction of, 158*

Campylotropous ovule, 363

Campylotropous ovule of Capsella, 345

Canal cells of archegonium, 289*

Capillitium of puffball, 271*

Capsella (shepherd's purse), 344, 345*, 346

Capsule, 295*

Capsule (compound ovary), 364*, 365

Capsule of moss, 294, 295*

Carduus (thistle), 413*

Carpel, 169*

Carpels, 363*

Carrot, root of, 356*

Caryopsis, 365, 434

Catalytic agent, 248

Caulescent, 353 
Celery, cells of, $46^{*}$

Cell, historical sketch of, 54,59

Cell, minute structure of, $72^{*}$

Cell, naming of, 54

Cell, summary of historical sketch of, 59

Cell and plant development, 57 *

Cell division, $73,75 *, 80$

Cell parts, functions of, 51,52

Cell parts, summary of, 52,53

Cell plate, 79

Cell sap, 52

Cell theory, 55

Cell wall, $46 *, 47 *, 48,54$

Cells, parts of, 46,51

Cells, thick-walled, of celery, $46 *$

Cells with plastids, $50 *$

Cells of root tips, 47 *

Cellular structure, 45,53

Centgener plots, 205*

Cherry, black, 407*

Cherry, flower and fruit of, 409*

Chlamydomonas, 222*

Chlorophyll, 50

Chloroplastids, $49,50 *$, 115

Chromatin, 73

Chromoplastids, $49,50 *$

Chromosome reduction, 311

Chromosomes, 74, 75*, 76

Chromosomes, reduction and division of, 80

Chromosomes and reproduction, 81*, 160*

Cineraria, body plan of, $18 *$

Circinate vernation, 298*, 299

Citranges, 183

Classification, 213

Claytonia, fern, 298*

Climate and water supply, 367

Close-fertilization defined, 174

Close-pollination defined, 167, 174

Clover, movements of, $37,39 *, 40$

Clover, pollination of, 172, 173

Clover, pulvini of, $39^{*}$

Clover, seasonal life of, 130, 131*

Cohesion theory, 145

Collateral vascular bundle, 301

Collecting hairs of locust, 171

Color of flowers, 362

Columbine, 399*

Columella, 262*

Commelinaceae, 417

Commercial relations of plants, 12

Complete flower, 360

Compositae, $410 *-413$

Compound pistils, 363*

Concentric vascular bundle, 301*
Conceptacles, 237*, 238*

Conduction in stems, 99,100

Cones of long-leaf pine, $379 *$

Cones of spruce, $331 *, 332 *$, 333*, 374*

Coniferales, 327

Conjugate nucleus, 228, 335

Contrasting characters, $188,189 *$

Convolvulus, leaf structure of, $149 *$

Coprinus comatus, number of spores in, 268-271

Cork bark, 85*, 86*, 101*

Cork cambium, 102

Cork layer, 91, 92

Corm of gladiolus, 157*

Corms, 354

Corn, breeding of, 178*, 179*, 193*, $194 *, 195 *$

Corn, hybridization of, 178*, 179*

Corn, inflorescence and flower, 193*, 433*

Corn, plant of, 193*

Corn, root system of, 112

Corn, stem structure of, 109*

Corn breeding, 193*, 194*, 195*

Corn kernels, oil and protein of, 195*

Corn roots, growth of, $61 *$

Corolla, 161

Cortex in roots, 110*

Cortex in Salvia, 105, 106*

Cortex of stems, $92 *$

Corymb, 358*

Cotyledon of pea; $24 *$

Couch grass, 431*

Crab apple, American, 408

Crenate leaf margin, 355*

Cross-fertilization defined, 174

Crossing and hybridizing, 174

Cross-pollination, 167, 174

Cruciferae, 400*, 401

Curly grain of maple, $88 *, 90$

Cycad, 321, 322*

Cycadales, 321, 325

Cyclic flowers, 358*

Cyclic leaf arrangement, 15*, 16*

Cypripedium, $427 *$

Cypripedium acaule, $427 *$, 428*

Cypripedium pubescens, $427 *$

Cytology, 59

Cytoplasm, 47*, 52*

Cytoplasmic sac, $47 *, 50 *, 53$

Czapek, experiment with roots, 36

Dandelion, flower and fruit, $412 *$.

Dandelion, habit and flowers, 410*

Dandelion, responses to stimuli, 40, $41 *, 42$

Darwin, Charles, 31, 207* 
Darwin, experiments in crossing, 175180

Darwin, experiments with roots, 34*

Decay, 255

Deciduous trees, 380

Dehiscence of anther, 162*

Dehiscent fruits, 364*, 365

Dentate leaf margin, 355*

Descriptive terms, 353

DeVries, Hugo, 201*

DeVries, mutation theory of, 199

Diageotropic, 20*, 30

Diaphototropic, 30

Diatropic, 30

Dichogamy, 361, 362*

Dichotomous, 300

Dichotomous branching, 235

Dichotomous venation, 300

Dicotyledons, flower morphology of, 339*

Dicotyledons, herbaceous and woody, 396

Dicotyledons, megasporangia, spores, and embryo of, 349*

Dicotyledons, megaspore and embryo of, $345 *$

Dicotyledons, microspore of, 348*

Dicotyledons, morphology of, 337, 349

Dicotyledons, sporophylls and sporangia, 341*

Dicotyledons, stem structure, 105, 108

Dicotyledons, structure of Salvia stem, 106*

Dicotyledons, summary of stem structure, 108

Dicotyledons and monocotyledons, 413, 414*, 415

Diffuse-porous wood, $382 *$

Digestion, 124, 125

Digestion in the bean, 126, 127*

Dimorphic flowers, 362*

Diœcious, 226, 237

Diœecious moss, 294*

Diœcious poplars, $389 *$

Diœcious willows, 388*

Dioön edule, cycad, 322*

Disease caused by bacteria, 256

Discase caused by fungi, 281-285

Dominance, Mendelian, 184*, 185

Dotted ducts, 94

Double fertilization, 346,347 *

Drupe, 364*, 365, 409* 。

Dry fruits, $364 *, 365$

Ducts, dotted, 93*, 94

Ducts, primitive, of fern, 305*

Ducts, water, 85 , 93*

Dwarf shoots, 380
East and corn crossing, 178*, 179

Ecology, 146-153, 435-445

Ecology defined, 214

Egg, 165*

Egg apparatus, 164

Elaeagnus, hairs of, 149*

Elaters, 312*, 313

Elm, flowers of, 395*

Elm, slippery, 395

Elm, white, 392*, 393*

Elms, 394*

Elodea, 49, 152

Elongation zone of root, $63^{*}, 64,65^{*}$

Emarginate leaf apex, $355^{*}$

Embryo of Capsella, 345*

Embryo of mandrake, 165*, 166*

Embryo sac, 164, 165*

Embryology, 57, 214

Endodermis of fern rhizome, 305*.

Endodermis of roots, $110 \%$

Endoenzyme, 247

Endosperm, 165,* 166

Endothia parasitica, 281

Entomophilous, 361

Environment, 3

Environment, adjustments to, 14, 23

Environment, animate, 7

Environment of animals, 11

Epicotyl of pea, 24

Epidermis of leaf, 113*, 114, 115*

Epidermis of root, 110*

Epidermis of Salvia, 106*

Epidermis of stem, 84, 85*, 91

Epigynous flower of apple, $409 *$

Epigynous flower of yarrow, 411*

Epigynous flowers, 358*, 359

Equisetales, 311, 315

Equisetum, life history of, 315*

Equisetum arvense, 312*

Erect tree type, 19, 20*

Erosion, 443*

Erythronium, 421*

Erythronium, bulb development of, 422*

Fvolution, 211, 215, 218

Exoenzyme, 247

Exoskeleton of stem, 300

Fagopymum (buckwheat), 17*

False whorls, 19, 20*

Fermentation, 246

Ferments, digestive, 248

Ferments, energy-forming, 248

Fertilization, 57*, 160

Fertilization, double, 347*

Fibers, central spindle, 77

Fibers, phloëm, 94* 
Fibers, traction, 77

Fibers, wood, 95*, 96

Fibrous roots, 357*

Filament of anther, 162

Filicales, 298-311

Filicales, anatomy of, 301*, 303*, 304*, $305 *$

Filicales, gametophyte, 308*

Filicales, gametophyte and embryo, 309*

Flagella, 222*

Fleshy fruits, $364 *, 365$

Floods, forest control of, 368

Floral plans, 361*

Flower, morphology of, 339*

Flower, parts of, 161, 162*, 163

Flower buds, 356

Flowers, clustered, 357*

Flowers, solitary, 357*

Follicle, 364*, 365

Food cycle, organic, 11*

Food relations of plants and animals, 9

Food storage, 98

Forces of environment, 3

Forest, central hardwood, 383

Forest control of rainfall, 368

Forest products, 370

Forest trees, groups of, 371

Forests, national, 366, 368*

Form of herbaceous plants, 18*

Form of plant body, 14

Form of trees, 19*, 20*, 21*, 22, 43*

Fragaria (strawberry), 403*, 404*

Fruit of mandrake, $166 *$

Fruits, classified, 365, 366

Fruits, kinds of, $364 *, 366$

Fucus vesiculosus, 235, 241

Fucus vesiculosus, habit of, 236*

Fucus vesiculosus, life history of, 240 , 241

Fucus vesiculosus, reproduction, 237*, $238 *, 239 *, 240 *$

Funaria, 293-297

Funaria, gametophyte and sex organs, 294*

Funaria, sporophyte, 295*

Fungi, 242-285

Fungi, classification of, 243

Fungi and disease, 281, 285

Funiculus of ovule, 162*, 165*, 169*

Gametangium in Spirogyra, 227

Gametes, 57*, 159, 160*, 163*

Gametes, female, 164

Gametes, male, 164*

Gametic purity, 187

Gametogenesis, 163
Gametophyte in algæ (Spirogyra), 226

Gametophyte in angiosperms, 343*, $348 * 349 *$

Gametophyte in bryophytes, 288, 289*

Gametophyte in gymnosperms, 322, $323 *, 334 * 335 *$

Gametophyte in pteridophytes, 308*, 309*, 319*

Gap, leaf, of ferns, 300*, 301*

Gap, leaf, of pines, 327*, 329*

Garden pea, Darwin's experiments with, 177

Generative cell of cycad, $323 *$

Generative cell of pollen, 164*

Generative cell of spruce, $334 *$

Geotropic, 30

Geum triflorum, 410

Gladiolus, corms of, 157*

Gleba of puffball, 271*, 272

Glumes of oat flower, 434

Grain of wood, $87 *, 88,90$

Gramineae, 430,435

Grass, couch, 431*

Grasses, 430

Gravity, root response to, 33*, 34*, $36 *$

Gravity sense, 31

Growth, 67, 72

Growth, summary of, 69, 72

Growth of buckwheat, 60

Growth of corn root, $61 *$

Growth of herbaceous stems, 67*

Growth of leaves, $66 *$

Growth of lilac bud, 68*

Growth of root-tip, $61 *, 64,65 *, 67 *$

Growth of roots and stems; 70*

Growth of root-tip cells, 63*

Guard cells, 113*, 114, 115*

Gymnosperms, 321-336

Gymnosperms, Coniferales, 325

Gymnosperms, Cycadales, 321

Gymnosperms, forest trees, 371-380

Habit, 353

Habitat, 353

Hairs, protective, 149*

Halberd-shaped leaf, 355*

Halophytic association, 435

Hanstein, 57

Hardwood trees, 380, 381*, 382*

Head, 358*

Heart-shaped leaf, 355*

Heartwood, 86

Hepaticae, 28t-293

Herbaceous plants, body plan and form of, 18* 
Herbaceous stem, growth of, 67*, 70*

Herbaceous stems, dicotyledons, 105108

Herbaceous stems, monocotyledons, $108,109 *$

Herbaceous stems, structure, 106*

Herbaceous stems, summary of structure, 108

Heterosporous, 314, 316, 318*

Heterostylous, 362*

Histology, 214

Holly, wood of, 382*

Homogamous, 362

Homosporous, 313, 316

Hooke, Robert, 54

Hyacinth, plant of, 418*

Hybridization of corn, 178*, 179*

Hybridization and new varieties, 180

Hybridization of plums, 182*

Hybridization of wheat, 180*, 181*

Hydrophytes, 152*, 153

Hydrophytic association, 435, 440*

Hymenium of mushroom, 269*, 270

Hypha, 258

Hypocotyl of pea, 24*

Hypogynous flowers, 358*, 359*

Imperfect flowers, 360*

Inanimate environment, 3

Inanimate environment, forces of, 3

Inanimate environment, materials of, 5

Inbreeding and crossing, $\mathbf{1 7 5}$

Inbreeding defined, 174

Income and outgo of animals, 9

Income and outgo of plants, 8

Incomplete flowers, $360 *$

Indehiscent fruits, 365, 368*

Industrial biology, 215

Industrial relations of plants, 12

Inflorescence, nature and kinds of, $357 *, 358 *$

Inflorescence and pollination, 107, 108

Inorganic foods, 9

Integuments, $162 *, 164,165 *$

Internode, 4*, 15*, 16*, 17*

Invasion, ecological, 438*, 439*, 463

Invasion by fungi, 283

Invertase, 248

Ipomoea (sweet potato), vegetative reproduction of, 158*

Iridaceae, 423-424*

Iris, ovule and pollen tube of, 347*

Iris versicolor, 424*

Irregular flowers, 360

Jacket cells, 334*

Jack-in-the-pulpit, 426*
Keel petals, 168, 169*

Knight, experiment of, 32*

Knight, Thomas Andrew, 31

Labellum of Cypripedium, 428*

Lady's-slipper, $427 *$

Lamellæ of mushroom, 268*, 269*

Lanceolate leaf, 355*

Lateral bud, 356

Leaf, epidermis of, 113*

Leaf, section of, 115*

Leaf arrangement, 15*, 16*

Leaf blade, $4 *, 15 *, 17 *$

Leaf gaps, 300*, 301*, 327*, 329*

Leaf petiole, $4 *$, 15*, 17 *

Leáf scars, 69*, 84*

Leaf structure, 113, 116

Leaf trace, 300*, 301*, 302*, 327* 329*

Leaves, cyclic and spiral arrangements, 16*

Leaves, form, venation, margins, and apex, 355*

Legume, 171*, 364*, 365

Leguminosce, 39

Lemma of oat flower, 434*

Leucoplastids, 49, 50*

Lichens, 277, 278*, 279*, 280

Life histories of algæ, 228-229

Life history of Fucus, 240, 241

Life history of Edogonium, 235

Life history of Vaucheria, 233

Lilac, body plan of, 15*, 16*

Lilac, bud growth, 68*, 71*

Lilac, leaf growth, 66

Lilac, stem structure, 85 *

Lilac twigs, 69*, 84*

Liliaceae, 418, 423

Lily, anther and pollen of, 343*

Lily, double fertilization in, $347 *$

Lily, microsporangium and spores, $343 *$

Lily, pond association, 440

Linear leaf, 355*

Lip cells of Sporangia, $306 *$

Lobed leaf margin, 355*

Locust, inflorescence and flower of, $171^{*}$

Locust, pollination of, 170, 173

Locust, seasonal life of, 133*

Lodicules of oat flower, $434 *$

Lupine, Darwin's experiments with, 177

Lycopodiales, 315,317

Lycopodium, 316*

Maidenhair fern, 297*, 299*

Malt, 250

Maltase, 248 
Malus (apple), 408

Mandrake, flower of, 161, 162*

Mandrake, fruit of, 166

Mandrake, ovules and seed of, $165 *$

Mandrake, pollen and ovary of, 164*

Mandrake, seedlings and fruit of, 166*

Map of forest areas, 368*

Maple, 390*, 391*

Maple, bird's-eye, 392

Maple, curly, 392

Maple, flowers of, 390*

Marigold, marsh, 307*, 339*

Mass culture, 205

Materials of environment, 5

Mechanism of movements, 28

Megasporangium, 317, 318*

Megaspore, 165*, 317, 318*, 319*

Megaspores, homology of, 349*

Megasporophyll, 317, 318*

Megasporophylls, morphology of, 341*

Mendel, Gregor, 185

Mendelian ratio, 186

Mendel's laws, 184*, 188*, 189*, 190*

Mendel's principles of heredity, 184*

Meristem, 61, 62*, 63*

Mesophyll of leaf, 114, 115*

Mesophytes, 146, 147*

Mesophytic association, 435, 436*

Mesophytic vegetation, 147*

Metaphase, $75 *, 77$

Metaplasmic bodies, 52

Micropyle, 162*, 164, 165*

Microsporangium, 317, 318*

Microspore, 317, 318*, 319*

Microspores of Selaginella, spruce, and mandrake, 348

Microsporophyll, 317, 318*

Microsporophylls, morphology of, 341*

Midvein of leaf, 113*, 115*

Migration and invasion, 438

Migration of plants, 436

Milkweed, leaf structure of, 113*-116*

Mimosa (sensitive plant), 58 .

Mirabilis, crossing of, 186*

Mitosis, $73-80$

Mitosis, function of, 73

Mitosis, phases of, $75 *-80$

Mitosis, process of, 74

Mold, Aspergillus, 266*

Mold, black, 259*, 260, 261*

Mold, blue, 264*, 265*

Molds, 258-267

Monocotyledons, 416

Monocotyledons, structure of, 108,109*

Monocotyledons and dicotyledons, 413, 414*, 415

Monœcious, $360,385 *$
Morning-glory, Darwin's experiments with, 176

Morphology, 213

Moss, 292*

Mosses, 293, 296

Mosses, Funaria, 293, 294*, 295*

Motor organs, pulvini, 29*, 39*

Mucronate leaf point, 355*

Musci, 293, 296

Mushrooms, 267*-271

Mushrooms, Amanita, 267*

Mushrooms, spore formation of, 268*, $269 *$

Mustard family, 400

Mutations, 199

Mutations in Enothera, 200*, 202*, 203*

Mycelium, 258

Nägeli, 55, 56

Nasturtium, phototropic response of, 26*

Neck-canal cells, 289*

Nectar of flowers, 362

Node, $4^{*}, 15^{*}, 16^{*}, 17^{*}$

Nuclear division, $73,75^{*}, 80$

Nucleolus, $47 *$, 53

Nucleus, $47 *, 53,55$

Nursery of yellow pine, 377 *

Nut, 364*, 365, 385*

Nutrition, 117

Nutrition of animal, $9 *$

Nutrition of green ylant, $8 *$

Nyctitropic (sleep) position, 38, 40

Oak, sections of, $89 *$

Oak, white, $383^{*}$, 384*, 385*

Oak stem, structure of, 86 *

Oat, inflorescence and flowers of, 433*, 434*

Oblanceolate leaf, 355*

Oblong leaf, 355*

Obovate leaf, 355*

Obtuse leaf apex, 355*

Odor of flowers, 362

EEdogonium, 233, 235

Edogonium, asexual reproduction, 235*

CEdogonium, sexual reproduction, 234*

Enothera, seedlings of, 202

Enothera gigas, 203*

Enothera lamarckiana, 199*, 200*, 203*

Enothera, lata and nanella, 200

Oögonium, 231*

Oögonium in Vaucheria, 230

Operculum of moss, 295* 
Orbicular leaf, $355^{*}$

Orchidaceae, $427 *, 428$

Organic food cycle, 11*, 285*

Organic foods, 9

Ornithogalum, 419*

Orthotropous ovule, 363

Osmosis, 137, 139*

Osmosis, experiment in, 138*

Osmotic pressure, 138

Outgo of animal, $9^{*}$

Outgo of plant, $8^{*}$

Oval leaf, $355 *$

Ovary, simple and compound, $363^{*}$

Oviate leaf, $355^{*}$

Ovules, 164, 165*

Ovules, campylotropous, 363

Ovules, kinds of, 363

Ovules, orthotropous, 363

Palet of oat flower, $434^{*}$

Palisade tissue of leaf, 113*, 115*

Palmate venation, 354, 355*

Panicle, 358*

Panicle of oat, $433^{*}$

Papilionaceous flowers, pollination of, 168

Pappus of dandelion, 412*

Parallel venation, 354,355

Paraphyses of moss, 293, 294*

Paraphyses of mushroom, 269*, 270

Parasites, 242

Parenchyma, palisade, 113*, 115*

Parenchyma, phloëm, 92*, 93*

Parenchyma, spongy, of leaf, 115*

Parenchyma, wood, 93*, 94

Parenchyma of cortex, 92

Parmelia, 279*

Pasteurization, 255

Path of water, $141 *$

Pathology, plant and animal, 215

Pea, crossing of, 178

Pea, flower of, 169*

Pea, tropisms of, 24*

Peas and Mendel's laws. 184*, 188*, $189 *, 190 *$

Pedigree culture, 205

Peduncle, 161, 357*

Penicillium, 264*, 265*

Perennial, seasonal life of, $133 *$

Perfect flowers, 360*

Perianth of flower, 161

Pericycle, 111

Pericycle of fern rhizome, $305^{*}$

Pericycle of roots, 111

Peridium of puffball, 271

Perigynous flowers, 358*, 409*

Peristome of moss, 295*
Permanent zone of root, $62,63^{*}$

Petiole, 15*, 17*

Phloëm, 84, 85*, 92*

Phloëm, elements of, 96*, 97

Phloëm, fibers of, $92^{*}$

Phloëm, parenchyma of, $92 *$

Photosynthesis, 117-120, 127*

Photosynthesis and respiration, 123

Phototropic response, 30

Phototropism of nasturtium, 26*

Physcia stellaris, 278*

Physiology, 58, 214

Physiology of bean plant, 127 *

Picea (spruce), forest of, 325,326 *

Picea (spruce), gametophytes of, 334*

Picea (spruce), ovule and seed of, 335

Picea (spruce), sporangia and spores of, 333

Picea (spruce), strobili of, 331*, 332*

Picea (spruce), wood structure of, $327 *, 328 * 329 *$

Pileus of mushroom, 268*, 269*

Pinales, 325, 327

Pine, cones of long-leaf, $379^{*}$

Pine, section of, 378*

Pine nursery, $377^{*}$

Pines, erect type, 19, 20*

Pines, forest trees, 378-380

Pinnate venation, $300,354,355$

Pinnule, 300

Pinus palustris, cones of, $379 *, 380$

Pistil, 162*, 169*

Pistils, compound, $363^{*}$

Pistils, kinds of, $363^{*}$

Pistils, simple, $363^{*}$

Pisum sativum, crossed and self-fertilized plants of, 178

Pith, 85

Pits, bordered, 327*, 328*

Placenta, 162*, 163

Placentæ, kinds of, $363^{*}$

Plankton, 219

Plant associations, 435,445

Plant associations, kinds of, 435

Plant associations, origin of, 436

Plant body, plan of, 14

Plant disease, 281

Plantain, vegetative reproduction, $156^{*}$

Plants, industrial and commercial relations of, 12

Plants, relations to animals, 8,9

Plastids, $49,50 *, 51$

Pleurotus ulmarius, 272

Plums, hybridization of, $182 *$

Plumule of pea, 24*

Polar nuclei, $164,165 *, 347^{*}$

Pollen, 163, 164* 
Pollen chamber of Zamia, 323*

Pollen formation, 343*

Pollen germination, $343 *$

Pollen tube, 164*, 323*, 325, 334*, $347 *$

Pollen tube of Iris, $424 *$

Pollination, $166-173$

Pollination, kinds of, 167

Pollination and inflorescence, 167

Pollination in locust, 170, 171*

Pollination in papilionaceous flowers, 168, 169*

Pollination in red clover, 172,173

Polyporus squamosus, 273*

Pome, 364*, 365, 409*

Pomelo, 183

Pond lilies, 152*

Pond-lily association, 440*

Pontederia, 429*

Pontederiaceae, 428

Poplars, 389*

Populus deltoides, 389

Potamogeton, 152

Potato, vegetative reproduction of, $150 *, 156$

Potato (sweet), vegetative reproduction of, 158*

Primary root, 356

Primrose, Lamarck's, 199*

Proembryo, 335

Progeotropic, 20*, 30

Pronuclei, 160, 228

Prop roots, 193*, 357

Prophase, 74, 75*

Prophototropic, 30

Protandrous flowers, 362

Protococus, 220, 221*

Protogynous, 362, 404*

Protonema of moss, $296^{*}$

Protoplasm, 47*

Protoplasm, naming of, 55

Protoplast, $47, * 52$

Protropic, 30

Prunus (cherry), 407

Prunus serotina, $407 *$

Pteris aquilina, 302-305

Pteris aquilina, anatomy of, $303^{*}, 304 *$, 305*

Ptomaine poisoning, 258

Ptomaines, 257

Puccinia graminis, 274, 275*

Puffballs, $270^{*}, 271^{*}, 272$

Pulvini, 28*, 29*, 37, 39*

Pulvini of bean leaf, 28*, 29*

Pulvini of clover, $39^{*}$

Pürkinge, 55

Putrefaction, 255
Pyrenoids of Spirogyra, 224*

Pyrus coronaria, $408^{*}$

Quarter-sawed oak, 87*, 88*, 89*

Quercus alba, 383*-386

Quercus alba, flowers and fruit of, $385^{*}$

Quercus rubra, wood of, 89*

Raceme, $357 *$

Rachis of fern leaf, 299*

Radish, 401

Rainfall, forest control of, 368

Ramentum, 299

Ranunculaceae, 397*, 399

Raphe, 364

Raspberries, 405

Rays, wood, $86 *, 87 *, 92 *, 93 *, 94$

Receptacle of flower, $161,339^{*}, 340$

Receptacle of Fucus, 237*

Red clover, pollination of, 172,173

Red clover, root system of, 111

Red oak, cuts of, $89^{*}$

Reduction division, $80^{*}, 81^{*}, 82$

Regular flowers, 360

Reproduction, sexual, 159, 160*, 161

Reproduction, vegetative, 154

Resin canals, 326

Respiration, 120-124, 127*

Respiration and photosynthesis, 123

Rhizoids of Ricciocarpus, 287, 289*

Rhizome, 299,* 353, 354*

Rhizophore, $317 *$

Rhizopus, 259*, 260*, 261*, 262*, 263*

Ricciocarpus, 288-293

Ricciocarpus, life history of, 291*

Ricciocarpus, reproductive organs of, 289*

Ricciocarpus, sporophyte and spores of, $290^{*}$

Ring-porous wood, 381*

Root, functions of, 112

Root, hairs of, $110^{*}$

Root cap, $61 *-62 *, 63 *$

Root hairs and absorption, 140*, 141*

Root pressure, 138*

Root system of clover, 111*

Root system of corn, 112*, 193*, 357*

Root tip, cell structure of, $62 *, 63^{*}$

Roots, anatomy of, 110*

Roots, arrangement of, 18, 19

Roots, fleshy, fibrous, 357

Roots, growth of, $61^{*}, 63^{*}, 65^{*}, 70^{*}$

Roots, primary and secondary, $4^{*}, 17 *$, $193^{*}$

Roots, prop, of corn, 193*, 357

Roots, sensitive zone of, $34^{*}, 36^{*}$

Roots, soil, water, aërial, 357 
Roots, tap, 4*, 356*

Rootstock of Solomon's seal, 354

Rosa (rose), 406

Rosaceae, 403-410

Rose, flower structure of, 405*

Rubus (raspberries and blackberries), 405

Runners, 354

Runners of Rhizopus, 261

Runners of strawberry, 155

Rusts, 274-275*

Sachs, experiment by, 33*

Sachs, Julius von, 31, 59

Sagittaria, 429*

Salicaceae, 387-390

Salix discolor, 388

Salvia, bark of, 105

Salvia, cortex of, 105

Salvia, stem structure of, 105, 106*, 107

Salvia, summary of structure of, 108

Salvia, vascular cylinder of, 105, 106*

Samara, 364*, 365, 390*

Saprolegnia, 282

Saprophytes, 242

Sapwood, 86

Sassafras, wood of, $381 *$

Scale, ovuliferous, 332

Scales of bud, $68 *$

Scape of dandelion, $40,41 *, 42$

Scars, bud-scale, $69 *, 84 *$

Schizomycetes, 250

Schleiden, M. J., 55, 56

Schultze, Max, 56

Schwann, 55

Seasonal life, 125

Seasonal life, summary of, 135

Seasonal life of Adiantum, 300

Seasonal life of annual, 126-130

Seasonal life of bean, 129*

Seasonal life of biennial, 130, 131*, 132

Seasonal life of perennial, 133*, 134

Secondary roots, 356*

Sedges, 430

Seed, 165*, 166, 169*

Seed of mandrake, 165

Seed of mandrake and spruce, 349

Seed of pea, $24^{*}$

Seed of spruce, $335^{*}$

Seed production in forest, 373

Seedling of bean, 4*, 129*

Seedling of mandrake, 166

Seedling of pea, $24 *$

Seedling of spruce, $335^{*}$

Selaginella, $317 *-320$

Selaginella, gametophytes and embryo of, $319^{*}$
Selaginella, life history of, $320 *$

Selaginella, sporangia and spores of, 318*

Selection, plant improvement by, 192

Selection in corn, 194*

Selection of fluctuating variations, 208-210

Selection of mutations, 210

Selection in tobacco, 198*

Self-fertilization, 174

Self-pollination, 167

Sepals, 161

Serrate leaf margin, 355*

Seta of moss, 293, 295*

Sexual reproduction, 159

Shásta daisy, 182, 183

Shull, George H., and corn crossing, 179

Shull and seedlings of Enothera lamarckiana, 202*

Sieve plates, $92,93 *$, 305*

Sieve tubes, 92, 93*, 305*

Silique, 363*, 365, 400*

Silver grain of wood, 88-90

Simple pistils, 363*

Sinuate leaf margin, 355*

'Skeletal tissue, 86*, 301*

Skeleton of plants, 49

Smilacina, 420

Smilacina, root structure, 62

Smilacina stellata, 421*

Smuts, 276, 277*

Soil, composition of, 141*

Soil and root hairs, 140*, 141*

Solitary flowers, $357 *$

Solute, $137 *$

Solvent, 138

Sori, 274, 275*, 306*, 307*

Sorus of fern, 306, 307*

Spadix, 426*

Spanish needles, 413

Spathe, 426*

Spatulate leaf, 355*

Spiderwort (Tradescantia), 417*

Spike, 358*

Spikelet, $433^{*}$

Spindle, $75^{*}, 76$

Spiral, leaf arrangement, 15*, 16*

Spiral flowers, 358

Spirogyra, 223-229

Spirogyra, fertilization discovered in, 57

Spirogyra, life history of, 229

Spirogyra, reproduction in, 227*

Spirogyra, structure of, 224*, 225*

Spongy tissue of leaf, 115*

Sporangiophore of Equisetum, 312*, $313^{*}$ 
Sporangiophore of molds, 261*

Sporangium of fern, $306 *, 307 *$

Sporangium of mold, $261 *, 262 *$

Spore chambers of puffball, 271

Spore tetrads, 290*, 314

Sporidia of rust, $275^{*}$

Sporidia of smuts, 277

Sporophore of puffball, 271*

Sporophyte in angiosperms, 337

Sporophyte in bryophytes, $290 *, 295 *$

Sporophyte in gymnosperms, 321, 325

Sporophyte in pteridophytes, 299,309*, $315^{*}$

Spreading tree type, 21*, 22*

Spring wood, $85^{*}, 86^{*}, 87^{*}$

Spruce, 325-337, 326*, 371-378

Spruce, anatomy of, $327 *$, 328*, 329* 330

Spruce, commercial importance of, 375

Spruce, embryo and seed of, 335*

Spruce, gametophytes in, 334*, 335*

Spruce, life history of, 336

Spruce, regrowth of, $376^{*}, 378$

Spruce, second growth of, $375^{*}$

Spruce, seed production in, $373,374 *$

Spruce, strobili of, 331*, 332*, 333*

Spruce, tolerance of, $372 *$

Spruce and balsam, $373^{*}$

Spur. shoots of apple, 409

Stalk cell, 323*

Stamens, $162 *$

Standard of pea flower, 168, 169*

Starch grains, 50*

Starch sheath, 224*

Stem structure of alder, $91 *$

Stem structure of corn, $109^{*}$

Stem structure of dicotyledons, 105, 106*

Stem structure of lilac, $85^{*}$

Stem structure of monocotyledons, $108,109 *$

Stem structure of oak, $86^{*}$

Stem structure of Salvia, 106*

Stems, function of tissues, 98

Stems, functions of, 83

Stems, growth of, $67 *, 70^{*}, 71 *$

Stems, kinds of, 353,354

Stems, multiple, of plantain, 156*

Stems, vegetative reproduction of, 154-157

Sterigmata of mushroom, 269*, 270

Sterilization, 254

Stigma, 163, 164*, 169*

Stimuli, internal, 29

Stimuli, kinds of, 30

Stimulus, 27

Stipe of mushroom, 268*
Stolon, 354

Stolon of Rhizopus, 261*

Stoma of fern sporangium, 306*

Stomata, 113*, 114, 115*

Storage in stems, 98, 99

Strasburger, Eduard, 56, 59

Strawberry, flower and fruit of, 404*

Strawberry, vegetative reproduction of, 155*

Strawberry plants, 403*

Strobili, 313

Structure of stems, roots, leaves, 83, 117

Stylar brush, 412*

Style, 163, 169

Subhymenium of mushroom, 269*

Succession, 440, 442*

Sugar cane, $430 *$

Sugar maples, grove of, 391*

Summaries :

Relations of plants to environment, 13

Body plan, 23

Adjustments by tropisms, 44

The parts of the cell, 52,53

The cell and the cell theory, 59

Growth and cell division, 69-72

Structure and physiology of trees, 100-103

Structure of herbaceous stems, 108

Seasonal life of bean, 130

Seasonal life, 135, 136

Variation and selection, 206-208

Evolution, 211

Biological sciences, 216

Bacteria, 258

Fern anatomy, 305

Spruce anatomy, 330

Angiosperms, 348, 349*, 350

Plant associations, 445

Summer wood, $85 *, 86 *$

Supernumerary buds, 356

Suspensor, 319*, 335*

Sweet potato, vegetative reproduction in, 158*

Sycamore, wood of, 382

Symbiosis of lichen, $280 *$

Synergidæ, 164, 347*

Tangelo, 183

Tangerine, 183

Taproot, 4*, 17*, 356*

Taraxacum (dandelion), $410^{*}$

Taxonomy, 212

Teliospores (teleutospores), 275*, 276

Telophase, $75 *, 78$

Terminal bud, 356 
Tetrads, 81, 290*

Tetrads in lily, 343*

Tetrads in Ricciocarpus, 290*

Thallophyta, 287

Thallus, 287

Thistle, 413

Timothy, variation in, 204*

'Tobacco, Connecticut broad-leaf, 197*

'Tobacco, Uncle Sam Sumatra, 198*

Tolerance in forest trees, $372,373 *$

Tracheæ, 93

'Tracheids, 304, 327, 328*

Tradescantia (spiderwort), 417*

Trama, 270

Transpiration, 127*, 142-146

Transpiration, control of, 143

Trees, erect type, 19, 20*

Trees, form and development of, 19

Trees, growth and bark formation, 102

Trees, hardwood, 380

Trees, importance and use of, 366

Trees, long and transverse sections of, $101 *$

Trees, longevity of, 103

Trees, physiology of, 104

Trees, poplar, 389*

Trees, responses to stimuli, 43*

Trees, shrubs, and forests, 366

Trees, spreading type, 21*, 22, 43*

Trees, structure and physiology of, 100-105

Trees, sugar maple, 390*, 391*

Trees, white elm, $393 *-395$

Trees, white oak, $383 *, 387$

Trees, willow, $387 *-389$

Trees and forests, $366-380$

Trifolium pratense, 37, 39*

Trifolium pratense, pollination of, 172 173

Trimorphic flowers, 362

Tropisms, 23, 27

Tropophytes, 150

Tube cell, 334*

Tube nucleus, 164*, 347*

Tubers, 354

Tubers of potato, $156 *, 334$

Turnip, 401

Twig, lilac, 69*, 84*

Ulmaceae, 393-396

Ulmus Americana, 392*, 395*

Ulmus fulva, 393, 335*

Ulmus racemosa, 393

Umbel, 358*

Undiilate leaf margin, 355*
Urediniospores (uredospores), 274, 275*

Ustilago zeae, 276, 277*

Vacuoles, 47*, 52

Variation, fluctuating, 197

Variation, summary, 206

Variation in apples, 192*

Variation in corn, 194*

Variation and selection, 192

Variation in timothy, 204

Variation in tobacco, 197

Vascular cylinder in Salvia, 105, 106*

Vascular plants, 299*

Vascular system of fern, 301*, 302

Vaucheria, 230-233

Vaucheria, asexual reproduction in, $233^{*}$

Vaucheria, sexual reproduction in, 230 , $231 *, 232 *$

Vegetation, instability of, 444

Vegetative reproduction, 154-159

Vegetative reproduction in fern, 158*

Vegetative reproduction in gladiolus, 157*

Vegetative reproduction by leaves, $157,158^{*}$

Vegetative reproduction in plantain, $156^{*}$

Vegetative reproduction in potato, 156*

Vegetative reproduction in raspberry, $155^{*}$

Vegetative reproduction by roots, $158^{*}$

Vegetative reproduction by stems, 154 , $155^{*}, 156 *, 157 *$

Vegetative reproduction in strawberry, 155*

Vegetative reproduction in sweet potato, $158^{*}$

Vegetative reproduction in tulip, 157*

Veil, 268

Veins of leaf, $113 *, 115^{*}, 116$

Venation of leaves, 355*

Venter of archegonium, 289*

Ventral-canal cells, 289*

Vicia faba, 34

Viola pinnata, flower structure of, $402 *$

Violaceae, 401*-403

Violet flowers, 401*, 402*

Von Mohl, 55, 56

Walking leaf fern, 158

Water, ecological relations of plants to, 146-153

Water, relation of plants to, 137-153 
Water ascent, 144-146

Water ascent, path of, 141*

Water ascent, rate of, 145

Water ducts, $93^{*}$

Wheat, rust of, $274,275 *$

White oak, 383*-386

White oak, inflorescence and flowers of, $385^{*}$

White oak timber, $384^{*}$

White sweet clover, seasonal life of, $130,131 *$

Willow, 387, 388*

Willow, reproduction of, $388^{*}$

Wing petals, 168, 169*

Wood, destruction by fungi, 273*

Wood, diffuse-porous, $382^{*}$

Wood, ring-porous, 381*

Wood, sections of, $87 *, 88 *, 89 *$

Wood, spring and summer, $85 *, 86$

Wood of alder, 91*, 92*, 93*

Wood of lilac, $85^{*}$

Wood of maple, $88^{*}$

Wood of oak, 86*, 101*

Wood of red oak, 89*

Wood cylinder, 84

Wood fibers, 93*, 94

Wood parenchyma, 92*, 93*, 94

Wood rays, $87 *, 94$

Xerophytes, 148*
Xerophytes, leaf structures of, $149 *$

Xerophytic association, 435, 438*

Xylem, 92*, 93*

Xylem elements, $96 *, 97,98$

Yarrow, inflorescence and flowers of, $411 *, 412 *$

Yeast, spore formation in, 247

Yeast cells, $245^{*}$

Yeasts, 234-250

Yeasts, budding of, $245 *, 246^{*}$

Yeasts, top and bottom, 244*

Yeasts, wild and cultivated, 244*

Yeasts, wine and beer, 244*

Zamia, 321-325

Zamia, life history of, 324*

Zamia, strobilus, sporangia, and gametophytes, 323

Zea mays, root system of, 112*, 193*

Zea mays, stem structure of, 109*

Zonation, 441*, 442*, 444

Zoösporangium of Vaucheria, 233*

Zoöspore of $E$ dogonium, 235*

Zoöspore of Vaucheria, 232, 233*

Zoöspores of Chlamydomonas, 222

Zoöspores of $E$ Edogonium, 235

Zoöspores of Vaucheria, 233

Zygote, 57, 159, 160*

Zymase, 246 

ANNOUNCEMENTS 



\section{TEXTBOOKS IN BIOLOGY}

\section{FOR HIGH SCHOOLS AND COLLEGES}

\section{BOTANY}

Bergen: Botanies (For list see High-School and College Catalogue)

Bergen and Caldwell : Introduction to Botany

With or without Key and Flora

Bergen and Caldwell : Practical Botany

Clute: Agronomy

Clute: Laboratory Botany

Clute: Laboratory Manual and Notebook in Botany

Densmore: General Botany

Duggar: Fungous Diseases of Plants

Eikenberry: Problems in Botany

Frye and Rigg: Laboratory Exercises in Elementary Botany

Gruenberg: Elementary Biology

Hodge and Dawson: Civic Biology

Meier: Herbarium and Plant Description

Meier: Plant Study (Revised Edition)

Penhallow: Manual of North American Gymnosperms

Roth: First Book of Forestry

\section{BACTERIOLOGY}

Conn: Bacteria, Yeasts, and Molds in the Home (Rev. Ed.) Moore: Laboratory Directions for Beginners in Bacteriology Reed: Manual of Bacteriology

Russell and Hastings : Experimental Dairy Bacteriology

\section{ZOÖLOGY}

Linville and Kelly: Laboratory and Field Work in Zoölogy

Linville and Kelly: Textbook in General Zoölogy

Meier: Animal Study

Pratt: Course in Invertebrate Zoölogy (Rev. Ed.)

Pratt: Course in Vertebrate Zoölogy

Sanderson and Jackson: Elementary Entomology

\section{PHYSIOLOGY}

Blaisdell: Life and Health

Blaisdell : Practical Physiology

Brown: Physiology for the Laboratory

Bussey: A Manual of Personal Hygiene

Hough and Sedgwick: The Human Mechanism (Rev. Ed.)

Jewett: The Next Generation

\section{GINN AND COMPANY PUblishers}




\section{PRINCIPLES OF RURAL ECONOMICS}

By Thomas Nixon CARver, Harvard University

$\mathbf{x x}+386$ pages

"Principles of Rural Economics" takes up such questions as farm management, coöperative buying and selling, and the place of agriculture in national economy.

What is good agriculture in its national significance; why rural migrations are from densely to sparsely populated areas; why agriculture is necessarily an industry of small units; why rural people are more generally self-employed than urban people; why, and under what conditions, agricultural coöperation is desirable and possible - these and a number of other questions of tremendous practical importance in rural life are carefully worked out in the text. Chapters are included on the history of modern agriculture, the factors of agricultural production, management, the distribution of the agricultural income, and problems of the rural social life.

\section{SELECTED READINGS IN RURAL ECONOMICS}

Edited by Thomas Nixon Carver, Harvard University

974 pages

This volume makes available a great deal of excellent supplementary material otherwise inconvenient of access. Considerable space is devoted to the historical matter necessary for a thorough understanding of the subject, and such questions as agricultural labor, rural marketing, and the farmer's business are fully discussed. The book contains in all forty-two selections by recognized authorities.

\section{GINN AND COMPANY Publishers}




\title{
BACTERIA, YEASTS, AND MOLDS IN THE HOME
}

\author{
(SECOND REVISED EDITION)
}

By H. W. Conn, late Professor of Biology in Wesleyan University

I $2 \mathrm{mo}$, cloth, 293 pages, illustrated

N

HE book contains an important summary of the facts which have rapidly accumulated in recent years concerning the relation of microörganisms to all matters connected with the home. The work is a popular and not a scientific discussion, free from many technical terms, and admirably adapted to the needs of the housewife, the student of domestic science, and all others interested in home economics.

Molds, which are the cause of mildew, the spoiling of many foods, and the decay of fruits; yeasts, which are the foundation of fermentation in the raising of bread ; and bacteria, which cause food to spoil, meat to decay, and contagious diseases to spread, all these phenomena which are of the most vital importance are presented in an interesting and helpful manner. The author explains the various actions of bacteria, and points out the sources of trouble and the principles which underlie the methods to be adopted for avoiding their effects. Special attention is paid to the problems of food preservation and to the practical methods which can be used in the home for preventing the distribution of contagious diseases.

To render the work more useful for classes in domestic science there is added an appendix containing directions for a series of simple experiments which will give to the student a practical knowledge of the most important properties of microörganisms. 


\section{COUNTRY LIFE EDUCATION SERIES}

A SERIES of practical texts for the student and the professional farmer, written by experts in their respective lines. These books aim to give a thorough exposition of both the theory and the practice of the various branches of farming and breeding.

\section{TYPES AND BREEDS OF FARM ANIMALS (Revised)}

By Charles S. Plumb, Professor of Arimal Husbandry in the College of Agriculture, Ohio State University. 820 pages, illustrated

\section{PRINCIPLES OF BREEDING}

By Eugene Davenport, Dean of the College of Agriculture, Director of the Agricultural Experiment Station, and Professor of Thremmatology in the University of Illinois. 727 pages, illustrated

\section{FUNGOUS DISEASES OF PLANTS}

By Benjamin Minge Duggar, Professor of Plant Physiology, Washington University, St. Louis. 508 pages, illustrated

\section{SOIL FERTILITY AND PERMANENT AGRICULTURE}

By Cyril George Hopkins, late Professor of Agronomy in the University of Illinois. xxiii +653 pages

PRINCIPLES AND PRACTICE OF POULTRY CULTURE By JонN H. Roвinsor. xvi +611 pages, illustrated

\section{GARDEN FARMING}

By Lee Cleveland Corbett, Horticulturist in the Bureau of Plant Industry, United States Department of Agriculture. 462 pages, illustrated

\section{THE APPLE}

By Albert E. Wilkinson, formerly of the Department of Horticulture in Cornell University. 492 pages, illustrated

\section{EQUIPMENT FOR THE FARM AND THE FARMSTEAD}

By HARry C. RAmsower, Director of Agricultural Extension Service, Ohio State University. 523 pages, illustrated

\section{Other volumes in preparation}





THIS BOOK IS DUE ON THE LAST DATE STAMPED BELOW

AN INITIAL FINE OF 25 CENTS WILL BE ASSESSED FOR FAILURE TO RETURN THIS BOOK ON THE DATE DUE. THE PENALTY WILL INCREASE TO 50 CENTS ON THE FOURTH DAY AND TO \$1.00 ON THE SEVENTH DAY OVERDUE.

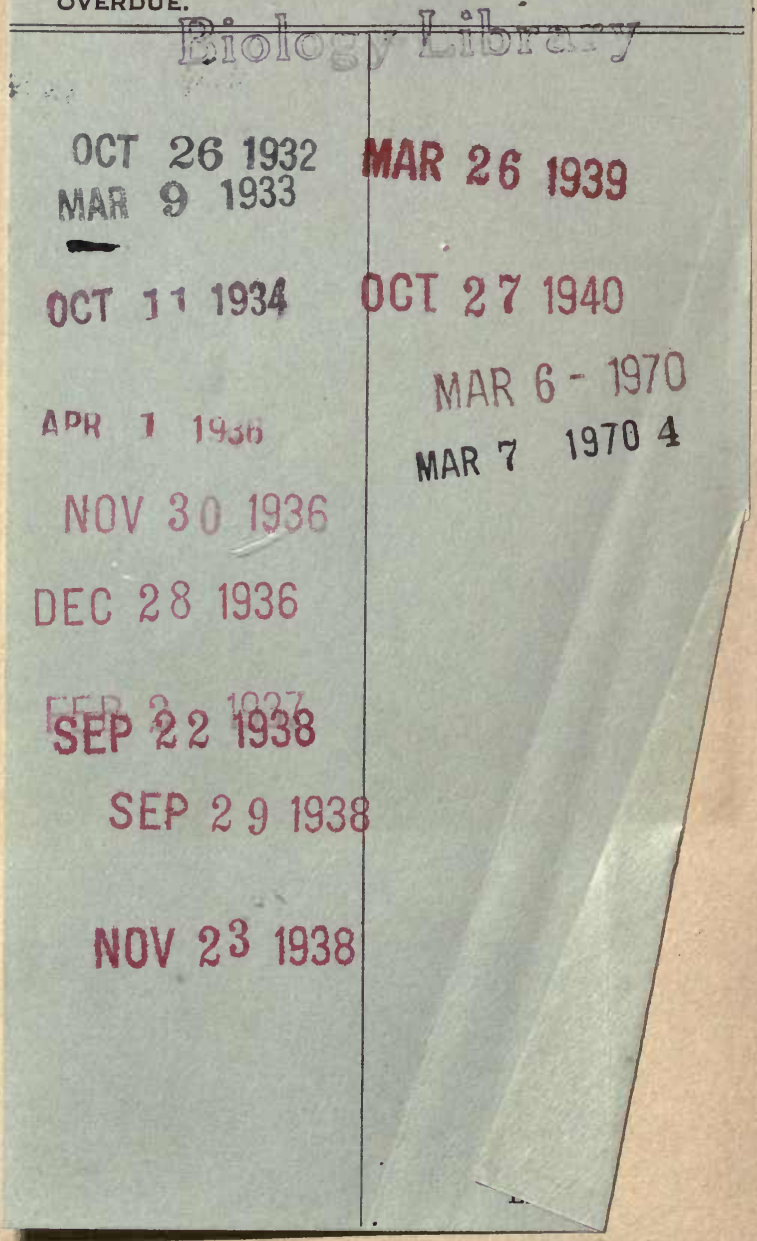


U.C. BERKELEY LIBRARIES

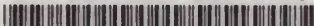

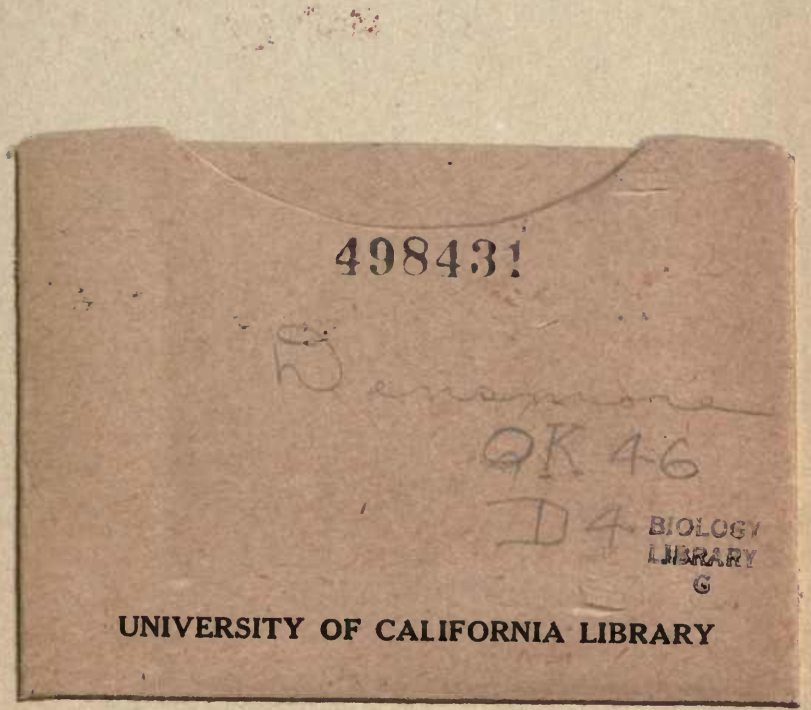


